\title{
UNTERSUCHUNG DES LOKALEN WÄRMEÜBERGANGS IN SEITENRÄUMEN VON TURBINENGEHÄUSEN AM BEISPIEL VON INDUSTRIEDAMPFTURBINEN
}

\author{
Der Fakultät Maschinenwesen \\ der Technischen Universität Dresden \\ zur Erlangung des akademischen Grades \\ Doktor-Ingenieur (Dr.-Ing.) eingereichte
}

Dissertation

vorgelegt von Dipl.-Ing. David Spura

Erster Gutachter:

Zweiter Gutachter:

Tag der Einreichung:

Tag der Verteidigung:
Prof. Dr.-Ing. Uwe Gampe

Prof. Dr.-Ing. Dieter Brillert

17.12.2020

22.07.2021 



\section{DANKSAGUNG}

Die der vorliegenden Dissertation zu Grunde liegenden Arbeiten und Untersuchungen wurden im Rahmen der beiden AG-Turbo-Forschungsverbundvorhaben COOREFLEX-turbo 4.3.6 („Thermisches und mechanisches Verhalten von Turbinengehäusen“, Laufzeit: 01.12.2013 bis 31.11.2016) und ECOFLEX 4.3.4 A („Untersuchung von Wärmeübergang in Dampfturbinenkomponenten Generisch variable Geometrien von Turbinenseitenräumen“, Laufzeit: 01.08.2017 bis 31.07.2020) durchgeführt. Die Projekte wurden jeweils hälftig durch die Siemens AG, Power and Gas Division, Görlitz, sowie vom Bundesministerium für Wirtschaft und Technologie (BMWi) auf Grund eines Beschlusses des Deutschen Bundestages unter den Förderkennzeichen (FKZ) 03ET7021H bzw. 03ET7092N gefördert. Ein herzlicher Dank gilt daher der Siemens AG und dort vor allem den Ansprechpartnern Dr.-Ing. Detlef Haje (2012/13), Dipl.-Ing. Thomas Knobloch (2013-2016), Dipl.-Ing. Alexander Blessing (2016-2018) sowie Dipl.-Ing. Norman Worlitz (seit 2018) für das Interesse an der Themenstellung, für die langjährige, vertrauensvolle und erfolgreiche Zusammenarbeit, für die sehr enge und konstruktive Projektbetreuung, für die finanzielle, technische und ideelle Unterstützung sowie nicht zuletzt für die Erlaubnis zur Veröffentlichung der Ergebnisse in dieser Arbeit. Ferner möchte ich meinen Dank der AG Turbo-Programmleitung und dort ganz besonders dem Leiter des Teilverbundprojektes (TVP) „Expansion“, Herrn Dr.-Ing. Alexander Wiedermann, für die wissenschaftliche Koordination der Verbundvorhaben sowie dem BMWi, vertreten durch den Projektträger Jülich (PtJ), für die Zuwendung und die wissenschaftliche und wirtschaftliche Betreuung aussprechen.

Mein besonderer Dank gilt meinem betreuenden Hochschullehrer Herrn Prof. Dr.-Ing. Uwe Gampe, Leiter der Professur für Thermische Energiemaschinen und -anlagen (TEA) sowie Direktor des Institutes und Zentrums für Energietechnik (IET/ ZET) an der TU Dresden, für die ausgezeichnete wissenschaftliche Betreuung der vergangenen Jahre sowie die Möglichkeit der Promotion auf einem meiner größten Interessensgebiete. Für die Übernahme des Zweitgutachtens möchte ich mich herzlich bei Herrn Prof. Dr.-Ing. Dieter Brillert, Leiter des Lehrstuhls für Strömungsmaschinen an der Universität Duisburg-Essen, bedanken.

Ein großer Dank geht an die Mitarbeiter unserer Professur für die zahlreichen Anregungen, endlosen angeregten Diskussionen („Input") sowie das zu jeder Zeit angenehme und familiäre Arbeitsumfeld. Hervorheben möchte ich Frau Gudrun Heidenreich, die „Gute Seele“ der Professur, ohne deren zuverlässige, hilfsbereite, geduldige und besonnene Art so mancher Irrweg durch den Verwaltungsdschungel der Universität nicht möglich gewesen wäre, weiterhin Frau Dr.-Ing. Sophie Schöne für die Initiierung des Projektes, meinem „Mentor“ Herrn Dr.-Ing. Guntram Buchheim, 
Projektkoordinator im ZET, sowie Dipl.-Ing. Michael Christen, dem weltbesten Zimmerkollegen, für die vielen kurzweiligen Stunden „zu zweit“. Meinem Kollegen und Projektmitarbeiter Dipl.-Ing. Oliver Paulick danke ich für die feine Zusammenarbeit seit März 2019 und wünsche ihm für die weitere Projektbearbeitung und für seine Promotion weiterhin viel Erfolg und ein gutes Händchen für die Versuche bei Dampfatmosphäre.

Ebenso bedanken möchte ich mich bei meinen Kollegen von der Professur für Magnetofluiddynamik, Mess- und Automatisierungstechnik (MFD) von Prof. Dr. rer. nat. habil. Stefan Odenbach und der dortigen Arbeitsgruppe „Messtechnik für thermische Energiemaschinen“ unter Leitung von Herrn Dr.-Ing. habil. Wieland Uffrecht für die enge Zusammenarbeit „Schulter an Schulter“, die vielen Diskussionen und den gegenseitigen Erfahrungsaustausch, für die Entwicklung und Bereitstellung der messtechnischen Ausstattung für Versuchsstand und -anlage sowie für die Unterstützung in allen messtechnischen Fragen. Ganz besonders möchte ich meinen langjährigen Weggefährten und treuen Mitstreiter Dipl.-Ing. Gunter Eschmann herausheben, ohne den die beiden Projekte nicht realisierbar gewesen wären. Ich wünsche ihm und seinem Kollegen Dipl.-Ing. Bernhard Weigel, zuständig für die messtechnische Instrumentierung des Dampfversuchsstandes, alles Gute für die Promotion und die jeweiligen (Nachfolge-) Projekte.

Ohne die enge Abstimmung und die rege Diskussion mit den technischen Mitarbeitern ist die Implementierung eines Versuchsstandes nicht möglich. Ich danke daher den beiden technischen Leitern des ZET, Herrn Dipl.-Ing. Danilo Höhne sowie Herrn Dipl.-Ing (FH) Daniel Voigt, sowie unserem Kesselwärter und „Mann für alle Fälle“ Martin Richter für die vielen gemeinsamen Stunden „in der Anlage“. Weiterhin gebührt der Dank dem Labor- und Versuchsfeldverbund der Fakultät Maschinenwesen unter der Leitung von Frau Dipl.-Ing. (FH) Christiana Sperling und Herrn Dipl.Wirt.-Ing. (FH) Lars Gladrow sowie den Männer um die Werkstattmeister Joachim Koch und Ralf Sterzik, Andreas Steudner, René Kramer, Mario Ulbricht, Matthias Opperskalski, Egbert Kretzschmar und allen anderen, die am Versuchsstand mitgewirkt haben, für die gewissenhafte und verlässliche Fertigung und Erweiterung unseres Totraumversuchsstandes SiSTeR.

Der Professur für Turbomaschinen und Flugantriebe, ehemals vertreten durch Prof. Dr.-Ing. Konrad Vogeler und nun durch Prof. Dr.-Ing. habil. Ronald Mailach, danke ich dafür, dass ich die beiden Schraubenverdichter und die Druckluftinfrastruktur im Zentrum für Energietechnik für den Versuchsbetrieb mit benutzen durfte. Für die Bereitstellung der Klimakammer und der Versuchsinfrastruktur zur Messung der Wärmeleitfähigkeit der PTFE-Probekörper danke ich Herrn Dipl.-Ing. Matthias Mischke von der Professur für Technische Thermodynamik unter Leitung von Frau Prof. Dr. rer. nat. habil. Cornelia Breitkopf. Weiterhin gilt mein Dank Herrn Dipl.-Ing. Jürgen Frey von der Arbeitsgruppe für Experimentelle Aerodynamik (EAD) am Institut für Luft- und Raumfahrt 
(ILR) der TU Dresden für die Möglichkeit, die dortige Ringkammerdüse für die Kalibrierung der beiden Fünflochsonden verwenden zu dürfen.

Den beiden Professoren Dr.-Ing. habil. Michał Ciałkowski und Dr.-Ing. habil. Andrzej Frackowiak vom Lehrstuhl für Wärmetechnik an der Technischen Universität Posen in Polen danke ich für ihren Rat und ihre Unterstützung bei der inversen Berechnung von Wärmeübergangkoeffizienten auf Basis finiter Elemente.

Weiterhin soll die hervorragende Wissenschafts- und Rechercheinfrastruktur der Sächsischen Landesbibliothek - Staats- und Universitätsbibliothek (SLUB) in Dresden hervorgehoben werden, ohne deren emsige Fernleihabteilung so manche russisch- und ukrainisch-sprachige Quelle in den Tiefen der Fachliteratur verschollen geblieben wäre. Als exzellent in jeder Hinsicht ist ebenfalls die Hochleistungsrechnerinfrastruktur (HPC) des Zentrums für Informationsdienste und Hochleistungsrechnen (ZIH) an der TU Dresden zu würdigen, auf deren Forschungscluster „Taurus“ etliche Prozessorstunden und -monate für die hier durchgeführten CFD-Untersuchungen angefallen sind.

Zu guter Letzt möchte ich einen ganz besonderen Dank an meine Familie richten, allen voran meinem Vater Mike, meiner Mutter Katrin und meiner Schwester Jessie, die mich immer gefördert, ermutigt und unterstützt haben meinen Weg zu gehen und mir zu jeder Zeit den Rücken freigehalten haben. Ohne euch wäre ich nie bis zu diesem Punkt gekommen! Ganz lieben Dank gebührt auch meiner Freundin Loreen für Ihre engelsgleiche Geduld und Ihr Verständnis, wenn es abends mal wieder später geworden ist, sowie für die fortwährende Motivation, immer durchzuhalten und die Arbeit so zu vollenden, wie sie hier ist. Danke an Euch alle.

Glück auf! 



\section{INHALT}

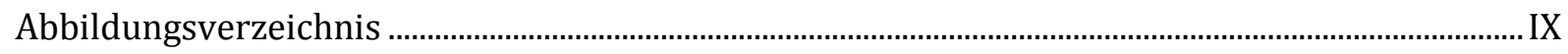

Tabellenverzeichnis ............................................................................................................................XVII

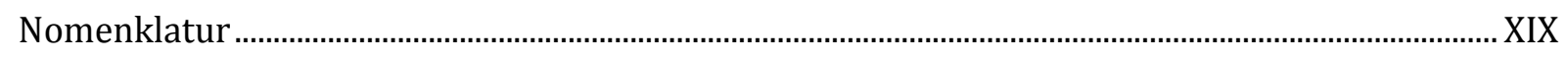

Lateinische Buchstaben.......................................................................................................................... XIX

Griechische Buchstaben ................................................................................................................. XXI

Hochgestellte Indizes .............................................................................................................XXII

Tiefgestellte Indizes .......................................................................................................................XXIII

Abkürzungen und Kurzbezeichnungen .................................................................................. XXVI

1 Einleitung und Motivation................................................................................................................

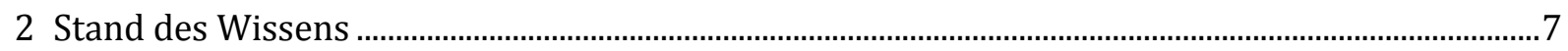

2.1 Strömung und Wärmeübergang in Seitenräumen von Dampfturbinen.....................................7

2.2 Strömung in Kavitäten mit T- und L-förmigem Querschnitt........................................................ 15

2.3 Wärmeübergang in Kavitäten mit quadratischem Querschnitt ..............................................17

2.4 Notwendigkeit und Ausgangslage der Untersuchungen ……………………………………... 18

2.5 Überblick über aktuelle Forschungstätigkeiten auf dem Gebiet..................................................20

3 Zielstellung, Methodik und Lösungsweg .......................................................................................... 23

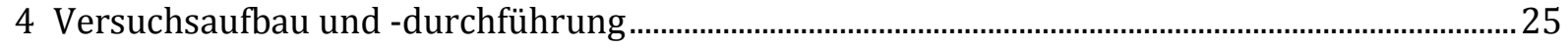

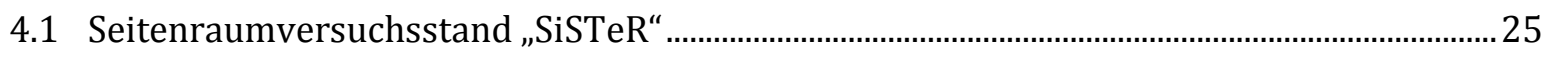

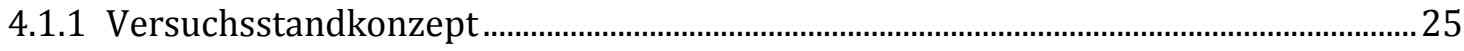

4.1.2 Dimensionierung und Skalierung der Modellgeometrie..................................................... 27

4.1.3 Experimentelle Implementierung des Versuchsstandes.................................................2 29

4.1.4 Versuchsanlage zur geregelten Druckluftbereitstellung für den Versuchsstand .....35

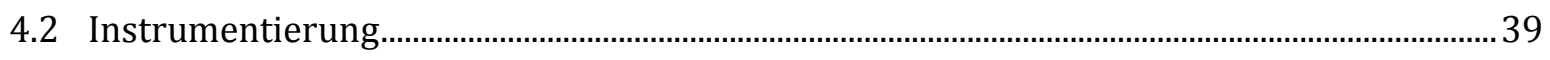

4.2.1 Wärmeübergangsmessung im Seitenraum ........................................................................... 39

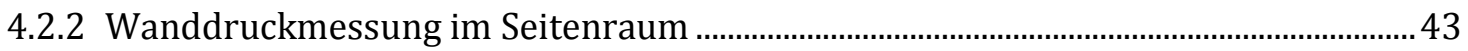

4.2.3 Strömungsmessung im konzentrischen Ringspalt des Hauptströmungskanals ....... 43

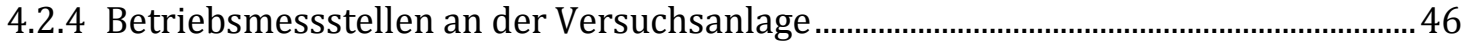

4.3 Messdatenerfassung und -verarbeitung..................................................................................... 47

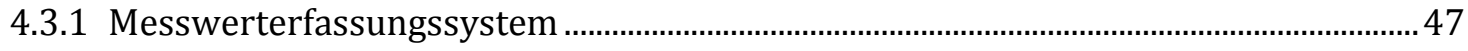


4.3.2 Datenverarbeitung und -synchronisierung ………............................................................. 48

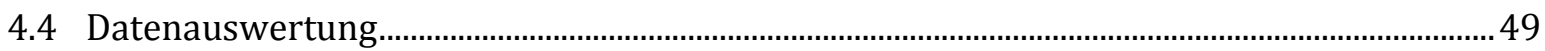

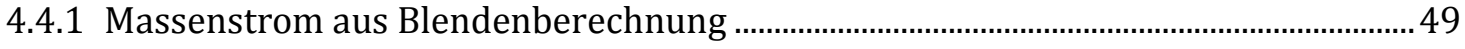

4.4.2 Bezugstemperatur, Bezugsdruck und charakteristische Strömungsgrößen im Versuchsstand

4.4.3 Thermisches Netzwerkmodell zur Abschätzung lokaler

Wärmeübergangskoeffizienten .............................................................................................. 52

4.4.4 Rückwärtsrechnung mittels gradientenbasiertem Optimierungsverfahren...............55

4.4.5 Ableitungsfreie Optimierung mittels evolutionärem Algorithmus .....................................60

4.4.6 Inverse Berechnung mittels Trefftz-Funktionen und Regularisierung..........................62

4.4.7 Vergleich der mit den Rechenmodellen ermittelten WÜK-Verläufe ............................... 71

4.5 Versuchsplanung und -durchführung ....................................................................................... 72

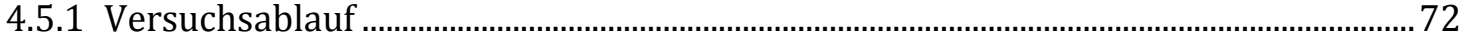

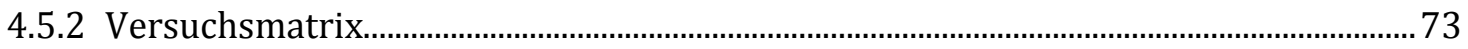

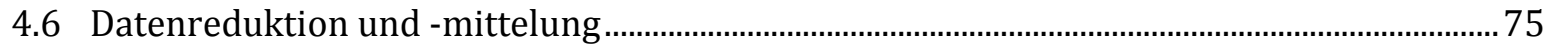

5 Ergebnisse und Diskussion ............................................................................................................

5.1 Betriebscharakteristik der Versuchsanlage

5.2 Kalibrierung der Fünflochsonde an der Freistrahldüse ……………………………………...... 77

5.3 Strömungsfeld im konzentrischen Ringspaltkanal .................................................................... 81

5.4 Druckverteilung an der Außenwandinnenoberfläche..................................................................91

5.5 Thermodynamische Stoffwerte der Wandmaterialien...................................................................97

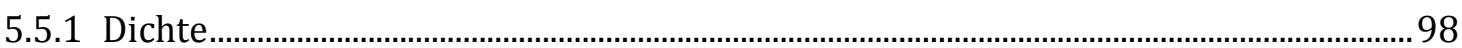

5.5.2 Wärmeleitfähigkeit, Temperaturleitfähigkeit, spezifische Wärmekapazität .......... 100

5.6 Kalibrierung des Messaufbaus an der Saugrohrstrecke ......................................................... 109

5.7 Messergebnisse zum lokalen Wärmeübergang in generischen Seitenraumgeometrien 114

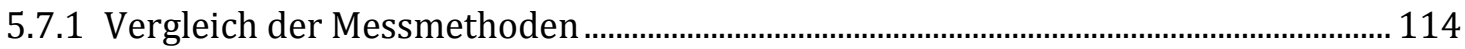

5.7.2 Reproduzierbarkeit und Streuung der Messwerte......................................................... 115

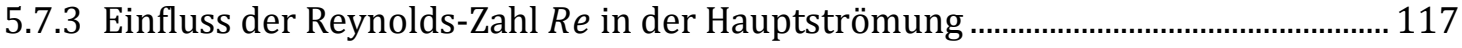

5.7.4 Einfluss der Einströmbreite $s$...................................................................................... 118

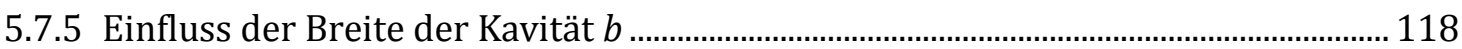

5.7.6 Einfluss der Exzentrizität der Kavität e e......................................................................... 119

5.7.7 Einfluss des Drallwinkels $\alpha$ in der Anströmung zum Seitenraum ............................... 119 
5.8 Analyse und Abschätzung von Messunsicherheiten ............................................................... 122

5.8.1 Unsicherheit der gemessenen Absolut- und Differenzdrücke ..................................... 122

5.8.2 Unsicherheit der gemessenen Temperaturen ................................................................. 123

5.8.3 Unsicherheit der berechneten Wärmeübergangskoeffizienten .................................... 124

5.8.4 Unsicherheit der geometrischen Maße von Seitenraum und Strömungskanal ...... 133

5.8.5 Unsicherheit des Massenstromes an der Blendenmessstrecke ………........................ 136

5.8.6 Unsicherheit der Reynolds-Zahl..................................................................................... 138

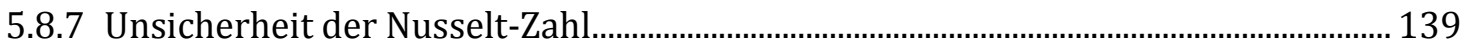

5.8.8 Unsicherheit der Strömungswinkel und Geschwindigkeitskomponenten ............... 140

5.9 Verallgemeinerung der Ergebnisse als Nusselt-Korrelation .................................................. 143

6 Numerische Nachrechnung ausgewählter Konfigurationen mittels CFD-Simulation................ 151

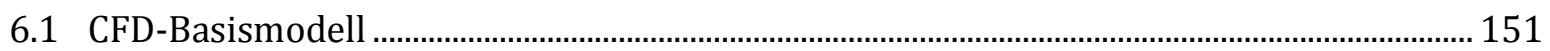

6.1.1 Geometrie

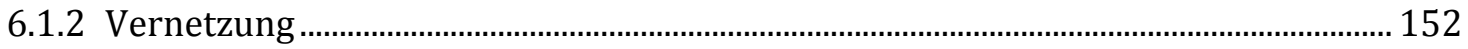

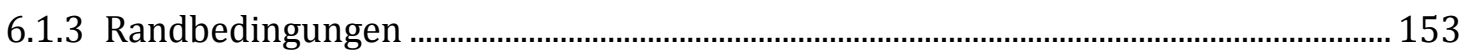

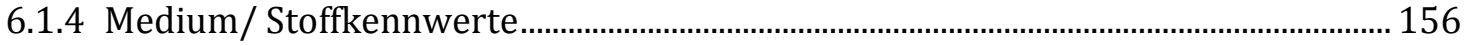

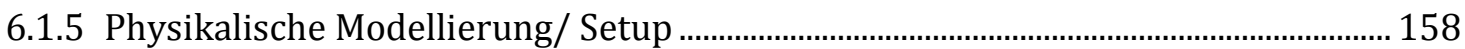

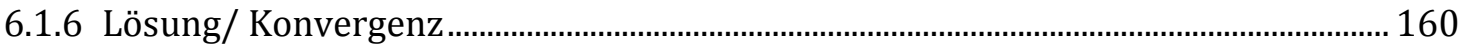

6.1.7 Auswertung und Ergebnisse ......................................................................................... 165

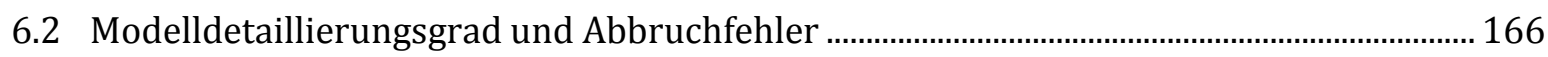

6.3 Netzunabhängigkeitsstudie ................................................................................................... 170

6.4 Einfluss der Randbedingungen und der Modellierung............................................................ 172

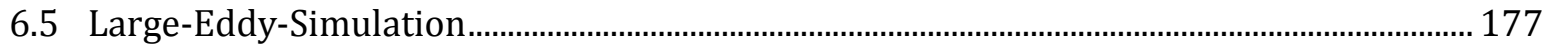

6.6 Ergebnisse der systematischen Nachrechnung ............................................................................. 183

7 Übertragung der Ergebnisse auf reale Turbinenverhältnisse.............................................................. 187

8 Zusammenfassung und Schlussfolgerungen..................................................................................... 189

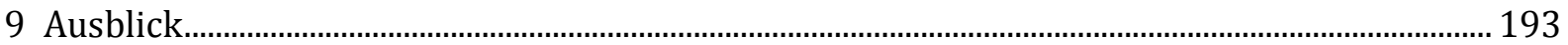

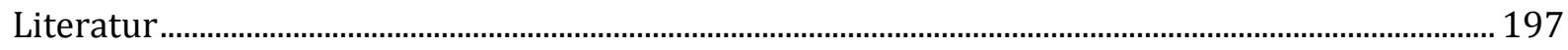

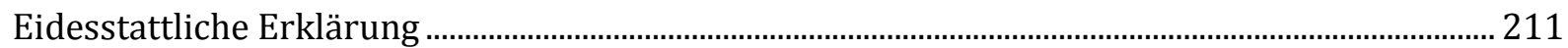





\section{ABBILDUNGSVERZEICHNIS}

Abb. 1.1 Veränderte Anforderungen an die lastflexible Fahrweise von Dampfturbinenanlagen.2

Abb. 1.2 Viertelschnittdarstellung einer Industriedampfturbine . .3

Abb. 1.3 Typische thermische Verformungen an Dampfturbinengehäusen ........................................ 4

Abb. 1.4 Schematische Strömungsstruktur in einem Seitenraum mit Anzapfung.............................6

Abb. 2.1 Berechnung der Wärmeübergangskoeffizienten in einer Dampfkammer ohne Anzapfung im Gehäuse einer HD-Turbine vom Typ K-200-130.............................................8

Abb. 2.2 Versuchsstand zur Untersuchung der Gasdynamik in Entnahmekammern ......................9

Abb. 2.3 Experimenteller Aufbau zur Untersuchung von Strömung und Wärmeübertragung in einer Entnahmekammer und im nachfolgenden Strömungskanal........................................ 9

Abb. 2.4 Prallstrahlanordnung an einer parallel zu einem überströmten Spalt versetzen Wand ... 10

Abb. 2.5 Verteilung der Wärmeübergangskoeffizienten an den Oberflächen der Anzapfkammer I einer russischen Turbine vom Typ K-500-240 PO HTZ in verschiedenen Lastpunkten 11

Abb. 2.6 Verteilung der lokalen und vom Staupunkt des Prallstrahls aus gemittelten Wärmeübergangskoeffizienten an der Außenoberfläche einer Anzapfkammer. 12

Abb. 2.7 Strömungsschema des Sekundärwirbels in der linken Hälfte eines Seitenraumes sowie gemessene und approximierte Geschwindigkeitsverteilung in der Grenzschicht

Abb. 2.8 Messsektion des sattdampfbetriebenen Versuchsstandmodelles einer Dampfentnahmekammer.

Abb. 2.9 Wasserversuchsstrecke zur experimentellen Bestimmung des Strömungsfeldes in einer L-förmigen Kavität

Abb. 2.10 Strömungsfeld in überströmten Kavitäten mit T-förmigem Querschnitt und unterschiedlichen Abmessungsverhältnissen

Abb. 2.11 Strömungsfeld in überströmten Kavitäten mit L-förmigem Querschnitt und unterschiedlicher Orientierung.

Abb. 2.12 Nomenklatur der geometrischen Abmessungen einer ebenen quadratischen Kavität17

Abb. 2.13 Lokale Nusselt-Zahl-Verläufe in ebenen quadratischen Kavitäten bei variierten Reynolds-Zahlen in der Hauptströmung.

Abb. 4.1 Versuchsstandkonzept.

Abb. 4.2 Geometrische Abmessungen des Seitenraumes und des Hauptströmungskanals. 26 
Abb. 4.3 Einstelloptionen der Seitenraumgeometrie..........................................................................27

Abb. 4.4 Realisierbare Abmessungen des Seitenraumes..................................................................... 28

Abb. 4.5 Digitaler Zwilling (Digital Twin) des Totraumversuchsstandes..........................................30

Abb. 4.6 Experimenteller Aufbau des Totraummodules ……................................................................ 33

Abb. 4.7 Experimenteller Aufbau des Kompensationsmodules ............................................................ 34

Abb. 4.8 Vereinfachtes Schaltschema der erweiterten Druckluftversuchsanlage ........................... 35

Abb. 4.9 Scheibenaufbau der Seitenraumaußenwand aus acht baugleichen, instrumentierten Messringen .40

Abb. 4.10 Elektrisch-thermisches Netzwerkmodell des WÜK-Sensors .............................................. 41

Abb. 4.11 Prinzipskizze zur Funktionsweise der beiden eingesetzten Messverfahren ...................42

Abb. 4.12 Pitot-Sonde zur Geschwindigkeitsprofilbestimmung ............................................................ 43

Abb. 4.13 Fünfloch-Pyramidensonde zur 3D-Geschwindigkeitsmessung .......................................... 44

Abb. 4.14 Mögliche Einbaupositionen der Fünfloch-Pyramidensonde im Versuchsstand ............. 44

Abb. 4.15 Sondenverstellgerät zur Höhen- und Winkeltraversierung einer Sonde im Hauptströmungskanal

Abb. 4.16 Winkelkonvention und Quadranten bei Verdrehung der Außenwand um ihre Längsachse 45

Abb. 4.17 Dynamische Anzeige der zeitlichen Messwertverläufe. 49

Abb. 4.18 Sankey-Diagramm der mittels Netzwerkmodell ermittelten axialen und radialen

Wärmeströme

Abb. 4.19 Abmessungen, Randbedingungen, Material- und Fluidparameter zweier benachbarter Messringe für die Modellierung des thermischen Ersatznetzwerkes 54

Abb. 4.20 Thermisches Ersatznetzwerk zur analytischen Abschätzung der Wärmeübergangskoeffizienten im Seitenraum 54

Abb. 4.21 Strukturiertes Rechennetz und Materialien des 2D-FE-Modells. . .57

Abb. 4.22 Randbedingungen des 2D-FE-Modells . .57

Abb. 4.23 Konvergenzverlauf der Designoptimierung für die inverse Bestimmung von WÜK mit dem FE-Modell .59

Abb. 4.24 Temperaturfeld der Seitenraumstrukturen für das konvergierte FE-Modell 60

Abb. 4.25 Design-Sets und Zielfunktion für die gradientenfreie Designoptimierung mittels evolutionärem Algorithmus zur inversen Bestimmung von WÜK 
Abb. 4.26 Form des Rechengebietes $\Omega$, seines Randes $\Gamma=\Gamma_{\mathrm{i}} \cup \Gamma_{\mathrm{v}} \cup \Gamma_{\mathrm{h}} \cup \Gamma_{\mathrm{inv}}$ und Position der Temperaturmessstellen $T_{k}{ }^{*}$ und der Wärmequellen $\tilde{q}$............................................................ 62

Abb. 4.27 Approximation der Wärmestromdichte auf dem Rand des Seitenraumes $\Gamma_{\text {inv }}$

Abb. 4.28 Basisfunktion $T_{0}$ und ausgewählte Trefftz-Funktionen $\theta_{j}$ als Teillösungen des inversen

Problems für den Beispielfall

Abb. 4.29 Resultierende Temperaturverteilung der Superpositionslösung. 67

Abb. 4.30 Einfluss der Wahl des Regularisierungsparameters $\beta$ auf die Genauigkeit und die Stabilität der Lösung des inversen Problems

Abb. 4.31 Vergleich der invers berechneten WÜK am oberen Rand der Kavität für die verschiedenen Teilungen

Abb. 4.32 Unstrukturiertes Rechennetz des FE-Modells für die inverse Berechnung in FreeFEM++ 70

Abb. 4.33 Vergleich der mittels der Rechenmodelle ermittelten lokalen Wärmeübergangskoeffizienten für den vorliegenden Beispielfall.

Abb. 4.34 Vergleich der mittels der Rechenmodelle ermittelten lokalen

Wärmeübergangskoeffizienten für einen weiteren Beispielfall . .72

Abb. 4.35 Zeitlicher Verlauf der mittels thermischen Netzwerkmodell in Echtzeit berechneten WÜK 73

Abb. 4.36 Auswertung der über ein festgelegtes Intervall gemittelten, gemessenen Temperaturen zur Bestimmung von WÜK mittels thermischem Netzwerkmodell ...... 75

Abb. 5.1 Netzkennlinien bei variiertem Hub der beiden Hauptventile im Druckluftsystem....... 77

Abb. 5.2 Versuchsaufbau zur automatisierten Winkeltraversierung und Kalibrierung der Fünflochsonde in einer Düsenströmung . .78

Abb. 5.3 Nomenklatur, Winkelkonventionen und Geschwindigkeitskomponenten an der kalibrierten Fünflochsonde .80

Abb. 5.4 Axialgeschwindigkeitsprofil im kreisringspaltförmigen Hauptströmungskanal . .82

Abb. 5.5 Strömungswinkel und Geschwindigkeitsverteilung in der Hauptströmung am Einlass in den Seitenraum, mit Stützgitter. . .84

Abb. 5.6 Drallwinkel $\alpha$ und Nickwinkel $\beta$ in der Hauptströmung am Einlass in den Seitenraum

Abb. 5.7 Axial- und Achsnormalgeschwindigkeit $c_{\mathrm{ax}}$ bzw. $c_{\mathrm{res}}$ in der Hauptströmung am Einlass in den Seitenraum. . .88 
Abb. 5.8 Umfangsgemittelte Strömungswinkel und Geschwindigkeitskomponenten über der Kanalhöhe in der Hauptströmung am Einlass in den Seitenraum 89

Abb. 5.9 Arithmetisch über den 4. Quadranten gemittelter und an einem Referenzpunkt gemessener Drallwinkel in der Strömung für verschiedene mittels Drallgitter eingestellte Blechwinkel 90

Abb. 5.10 Drallwinkel am Referenzpunkt für verschiedene mittels Drallgitter eingestellte Blechwinkel und erhöhte Reynolds-Zahlen 90

Abb. 5.11 Wanddruckverteilung entlang der Innenoberfläche der Außenwand für $360^{\circ}$ -

Umfangstraversierung, mit Stützgitter

Abb. 5.12 Wanddruckverteilung am Umfang und entlang der Achse der Außenwand für $360^{\circ}$ Umfangstraversierung, mit Stützgitter

Abb. 5.13 Vergleich der gemessenen Wanddruckverteilung entlang der Innenoberfläche der Außenwand zur Untersuchung des Einflusses einer eventuellen Sondenverschattung

Abb. 5.14 Einfluss des Blechwinkels $\gamma$ in der Hauptströmung auf die Wanddruckverteilung entlang der Innenoberfläche der Außenwand.

Abb. 5.15 Einfluss des am Drallgitter eingestellten Blechwinkels $\gamma$ auf die axiale Druckverteilung an der Innenoberfläche der Außenwand

Abb. 5.16 Einfluss des Drucks bzw. der Geschwindigkeit in der Hauptströmung bei gleicher Reynolds-Zahl auf die Druckverteilung an der Innenoberfläche der Außenwand bei $\varphi=0^{\circ}$ für unterschiedliche Blechwinkel $\gamma$.

Abb. 5.17 Einfluss der Reynolds-Zahl in der Hauptströmung auf die Druckverteilung bei $\varphi=0^{\circ}$

für unterschiedliche Blechwinkel $\gamma$ .97

Abb. 5.18 Probennahme aus PTFE-Platte . .98

Abb. 5.19 Hot-Disk-Sonden 101

Abb. 5.20 Aufbau der Referenz-Platten-Apparatur 103

Abb. 5.21 Versuchsaufbau zur Bestimmung der Wärmeleitfähigkeit einer PTFE-Flachprobe mittels Wärmestrommessplattenapparatur

Abb. 5.22 Abhängigkeit der mittels Plattenapparatur gemessenen Wärmeleitfähigkeit der PTFEFlachprobe von der tatsächlichen Temperatur der Klimakammer. 106

Abb. 5.23 Saugrohrstrecke zur Kalibrierung der Wärmeübergangssensorik in einer Rohrströmung. 109

Abb. 5.24 Berechnete WÜK-Verläufe für die Saugrohrströmung bei $R e=2,7 \cdot 10^{5}$ nach verschiedenen Literaturansätzen. 
Abb. 5.25 In den verschiedenen Versuchsreihen mittels stationärer inverser Methode bestimmte lokale WÜK-Verläufe für die Saugrohrströmung bei unterschiedlichen Reynolds-Zahlen.

Abb. 5.26 Überblick über die mittels stationärer inverser Methode bestimmten lokalen WÜKVerläufe für die Saugrohrströmung bei unterschiedlichen Reynolds-Zahlen

Abb. 5.27 Vergleich der mittels beider Messmethoden ermittelten lokalen WÜK-Verteilungen

Abb. 5.28 Einfluss von Variationen bei der Versuchsdurchführung auf die Reproduzierbarkeit und Streuung der lokalen, umfangsgemittelten WÜK-Messwerte

Abb. 5.29 Einfluss der Einströmbreite auf die WÜK-Verteilung im Seitenraum 120

Abb. 5.30 Einfluss der Breite der Kavität auf die WÜK-Verteilung im Seitenraum 120

Abb. 5.31 Einfluss der Exzentrizität der Kavität auf die WÜK-Verteilung im Seitenraum

Abb. 5.32 Einfluss des Blechwinkels auf die WÜK-Verteilung im Seitenraum 121

Abb. 5.33 Häufigkeitsverteilung der Eingabeparameter der Monte-Carlo-Simulation 128

Abb. 5.34 Häufigkeitsverteilung der Ausgabeparameter der Monte-Carlo-Simulation 129

Abb. 5.35 Auflösung des Datenerfassungssystems.

Abb. 5.36 Äußere axiale Referenzmaße zur Einstellung der lateralen Seitenraumabmessungen und der Außenwandposition.

Abb. 5.37 Relative Gesamtunsicherheit der Reynolds-Zahl im Versuchsstand und Einzelunsicherheiten der zugrunde liegenden Messgrößen

Abb. 5.38 Approximation des WÜK-Verlaufes mittels einer Gauß'schen Glockenkurve

Abb. 5.39 Experimentell bestimmte Nusselt-Zahl-Verläufe im Seitenraum mit $s=b=46,3 \mathrm{~mm}$ im Vergleich zu Literaturwerten für ebene quadratische Kavitäten

Abb. 5.40 Streudiagramm der mittels Korrelation für alle Messpunkte und Konfigurationen berechneten über den gemessenen lokalen Nusselt-Zahlen.

Abb. 5.41 Vergleich der Nusselt-Korrelation mit der gemessenen WÜK-Verteilung im Seitenraum für variierte Einströmbreite $s$...

Abb. 5.42 Vergleich der Nusselt-Korrelation mit der gemessenen WÜK-Verteilung im Seitenraum für variierte Totraumbreite $b$.

Abb. 5.43 Vergleich der Nusselt-Korrelation mit der gemessenen WÜK-Verteilung im Seitenraum für variierte Exzentrizität $e$. 150

Abb. 5.44 Vergleich der Nusselt-Korrelation mit der gemessenen WÜK-Verteilung im Seitenraum für variierten Blechwinkel $\gamma$. 
Abb. 6.1 Geometrie, Ein- und Auslaufstrecken des CFD-Basismodells .............................................. 152

Abb. 6.2 Rechennetz des Basismodells …………….............................................................................. 152

Abb. 6.3 Rechennetz des Modelles für die konjugierte WÜ-Simulation ........................................... 153

Abb. 6.4 Position der Monitorpunkte innerhalb der Domain .............................................................. 163

Abb. 6.5 Typischer Konvergenzverlauf der Residuen sowie des WÜK-Konvergenzkriteriums für

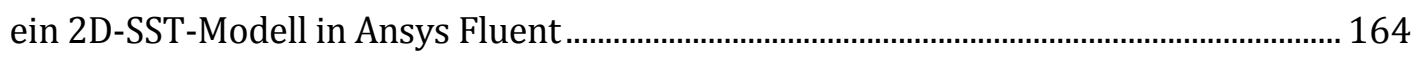

Abb. 6.6 Typischer Konvergenzverlauf der WÜK-Monitorpunkte für ein 2D-SST-Modell in

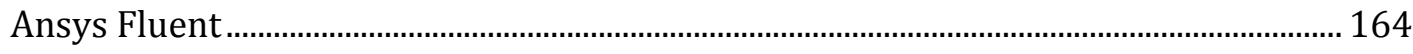

Abb. 6.7 Sektormodell, Mantellinie und Zentriwinkel............................................................................. 166

Abb. 6.8 Einfluss des modellierten Sektorwinkels auf die berechneten lokalen WÜK .................. 167

Abb. 6.9 Statistische Kenndaten der mittels CFD berechneten WÜK an der Außenwand ........ 169

Abb. 6.10 Zeitlich und umfangsgemittelter WÜK-Verlauf, zugehörige Streuungen (Standardabweichungen) und Konfidenzintervalle 170

Abb. 6.11 Einfluss verschiedener Vernetzungsparameter auf die berechneten lokalen WÜK...... 171

Abb. 6.12 Einfluss der Wandmodellierung auf den mit Ansys Fluent berechneten WÜK-Verlauf

Abb. 6.13 Einfluss der Ein- und Auslassrandbedingungen auf den mit Ansys Fluent berechneten WÜK-Verlauf

Abb. 6.14 Einfluss der Stoffwerte sowie der Turbulenzmodellierung auf den mit Ansys Fluent berechneten WÜK-Verlauf.

Abb. 6.15 Zeitlicher Verlauf und invers aufgetragener, auflaufender, harmonischer Mittelwert des WÜK im Monitorpunkt P07.

Abb. 6.16 Invers aufgetragene, auflaufende, harmonische Mittelwerte des WÜK in allen Monitorpunkten 180

Abb. 6.17 Vorwärts aufgetragene, über $n=50.000$ Iterationen gleitende harmonische Mittelwerte des WÜK in allen Monitorpunkten 180

Abb. 6.18 Harmonischer Mittelwert der lokalen WÜK-Verteilung an der Außenwand des $90^{\circ}$ LES-Modells

Abb. 6.19 Arithmetischer Mittelwert der lokalen WÜK-Verteilung an der Außenwand des $90^{\circ}$ LES-Modells

Abb. 6.20 Harmonischer Mittelwert der lokalen WÜK-Verteilung an der Außenwand des 2D-

LES-Modells 
Abb. 6.21 Arithmetischer Mittelwert der lokalen WÜK-Verteilung an der Außenwand des 2DLES-Modells 182

Abb. 6.22 CFD-Nachrechnung zum Einfluss der Einströmbreite auf die WÜK-Verteilung im Seitenraum.

Abb. 6.23 CFD-Nachrechnung zum Einfluss der Breite der Kavität auf die WÜK-Verteilung im Seitenraum 185

Abb. 6.24 CFD-Nachrechnung zum Einfluss der Exzentrizität der Kavität auf die WÜK-Verteilung im Seitenraum 186

Abb. 6.25 CFD-Nachrechnung zum Einfluss des Drallwinkels auf die WÜK-Verteilung im Seitenraum. 186

Abb. 7.1 Charakteristische thermodynamische Größen und Abmessungen am Längsschnitt eines Seitenraumes 



\section{TABELLENVERZEICHNIS}

Tab. 4.1 Realisierbare Parameter im Versuchsstand.

Tab. 4.2 Vergleich der eingesetzten Messmethoden zur rückwirkungsarmen Bestimmung des lokalen WÜK in jedem Messmodul

Tab. 4.3 Unterschiede zwischen den berechneten Wärmequellen aus dem Netzwerkmodell und den tatsächlich eingetragenen Heizleistungen

Tab. 4.4 Thermische Widerstände und zugehörige Querschnittsflächen für das

Netzwerkmodell . .55

Tab. 4.5 Wärmeleitfähigkeit der Materialien der Seitenraumstrukturen. .56

Tab. 4.6 Einteilung des Randes $\Gamma_{\text {inv }}$ in Unterabschnitte.

Tab. 4.7 Vergleich der berechneten Temperaturen $T_{k}$ mit den gemessenen Solltemperaturen $T_{k}^{*}$ für Variante 3 . .70

Tab. 4.8 Versuchsmatrix für die vorgesehene experimentelle Parameterstudie. . .74

Tab. 5.1 Form und Nennmaße der PTFE-Proben 98

Tab. 5.2 Berechnung der Mittelwerte und Abweichungen für die ermittelten Probenvolumina 98

Tab. 5.3 Abmessungen, Volumen, Masse und Dichte der PTFE-Proben. 100

Tab. 5.4 Abmessungen, Masse und Dichte der PTFE-Proben. 101

Tab. 5.5 Mittels Hot-Disk-Verfahren bestimmte spezifische Wärmekapazität, Temperatur- und Wärmeleitfähigkeit der PTFE-Proben

Tab. 5.6 Mittels Referenz-Platten-Apparatur bestimmte Wärmeleitfähigkeit der PTFE-Probe

Tab. 5.7 Mittels Wärmestrommessplattenapparatur bestimmte Wärmeleitfähigkeit der PTFE-

Probe. 106

Tab. 5.8 Literaturdaten zum Bridgman-Parameter für virginales PTFE

Tab. 5.9 Übersicht über Mittelwerte und Messunsicherheiten der mittels der verschiedenen Messverfahren ermittelten Wärmeleitfähigkeit von PTFE 108

Tab. 5.10 Symbolerklärung für Diagramme mit WÜK-Ergebnissen 114

Tab. 5.11 Teilunsicherheiten und Gesamtunsicherheit der eingesetzten Drucksensorgruppen 
Tab. 5.12 Teilunsicherheiten und Gesamtunsicherheit der eingesetzten Temperatursensoren 124

Tab. 5.13 Absolute und relative Abweichungen der WÜK aus dem Netzwerkmodell 125

Tab. 5.14 Rang-Korrelationsmatrix nach Spearman zwischen den Ein- und Ausgabeparametern der Probabilistik-Analyse.

Tab. 5.15 Absolute und relative Abweichungen der WÜK aus der Gradientenoptimierung

Tab. 5.16 Absolute und relative Abweichungen der WÜK aus der Rückwärtsrechnung mittels Trefftz-Funktionen

Tab. 5.17 Matrix der linearen Korrelationskoeffizienten nach Pearson zwischen den Ein- und Ausgabeparametern der idealen Glockenkurven-Fits.

Tab. 5.18 Matrix der Rang-Korrelationskoeffizienten nach Spearman zwischen den Ein- und Ausgabeparametern der idealen Glockenkurven-Fits 144

Tab. 6.1 Monitor-Punkte, deren Koordinaten und dort ausgewertete Größen. 162

Tab. 6.2 Randbedingungen und Modelleinstellungen des Fluent-Basismodells 172 


\section{NOMENKLATUR}

\section{LATEINISCHE BUCHSTABEN}

\begin{tabular}{|c|c|c|}
\hline$A$ & {$\left[\mathrm{~m}^{2}\right]$} & (Durchström-/ Querschnitts-) Fläche \\
\hline$B$ & {$[\mathrm{~mm}]$} & Breite \\
\hline$B i$ & {$[-]$} & Biot-Zahl \\
\hline C & {$[-]$} & Durchflusskoeffizient \\
\hline$C F L$ & {$[-]$} & Courant-(Friedrichs-Lewy-) Zahl \\
\hline$D$ & {$[\mathrm{~mm}]$} & (größerer/ Außen-) Durchmesser \\
\hline Dig & {$[-]$} & Dezimal-Ziffernschritt (Digit) \\
\hline E & {$[\mathrm{MPa}]$} & Elastizitätsmodul \\
\hline G & {$\left[\frac{1}{\mathrm{GPa}}\right]$} & Bridgman-Parameter \\
\hline$H$ & {$[\mathrm{~mm}]$} & Höhe; Hub \\
\hline$I$ & {$[\mathrm{~A}]$} & elektrische Stromstärke \\
\hline$J$ & {$\left[\mathrm{~K}^{2}\right]$} & Zielfunktional \\
\hline$L$ & {$[\mathrm{~m}]$} & (Lauf-) Länge \\
\hline$M$ & {$\left[\frac{\mathrm{kg}}{\mathrm{kmol}}\right]$} & molare Masse \\
\hline$M a$ & {$[-]$} & Mach-Zahl \\
\hline$N$ & {$[-]$} & Anzahl \\
\hline $\bar{N}$ & {$[-]$} & relative Leistung/ prozentuale Teillast \\
\hline$N u$ & {$[-]$} & Nusselt-Zahl \\
\hline$P$ & {$[\mathrm{~W}]$} & Leistung \\
\hline $\operatorname{Pr}$ & {$[-]$} & Prandtl-Zahl \\
\hline$\dot{Q}$ & {$[\mathrm{~W}]$} & Wärmestrom \\
\hline$R$ & {$\left[\frac{\mathrm{J}}{\operatorname{kg~K}}\right]$} & spezifische Gaskonstante \\
\hline$R$ & {$[\mathrm{~mm}]$} & (Außen-) Radius \\
\hline$R$ & $\left\lceil\frac{\mathrm{K}}{\mathrm{W}}\right]$ & thermischer Widerstand \\
\hline$R$ & {$[\Omega]$} & elektrischer Widerstand \\
\hline $\bar{R}$ & {$\left[\frac{\mathrm{J}}{\mathrm{mol} \mathrm{K}}\right]$} & molare Gaskonstante, $\bar{R}=8,314 \mathrm{~J} /(\mathrm{mol} \mathrm{K})$ \\
\hline$R e$ & {$[-]$} & Reynolds-Zahl \\
\hline
\end{tabular}


$R z \quad[\mu \mathrm{m}] \quad$ mittlere Rautiefe (Zehnpunkthöhe)

$T \quad[\mathrm{~K}],\left[{ }^{\circ} \mathrm{C}\right] \quad$ Temperatur

Tu [-] Turbulenzgrad/ -intensität

$U \quad[\mathrm{~mm}] \quad$ benetzter Umfang

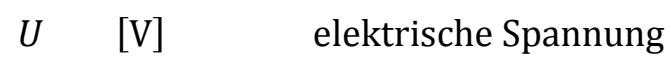

$V \quad\left[\mathrm{~m}^{3}\right] \quad$ Volumen/ Rauminhalt

$a \quad\left[\frac{\mathrm{m}^{2}}{\mathrm{~s}}\right] \quad$ Temperaturleitkoeffizient/ spezifische Temperaturleitfähigkeit

a [mm $]$ Maschenweite; Abstand

$b \quad[\mathrm{~mm}] \quad$ Breite (der Kavität)

c $\quad\left[\frac{\mathrm{J}}{\mathrm{kg} \mathrm{K}}\right] \quad$ spezifische Wärmekapazität

$c, \vec{c} \quad\left[\frac{\mathrm{m}}{\mathrm{s}}\right] \quad$ (Absolut-) Geschwindigkeit

$d \quad[\mathrm{~mm}] \quad$ (kleinerer/Innen-) Durchmesser

$\operatorname{dTopt}\left[\mathrm{K}^{2}\right] \quad$ Zielfunktion

$e \quad[\mathrm{~mm}] \quad$ Exzentrizität (der Kavität)

$g \quad\left[\frac{\mathrm{m}}{\mathrm{s}}\right] \quad$ Erdbeschleunigung, $g=9,80665 \mathrm{~m} / \mathrm{s}^{2}$

$h \quad[\mathrm{~mm}] \quad$ Höhe

$h \quad\left[\frac{\mathrm{J}}{\mathrm{kg}}\right] \quad$ spezifische Enthalpie

$i, j, k[-] \quad$ Laufvariablen (in $x-, y$-, bzw. $z$-Richtung)

$k \quad[-] \quad$ Korrekturfaktor

$k \quad\left[\frac{\mathrm{m}^{2}}{\mathrm{~s}^{2}}\right] \quad$ spezifische turbulente kinetische Energie

$k \quad\left[\frac{\mathrm{W}}{\mathrm{m}^{2} \mathrm{~K}}\right] \quad$ Wärmedurchgangskoeffizient

$k \quad[\mathrm{~mm}] \quad$ Abstand

$k \quad[\mu \mathrm{m}] \quad$ äquivalente Rauigkeit

$l \quad[\mathrm{~mm}] \quad$ (charakteristische) Länge

$m \quad$ [kg] Masse

$\dot{m} \quad\left[\frac{\mathrm{kg}}{\mathrm{s}}\right] \quad$ Massenstrom/-durchsatz

$n \quad[-] \quad$ Anzahl (an Iterationen)

$\vec{n} \quad[-] \quad$ Normalenvektor/ Einheitsvektor in Normalenrichtung

$n \quad\left[\frac{1}{\min }\right] \quad$ Drehzahl 


$\begin{array}{lll}p & {[\mathrm{~Pa}]} & \text { (statischer/ Absolut-) Druck } \\ q & {\left[\frac{\mathrm{m}}{\mathrm{s}}\right]} & \text { querschnittsgemittelte Geschwindigkeit } \\ \hat{\dot{q}}, \dot{q} & {\left[\frac{\mathrm{W}}{\mathrm{m}^{2}}\right]} & \text { (flächenspezifische) Wärmestromdichte } \\ \tilde{q} & {\left[\frac{\mathrm{W}}{\mathrm{m}^{3}}\right]} & \text { (volumetrische) Wärmequelldichte } \\ r & {[\mathrm{~mm}]} & \text { Radius; radiale Koordinate } \\ r & {[-]} & \text { Druck-/ Wärmerückgewinnungsfaktor } \\ r & {[-]} & \text { linearer Korrelationskoeffizient nach Pearson } \\ \tilde{r} & {[-]} & \text { Rangkorrelationskoeffizient nach Spearman } \\ s & {[\mathrm{~mm}]} & \text { Breite (der Einströmöffnung); Wandstärke; Dicke; Pfadlänge } \\ s & {\left[\frac{\mathrm{J}}{\mathrm{kg} \mathrm{K}}\right]} & \text { spezifische Entropie } \\ t & {[\mathrm{~s}]} & \text { Zeit } \\ u & {\left[\frac{\mathrm{m}}{\mathrm{s}}\right]} & \text { (Strömungs-/ Durchström-) Geschwindigkeit } \\ u & {[\mu \mathrm{mm}]} & \text { Verschiebung } \\ x & {[\mathrm{~mm}]} & \text { Breite; axiale Koordinate } \\ x, y & {[-]} & \text { dimensionslose Druckdifferenz } \\ y & {[\mathrm{~mm}]} & \text { Durchbiegung; Höhenkoordinate } \\ y & {[\%]} & \text { relativer Ventilhub } \\ z & {[\mathrm{~mm}]} & \text { Tiefenkoordinate }\end{array}$

\section{GRIECHISCHE BUCHSTABEN}

$\begin{array}{lll}\Gamma & {[-]} & \text { Rand } \\ \Delta & {[-]} & \text { absoluter Fehler/ Messabweichung/ Unsicherheit/ Differenz } \\ \Lambda & {[-]} & \text { geometrischer/ Skalierungsfaktor/ Maßstab } \\ \Omega & {[-]} & \text { Rechengebiet } \\ & \\ \alpha & {\left[\frac{\mathrm{W}}{\mathrm{m}^{2} \mathrm{~K}}\right]} & \text { Wärmeübergangskoeffizient } \\ \alpha & {\left[{ }^{\circ}\right]} & \text { Drallwinkel } \\ \alpha & {[\%]} & \text { Unterrelaxationsfaktor } \\ \beta & {\left[\mathrm{K}^{2}\right]} & \text { Regularisierungsparameter } \\ \beta & {\left[{ }^{\circ}\right]} & \text { Nickwinkel }\end{array}$




\begin{tabular}{|c|c|c|}
\hline$\beta$ & {$[-]$} & Blendenöffnungsverhältnis \\
\hline$\gamma$ & {$\left[{ }^{\circ}\right]$} & Blechwinkel \\
\hline$\delta$ & {$[\mu \mathrm{m}]$} & Grenzschichtdicke \\
\hline$\delta$ & {$[\%]$} & relativer Fehler/ Messabweichung/ Unsicherheit \\
\hline$\varepsilon$ & {$[-]$} & Expansionszahl \\
\hline$\varepsilon$ & {$[-]$} & relative Exzentrizität \\
\hline$\varepsilon$ & {$\left[\frac{\mathrm{m}^{2}}{\mathrm{~s}^{3}}\right]$} & spezifische turbulente Dissipationsrate \\
\hline$\zeta$ & {$[-]$} & Druckverlustbeiwert \\
\hline$\eta, \mu$ & {$[\mathrm{Pa} s]$} & dynamische Viskosität \\
\hline$\theta$ & {$[\mathrm{K}]$} & Trefftz-Funktion \\
\hline$\theta$ & {$\left[{ }^{\circ}\right]$} & Kreissektor-/ Zentriwinkel \\
\hline$\kappa$ & {$[-]$} & Isentropenexponent \\
\hline$\lambda$ & {$[\mathrm{mm}]$} & Wirbellänge(nskala) \\
\hline$\lambda$ & {$\left[\frac{\mathrm{W}}{\mathrm{m} \mathrm{K}}\right]$} & Wärmeleitkoeffizient/ spezifische Wärmeleitfähigkeit \\
\hline$\lambda$ & {$[-]$} & Rohrreibungsbeiwert/ Rohrreibungszahl \\
\hline$\mu$ & {$[-]$} & Mittel-/ Erwartungswert \\
\hline$v$ & {$[-]$} & Querkontraktionszahl/ Poisson-Zahl \\
\hline$v$ & {$\left[\frac{\mathrm{m}^{2}}{\mathrm{~s}}\right]$} & kinematische Viskosität \\
\hline$\xi$ & {$[-]$} & Druckverlustbeiwert \\
\hline$\rho$ & {$\left[\frac{\mathrm{kg}}{\mathrm{m}^{3}}\right]$} & (Massen-) Dichte \\
\hline$\sigma$ & {$[-]$} & (Stichproben-) Standardabweichung \\
\hline$\sigma$ & {$[\mathrm{MPa}]$} & Mechanische Spannung \\
\hline$\tau$ & {$[\mathrm{Pa}]$} & Schub-/ Reibspannung \\
\hline$\varphi$ & {$[\%]$} & relative Luftfeuchtigkeit \\
\hline$\varphi$ & {$\left[{ }^{\circ}\right]$} & (Verdreh-/ Dreh-) Winkel, Umfangskoordinate \\
\hline$\omega$ & {$\left[\frac{1}{s}\right]$} & turbulente Wirbelfrequenz/ spezifische Dissipationsrate \\
\hline
\end{tabular}

\section{HOCHGESTELLTE INDIZES}

$\begin{array}{ll}+, * & \text { dimensionsloses Wandprofil } \\ * & \text { gemessen }\end{array}$




\section{TIEFGESTELLTE INDIZES}

0

0

1

1

1

1

2

2

2

3

100

$\infty$

Abs

AR

AW

B

$\mathrm{B}, \mathrm{Bl}$

D

F

Fl

$\mathrm{H}$

HS

IR

Iso

K

Kalib

KK

Korr.

$\mathrm{L}$
Anfangs-/ Ausgangs-; Standard-/ Basis-

homogen

Einströmöffnung

Eintritts-/ Anström-/ Einström-/ Vor-/ Anfangs-/ Eingangs-

Innenring

Einheits-

Totraum

Austritts-/ Abström-/ Ausström-/ Nach-/ End-/ Ausgangs-

Zwischenring

Außenring

$100 \%$

Freiströmung; stationär

Absatz; Abschnitt

Außenring

Außenwand

Bezugs-/ Referenz- (Bulk)

Blende

Dichtung

Fluid

Flansch

Heizseite

Hauptströmung

Innenring

Isolation

Kammer; Kühlseite

Kalibrierung

Klimakammer

Korrelation

Luft; links 
M

$\mathrm{N}$

0

Pr

$\mathrm{R}$

S

S

S

Streu

Sty

SW

$\mathrm{T}$

Th

TR

U, Umg, Umgeb

U

Ü

V

V

VS

W

W

Z

a

abs

arith

aus

ax

diff

dyn
Mitte; Modell

Norm-/ Standard-/ Referenzbedingungen

oben

Probe/ Prüfkörper

rechts

Saugstutzen

Intervallmittelpunkt

Staupunkt

Streuung

Sytropor

Seitenwand

Temperaturmessstelle; Total-; Turbine

Thermistor

Totraum

Umgebungs-

Umfangs-; unten

Überdruck

Vergleichs-

Verlust-/ -verlust

Versuchsstand

Wand-

Wirk-

zeitlich

außen; Außen-

absolut

arithmetisch

Ausgangs-/ Ausström-

axial

Differenz-

dynamisch 


\begin{tabular}{|c|c|}
\hline e & extra \\
\hline eff & effektiv \\
\hline eig & Eigen- \\
\hline ein & Eingangs-/ Einström- \\
\hline el & elektrisch \\
\hline en & Energie- \\
\hline $\mathrm{f}$ & frei; wandnächster Knoten \\
\hline ges & Gesamt- \\
\hline gleit & gleitend \\
\hline $\mathrm{h}$ & horizontal \\
\hline $\mathrm{h}$ & hydraulisch/ hydrodynamisch \\
\hline harm & harmonisch \\
\hline $\mathrm{i}$ & innen; Innen- \\
\hline int & integriert/ integral \\
\hline inv & invers \\
\hline korr & korrigiert \\
\hline $\mathrm{m}$ & mittlere(r/s) \\
\hline $\max$ & maximal \\
\hline mess & gemessen \\
\hline $\min$ & minimal \\
\hline $\mathrm{n}$ & nach \\
\hline $\mathrm{p}$ & isobar \\
\hline $\mathrm{r}$ & radial \\
\hline red & reduziert \\
\hline ref & Referenz- \\
\hline rel & relativ \\
\hline res & resultierend \\
\hline s & Spalt \\
\hline $\mathrm{t}$ & turbulent \\
\hline $\mathrm{v}$ & vertikal; vor; isochor \\
\hline
\end{tabular}




\section{ABKÜRZUNGEN UND KURZBEZEICHNUNGEN}

2D

zweidimensional

3D

dreidimensional

3WV

Dreiwegeventil

$\mathrm{A} / \mathrm{D}$

Analog-Digital

ADU

Analog-Digital-Umwandler

Abb.

Abbildung

AG

Arbeitsgemeinschaft

AG

Aktiengesellschaft

APDL

Ansys Parametric Design Language

ASME

American Society of Mechanical Engineers

AW

Außenwand

BMWi

Bundesministerium für Wirtschaft und Energie

CAD

rechnergestützte Konstruktion (Computer Aided Design)

CCL

Ansys CFX Command Language

CFD

numerische Strömungsmechanik (Computational Fluid Dynamics)

CGNS

CFD General Notation System

CHT

konjugierter Wärmeübergang (Conjugate Heat Transfer)

COM

Communication Port

COOREFLEX $\quad \mathrm{CO}_{2}$-Reduktion durch Flexibilität

CPU

Prozessorkern (Central Processing Unit)

DACE

numerische Parameterstudie (Design and Analysis of Computer Experiments)

DDE

Dynamic Data Exchange

$\mathrm{DF}(\mathrm{Z} / \mathrm{M})$

Durchfluss (-zähler/ -messgerät)

DGRL

Druckgeräterichtlinie

DRV

Druckreduzierventil/ Druckminderer

Diff.

Differenz

DIN

Deutsches Institut für Normung e. V.

DN

Nenndurchmesser/ -weite

DV

Design-Variable 
EA

EAD

ECOFLEX

EMSR

EN

FD

FE

FEM

FF

FKZ

FSI

Gl(n).

$\mathrm{GmbH}$

GuD

HD

HeiKo

HPC

HTC

hydr.

IET

IFAM

ILR

ISO

Kap.

Korr.

LDA

Leg.

LES

LSF
Evolutionärer Algorithmus

Arbeitsgruppe für Experimentelle Aerodynamik

Turbomaschinen-Schlüsseltechnologien für flexible Kraftwerke und eine er-

folgreiche Energiewende

Elektrische Mess-, Steuer- und Regelungstechnik

Europäische Norm

Frischdampf

finite Elemente

Finite-Elemente-Methoden

Vollausstattung (Full Feature)

Förderkennzeichen

Fluid-Struktur-Kopplung (Fluid-Solid Interaction)

Gleichung(en)

Gesellschaft mit beschränkter Haftung

Gas-und-Dampf

Hochdruck

Heizkondensator

Hochleistungsrechnerinfrastruktur (High-Performance Computing)

Wärmeübergangskoeffizient (Heat Transfer Coefficient)

hydraulisch

Institut für Energietechnik

Fraunhofer-Institut für Fertigungstechnik und Angewandte Materialforschung

Institut für Luft- und Raumfahrt

International Organization for Standardization

Kapitel

Korrelation

Laser-Doppler-Anemometrie

Legierung

Grobstruktursimulation (Large Eddy Simulation)

Approximation nach Methode der kleinsten Fehlerquadrate (Least-square Fit) 
MFD

MSV

NBR

NP

NTC

OptiSysKom

PET

PIV

PLS

PN

Pos.

PTB

PtJ

PTFE

PVC

RANS

RB

RDS-PP

RMS

RSM

RV

SichV

SIMPLE(C)

SiSTeR

SLUB

SPI

SST

SST

SVG
Professur für Magnetofluiddynamik, Mess- und Automatisierungstechnik Motorstellventil

Acrylnitril-Butadien-Kautschuk (Nitrile Butadiene Rubber)

Normalposition

negativer Temperaturkoeffizient (Negative Temperatur Coefficient)

Optimierung der Prozesse und Systeme sowie der Lebensdauer der Gesamtanlage und ihrer Komponenten

Polyethylenterephthalat

Partikelbildgeschwindigkeitsmessung (Particle Image Velocimetry)

Prozessleitsystem

Nenndruck

Position

Physikalisch-Technische Bundesanstalt

Projektträger Jülich

Polytetrafluorethylen

Polyvinylchlorid

(Stationäre) Reynolds-gemittelte Simulation (Reynolds-Avgeraged Navier-Stokes)

Randbedingung

Kraftwerkskennzeichnungssystem (Reference Designation System for Power Plants)

quadratisches Mittel (Root Mean Square)

Reynolds-Stress-Modell

Regelventil

Sicherheitsventil

Semi-Implicit Method for Pressure Linked Equations (consistent)

Totraumversuchsstand (Side Space Test Rig)

Sächsische Landesbibliothek - Staats- und Universitätsbibliothek

Serial Peripheral Interface

Shear Stress Transport

Siemens Dampfturbine (Siemens Steam Turbine)

Sondenverstellgerät 
Tab. Tabelle

TEA Professur für Thermische Energiemaschinen und -anlagen

TRV Totraumversuchsstand

TU Technische Universität

TVP Teilverbundprojekt

URANS (Instationäre) Reynolds-gemittelte Simulation (Unsteady RANS)

URF Unterrelaxationsfaktor

VBA Visual Basic for Application

VDI Verein Deutscher Ingenieure

VGB Vereinigung der Großkesselbesitzer

VGV Variables Drallgitter (Variable Guide Vane)

VSD Drehzahlregelung (Variable Speed Drive)

WALE Wall-Adapting Local Eddy-Viscosity

WÜ Wärmeübergang

WÜK Wärmeübergangskoeffizient

ZET Zentrum für Energietechnik

ZIH Zentrum für Informationsdienste und Hochleistungsrechnen

ZP

Zwischenposition 



\section{EINLEITUNG UND MOTIVATION}

Seit ihrer Erfindung im Jahre 1884 durch den britischen Ingenieur Sir Charles Algernon Parsons $[1,2]$ hat die axial durchströmte, mehrstufige Reaktionsdampfturbine bis heutig stetig an ihrer Bedeutung als thermische Turbokraftmaschine gewonnen. In ihrer über 130-jährigen Geschichte hat sich dabei ihr Anwendungsgebiet - nicht zuletzt wegen der universellen Bereitstellung ihres Arbeitsmediums aus vielfältigen verschiedenen Energieträgern - noch deutlich vergrößert. Neben ihrem klassischen Einsatzgebiet als Heavy-Duty-Maschinen in Schwerbauweise mit größten Leistungseinheiten bis zu 1900 MW (Siemens SST5-9000, [3]), wie sie etwa zur elektrischen Energieversorgung in fossilen (Kohle, Öl, Gas) und nuklearen (Kernenergie) Großkraftwerken als Anlagenbestandteil von Dampfturbinenanlagen (zum Beispiel in Verbindung mit der Kraft-WärmeKopplung in Gas-und-Dampf- (GuD-) Kombi-/ Heizkraftwerken) zum Einsatz kommen, haben sich Dampfturbinen heute auch in kleineren Leistungseinheiten zur Energiebereitstellung aus alternativen (Abfallverbrennungsanlagen) und regenerativen Energiequellen (Geothermie-, Biomasse-, solarthermische Kraftwerke) etabliert. Auch für Industrieanwendungen, bei denen Abdampf entsteht, Prozess- oder Heizdampf mit bestimmten Parametern (Druck, Temperatur) benötigt wird oder Arbeitsmaschinen angetrieben werden müssen, stellen heute kleinere, maßgeschneiderte Industriedampfturbinen eine sinnvolle Alternative dar, um Energie und Kosten zu sparen. Typische Abnehmer sind hierbei etwa die Grundstoff- und Schwerindustrie (Bergbau, Verhüttung, Eisen- und Stahlwerke, Metallindustrie), die (petro-) chemische Industrie (Erdöl- und Erdgasveredlung, Raffinerien), die Verfahrenstechnik (Lebensmittelindustrie; Holzverarbeitung, Papier- und Zellstoffindustrie; Zementwerke), Meerwasserentsalzungsanlagen sowie kleine und größere, private wie regionale Stromversorger [3].

Im Gegensatz zu den Großkraftwerksturbinen liegen bei Industriedampfturbinen die primären Entwicklungsziele nicht ausschließlich in der Steigerung der Effizienz (höhere Volllastwirkungsgrade) bei gleichbleibend hohen Einheitenleistungen und langen Lebensdauern. Auf Grund ihres variablen Einsatzes kommen noch zusätzliche Anforderungen hinsichtlich der Last- und Betriebsflexibilität hinzu, die sich in Zeiten volatiler Energieeinspeisung im Zuge der Energiewende in Deutschland noch deutlich verschärfen ( Abb. 1.1). Dazu zählt - neben dem beschleunigten Anund Abfahren sowie schnellen Lastwechseln - vor allem die Realisierung variabler Eintrittsparameter, Einspeise-, Anzapf- bzw. Entnahmedampfmengen zur Abdeckung des aktuellen Wärme-, Leistungs- und Drehzahlbedarfs bei noch akzeptablen Teillastwirkungsgraden. 


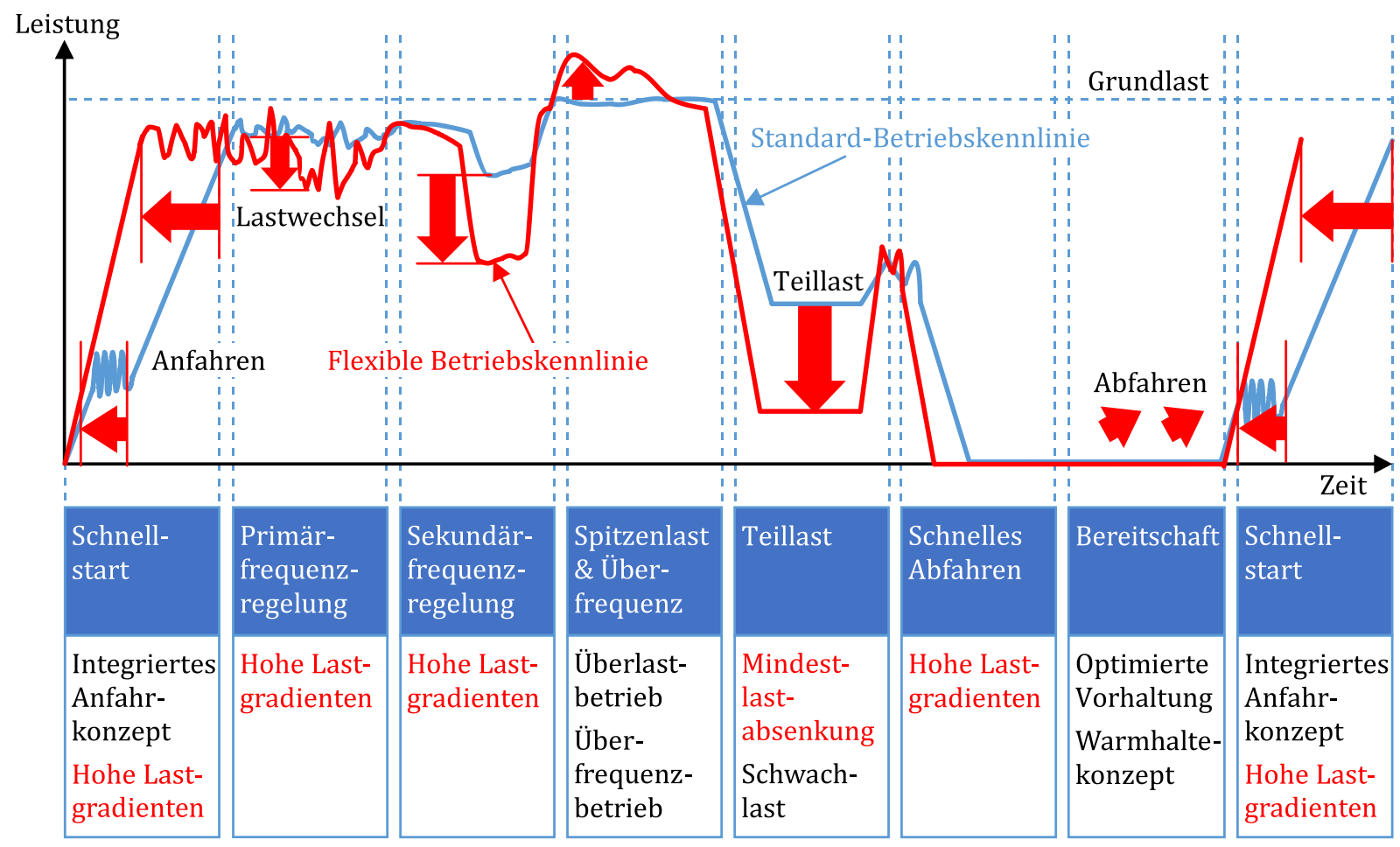

Abb. 1.1 Veränderte Anforderungen an die lastflexible Fahrweise von Dampfturbinenanlagen [4].

Dies ist der Grund dafür, weshalb Industriedampfturbinen stets auf ihren Anwendungsfall abgestimmt und auf die Bedürfnisse des Kunden angepasst werden müssen. Das hat Auswirkungen auf den gesamten Entwicklungsprozess, beginnt bereits in der Angebotsphase und zieht sich über Projektierung und Konstruktion bis hin zur Optimierung für den nächsten Produktlebenszyklus. Entsprechend unterscheiden sich die ausgelieferten Maschinen hinsichtlich Aufbau, Größe, Lage und Anzahl von Einbauten wie Anzapfungen oder Entnahmen sehr stark voneinander.

Um die Entwicklungs- und Herstellungskosten dennoch in einem vertretbaren Rahmen zu halten, kommen Baukastensysteme zum Einsatz. Dabei wird auf vorentwickelte modularisierte und standardisierte Baureihen zurückgegriffen, bei denen die einzelnen Module je nach Anwendungsfall zusammengestellt werden [5]. Die Siemens AG bietet beispielsweise neun verschiedene Grundtypen von Industriedampfturbinen (SST-200 bis SST-900) mit einem Leistungsspektrum von 2 bis $250 \mathrm{MW}$ bei Drehzahlen zwischen 3000 und $20.000 \mathrm{~min}^{-1}$ und Frischdampfparametern von 30 bis 180 bar bzw. 400 bis $565^{\circ} \mathrm{C}$ [3] an. Diese können sowohl als Kondensations- als auch als Gegendruckturbinen ausgeführt sein und Dampfzuführungen, -entnahmen (geregelt) und -anzapfungen (ungeregelt) bei verschiedenen Zuständen (Drücken und Temperaturen) besitzen.

Abb. 1.2 zeigt den Viertelschnitt durch eine Industriedampfturbine vom Typ Siemens SST-600 in der Ausführung einer einflutigen Entnahme-Gegendruckturbine. 
Wegen ihrer kompakten, zumeist eingehäusigen Ausführung mit druckbedingt großen Gehäusewanddicken sind Industriedampfturbinen hohen thermomechanischen Belastungen, Beanspruchungen und Verformungen ausgesetzt, zu denen es immer dann kommt, wenn die Temperaturen zwischen und innerhalb von Bauteilen unterschiedlich sind. Sie sind also unvermeidbar, dürfen jedoch zu keinem Zeitpunkt die zulässigen konstruktions- und werkstoffabhängigen Grenzen überschreiten, die die Betriebssicherheit gefährden könnten.

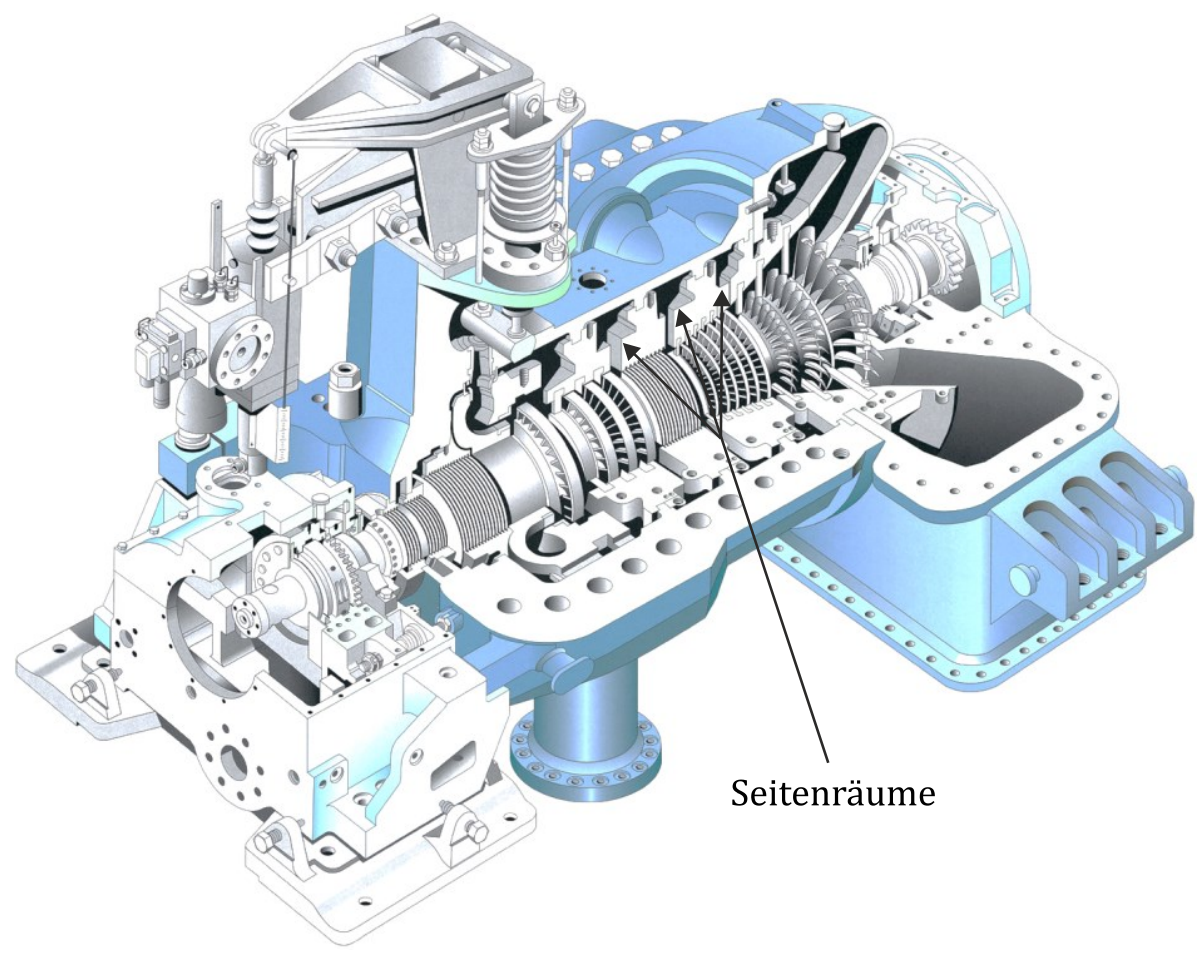

Abb. 1.2 Viertelschnittdarstellung einer Industriedampfturbine vom Typ Siemens SST-600 [6].

Große lokale Temperaturgradienten, die vor allem in Betriebsphasen mit instationären Dampfparametern auftreten, führen besonders an Stellen mit starker Abweichung von der Rotationssymmetrie zu Gehäuseverkrümmungen (vgl. $>$ Abb. 1.3), die sich zum einen negativ auf die Teilfugendichtheit auswirken und zum anderen Einfluss auf die Axial- und Radialspiele zwischen Rotor und Stator haben können. Bei zu kleinen Spielen entstehen Anstreifschäden, die Beschädigungen an den schleifenden Teilen nach sich ziehen. Die Bauteillebensdauer wird dabei stark beeinträchtigt, sodass durch Reparaturen häufigere Revisionsintervalle notwendig werden, die mit ungewünschten Stillstandzeiten einhergehen. Zu große Spalte wiederum erhöhen die Leckageverluste in den Stufen, die sich in der Verminderung des inneren Turbinenwirkungsgrades niederschlagen.

Werden thermische Verformungen (Aufweitungen, Dehnungen, Verzerrungen) behindert, so können die resultierenden Spannungen zu Werkstoffermüdung, Rissausbildung bis hin zum Bauteilversagen führen. 

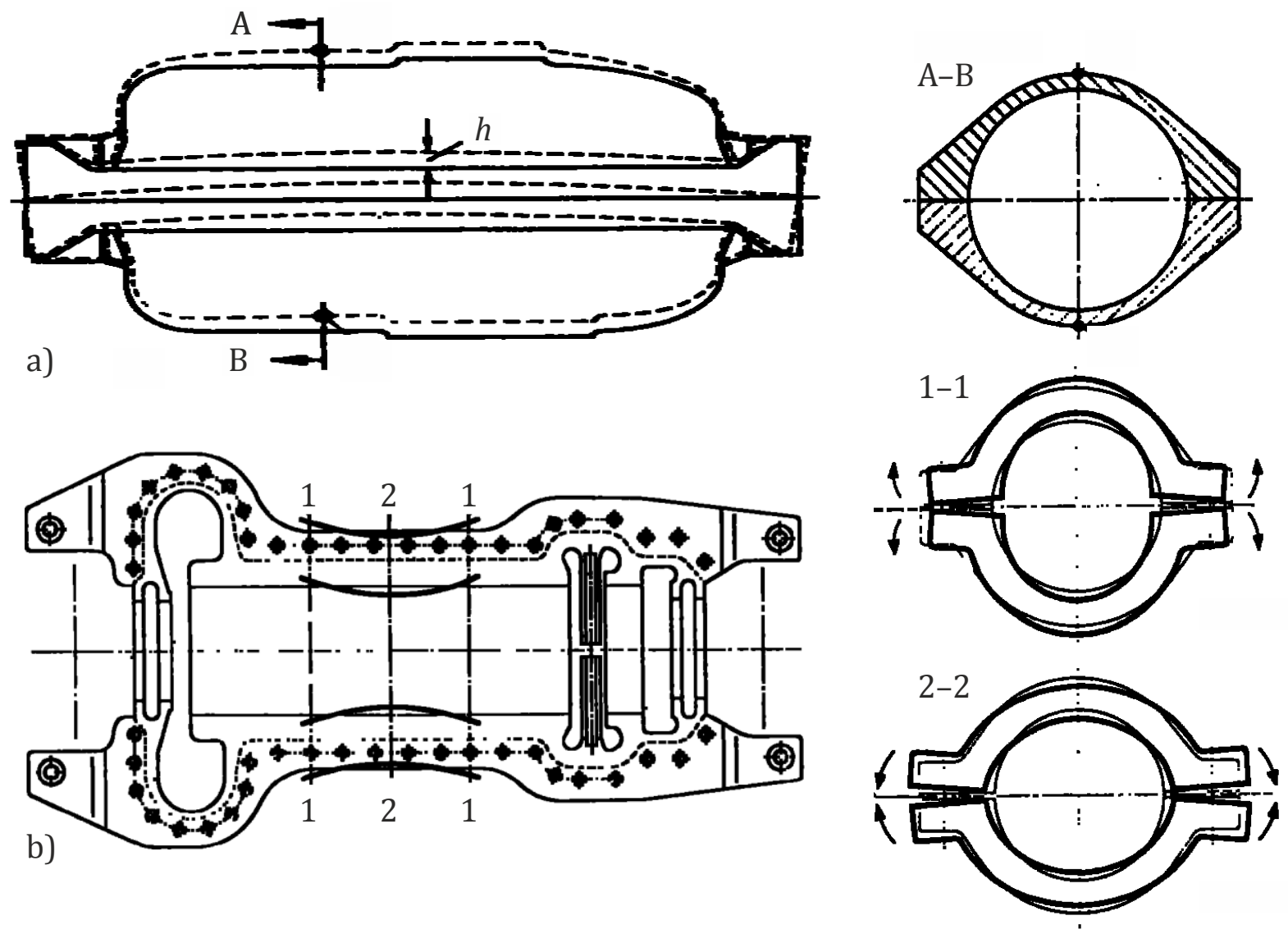

Abb. 1.3 Typische thermische Verformungen an Dampfturbinengehäusen [7].

a) Gehäuseverkrümmung ohne Querschnittsdeformation („Katzbuckeln“);

b) Ovalitätsverformungen von Gehäusequerschnitten („Unrundwerden“).

Um all diese Effekte bei der Auslegung und beim lastflexiblen Betrieb der Industriedampfturbinen zu berücksichtigen, müssen für jede einzelne Maschine die Temperaturfelder und das daraus resultierende thermische Spannungs- und Verformungsverhalten verlässlich vorausbestimmt werden. Dazu kommen heute in der Regel numerische Simulationen auf Basis der Finite-ElementeMethode (FEM) zum Einsatz. Während der Angebotsphase, in der noch kein finales Gehäusemodell vorliegt, können mithilfe semianalytischer Verfahren auf Basis eines Stufenkörpermodells [7] thermische Gehäuseverkrümmungen schnell und mit geringem Rechenaufwand berechnet werden. Um möglichst realitätsnahe Ergebnisse abbilden zu können, ist in beiden Fällen - neben der genauen Kenntnis der Geometrie und der zeitlich veränderlichen Fluidparameter - auch das Wissen um die thermischen Randbedingungen in Gestalt des Wärmeübergangsverhaltens zwischen Fluid und Gehäuse von entscheidender Bedeutung. In der Praxis werden je nach Geometrie der wärmeübertragenden Oberfläche verschiedene Nusselt-Korrelationen, empirische Größengleichungen oder konstante Erfahrungs- bzw. Richtwerte angesetzt. Einzelnachrechnungen, bei denen in einem iterativen Prozess die Wärmeübergangsbedingungen im Modell solange angepasst 
werden, bis simulierte mit punktuell unter Prüfstandbedingungen gemessenen Gehäusetemperaturen und -verschiebungen an der Referenzturbine hinreichend gut übereinstimmen, zeigen, dass die iterierten Wärmeübergangskoeffizienten vor allem in den nicht direkt oder nur gering durchströmten Bereichen und bei höheren Abdampfparametern (Druck und Temperatur) teilweise recht deutlich von den anfangs angenommenen, dem Wissensstand entnommenen Wärmeübergangskoeffizienten abweichen [7] - also dort, wo die Strömungssituation zu stark von den zugrunde gelegten Korrelationen (z. B. für Rohr-, Ringspalt- oder Plattenüberströmung) abweichen. Neben Einströmkasten, Radkammer und Abdampfgehäuse tritt dieser Effekt in besonderem Maße in den Seitenräumen (vgl. A Abb. 1.2) zwischen den Leitgitterträgern („Trommeln“) und dem Außengehäuse auf. Diese Toträume entstehen, da auch die Leitgitterträger nach dem Baukastenprinzip aus einem Pool an vorkonstruierten Teilen ausgewählt, angepasst und in das ebenfalls modularisierte Gehäuse eingesetzt werden. Die Einbaulängen können somit variieren.

Zwischen der Ein- und der Ausgangsseite der in das Gehäuse eingehängten Leitgitterträger herrschen auf Grund der Entspannung des Dampfes in den zugehörigen Laufschaufelreihen hohe Druckdifferenzen, weshalb die Anzahl der Stufen eines Leitgitters und damit auch seine Länge begrenzt sind, um die Flanschverbindung dicht zu halten und Leckageströme zu verhindern. Um einen Ausgleich für die thermische Dehnung der Struktur gewährleisten zu können, werden zudem Abstände zwischen den Leitgitterträgern gelassen (wärmeelastische Bauweise). Soll in den Seitenräumen bei Bedarf zusätzlich die Möglichkeit bestehen, Dampf zu entnehmen oder einzuspeisen, müssen die Spalte entsprechend größer vorgesehen werden.

Durch die äußere Hauptströmung im Schaufelkanal wird das Fluid in diesen T- oder L-förmigen Umfangskavitäten angetrieben. Die sich ausbildenden, mehrdimensionalen Wirbelstrukturen werden durch Dampfentnahmen oder -einkopplungen zusätzlich beeinflusst (Abb. 1.4) und bestimmen das Wärmeübergangsverhalten zwischen Fluid und Außenwand maßgeblich. Während sowohl freie Konvektion als auch Kondensation weitgehend modellierbar sind, existieren erhebliche Defizite im Wissenstand zur einphasigen erzwungenen Konvektion in den Seitenräumen [7], die einen großen Teil der Innenoberfläche des Gehäusemantels überdecken und damit dessen Verformungsverhalten maßgeblich mitbestimmen. 


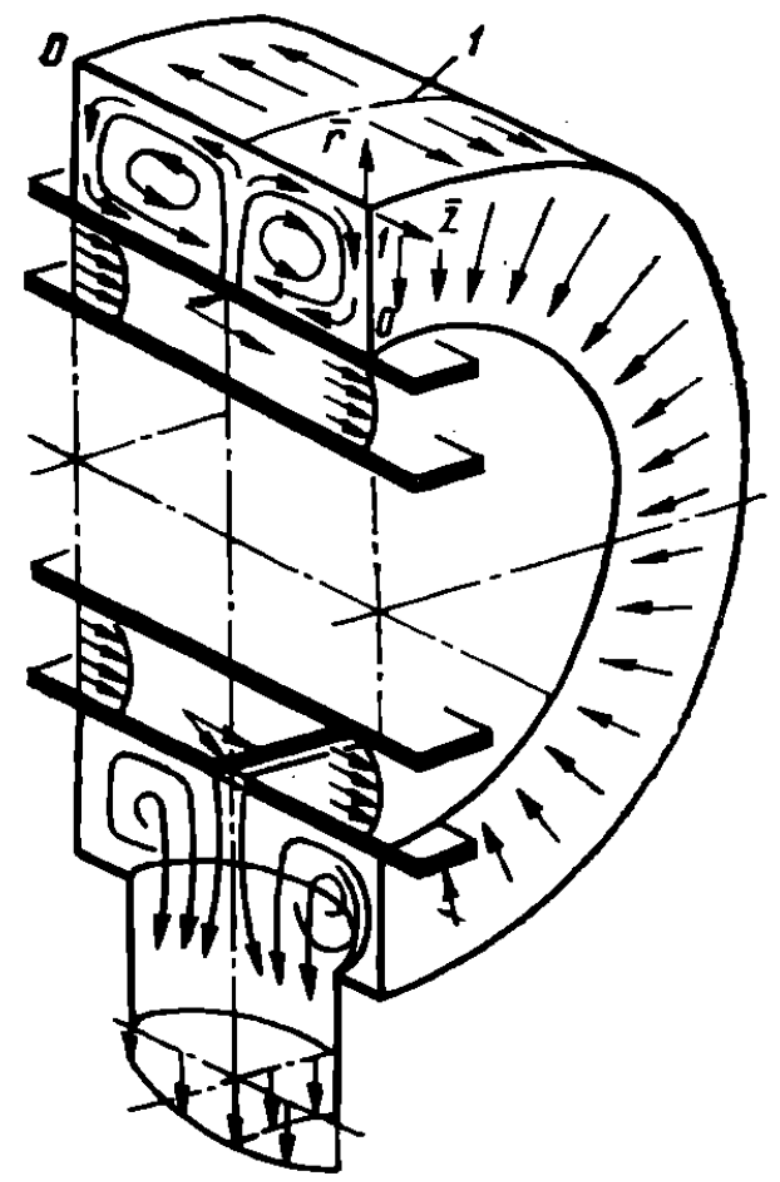

Abb. 1.4 Schematische Strömungsstruktur in einem Seitenraum mit Anzapfung [8]. 


\section{STAND DES WISSENS}

\subsection{STRÖMUNG UND WÄRMEÜBERGANG IN SEITENRÄUMEN VON DAMPFTURBINEN}

Während der 1970er- und 1980er-Jahre sind vor allem im russischsprachigen Raum zahlreiche Untersuchungen zur Thermofluiddynamik in Seitenräumen von Dampfturbinengehäusen, zumeist mit Anzapfung oder Entnahme, durchgeführt worden. Einen umfassenden Überblick über die Arbeiten auf dem Gebiet bietet [8]. Im Folgenden sollen die wesentlichen Erkenntnisse und Ergebnisse der Sowjetforschung zusammengefasst werden:

Häufigste Grundlage für die Rückwärtsberechnung von Wärmeübergangskoeffizienten in Anzapfkammern bildeten Temperaturmessungen an ausgeführten Dampfturbinen russischen Typs (u. a. K-200-130, K-300-240, K-500-60/150, VK-100-2, VK-100-6, VPT-50-3) beim Anfahren. Neben numerischen Methoden kamen hierbei auch analytische Ansätze zur Anwendung, beispielsweise Wärmebilanzmethoden [9,10], bei denen die Gehäuse als Wärmespeicher $(B i \leq 2$, Dicke $H$, Radius $R$ ) modelliert und unter Annahme quasistationärer Wärmeleitung die gespeicherte mit der konvektiv eingetragenen Wärme gleichsetzt wird. Es ergibt sich dann für den über der gesamten Fläche als konstant angenommenen WÜK:

$$
\alpha=\frac{\rho c H}{\left(T_{\mathrm{F}}-T_{\mathrm{W}, \mathrm{i}}\right) \cdot \Delta t} k_{r} k_{\mathrm{z}} k_{\delta}
$$

mit den Korrekturfaktoren für die radiale $(r)$ bzw. axiale $(z)$ Temperaturverteilung

$$
k_{r}=1+\frac{H}{2 R}, \quad k_{z}=1-a \cdot \frac{\partial^{2} T}{\partial z^{2}} \cdot\left(\frac{\partial T}{\partial t}\right)^{-1}
$$

sowie für den Abstand $\delta$ der Wandtemperaturmessung von der Innenoberfläche

$$
k_{\delta}=\left[1-\frac{T_{\mathrm{W}, \mathrm{i}}-T_{\mathrm{W}, \mathrm{a}}}{T_{\mathrm{F}}-T_{\mathrm{W}, \mathrm{i}}} \cdot \frac{2 \delta H-\delta^{2}}{(H-\delta)^{2}}\right]^{-1}
$$

Auf diese Weise wurde die Abhängigkeit des WÜK in ausgewählten Anzapfkammern (камерах отбора) mit und ohne Anzapfung von der Nennlast $P_{\mathrm{N}}$ [10] bzw. von der relativen Turbinenleistung $\bar{N}=P / P_{\mathrm{N}}[9]$ ermittelt und in empirische Korrelationen der Form $\alpha=A+B \cdot \bar{N}^{C}(C \leq 1)$ gebracht ( Abb. 2.1). 

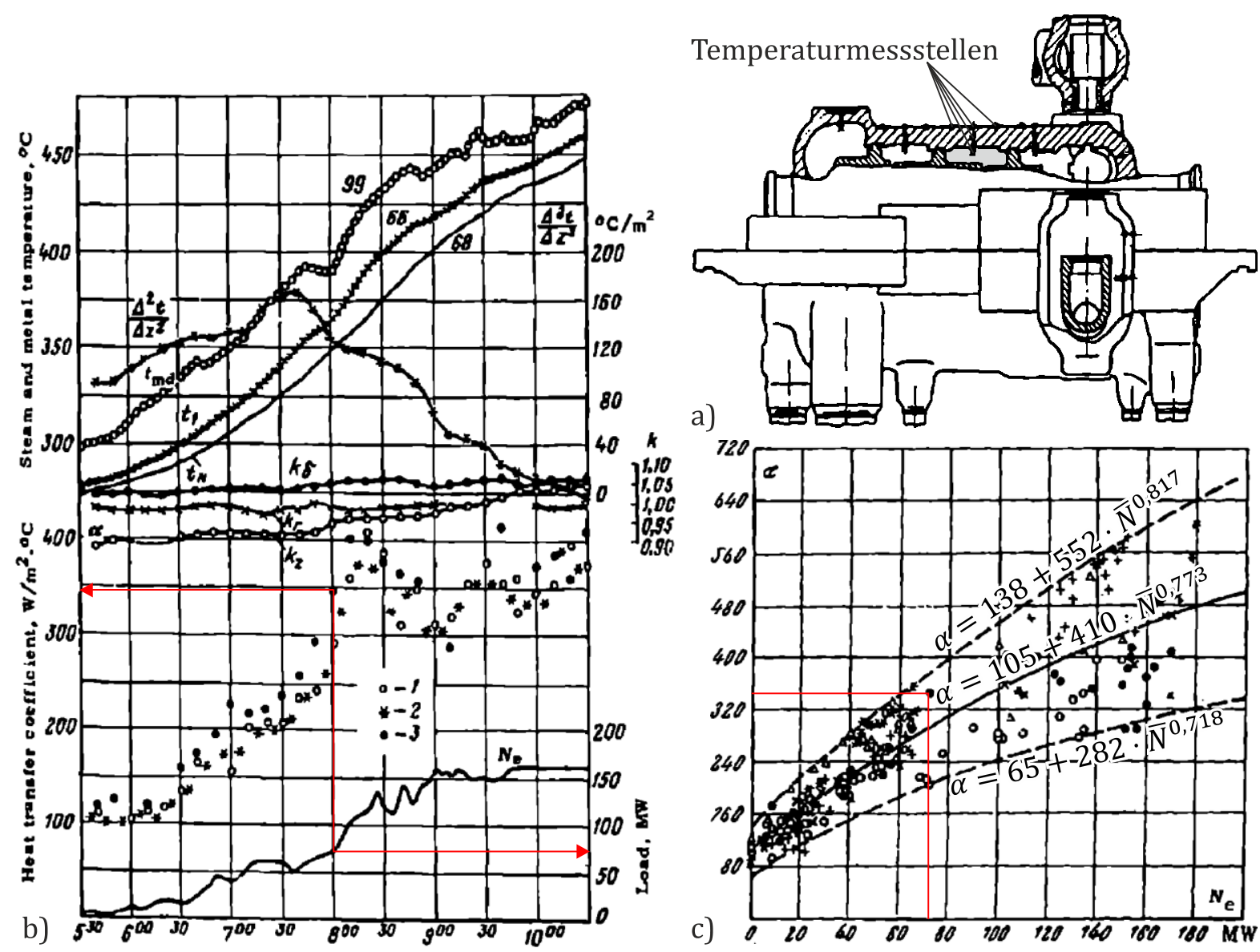

Abb. 2.1 Berechnung der Wärmeübergangskoeffizienten in einer Dampfkammer ohne Anzapfung im Gehäuse einer HD-Turbine vom Typ K-200-130 während des Startvorganges in Abhängigkeit von der Leistung [9]; a) Lage der Kammer und der Temperaturmessstellen; b) zeitlicher Verlauf der gemessenen Temperaturen, der Turbinenleistung und der WÜK; c) WÜK in Korrelation zur Turbinenleistung;

1,2 - Numerische Berechnung; 3 - Berechnung nach Wärmebilanzmethode.

Unter Anwendung mehrerer über das Gehäuse verteilter Temperaturmessstellen konnten mit dieser Methode auch lokale Werte des WÜK in ausgeführten Dampfkammern aufgelöst werden: In $[8,11]$ ist beispielsweise der Einfluss des Betriebszustandes in Form des Kammerdruckes auf den WÜK in den verschiedenen Viertelsektoren um den Umfang einer Anzapfkammer mit und ohne Dampfanzapfung für einen Speisewasservorwärmer und mit Verengung durch den Teilfugenflansch am Innengehäuse angegeben.

Als Grundlage für weiterführende Betrachtungen zum Wärmeübergang sind weiterhin Untersuchungen zur Strömung in Seitenräumen durchgeführt worden, und zwar nicht nur an ausgeführten Maschinen wie bei [11] in Entnahmekammern und den zugehörigen Einströmspalten. Auch Versuchsstände kamen zum Einsatz. Mit zwei verschiedenen druckluftbetriebenen Apparaturen sind in [11] (Abb. 2.2) und wenig später in [8,12,13] ( Abb. 2.3) unabhängig voneinander Strömungsprofile und -strukturen in axialsymmetrischen Seitenraummodellen mit Fluidentnahme bei unterschiedlichem Drall in der kreisringspaltförmigen Hauptströmung aufgezeichnet worden [14]. 


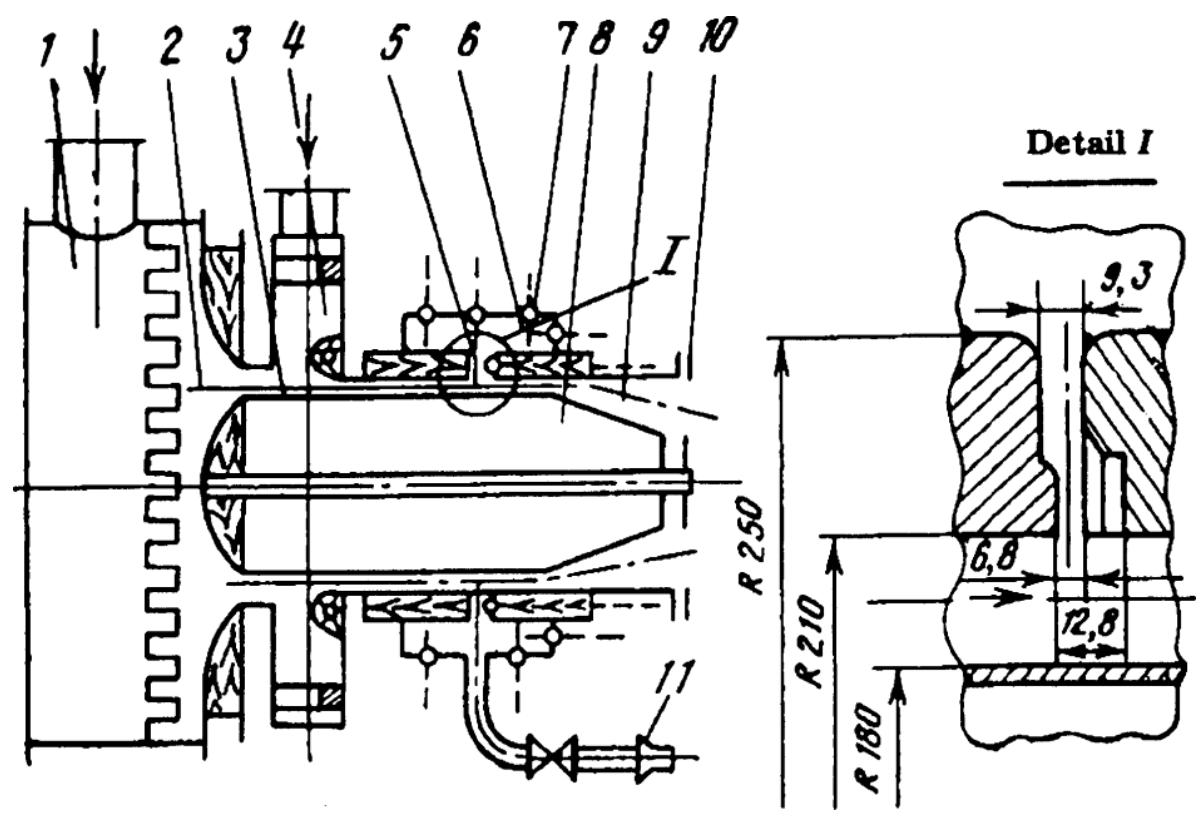

Abb. 2.2 Versuchsstand zur Untersuchung der Gasdynamik in Entnahmekammern [11]. 1 - Beruhigungskammer; 2 - Einlauf; 3 - Ringkanal; 4 - Wirbelkammer; 5 - Ringspalt;

6 - Entnahmekammer; 7 - Messpunkte für Sonden und statische Druckmessungen (gestrichelte Linie - Sondenachse, sechs Punkte gleichmäßig um den Umfang verteilt in jeder Traverse); 8 - Verdränger; 9 - Auslass; 10 - Leiteinrichtung; 11 - Messdüse.

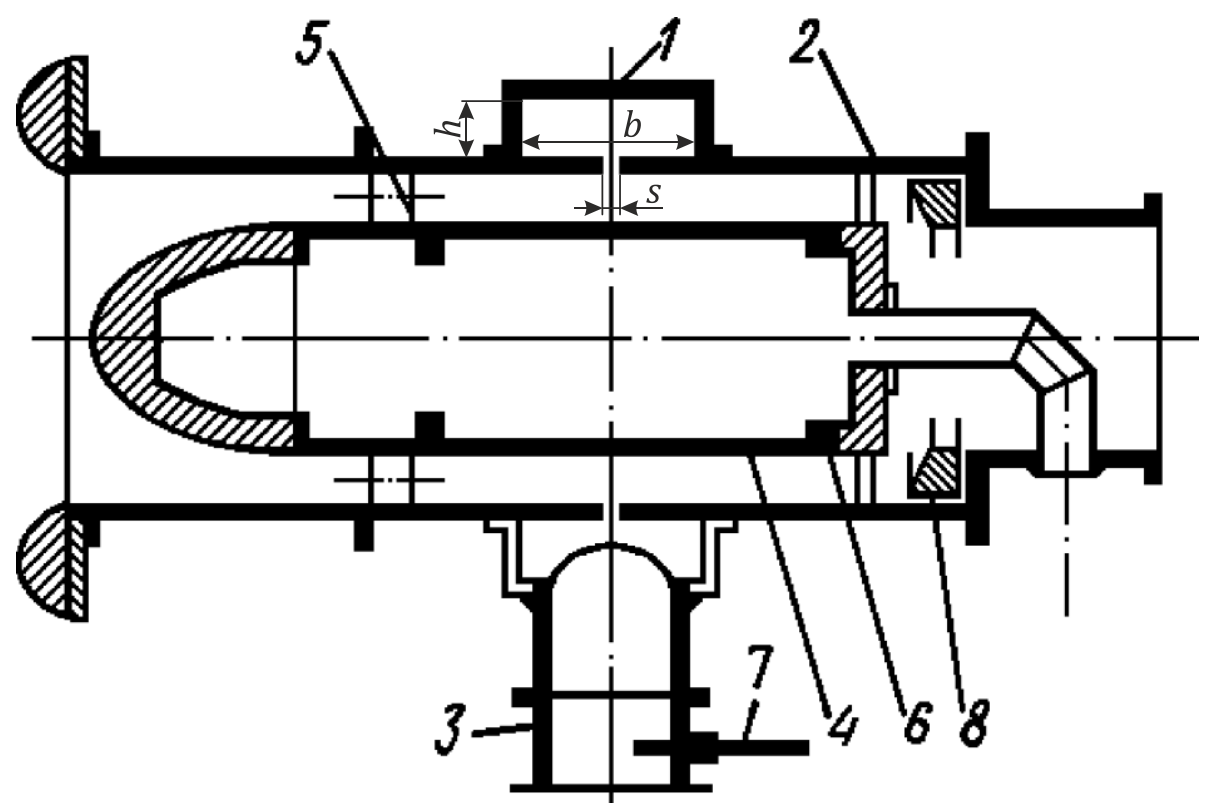

Abb. 2.3 Experimenteller Aufbau zur Untersuchung von Strömung und Wärmeübertragung in einer Entnahmekammer und im nachfolgenden Strömungskanal $[8,12]$.

1 - Entnahmekammer (axial und am Umfang verschiebbar; transparent, $h / b=1: 3, s / h=1: 8$ );

2 - Gehäuse; 3 - Entnahmestutzen; 4 - Ringkanal; 5 - Leitgitter ( $\alpha=30 ; 50 ; 70^{\circ}$ );

6 - Austauschbare Ringe zur Variation der Breite des Einströmspaltes in die Kammer;

7 - Klappe zur Regelung des Entnahmemassenstromes; 8 - konischer Ring (axial verschiebbar) zur Einstellung des Durchsatzes durch den Strömungsteil. 
Separat dazu wurden in einer flachen Versuchsanordnung ( Abb. 2.4) die Strömungsparameter entlang der Ausbreitungsachse eines Prallstrahls erforscht, der durch Überströmung eines Spaltes entsteht und an eine parallel zur Hauptströmung hinter dem Spalt angeordneten Wand aufprallt. Der Spalt modelliert hierbei die Einströmöffnung zum Seitenraum. Die Wand, auf der der Strahl auftrifft, stellt die Außenwand der Kammer dar. Bestimmt wurde die Abhängigkeit der Druckverteilung im Strahl von der Überströmgeschwindigkeit $v$, der Strahllänge $h$ zwischen Öffnung und Wand sowie der Anfangsbreite $b_{0}$ des Strahls $[8,15]$. Der Einfluss der Form der Einströmöffnung zum Seitenraum sowie deren Anstellwinkel zum Hauptströmungskanal auf das Strömungsprofil des Strahls sowie auf die Ausbildung von Rezirkulationsgebieten in der Einströmöffnung werden in [8] diskutiert.

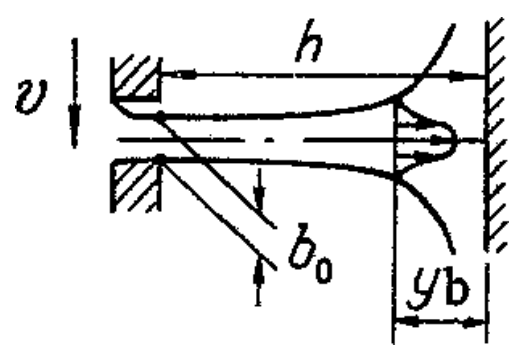

Abb. 2.4 Prallstrahlanordnung an einer parallel zu einem überströmten Spalt versetzen Wand $[8,15]$.

Darauf aufbauend wurde erstmals auch eine Nusselt-Korrelation für die axiale und periphere WÜK-Verteilung in einer Anzapfkammer einer Dampfturbine vom Typ K-300-240 aus Temperaturmessdaten entwickelt, bei der die Abmessungsverhältnisse der Kammer, die Reynolds-Zahl, die axiale und radiale Position und der Drallwinkel des Wandstrahls Berücksichtigung finden $[8,16,17]$. Die Korrelationen bilden laut Autoren die experimentellen Werte mit einer mittleren Abweichung von $\pm 6 \%$ ab und sind im Anhang A1 zusammengetragen. Der Algorithmus wurde auch auf zwei Kammern einer Dampfturbine vom Typ K-500-240 PO HTZ während eines Anfahrvorganges angewandt und mit den Ergebnissen einer Rückwärtsrechnung basierend auf Temperaturmessungen verglichen. Für die Hochdruckkammer sind die so ermittelten lokalen WÜK an allen Seitenraumoberflächen bei verschiedenen Lastpunkten in Abb. 2.5 dargestellt. Es wurde festgestellt, dass zur Berechnung des Temperaturfeldes für Dampfturbinenbauteile mit hoher Wärmekapazität und hoher Wärmeleitfähigkeit, wozu auch Gehäuse zählen, instationäre Wärmeübergangsprozesse nur in der sehr kurzen Phase zu Beginn des Anfahrens auftreten, in denen die Wandtemperaturen praktisch noch unverändert bleiben. Ansonsten kann mit quasistationären WÜK, wie sie die angegebenen empirischen Korrelationen liefern, gerechnet werden [8]. 


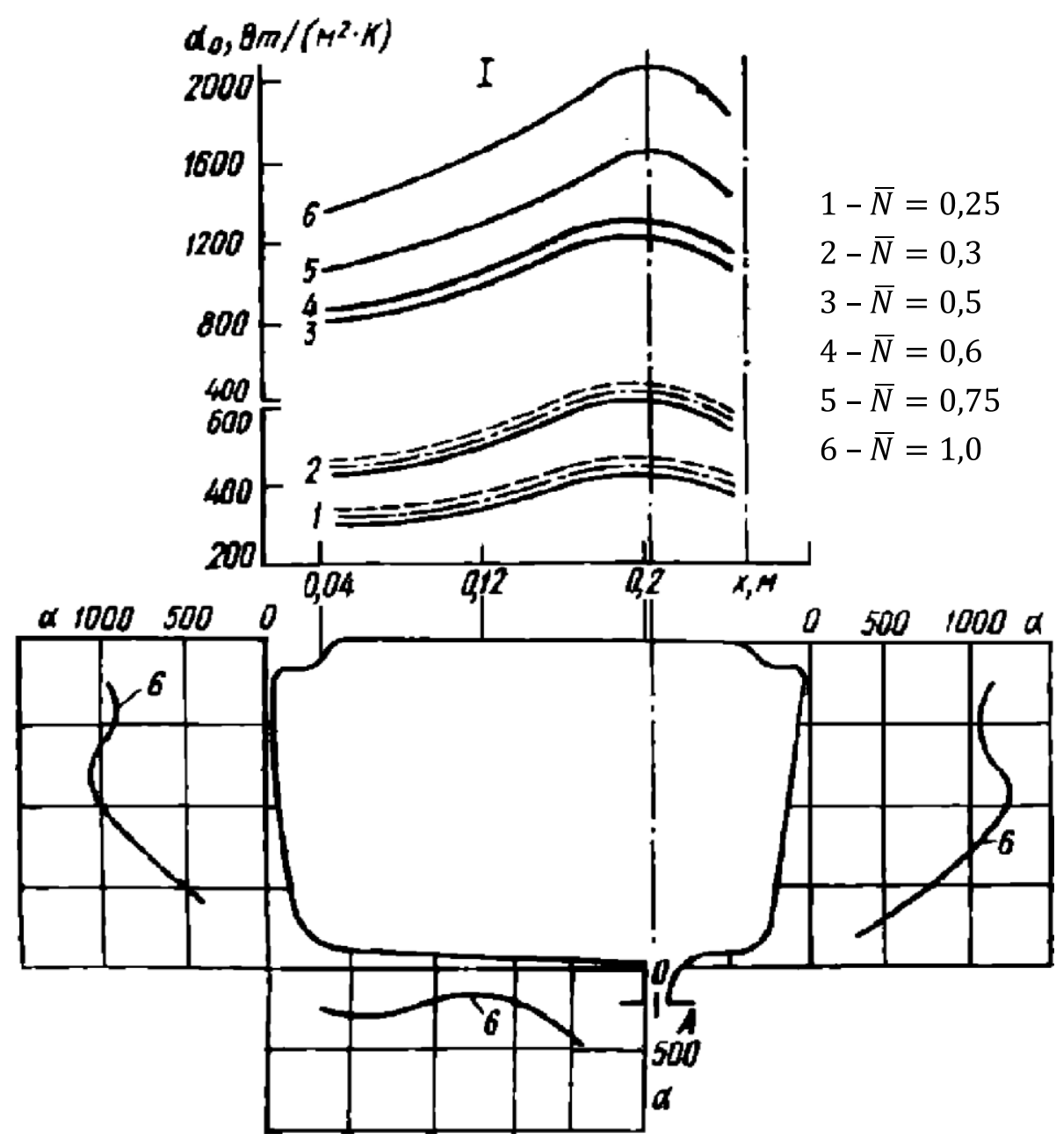

Abb. 2.5 Verteilung der Wärmeübergangskoeffizienten an den Oberflächen der Anzapfkammer I einer russischen Turbine vom Typ K-500-240 PO HTZ in verschiedenen Lastpunkten beim Anfahren [8,16]. Modifizierte Darstellung mit nach außen geklappten Diagrammen für die WÜKVerteilung an den Seitenwänden zur besseren Verständlichkeit. $[\alpha]=\mathrm{W} /\left(\mathrm{m}^{2} \mathrm{~K}\right) ; \bar{N}=P / P_{\mathrm{N}}$.

In $[18,19]$ wird (anstelle von Gleichung A.6) eine glockenförmige Verteilung des axialen Wärmeübergangskoeffizienten in einer Kammer ohne Anzapfung („Totraum“) vorgeschlagen:

$$
\alpha(x)=\alpha_{0} e^{-b \tilde{x}^{c}}
$$

mit dem maximalen WÜK $\alpha_{0}$ im Staupunkt des Prallstrahls an der hinteren Wand und dem doppelten dimensionslosen Abstand $\tilde{x}=2 x / b_{0}$ vom Staupunkt bezogen auf die Anfangsbreite $b_{0}$ des Prallstrahls. Die Größe der experimentell zu bestimmenden Konstanten $b$ und $c$ ist nicht dokumentiert. Auf Basis der empirischen Korrelationen, wie Malyarenko, Barsukov et al. sie veröffentlicht haben $[8,16,17]$, haben Goloshchapov und Mitarbeiter [20,21] daraus Gleichungen zur Bestimmung des (vom Staupunkt des Prallstrahls aus) über der „Lauflänge“ $L$ gemittelten Wärmeübergangskoeffizientenverlaufs

$$
\bar{\alpha}(L)=\frac{1}{L} \int_{0}^{L} \alpha(x) d x
$$


an der Außenoberfläche einer Anzapfkammer (Abb. 2.6) gebildet, die sie hierzu in zwei axiale Sektoren untergliedern: einen in unmittelbarer Umgebung der Stauströmung (I) und einen weiteren im Bereich der Ecken der Kammer (II), in dem wandparallele Strömung vorherrscht. Des Weiteren wird auch eine periphere Verteilung der über der gesamten axialen Breite gemittelten WÜK präsentiert, und zwar ausgehend vom „neutralen“ Querschnitt $\left(c_{\mathrm{u}}=0\right)$ bis hin zur gegenüberliegenden Anzapfung. Darüber hinaus wurden Korrekturfaktoren angegeben, mit denen der Einfluss von Drall in der Strömung sowie der Verengung des Umfangsquerschnittes der Kammer durch Teilfugenflansche auf die axialen bzw. Kammermittelwerte berücksichtigt werden können. Alle Korrelationen hierzu sind in Anhang A2 hinterlegt.

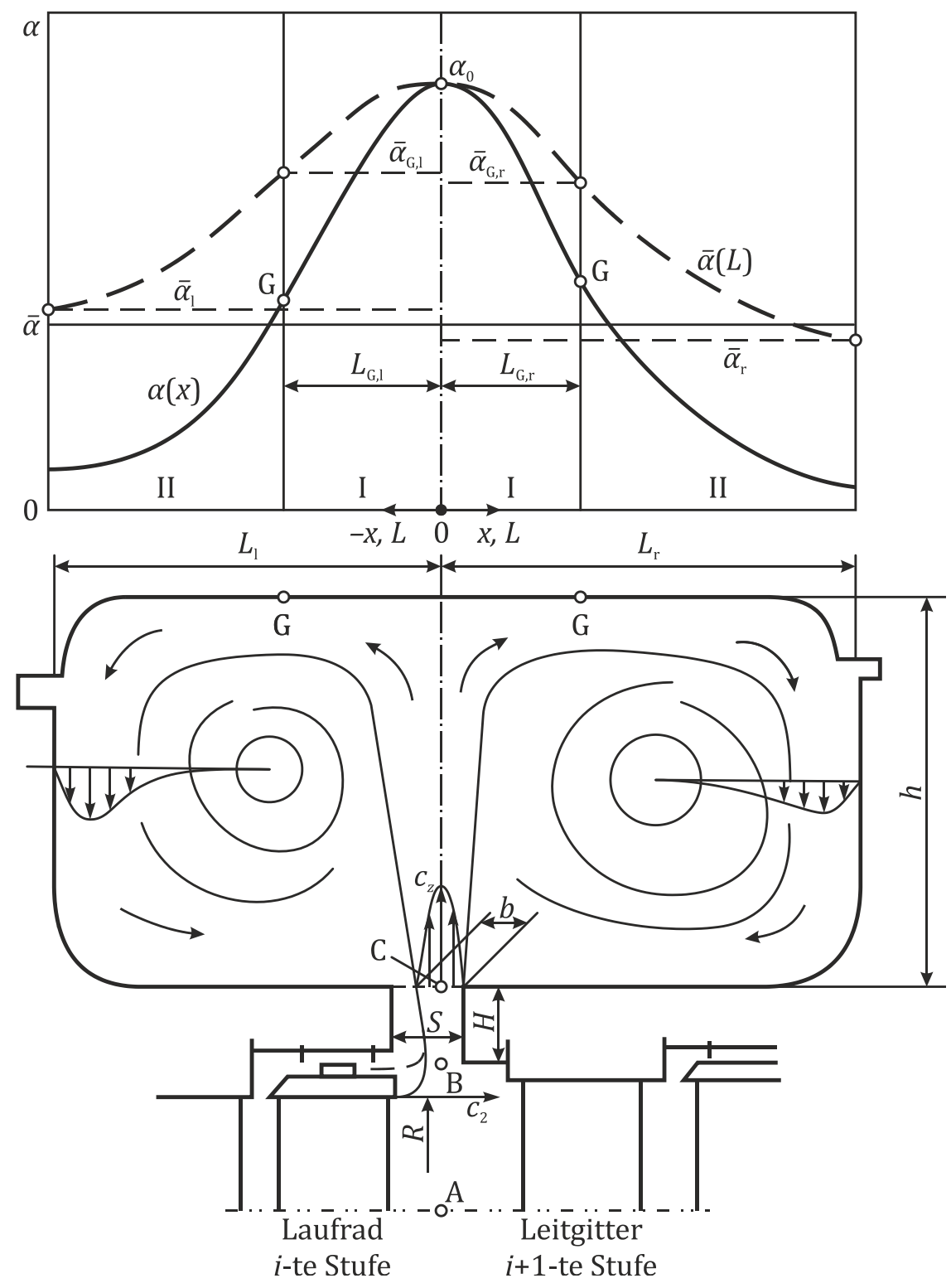

Abb. 2.6 Verteilung der lokalen und vom Staupunkt des Prallstrahls aus gemittelten Wärmeübergangskoeffizienten an der Außenoberfläche einer Anzapfkammer [20,21]. Darstellung digitalisiert und transkribiert sowie erweitert durch Graphen für mittlere WÜK $\bar{\alpha}(L)$. 
Eine alternative Herangehensweise besteht in der Ableitung lokaler WÜK von der Kinematik der Sekundärwirbel im Seitenraum [8,17] unter Zuhilfenahme eines Grenzschichtansatzes für eine längsüberströmte Wand nach $[22,23]$. Diese geht von einer quadratischen Geschwindigkeitsverteilung in der Grenzschicht entlang der Strahlausbreitungsrichtung zwischen Staupunkt und der Ecke des Seitenraumes aus (Abb. 2.7). Die Sekundärwirbel werden dabei als Starrkörperwirbel betrachtet. Im Gegensatz zu den oben vorgestellten Methoden ermöglicht dieser Ansatz nicht nur die Betrachtung der Außenwand. Er gestattet ferner auch die Berechnung lokaler WÜK für die Seitenwände sowie für die Innenoberflächen des Seitenraumes ( Abb. 2.5). Der Algorithmus berücksichtigt außerdem die Transition zwischen laminarer und turbulenter Grenzschicht und unterscheidet Regionen beschleunigter und verzögerter Wandströmung ( Anhang A3). In den äußersten Ecken werden auf Grund der Eckwirbel (Tertiärwirbel) erhöhte WÜK berechnet.

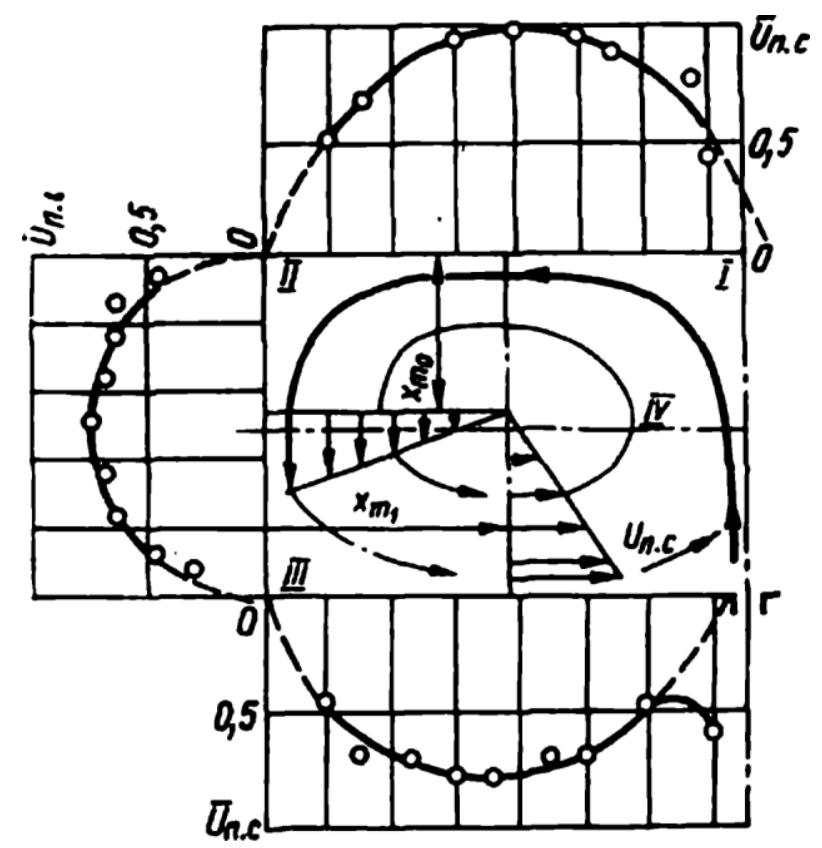

Abb. 2.7 Strömungsschema des Sekundärwirbels in der linken Hälfte eines Seitenraumes sowie gemessene und approximierte Geschwindigkeitsverteilung in der Grenzschicht [8,17].

Mittels eines sattdampfbetriebenen Versuchsstandes ( Abb. 2.8) wurden erstmalig auch Wärmeübergangsverteilungen über dem Umfang einer Dampfentnahmekammer bei verschiedenen Anzapfmengen ermittelt [8]. Die Ergebnisse sind jedoch wesentlich von Auftrieb und Kondensation beeinflusst und für die in der vorliegenden Arbeit betrachteten Strömungsverhältnisse nicht nutzbar. 


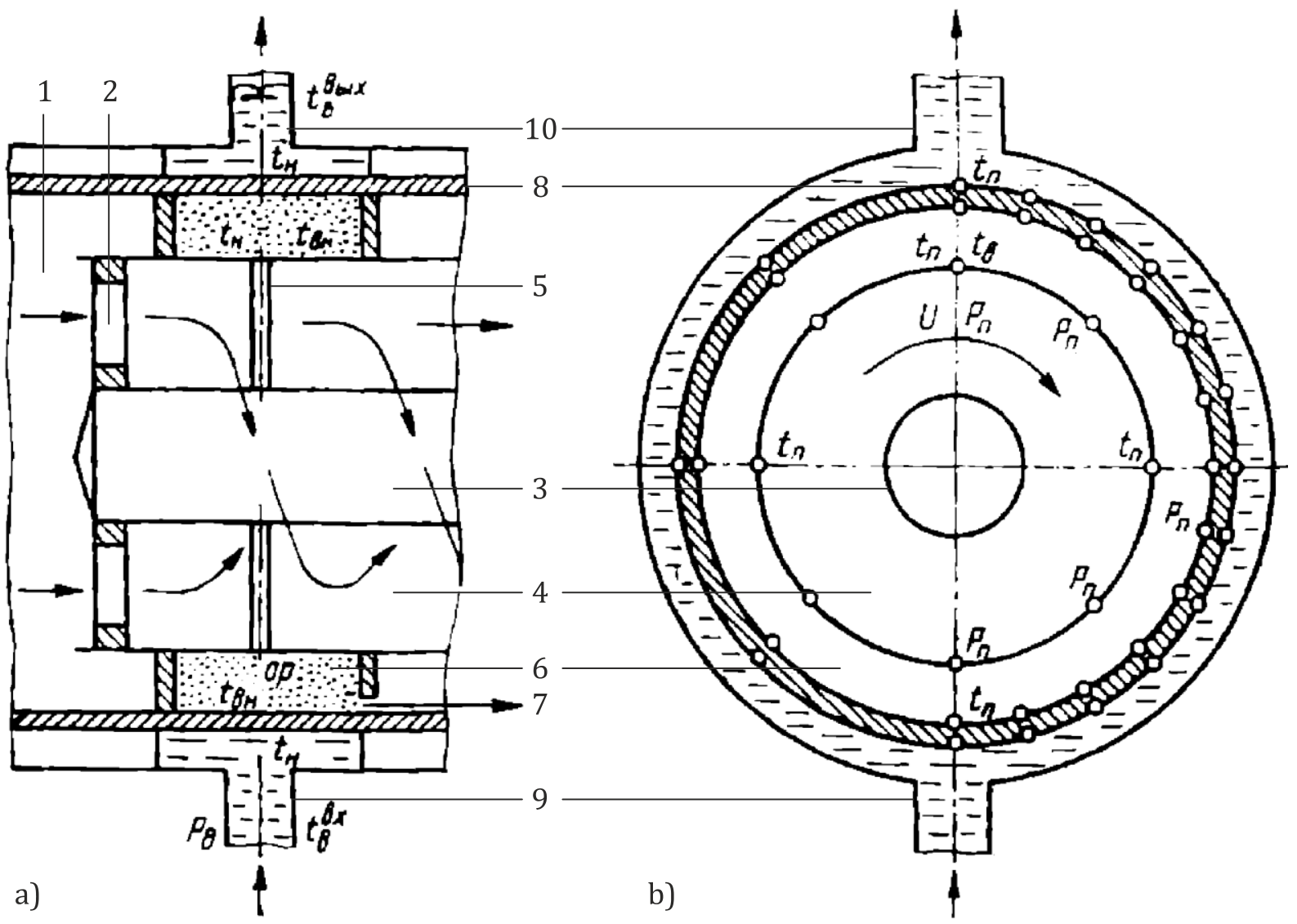

Abb. 2.8 Messsektion des sattdampfbetriebenen Versuchsstandmodelles einer Dampfentnahmekammer [8]. a) Schnittansicht; b) Messstellen am Umfang der Entnahmekammer; 1 - Beruhigungskammer; 2 - Dralleinrichtung; 3 - Verdrängungskörpoer; 4 - Ringkanal; 5 - Ringspalt; 6 - Entnahmekammer; 7 - Entnahme; 8 - Wand; 9 - Kühlwasser-Vorlauf; 10 - Kühlwasser-Rücklauf.

Daneben kamen auch numerische Strömungssimulationen für Seitenräume mit Anzapfung zum Einsatz, bei denen die benachbarten Stufen im Hauptströmungskanal mit modelliert wurden $[24,25]$. Diese erfolgten jedoch ohne Betrachtung des lokalen Wärmeüberganges sondern dienten vielmehr der Abklärung der Fluidverteilung und des Druckverlustes im Seitenraum sowie der Interaktion zwischen Seitenraum- und Hauptströmung.

Weiterentwicklungen in der zur Verfügung stehenden Rechentechnik gestatten es heute, den Wärmeübergang durch eine gleichzeitige Simulation von Strömung und Wandstrukturen konjugiert zu berechnen. In neueren Studien $[26,27]$ wurde auf Basis einer solchen transienten CHTSimulation das Aufheiz- und Verformungsverhaltens einer Dampfturbine während des Anfahrens simuliert, die in ihrem Gehäuse Seitenräume beherbergt. Nach wie vor werden jedoch auch empirische Ansätze und Nusselt-Korrelationen für die wärmeübertragenden Oberflächen in Dampfturbinenseitenräumen genutzt [28-30]. 
Dem Großteil der oben aufgeführten Referenzen ist gemein, dass sie sich nur auf bestimmte Seitenraumgeometrien beziehen, wie sie in den jeweils untersuchten Dampfturbinentypen (zumeist russischen Typs) auftreten. Zwar wurde mit verschiedenen Ansätzen teilweise sogar die räumliche Verteilung der WÜK - axial und in Umfangsrichtung - ermittelt, hauptsächlich für klassische Anzapf-, Entnahme- oder Zudampfkammern. Mitunter sind sogar Korrelationen gebildet worden, die zum Teil auch semi-analytisch hergeleitet worden sind. Verallgemeinerungsfähig für beliebige andere Seitenräume und Kavitäten sind die Erkenntnisse aber nicht [31].

\subsection{STRÖMUNG IN KAVITÄTEN MIT T- UND L-FÖRMIGEM QUERSCHNITT}

Desweiteren wurden in der Vergangenheit allgemeine Kavitäten hinsichtlich des Strömungsfeldes experimentell erforscht. Meist dienten die Ergebnisse zur Validierung numerischer Simulationen, anfangs noch mit vereinfachten Turbulenzansätzen, später dann mit Hilfe komplexerer CFD-Modelle. So studierten beispielsweise Aroussi und Mitarbeiter in den späten 1980er-und frühen 1990er-Jahren die Wasserströmung in überströmten Kavitäten mit L- oder T-förmigem Querschnitt [32-38] (Abb. 2.9), die in ihrer Form sehr stark den Gehäuseseitenräumen in Dampfturbinen ähneln. Zur Anwendung kamen Messungen mittels Laser-Doppler-Anemometrie (LDA;

Abb. 2.9) und optischer Strömungsverfolgung (Particle Image Velocimetry, PIV; Abb. 2.11) sowie numerische Nachrechnungen mittels einfacher Wirbelviskositätsmodelle, die zum großen Teil die Sekundärwirbel in den äußeren Ecken der Kavität nicht vorhersagen konnten. Wärmeübergang wurde nicht betrachtet.

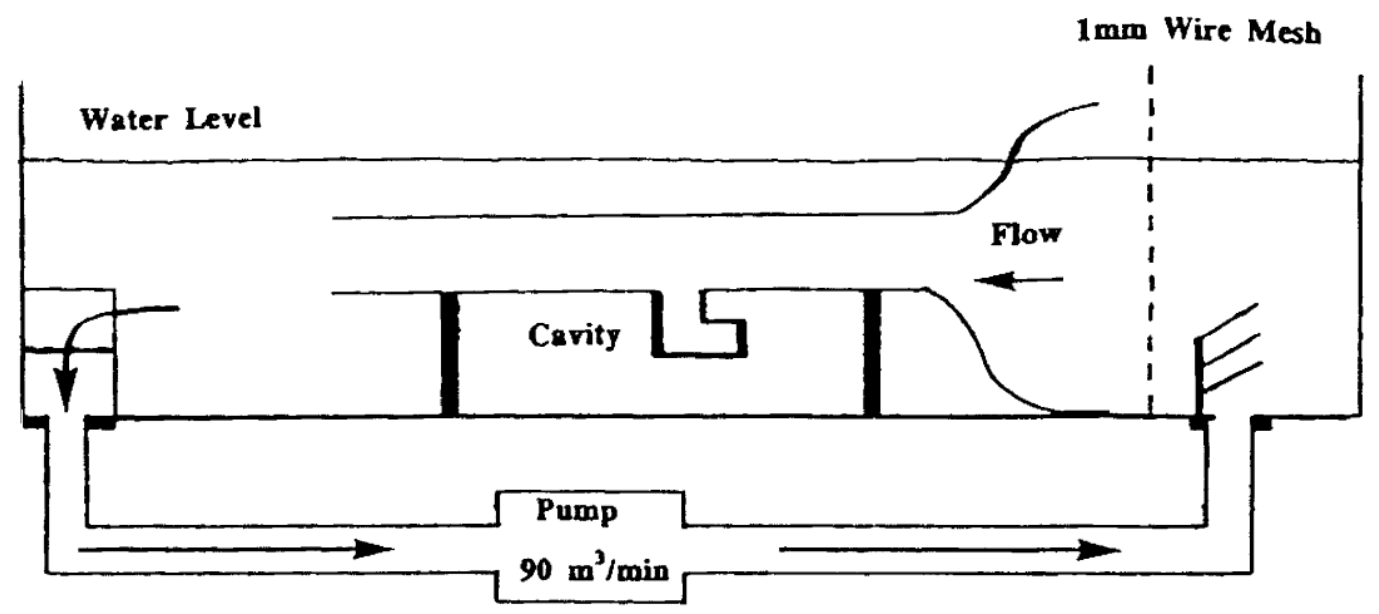

Abb. 2.9 Wasserversuchsstrecke zur experimentellen Bestimmung des Strömungsfeldes in einer L-förmigen Kavität [37]. 


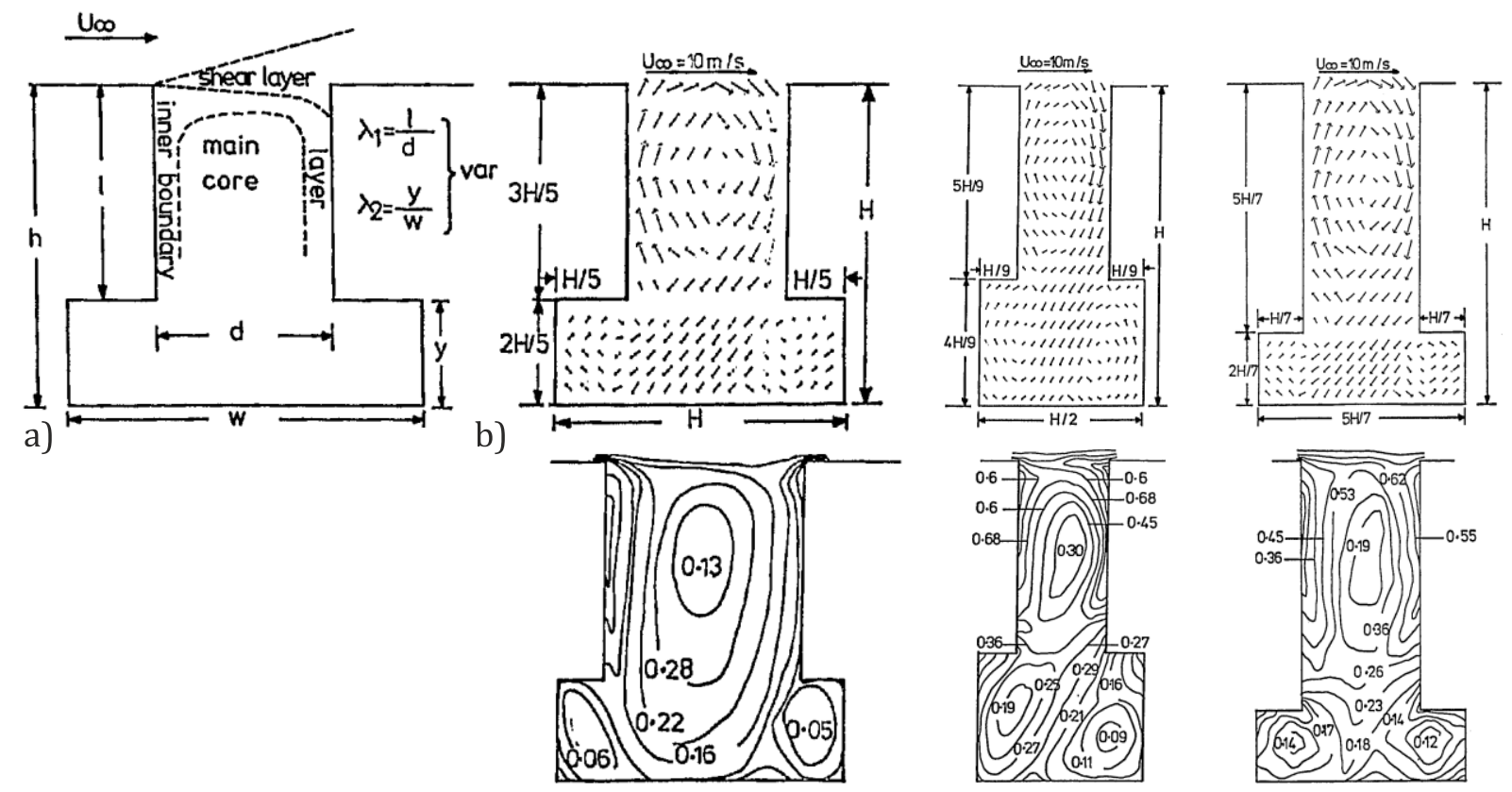

Abb. 2.10 Strömungsfeld in überströmten Kavitäten mit T-förmigem Querschnitt und unterschiedlichen Abmessungsverhältnissen [34].

a) Strömungsgebiete; b) Ergebnisse aus LDA-Messungen; $H=70 \mathrm{~mm}$.
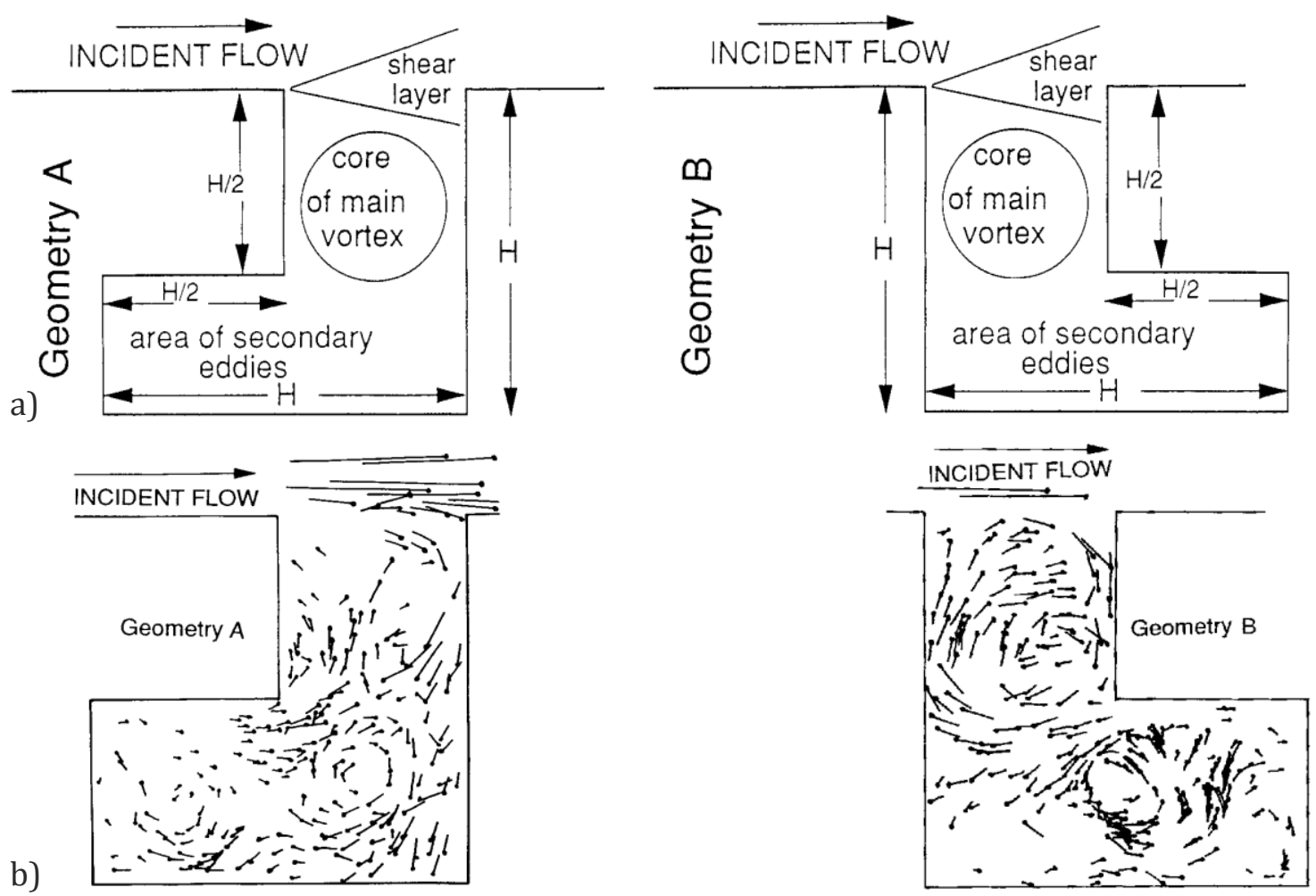

Abb. 2.11 Strömungsfeld in überströmten Kavitäten mit L-förmigem Querschnitt und unterschiedlicher Orientierung (nach [37,38], Bilder $\mathrm{z}$. T. gedreht und Beschriftungen verschoben).

a) Strömungsgebiete; b) Ergebnisse aus PIV-Messungen; $H=70 \mathrm{~mm}$. 


\subsection{WÄRMEÜBERGANG IN KAVITÄTEN MIT QUADRATISCHEM QUER- SCHNITT}

Für den einfachen Grenzfall der rechteckigen und quadratischen Kavitäten wiederum existiert eine Vielzahl an Veröffentlichungen aus den letzten 50 Jahren, die sich mit dem lokalen Wärmeübergang zu bzw. von einer oder mehreren beheizten oder gekühlten Wänden befassen [39-59], wobei auch hier die Ergebnisse hauptsächlich zur Validierung für CFD-Codes eingesetzt wurden und noch immer eingesetzt werden.

Für den Fall der reinen erzwungenen Konvektion in ebenen Kavitäten ( Abb. 2.12) mit quadratischem Querschnitt $(B / H=1)$ sind in $-A b b .2 .13$ von verschiedenen Autoren $[46,55,56,60]$ experimentell bestimmte lokale WÜK in Form von Nusselt-Zahl-Verläufen entlang des Bodens zusammengetragen. Dabei wurde sowohl die Nusselt-Zahl $N u_{l}(x)=\alpha(x) l / \lambda$ als auch die Reynolds-Zahl $R e_{l}=u_{\infty} l / v$ einheitlich auf die Kanalhöhe $l$ als charakteristische Länge umgerechnet, und nicht, wie zum Teil geläufig, auf Kavitätbreite $B$ oder -höhe $H$ bezogen. Die Reynolds-Zahlen in der Hauptströmung reichen vom kritischen Wert 2.300 bis in den Bereich sehr starker Turbulenz $(R e=686.000)$. In dieser Form reihen sich die Ergebnisse aus den unterschiedlichen Quellen exzellent untereinander ein. Es zeigt sich, dass die Reynolds-Zahl einen wesentlichen Einfluss hat, und zwar sowohl auf den Mittelwert der Nusselt-Zahlen, der um mehrere Größenordnungen von 5 auf 8000 wächst, als auch auf deren lokale Verteilung, die sich mit steigenden Reynolds-Zahlen stärker ausprägt. Interessant ist in diesem Zusammenhang auch, dass unterschiedliche Quellen auch einen Einfluss der Wärmeübertragungsrandbedingungen (konstante Wandtemperatur bzw. konstanter -wärmestrom/ Kühlen bzw. Heizen) sowie der Grenzschichtdicke $\delta$ in der Anströmung auf den lokalen Wärmeübergang feststellen.

Auf Grund der einfacheren Strömungsverhältnisse lassen sich diese Erkenntnisse nicht 1:1 auf Kavitäten komplexerer Geometrie übertragen. Dennoch können die in der Literatur festgestellten prinzipiellen Gesetzmäßigkeiten gut auf experimentellem Wege qualitativ nachgeprüft werden (siehe Kap. 5.9).

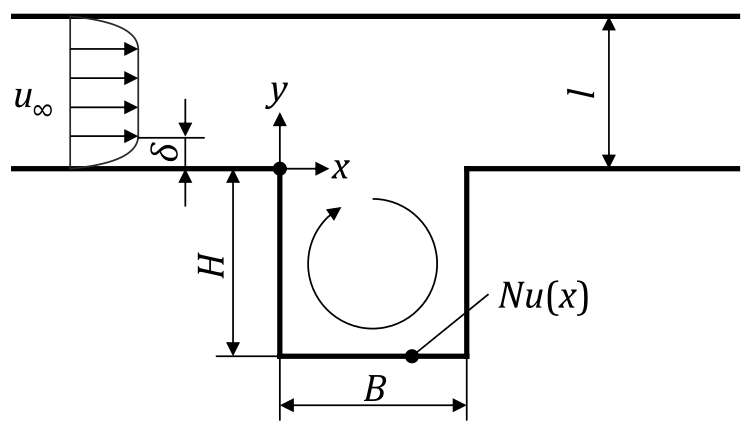

Abb. 2.12 Nomenklatur der geometrischen Abmessungen einer ebenen quadratischen Kavität 


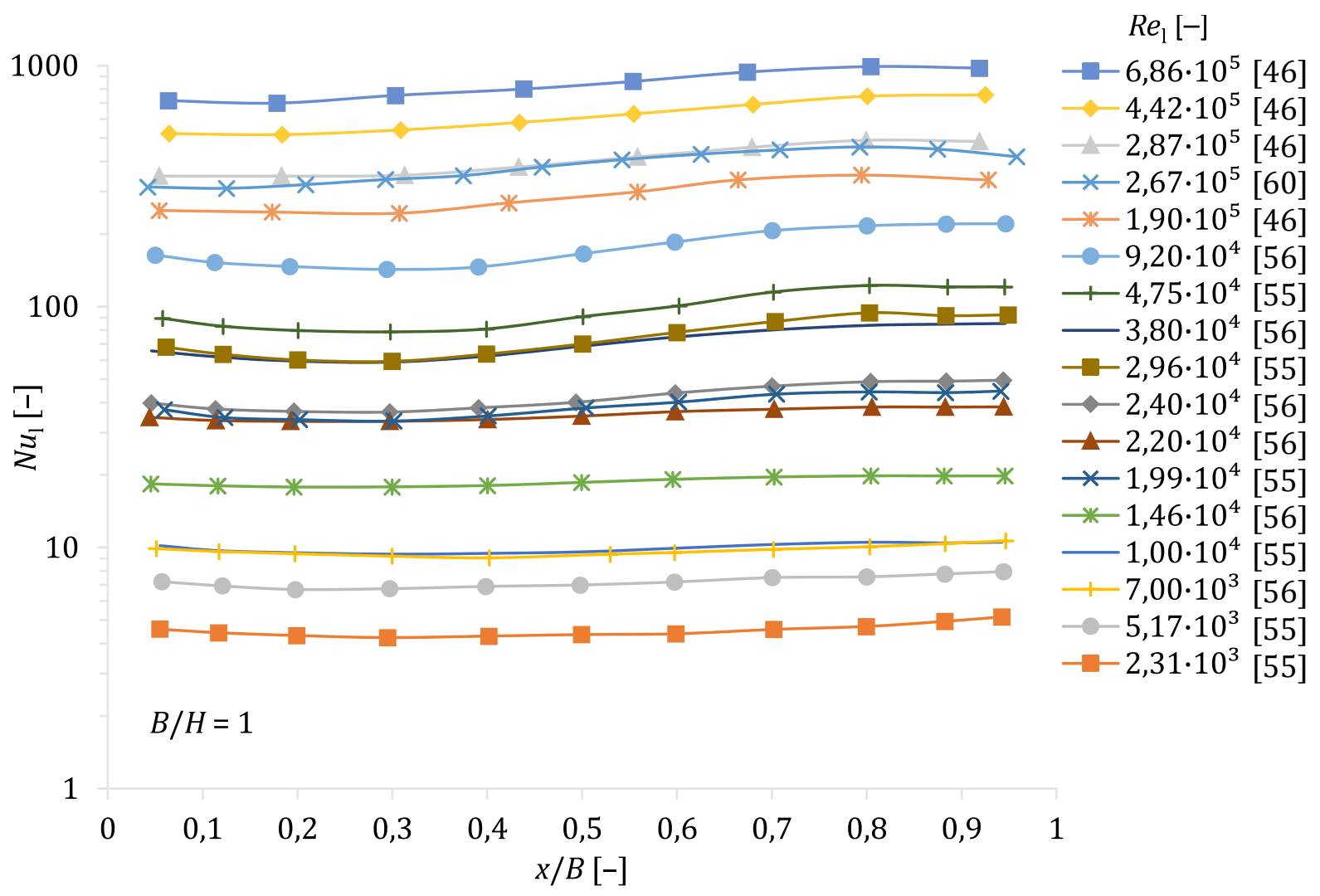
Abb. 2.13 Lokale Nusselt-Zahl-Verläufe in ebenen quadratischen Kavitäten bei variierten Rey-
nolds-Zahlen in der Hauptströmung. Daten aus [46,55,56,60]. Ähnlichkeitskennzahlen
umgerechnet auf Kanalhöhe $l$ als charakteristische Länge (vgl. $>$ Abb. 2.12).

\subsection{NOTWENDIGKEIT UND AUSGANGSLAGE DER UNTERSUCHUNGEN}

Trotz der zahlreichen Forschungstätigkeiten, von denen die relevantesten oben aufgezählt worden sind, beklagen viele Autoren und Wissenschaftler nach wie vor den Mangel an Wissen zum Wärmeübergang in diesen speziellen Bereichen der Turbine [7,9-11,26,28,29,31,61-63].

So schreibt auch Leizerovich, der damals bereits aktiv an den sowjetischen Forschungstätigkeiten mitgewirkt hat, in seinem Buch „Steam turbines for modern fossil-fuel power plants“ [31] aus dem Jahre 2008 (!):

„Contrary to the steam-lines and turbine rotors, with their pretty definite patterns of the steam flow relating to the heated surfaces, for the valve steam-chests and cylinder casings, their steam flow patterns are much more variable and intricate, less studied and need special experimental researches (...) Our knowledge of the heat transfer conditions for the stator turbine elements mainly comes from assessments based on temperature measurements of the heating steam and heated metal (...) [I]n many cases there do not exist any criterion equations that can be used for estimating the heat transfer conditions for steam turbine stator elements, especially for turbine casings" ([31], S. $231 \mathrm{f}$.) 
und weiter:

"Unfortunately, all the existent data on heat transfer for turbine casings are not still sufficient for their generalizing, and new experimental researches are needed (...) Of special interest would be the heat transfer conditions for the intercasing space of the integrated HP-IP [high-/ intermediate-pressure] cylinders of different types (...) It should be supplemented that for some intercasing chambers of such cylinders without a forced steam flow it is also difficult to set definitely the dependency between the steam temperatures in the mainstream and within the chamber. All these circumstances need thorough investigations" ([31], S. 233 f.).

Selbiges stellt auch Topel in Ihren Arbeiten von 2014 und 2015 zur numerischen Modellierung der Relativverformung zwischen Rotor und Stator einer Industriedampfturbine von Siemens während des Anfahrens [28,29] fest:

"The BCs [boundary conditions] that were found to have the largest influence towards capturing the differential expansion (...) were those related to the bearings and the inter-casing cavity. Their effects were most influential in the loading phase of the start in which the casing begins to grow faster than the rotor" ([28], S. 41).

"The assumption of the temperature and HTC of the steam that leaks into the inter-casing cavity also plays an important part in the expansion rate of the casing during the loading stage (...) There is a certain level of uncertainty when it comes to defining the heat transfer conditions in this region. Presently, all existing data is not yet sufficient for accurate generalized correlations. One of the main challenges is to set the dependency between the steam temperatures in the mainstream and within the chamber" ([29], S. 7).

Motivation und Ausgangspunkt für die vorliegende Arbeit waren die Erkenntnisse von Heße [7,64], der bei der Validierung seines neu entwickelten semi-analytischen Tools zur Berechnung der thermischen Gehäuseverkrümmung und zur Radialspielauslegung von Industriedampfturbinen auf dasselbe Problem gestoßen ist:

„Die Berechnung thermischer Gehäuseverformungen erweist sich oft als schwierig, weil ein umfangreicher Kenntnisstand insbesondere zu den thermischen Randbedingungen erforderlich ist, um zuverlässige Ergebnisse zu erhalten“ ([7], S. 13).

„Eine besondere Bedeutung kommt den nicht durchströmten Bereichen zwischen Innenbauteilen und Außengehäuse zu. Aus den FE-Analysen wird deutlich, dass der Wärmeübergang mit geringer Intensität abläuft, die Anwendung der Nusselt-Beziehung für natürliche Konvektion jedoch teilweise stark abweichende Wärmeübergangskoeffizienten ergibt. Offensichtlich tre- 
ten Phasen mit überwiegend natürlicher Konvektion auf, die zeitweise von erzwungener Konvektion überlagert werden. Eine möglichst genaue Kenntnis der Wärmeübertragungsmechanismen in diesem Bereich ist wichtig, da je nach Turbine große Abschnitte des Außengehäuses von diesen Randbedingungen betroffen sein können, so dass sich Unsicherheiten entsprechend stark auf das simulierte Temperaturfeld auswirken. Weitergehende Messungen z. B. mit einem Prüfstand unter Laborbedingungen sind zu empfehlen. Dadurch ergeben sich Möglichkeiten, die Einflussgrößen auf den Wärmeübergang systematisch zu untersuchen. Es kann erwartet werden, dass sich sowohl geometrische als auch strömungsbedingte, d. h. lastpunktabhängige Einflüsse ergeben“ ([7], S. 53).

Hieraus ergibt sich unmittelbar die Notwendigkeit der beabsichtigten Untersuchungen.

\section{5 ÜBERBLICK ÜBER AKTUELLE FORSCHUNGSTÄTIGKEITEN AUF DEM GEBIET}

Nachdem über 20 Jahre seit den letzten Forschungstätigkeiten vergangen sind, widmen sich neben den beiden eingangs erwähnten AG-Turbo-Projekten COOREFLEX-turbo 4.3.6 und ECOFLEX 4.3.4 A, welche die Professuren TEA und MFD gemeinsam mit der Siemens AG, Görlitz, bearbeiten und in deren Rahmen die vorliegende Dissertation entstanden ist, aktuell auch drei weitere Projekte in Deutschland und Italien dem Wärmeübergang in Gehäuseseitenräumen von Turbinen:

Das ECOFLEX-Teilprojekt 4.3.4 C („Untersuchung von Wärmeübergang in Dampfturbinenkomponenten - Messungen in realer Dampfturbine“; FKZ 03ET7092L, Laufzeit 01.08.2017 bis 31.01.2021), welches die Professur MFD gemeinsam mit der Universität Darmstadt und der MAN Energy Solutions durchführt, beschäftigt sich unter anderem mit der lokalen Messung von Wärmeübergangskoeffizienten an 14 ausgewählten Positionen an der Gehäuseinnenoberfläche sowie an den Leitgitterträgern einer Versuchsdampfturbine der MAN in Oberhausen. Zum Einsatz kommen hierbei speziell entwickelte, für die Anwendung in Dampfatmosphäre (Sattdampf bis 3 bar und $170{ }^{\circ} \mathrm{C}$ ) angepasste Wärmestromsonden/ -lanzen, die auf der Wärmestrom-Temperaturdifferenz-Methode basieren, sowie neuartige WÜK-Sensoren, die nach dem Übertemperaturprinzip funktionieren.

Im ECOFLEX-Teilprojekt 4.3.4 B („Untersuchung von Wärmeübergang in Dampfturbinenkomponenten - Turbinenseitenräume unter Nassdampfbedingungen“; FKZ 03ET7092I, Laufzeit 01.11.2017 bis 31.10.2020) untersucht der an der Universität Duisburg-Essen angesiedelte Lehrstuhl für Strömungsmaschinen gemeinsam mit der Siemens AG in Mühlheim an der Ruhr den Wärmeübergang bei Kondensation bzw. Verdampfung an den Leitgitterstegen zweier benachbarter Gehäuseseitenräume in Niederdruck-Dampfturbinen. Die Untersuchungen werden dabei mit Hilfe eines 
generischen Dampfversuchsstandes durchgeführt, bei dem der Dampf unter nahezu atmosphärischen Bedingungen (max. 1,5 bar, $120^{\circ} \mathrm{C}, 650 \mathrm{~kg} / \mathrm{h}$ ) über eine elektrisch beheizte oder mit temperiertem Wasser gekühlte, waagerecht in einem ebenen Kanal angeordnete ebene Platte bzw. eine rückwärtsgewandte Stufe (backward-facing step) strömt. Der Wärmestrom wird dabei über Thermoelemente ermittelt, die in verschiedenen Tiefen über der Wand angeordnet sind, welche aus mehreren, voneinander isolierten Gliedern besteht. Parallel erfolgt eine Dickenbestimmung des Kondensatfilms mittels zweier optischer Messverfahren [65,66].

Mit beiden AG-Turbo-Projekten besteht eine enge Zusammenarbeit sowie ein reger wissenschaftlicher Austausch.

Im Rahmen des regionalen Forschungsprogrammes STech (Smart Turbine Technologies), welches innerhalb der Förderinitiative FAR-FAS 2014 (Fonds für Forschungsförderung und für strukturschwache Regionen) der Region Toskana finanziert wird, führt die Universität Florenz in Zusammenarbeit mit Baker Hughes GE, Florenz, eine numerische Parameterstudie (DACE) zur Aerodynamik einer in ihrer Geometrie variablen Dampfturbinengehäusekavität mit T-förmigem Querschnitt sowie zur Wärmeübertragung an deren Außenwand durch. Innerhalb des Projektes mit dem Titel „Entwicklung numerischer Modelle zur Untersuchung von instationären Wechselwirkungen in innovativen Komponenten axialer Turbomaschinen" (Sviluppo di modelli numerici per lo studio dell'interazione non stazionaria nei componenti innovativi delle turbomacchine assiali) gelang es der dortigen Forschungsgruppe „Wärmeübertragung und Verbrennung“ (Heat Transfer and Combustion Group), auf Basis von insgesamt 320 numerischen Einzelsimulationen für 64 verschiedene Geomerien eine Korrelation für den flächengemitelten WÜK zu entwickeln, die die geometrischen Abmessungen sowie den Massestrom, den Druck und die Temperatur in der Hauptströmung und in der Kavität berücksichtigt [67]. Für die stationären RANS-Simulationen wurde überhitzter Dampf verwendet. Die genaue Form sowie die Werte für die Koeffizienten innerhalb der Korrelation wurden nicht publiziert. Die Ergebnisse sind daher weder reproduzier- noch verwertbar.

Weitere als die hier benannten Forschungsprojekte, an denen allesamt öffentliche Forschungseinrichtungen beteiligt sind, sind bisher nicht bekannt geworden. Nach Aussage von Industrievertretern bei der AG Turbo beschäftigen sich jedoch viele Turbinenhersteller (intern) mit dem Thema. Da es sich hierbei allerdings häufig um firmeninternes Spezialwissen handelt, von dessen Exklusivität sich die Unternehmen einen Wettbewerbsvorteil in Hinblick auf eine höhere Lastflexibilität bzw. auf den Abbau von Konservativitäten bei der Auslegung ihrer Turbinen gegenüber ihren Mitbewerbern erhoffen, werden weder die Tätigkeiten noch die Ergebnisse publik gemacht. 



\section{ZIELSTELLUNG, METHODIK UND LÖSUNGSWEG}

Ziel der vorliegenden Arbeit ist es, den Wissensstand zum Wärmeübergang in Gehäuseseitenräumen am Beispiel von Industriedampfturbinen wie folgt weiterzuentwickeln:

- Zum ersten Mal soll im Modellmaßstab auf experimentellem Weg die lokale Verteilung des Wärmeübergangskoeffzienten (WÜK) in Seitenräumen bei reiner erzwungener Konvektion (d. h. unter Ausschluss von Auftriebseffekten und Phasenübergängen) untersucht werden.

- Dabei sollen nicht wie bisher nur einzelne, in der Praxis ausgeführte Turbinenseitenräume sondern viele verschiedene Seitenraumkonfigurationen betrachtet werden.

- Bei der Wahl der zu untersuchenden Versuchskonfigurationen soll ein möglichst großer, praxisrelevanter Parameterbereich wie in Industriedampfturbinen realisiert werden.

- Erstmals sollen die experimentellen Untersuchungen auch durch vergleichende numerische Analysen mittels moderner Strömungslöser begleitet werden. Bisherige Betrachtungen erfolgten häufig losgelöst voneinander.

- Die Ergebnisse sollen in Form einer einfach zu handhabenden Nusselt-Korrelation verallgemeinert werden.

Zur Erreichung der Zielstellung soll ein komplett neuer, druckluftbetriebener, modularer Versuchsstand mit skalierter, generisch variabler Seitenraumgeometrie nebst Versorgungsinfrastruktur zur geregelten Druckluftbereitstellung entwickelt und errichtet werden. Mit der Versuchseinrichtung sollen erstmalig systematische experimentelle Untersuchungen zum Wärmeübergang bei reiner erzwungener Konvektion zwischen Außenwand und Fluid in verschiedenen Totraumkonfigurationen (ohne Fluidzufuhr oder -entnahme) in Abhängigkeit von deren Abmessungsverhältnissen und von den Strömungsverhältnissen in der Hauptströmung (Reynolds-Zahl, Drall, Geschwindigkeit) durchgeführt werden.

Für die rückwirkungsarme Bestimmung der lokalen Wärmeübergangskoeffizienten entlang der Innenoberfläche der Totraumaußenwand sollen gleichzeitig zwei unabhängige Messverfahren mit möglichst hoher örtlicher Auflösung und nur sehr geringem Wärmeeintrag zur Anwendung kommen, für die die Instrumentierung entsprechend ausgelegt, gefertigt und kalibriert werden muss. Im Zentrum der vorliegenden Arbeit steht dabei die inverse WÜK-Bestimmung durch Rückwärtsrechnung auf Basis stationärer gemessener Materialtemperaturen in der den Seitenraum nach außen hin begrenzenden Außenwand - ein Verfahren, welches am Lehrstuhl für Thermische Energiemaschinen und -anlagen schon seit vielen Jahren etabliert ist [68-72]. Demgegenüber soll die lokale Übertemperaturmethode, welche auf einem miniaturisierten Sensorkonzept basiert und an 
der Professur für Magnetofluiddynamik, Mess- und Automatisierungstechnik (MFD) entwickelt und auch dort ausgewertet wird, nur für Validierungs- und Vergleichszwecke dienen. Für weiter führende Informationen hierzu sei auf [73] verwiesen. Für die stationäre inverse Methode, für die die genaue Bestimmung der thermodynamischen Stoffwerte der Wandmaterialien eine wichtige Rolle spielt, sollen verschiedene numerische und analytische Verfahren zur Rückwärtsrechnung getestet und hinsichtlich ihrer Genauigkeit und ihrer Rechendauer miteinander verglichen werden. In einer umfassenden, experimentellen Parameterstudie soll anschließend mit Hilfe des Versuchstandes die Sensitivität des Wärmeübergangs im Seitenraum von den verschiedenen Einflussgrößen feststellt und quantifiziert werden. Die gefundenen Erkenntnisse sollen verallgemeinert und nach Möglichkeit in einer ersten Nusselt-Korrelation zusammengefasst werden, wobei die Unsicherheiten der Messgrößen sowie der Korrelation abzuschätzen sind sowie eine Strategie zur Übertragung der Ergebnisse auf reale Turbinenverhältnisse erarbeitet werden soll.

Neben den Arbeiten zum Wärmeübergang sollen begleitende experimentelle Untersuchungen zum dreidimensionalen Strömungsprofil in der Hauptströmung durch Traversierung mit einer auf geeignete Weise zu kalibrierenden Fünflochsonde sowie zur Wanddruckverteilung entlang der Innenoberfläche der Außenwand des Seitenraumes über Wandanbohrungen erfolgen. Beide Maßnahmen sollen Aufschluss über die Gleichmäßigkeit der Strömung über den Umfang bzw. der tatsächlichen Drallverteilung hinter dem Drallgitter geben und sicherstellen, dass eventuelle Nachlaufdellen der Einbauten weitgehend abgeklungen sind.

Abschließend soll geprüft werden, ob und inwieweit die im Modell gemessenen WÜK-Verläufe mittels kommerziell verfügbarer CFD-Codes auf numerischen Wege strömungsmechanisch nachgerechnet werden können. Der Fokus liegt dabei auf stationären Simulationen unter Anwendung moderner, dem Stand der Technik entsprechender Turbulenzmodelle. Eine Grundlagenuntersuchung soll jedoch explizit nicht durchgeführt werden, da dies den Rahmen der vorliegenden Dissertation sprengen würde. Für eine ausgewählte Versuchskonfiguration soll anhand des Vergleichs mit den gemessenen Werten zunächst in verschiedenen Sensitivitätsstudien der Einfluss des Rechengitters, des Modelldetaillierungsgrades sowie ausgewählter Randbedingungen und Modelleinstellungen auf den berechneten lokalen WÜK-Verlauf festgestellt werden. Dabei ist auch der Einsatz einer instationären Grobstruktursimulation (LES) zu diskutieren. Mit Hilfe eines geeigneten Einstellungs- und Parametersatzes sollen anschließend alle experimentell untersuchten Versuchskonfigurationen mit dem validierten CFD-Modell nachgerechnet werden.

Zum Schluss der Arbeit soll in einer Zusammenfassung noch einmal eine Übersicht über das bearbeitete Thema in ganzer Breite geschaffen werden, um mit einem Ausblick auf ausstehende Arbeiten und auf die nächsten anstehenden bzw. weiterführenden Schritte zu schließen. 


\section{VERSUCHSAUFBAU UND -DURCHFÜHRUNG}

\subsection{SEITENRAUMVERSUCHSSTAND „SISTeR“}

Zur systematischen Untersuchung des lokalen erzwungenen Wärmeübergangs in skalierten Modellen von Gehäuseseitenräumen in Dampfturbinen wurde ein generisch variabler Versuchsstand entwickelt. Dieser Seitenraumversuchsstand trägt den Namen „SiSTeR“ (idie $\underline{\text { Space }}$ Test $\underline{\text { Rig) }}$ und wurde am Zentrum für Energietechnik (ZET) an der TU Dresden errichtet.

\subsubsection{Versuchsstandkonzept}

Der Versuchsstand wird mit Druckluft als Arbeitsfluid betrieben und bildet den kreisringspaltförmigen Hauptströmungskanal einer repräsentativen Dampfturbine mit einem radial nach außen hin anschließenden axialsymmetrischen Seitenraum ab (Abb. 4.1). Die Luft strömt von links in den Versuchsstand ein (1) und wird über einen Verdrängungskörper (11) auf einen Kreisringspalt (annulus; 2; Abb. 4.2) mit der Breite $\Delta r$ und dem Innenradius $r$ gelenkt. In diesem kann optional ein festes oder verstellbares Drallgitter (3) eingesetzt werden, um der Strömung bei Bedarf einen definierten Drallwinkel $\alpha$ in Umfangsrichtung aufzuprägen. Dadurch kann die drallbehaftete Fehlabströmung aus dem Laufrad unmittelbar vor dem Gehäuseseitenraum in Folge von Mehr- oder Minderumlenkung imitiert werden, wie sie beispielsweise im Teil- bzw. Überlastlastbetrieb einer Turbine auftreten kann. Es schließt sich eine Beruhigungsstrecke (4) mit einer dimensionslosen Anströmlänge von $L_{\mathrm{f}} / d_{\mathrm{h}}=15$ an, in der sich die Strömung gleichmäßig ausbilden kann. Der rotationssymmetrische Modellseitenraum („Ringkammer“) weist eine T-förmige Meridianschnittkontur auf und besteht aus der Einlassöffnung (9), die den Zugang zur äußeren Überströmung darstellt und in axialer Richtung durch die beiden Einströmseitenwände (5) berandet ist, sowie der eigentlichen (Umfangs-) Kavität (cavity; 8), die durch die beiden Seitenwände (6) sowie der Außenwand mit der WÜK-Instrumentierung (7) begrenzt wird. Nach der Durchströmung der Messzelle wird das Fluid wieder auf einen Rohrquerschnitt (11) geleitet und verlässt den Versuchsstand (12).

Die Begrenzung des Seitenraumes wurde so gestaltet, dass dessen Abmessungen ( Abb. 4.2) in axialer Richtung flexibel variiert werden können. Durch axiales Verschieben der rechten Einströmseitenwand ( Abb. 4.3 a) sowie der beiden Seitenwände (Abb. 4.3 b) entlang der Versuchsstandsachse können die Breite der Kavität $b$ und der Einströmöffnung $s$ sowie die Exzentrizität $e$ zwischen deren Mittellinien eingestellt werden. 


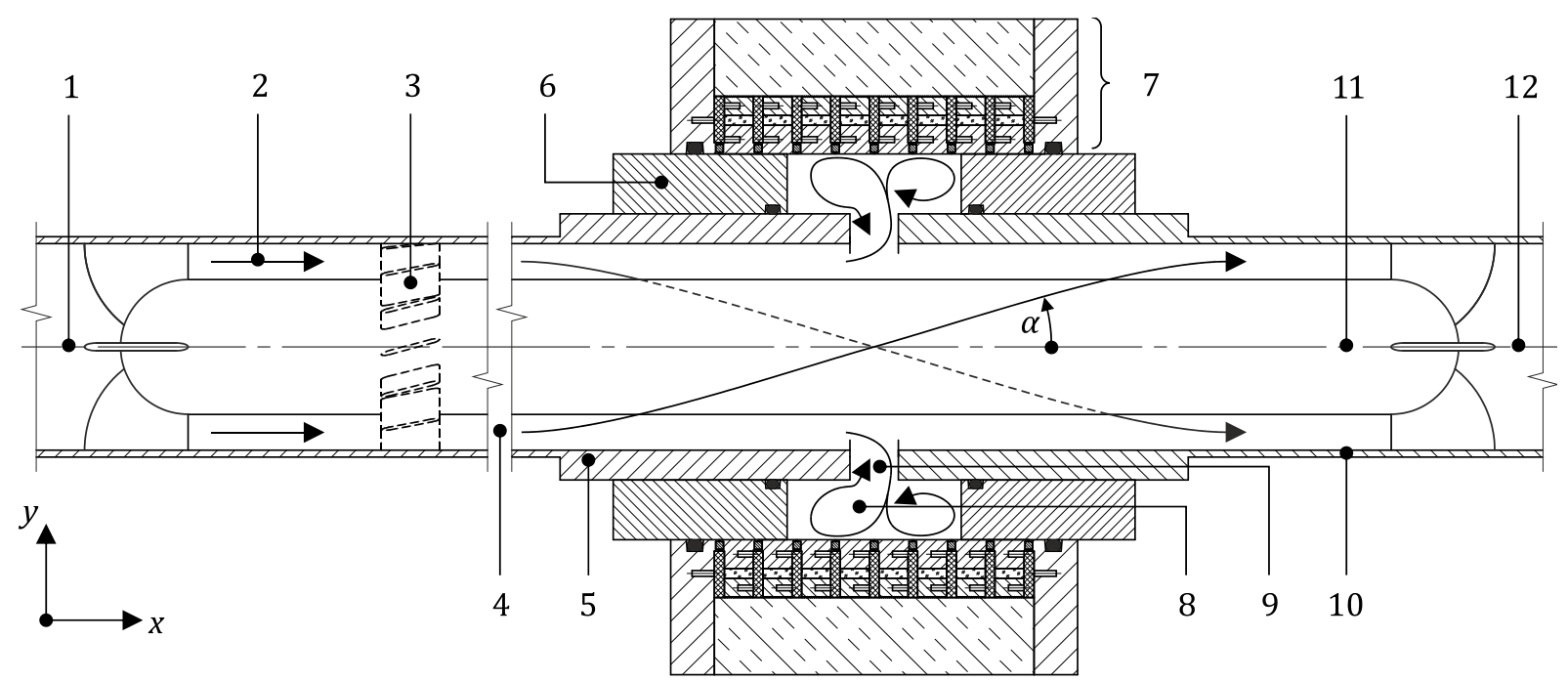

Abb. 4.1 Versuchsstandkonzept:

1 - Einströmung; 2 - Hauptströmungskanal (Ringspalt); 3 - Drallgitter (optional);

4 - Anströmstrecke; 5 - Einströmseitenwand (rechte Seite axial verschiebbar);

6 - Seitenwand (axial verschiebbar); 7 - Außenwand (mit WÜK-Instrumentierung, axial verschiebbar, drehbar); 8 - Seitenraum/ Kavität (ohne Anzapfung: „Totraum“);

9 - Einlassöffnung; 10 - Außenrohr; 11 - Verdrängungskörper/ Inliner; 12 - Abströmung.

Letztere Größe bestimmt die Form des Seitenraumes, welche von symmetrischem („T-Form“; $e=0$ ) bis hin zu asymmetrischem Querschnitt („L-Form“) mit stromaufwärts $(e<0)$ bzw. stromabwärts $(e>0)$ versetzter Kavität verändert werden kann. Auf diese Weise können nicht nur die Seitenräume verschiedener Dampfturbinentypen modelliert werden, sondern auch rechteckige, quadratische oder sonstige T- oder L-förmige Kavitäten, wie sie in vielen Einsatzbereichen der thermischen Energietechnik vorkommen. Die lokale Verteilung der WÜK wird an mehreren Stellen entlang der Innenoberfläche der Seitenraumaußenwand bestimmt ( Abb. 4.22). Durch Verschieben und Verdrehen der Außenwand kann zusätzlich die Messstellendichte in axialer sowie in Umfangsrichtung weiter erhöht werden ( Abb. 4.3 c).

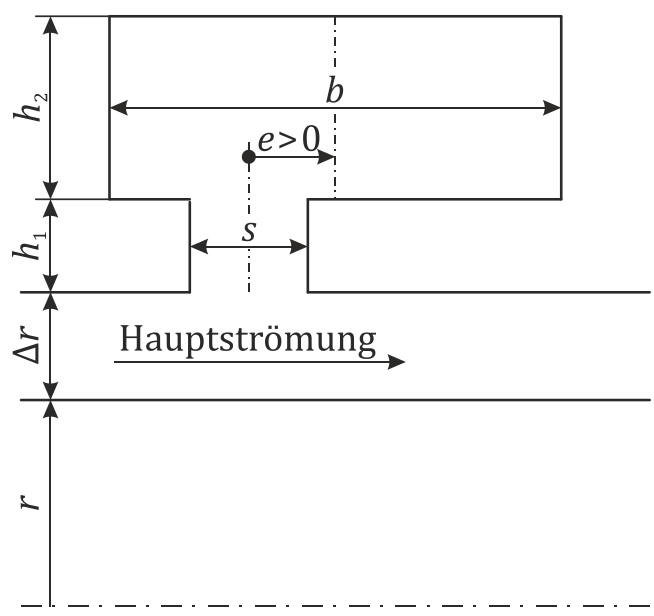

Abb. 4.2 Geometrische Abmessungen des Seitenraumes und des Hauptströmungskanals. 
a)

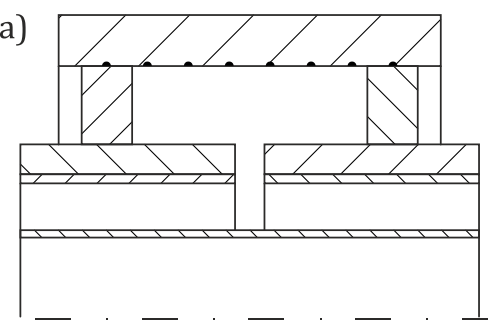

b)

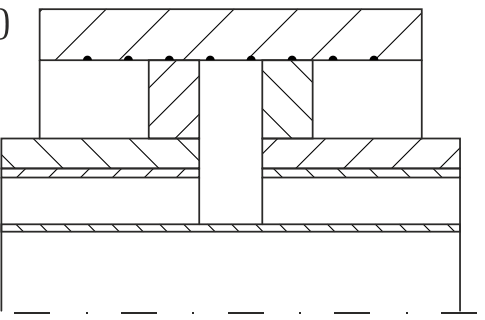

c)

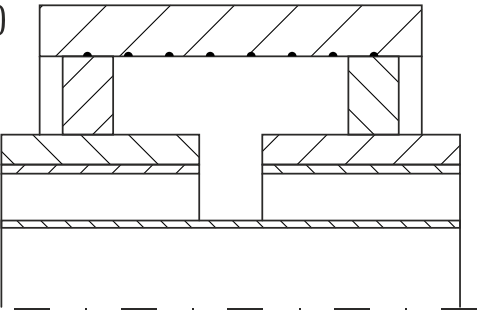

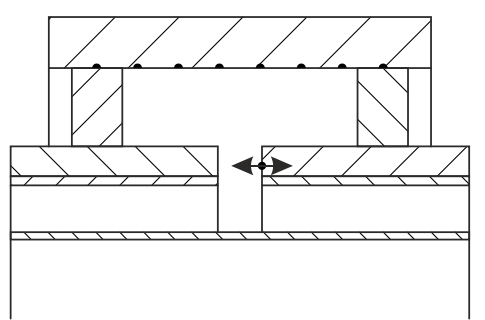
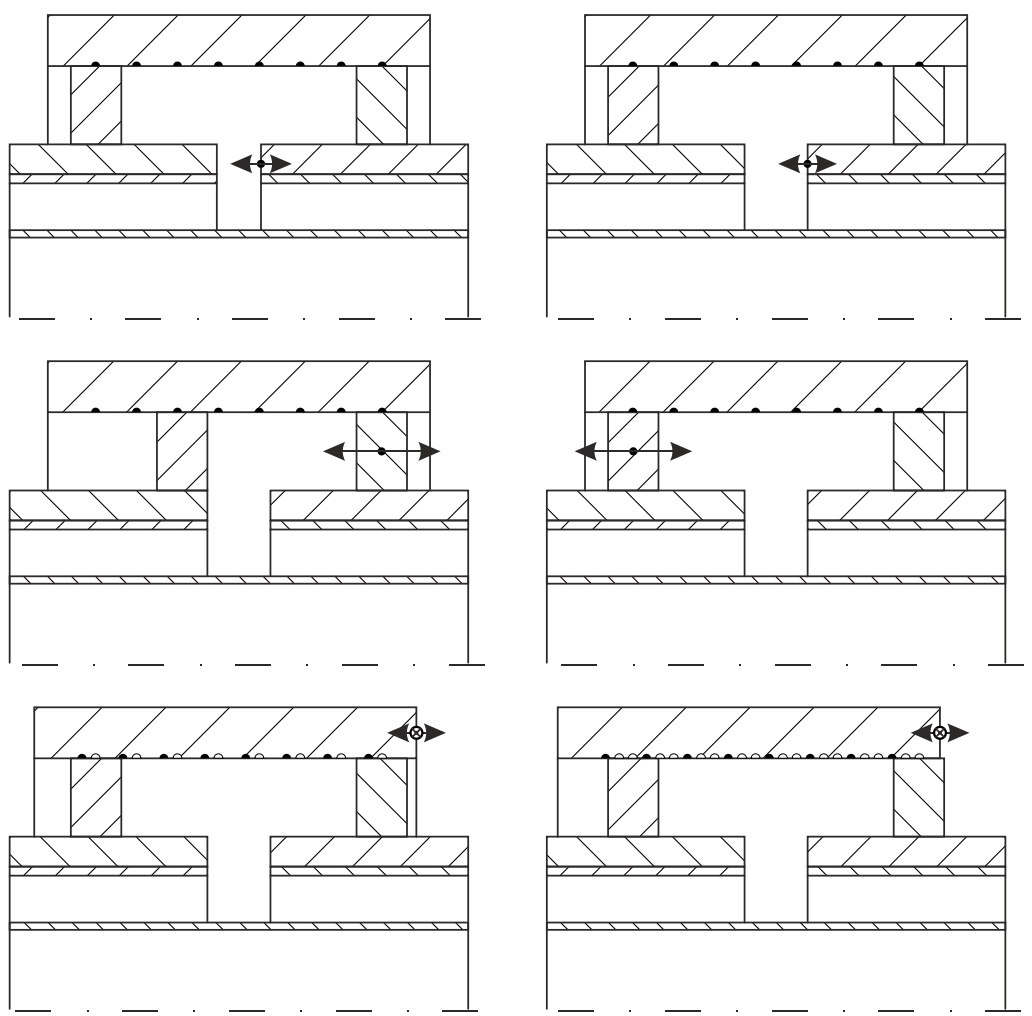

Abb. 4.3 Einstelloptionen der Seitenraumgeometrie:

a) Variation der Einströmbreite $s$ durch axiale Verschiebung der rechten Einströmseitenwand;

b) Variation der Breite $b$ und der Exzentrizität $e$ der Kavität durch axiale Verschiebung der beiden Seitenwände;

c) Erhöhung der Messstellendichte durch axiale Verschiebung der Außenwand.

\subsubsection{Dimensionierung und Skalierung der Modellgeometrie}

Aufbauend auf umfangreichen Parameterstudien zu den Abmessungen ausgeführter Seitenräume in Industriedampfturbinen des Industriepartners sowie zu den thermodynamischen Parametern im unmittelbar angrenzenden Schaufelkanal [74] wurde in einer vorbetrachtenden Auslegung [75,76] die Geometrie des Versuchsstandes auf der Grundlage der Reynolds-Ähnlichkeit und unter Berücksichtigung der Druckgeräterichtlinie (DGRL, Art. 4 Abs. 3) dimensioniert. Sowohl der Strömungskanal als auch die Seitenraumabmessungen weisen einen Skalierungsfaktor von $\Lambda=1: 3,81 \mathrm{bzgl}$. durchschnittlicher Turbinengeometrie auf [75,76]. Es ergeben sich die in $\$$ Abb. 4.4 abgebildeten, realisierbaren Abmessungen.

Der hydraulische Durchmesser des ringspaltförmigen Hauptströmungskanals entspricht

$$
d_{\mathrm{h}}=l=\frac{4 A}{U}=\frac{4 \cdot \frac{\pi}{4}\left(D^{2}-d^{2}\right)}{\pi \cdot(D+d)}=D-d=\Delta d
$$

mit der durchströmten Querschnittsfläche $A=\frac{\pi}{4}\left(D^{2}-d^{2}\right)$ und dem benetzten Umfang $U=\pi \cdot(D+d)$. 


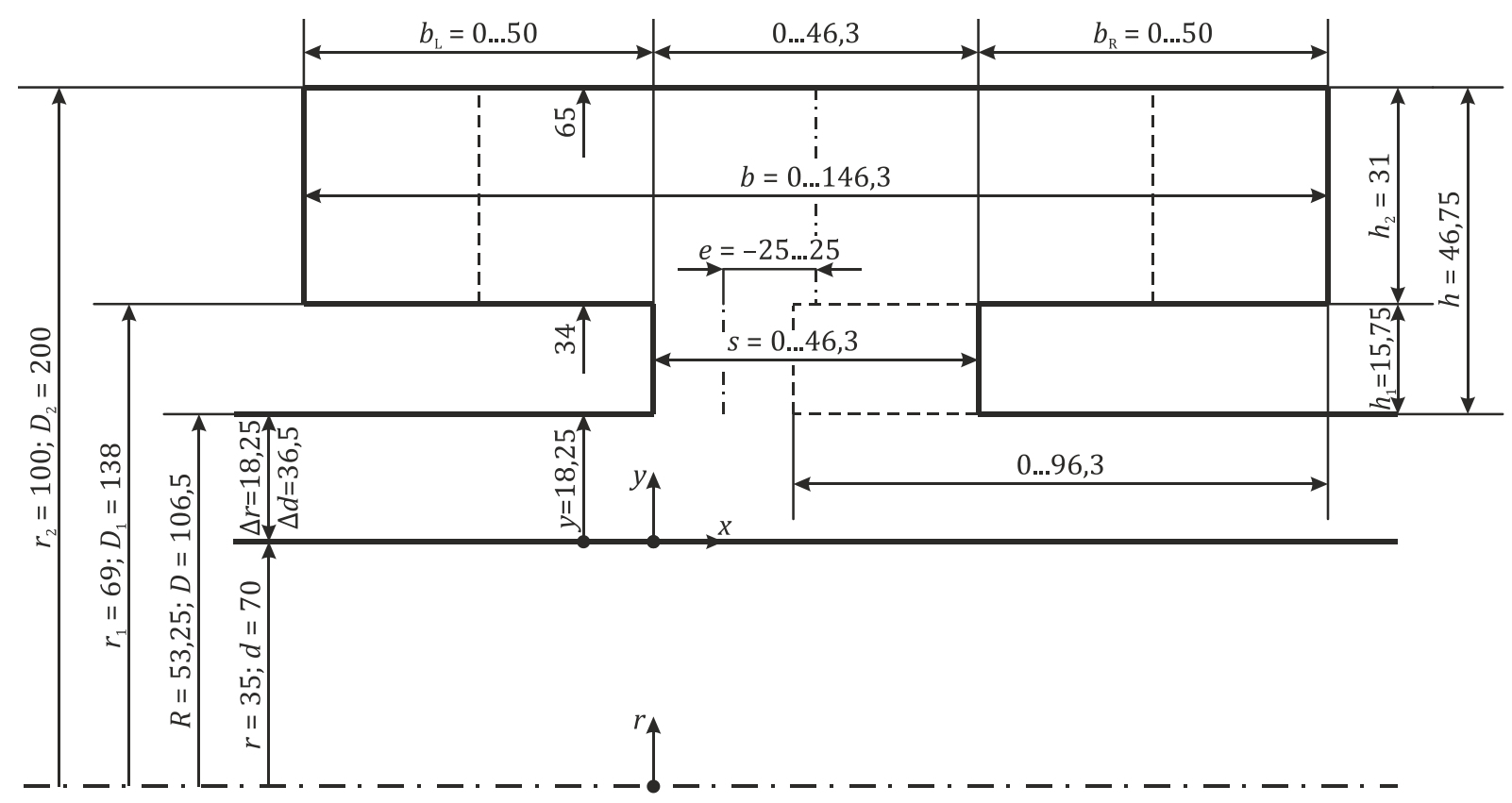

Abb. 4.4 Realisierbare Abmessungen des Seitenraumes (alle Angaben in mm).

Sämtliche Bezugsgrößen (siehe Kap. 4.4.2) und Ähnlichkeitskennzahlen werden auf diese charakteristische Länge $l$ und die (im Gegensatz zum Seitenraum) wohl definierten und gut messbaren Zustände im Hauptströmungskanal bezogen. Bei Drücken $p=(2,1 \ldots 3,4)$ bar und Temperaturen $T=(25 \ldots 45)^{\circ} \mathrm{C}$ werden dort bei einem maximalen Durchsatz von $\dot{m}=1,16 \mathrm{~kg} / \mathrm{s} \mathrm{im} \mathrm{Paral-}$ lelbetrieb der beiden Verdichter (Kap.4.1.4) querschnittsgemittelte axiale Strömungsgeschwindigkeiten

$$
q=\frac{\dot{m}}{\rho(p, T) \cdot A}
$$

von bis zu 85 m/s und Reynolds-Zahlen

$$
R e=\frac{q \cdot l}{v}=\frac{\dot{m} \cdot l}{A \cdot \eta(T)}=\frac{4 \dot{m}}{\eta(T) \cdot U}=\frac{4 \dot{m}}{\eta(T) \cdot \pi(D+d)}
$$

von maximal 430.000 erreicht ( Tab. 4.1) erreicht, was einer mit Dampfturbinenpfaden vergleichbaren hochturbulenten Strömung im Ringspalt entspricht.

Druckeffekte wie Druckverlust können auf Grund der zu geringen Euler-Zahl nicht äquivalent abgebildet werden, stehen jedoch auch nicht im Fokus der hier durchgeführten Forschungstätigkeiten. Auch schwerkraftbedingte Effekte wie Auftrieb und freie Konvektion werden in Folge der zu hohen Froude-Zahl im Versuchsstand zu klein modelliert [75], können jedoch mit dem bisherigen Wissensstand bereits verlässlich vorausgesagt werden [7]. Um freie Konvektion sogar weitestgehend auszuschließen und nur reine erzwungene Konvektion in den Seitenräumen zu untersuchen, 
werden bei den beiden zu verwendenden Messmethoden ( Kap. 4.2.1) die Temperaturunterschiede zwischen Wand und Fluid gering gehalten (maximale Übertemperatur ca. $6 \mathrm{~K}$ ). Die (partielle) Ähnlichkeit zwischen Modell und Original gewährleistet die spätere Ergebnisübertragung nach der Ähnlichkeitstheorie [7] (siehe Kap. 7).

Tab. 4.1 Realisierbare Parameter im Versuchsstand

\begin{tabular}{lcc}
\hline Größe & $\min$ & $\max$ \\
\hline$p[\mathrm{bar}]$ & 2,1 & 3,4 \\
$T\left[{ }^{\circ} \mathrm{C}\right]$ & 25 & 45 \\
$\dot{m}[\mathrm{~kg} / \mathrm{s}]$ & 0 & 1,16 \\
$q[\mathrm{~m} / \mathrm{s}]$ & 0 & 85 \\
$R e_{\Delta d}[-]$ & 0 & 430.000 \\
\hline
\end{tabular}

\subsubsection{Experimentelle Implementierung des Versuchsstandes}

Neben der präzisen Modellierung der Ähnlichkeitsverhältnisse zur Einhaltung der Ähnlichkeitsanforderungen und den Vorgaben der Druckgeräterichtlinie ( Kap. 4.1.2) sowie einer möglichst großen Variabilität der Seitenraumgeometrie ( Kap. 4.1.1) wurde bei der Konstruktion des Versuchsstandes großer Wert auf einen modularen Aufbau sowie auf einen möglichst großen Anteil von Norm- und Standardteilen gelegt, um jederzeit die einfache Auf-, Um- und Nachrüstbarkeit durch das Auswechseln einzelner Teilmodule zu ermöglichen. Der Digitale Zwilling (Digital Twin) des Versuchsstandes ist in Abb. 4.5 dargestellt. Das komplette, mit SolidWorks 2012 und 2016 erstellte CAD-Modell umfasst über 1200 Einzelkomponenten und diente als Grundlage zur Ableitung aller ca. 150 Fertigungs- und Montagezeichnungen.

Die Verbindung der Module erfolgt zum einen über die Verschraubung der Außenrohrbauteile (nahtlos gezogen; DN 100/ PN 6; $\emptyset 114,3 \times 3,6$ ) durch genormte Vorschweißflansche (Typ 11) mit Dichtleiste (Form B1) nach DIN EN 1029-1 und nachträglich eingefrästen Dichtnuten für O-Ringe aus NBR 70 nach DIN 3771/ DIN ISO 3601, zum anderen über eine schraubengesicherte und mit O-Ringen gedichtete Nippel-Muffe-Steckverbindung am ebenfalls nahtlosen Innenrohr (DN 70/ PN 6; $\varnothing 70 \times 2$,9). Alle Rohrbauteile und auch ein Großteil der sonstigen Komponenten der Module bestehen - sofern nicht anders angegeben - aus nichtrostendem austenitischem Chrom-Nickel-Stahl („V2A“; Werkstoffgruppe A2), im Wesentlichen 1.4301 (X5CrNi18-10) und 1.4541 (X6CrNiTi18-10), oder noch höher legiertem Chrom-Nickel-Molybdän-Stahl („V4A“), hauptsächlich 1.4571 (X6CrNiMoTi17-12-2; A5) und 1.4401 (X5CrNiMo17-12-2; A4). 


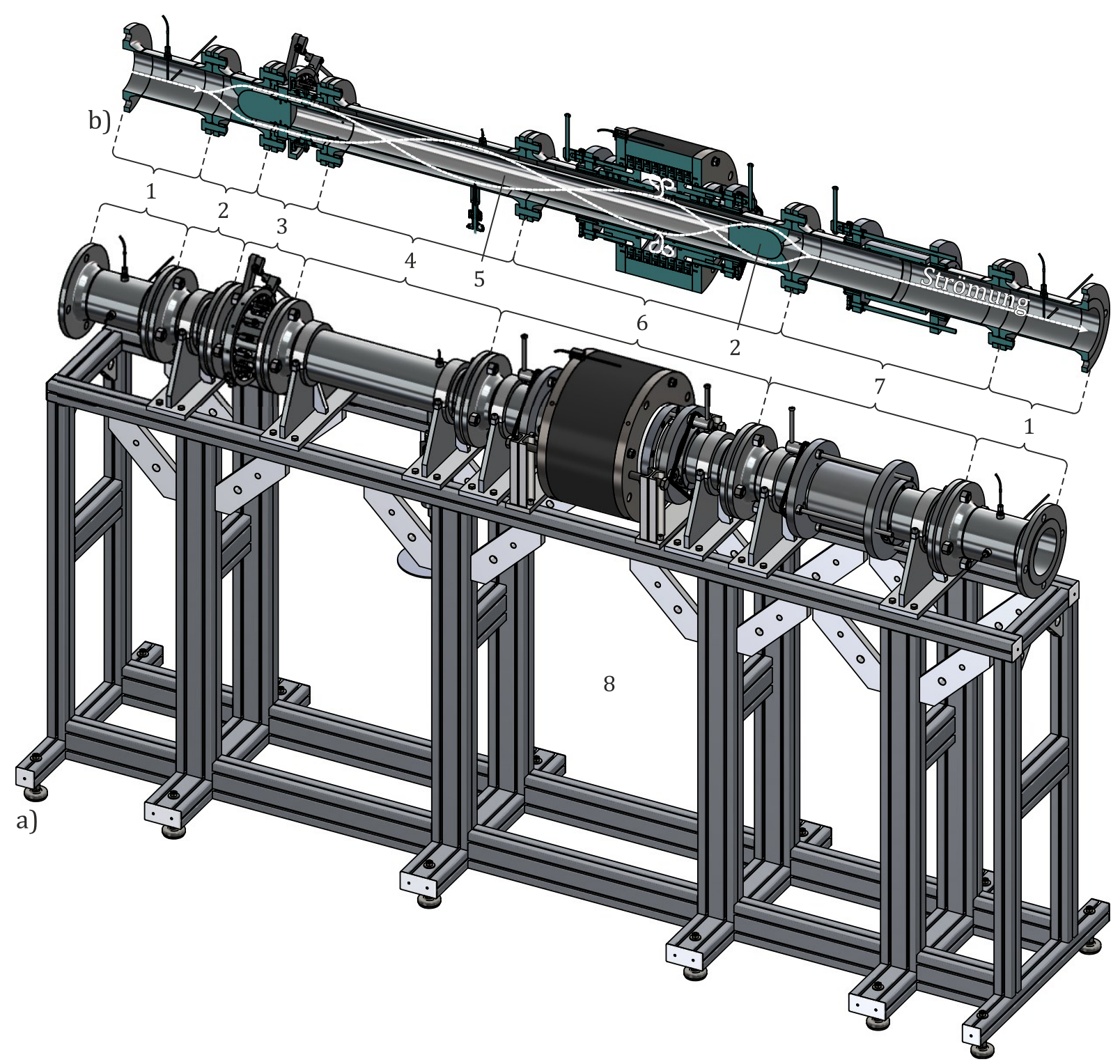

Abb. 4.5 Digitaler Zwilling (Digital Twin) des Totraumversuchsstandes.

a) Gesamtdarstellung; b) Halbschnitt des Strömungskanals;

1 - Demontagemodul (mit Wanddruckmessung, Thermistor- und Pitotsonde);

2 - Ein-/ Abströmmodul (Überführung der Strömung von Rohrquerschnitt in kreisringspaltförmigen Hauptströmungskanal und umgekehrt);

3 - Leitgittermodul - als Stützgitter mit vier Blechen $\left(\gamma=0^{\circ}\right)$, als festes Drallgitter mit 12 Blechen $\left(\gamma=15^{\circ}\right)$ oder (wie dargestellt) als verstellbares Drallgitter mit 12 Blechen $\left(\gamma=-90 \ldots 90^{\circ}\right)$;

4 - Anströmmodul (mit Referenzdruckmessstelle und Fünflochsonde);

5 - Innenrohrmodul;

6 - Totraummodul (mit verstellbaren Seitenwänden und instrumentierter Außenwand);

7 - Kompensationsmodul (zur Verstellung der Breite der Einströmöffnung zum Seitenraum);

8 - Abstützung (mit Rohrlagerschalen). 
In der aktuellen Ausbaustufe besteht der Versuchsstand aus insgesamt acht verschiedenen Modulen (Nummerierung vgl. $>$ Abb. 4.5):

Am Ein- und Auslass des Versuchsstandes befindet sich jeweils ein Demontagemodul (1), welches aus einem beidseitig geflanschten Außenrohr besteht und eine axiale Länge von 220 mm aufweist. Beide Module dienen, wie ihre Bezeichnung bereits vermuten lässt, der Demontage und dem Umbau des Versuchsstandes. Außerdem befinden sich mittig entlang des Umfangs der Mantelfläche der Demontagemodule verteilt jeweils eine Wanddruckbohrung zur Messung des statischen Druckes, eine Pitot-Sonde zur Ermittlung des Staudrucks sowie eine Thermistorsonde für die Bestimmung der Strömungstemperatur an der 3-, 9- bzw. 12-Uhr-Position $\$$ Abb. A.17; Nomenklatur gemäß Abb. 4.16).

Mit Hilfe des Ein- und Abströmmoduls (2) wird die anströmende Luft vom Rohrquerschnitt in den Kreisringspalt übergeleitet und umgekehrt ( Abb. A.18b). Der Querschnittsübergang erfolgt nach dem Vorbild von Turbinenrad-Gaszählern (PTB-Richtlinie G13/ DIN EN 12261) kontinuierlich mittels eines halbkugelförmigen Verdrängungskörpers, an dem vier radiale, viertelkreisförmige, beidseitig elliptisch abgerundete Stützrippen (Blechstärke $4 \mathrm{~mm}$, Halbachsen der elliptischen Verrundung: $2 \times 6$ ) angeschweißt sind. Beim Einströmmodul sind diese wiederum mit dem Außenrohr verschweißt und fungieren als Festlager der angeschlossenen Innenrohrbauteile. Beim Abströmmodul sind die Streben nicht mit dem Außenrohr verbunden sondern können axial in ihm gleiten und bilden somit das Innenrohrloslager.

In einem nachgeschalteten Leitgittermodul (3) kann der Ringspaltströmung optional ein Drall aufgeprägt werden. Während in der Anfangsphase zur Untersuchung drallfreier Strömung zunächst nur ein Stützgitter mit vier rechteckigen Blechen (Blechstärke $2 \mathrm{~mm}$, Halbachsen: $2 \times 4$ ) ohne Anstellwinkel $\left(\gamma=0^{\circ}\right)$ einbaut war, wurde für die Versuche zur Untersuchung des Einflusses von Fehlabströmung ein verstellbares Drallgitter (ähnlich eines Chevron-Drallerzeugers gemäß DIN EN ISO 5167-1) mit zwölf geraden (nicht konturierten), beidseitig elliptisch „angespitzten“, trapezförmigen Blechen mit derselben Blechstärke und elliptischen Verrundung wie das Stützgitter eingesetzt, bei dem der Blechwinkel $\gamma$ über einen als zweigliedriges räumliches Kugelgelenkgetriebe (Doppelschwinge/ Kurbeltrieb) mit umlaufendem Antrieb ausgeführten Verstellmechanismus (vgl. a. [77]) stufenlos zwischen $\gamma=0^{\circ}$ (keine Anstellung, d. h. rein axiale Ausrichtung) und $\pm 90^{\circ}$ (voll geschlossen) eingestellt werden kann ( Abb. A.19 a, b).

Das Anströmmodul (4) bildet gemeinsam mit dem Innenrohrmodul (5), welches Leitgitter- und Abströmmodul miteinander verbindet, eine freie Anströmstrecke ohne Einbauten, entlang derer sich die Ringspaltströmung gleichmäßig ausbilden kann und Nachlaufeffekte nach dem Leitgitter ausgeglichen werden können. 
Herzstück des Versuchsstandes ist das Totraummodul ( Abb. 4.6). Es ist in seiner Grundkonfiguration symmetrisch aufgebaut und besteht im Wesentlichen aus fünf Teilmodulen bzw. -baugruppen:

- zwei einseitig geflanschte Außenrohre zum Anschluss an das Anströmmodul (links) und an das Kompensationsmodul (rechts),

- zwei Seitenwände inklusive Verstellmechanismus und

- die Außenwand mit der Wärmeübergangsinstrumentierung.

Das Seitenwandmodul ist über eine Klemmverbindung fest auf dem Außenrohr (Pos. 1 in Abb. 4.6) verspannt. Über einen tangential angezogenen Klemmring (2), welcher aus einer gut spanbaren Aluminiumlegierung (3.1325; EN AW-2017 A; AlCuMg1) gefertigt ist, und Zylinderschrauben (3) wird ein Aluminium-Hohlzylinder (4) aus 3.1645 (EN-AW 2007; AlCu4MgPb), der an der Totraumöffnung bündig mit dem Rohrende abschließt und gemeinsam mit diesem die Höhe der Einstromöffnung $h_{1}$ herstellt, fest mit dem Grundkörper (5; Werkstoff: 3.1325) verbunden, der seinerseits den Bewegungsmechanismus für die Seitenwandverstellung im Totraum aufnimmt („Spindellager"). Dieser besteht aus vier über dem Umfang verteilte Bewegungsschrauben (sog. "Gewindespindeln“, 6) mit metrischem ISO-Gewinde (M10), deren Drehbewegung über quergestiftete Kettenräder („Ritzel“; 7) und einen Rollenkettentrieb (8) nach DIN 8192 synchronisiert wird. An einer der Spindeln ist - ebenfalls über eine Querstiftverbindung -eine Handkurbel (9) angebracht, über welche manuell die Spindeln gedreht werden können. Über die Gewindebohrungen in einem mit der Seitenwand verschraubten Aufnehmer (10; 3.1645) wird die Rotation der Spindeln in eine Linearbewegung der Seitenwand (11) umgewandelt, welche über eine schraubengesicherte Gleitfeder (12) axial geführt wird. Die Seitenwand (11) besteht aus dem Kunststoff PET, der eine geringe Wärmeleitfähigkeit und thermische Ausdehnung aufweist. Die Totraumaußenwand (13) wird über zwei Anschläge (nicht in Abb. 4.6 dargestellt) in ihrer axialen Stellung gehalten und kann bei Bedarf über je zwei Stellschrauben (M16) mit Messing-Backenmuttern axial verschoben werden (Abb. 4.5). Die Verdrehung der Außenwand erfolgt über zwei eigens angefertigte Verstellhebel mit einer Hebellänge von $60 \mathrm{~cm}$, die über Gewindestifte in radiale Bohrungen in den beiden Spannscheiben der Außenwand ( Kap. 4.2.2) eingreifen. Der Totraum ist über radial verpresste, innendichtende 0-Ringe in allen beweglichen und ruhenden Elementen pneumatisch abgedichtet. Alle 0-Ringe sind nach DIN 3771 bzw. DIN ISO 3601 ausgelegt und aus Acrylnitril-Butadien-Kautschuk mit einer Härte von 70 Shore A (NBR 70) gefertigt. 


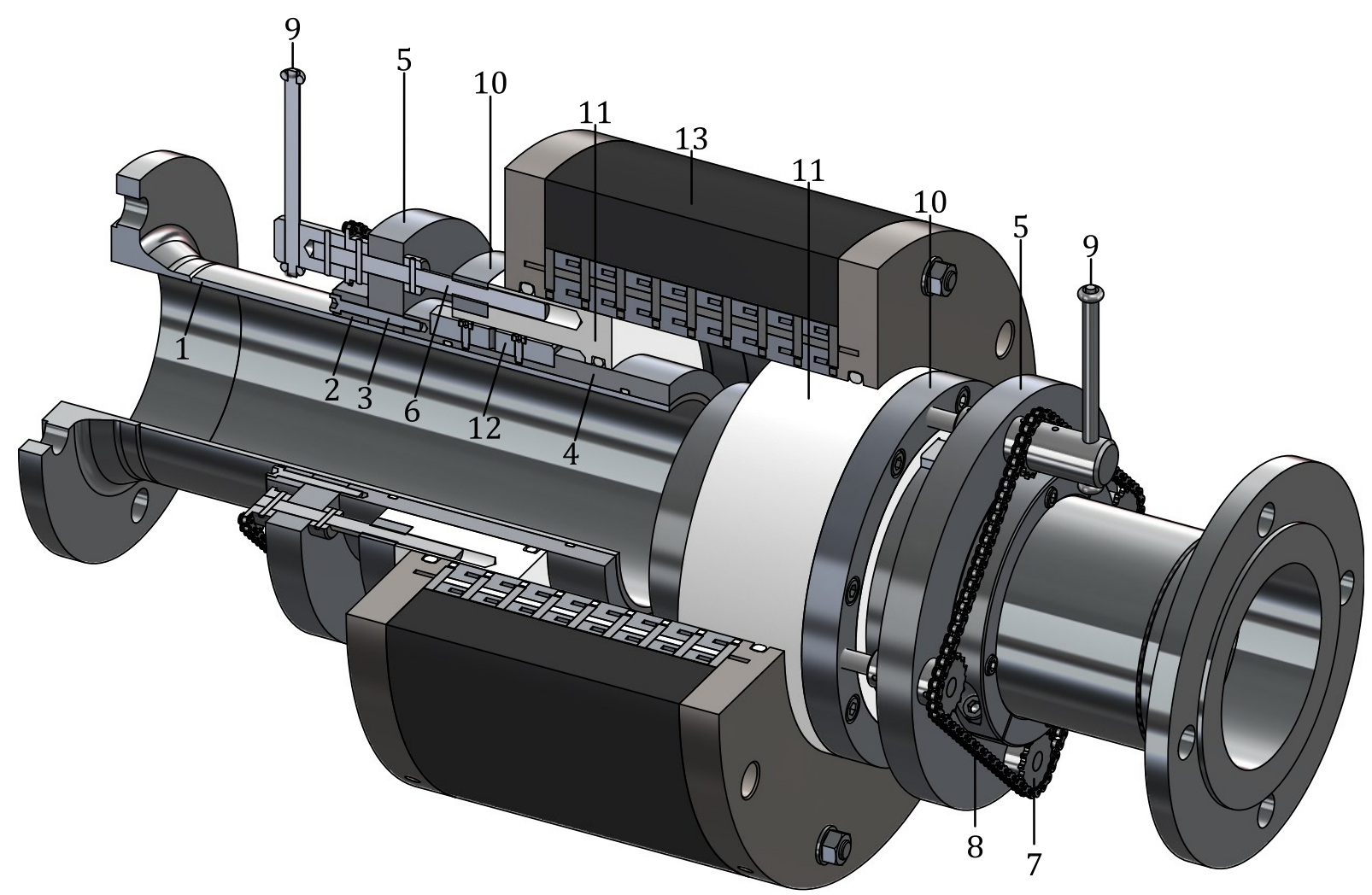

Abb. 4.6 Experimenteller Aufbau des Totraummodules (Viertelschnittdarstellung).

1 - Außenwand; 2 - Klemmring; 3 - Schraubverbindung; 4 - Seitenwand der Einströmöffnung; 5 - Grundkörper Spindellager; 6 - Gewindespindel; 7 - Kettenrad („Ritzel“); 8 - Rollenkette; 9 - Kurbel; 10 - Aufnehmer; 11 - Seitenwand; 12 - Gleitfeder; 13 - Außenwand.

Das Kompensationsmodul (Pos. 7 in Abb. 4.5) dient der Verstellung der Breite der Einströmöffnung des Totraumes, indem es den axialen Versatz aufnimmt („,kompensiert“), da die Gesamtlänge des Versuchsstandes festgelegt ist. Der Verstellmechanismus ist dabei nahezu identisch mit dem der Seitenwand des Totraummoduls. Über längere Bewegungsschrauben (Pos. 6 in Abb. 4.7) wird der Abstand zu einem auf dem gegenüberliegenden Außenrohrabschnitt befindlichen, ebenfalls über eine Klemmverbindung (2) arretierten Aufnehmer (10) verändert, wodurch der linke Außenrohrteil (1) inklusive Flansch (und inklusive des rechten Außenrohrteils des Totraummoduls) herangezogen oder abgestoßen wird. Der Innenraum des Rohres wird über eine gleitend gelagerte Führungshülse (4) abgedeckt. Die Abdichtung ist erneut mit 0-Ringen aus NBR 70 realisiert. 


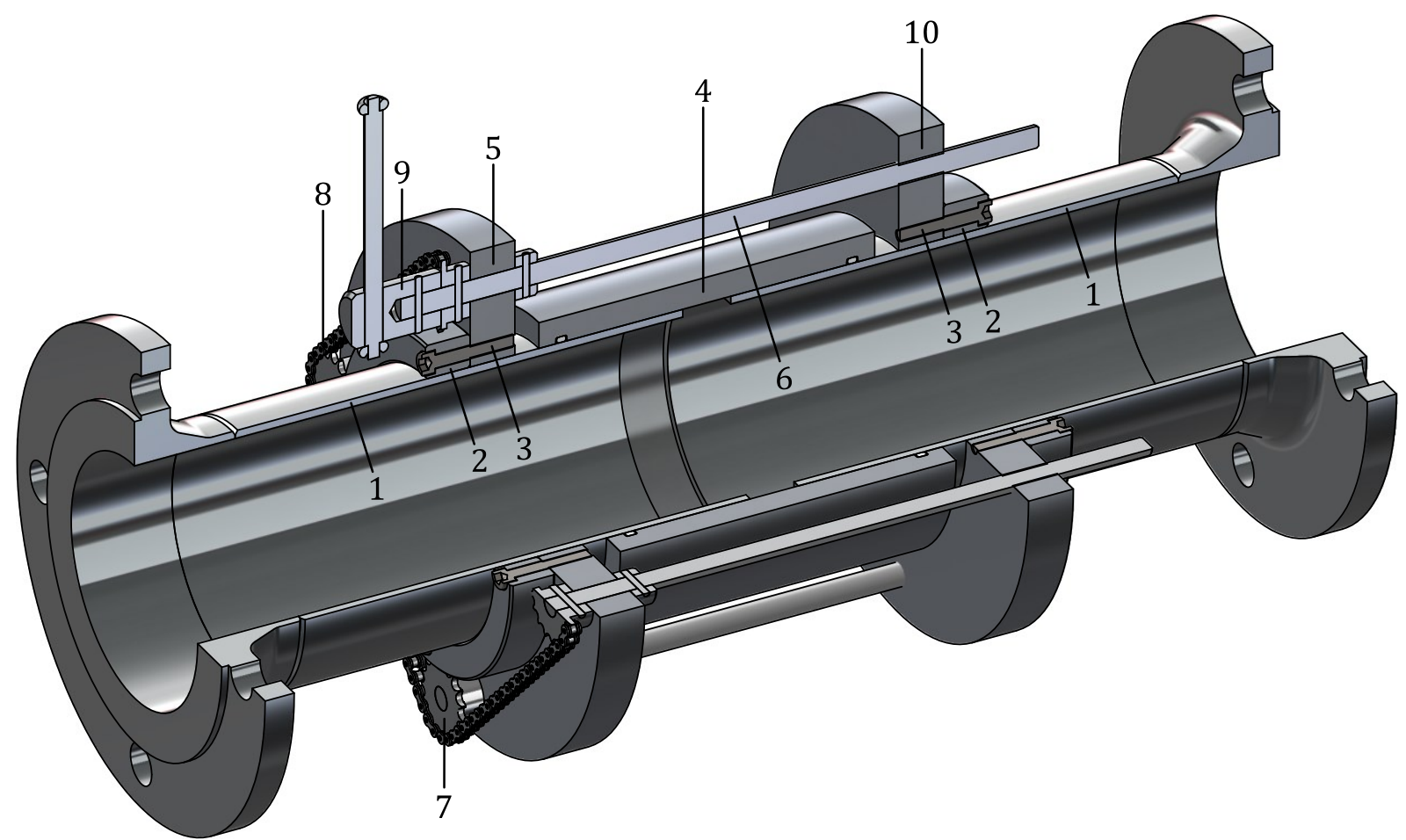

Abb. 4.7 Experimenteller Aufbau des Kompensationsmodules (Viertelschnittdarstellung).

1 - Außenwand; 2 - Klemmring; 3 - Schraubverbindung; 4 - Führungshülse;

5 - Grundkörper Spindellager; 6 - Gewindespindel; 7 - Kettenrad („Ritzel“); 8 - Rollenkette;

9 - Kurbel; 10 - Aufnehmer.

Die Abstützung der Rohrstrukturen des Versuchsstandes (Pos. 8 in Abb. 4.5) erfolgt durch eine Konstruktion aus Aluminiumprofilen $(45 \times 45$ bzw. $45 \times 90)$. Die Rohrleitung ist dabei an mehreren Stellen in Rohrlagerschalen eingelegt, um eine nennenswerte Eigenlastdurchbiegung der Rohre infolge ihrer Schwerkraft auszuschließen. Die Rohrmitte liegt dabei in einer ergonomischen Arbeitshöhe von 1,10 m über dem Boden. Die Rohrlagerschalen können bei Bedarf in ihrer axialen Position entlang der beiden Führungsschienen verschoben werden. Damit die einzelnen Module exakt fluchten und der Verstellmechanismus der Seitenraumöffnung ohne Verkanten gleiten kann, wurden die Lagersitze der Rohrschalen CNC-gefräst und die Außenrohre an allen Auflagerpunkten auf exakten Rundlauf überdreht, sodass sich eine enge Spielpassung, eine hohe Konzentrizität und damit ein hohes Maß an Geradheit der montierten Rohrleitung ergeben. Durch das nachträgliche Abdrehen der Vorschweißflansche aller Module wird ein Verziehen der Rohrleitung beim Verschrauben vermieden. Das Rohr bleibt so im montierten Zustand kraftfrei. Dieses wird abschließend an exponierten Lagerpunkten durch aufgesetzte Rohrschellen in seiner axialen Lage gesichert. 


\subsubsection{Versuchsanlage zur geregelten Druckluftbereitstellung für den Versuchsstand}

Für den Anschluss des neu konzipierten Versuchsstandes wurde die bestehende Druckluft-Versuchsinfrastruktur um einen neuen Strang erweitert und entsprechende Armaturen und Messstellen vorgesehen. Ein vereinfachtes Schaltschema findet sich in Abb. 4.8, ein vollständiges Rohrleitungs- und Instrumentenfließschema in Abb. A.8 und ein Installationsplan des NeubauStranges in Abb. A.9 im Anhang A6.

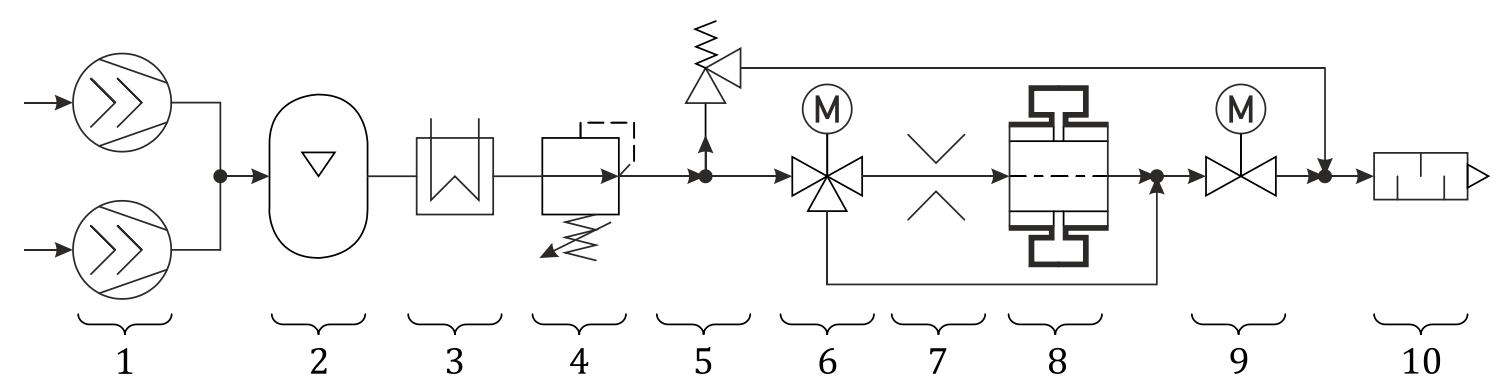

Abb. 4.8 Vereinfachtes Schaltschema der erweiterten Druckluftversuchsanlage mit Druck-Durchfluss-Regelung und Massenstrommessung zum Betrieb des Seitenraumversuchsstandes: 1 - Schraubenverdichter; 2 - Druckluftspeicher; 3 - Druckluftnachkühler;

4 - Druckreduzierventil; 5 - Sicherheitsabblaseventil; 6 - Dreiwegeventil; 7 - Messblende;

8 - Versuchsstand; 9 - Motorstellventil; 10 - Ausblaseschalldämpfer.

Als Druckluftquelle stehen zwei öleingespritzte Schraubenkompressoren zur Verfügung ( Abb. A.10). Beide Verdichter sind standardmäßig luftgekühlt und besitzen einen Ölabscheider sowie einen Absorptionskältetrockner, der die ins Netz gespeiste Druckluft entfeuchtet.

Der kleinere der beiden Verdichter ist drehzahlgeregelt (variable speed drive) und liefert je nach Abnahmemenge bis zu 0,571 kg/s Luft. Dies entspricht einem auf Standardansaugbedingungen $\left(p_{\mathrm{S}, \mathrm{N}}=1 \mathrm{bar}, T_{\mathrm{S}, \mathrm{N}}=20^{\circ} \mathrm{C}, \varphi_{\mathrm{S}, \mathrm{N}}=0 \%\right)$ bezogenen Referenzvolumenstrom nach ISO 1217 von $0,481 \mathrm{~m}_{\mathrm{N}}^{3} / \mathrm{s}$. Durch das kontinuierliche Anpassen der Motordrehzahl (600 bis $3085 \mathrm{~min}^{-1}$ ) kann der Netzdruck auf einem eingestellten Sollwert zwischen 4 und 12,5 bar $_{\ddot{U}}$ konstant gehalten werden. Der größere Verdichter kann für Betriebsüberdrücke zwischen 4 und 7,5 barü eingesetzt werden. Da der Verdichter nicht drehzahlregelbar ist, speist er unter konstanter Drehzahl von $n \equiv 1923 \mathrm{~min}^{-1}$ einen Massenstrom von $0,716 \mathrm{~kg} / \mathrm{s}$ (entsprechend 0,603 $\mathrm{m}_{\mathrm{N}}^{3} / \mathrm{s}$ ) ins System. Durch eine Ein- und Abschaltsteuerung wird durch automatische Be- und Entlastung des Verdichters der Netzdruck innerhalb programmierbarer Druckgrenzen ( $p_{\min }$ und $p_{\max }$ ) gehalten (Dreipunktregelung durch „intermittierende Betriebsweise“).

Beide Verdichter können einzeln im „Solobetrieb“ oder auch parallel betrieben werden, um den Durchsatz weiter zu steigern. $\$$ Tab. A.1 in $>$ Anhang A6 fasst alle Einstellungen an den Steuerungen der Schraubenverdichter zusammen. 
Beim Parallelbetrieb der beiden Kompressoren war es in Folge zu geringer Zu- und Abluftquerschnitte jedoch innerhalb kürzester Betriebsdauer (ca. 1 h) zu einer unzulässig hohen Aufheizung des Raumes und damit zur Überhitzung und Sicherheitsabschaltung der Verdichterelemente gekommen, was einen Messbetrieb über mehrere Stunden unmöglich machte. Im Zuge dieser Arbeit wurde daher je ein zusätzlicher wasserbetriebener Ölkühler („Energierückgewinnungssystem“) in die Ölkreisläufe der beiden Verdichter eingebracht ( Abb. A.10), um einen Großteil (bis zu 75 \%) der Kompressionswärme über das gebäudeinterne Kühlwassersystem abzuführen. Durch diese Maßnahme konnte der Prüfstand dauerhaft unter kontrollierten Bedingungen gefahren werden. Über eine gemeinsame Druckleitung (DN 100/ PN 16, innen und außen verzinkt) strömt die Verdichterluft mit einer Temperatur von ca. $25{ }^{\circ} \mathrm{C}$ durch einen nachgeschalteten Druckluftspeicher mit einem Fassungsvermögen von $6 \mathrm{~m}^{3}$, der auf Durchzug geschaltet ist. Dieser Windkessel reduziert Druckschwankungen in dem offenen Luftsystem (open-type system) und glättet damit den zeitlichen Verlauf des Massenstromes. Ein weiterer nachgeschalteter Luftfilter scheidet die letzten verbliebenen Ölpartikel ab, wodurch technisch ölfreie Druckluft für den Versuchsstandbetrieb zur Verfügung steht.

Mittels eines zusätzlich nachgeschalteten wassergekühlten Druckluftnachkühlers in Gestalt eines Rippenrohrbündelwärmeübertragers ( Abb. A.10) kann die restliche überschüssige Wärmemenge aus der Strömung abgeführt und die Temperatur der Druckluft durch entsprechende Ansteuerung des wasserseitigen Zulaufventils über das Prozessleitsystem auf einen anzugebenden Zielwert ausgeregelt werden. Dieser Nachkühler stand erst in einer späteren Projektphase zur Verfügung, sodass die ersten Versuche noch ohne Temperierung stattgefunden haben (siehe Kap. 5.7.2).

Während zuvor nur ein Prüfstand an die Druckluftinfrastruktur angeschlossen war, wurde die bestehende Anlage für den Betrieb des neu zu errichtenden Seitenraumversuchsstandes über einen zweiten Strang wie folgt erweitert ( Abb. A.9): direkt nach dem Durchbruch der Abzweigleitung (Abb. A.11 a) in den Raum, in dem auch der Versuchsstand platziert wird, ist eine elektrisch betätigte Absperreinrichtung mit Stellantrieb in Form eines Motorkugelhahns angeordnet, mit der der Strang bei Nichtbenutzung vom Betriebspanel aus ( Abb. A.12) vollständig abgesperrt werden kann. Er ist zugleich wesentlicher Bestandteil einer neu etablierten Sicherheitsschaltung, zu der noch ein weiterer baugleicher Motorkugelhahn im bereits bestehenden Versuchsstrang gehört. Das Sicherheitskonzept sieht vor, dass immer nur ein Strang betrieben werden kann, während der andere vollständig abgesperrt sein muss. Anderenfalls können die Verdichter nicht in Betrieb genommen werden. Die Freigabe der Stränge erfolgt zentral von der Messwarte aus. Ein Rücksetzen und Umschalten ist immer nur bei geschlossenen Motorkugelhähnen 
und ausgeschalteten Verdichtern möglich. Vor dem erneuten Anfahren der Anlage muss der automatisch ausgelöste Notauszustand an den zu betreibenden Verdichtern aufgehoben werden.

Weil beide Verdichter nur bei minimal 5 bar betrieben werden können, um den internen Ölkreislauf aufrecht zu erhalten, ist eine Absenkung des Druckniveaus notwendig, um beim Betrieb des Seitenraumversuchsstandes nicht mit der Druckgeräterichtlinie (DGRL 2014/68/EU) in Berührung zu kommen (Art. 4, Abs. 3). Dies wird durch ein selbsttätiges, federbelastetes Druckreduzierventil realisiert (Abb. A.11 a), welches den Vordruck $p_{1}$ von 6 bar auf einen einstellbaren Sollwert $p_{2}$ zwischen 2,1 und 3,4 bar drosselt. Der Druckminderer ist demnach als überkritisches Ventil mit

$$
\frac{p_{2}}{p_{1}} \leq\left(\frac{2}{\kappa+1}\right)^{\frac{\kappa}{\kappa-1}}=0,528
$$

ausgeführt und begrenzt über seine kritische Querschnittsfläche (Vena contracta) den maximal möglichen Durchsatz durch den Strang. Je nach gewünschter Abnahmemenge kann durch Eindrehen der Stellschraube die Drosselung verkleinert und damit der Hinterdruck und der Durchsatz vergrößert werden.

Ein weiterer Handkugelhahn dient der zur manuellen Nachdrosselung des Luftstroms und optional zur Druckhaltung des Druckminderers bei ausgeschalteten Verdichtern und abgesperrtem System, wird jedoch nicht genutzt, sondern ausschließlich im Durchgang betrieben.

Um das „Niederdruck“-System vor einem unzulässigen Druckanstieg (wie etwa im sehr unwahrscheinlichen Fall des Membranrisses im Druckminderer) zu schützen, wird ein Sicherheitsüberdruckventil nachgeschaltet ( Abb. A.11 a), welches bei Überschreitung eines Auslösedruckes von 4,5 bar proportional öffnet und einen Teil des Luftstromes via der Ausblasleitung über Dach nach außen abführt.

Parallel dazu wurde noch eine weitere redundante Sicherheitsmaßnahme vorgesehen. Mittels eines Piezosensors wird dauerhaft der Druck nach dem Druckminderer gemessen. Bei Überschreiten eines Grenzwertes von 3,7 bar wird über eine integrierte elektronische Schaltung ein potenzialfreier Kontakt geschaltet, der den Motorkugelhahn schließt und die Verdichter ausschaltet und verriegelt, bis der Druck wieder unter die Schaltgrenze gefallen ist. Das mechanische Sicherheitsventil dient demnach nur als Ultima Ratio, wenn die Druckwächterschaltung ausfallen sollte. Der Massenstrom durch den Hauptstrang, in dem sich der Versuchsstand befindet, kann über ein zweites Betriebspanel ( Abb. A.12) indirekt mittels des nachfolgenden elektrisch angetriebenen Dreiwegeventils („3WV“) eingestellt werden, welches zwischen 0 und $100 \%$ der Abnahmeluftmenge über eine Bypass-Leitung an ihm vorbei fördern kann. 
Die Messung erfolgt direkt über eine Normblende ( Abb. A.13). Die Ringkammerblende mit Eckdruckentnahme und die Blendenmessstrecke wurden nach DIN EN ISO 5167-1 und 2 für minimale Messunsicherheiten ausgelegt [75]. Daher wurde auf ausreichend lange An- und Abströmstrecken geachtet. Durch einen zusätzlichen Zanker-Strömungsumformer nach DIN EN ISO 5167-2 stromaufwärts vor der Blende wird das Strömungsprofil in der Anströmung noch weiter vergleichmäßigt. Über den Austausch der Blendeneinsätze mit unterschiedlichen Bohrungsdurchmessern $(\varnothing d=40$ bzw. $71 \mathrm{~mm}$ ) kann der Messbereich der Blende für hohe und niedrige Durchfluss-Reynolds-Zahlen gewechselt werden. Für alle im Rahmen dieser Arbeit durchgeführten Versuche wurde durchgängig die 71-mm-Blende verwendet.

Bevor die Luft in den Versuchsstand einströmt (Abb. A.14), wird mithilfe eines weiteren, unmittelbar nach dem letzten Rohrkrümmer vor der Testsektion angeordneten ZwischenflanschStrömungsumformers mit Wabenstruktur und herausgebogenen Blechkrampen, vor dem ein Turbulenzsieb mit rechteckigem Gitter (Drahtdurchmesser $d=0,32 \mathrm{~mm}$, Maschenweite $a=2 \mathrm{~mm}$ ) eingelegt ist ( Abb. A.18 a) und dem eine Beruhigungsstrecke $\left(L / d_{\mathrm{h}}=10\right)$ folgt, ein voll entwickeltes, turbulentes Strömungsprofil erzeugt. Vor und nach dem Versuchsstand sind jeweils Wellrohrkompensatoren mit Führungsrohr (Innendurchmesser $106 \mathrm{~mm}$ ) angeordnet. Diese gleichen möglichen Axialversatz während der Montage sowie eventuell auftretende thermische Dehnungen im Betrieb aus (die Umgebungstemperatur kann je nach Jahreszeit zwischen 15 und $35^{\circ} \mathrm{C}$ variieren) und stellen somit den kraft- und momentfreien Anschluss des Versuchsstandes sicher. Der über die Faltenbälge wirkende Axialschub infolge Innendruck ist demgegenüber als gering einzuschätzen. Direkt nach dem letzten Kompensator strömt die Luft nach hinten ab ( Abb. A.14), bevor sich der Hauptstrang mit dem Bypass wieder vereint ( Abb. A.11 b).

Am Ende von Bypassleitung und Hauptstrang befinden sich noch zwei weitere Handkugelhähne, mit denen bei Bedarf beide Stränge manuell nachgedrosselt oder abgesperrt werden können, etwa zur Druckdichtheitsprüfung. Unmittelbar nach der Wiedereinmündung der Bypassleitung ist ein Motorstellventil („MSV“) angeordnet, mit dem das Druckniveau im Niederdrucksystem und damit auch im Versuchsstand eingestellt werden kann. Dahinter wird die Luft über Dach in die Umgebung abgeblasen, an dessen Ende noch ein Ausblaseschalldämpfer angeordnet ist ( Abb. A.15), der nach eigenen Messungen den A-bewerteten Schallpegel unmittelbar an der Mündung (Kugelradius $S R=1 \mathrm{~m})$ bei maximalem Massenstrom von vorher $115 \mathrm{~dB}(\mathrm{~A})$ auf unter $48 \mathrm{~dB}(\mathrm{~A})$ senkt. 


\subsection{INSTRUMENTIERUNG}

\subsubsection{Wärmeübergangsmessung im Seitenraum}

Zur Bestimmung der lokalen Wärmeübergangskoeffizienten entlang der Innenoberfläche der den Seitenraum radial nach außen hin begrenzenden Außenwand kommt ein speziell entwickelter Scheibenaufbau (Abb. 4.9) aus acht identischen Messmodulen (sog. „Messringen“) zur Anwendung, die zwischen zwei Stahlflanschen mit Gewindespindeln verspannt sind. Jeder dieser Messringe beherbergt jeweils die Instrumentierung für die beiden zu verwendenden, rückwirkungsarmen Messmethoden:

Für die stationäre inverse Methode besteht jedes WÜK-Messmodul aus drei aufeinander geschrumpften konzentrischen Ringen und besitzt eine eigene, bifilare Heizwicklung aus eloxiertem Konstantandraht an der Außenoberfläche, welcher in einer eingefrästen und geschwärzten Gewindenut eingeklebt ist ( Abb. A.20 a und Abb. A.21 b) und über den durch Bestromen für jeden Ring ein einstellbarer, flächiger Wärmestrom im Watt-Bereich appliziert werden kann. Innenund Außenring bestehen aus einer Aluminiumlegierung (EN-AW 2007/ 3.1645) mit hoher Wärmeleitfähigkeit, um eine homogene Temperatur in den Ringen zu gewährleisten. Der Mittelring wurde aus weniger thermisch leitfähigem PTFE gefertigt und dient als sog. Hilfswand, um eine ausreichend hohe und daher gut messbare Temperaturdifferenz zwischen Innen- und Außenring zu generieren. Um Wärmeverluste zu den benachbarten Ringen oder Flanschen zu minimieren, sind die Messmodule durch Polystyrolhalbschalen ( Abb. A.21 a) und PTFE-Dichtringe zueinander thermisch isoliert sind. Letztere dienen auch der Zentrierung der Module untereinander sowie als Abstandshalter. Dadurch wird ein metallischer Kontakt zwischen den Innenringen vermieden. Die verbleibenden schmalen Spalte sind mit Luft gefüllt (siehe Abb. 4.9). Eine Isolation aus einem geschlossenporigen Elastomerschaum bildet eine Wärmebarriere des Scheibenaufbaus gegenüber der Umgebung ( Abb. A.22). Materialtemperaturen werden mit Hilfe eingeklebter, kalibrierter NTC-Thermistoren (Kaltleiter) an jeweils vier äquidistanten Umfangspositionen $\left(4 \times 90^{\circ}\right)$ mittig in jedem Aluminiumring sowie in beiden Stahlflaschen gemessen. Zur Verringerung des Ableitfehlers (Wärmeaustrag am Messpunkt über die Messleitungen) werden die Drähte erst noch über einen Winkel von $45^{\circ}$ (entlang der Isothermen) in Umfangsnuten verlegt, bevor sie nach außen durchgeführt werden (Abb. A.20 a). Thermistoren bieten als elektrische Widerstände einen höheren Messeffekt und als passive Bauteile ein geringeres Rauschen gegenüber konventionell eingesetzten Thermoelementen. Zur Bilanzierung der axialen und radialen Verlustwärmeströme sowie der tatsächlich in das Fluid abgegebenen Wärmemenge kommen verschiedene Ansätze in Frage (siehe Kap. 4.4). 


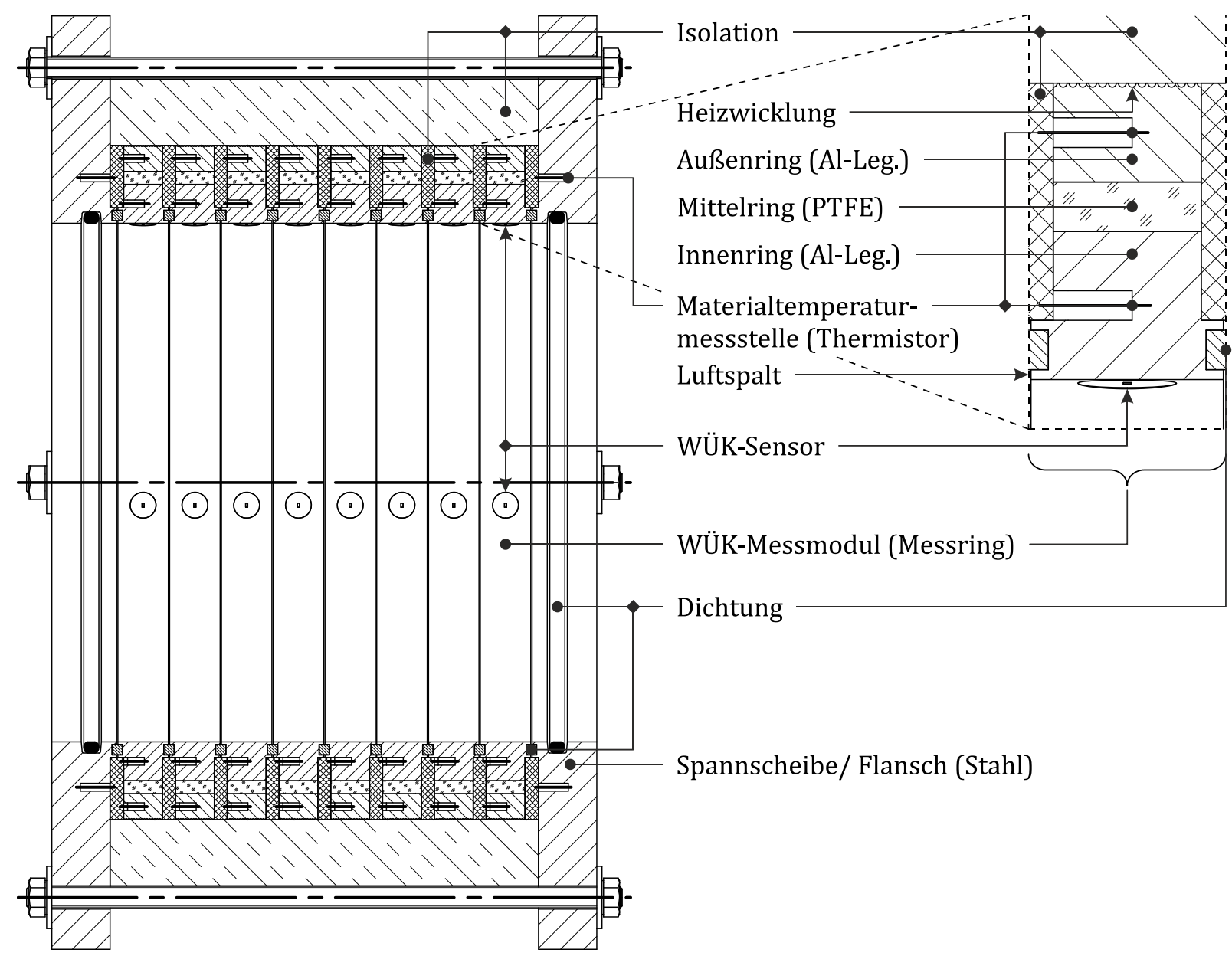

Abb. 4.9 Scheibenaufbau der Seitenraumaußenwand aus acht baugleichen, instrumentierten Messringen zur rückwirkungsarmen Bestimmung lokaler Wärmeübergangskoeffizienten im Seitenraum mittels stationärer inverser und lokaler Übertemperaturmethode.

Speziell angefertigte WÜK-Sensoren (auch WÜ- oder kurz $\alpha$-Sensoren genannt) kommen für die Anwendung der lokalen Übertemperaturmethode zum Einsatz [78,79]. Diese werden von der Professur für Magnetofluiddynamik, Mess- und Automatisierungstechnik der TU Dresden und der dortigen Arbeitsgruppe „Messtechnik für thermische Energiemaschinen“ unter Leitung von Dr.-Ing. habil. Wieland Uffrecht entwickelt und seit Jahren erfolgreich für unterschiedliche Anwendungsfälle eingesetzt, unter anderem in Prallstrahlanordnungen [80] und in rotieren Scheiben und Kavitäten bis $n=11.000$ min $^{-1}$ [81-85] unter Verwendung von Telemetrie.

Der hier verwendete WÜK-Sensor besteht aus einem NTC-Thermistor mit einer effektiven Sensoroberfläche von $A_{\mathrm{Th}}=0,8 \times 1,6 \mathrm{~mm}^{2}$, der in eine offenporige Aerogel-Tablette $(\varnothing 10 \times 5)$ aus Phenolharzschaum eingebettet ist (Abb. A.20 b, c). Die Sensortabletten werden bündig in die Innenoberfläche der Aluminium-Innenringe eingesetzt und in zwei axialen Reihen in einem Abstand von $90^{\circ}$ angeordnet, wobei der Winkelversatz relativ zu den Materialtemperaturmessstellen $5^{\circ}$ beträgt ( Abb. A.20 a und Abb. A.21 a). 
Solange eine Spannung an den Thermistor angelegt wird, dissipiert er aufgrund seines ohmschen Widerstandes $R$ einen kleinen Wärmestrom (elektrische Verlustleistung $P$ ) im Milliwatt-Bereich ( Abb. 4.10). Durch Eigenerwärmung steigt seine Temperatur $T_{\mathrm{Th}}$ an, wodurch in Folge des negativen Temperaturkoeffizienten des Halbleitermaterials sein Widerstand abnimmt. Je nach Größe des lokal vorherrschenden WÜK stellt sich ein mehr oder weniger großer, punktueller Wärmestrom $\dot{Q}(t)$ ein, der ans Fluid abgegeben wird. Durch die Schaumstoffisolation fließt nur ein geringer Teil der Wärme durch die Isolation in die Wand.

Aus dem zeitlichen Verlauf (Sprungantwort) der Thermistortemperatur $T_{\mathrm{Th}}(t)$, welche sich aus der Temperaurabhängigkeit des elektrischen Widerstandes $(R=U / I)$ des Halbleiters ergibt, und der Wirkleistung $(P=U \cdot I)$ kann zum Beispiel durch Kurvenregression unter Zuhilfenahme eines Netzwerkmodelles aus thermischen Widerständen und Kapazitäten direkt der lokale Wärmeübergangwiderstand $R_{\alpha}$ approximiert und aus ihm der lokale WÜK $\alpha$ berechnet werden. Eine separate Bestimmung der Bezugstemperatur entfällt. Als Referenz dient die Temperatur des Thermistors zum Einschaltzeitpunkt („adiabate Wandtemperatur“) [73].

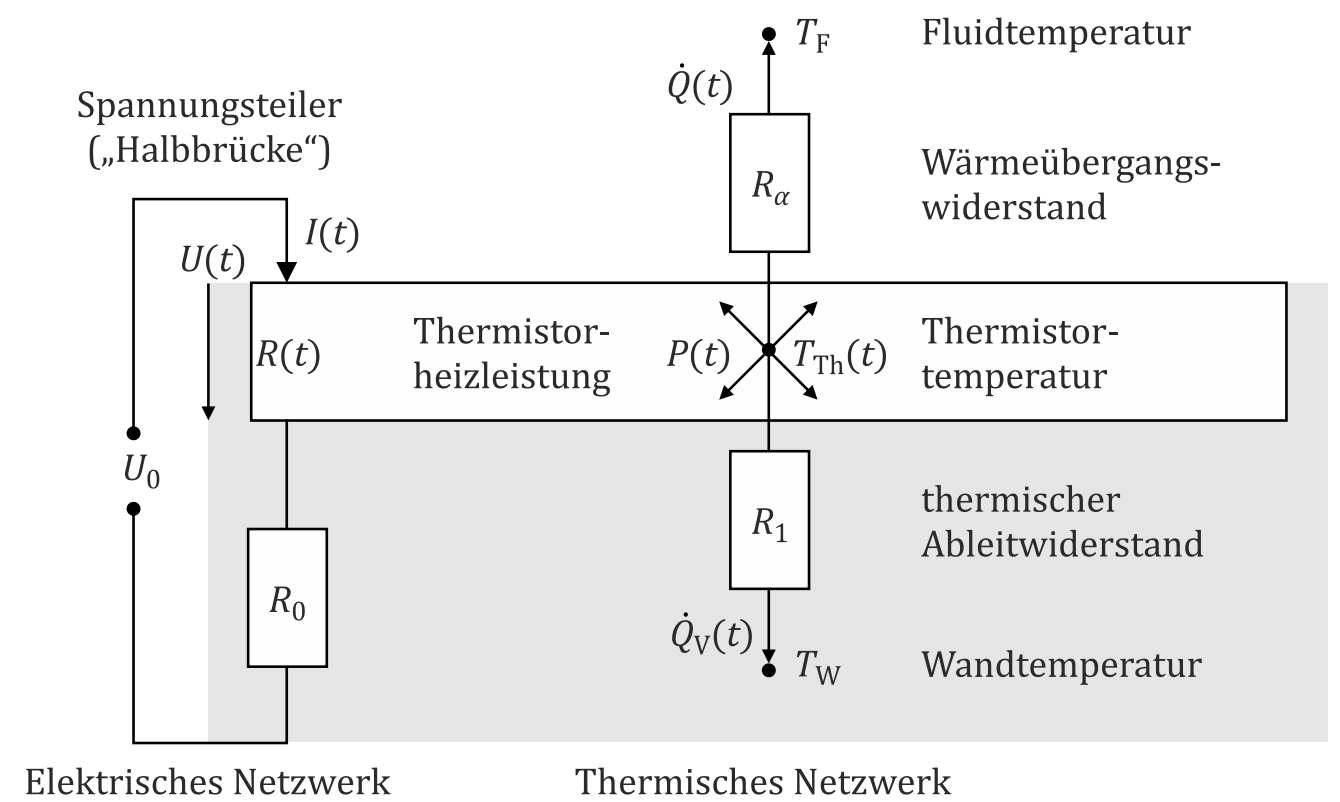

Abb. 4.10 Elektrisch-thermisches Netzwerkmodell des WÜK-Sensors (vereinfacht).

Wärmeabgabe eines in einer Messbrücke verschalteten Thermistors an Fluid und Wand 
Auf Grund der geringen Abmessungen der aktiven Fläche erfasst der Sensor den Wärmeübergang nur in der unmittelbaren Umgebung des Thermistors. Dies gestattet die Auflösung lokal begrenzter WÜ-Effekte und eine hohe Messstellendichte. Wegen der geringen Aufheizung hat der Sensor nahezu keinen Einfluss auf das Strömungsfeld und auf die Temperatursituation der Wandstrukturen [73]. Dadurch können beide Verfahren gleichzeitig eingesetzt werden, ohne dass sie sich gegenseitig beeinflussen.

Tab. 4.2 zeigt noch einmal einen zusammenfassenden Vergleich der beiden Messmethoden (vgl. hierzu auch Abb. 4.11). Im Fokus dieser Arbeit soll die stationäre inverse Methode stehen. Die lokale Übertemperaturmethode wird ausführlich in der Dissertation von Dipl.-Ing. Gunter Eschmann beschrieben [73] und soll hier nur zu Validierungszwecken herangezogen werden.

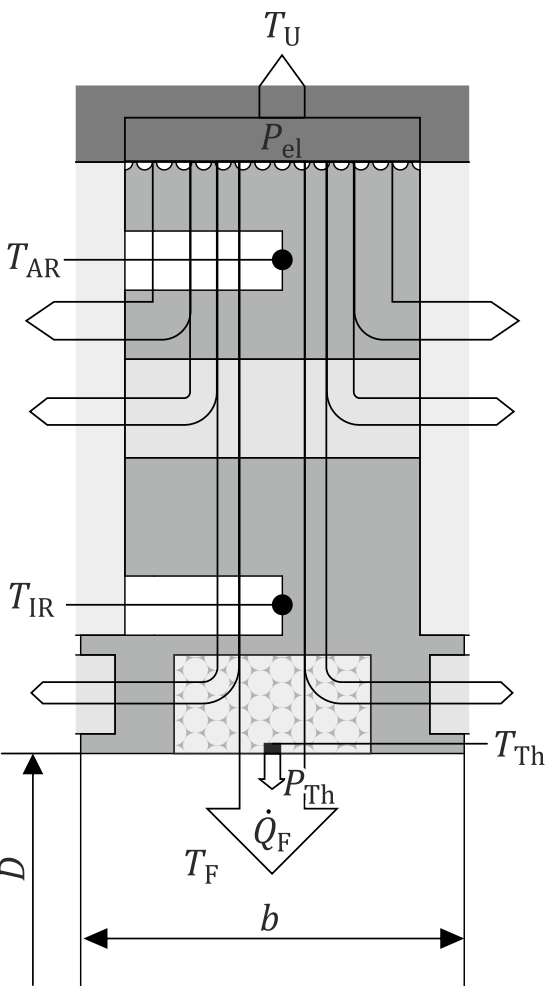

Abb. 4.11 Prinzipskizze zur Funktionsweise der beiden eingesetzten Messverfahren

Tab. 4.2 Vergleich der eingesetzten Messmethoden zur rückwirkungsarmen Bestimmung des lokalen WÜK in jedem Messmodul

\begin{tabular}{|c|c|c|}
\hline & Stationäre inverse Methode & Lokale Übertemperaturmethode \\
\hline $\begin{array}{l}\text { Aufprägen des } \\
\text { Wärmestroms }\end{array}$ & $\begin{array}{l}\text { Äußerer, flächiger Wärmestrom } P_{\mathrm{el}} \\
\text { über Heizwicklung am Außenring (AR) }\end{array}$ & $\begin{array}{l}\text { Punktueller Wärmestrom } P_{\text {Th }} \text { auf der } \\
\text { Wandinnenseite durch Aufheizung ei- } \\
\text { nes Thermistors }\end{array}$ \\
\hline $\begin{array}{l}\text { ermittelte Mess- } \\
\text { größen }\end{array}$ & $\begin{array}{l}\text { Materialtemperaturen am Außenring } \\
\left(T_{\mathrm{AR}}\right) \text { und Innenring }\left(T_{\mathrm{IR}}\right) \text { sowie Fluid- } \\
\left(T_{\mathrm{F}}\right) \text { und Umgebungstemperatur }\left(T_{\mathrm{U}}\right)\end{array}$ & $\begin{array}{l}\text { Aufheizkurven von Thermistor- } \\
\text { (über)temperatur } T_{\mathrm{Th}} \text { und -wär- } \\
\text { mestrom } P_{\mathrm{Th}} \text { aus elektrischem Wider- } \\
\text { stand }(U / I) \text { bzw. Leistung }(U \cdot I)\end{array}$ \\
\hline \multirow[t]{3}{*}{$\begin{array}{l}\text { Berechnung des } \\
\text { WÜK }\end{array}$} & $\begin{array}{l}\text { Berechnung des tatsächlichen Wär- } \\
\text { mestroms an Fluid } \dot{Q}_{\text {F }} \text { und des WÜK für } \\
\text { jeden Ring mittels 1D-Netzwerk- oder } \\
\text { 2D-FE-Modells (invers) }\end{array}$ & $\begin{array}{l}\text { Netzwerkmodell aus thermischen Wi- } \\
\text { derständen und Kapazitäten zur Er- } \\
\text { mittlung des lokalen WÜ-Widerstandes } \\
\text { an jedem Sensor }\end{array}$ \\
\hline & & $P_{\mathrm{Th}}=\left(T_{\mathrm{Th}, \infty}-T_{\mathrm{Th}, 0}\right) \cdot\left(\frac{1}{R_{\alpha}}+\frac{1}{R_{1}}\right)$ \\
\hline & $\alpha=\frac{\dot{Q}_{\mathrm{F}}}{\pi \cdot D \cdot b \cdot\left(T_{\mathrm{IR}}-T_{\mathrm{F}}\right)}$ & $\alpha=\frac{1}{R_{\alpha} \cdot A_{\mathrm{Th}}}$ \\
\hline $\begin{array}{l}\text { Größe des Wär- } \\
\text { mestromes }\end{array}$ & Watt-Bereich (ca. 4 W) & Milliwatt-Bereich \\
\hline $\begin{array}{l}\text { Größe der Über- } \\
\text { temperatur }\end{array}$ & $(2 \ldots 8) \mathrm{K}$ & $(2 \ldots 8) \mathrm{K}$ \\
\hline Beurteilung & \multicolumn{2}{|c|}{$\begin{array}{l}\text { Rückwirkungsarme Messmethoden mit geringem Wärmeeintrag zur Bestimmung } \\
\text { des lokalen WÜK bei reiner erzwungener Konvektion }\end{array}$} \\
\hline
\end{tabular}




\subsubsection{Wanddruckmessung im Seitenraum}

Zur Charakterisierung der wandnahen Strömungsverhältnisse, die in unmittelbarem Zusammenhang mit den örtlichen Wärmeübergangsmechanismen stehen, kommt eine alternative Totraumaußenwand aus PVC zur Anwendung, die mit insgesamt 17 in einer axialen Reihe angeordneten Wandanbohrungen versehen ist ( Abb. A.23). Die Bohrungen besitzen einen Durchmesser von $1 \mathrm{~mm}$ und einen Achsabstand von 10 mm für eine hohe örtliche Auflösung. Durch Verdrehen der Außenwand (Abb. 4.16) kann die Wanddruckverteilung am Umfang bestimmt werden. So lassen sich beispielweise eventuelle Nachlaufdellen oder Ungleichmäßigkeiten in der Totraumströmung feststellen und weitere Referenzwerte für den Vergleich mit numerischen Nachrechnung generieren.

\subsubsection{Strömungsmessung im konzentrischen Ringspalt des Hauptströmungskanals}

Zur Messung des Strömungsprofiles im kreisringspaltförmigen Hauptströmungskanal des Versuchsstandes kommen zwei verschiedene Sondentypen zum Einsatz. Mittels einer gekröpften Pitot-Sonde ( Abb. 4.12), deren Staurohr einen Außendurchmesser von nur 1 mm aufweist, kann das rein axiale Strömungsprofil sehr genau erfasst werden. Für potenziell drallbehaftete und dreidimensionale Strömungen wird eine gekröpfte Fünflochsonde ( Abb. 4.13) eingesetzt. Der pyramidenstumpfförmige Sondenkopf hat einen Durchmesser von 2,4 mm, der Sondenschaftdurchmesser beträgt $6 \mathrm{~mm}$. Im Totraumversuchsstand wird die Fünflochsonde an zwei verschiedenen Stellen eingesetzt: Zum einen wird sie durch die Außenwand, die auch für Wanddruckmessungen verwendet wird (Abb. A.23), eingeführt, wo sie zur Traversierung der Hauptströmung unmittelbar 0,7 mm vor der Einströmöffnung zum Totraum dient (Pos. 1 in Abb. 4.14). Während der Wärmeübergangsmessungen, bei denen der Einfluss des Dralls untersucht werden soll ( Kap. 5.7.7) und die Messring-Außenwand (Abb. 4.9) verbaut ist, wird die Sonde von unten in den Hauptströmungskanal geführt (Pos. 2 in $>$ Abb. 4.14), und zwar 445 mm stromauf der Einströmöffnung zum Seitenraum und damit in etwa an derselben axialen Position wie die Referenzdruckbohrung.

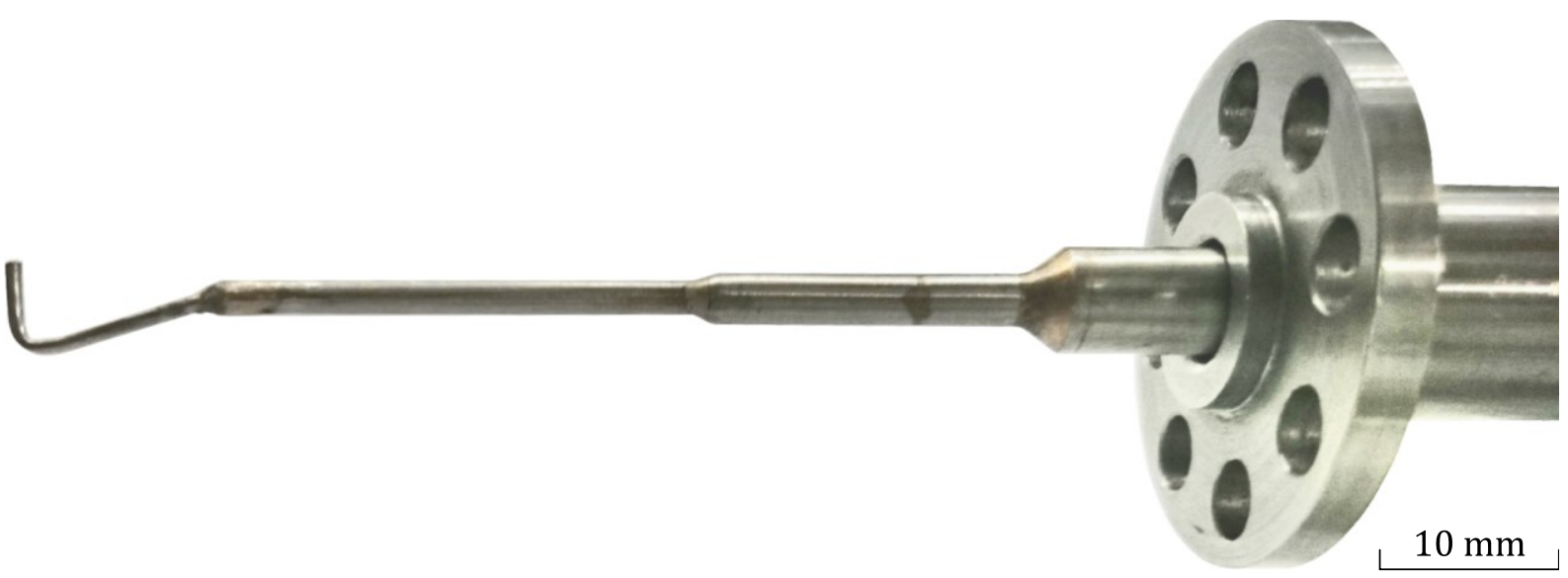

Abb. 4.12 Pitot-Sonde zur Geschwindigkeitsprofilbestimmung im Hauptströmungskanal. 


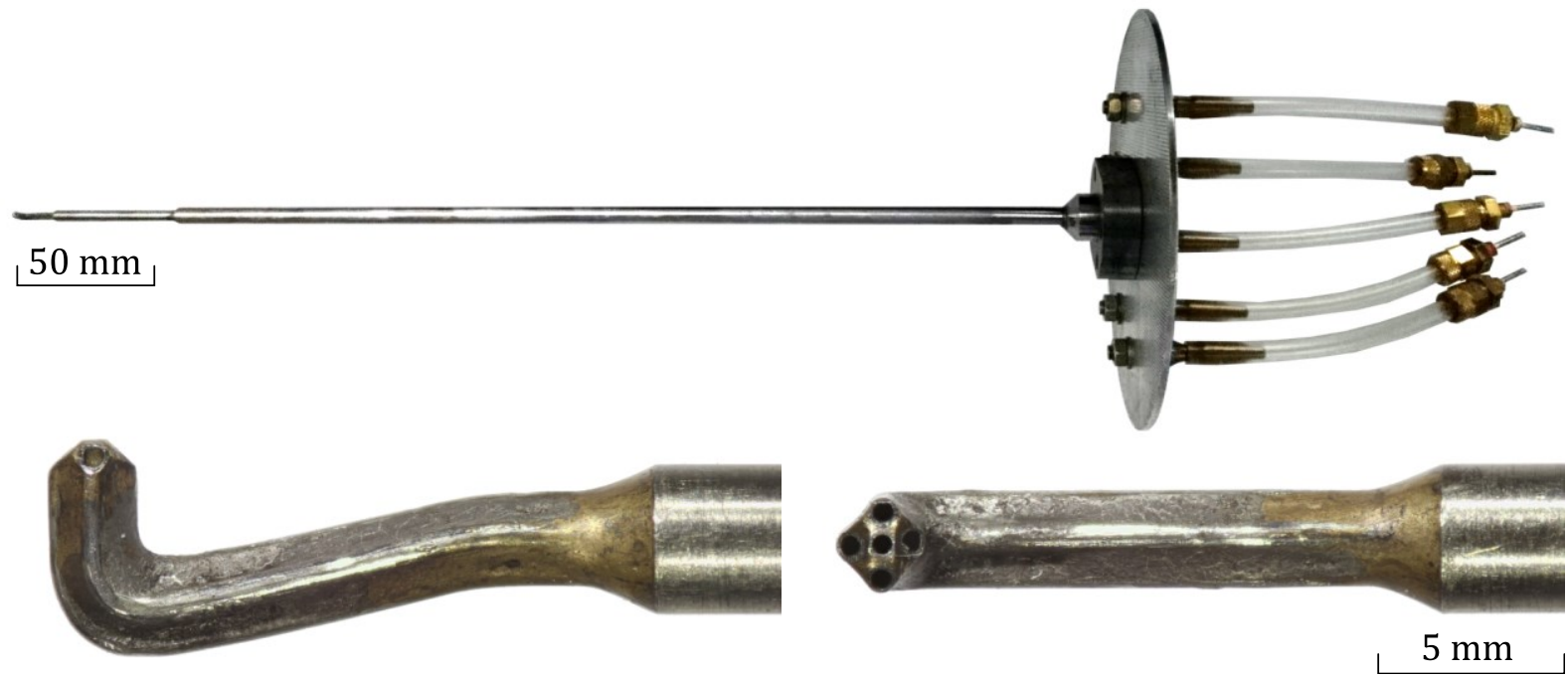

Abb. 4.13 Fünfloch-Pyramidensonde zur 3D-Geschwindigkeitsmessung im Hauptströmungskanal. Die beiden Detailabbildungen des Sondenkopfes wurden durch Focus stacking mehrerer Mikroskopaufnahmen mit unterschiedlicher Brennweite mittels des Pyramidenverfahrens „PMax“ erstellt.

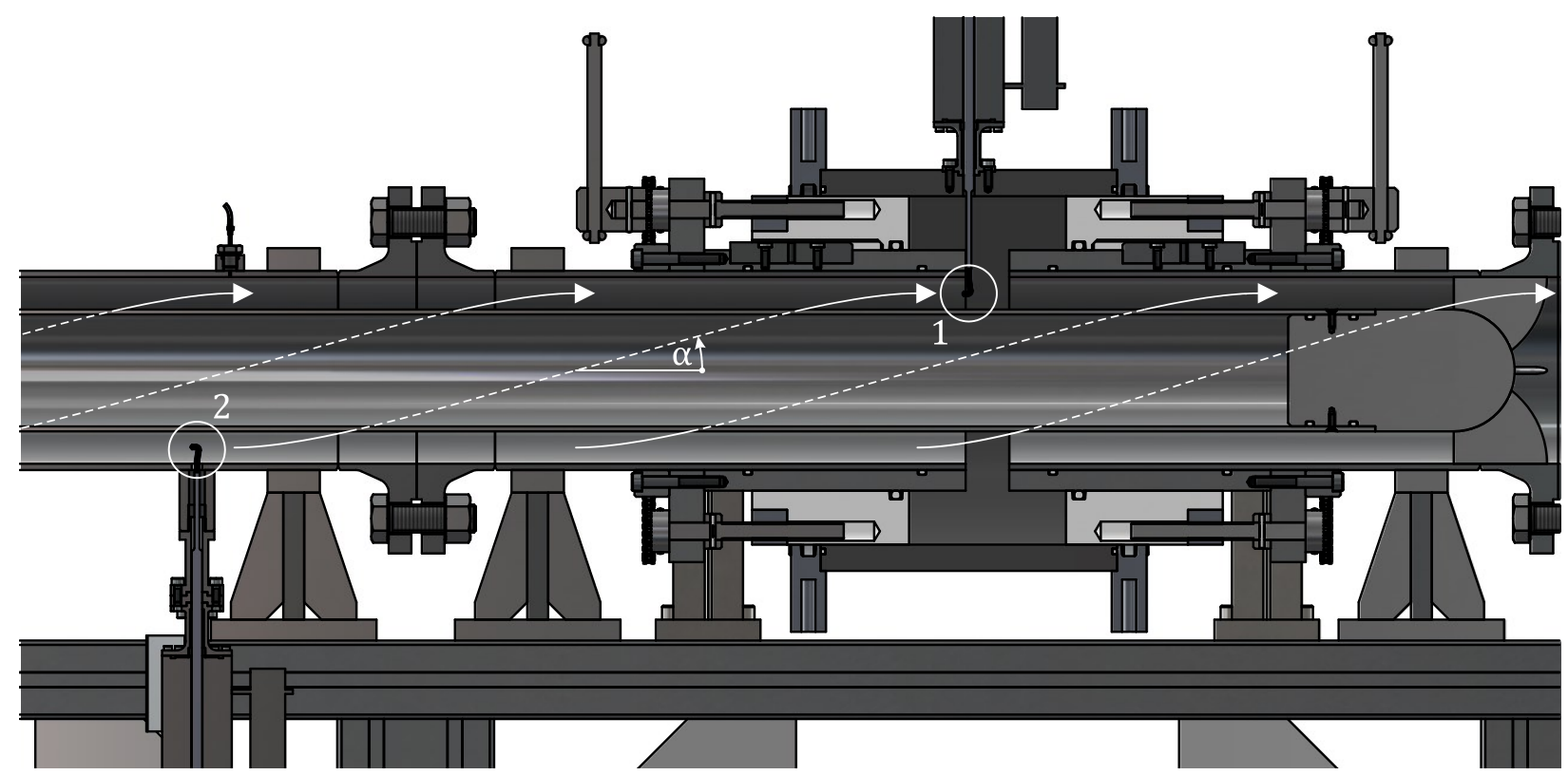

Abb. 4.14 Mögliche Einbaupositionen der Fünfloch-Pyramidensonde im Versuchsstand:

1 - unmittelbar vor der Einströmöffnung zum Totraum; 2 - in der Nähe der Referenzdruckbohrung. Da Sonde und -verstellgerät nur einmal existieren, können sie nur an einer der beiden Positionen eingesetzt werden.

In beiden Fällen kommt ein Sondenverstellgerät (SVG) zur Anwendung, welches an der Professur für Mess- und Automatisierungstechnik entwickelt wurde und mit dem die Höhe/ Eintauchtiefe der Sonde in die Strömung (radiale Position) sowie eine Verdrehung der Sonden um die Sondenschaftlängsachse (Gierwinkeloffset $\Delta \alpha$ ) zur Erweiterung des Drallwinkelmessbereiches eingestellt werden kann ( Abb. 4.15). 
Durch Verdrehung der Außenwand kann die Sonde auch über dem Umfang traversiert werden, um das Strömungsprofil in einem ganzen Viertelsektor (Quadrant) des Hauptströmungskanals zu ermitteln ( Abb. 4.16).

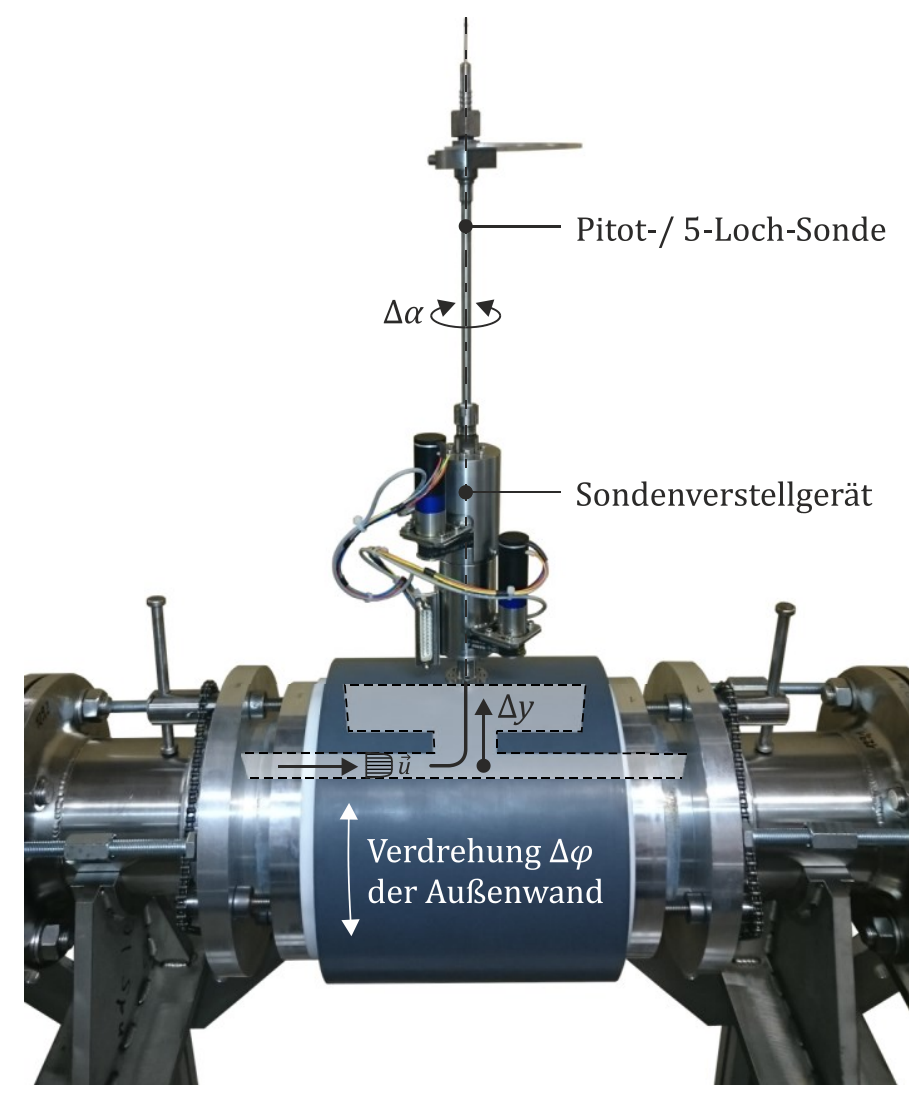

Abb. 4.15 Sondenverstellgerät zur Höhen- und Winkeltraversierung einer Sonde im Hauptströmungskanal

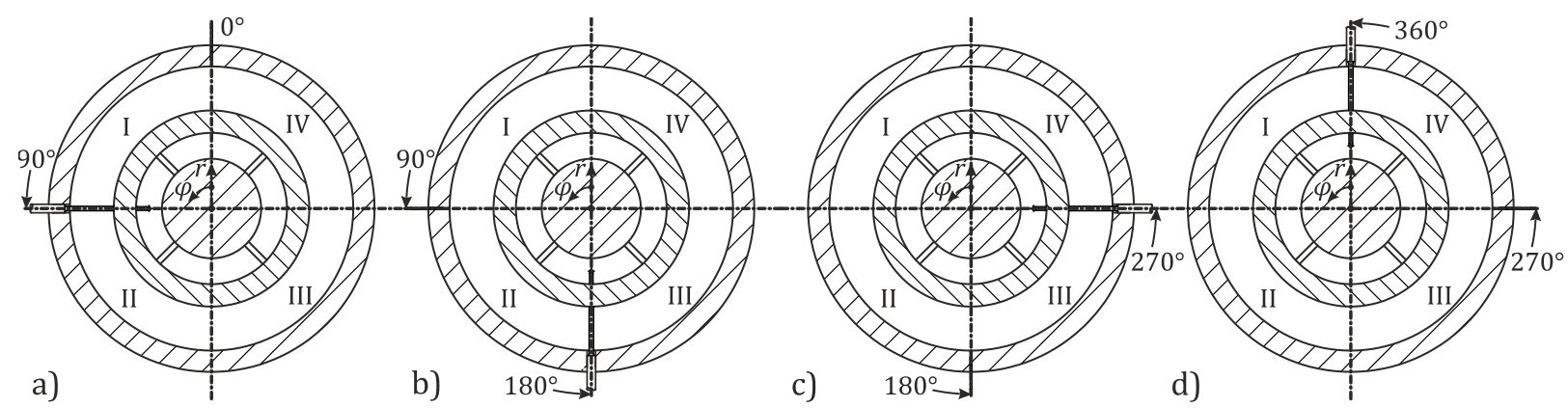

Abb. 4.16 Winkelkonvention und Quadranten bei Verdrehung der Außenwand um ihre Längsachse um den Winkel $\Delta \varphi$ zur Umfangstraversierung des Strömungsfeldes in der Hauptströmung mittels Fünflochsonde sowie der Wanddruckverteilung entlang der Außenwand (Blick in Strömungsrichtung). a) $\Delta \varphi=0^{\circ}=360^{\circ}$, b) $\Delta \varphi=90^{\circ}$, c) $\Delta \varphi=180^{\circ}$, d) $\Delta \varphi=270^{\circ}$. 


\subsubsection{Betriebsmessstellen an der Versuchsanlage}

Zur Überwachung des sicheren Betriebes der Versuchsanlage sind über das gesamte Rohrleitungssystem hinweg verteilt verschiedene Betriebsmessstellen für Druck, Temperatur, Durchsatz, Ventilhub usw. angeordnet. Einige davon werden während der Versuchsdurchführung durchgehend aufgezeichnet, um die funktionsgemäße und stationäre Betriebsweise der Anlage sicherzustellen, eventuelle Veränderungen frühzeitig zu erkennen und zu beheben oder in der Auswertung zu berücksichtigen. Eine Übersicht aller Betriebsmessstellen ist in Abb. A.8 dargestellt sowie in $\$ Tab. A.2 im $\gg$ Anhang A7 aufgelistet.

So werden an verschiedenen Stellen statische Betriebsdrücke über senkrechte Wandanbohrungen in der Rohrleitung abgegriffen und über Scanivalve-Druckschläuche (Vinyl bzw. PTFE; Innendurchmesser 0,063 in =1,59 $\mathrm{mm}$ ) in die Messgeräte geführt, wo sie über die dort integrierten Piezosensoren erfasst werden, die im Vorfeld mittels eines Druckkalibrators auf den jeweils zu erwartenden Wertebereich kalibriert worden sind ( Kap. 5.8.1). Druckbohrungen befinden sich unter anderem vor dem Druckminderer (P1_Vordruck), der im Wesentlichen mit dem Druck im Druckluftspeicher (abzüglich der Druckverluste von ca. 0,2 bar) korrespondiert, nach dem Druckminderer (P2_n_DRV) sowie nach dem Sicherheitsventil (P3_n_SichV). Die letzteren beiden Messpunkte dienen der in Kap. 4.1.4 beschriebenen Überwachung des funktionsgemäßen Betriebs des Druckminderers. Weiterhin wird der Druck vor (P5_V_MSV) und nach dem Motorstellventil (P6_n_MSV) bestimmt, um die ordnungsgemäße Funktion der Ausblasung zu observieren. Die Berechnung des Blendenmassenstroms (Kap.4.4.1) erfordert die Bestimmung des Blendenvordrucks (p_B1_vor), des -differenzdrucks (p_B1_diff) sowie der Strömungstemperatur vor der Blende (TE_B1). Letztere wird mittels einer Thermoelementsonde in der Rohrmitte gemessen.

Auf eine höhere Messgenauigkeit wird bei den Messstellen im Versuchsstand geachtet. So werden die Strömungstemperaturen vor (T_v_TRV) und nach dem Versuchsstand (T_n_TRV) mittels kalibrierter Thermistorsonden erfasst. In unmittelbarer Nähe zum Thermistorsondenkopf wird außerdem jeweils mittels einer Pitotsonde der dynamische Druck in der Strömung (P4_V_TRV_diff bzw. P8_n_TRV_diff) gegenüber dem statischen Wanddruck vor Ort (P4_v_TRV bzw. P8_n_TRV) gemessen ( Abb. A.17). Eine dritte Thermistorsonde wird verwendet, um die Umgebungstemperatur am Versuchsstand (T_Umgeb) unmittelbar oberhalb des Totraummoduls ( Abb. A.22) aufzunehmen.

Ferner werden die Betriebstemperatur der Datenerfassungsgeräte (T_14_1, T_14_2 usw.; - Kap 4.3.1) sowie der Hub des Dreiwegeventils (3WV) und des Motorstellventil (MSV) erfasst. Zusammen mit den eigentlichen Versuchsmessdaten (siehe nachfolgende Teilkapitel) werden insgesamt bis zu 131 Betriebs- und Versuchsmessstellen zur selben Zeit synchron aufgezeichnet 
( Tab. A.2). Weitere versuchsrelevante Betriebsmessstellen, die nicht mittels der in Kap. 4.2.4 dargestellten Datenaufnahme erfasst werden, für die Steuerung und Überwachung der Anlage dennoch wesentlich sind, sind in $\$$ Tab. A.2 ( Anhang A7) angegeben.

Alle Messstellen sowie Leitungsstränge, Armaturen und Geräte wurden gemäß Kraftwerkskennzeichnungssystem RDS-PP nach DIN 6779-10, DIN EN 81346-1 sowie VGB-B 101 und 102 gekennzeichnet und beschriftet (vgl. Abb. A.8 sowie $>$ Tab. A.3 und Tab. A.4 in Anhang A7). Dadurch ist eine eindeutige Dokumentation und ein schnelles Auffinden der Messstellen in der Anlage möglich (siehe hierzu auch [86]).

\subsection{MESSDATENERFASSUNG UND -VERARBEITUNG}

\subsubsection{Messwerterfassungssystem}

Zur Aufnahme der Messdaten kommt ein ebenfalls an der Professur für Magnetofluiddynamik, Mess- und Automatisierungstechnik entwickeltes Messwerterfassungsystem zum Einsatz. Dieses besteht aus den beiden Messwertaufnahmegeräten G14.1 und G14.2. Bei den Geräten handelt es sich jeweils um 19-Zoll-Einschübe, die die zu benutzenden Drucksensoren für die verschiedenen Druckbereiche sowie die Platinen zur Beschaltung und Messung der Widerstände der Thermistoren und WÜK-Sensoren beinhalten (siehe Abb. A.24). Außerdem stehen zusätzliche RS-232-Anschlüsse für weitere analoge Signale zur Verfügung, wie sie z. B. für die Thermoelemente an der Blendenmessstrecke oder für Widerstandferngeber der elektrischen Antriebe von Dreiwege- und Motorstellventil benötigt werden.

Insgesamt verfügen die beiden Messwerterfassungsgeräte unter anderem über

- 28 Druckmessstellen (inkl. integriertem Barometer für Umgebungsdruck),

- 90 Temperaturmessstellen für Thermistoren (inkl. Gerätetemperaturen),

- 16 RS-232-Anschlüsse für weitere analoge Eingangssignale (darunter 2 analoge Eingänge für Motorstellventilhübe sowie2 Temperaturmessstellen für Thermoelemente),

Der ausgangseitig angeordnete A/D-Wandler wandelt die analogen in digitale Signale. Die Datenübertragung zwischen Messwerterfassung und Messrechner erfolgt über DDE-Protokoll mittels serieller RS-232-Schnittstelle (COM-Port). Durch ihre kompakte Bauweise sind die Geräte sehr leicht transportfähig. Somit können etwa beim Kalibrieren nicht nur die Übertragungseigenschaften der einzelnen Sensoren sondern die der gesamten Messketten berücksichtigt werden, zu denen noch die Verlötung, Verdrahtung und der A/D-Wandler gehören. Dadurch ist eine direkte Korrelation des digitalen Ausgangssignals („Digit“) mit der physikalischen Messgröße möglich. 


\subsubsection{Datenverarbeitung und -synchronisierung}

Den Abgriff der digitalen Signale auf dem Messrechner übernimmt ein In-House-Telemetrieprogramm der Messtechnik-Kollegen (Abb. A.25 a). Dabei werden alle Datenkanäle mit einer Abtastrate von $20 \mathrm{~Hz}$ seriell abgetastet. Das entspricht einer Symbolrate von 38.400 Symbolen/s („Baud-Rate“). Zusätzlich ist es optional möglich, die einzelnen Daten über eine bestimmte Anzahl an aufeinanderfolgenden Datensätzen zu mitteln oder sich Minimal- bzw. Maximalwerte für dieses Intervall ausgeben zu lassen. Für das Auslesen der Betriebsmessdaten wird eine zeitliche Mittelung über zehn aufeinander folgende Messwerte gewählt. Entsprechend ergibt sich eine tatsächliche Aufnahmerate von $1 \mathrm{Messwertsatz} / 0,5 \mathrm{~s}=2 \mathrm{~Hz}$.

Die weitere Datenverarbeitung und -speicherung erfolgt über Microsoft Excel 2013 und VBA (Visual Basic for Application). Eine VBA-Datensammelroutine spricht dabei das Telemetrieprogramm an, holt in einem frei wählbaren, festgelegten Zeitintervall, üblicherweise aller zwei Sekunden, einen kompletten Datensatz ab und trägt die Werte in ein vorbereitetes Tabellenblatt ein ( Abb. A.25 b). Dieser wird mit einem Windows-Zeitstempel versehen, der bei jedem neuen Durchlauf neu generiert und mitgeschrieben wird. Damit ist eine eindeutige, millisekundengenaue Zuordnung und Chronologisierung der Messdaten möglich (Data Logging).

Über Kalibrierpolynome und Berechnungsprogramme, die ebenfalls in Form von VBA-Makros hinterlegt sind (siehe auch Kap. 4.4), werden gleichzeitig bei jedem Zeitschritt die Digits in die physikalischen Zielgrößen (Druck, Temperatur, Durchsatz, Geschwindigkeiten, Reynolds-Zahlen etc.) umgerechnet und mitgeschrieben ( Abb. A.25 b). Zusätzlich wird je ein Messschrieb (Log) für die Rohdaten und für die umgewerteten Messwerte angelegt und mit jedem neuen Datensatz fortgeschrieben ( Abb. A.25 c). Dies erleichtert die anschließende Auswertung deutlich. Außerdem werden die zeitlichen Verläufe aller relevanten Messwerte in dynamischen Diagrammen dargestellt ( Abb. 4.17). Diese „Live-Anzeige“ ermöglicht eine schnelle und präzise Überwachung und Steuerung des Versuchsanlagenbetriebes. 

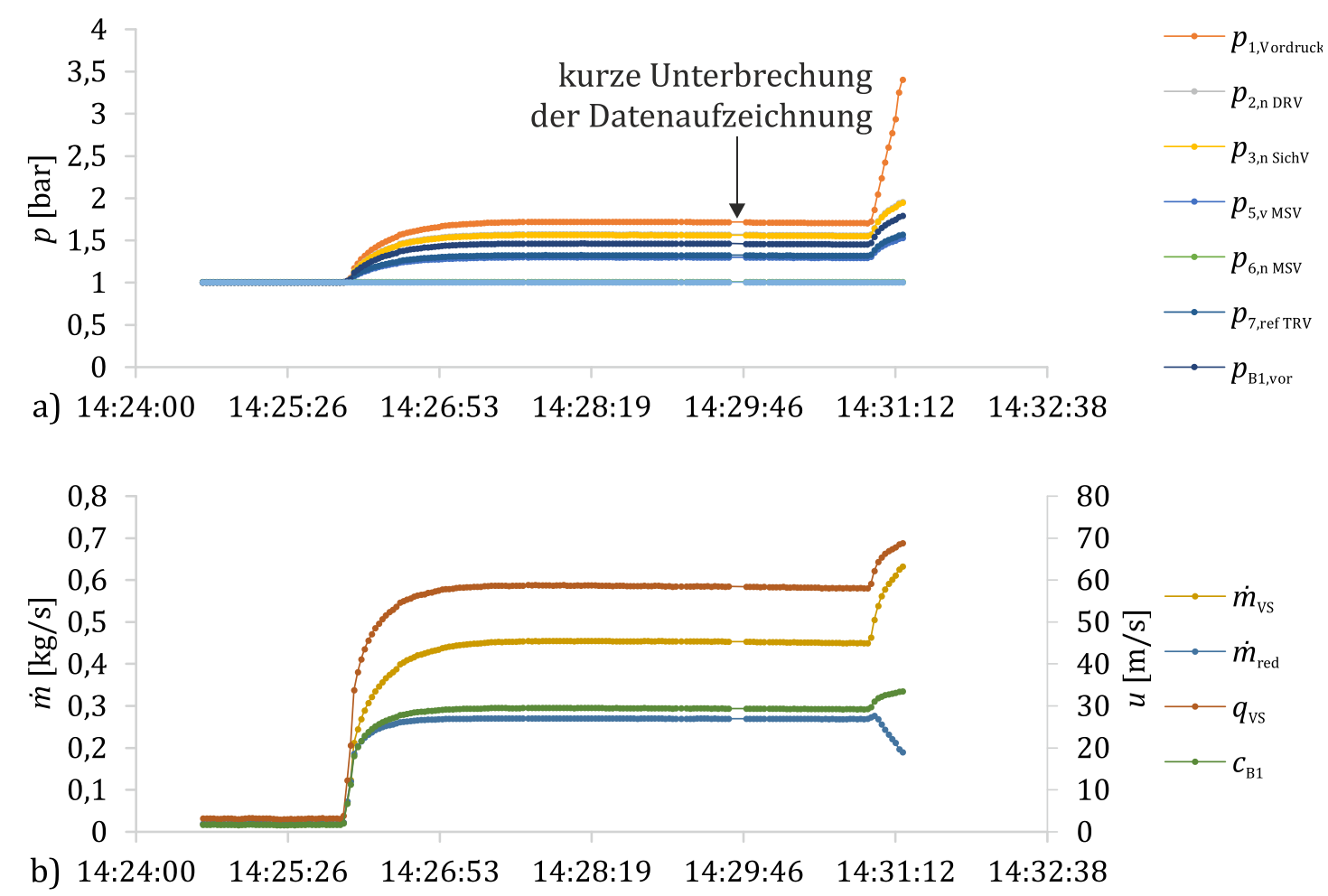

Abb. 4.17 Dynamische Anzeige der zeitlichen Messwertverläufe (Auswahl).

a) Druck- und b) Durchsatzkenngrößen.

\subsection{DATENAUSWERTUNG}

\subsubsection{Massenstrom aus Blendenberechnung}

Zur Berechnung des durch den Hauptstrang fließenden Massenstromes aus den gemessenen Werten für Druck $p_{1}=$ p_B1_vor und Temperatur $T_{1}=$ TE_B1 vor der Blende sowie der Wirkdruckdifferenz $\Delta p_{\mathrm{W}}=$ p_B1_diff über der Blende kommt der in DIN EN ISO 5167-2 genormte Algorithmus zur Anwendung (siehe auch [75]):

Gemäß der Drosselgleichung berechnet sich der Massenstrom zu:

$$
\dot{m}=\frac{C}{\sqrt{1-\beta^{4}}} \cdot \varepsilon \cdot \frac{\pi}{4} d^{2} \cdot \sqrt{2 \cdot \Delta p_{\mathrm{W}} \cdot \rho_{1}}
$$

mit dem Durchmesserverhältnis (Blendenöffnungsverhältnis)

$$
\beta=\frac{d}{D}
$$

welches für den vorliegenden Fall (Rohrinnendurchmesser $D=D_{\mathrm{i}}=107,1 \mathrm{~mm}$, Blendenöffnungsdurchmesser $d=71 \mathrm{~mm}$ ) bei $\beta=0,663$ liegt, der Expansionszahl 


$$
\varepsilon=1-\left(0,351+0,256 \beta^{4}+0,93 \beta^{8}\right) \cdot\left[1-\left(\frac{p_{2}}{p_{1}}\right)^{\frac{1}{\kappa}}\right]
$$

mit $p_{2}=p_{1}-\Delta p_{\mathrm{W}}$ und dem Durchflusskoeffizienten $C$, der gemäß der Reader-Harris/GallagherGleichung für Blenden mit Eckdruckentnahme wie folgt ermittelt wird:

$C=0,5961+0,0261 \beta^{2}-0,216 \beta^{8}+0,000521\left(\frac{10^{6} \beta}{R e_{D}}\right)^{0,7}+(0,0188+0,0063 A) \beta^{3,5}\left(\frac{10^{6}}{R e_{D}}\right)^{0,3}$

wobei

$$
A=\left(\frac{19000 \beta}{R e_{D}}\right)^{0,8}
$$

Wie sich zeigt, hängt der Durchflusskoeffizient von der Reynolds-Zahl in der Rohrströmung vor der Blende

$$
R e_{D}=\frac{u_{1} \cdot D}{v_{1}}=\frac{4 \dot{m}}{\pi \eta_{1} D}
$$

ab, weshalb der Massenstrom iterativ bestimmt werden muss. Zugleich wird auch die gemessene Temperatur $T_{1}$ unter Beachtung des kinetisch gebundenen („dynamischen“) Energieanteils mittels eines als konstant angenommenen Temperatur-Recovery-Faktors von $r=0,7$ für blanke, querangeströmte, zylindrische Temperaturmessstellen [87] auf den reinen statischen Wert $T_{1 \text {,korr }}$ korrigiert:

$$
T_{1, \text { korr }}=T_{1}-r \cdot \frac{u_{1}^{2}}{2 c_{p}}
$$

Dabei entspricht $u_{1}$ der querschnittsgemittelten Geschwindigkeit in der Blendenmessstrecke:

$$
u_{1} \equiv q_{1}=\frac{\dot{m}}{\rho_{1} \cdot \frac{\pi}{4} D^{2}}
$$

Die benötigten Stoffwerte werden dabei bei $p_{1}$ und $T_{1, \text { korr }}$ bestimmt:

$$
\begin{gathered}
\rho_{1}=\rho\left(p_{1}, T_{1, \mathrm{korr}}\right) \\
\eta_{1}=\eta\left(T_{1, \mathrm{korr}}\right) \\
\kappa=\kappa\left(p_{1}, T_{1, \mathrm{korr}}\right) \equiv 1,4
\end{gathered}
$$

Als Startwerte dienen $C=0,605$ und $T_{1, \text { korr }}=T_{1}$. 


\subsubsection{Bezugstemperatur, Bezugsdruck und charakteristische Strömungsgrößen im Versuchsstand}

Nicht nur zur Berechnung der Stoffdaten im Versuchsstand sondern auch für die zu messenden WÜK ( Kap. 4.2.1), Wanddrücke ( Kap. 4.2.2) und Strömungsgeschwindigkeiten ( Kap. 4.2.3) ist die präzise Bestimmung einer charakteristischen Bezugstemperatur sowie eines Bezugsdruckes notwendig. Dazu werden in einem ersten Schritt zunächst die in Kap. 4.2.4 beschriebenen, mittels Thermistorsonden gemessenen Strömungstemperaturen („Eigentemperaturen“) an der Ein- und Ausströmung des Versuchsstandes wie bei der Blendenberechnung (Gleichung 4.8) um den dynamischen Anteil korrigiert, und zwar unter Zuhilfenahme der mittels der Pitotsonden vor Ort gemessenen dynamischen Drücke $\Delta p=\frac{\rho}{2} u^{2}$ :

$$
T_{\mathrm{korr}}=T_{\mathrm{eig}}-r \cdot \frac{u^{2}}{2 c_{p}}=T_{\mathrm{eig}}-r \cdot \frac{\Delta p}{\rho\left(p, T_{\mathrm{korr}}\right) c_{p}\left(p, T_{\mathrm{korr}}\right)}
$$

Gleichung 4.9 wird iterativ ausgewertet. Als Startwert empfiehlt sich $T_{\text {korr }}=T_{\text {eig }}$. Aus den so ermittelten Werten vor und nach dem Versuchsstand wird dann die Bezugstemperatur berechnet:

$$
T_{\text {ref }}=\frac{1}{2}\left(T_{\mathrm{v}_{-} \mathrm{TRV}, \mathrm{korr}}+T_{\mathrm{n}_{-} \mathrm{TRV}, \mathrm{korr}}\right)
$$

Der statische Referenzdruck (P7_ref_TRV) hingegen wird direkt im kreisringspaltförmigen Strömungskanal unmittelbar $L=422 \mathrm{~mm}\left(L / d_{\mathrm{h}}=11,5\right)$ vor der Einströmöffnung zum Seitenraum gemessen und um den geringen Druckverlustanteil, der bis dahin noch entsteht, korrigiert:

$$
p_{\text {ref,korr }}=p_{\text {ref }}-\zeta \cdot \frac{\rho\left(p_{\text {ref,korr }}, T_{\text {ref }}\right)}{2} q^{2}
$$

Diese Gleichung wird komplettiert mit der querschnittsgemittelten (Axial-) Geschwindigkeit

$$
q=\frac{\dot{m}}{\rho\left(p_{\mathrm{ref}, \mathrm{korr}}, T_{\mathrm{ref}}\right) \cdot A}
$$

der durchströmten Querschnittsfläche $A=\frac{\pi}{4}\left(D^{2}-d^{2}\right)$, dem Druckverlustbeiwert $\zeta=\lambda \cdot L / d_{\mathrm{h}}$, dem Rohrreibungsbeiwert für turbulente Strömung in hydraulisch glatten Rohren nach Nikuradse

$$
\frac{1}{\sqrt{\lambda}}=-2 \log \left(\frac{2,51}{\operatorname{Re} \sqrt{\lambda}}\right)
$$

und der Reynolds-Zahl

$$
R e=\frac{q \cdot d_{\mathrm{h}}}{v}=\frac{\dot{m} \cdot d_{\mathrm{h}}}{A \cdot \eta\left(T_{\mathrm{ref}}\right)}
$$

die für ideale Gase nur eine Funktion der Temperatur ist (s. a. [75]). Als Startwerte für die Iteration werden hier $\lambda=0,02$ und $p_{\text {ref,korr }}=p_{\text {ref }}$ gesetzt. 


\subsubsection{Thermisches Netzwerkmodell zur Abschätzung lokaler Wärmeübergangskoeffi- zienten}

Für eine schnelle Analyse der auflaufenden Messdaten bereits während der Messung, zum Beispiel für das Online-Monitoring ( Kap. 4.3.2 und 4.5.1), ist es wünschenswert, lokale WÜK direkt auf Basis der gemessenen Temperaturen und ohne numerisches Modell abschätzen zu können. Dazu sollen zwei benachbarte Messringe in Abb. 4.19 betrachtet werden. Durch Bestromen der Heizwicklung steigt für jeden Ring die Temperatur im Außenring an. Die eingetragene Wärme fließt jedoch nur teilweise über den Zwischenring, welcher als Hilfswand dient ( Abb. 4.9), zu dem jeweiligen Innenring. Zu einem geringen Teil strömt die Wärme über die Styropor-Isolation zu den beiden benachbarten Außenringen sowie über die Armaflex-Isolation nach außen. Der Teil der Wärme, welcher den Innenring erreicht, teilt sich wiederum auf in axiale Wärmeverluste zu den beiden benachbarten Innenringen sowie in den Anteil, welcher in Folge von erzwungener Konvektion tatsächlich in das Fluid abgegeben wird.

Auf Grund der hohen Wärmeleitfähigkeit der eingesetzten Aluminiumlegierung sowie von Baustahl ( Tab. 4.5) besitzen sowohl die Innen- und die Außenringe als auch die beiden Flansche eine einheitliche Temperatur und können daher als quasi-isotherm angesehen werden ( Abb. 4.24). Aus diesem Grund können sie als Knoten in einem thermischen Netzwerk modelliert werden

Abb. 4.20, siehe auch $[88,89])$. Unter der Annahme eines perfekten thermischen Kontaktes zwischen Heizwicklung und Gewinderille kann der Wärmeeintrag an den Außenringen durch eine Wärmequelle in den Knoten abgebildet werden. Die Wärmebarrieren werden als thermische Ersatzwiderstände modelliert: in Form von axialen und radialen Wärmeleitwiderständen für die Zwischenringe, die Isolation, die PTFE-Dichtringe und die Luftspalte bzw. als Wärmeübergangswiderstände für die innere und äußere Konvektion (siehe $>$ Tab. 4.4). Die Anwendung des 1. Kirchhoff'schen Gesetzes für die Knoten $(1, j)$

$$
0=\frac{T_{1, j-1}-T_{1, j}}{R_{\lambda, \mathrm{s} 1, \mathrm{ges}}}+\frac{T_{1, j+1}-T_{1, j}}{R_{\lambda, \mathrm{s} 1, \mathrm{ges}}}+\frac{T_{\mathrm{F}}-T_{1, j}}{R_{\alpha, j}}+\frac{T_{3, j}-T_{1, j}}{R_{\lambda, 2}}
$$

liefert die Wärmeübergangswiderstände

$$
R_{\alpha, j}=\frac{T_{1, j}-T_{\mathrm{F}}}{\frac{T_{1, j-1}-T_{1, j}}{R_{\lambda, \mathrm{s} 1, \mathrm{ges}}}+\frac{T_{1, j+1}-T_{1, j}}{R_{\lambda, \mathrm{s} 1, \mathrm{ges}}}+\frac{T_{3, j}-T_{1, j}}{R_{\lambda, 2}}}
$$

aus denen schließlich die mittleren Wärmeübergangskoeffizienten über jeden Ring

$$
\alpha_{j}=\frac{1}{R_{\alpha, j} \cdot A_{\mathrm{i}}}
$$

mit der wärmeübertragenden Fläche $A_{\mathrm{i}}=\pi \cdot D_{\mathrm{i}} \cdot b_{\mathrm{i}}$ berechnet werden können. 
Auf dieselbe Weise können durch Aufstellen des 1. Kirchhoff'schen Gesetzes für die Knoten $(3, j)$ auch die Werte der nodalen Wärmequellen $\dot{Q}_{\mathrm{a}, j}$ verifiziert werden:

$$
\begin{aligned}
& 0=\dot{Q}_{\mathrm{a}, j}+\frac{T_{3, j-1}-T_{3, j}}{R_{\lambda, \mathrm{s} 3}}+\frac{T_{3, j+1}-T_{3, j}}{R_{\lambda, \mathrm{s} 3}}+\frac{T_{\mathrm{U}}-T_{3, j}}{R_{k, \mathrm{Iso}}}+\frac{T_{1, j}-T_{3, j}}{R_{\lambda, 2}} \\
& \dot{Q}_{\mathrm{a}, j}=\left(\frac{1}{R_{k, \mathrm{Iso}}}+\frac{2}{R_{\lambda, \mathrm{s} 3}}+\frac{1}{R_{\lambda, 2}}\right) \cdot T_{3, j}-\left(\frac{T_{3, j-1}+T_{3, j+1}}{R_{\lambda, \mathrm{s} 3}}+\frac{T_{\mathrm{U}}}{R_{k, \mathrm{Iso}}}+\frac{T_{1, j}}{R_{\lambda, 2}}\right)
\end{aligned}
$$

Da das thermische Netzwerk nur in der Lage ist, rein axiale und radiale Wärmeströme ( Abb. 4.18) abzubilden, können diagonale Flüsse durch das Styropor z. B. von einem Außenring zu einem benachbarten Innenring oder zwischen zwei gegenüber liegenden Zwischenringen nicht berücksichtigt werden. Außerdem wird angenommen, dass Messringe, die teilweise von den Seitenwänden überdeckt sind (siehe z. B. Abb. 4.22), voll in Kontakt mit dem Fluid stehen. Daher können die berechneten Werte der Wärmequellen $\dot{Q}_{\mathrm{a}, j}$ geringfügig von der tatsächlich eingetragenen elektrischen Leistung $P_{\mathrm{el}, j}$ abweichen. Wie $>$ Tab. 4.3 zeigt, liegen die sich ergebenden maximalen absoluten Abweichungen für den im nachfolgenden Kapitel dargestellten Beispielfall unter 3,5 \% für die Ringe 2 bis 7 und bei 4,1 \% bzw. 6,6 \% für die beiden äußeren Ringe, was als ausreichend kleine Unsicherheit des analytischen Modells einzuschätzen ist. Es eignet sich daher bestens für die Bestimmung des thermischen Gleichgewichtes der Messung ( Kap. 4.5.1).

Tab. 4.3 Unterschiede zwischen den berechneten Wärmequellen aus dem Netzwerkmodell und den tatsächlich eingetragenen Heizleistungen

\begin{tabular}{ccccc}
\hline Modul $j$ & $\dot{Q}_{\mathrm{a}, j}[\mathrm{~W}]$ & $P_{\mathrm{el}, j}[\mathrm{~W}]$ & Diff. [W] & Diff. [\%] \\
\hline 1 & 3,827 & 3,990 & $-0,163$ & $4,1 \%$ \\
2 & 3,845 & 3,987 & $-0,141$ & $-3,5 \%$ \\
3 & 4,112 & 4,047 & 0,065 & $1,6 \%$ \\
4 & 4,143 & 4,008 & 0,135 & $3,4 \%$ \\
5 & 4,075 & 4,001 & 0,074 & $1,8 \%$ \\
6 & 4,021 & 4,013 & 0,008 & $0,2 \%$ \\
7 & 3,997 & 4,009 & $-0,012$ & $-0,3 \%$ \\
8 & 3,737 & 4,001 & $-0,264$ & $-6,6 \%$ \\
\hline
\end{tabular}

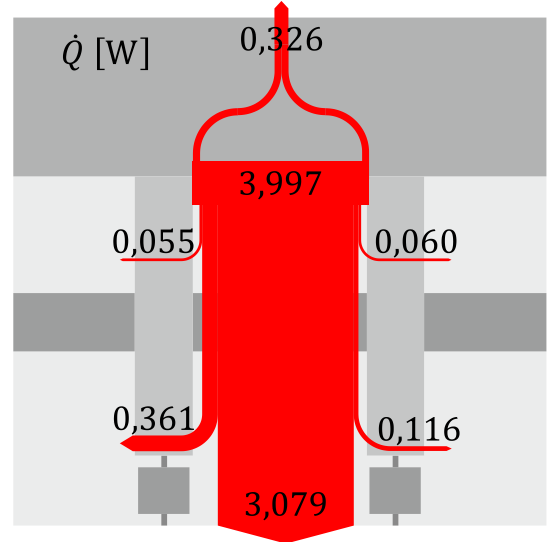

Abb. 4.18 Sankey-Diagramm der mittels Netzwerkmodell ermittelten axialen und radialen Wärmeströme für Ring 7. 


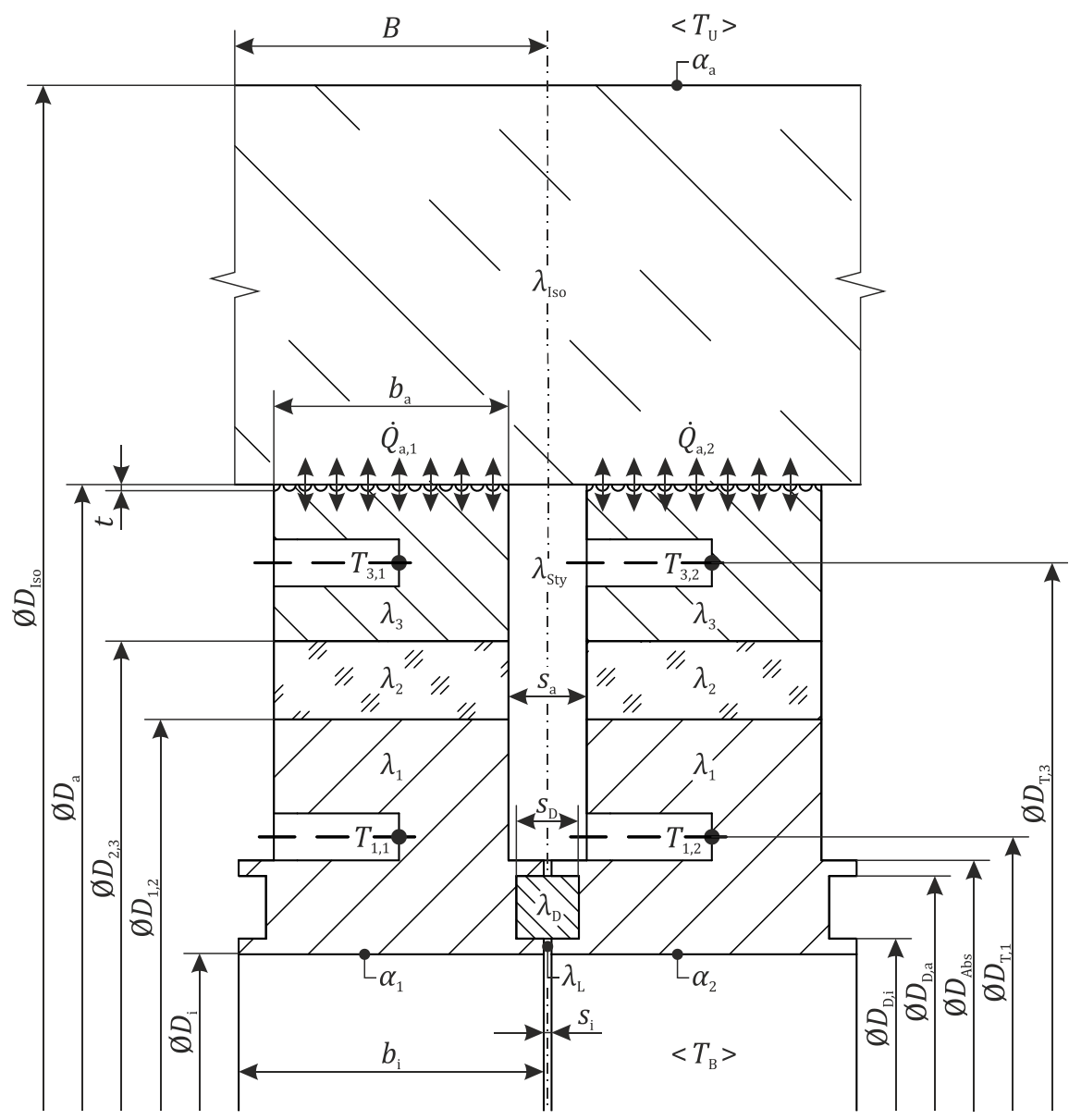

Abb. 4.19 Abmessungen, Randbedingungen, Material- und Fluidparameter zweier benachbarter Messringe für die Modellierung des thermischen Ersatznetzwerkes.

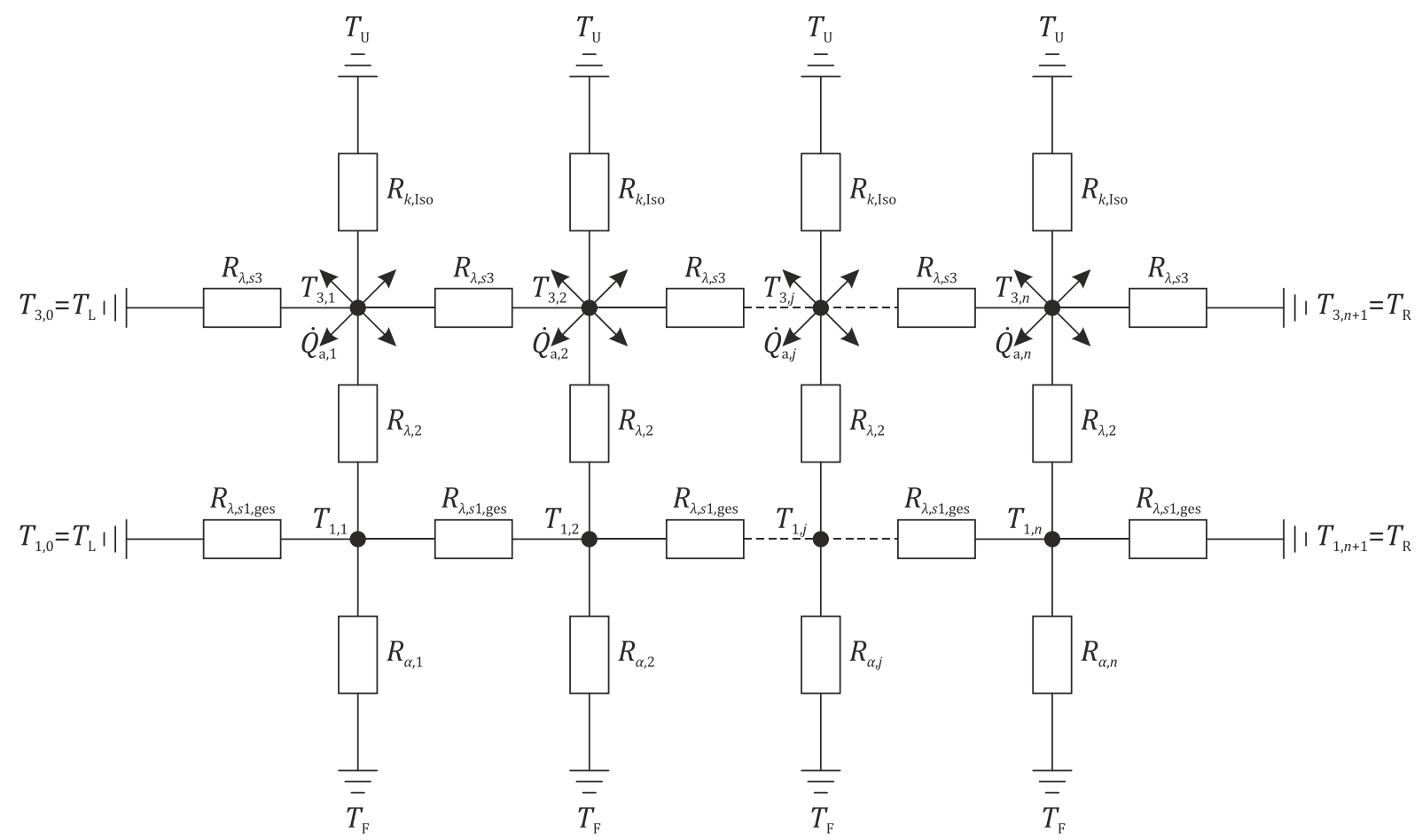

Abb. 4.20 Thermisches Ersatznetzwerk zur analytischen Abschätzung der Wärmeübergangskoeffizienten im Seitenraum. 
Tab. 4.4 Thermische Widerstände und zugehörige Querschnittsflächen für das Netzwerkmodell

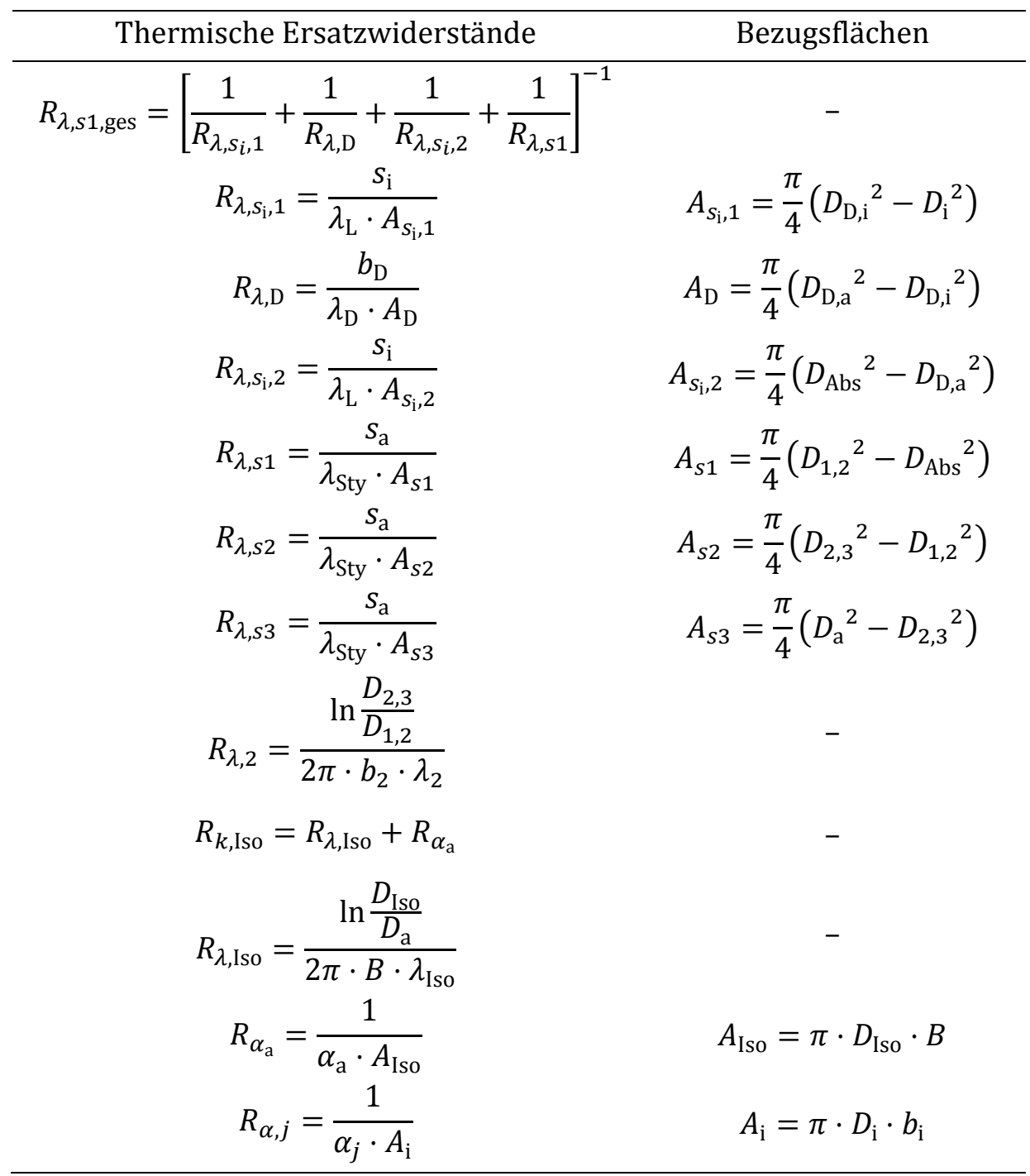

\subsubsection{Rückwärtsrechnung mittels gradientenbasiertem Optimierungsverfahren}

Für die genauere Berechnung der lokalen Wärmeübergangskoeffizienten auch in den Randbereichen des Seitenraumes, wo die Messringe infolge Überdeckung durch die Seitenwände nur teilweise im Kontakt mit dem Fluid stehen können, wurde ein vollparametrisches zweidimensionales FE-Modell der Seitenraumstrukturen in Ansys Mechanical APDL 17.2 implementiert ( Abb. 4.21). Dieses umfasst nicht nur die Außenwand mit den Messringen und der Isolation, sondern auch die beiden Seitenwände sowie die Einströmseitenwände, welche fest mit den Außenrohren aus nichtrostendem Edelstahl verbunden sind. Die beiden Außenrohre, welche den Strömungskanal nach außen hin begrenzen, wurden nach einer Länge von jeweils $200 \mathrm{~mm}$ abgeschnitten. Nicht mit modelliert wurden die vier Gewindespindeln, welche die Messringe zwischen den beiden Stahlflanschen verspannen, sowie der Verstellmechanismus für die beiden Seitenwände bestehend aus den beiden Aufnehmern, den Klemmverschraubungen, den jeweils vier Spindeln und Ritzeln, dem 
Kurbelrad und der Kette. Ferner wurden die Gewindebohrungen und die Passfedernut in den Seitenwänden, die Bohrungen und Nuten für die Thermistoren in den Messringen und den Stahlflanschen sowie die 0-Ringe und -nuten im Modell weg gelassen. Es wird erwartet, dass alle diese Vereinfachungen keinen signifikanten Einfluss auf die thermische Situation der interessierenden seitenraumnahen Strukturen haben.

Die Abmessungen des Seitenraumes (Abb. 4.2) als auch die axiale Position der Außenwand dienen als geometrische Eingabeparameter. Das strukturierte Rechennetz ( Abb. 4.21) wird für jede geometrische Konfiguration automatisch erzeugt und besteht aus ca. 30.000 rechteckigen thermischen 2D-Strukturelementen (PLANE77). Die Netze der einzelnen Komponenten sind entlang ihrer Berührungslinien direkt verbunden, um nichtlineare Kontaktelemente zu vermeiden, welche sich negativ auf die Konvergenz der Lösung auswirken. Das Material und die Wärmeleitfähigkeit für alle Komponenten ist in Abb. 4.21 dargestellt und in $>$ Tab. 4.5 zusammengefasst. Der Wärmeeintrag durch die Heizwicklungen in jedem Messring $j$ wird durch eine konstante volumetrische Wärmequelle in der äußeren Elementreihe eines jeden Außenrings realisiert. Die Enden der Außenrohre werden als adiabat angenommen, da auf Grund der ausreichend großen Rohrlänge der axiale Wärmestrom an diesen Stellen als vernachlässigbar einzuschätzen ist. Für alle anderen Oberflächen wurden konvektive Randbedingungen 3. Art (Wärmeübergangskoeffizienten und Bezugstemperaturen) angesetzt. In $>$ Abb. 4.22 werden gemessene Daten schwarz dargestellt. Blaue Werte sind mit Annahmen (Korrelationen, Erfahrungswerte) abgedeckt. Orange Größen sind unbekannt und sollen aus der inversen Berechnung ermittelt werden.

Tab. 4.5 Wärmeleitfähigkeit der Materialien der Seitenraumstrukturen

\begin{tabular}{llc}
\hline Komponente & Material & $\lambda[\mathrm{W} /(\mathrm{m} \mathrm{K})]$ \\
\hline Innenring & AlCu4MgPb (3.1645) & 150 \\
Zwischenring & PTFE & 0,31 \\
Außenring & AlCu4MgPb (3.1645) & 150 \\
Dichtring & PTFE & 0,31 \\
Zwischenringisolation & Polyurethanschaum (Styropor $\left.{ }^{\circledR}\right)$ & 0,035 \\
Außenisolation & Armaflex Protect R-90 & 0,05 \\
Luftspalt & Luft (Idealgas) & 0,025 \\
Spannscheibe (Flansch) & Baustahl & 50 \\
Seitenraum Totraum & PET & 0,24 \\
Spindelaufnehmer & AlCu4MgPb (3.1645) & 150 \\
Seitenraum Einströmöffnung & AlCu4MgPb (3.1645) & 150 \\
Außenrohr & X6CrNiMoTi17-12-2 (1.4571) & 15 \\
\hline
\end{tabular}




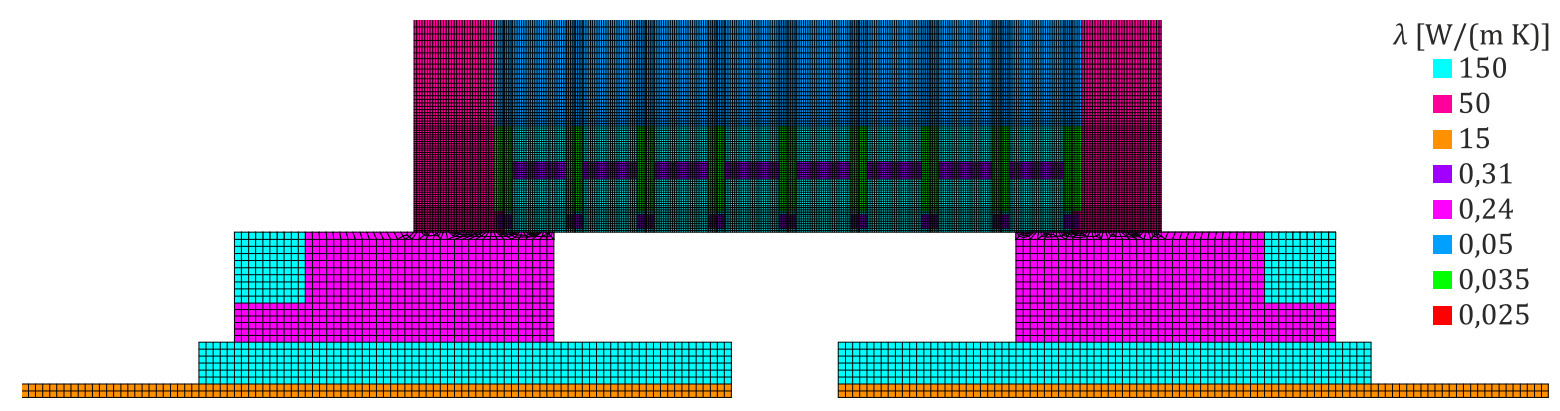

Abb. 4.21 Strukturiertes Rechennetz und Materialien des 2D-FE-Modells.

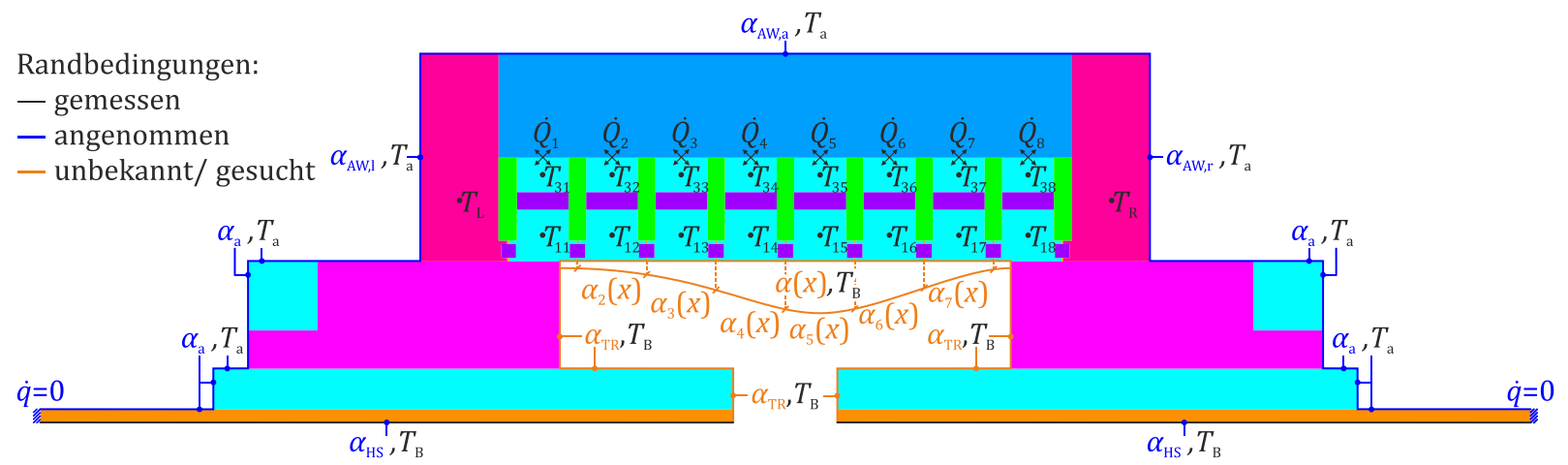

Abb. 4.22 Randbedingungen des 2D-FE-Modells, Position der Temperaturmessstellen $T_{k}^{*}$ und der Wärmequellen $\dot{Q}_{j}$.

Die Wärmeübergangskoeffizienten $\alpha_{\mathrm{HS}}$ zwischen der Hauptströmung und dem Außenrohr wurden mit der Nusselt-Korrelation für erzwungene Konvektion bei einphasiger, vollentwickelter turbulenter Strömung in einem konzentrischen Ringspalt mit äußerer Kühlung gemäß VDI-Wärmeatlas [90] aus der Reynolds-Zahl in der Hauptströmung berechnet. Für die innere Begrenzung des Seitenraumes - mit Ausnahme der Innenoberfläche der Außenwand - wurde ein Startwert von $\alpha_{\mathrm{TR}}=\alpha_{\mathrm{HS}} / 4$ angesetzt. Bezugstemperatur für den inneren Wärmeübergang ist der Mittelwert der gemessenen Totaltemperaturen in der $\mathrm{Zu}$ - und Abströmung des Versuchsstandes (vgl.

Kap. 4.4.2):

$$
T_{\mathrm{B}}=\frac{1}{2}\left(T_{\text {ein }}+T_{\text {aus }}\right)
$$

für die freie Konvektion an den äußeren Oberflächen wurde ein konstanter Wärmeübergangskoeffizient von $\alpha_{\mathrm{a}}=\alpha_{\mathrm{AW}}=10 \mathrm{~W} /\left(\mathrm{m}^{2} \mathrm{~K}\right)$ angenommen. Referenztemperatur hier ist die gemessenen Umgebungstemperatur $T_{\mathrm{U}}$. Beide Werte für $\alpha_{\mathrm{TR}}$ und $\alpha_{\mathrm{AW}}$ werden im anschließenden Optimierungsprozess angepasst. Ihr Einfluss auf das Temperaturfeld in der Außenwand wird jedoch als gering eingeschätzt. 
Die Verteilung der lokalen Wärmeübergangskoeffizienten entlang der Innenoberfläche der Außenwand wird als glatter quadratischer Spline angesetzt, welcher aus $N$ stückweise stetig-differenzierbaren Polynomfunktionen zweiten Grades zusammengesetzt ist, und zwar eine für jeden der $N$ Messringe, welche (zumindest teilweise) im Kontakt mit dem Fluid in der Kavität stehen:

$$
\alpha_{j}(x)=a_{j} \cdot x^{2}+b_{j} \cdot x+c_{j}, x \in\left[x_{j}, x_{j+1}\right], j \in[0 ; N-1]
$$

Dabei kennzeichnet $\left[x_{j}, x_{j+1}\right]$ das Kontaktintervall und $x_{\mathrm{S}, j}=\left(x_{j}+x_{j+1}\right) / 2$ den Intervallmittelpunkt der wärmeübertragenden Innenoberfläche von Ring $j$. Mit den Übergangs- und Randbedingungen für den Spline

$$
\begin{array}{llr}
\alpha_{j-1}\left(x_{j}\right)=\alpha_{j}\left(x_{j}\right) & j \in[1 ; N-1] & \text { (stetig) } \\
\left.\frac{d \alpha_{j-1}}{d x}\right|_{x_{j}}=\left.\frac{d \alpha_{j}}{d x}\right|_{x_{j}} & j \in[1 ; N-1] & \text { (differenzierbar) } \\
\left.\frac{d \alpha_{0}}{d x}\right|_{x_{0}}=\left.\frac{d \alpha_{N-1}}{d x}\right|_{x_{N}}=0 & & \text { (linker und rechter Rand) }
\end{array}
$$

verbleiben die über die einzelnen Intervalle gemittelten Wärmeübergangskoeffizienten $\bar{\alpha}_{j}$ als Freiheitsgrade für den Spline übrig:

$$
\int_{x_{j}}^{x_{j+1}} \alpha_{j}(x) d x=\bar{\alpha}_{j} \cdot\left(x_{j+1}-x_{j}\right), j \in[0 ; N-1]
$$

Als Referenztemperatur für den Wärmeübergang im Seitenraum wird erneut die zwischen Einund Auslass des Versuchsstandes gemittelte Ruhetemperatur der Hauptströmung (Gleichung 4.18) gewählt. Aufbau und Lösung des FE-Modelles erfolgen vollautomatisch. Im Batch-Modus (Kommandozeilenmodus) dauert ein kompletter Rechendurchlauf auf einem Knoten des Hochleistungsrechner Taurus der TU Dresden mit 8 Kernen (CPU) ca. 6 s. Anschließend werden Knotentemperaturen an den Punkten evaluiert, an denen im Versuchsstand die Materialtemperaturen in den Innen- und Außenringen sowie im linken und rechten Stahlflansch gemessen werden. Für die inverse Berechnung werden die gesuchten WÜK („Designvariablen“)

$$
D V:=\left\{\bar{\alpha}_{j}(j \in[1 \ldots N]) ; \alpha_{\mathrm{TR}} ; \alpha_{\mathrm{AW}, \mathrm{l}} ; \alpha_{\mathrm{AW}, \mathrm{r}} ; \alpha_{\mathrm{AW}, \mathrm{a}}\right\}
$$

solange variiert, bis die simulierten Temperaturen $T_{k}$ mit den gemessenen Werten $T_{k}^{*}$ bestmöglich übereinstimmen. Die Optimierungsaufgabe besteht also in der Minimierung der Quadratsumme der Abweichungen zwischen berechneten und gemessenen (Soll-) Temperaturen in folgender Zielfunktion (objective function):

$$
\mathrm{dTopt}=\sum_{k}\left(T_{k}-T_{k}^{*}\right)^{2} \stackrel{!}{\rightarrow} \min , \quad k \in[11 \ldots 18 ; 31 \ldots 38 ; \mathrm{r} ; \mathrm{l}]
$$

Startwerte für $\bar{\alpha}_{j}$ werden dem thermischen Netzwerkmodell ( $\$$ Kap. 4.4.3) entnommen. 
Für diese sogenannte Designoptimierung wurde das in Ansys Mechanical APDL integrierte gleichnamige Tool verwendet. Die Optimierung wurde mit einem Gradientenverfahren 1. Ordnung firstorder gradient optimization) durchgeführt. Wie Abb. 4.23 zeigt, konvergiert der Optimierungsprozess nach 15 bis 20 Iterationen. Alle Abweichungen zwischen kalkulierten und Solltemperaturen liegen unterhalb $\pm 0,15 \mathrm{~K}$ (schwarze horizontale Linien in $>$ Abb. $4.23 \mathrm{~b}$ ), was gleichzeitig mit der maximalen beobachteten Abweichung der Temperaturmessdaten an den vier Umfangspositionen eines jeden Ringes übereinstimmt. Das entspricht einem Wert von dTopt $\approx 0,1 \mathrm{~K}^{2}$ ( Abb. $4.23 \mathrm{a}$ ). Gleichzeitig erreichen die iterierten WÜK nach 20 Durchläufen eine stationäre Lösung. Die konvergierten Werte (schwarzer Rahmen in Abb. 4.23 c) ergeben zusammen mit den Positionen $x_{\mathrm{S}, j}$ der Messringe die lokale WÜK-Verteilung in Abb. 4.23 d. Die schwarze Kurve kennzeichnet den berechneten Spline (Gleichung 4.19) und die horizontalen Linien die Oberflächen $\left[x_{j} ; x_{j+1}\right]$ der Innenringe, die in Kontakt mit dem Fluid sind. Zu Illustrationszwecken wurde das Strömungsfeld im Totraum aus der systematischen CFD-Nachrechnung in $>$ Kap. 6.6 hinterlegt.
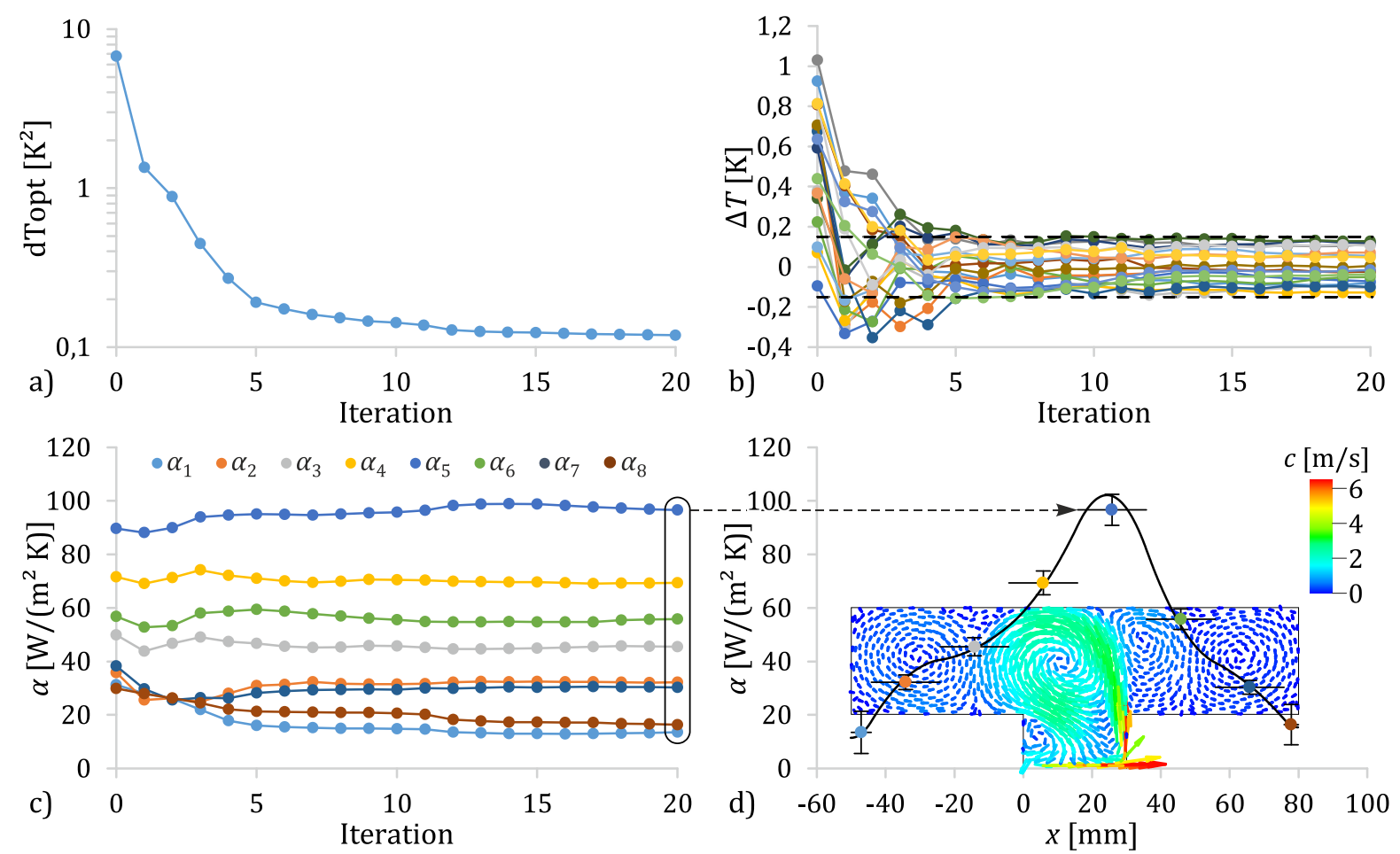

Abb. 4.23 Konvergenzverlauf der Designoptimierung für die inverse Bestimmung von WÜK mit dem FE-Modell für einen Beispielfall ( $s=30 \mathrm{~mm}, b=130 \mathrm{~mm}, e=0, R e=118.000)$.

a) Zielfunktion dTopt;

b) Differenzen zwischen simulierten und gemessenen Temperaturen $T_{k}-T_{k}^{*}$;

c) iterierte WÜK-Intervallmittelwerte $\bar{\alpha}_{j}$;

d) Resultierende WÜK-Verteilung entlang der Außenwand für das konvergierte FE-Modell. Vertikale Fehlerbalken kennzeichnen die Standardabweichung aus der Probabilistikanalyse ( Kap. 5.8.3.2). Horizontale Linien symbolisieren die Ringoberflächen, die in Kontakt mit dem Fluid im Seitenraum sind. 
Das resultierende Temperaturfeld der Seitenraumstrukturen ist in Abb. 4.24 dargestellt. Wie erwartet sind bei $x \approx 30 \mathrm{~mm}$, also im Bereich des Staupunktes, an dem der Prallstrahl auf die Außenwand auftrifft, die maximalen WÜK und damit die größte Kühlwirkung festzustellen. So weist der Innenring 5 eine deutlich geringere Temperatur auf als seine Nachbarn.

Das Modell sowie die Ergebnisse für einen weiteren Anwendungsfall $(s=46,3 \mathrm{~mm}, b=146,3 \mathrm{~mm}$, $e=0, R e=107.000$ ) wurden bei der ASME Turbo Expo 2018, welche vom 11. bis 15. Juni 2018 in Oslo, Norwegen, stattfand, der Fachwelt präsentiert [91] und im Journal of Engineering for Gas Turbines and Power veröffentlicht [92].

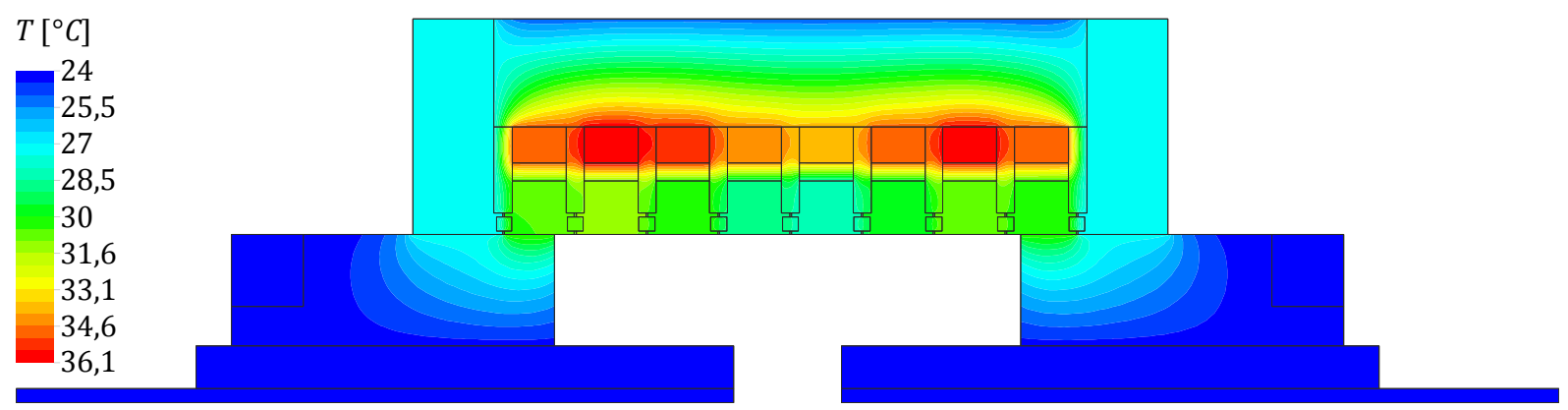

Abb. 4.24 Temperaturfeld der Seitenraumstrukturen für das konvergierte FE-Modell.

\subsubsection{Ableitungsfreie Optimierung mittels evolutionärem Algorithmus}

Zur Abklärung, ob mit dem oben beschriebenen Verfahren auch tatsächlich das globale Optimum getroffen worden ist, wurde die Optimierungsrechnung nochmals mit einem evolutionärer Algorithmus (EA) unter Verwendung der Optimierungsumgebung Dakota 6.9.0 durchgeführt. Das Simulationsmodell bleibt unberührt und wird weiterhin in Ansys Mechanical APDL 17.2 als „analysis driver" für Dakota gelöst.

Nicht gradientenbasierte (sog. „ableitungsfreie“) Optimierungsalgorithmen haben den Vorteil, dass sie prinzipiell das Auffinden eines globalen Optimums ermöglichen. Einen Nachweis, ob das globale Optimum tatsächlich erreicht wurde, gibt es jedoch auch beim EA nicht, die Wahrscheinlichkeit ist allerdings sehr hoch. Evolutionäre Algorithmen arbeiten mit verschiedenen Merkmalen und Mechanismen, die der Genetikforschung entlehnt sind. Ausgehend von einer Startpopulation, im vorliegenden Fall die Startwerte für alle Designvariablen, werden in einem sogenannten Reproduktionsschritt einzelne Individuen (Design-Parameter) zufällig verändert (Mutation) oder deren Werte miteinander vertauscht (Crossover) und anschließend deren Stärke (Fitness), das heißt der sich ergebende Wert der Zielfunktion bestimmt. Nach einer gewissen Anzahl zufälliger Variationen (Populationsgröße) werden die Parametersets mit der größten Fitness (best clones/individuals) ausgewählt und als Ausgangsbasis (Kind) für eine neue Generation ausgewählt (survival of the fittest). Die Art und 
Weise, wie stark die Fitness für die Auswahl der neuen Generation gewichtet wird (fitness type), wie die Individuen für die neue Generation ausgewählt werden und wie stark sie mutiert und gekreuzt werden, ist neben anderen verwendeten Einstellungen in $\mathbf{T a b}$. A.7 zusammengefasst.

Für die Designparameter (Gl. 4.24) wurden dieselben Startwerte wie für die Rückwärtsrechnung mittels Gradientenoptimierung verwendet. Als untere zulässige Grenze wurde durchgängig $0,001 \mathrm{~W} /\left(\mathrm{m}^{2} \mathrm{~K}\right)$ vorgegeben, als obere Grenze $200 \mathrm{~W} /\left(\mathrm{m}^{2} \mathrm{~K}\right)$ für die WÜK im Totraum sowie in der Hauptströmung bzw. $30 \mathrm{~W} /\left(\mathrm{m}^{2} \mathrm{~K}\right)$ für die äußere Konvektion.

Wie in Abb. 4.25 zu sehen ist, hat der Optimierer erst nach ca. 20.000 Iterationen ein weitgehend stabiles Minimum gefunden, nachdem er gerade zu Beginn für die ersten 5.000 bis 10.000 Iterationen noch größere Streuungen in den Designvariablen zugelassen hat. Der Wert der Zielfunktion dTopt (in Dakota obj_fun bezeichnet) liegt nach 20.000 Iterationen bei 0,1611 $\mathrm{K}^{2}$ und kommt damit noch nicht einmal an den Wert des (lokalen) Minimums des gradientenbasierten Algorithmus $\left(0,1189 \mathrm{~K}^{2}\right)$ heran, sondern entspricht in etwa dessen Wert nach der fünften bis sechsten (!) Iteration. In Anbetracht der sehr langen Laufzeit (ca. 56 h für 20.000 Durchläufe auf einer lokalen CPU) ist der Einsatz des evolutionären Algorithmus nicht zu empfehlen, wenn bereits sinnvolle Startwerte vorhanden sind, da er beispielsweise gegenüber dem gradientenbasierten Optimierer ein Vielfaches an Zeit benötigt und trotzdem nicht das exakte (globale) Minimum findet. Falls jedoch überhaupt keine Informationen zu (ungefähren) Startwerten bekannt sind, kann die Anwendung solcher stochastischer, metaheuristischer Methoden als erster Schritt sinnvoll sein. Im zweiten Schritt sollte dann aber immer eine gradientenbasierte Optimierung folgen.

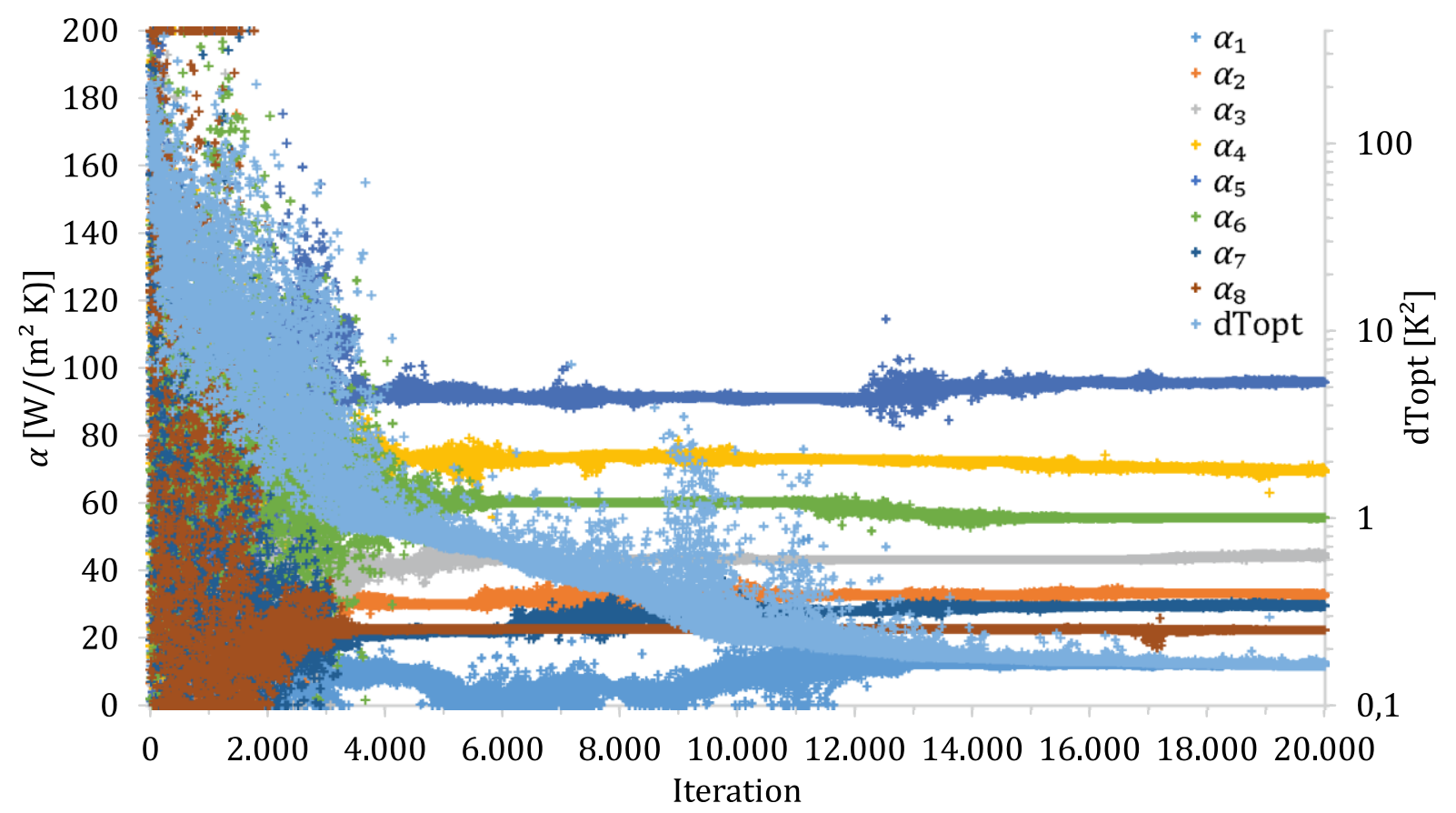

Abb. 4.25 Design-Sets und Zielfunktion für die gradientenfreie Designoptimierung mittels evolutionärem Algorithmus zur inversen Bestimmung von WÜK. 


\subsubsection{Inverse Berechnung mittels Trefftz-Funktionen und Regularisierung}

Der Nachteil iterativer Optimierungsverfahren besteht in der langsamen Konvergenz des Problems sowie im verbleibenden Abbruchfehler (Residuum). Außerdem ist nicht klar, ob die konvergierte Lösung nur ein lokales Optimum (Minimum) darstellt und folglich von den eingesetzten Startwerten abhängt. Daher besteht der Wunsch nach einer schnellen, direkten (analytischen) Lösung des numerischen Problems. Die Bestimmung des (örtlich und ggf. auch zeitlich variablen) Wärmeübergangskoeffizienten am Rand eines Rechengebietes auf der Basis von gemessenen Temperaturen innerhalb des Rechengebietes stellt dabei ein klassisches inverses Wärmeleitungsproblem dar. Dieses kann wie folgt formuliert werden. Innerhalb des Rechengebietes $\Omega$ ( Abb. 4.26) ist die stationäre Wärmeleitungsgleichung mit inneren Wärmequellen (Fourier-Gleichung) zu lösen:

$$
\nabla \cdot(\lambda \nabla T)=\tilde{q}
$$

mit der örtlich verschiedenen Wärmeleitfähigkeit $\lambda(\vec{x})$ und der lokal begrenzten, inneren Wärmequelldichte $\tilde{q}(\vec{x})$. An den Rändern $\Gamma_{\mathrm{i}}, \Gamma_{\mathrm{v}}$ und $\Gamma_{\mathrm{h}}$ sind Randbedingungen 3. Art gegeben:

$$
\begin{array}{ll}
\Gamma_{\mathrm{i}}: & -\lambda \nabla T \cdot \vec{n}=\alpha_{\mathrm{i}}\left(T-T_{\mathrm{F}}\right) \\
\Gamma_{\mathrm{v}}: & -\lambda \nabla T \cdot \vec{n}=\alpha_{\mathrm{v}}\left(T-T_{\mathrm{U}}\right) \\
\Gamma_{\mathrm{h}}: & -\lambda \nabla T \cdot \vec{n}=\alpha_{\mathrm{h}}\left(T-T_{\mathrm{U}}\right)
\end{array}
$$

wobei $\alpha_{\mathrm{i}}, \alpha_{\mathrm{v}}$ und $\alpha_{\mathrm{h}}$ die Wärmeübergangskoeffizienten für innere bzw. (äußere) vertikale und horizontale Oberflächen und $T_{\mathrm{F}}$ bzw. $T_{\mathrm{U}}$ die dazugehörigen Referenztemperaturen kennzeichnen.

$\partial T / \partial n \equiv \nabla T \cdot \vec{n}$ entspricht der Ableitung des Temperaturfeldes in Normalenrichtung $\vec{n}$ zur Wand. Die unbekannten Werte der Wärmeübergangskoeffizienten $\alpha_{\text {inv }}$ auf dem Rand $\Gamma_{\text {inv }}$

$$
\Gamma_{\text {inv }}: \quad-\lambda \nabla T \cdot \vec{n}=\alpha_{\text {inv }}\left(T-T_{\mathrm{F}}\right)
$$

sind nach der inversen Methode zu bestimmen.

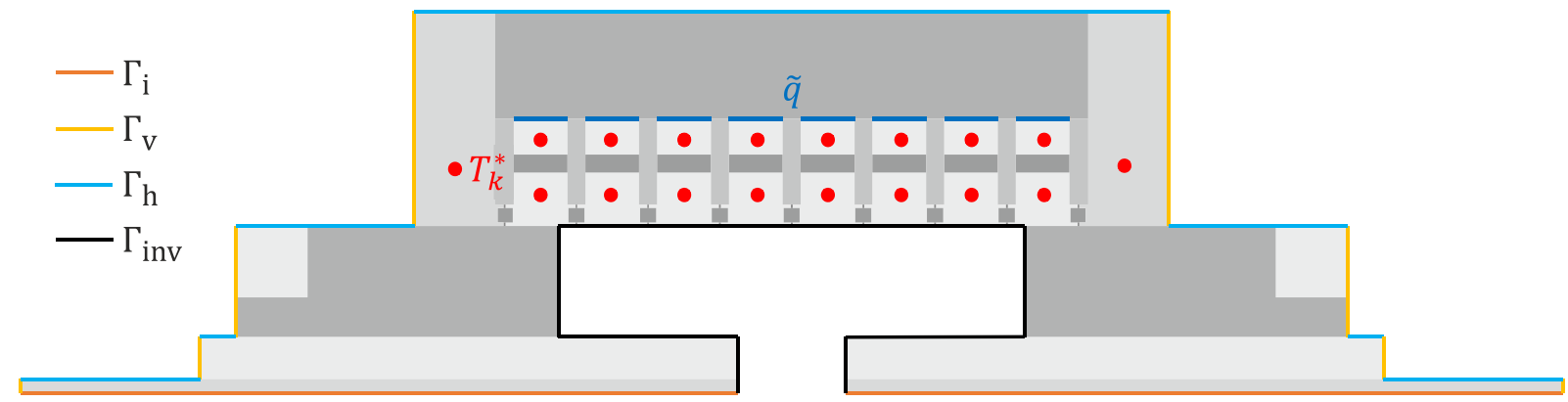

Abb. 4.26 Form des Rechengebietes $\Omega$, seines Randes $\Gamma=\Gamma_{i} \cup \Gamma_{v} \cup \Gamma_{h} \cup \Gamma_{\text {inv }}$ und Position der Temperaturmessstellen $T_{k}{ }^{*}$ und der Wärmequellen $\tilde{q}$. 
Wie schon beim gradientenbasierten Optimierungsverfahren besteht auch hierbei der Ansatz in der Minimierung der Summe der quadratischen Abweichungen zwischen berechneten Temperaturen $T_{k}=T\left(x_{k}, y_{k}\right)$ und den entsprechenden gemessenen Temperaturen $T_{k}^{*}$ (Positionen innerhalb des Rechengebietes $\Omega$ siehe Abb. 4.26) in Form des Funktionals (vgl. Gleichung 4.25)

$$
J[T]=\sum_{k=1}^{m}\left(T_{k}-T_{k}^{*}\right)^{2} \stackrel{!}{\rightarrow} \min
$$

Zur Lösung des inversen Problems kommt nun jedoch eine Methode zum Einsatz, welches sich das geschickte Anwenden des Superpositionsprinzips zu Nutze macht [93-107]. Demnach kann ein lineares Problem in mehrere Teilprobleme zerlegt werden ( Anhang A8), die separat gelöst werden können und später durch Linearkombination die Lösung des Gesamtproblems liefern. Die Lösung der Fourier-Gleichung als lineare partielle Differenzialgleichung ist ein solches lineares Problem [108-110]. Voraussetzung für das weitere Vorgehen ist, dass die unbekannten Randwerte auf $\Gamma_{\text {inv }}$ zunächst als Randbedingungen 2. Art (konstante Wandwärmestromdichten) gesucht werden müssen und erst später in die WÜK umgerechnet werden können.

Im vorliegenden Fall wird der Rand $\Gamma_{\text {inv }}$ in $n$ diskrete Teilintervalle $\Gamma_{\text {inv, } j}$ zerlegt. Die Wärmestromdichteverteilung $\dot{q}_{\text {inv }}$ auf dem Rand $\Gamma_{\text {inv }}$ kann dann rein formell als Linearkombination der Variablenfunktion $\dot{q}_{\mathbb{1}, j}(s)$ ausgedrückt werden:

$$
\Gamma_{\mathrm{inv}}: \quad-\lambda \nabla T \cdot \vec{n}=\dot{q}_{\mathrm{inv}}=\sum_{j=1}^{n} c_{j} \dot{q}_{\mathbb{1}, j}(s)
$$

In jedem Intervall $\Gamma_{\text {inv, } j}$ ist die Funktion $\dot{q}_{\mathbb{1}, j}$ Eins und außerhalb Null:

$$
\frac{\dot{q}_{1, j}(s)}{\mathrm{W} / \mathrm{m}^{2}}=\left\{\begin{array}{rrr}
-1 & \forall & s \in \Gamma_{\mathrm{inv}, j} \\
0 & \forall & s \notin \Gamma_{\mathrm{inv}, j}
\end{array} \quad j=1 \ldots n\right.
$$

Aus den Gleichungen 4.30 und 4.31 folgt, dass die unbekannten Koeffizienten $c_{j}$ die (über die Fläche konstante) Wärmestromdichte über den jeweiligen Intervallen, geteilt durch $\mathrm{W} / \mathrm{m}^{2}$, darstellen:

$$
\dot{q}_{\mathrm{inv}, j}=-c_{j} \frac{\mathrm{W}}{\mathrm{m}^{2}}
$$

Die Minuszeichen in den Gleichungen 4.31 und 4.32 wurden eingeführt, um in den Darstellungen in Abb. 4.28 die Wärmeabgabe von den Seitenwandstrukturen in das Fluid zu verdeutlichen, und heben sich gegenseitig auf. Die Verteilung der Wärmestromdichte auf dem Rand $\Gamma_{\text {inv }}$ wird also in Form einer Stufenfunktion gesucht (siehe Abb. 4.27). 


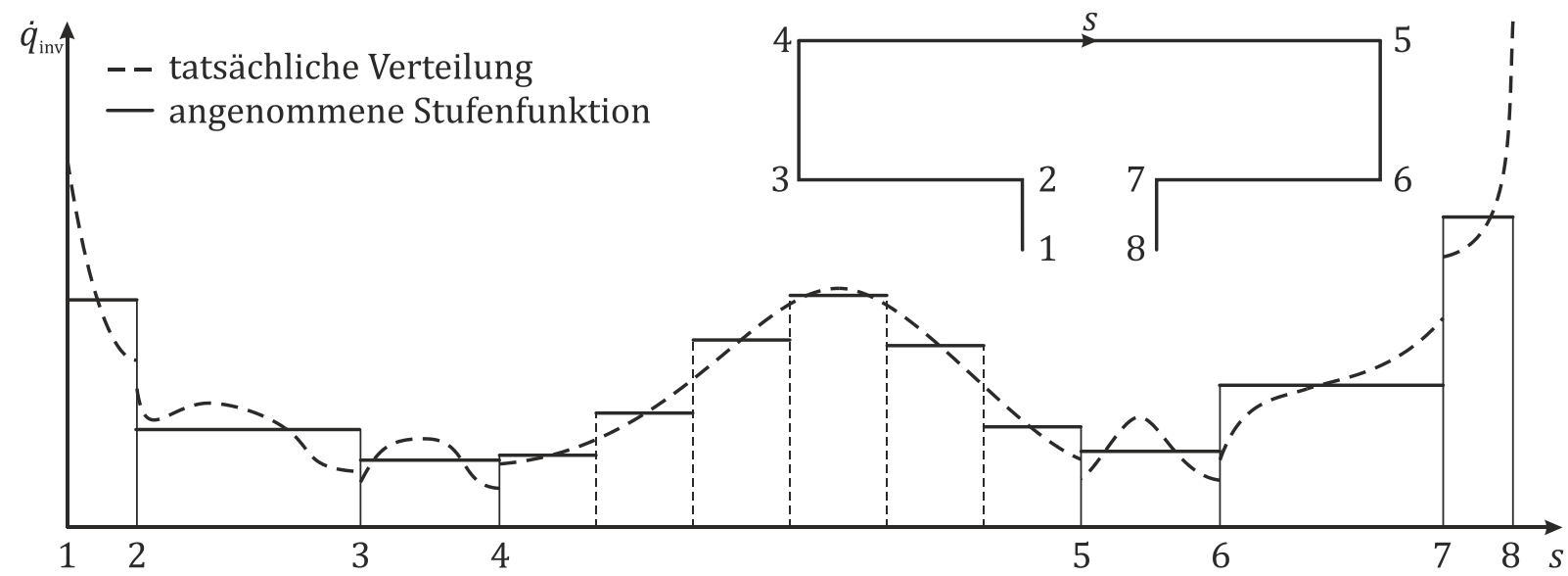

Abb. 4.27 Approximation der Wärmestromdichte auf dem Rand des Seitenraumes $\Gamma_{\text {inv. }}$

Die Lösung von Gleichung 4.26 mit den Randbedingungen 4.27 und 4.30 soll nun unter Einbeziehung der Koeffizienten $c_{j}$ als folgende Linearkombination einer „homogenen“ Lösung $T_{0}$ und den sogenannten Trefftz-Funktionen $\theta_{j}$ ausgedrückt werden („Kunstgriff“):

$$
\Omega: \quad T(x, y)=T_{0}(x, y)+\sum_{j=1}^{n} c_{j} \theta_{j}(x, y)
$$

Die Funktion $T_{0}$ ist dabei die Lösung der Differenzialgleichung 4.26 mit den Randbedingungen 4.27 und der Annahme, dass alle Ränder mit unbekannten Randbedingungen adiabat sind:

$$
\Gamma_{\mathrm{inv}}: \quad-\lambda \nabla T \cdot \vec{n}=0
$$

Die Funktionen $\theta_{j}(x, y)$ sind dann die Lösungen der Fourier-Gleichung ohne Wärmequellen

$$
\nabla \cdot(\lambda \nabla T)=0
$$

bezüglich der Randbedingungen

$$
\begin{array}{ll}
\Gamma_{\mathrm{i}}: & -\lambda \nabla \theta_{j} \cdot \vec{n}=\alpha_{\mathrm{i}} \theta_{j} \\
\Gamma_{\mathrm{v}}: & -\lambda \nabla \theta_{j} \cdot \vec{n}=\alpha_{\mathrm{v}} \theta_{j} \\
\Gamma_{\mathrm{h}}: & -\lambda \nabla \theta_{j} \cdot \vec{n}=\alpha_{\mathrm{h}} \theta_{j}
\end{array}
$$

sowie

$$
\Gamma_{\mathrm{inv}}: \quad-\lambda \nabla \theta_{j} \cdot \vec{n}=\dot{q}_{\mathbb{1}, j}
$$

d. h. jede Funktion $\theta_{j}$ wird bestimmt, indem angenommen wird, dass der Rand $\Gamma_{\mathrm{inv}, j}$ von $A b-$ schnitt $j$ Einheitswärmestrom aufweist und alle übrigen Abschnitte adiabat sind. Die Koeffizienten $c_{j}$ haben sich entsprechend heraus gekürzt (Herleitung siehe Anhang A8). Damit sind zunächst einmal alle Teilprobleme eindeutig lösbar. 
Als nächstes sollen die unbekannten Werte von $c_{j}$ in Gl. 4.30 ermittelt werden. Dafür wird Gleichung 4.33 in Gleichung 4.29 eingesetzt:

$$
J[c]=\sum_{k=1}^{m}\left(T_{0, k}+\sum_{j=1}^{n} c_{j} \theta_{j, k}-T_{k}^{*}\right)^{2} \stackrel{!}{\rightarrow} \min
$$

wobei $T_{0, k}=T_{0}\left(x_{k}, y_{k}\right)$ und $\theta_{j, k}=\theta_{j}\left(x_{k}, y_{k}\right)$ die berechneten Temperaturen in denjenigen Knoten $\left(x_{k}, y_{k}\right)$ darstellen, welche mit den Temperaturmesspunkten korrespondieren. Für die gesuchten Werte der Koeffizienten $c_{j}$ wird das Funktional 4.38 minimal. Dies ist genau dann der Fall, wenn alle Ableitungen von $J[c]$ nach $c_{j}$ verschwinden:

$$
\frac{\partial J[c]}{\partial c_{j}}=2 \sum_{k=1}^{m}\left(T_{0, k}+\sum_{j=1}^{n} c_{j} \theta_{j, k}-T_{k}^{*}\right) \theta_{j, k} \stackrel{!}{=} 0
$$

Es ergibt sich das folgende lineare Gleichungssystem:

$$
\sum_{k=1}^{m}\left(\theta_{j, k} \sum_{j=1}^{n} c_{j} \theta_{j, k}\right)=-\sum_{k=1}^{m}\left(T_{0, k}-T_{k}^{*}\right) \theta_{j, k}
$$

Das Gleichungssystem 4.40 kann bereits eindeutig gelöst werden. Es werden jedoch extrem hohe positive und negative Wärmeströme $\dot{q}_{\text {inv,j }}$ berechnet, die zwar das globale Minimum von Gleichung 4.38 mathematisch exakt abbilden, jedoch physikalisch unplausibel sind. Der Grund hierfür ist, dass inverse Probleme zu den schlecht gestellten Problemen (ill-posed problems) im Sinne von Hadamard [111] zählen. Dies bedeutet, dass kleinere Abweichungen in den gemessenen Temperaturen zu großen (lokalen und zeitlichen) Abweichungen in der Lösung des inversen Problems führen können.

Die Qualität der Lösung von Gleichung 4.40 kann jedoch durch eine sog. Tichonov-Regularisierung [112] verbessert werden. Dazu wird dem Funktional 4.29 ein Strafterm angefügt:

$$
J[c]=\sum_{k=1}^{m}\left(T_{k}-T_{k}^{*}\right)^{2}+\beta \sum_{j=1}^{n} c_{j}^{2}
$$

der den über die Grenzen mit unbekannten Randbedingungen fließenden Wärmestrom in die Optimierung mit einbezieht (Energiemethode). Durch Minimierung der Energiedissipation, die am Ende eines jeden physikalischen Prozess steht (1. Hauptsatz der Thermodynamik), werden örtliche Überhöhungen der gesuchten Wärmestromdichte, die sonst bei der Lösung auftreten könnten, geglättet. Dabei ist $\beta>0$ der zu wählende Regularisierungsparameter, der den Energieterm 
gegenüber dem Zielfunktional entsprechend wichtet. Setzt man nun Gleichung 4.33 in Gleichung 4.41 ein, erhält man

$$
J[c]=\sum_{k=1}^{m}\left(T_{0, k}+\sum_{j=1}^{n} c_{j} \theta_{j, k}-T_{k}^{*}\right)^{2}+\beta \sum_{j=1}^{n} c_{j}^{2}
$$

Ableitung von Gleichung 4.42 nach $c_{j}$ liefert

$$
\frac{\partial J[c]}{\partial c_{j}}=2 \sum_{k=1}^{m}\left(T_{0, k}+\sum_{j=1}^{n} c_{j} \theta_{j, k}-T_{k}^{*}\right) \theta_{j, k}+2 \beta c_{j} \stackrel{!}{=} 0
$$

Das sich ergebende lineare Gleichungssystem

$$
\sum_{k=1}^{m}\left(\theta_{j, k} \sum_{j=1}^{n} c_{j} \theta_{j, k}\right)+\beta c_{j}=-\sum_{k=1}^{m}\left(T_{0, k}-T_{k}^{*}\right) \theta_{j, k}
$$

mit $n$ Gleichungen für die $n$ Unbekannten $c_{j}(j=1 \ldots n)$ kann wieder direkt, d. h. ohne die Vorgabe von Startwerten, ohne Iterationen und Residuen, gelöst werden, beispielsweise mittels Gauß'schem Eliminierungsverfahren. Um ein Überanpassen (over-fitting) zu vermeiden, darf die Anzahl der Abschnitte $\Gamma_{\text {inv, } j}$ mit unbekanntem Wärmestrom nicht größer sein als die Anzahl der Temperaturmessstellen: $n \leq m$.

Zur Implementierung des obigen Algorithmus für die inverse Berechnung mittels Trefftz-Funktionen wurde das im vorangegangenen Kap. 4.4.4 beschriebene FE-Modell in Ansys Mechanical APDL 17.2 entsprechend erweitert. Wie in Abb. 4.27 dargestellt wird die innere Begrenzung der Kavität $\Gamma_{\text {inv }}$ sinnvollerweise so gegliedert, dass jede Kante ein eigenes Intervall $\Gamma_{\text {inv, } j}$ erhält. Die obere Kante (Linie 4-5) wird noch weiter unterteilt in eine festgelegte Zahl an Unterabschnitten, um dort örtliche Unterschiede in den WÜ-Verhältnissen auflösen zu können. Alle anderen Kanten werden nicht weiter zerlegt, da dort auf Grund fehlender Temperaturmessstellen in der Nähe eine Reproduktion der tatsächlichen WÜK ohnehin kaum möglich ist. Sechs verschiedene Varianten wurden hinsichtlich der Teilung des oberen Randes der Kavität untersucht, um den Mittelungseinfluss auf die berechnete Verteilung der lokalen WÜK feststellen zu können ( Tab. 4.6).

In den Varianten 1 bis 5 wurde die Linie 4-5 in $N_{\mathrm{Abs}}=6 ; 7 \ldots 10$ äquidistante Abschnitte unterteilt. Bei Variante 6 entspricht die Unterteilung den benetzten Oberflächen der einzelnen Messringe, wie das schon für die Spline-Funktion bei der Gradientenoptimierung so gehandhabt wurde (Abb. 4.22 und Gleichung 4.19). Zur Lösung des inversen Problems in Form von Gleichung 4.33 wurden für den vorliegenden Anwendungsfall ( Kap. 4.4.4) im ersten Schritt die Basisfunktion $T_{0}$ sowie die Trefftz-Funktionen $\psi_{j}, j=1 \ldots n$, auf direktem Wege ermittelt. Eine Auswahl dieser Funktionen ist in Abb. 4.28 dargestellt. Wie ersichtlich ist, weisen die Funktionen $\psi_{j}$ ein Minimum an demjenigen Segment $\Gamma_{\text {inv,j }}$ der inneren Begrenzung auf, an dem der Wärmefluss Eins ist. 
Tab. 4.6 Einteilung des Randes $\Gamma_{\text {inv }}$ in Unterabschnitte

\begin{tabular}{cccccccc}
\hline Anzahl der & \multicolumn{7}{c}{ Kante vom Rand $\Gamma_{\text {inv }}$} \\
\cline { 2 - 8 } Abschnitte & $1-2$ & $2-3$ & $3-4$ & $4-5$ & $5-6$ & $6-7$ & $7-8$ \\
\hline Var. 1 & 1 & 1 & 1 & $\mathbf{6}$ & 1 & 1 & 1 \\
Var. 2 & 1 & 1 & 1 & $\mathbf{7}$ & 1 & 1 & 1 \\
Var. 3 & 1 & 1 & 1 & $\mathbf{8}$ & 1 & 1 & 1 \\
Var. 4 & 1 & 1 & 1 & $\mathbf{9}$ & 1 & 1 & 1 \\
Var. 5 & 1 & 1 & 1 & $\mathbf{1 0}$ & 1 & 1 & 1 \\
Var. 6 & 1 & 1 & 1 & $\mathbf{8}^{*}$ & 1 & 1 & 1 \\
\hline
\end{tabular}

* Abschnitte gemäß Ringteilung

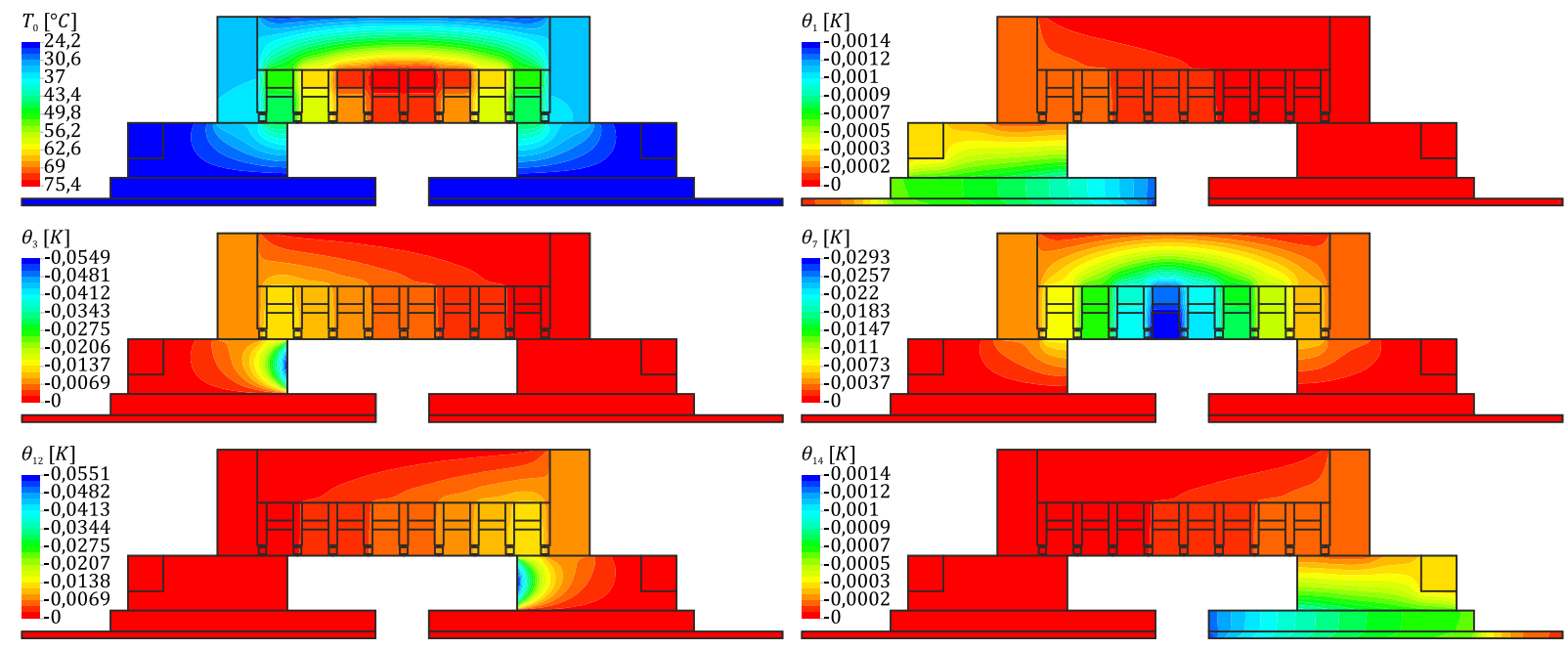

Abb. 4.28 Basisfunktion $T_{0}$ und ausgewählte Trefftz-Funktionen $\theta_{j}$ als Teillösungen des inversen Problems für den Beispielfall

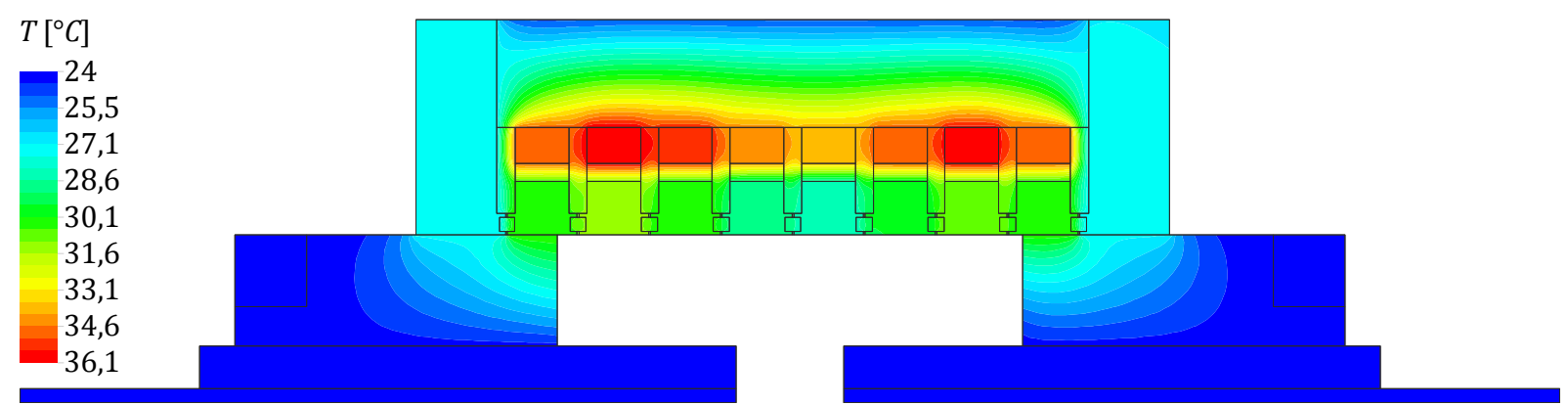

Abb. 4.29 Resultierende Temperaturverteilung der Superpositionslösung für Variante 3. Regularisierungsparameter: $\beta=10^{-5} \mathrm{~K}^{2}$. 
Wie erwartet liefert die Berechnung ohne Regularisierung gemäß Gleichung 4.40 keine stabile bzw. plausible Lösung für die Koeffizienten $c_{j}$. Daher muss das Problem regularisiert werden (Gleichung 4.44). Zur Bestimmung des optimalen Regularisierungsparameters $\beta$ und zur Abschätzung, wie gut sich die berechneten Werte aus der Lösung des Inversen Problems mit den gemessenen (Soll-) Temperaturen $T_{k}^{*}$ decken, wird die empirische Stichprobenstandardabweichung $\sigma$ definiert:

$$
\sigma=\sqrt{\frac{1}{m} \sum_{k=1}^{m}\left(T_{k}-T_{k}^{*}\right)^{2}}=\sqrt{\frac{J[T]}{m}}
$$

In Abb. 4.30 ist für Variante 3 der Einfluss des Regularisierungsparameters auf die Standardabweichung $\sigma$, die einzelnen Temperaturdifferenzen $(\Delta T)_{k}$, die berechneten Wärmestromdichten $\dot{q}_{\text {inv }, j}$ sowie den gesamten, integralen über $\Gamma_{\text {inv }}$ fließenden Wärmestrom $\dot{Q}_{\text {inv }}$ dargestellt.
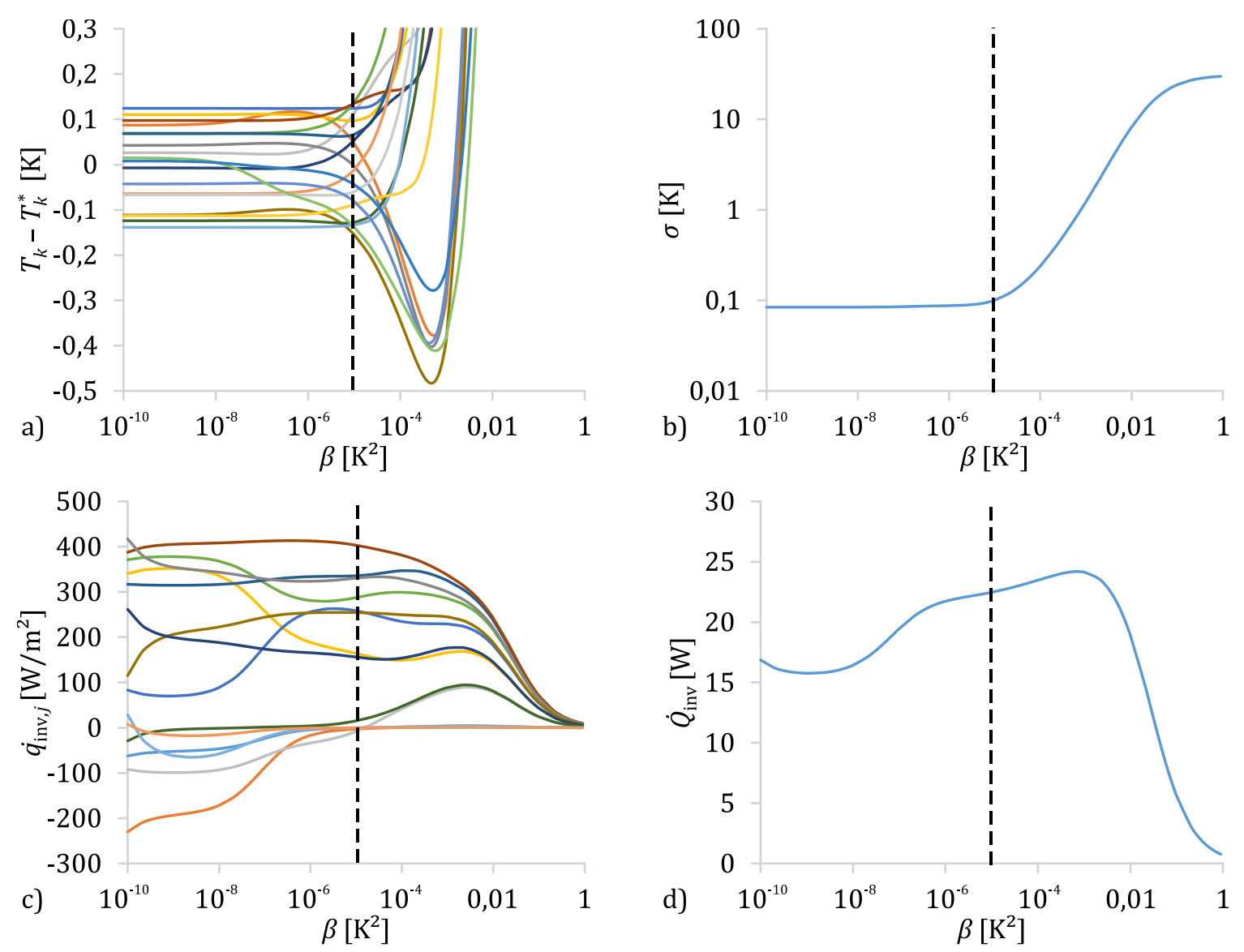

Abb. 4.30 Einfluss der Wahl des Regularisierungsparameters $\beta$ auf die Genauigkeit und die Stabilität der Lösung des inversen Problems für Variante 3:

a) Abweichungen zwischen berechneten und gemessenen Temperaturen $(\Delta T)_{k}=T_{k}-T_{k}^{*}$,

b) die daraus resultierende Stichproben-Standardabweichung $\sigma$ als Genauigkeitsparameter,

c) die berechneten Wärmestromdichten $\dot{q}_{\text {inv }, j}$ in den einzelnen Abschnitten und

d) der gesamte Wärmestrom $\dot{Q}_{\text {inv }}$ über alle Abschnitte $\Gamma_{\text {inv }}$ 
Für kleine Werte von $\beta \rightarrow 0$ wird $\sigma$ zwar minimal, d. h. die Solltemperaturen werden am besten approximiert, die dafür notwendigen Wärmestromdichten $\dot{q}_{\mathrm{inv}, j}$ nehmen allerdings teilweise unrealistische Werte an. Sehr große $\beta$-Werte wiederum minimieren zwar den über die Grenze fließenden Wärmestrom $\dot{Q}_{\text {inv }}$, jedoch nur auf Kosten von hohen Abweichungen zwischen berechneten und gemessenen Temperaturen $(\Delta T)_{k}$. Als besten Kompromiss wird daher $\beta=10^{-5} K^{2}$ für alle weiteren Betrachtungen und Auswertungen gewählt.

Die finale Lösung für die Temperaturverteilung für Variante 3 ist in Abb. 4.29 abgebildet. Der mittlere WÜK $\bar{\alpha}_{j}$ in jeder Sektion $j$ wird aus den mittleren Werten für die Wärmestromdichten $\dot{q}_{(\mathrm{inv},) j}$ und Wandtemperaturen $\bar{T}_{\mathrm{W}, j}$ sowie der Bezugstemperatur $T_{\mathrm{B}}$ berechnet:

$$
\bar{\alpha}_{j}=\frac{\dot{q}_{j}}{\bar{T}_{\mathrm{W}, j}-T_{\mathrm{B}}}
$$

In Abb. 4.31 sind die gemittelten WÜK auf dem Rand 4-5 über den Intervallmittelpunkten $x_{\mathrm{S}, j}$ für jeden Fall aufgetragen. Wie sich zeigt, haben Art und Anzahl der Unterteilungen von Linie 4-5 keinen Einfluss auf die berechnete WÜK-Verteilung und damit auf die Stabilität der Lösung. In allen Fällen konnte eine gute Konsistenz zwischen gemessenen und invers berechneten Temperaturen erreicht werden: Für Variante 3 etwa beträgt die maximale absolute Abweichung über alle Messringtemperaturen zwischen $T_{k}$ und $T_{k}^{*} 0,15 K$ ( Tab. 4.7). Dies entspricht wieder der maximal beobachteten Abweichung der gemessenen Temperatur am Umfang eines Ringes. Die Standardabweichung liegt bei $\sigma=0,1 K$, s. a. Abb. $4.30 \mathrm{~b}$.

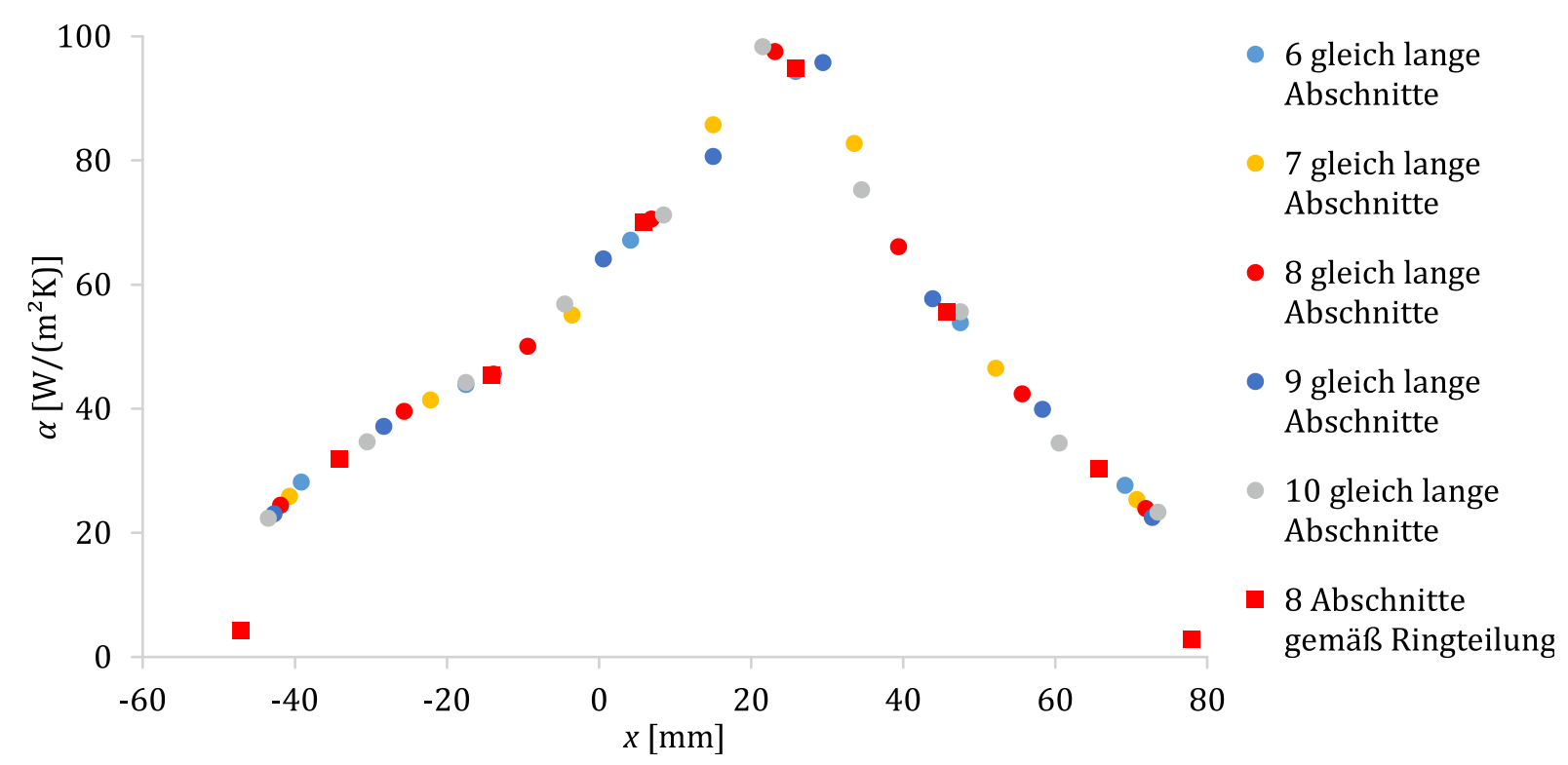

Abb. 4.31 Vergleich der invers berechneten WÜK am oberen Rand der Kavität für die verschiedenen Teilungen. $\beta=10^{-5} K^{2}$ 
Tab. 4.7 Vergleich der berechneten Temperaturen $T_{k}$ mit den gemessenen Solltemperaturen $\boldsymbol{T}_{k}{ }^{*}$ für Variante 3

\begin{tabular}{rcccc}
\hline$k$ & Name & $T_{k}^{*}\left[{ }^{\circ} \mathrm{C}\right]$ & $T_{k}\left[{ }^{\circ} \mathrm{C}\right]$ & $T_{k}-T_{k}^{*}[K]$ \\
\hline 0 & TL & 30,49 & 30,54 & 0,05 \\
1 & T11 & 31,07 & 31,18 & 0,11 \\
2 & T12 & 30,17 & 30,26 & 0,10 \\
3 & T13 & 28,87 & 28,99 & 0,12 \\
4 & T14 & 28,26 & 28,40 & 0,14 \\
5 & T15 & 29,54 & 29,61 & 0,07 \\
6 & T16 & 30,78 & 30,91 & 0,13 \\
7 & T17 & 30,38 & 30,37 & 0,00 \\
8 & T18 & 35,08 & 34,93 & $-0,15$ \\
9 & T31 & 36,07 & 36,13 & 0,05 \\
10 & T32 & 35,64 & 35,51 & $-0,13$ \\
11 & T33 & 34,53 & 34,39 & $-0,13$ \\
12 & T34 & 33,93 & 33,92 & $-0,01$ \\
13 & T35 & 34,96 & 34,90 & $-0,06$ \\
14 & T36 & 35,96 & 35,87 & $-0,09$ \\
15 & T37 & 34,86 & 34,78 & $-0,08$ \\
16 & T38 & 27,35 & 27,21 & $-0,14$ \\
17 & TR & 27,16 & 27,12 & $-0,04$ \\
\hline
\end{tabular}

In Zusammenarbeit mit Prof. Michał Ciałkowski und Prof. Andrzej Frąckowiak von der PUT Poznan ist ein weiteres zweidimensionales FE-Modell mit unstrukturiertem Dreiecksnetz ( Abb. 4.32) in der Entwicklungsumgebung FreeFem++ [113] entstanden, mit dessen Hilfe der vorliegende Anwendungsfall ebenfalls ausgewertet wurde. Die Ergebnisse wurden zusammen mit den theoretischen Betrachtungen vom Beginn dieses Teilkapitels bei der XI International Conference on Computational Heat, Mass and Momentum Transfer (ICCHMT) vom 21. bis 24. Mai 2018 in Krakau, Polen vorgestellt [88] und in einer gemeinsamen Publikation im International Journal of Numerical Methods for Heat \& Fluid Flow veröffentlicht [89]. Eine weitere Veröffentlichung erfolgte beim Symposium on Heat and Mass Transfer der Technischen Universität Köslin, Polen, die vom 16. bis 19. September 2019 in Kolberg, Polen, stattfand [114]. Die dort ermittelten WÜK werden im folgenden Teilkapitel mit diskutiert.

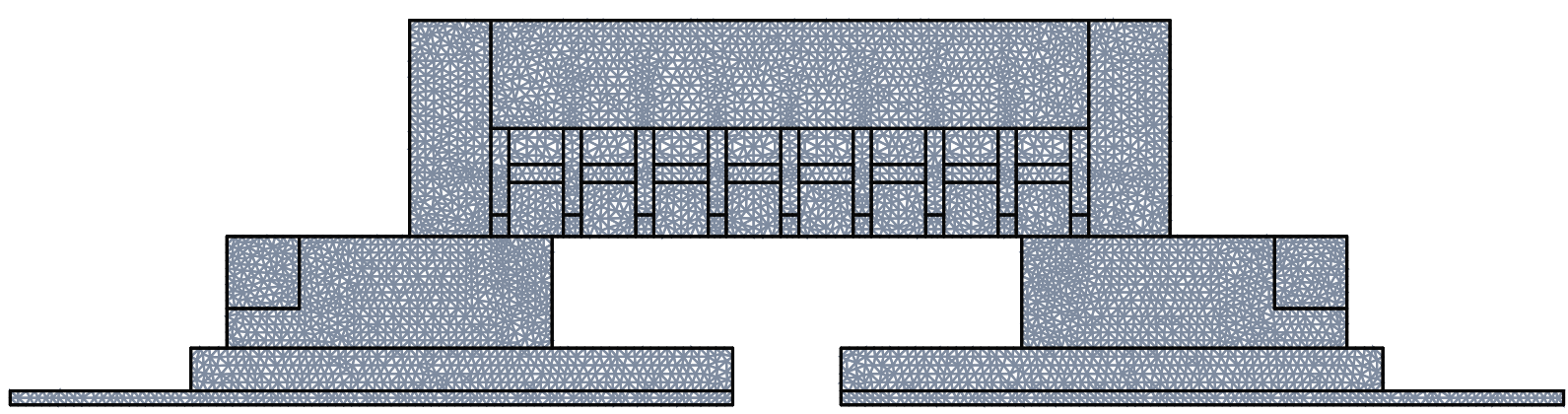

Abb. 4.32 Unstrukturiertes Rechennetz des FE-Modells für die inverse Berechnung in FreeFEM++. 


\subsubsection{Vergleich der mit den Rechenmodellen ermittelten WÜK-Verläufe}

Die für den vorliegenden Fall mittels der drei vorgestellten Berechnungsmodelle ermittelten lokalen Wärmeübergangkoeffizienten sind in Abb. 4.33 zusammengefasst. Wie sich zunächst zeigt, stimmen die mit den beiden FE-basierten inversen Verfahren sowie die mit dem analytischen thermischen Netzwerkmodell ermittelten Werte sehr gut überein. Die Ergebnisse des gradientenbasierten Optimierungsverfahrens sind - mit Ausnahme der beiden äußeren Punkte quasi identisch mit den Resultaten der Trefftz-Zerlegung für die Ringteilung (orange Symbole), obwohl bei ersterem eine Spline-basierte WÜK-Verteilung (RB 3. Art) angesetzt wird, während bei letzterem abschnittsweise konstante Wärmeströme (RB 2. Art) als Zielparameter gesucht werden. Das beweist zum einen, dass die Ergebnisse unabhängig von der Art und der Form der gesuchten thermischen Randbedingungen sind, und zum anderen, dass die Optimierung für den vorliegenden Fall vollständig konvergiert ist und das globale Minimum getroffen hat. Während zwischen den Ergebnissen aus dem FreeFEM- und dem Ansys-Modell zu den Rändern hin noch geringe Abweichungen auftreten, welche vermutlich aus dem Nichtmodellieren der metallischen Stege zwischen zwei benachbarten Innenringen (vgl. Abb. 4.32 mit Abb. 4.21) resultieren, stimmen die Werte für die inneren der acht gleich langen Abschnitte gut überein (blaue Symbole). Dies bestätigt die Validität des Algorithmus und dessen Implementierung sowie die weitgehende Unabhängigkeit der Ergebnisse vom Rechennetz und vom Simulationsprogramm.

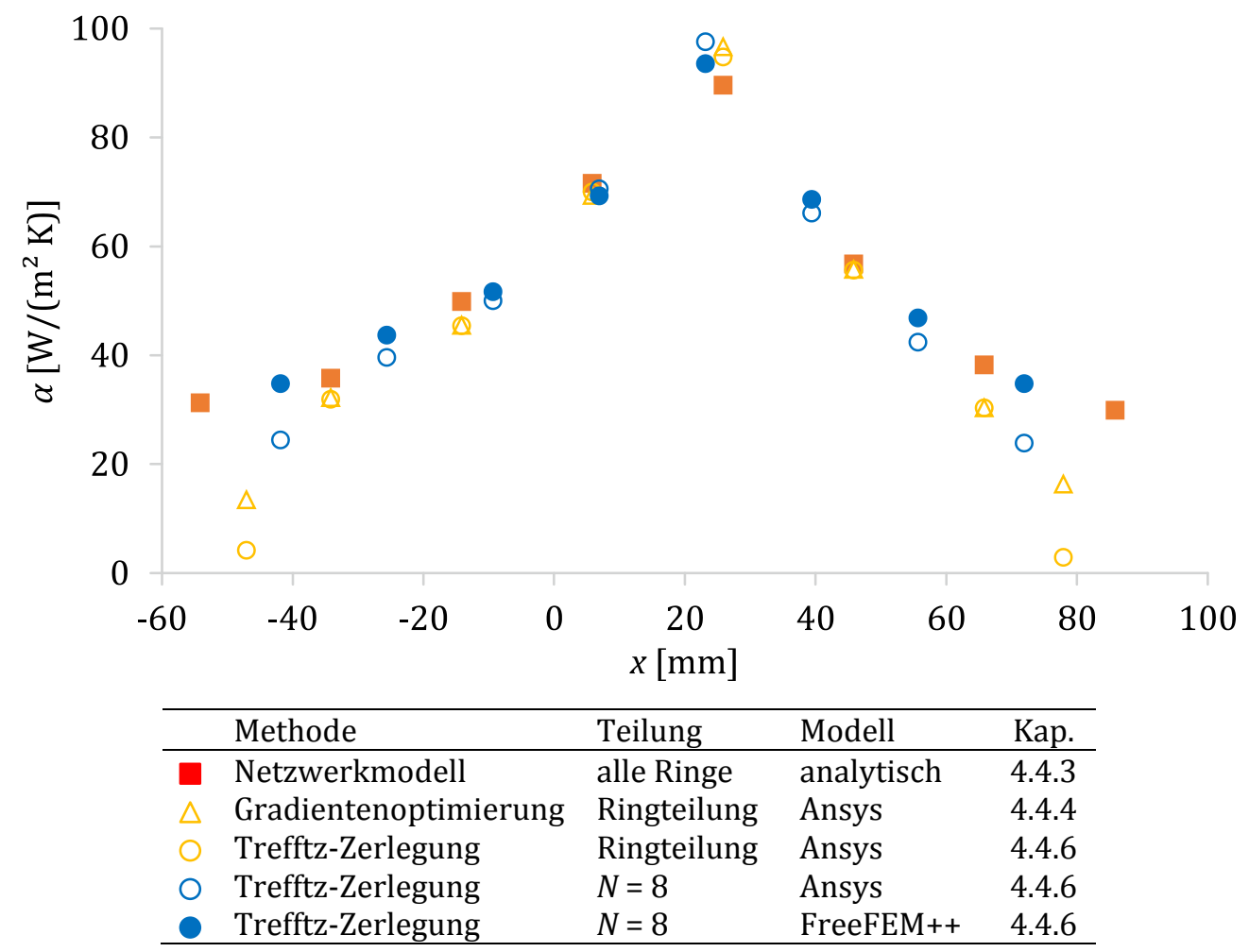

Abb. 4.33 Vergleich der mittels der Rechenmodelle ermittelten lokalen Wärmeübergangskoeffizienten für den vorliegenden Beispielfall:

$s=30 \mathrm{~mm}, b=130 \mathrm{~mm}, e=0, x_{\mathrm{SW}, \mathrm{l}}=4,2 \mathrm{~mm}, R e=114.000$. 
In den Randbereichen liegen die FreeFEM-Werte wiederum sehr nahe an dem WÜK-Verlauf, den das stark vereinfachte, analytische Netzwerkmodell liefert, welches sich daher ebenfalls bestens zur Abschätzung der WÜK an der Innenoberfläche der Außenwand eignet.

Insgesamt sind alle drei vorgestellten Rechenmethoden und -modelle valide und können für die inverse Berechnung der lokalen WÜK im Versuchsstand auf Basis der gemessenen Wandtemperaturen verwendet werden. Zu den Unsicherheiten der einzelnen Modelle siehe Kap. 5.8.3.

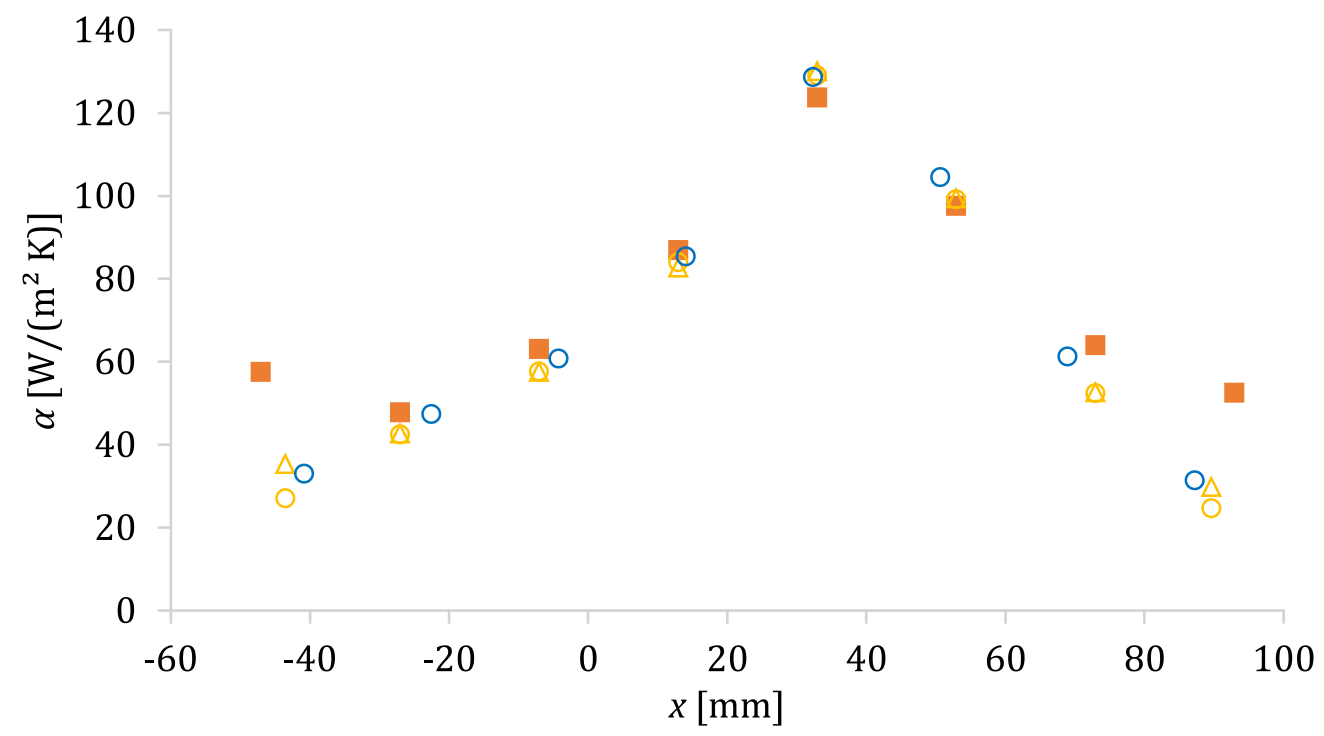

\footnotetext{
Abb. 4.34 Vergleich der mittels der Rechenmodelle ermittelten lokalen Wärmeübergangskoeffizienten für einen weiteren Beispielfall [88,89,114]:

$s=46,3 \mathrm{~mm}, b=146,3 \mathrm{~mm}, e=0, x_{\mathrm{SW}, 1}=-2,9 \mathrm{~mm}, R e=117.000$.
}

\subsection{VERSUCHSPLANUNG UND -DURCHFÜHRUNG}

\subsubsection{Versuchsablauf}

Nachdem die Abmessungen des Seitenraumes eingestellt worden sind und die Hauptströmung im Versuchsstand einen stationären Zustand erreicht hat, werden die Heizwicklungen aller acht Messringe eingeschaltet und für jedes Modul eine konstante elektrische Leistung über separate Laborspannungsversorgungsgeräte $(1 \times$ Voltcraft PS 2403 Pro; $6 \times$ Blanko PS-3003) eingestellt. Gleichzeitig wird die Messsequenz für die lokale Übertemperaturmethode gestartet. Dabei befinden sich immer zwei WÜK-Sensoren gleichzeitig für drei Minuten im Messbetrieb. Danach werden sie abgeschaltet und zwei weitere Sensoren folgen. Insgesamt werden drei aufeinander folgende Durchläufe durchgeführt, d. h. jeder Sensor wird dreimal betrieben. Druck-, Temperatur-, Geschwindigkeits- und andere über das Druckluftsystem verteilte Betriebsmessstellen werden alle zwei Sekunden mit Hilfe der beiden von der Professur MFD eigens entwickelten Datenerfassungsgeräte überwacht und aufgezeichnet. Nach zwei bis drei Stunden haben die Materialtemperaturen 
in der Totraumaußenwand das thermische Gleichgewicht (thermal equilibrium) erreicht. Das wird auch an den lokalen WÜK deutlich, welche mit dem in $\$$ Kap. 4.4 .3 beschriebenen Netzwerkmodell in Echtzeit bereits während der Messung berechnet und mitgeschrieben werden können. Wenn sich diese über einen längeren Zeitraum (mehrere Minuten) nicht weiter ändern ( Abb. 4.35), kann die Messung für die stationäre inverse Methode beendet werden. Der Versuchsablauf kann dann anschließend für eine andere Reynolds-Zahl oder eine andere geometrische Seitenraumkonfiguration wiederholt werden. Für weiterführende Informationen sowie einen detaillierten Versuchsablauf sei auf die Bedienungsanleitung des Totraumversuchsstandes verwiesen [115].

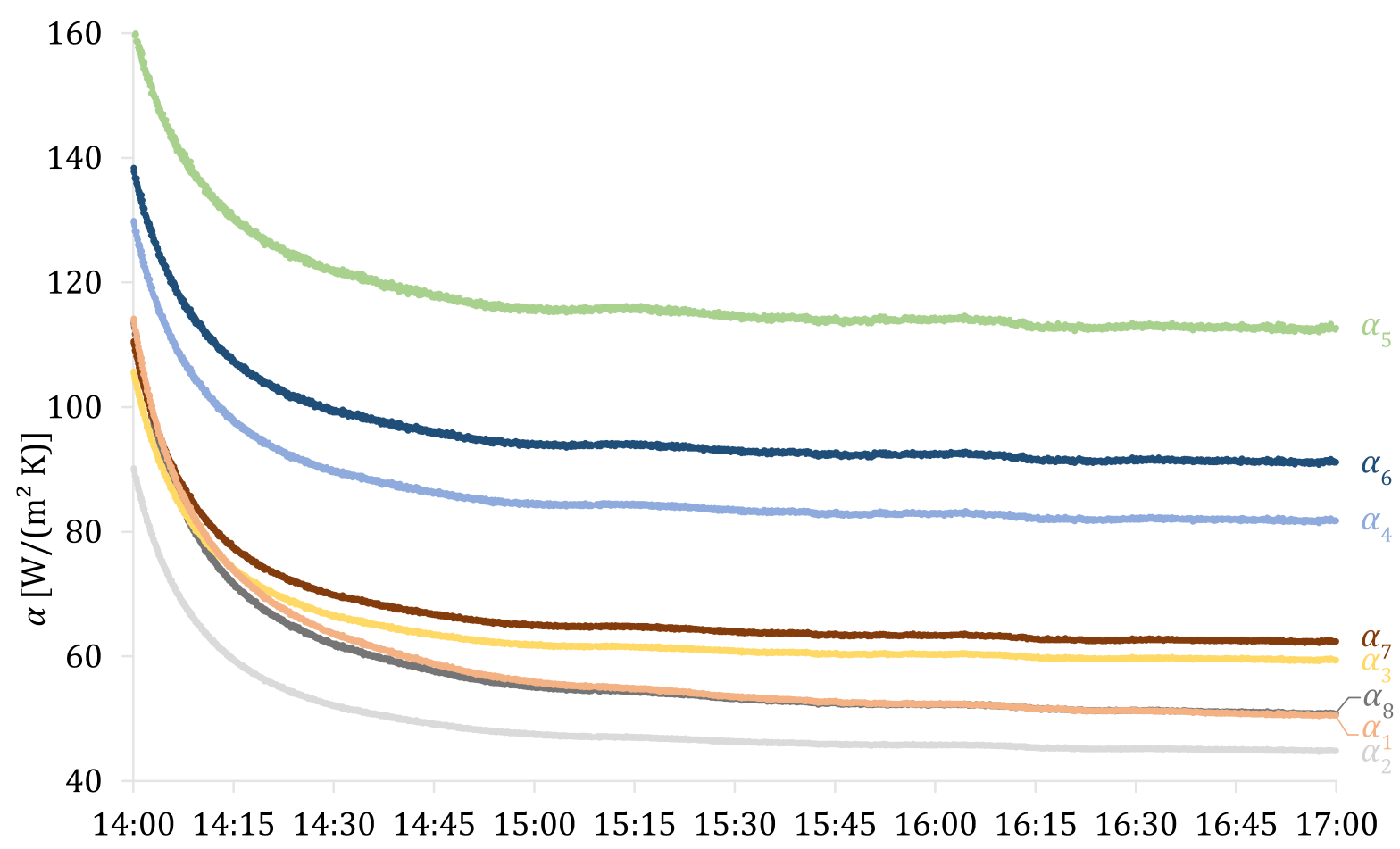

Abb. 4.35 Zeitlicher Verlauf der mittels thermischen Netzwerkmodell in Echtzeit berechneten WÜK während einer sich thermisch ausgleichenden Messung.

\subsubsection{Versuchsmatrix}

Innerhalb der vorliegenden Dissertation soll durch systematische Parametervariation der Einfluss folgender fluiddynamischer und geometrischer Parameter auf den lokalen Wärmeübergang untersucht werden:

- Reynolds-Zahl Re in der Hauptströmung,

- Drall (Blechwinkel $\gamma$ ) in der Hauptströmung,

- Breite der Einströmöffnung $s$,

- Breite der Kavität $b$,

- Exzentrizität der Kavität gegenüber der Einströmöffnung $e$. 
In vier verschiedenen Versuchsreihen soll dabei immer einer der vier Parameter $s, b, e$ bzw. $\varphi$ über einen möglichst großen Stellbereich variiert und alle anderen Parameter konstant gehalten werden ( Tab. 4.8). Für alle diese Konfigurationen werden die lokalen WÜK jeweils für vier verschiedene festgelegte Reynolds-Zahlen untersucht, die zwischen 40.000 und 110.000 liegen und allesamt mit dem drehzahlgeregelten Schraubenverdichter realisiert werden können. Zur Erhöhung der Messstellendichte wird zusätzlich für jede Geometrievariante und jede Reynolds-Zahl die Außenwand um eine halbe Ringteilung axial verschoben, sodass sowohl Werte an der „Normalposition“ (NP) der Außenwand, bei der ein Maximum der Messringe mit Fluid im Totraum in Kontakt sind, als auch an den „Zwischenpositionen“ (ZP) zur Verfügung stehen. Insgesamt ergeben sich somit 136 Versuche. Mit einer durchschnittlichen Versuchsdauer von 2,5 h entspricht dies einer reinen Messzeit von 260 h. Darin sind Reproduktions- und Testmessungen noch nicht enthalten.

Tab. 4.8 Versuchsmatrix für die vorgesehene experimentelle Parameterstudie

\begin{tabular}{|c|c|c|c|c|c|c|c|c|}
\hline \multicolumn{4}{|c|}{ Abmessungen } & \multirow{2}{*}{ Bemerkung } & \multicolumn{4}{|c|}{$\operatorname{Re}\left[10^{5}\right]$} \\
\hline$s[\mathrm{~mm}]$ & $b[\mathrm{~mm}]$ & $e[\mathrm{~mm}]$ & $\gamma\left[^{\circ}\right]$ & & 0,4 & 0,6 & 0,9 & 1,1 \\
\hline 10 & 110 & 0 & 0 & \multirow{5}{*}{$\begin{array}{c}\text { Einfluss } \\
\text { Einströmbreite } s\end{array}$} & $\mathrm{NP} / \mathrm{ZP}$ & $\mathrm{NP} / \mathrm{ZP}$ & $\mathrm{NP} / \mathrm{ZP}$ & NP/ZP \\
\hline 20 & 120 & 0 & 0 & & $\mathrm{NP} / \mathrm{ZP}$ & $\mathrm{NP} / \mathrm{ZP}$ & $\mathrm{NP} / \mathrm{ZP}$ & $\mathrm{NP} / \mathrm{ZP}$ \\
\hline 30 & 130 & 0 & 0 & & $\mathrm{NP} / \mathrm{ZP}$ & $\mathrm{NP} / \mathrm{ZP}$ & $\mathrm{NP} / \mathrm{ZP}$ & $\mathrm{NP} / \mathrm{ZP}$ \\
\hline 40 & 140 & 0 & 0 & & $\mathrm{NP} / \mathrm{ZP}$ & $\mathrm{NP} / \mathrm{ZP}$ & $\mathrm{NP} / \mathrm{ZP}$ & $\mathrm{NP} / \mathrm{ZP}$ \\
\hline 46,3 & 146,3 & 0 & 0 & & $\mathrm{NP} / \mathrm{ZP}$ & NP/ZP & $\mathrm{NP} / \mathrm{ZP}$ & NP/ZP \\
\hline 46,3 & 121,3 & 0 & 0 & \multirow{4}{*}{$\begin{array}{c}\text { Einfluss } \\
\text { Seitenraumbreite } b\end{array}$} & $\mathrm{NP} / \mathrm{ZP}$ & NP/ZP & $\mathrm{NP} / \mathrm{ZP}$ & NP/ZP \\
\hline 46,3 & 96,3 & 0 & 0 & & $\mathrm{NP} / \mathrm{ZP}$ & $\mathrm{NP} / \mathrm{ZP}$ & $\mathrm{NP} / \mathrm{ZP}$ & $\mathrm{NP} / \mathrm{ZP}$ \\
\hline 46,3 & 71,3 & 0 & 0 & & $\mathrm{NP} / \mathrm{ZP}$ & $\mathrm{NP} / \mathrm{ZP}$ & $\mathrm{NP} / \mathrm{ZP}$ & $\mathrm{NP} / \mathrm{ZP}$ \\
\hline 46,3 & 46,3 & 0 & 0 & & $\mathrm{NP} / \mathrm{ZP}$ & NP/ZP & NP/ZP & NP/ZP \\
\hline 46,3 & 96,3 & -25 & 0 & \multirow{4}{*}{$\begin{array}{c}\text { Einfluss } \\
\text { Exzentrizität } e\end{array}$} & $\mathrm{NP} / \mathrm{ZP}$ & $\mathrm{NP} / \mathrm{ZP}$ & $\mathrm{NP} / \mathrm{ZP}$ & NP/ZP \\
\hline 46,3 & 96,3 & $-12,5$ & 0 & & $\mathrm{NP} / \mathrm{ZP}$ & NP/ZP & $\mathrm{NP} / \mathrm{ZP}$ & NP/ZP \\
\hline 46,3 & 96,3 & 12,5 & 0 & & $\mathrm{NP} / \mathrm{ZP}$ & NP/ZP & $\mathrm{NP} / \mathrm{ZP}$ & NP/ZP \\
\hline 46,3 & 96,3 & 25 & 0 & & $\mathrm{NP} / \mathrm{ZP}$ & NP/ZP & $\mathrm{NP} / \mathrm{ZP}$ & NP/ZP \\
\hline 46,3 & 146,3 & 0 & 10 & \multirow{4}{*}{$\begin{array}{c}\text { Einfluss } \\
\text { Blechwinkel } \gamma\end{array}$} & $\mathrm{NP} / \mathrm{ZP}$ & NP/ZP & $\mathrm{NP} / \mathrm{ZP}$ & NP/ZP \\
\hline 46,3 & 146,3 & 0 & 20 & & $\mathrm{NP} / \mathrm{ZP}$ & NP/ZP & NP/ZP & NP/ZP \\
\hline 46,3 & 146,3 & 0 & 30 & & $\mathrm{NP} / \mathrm{ZP}$ & NP/ZP & $\mathrm{NP} / \mathrm{ZP}$ & NP/ZP \\
\hline 46,3 & 146,3 & 0 & 40 & & NP/ZP & $\mathrm{NP} / \mathrm{ZP}$ & $\mathrm{NP} / \mathrm{ZP}$ & NP/ZP \\
\hline
\end{tabular}




\subsection{DATENREDUKTION UND - MITTELUNG}

Zur Reduktion der Daten werden alle gemessenen und berechneten physikalischen Größen über ein für jede Messung neu zu spezifizierendes Zeitintervall von mindestens fünf Minuten arithmetisch gemittelt. Dazu ist in jeder Messdatei bereits ein entsprechendes Arbeitsblatt zur Auswertung („Inverse“) enthalten. Ausgangspunkt zur Festlegung des Mittelungszeitraums ist der vollständige thermodynamische Gleichgewichtszustand der Versuchsanordnung (vgl. $>$ Abb. 4.35). Für die an den vier Umfangspositionen gemessenen Materialtemperaturen in den beiden Flanschen F1 und F2 sowie den acht Ringen R1 bis R8 ergeben sich die in $>$ Abb. 4.36 a dargestellten Verläufe. Unterschiedliche Farbtöne symbolisieren hierbei Ergebnisse von unterschiedlichen Thermistorreihen: die Farbe wird beginnend bei Reihe A/E (12-Uhr-Position) entgegen des Uhrzeigersinns bis zur Reihe D/H (3-Uhr-Position) immer heller. Strichpunktlinien kennzeichnen die arithmetischen Umfangsmittelwerte, vertikale Fehlerbalken das dazugehörige Streuband der Werte über alle vier Reihen an den jeweiligen axialen Positionen. Die Spannweite zwischen größter und kleinster am Umfang gemessener Temperatur liegt für alle axialen und radialen Positionen bei einem Großteil der Messungen unter einem Wert von 0,3 K. Dies entspricht einer maximal beobachteten Abweichung von $\pm 0,15 \mathrm{~K}$. Die Standardabweichung, die aus der zeitlichen Schwankung der Einzelwerte resultiert, liegt demgegenüber lediglich bei Werten unterhalb von weniger als 0,01 K ( Kap. 5.8.2). Werden nun aus den umfangsgemittelten Temperaturen mit Hilfe des thermischen Netzwerkmodells die lokalen WÜK berechnet und diese mit den Resultaten verglichen, die sich jeweils separat aus den Temperaturen für jede Umfangsposition ergeben würden (Abb. 4.36 b), so kann als Streuung eine konstante Abweichung von $\pm 4 \mathrm{~W} /\left(\mathrm{m}^{2} \mathrm{~K}\right)$ als Richtwert angegeben werden (vertikale Fehlerbalken in Abb. 4.36 b).
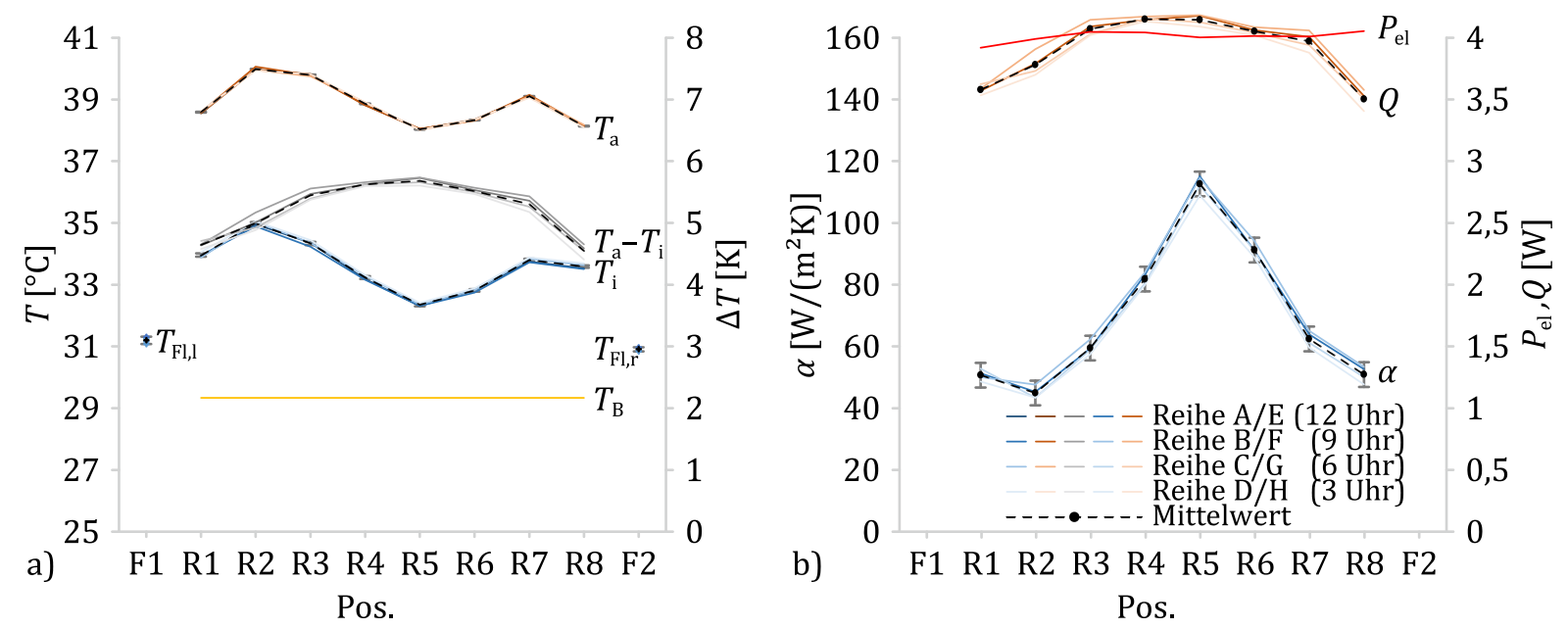

Abb. 4.36 Auswertung der über ein festgelegtes Intervall gemittelten, gemessenen Temperaturen zur Bestimmung von WÜK mittels thermischem Netzwerkmodell.

a) Material- und Bezugstemperaturen; b) gemessene elektrische Leistungen der Heizwicklungen sowie berechnete WÜK und Wärmeströme aus dem thermischen Netzwerkmodell. 


\section{ERGEBNISSE UND DISKUSSION}

\subsection{BETRIEBSCHARAKTERISTIK DER VERSUCHSANLAGE}

Um erste Betriebserfahrungen mit der Versuchsanlage zu sammeln und ein Gefühl für die implementierte Druck-Durchfluss-Regelung zu erhalten, wurde in einem ersten Schritt das Betriebskennfeld des erweiterten Druckluftsystems bestimmt. Dazu wurden für verschiedene relative Ventilhübe $y=\left(H-H_{0}\right) /\left(H_{100}-H_{0}\right)$ des Dreiwegeventils (3WV) sowie des Motorstellventils (MSV) der Massenstrom, welcher über den Hauptstrang durch den Versuchsstand fließt, sowie der statische Druck an verschiedenen über den Mitteldruckbereich $(p=2 \ldots 2,4$ bar) des Rohrleitungssystems verteilten Betriebsmessstellen ermittelt und als Netzkennlinien übereinander aufgetragen. In Abb. 5.1 ist ein solches Betriebskennfeld für den Solobetrieb des drehzahlgeregelten Verdichters und die Werkseinstellung des Druckminderers dargestellt.

Wie in Abb. 5.1 a zu sehen ist, nimmt der Massenstrom mit größerem Ventilhub des 3WV gemäß Kennlinie nahezu ideal linear zu. Die Betriebsdrücke im System ändern sich hingegen kaum: die etwas geringeren Drücke vor dem 3WV bei mittleren Ventilhüben lassen auf einen etwas geringen Druckverlust des Ventils bei etwa gleichmäßiger Aufteilung der Medienströme schließen.

Während im reinen Bypassbetrieb ( $\left.y_{3 w v}=0\right)$ der Druck vor der Messblende im Hauptstrang dem Druck an der Wiedervereinigung von Bypass und Hauptstrang kurz vor dem MSV entspricht, steigt ersterer mit zunehmender Durchströmung leicht an. Erwartungsgemäß generiert der Hauptstrang und dort insbesondere die Messblende einen höheren Druckverlust als der Bypass, der kürzer ist und keine weiteren Einbauten aufweist. Auf den Referenzdruck im Versuchsstand hat dies jedoch keinen wahrnehmbaren Einfluss, was für eine unabhängige Druck- und Durchflusseinstellung essentiell ist und die richtige Auslegung des Regelschemas bestätigt.

- Abb. 5.1 b zeigt den Einfluss des Ventilhubs des MSV auf Massenstrom und Betriebsdrücke bei voller Durchströmung des Versuchsstandes ( $y_{3 \mathrm{WV}}=100 \%$ ). Mit stärkerer Öffnung des MSV sinkt der Gesamtdruckverlust der Anlage. Der Durchsatz nimmt exponentiell zu und die Betriebsdrücke im vorgeschalteten Mitteldrucksystem linear ab. Beides ist charakteristisch für die gleichprozentige Kennlinie des Ventils. Bei Hüben von $y_{\mathrm{MSV}}>55 \%$ bricht der Druck im System zusammen. Der Verdichter ist im Solobetrieb dann nicht mehr in der Lage, ausreichend Fluid nachzuliefern. Der Mindestdruck im Ölkreislauf kann nicht aufrechterhalten werden, weshalb ein Dauerbetrieb in diesem Zustand nicht angebracht ist. Im Sinne der Betriebsstabilität wird daher - sofern nicht anders angegeben - für alle im Folgenden beschriebenen Versuche das MSV auf Mittelstellung $\left(y_{\mathrm{MSV}}=50 \%\right)$ betrieben. 
Wie für Regelventile üblich, lassen 3WV und MSV auch bei Nullhub $(y=0)$ noch eine kleine Leckagemenge passieren, was für den Versuchsbetrieb jedoch keine Auswirkungen hat. Beide Diagramme in Abb. 5.1 sind über den mit der gestrichelten Linie gekennzeichneten Betriebspunkt verknüpft.

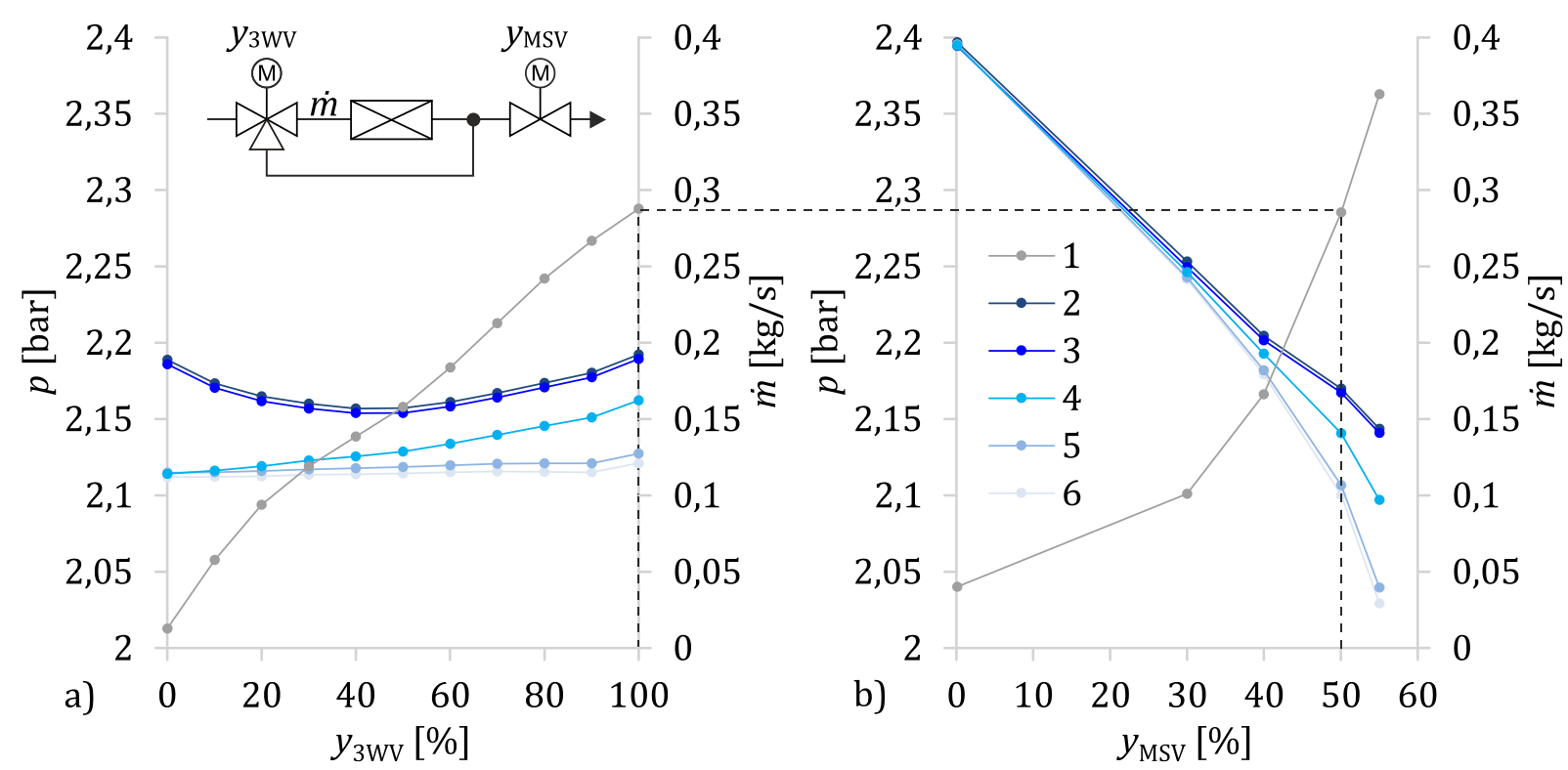

Abb. 5.1 Netzkennlinien bei variiertem Hub der beiden Hauptventile im Druckluftsystem:

Beeinflussung des Durchsatzes durch den Versuchsstand (Hauptstrang) sowie ausgewählter Systemdrücke bei Solobetrieb des drehzahlgeregelten Verdichters und variierter Ventilstellung a) des Dreiwegeventils $\left(y_{\mathrm{MSV}}=50 \%^{1}\right)$, b) des Motorstellventils $\left(y_{3 \mathrm{WV}}=100 \%^{2}\right)$; $p_{1, \text { Vordruck }}=(5,82 \ldots 5,89)$ bar, $p_{2, \text { n. DRV,Soll }}=2,4$ bar, $p_{\mathrm{U}}=(999,1 \ldots 1008,3) \mathrm{hPa}$; 1 - Durchsatz durch Versuchsstand $\dot{m}_{\mathrm{VS}} ; 2$ - Druck nach Druckreduzierventil $p_{2, \mathrm{n} \text {. DRV; }}$

3 - Druck nach Sicherheitsventil $p_{3, \mathrm{n} \text {. Sichv }} ; 4$ - Vordruck Messblende $p_{\mathrm{B} 1, \mathrm{vor}}$;

5 - Referenzdruck Versuchsstand $p_{7, \text { ref,TRV }} 6$ - Druck vor Motorstellventil $p_{5, \mathrm{v} \text {. MSV }}$.

\subsection{KALIBRIERUNG DER FÜNFLOCHSONDE AN DER FREISTRAHLDÜSE}

Vor der Verwendung im Totraumversuchsstand wurde die Fünflochsonde zunächst an der Freistrahlkalibrierstrecke des Instituts für Luft- und Raumfahrttechnik (ILR) der TU Dresden und der dortigen Arbeitsgruppe für Experimentelle Aerodynamik (EAD) (Leitung: Dr.-Ing. Veit Hildebrand) kalibriert. Mittels eines bei konstanter Drehzahl betriebenen Radialgebläses wird über eine Düse ein stationärer Luftfreistrahl generiert ( Abb. 5.2), in dem die Sonde mittels zweier Schrittmotoren definiert um ihre Sondenschaftlängsachse (Winkel $\alpha$ ) und diese wiederum normal zur Strahlachse um den Sondenkopf (Winkel $\beta$ ) gedreht wird.

\footnotetext{
${ }^{1} y_{\mathrm{MSV}}=50 \%$ bedeutet, dass das Motorstellventil zu $50 \%$ geöffnet ist.

${ }^{2} y_{3 \mathrm{WV}}=100 \%$ bedeutet, dass $100 \%$ des Gesamtdurchsatzes über den Hauptstrang fließt
} 


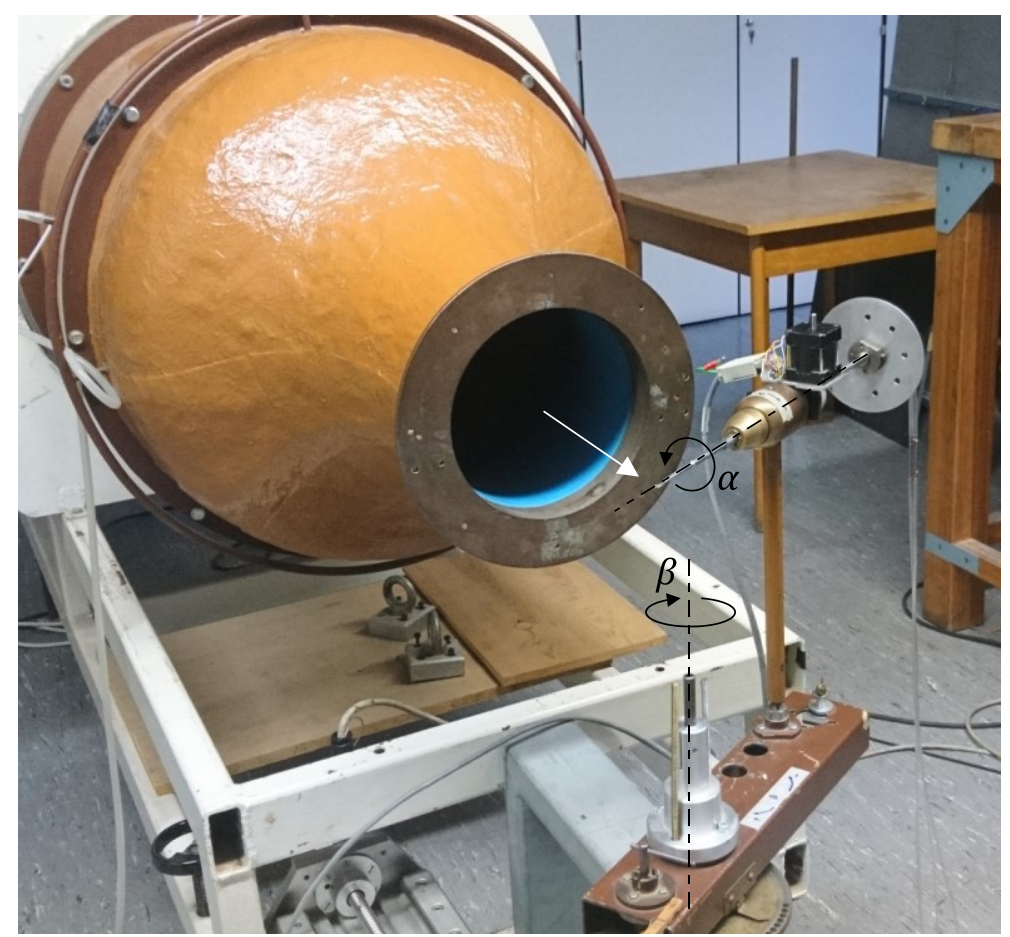

Abb. 5.2 Versuchsaufbau zur automatisierten Winkeltraversierung und Kalibrierung der Fünflochsonde in einer Düsenströmung am ILR-EAD der TU Dresden.

Die Verstellung des Gierwinkels $\alpha$ und des Nickwinkels $\beta$ der Sonde (Abb. 5.3 c) erfolgt dabei im sogenannten Yaw-Pitch-Mode automatisiert mittels zweier Schrittmotoren über eine ISELSteuerung. Pro Winkelkombination $(\alpha ; \beta)$ werden in einer Taktung von $2 s$ der Umgebungsdruck $p_{\text {Umg }}$, der Kammerdruck gegenüber Umgebung $p_{\mathrm{K}}-p_{\text {Umg }}$ sowie der Differenzdruck der Mittelbohrung der Sonde zu allen vier Seitenbohrungen, die gemäß ihrer Lage mit „oben“ ( $\left.p_{\mathrm{M}}-p_{0}\right)$, „unten“ $\left(p_{\mathrm{M}}-p_{\mathrm{U}}\right)$, „links“ ( $\left.p_{\mathrm{M}}-p_{\mathrm{L}}\right)$ und „rechts“ ( $\left.p_{\mathrm{M}}-p_{\mathrm{R}}\right)$ gekennzeichnet sind ( Abb. $\left.5.3 \mathrm{a}\right)$, gemessen und über jeweils 10 Werte gemittelt. Für die Kalibrierung werden daraus reduzierte (dimensionslose) Druckdifferenzen gebildet:

$$
\begin{gathered}
x=\frac{p_{\mathrm{O}}-p_{\mathrm{U}}}{\Delta p_{\mathrm{B}}}=\frac{\left(p_{\mathrm{M}}-p_{\mathrm{U}}\right)-\left(p_{\mathrm{M}}-p_{\mathrm{O}}\right)}{\Delta p_{\mathrm{B}}} \\
y=\frac{p_{\mathrm{L}}-p_{\mathrm{R}}}{\Delta p_{\mathrm{B}}}=\frac{\left(p_{\mathrm{M}}-p_{\mathrm{R}}\right)-\left(p_{\mathrm{M}}-p_{\mathrm{L}}\right)}{\Delta p_{\mathrm{B}}} \\
f_{Q}=\frac{\Delta p_{\mathrm{dyn}}}{\Delta p_{\mathrm{B}}}
\end{gathered}
$$

die sich auf den Bezugsdifferenzdruck

$$
\Delta p_{\mathrm{B}}=\left(p_{\mathrm{M}}-p_{\mathrm{O}}\right)+\left(p_{\mathrm{M}}-p_{\mathrm{U}}\right)+\left(p_{\mathrm{M}}-p_{\mathrm{L}}\right)+\left(p_{M}-p_{\mathrm{R}}\right)
$$

beziehen. 
Der dynamische Druck an der Sonde entspricht dem statischen Druckunterschied zwischen der Düsenkammer und der Umgebung

$$
\Delta p_{\text {dyn }}=p_{\mathrm{K}}-p_{\text {Umg }}=\frac{\rho_{\infty}}{2} u_{\infty}^{2}
$$

und betrug für alle Kalibriermessungen ca. $300 \mathrm{~Pa}$. Das entspricht einer Freistrahlgeschwindigkeit von $u_{\infty} \approx 22 \mathrm{~m} / \mathrm{s}$, wie man sie ungefähr auch in der Kanalmitte der Hauptströmung im Versuchsstand bei einer Reynolds-Zahl von $R e \approx 110.000$ erwarten würde. Eine Kalibrierung hinsichtlich variierter Strömungsgeschwindigkeiten/ Reynolds-Zahlen und Absolutdrücken wurde nicht durchgeführt. Bei inkompressiblen Strömungen $(M a \ll 0,3)$ ist deren Einfluss auf die gemessenen relativen Druckdifferenzen (Gleichungen 5.1 bis 5.3) vernachlässigbar [116].

Der Kalibrierbereich für beide Winkel beträgt jeweils $\pm 30^{\circ}$. Darüber hinaus ist durch Strömungsablösung am Sondenkopf im Bereich der Bohrungen keine Eindeutigkeit der Messergebnisse mehr gegeben. Anstell- und Schiebewinkel sowie der dynamische Druckkoeffizient $f_{Q}$ können als zweidimensionale Polynome vierter bis fünfter Ordnung der reduzierten Größen $x$ und $y$ dargestellt werden, welche auch Querempfindlichkeiten im geeigneten Maße abbilden können [117]:

$$
\begin{aligned}
& \alpha=\sum_{i=0}^{4} \sum_{k=0}^{5} A_{i, k} x^{i} y^{k} \\
& \beta=\sum_{i=0}^{5} \sum_{k=0}^{4} B_{i, k} x^{i} y^{k} \\
& f_{Q}=\sum_{i=0}^{4} \sum_{k=0}^{4} Q_{i, k} x^{i} y^{k}
\end{aligned}
$$

Die Bestimmung der Koeffizienten $A_{i, k}, B_{i, k}$ und $Q_{i, k}$ erfolgt durch Minimierung der Summe der Abweichungen zwischen den aus $x$ und $y$ mittels Polynom berechneten und den eingestellten Sollwerten:

$$
\left\{\sum_{m=0}^{N_{m}}\left[\sum_{i=0}^{N_{i}-1} \sum_{k=0}^{N_{k}-1}\left(A_{i, k} x_{m}^{i} y_{m}^{k}\right)-\alpha_{\text {Soll }, m}\right]\right\}^{2} \rightarrow \text { min usw. }
$$

Auf Grund der Linearität der Polynome existiert genau eine eindeutige Lösung, welche analytisch berechnet werden kann (Lösungsschema siehe Anhang A11). Die Approximationsgenauigkeit der Polynome ist in Anhang A12 dargestellt. Die mittlere quadratische Abweichung zum Sollwert beträgt im Kalibrierbereich demnach $\sigma_{\alpha}=0,29^{\circ}, \sigma_{\beta}=0,24^{\circ}$ und $\sigma_{f_{Q}}=0,097$.

Mit Hilfe der Kalibrierfunktionen 5.6 bis 5.8 lassen sich im Anwendungsfall aus den gemessenen Druckdifferenzen an der Sonde die Winkel $\alpha$ und $\beta$ sowie nach Gl. 5.5 die Absolutgeschwindigkeit 


$$
c=|\vec{c}|=\sqrt{\frac{2 \Delta p_{\mathrm{dyn}}}{\rho}}=\sqrt{\frac{2 f_{Q} \Delta p_{\mathrm{B}}}{\rho}}
$$

berechnen, aus denen sich wiederum die Geschwindigkeitskomponenten im Zylinderkoordinatensystem ( Abb. 5.3 b) ergeben:

$$
\begin{gathered}
c_{\mathrm{r}}=c \cdot \sin \beta \\
c_{\mathrm{u}}=c \cdot \cos \beta \cdot \sin \alpha \\
c_{\mathrm{ax}}=c \cdot \cos \beta \cdot \cos \alpha
\end{gathered}
$$

Blickt man in Strömungsrichtung von vorn auf den Sondenkopf und zeigt der Sondenschaft nach oben, so gilt:

$\beta>0 \Rightarrow c_{\mathrm{r}}>0$ : Strömung radial nach außen (Nickwinkel)

$\alpha>0 \Rightarrow c_{\mathrm{u}}>0$ : Strömung gegen Uhrzeigersinn/ in mathematisch positive Richtung (Drallwinkel)

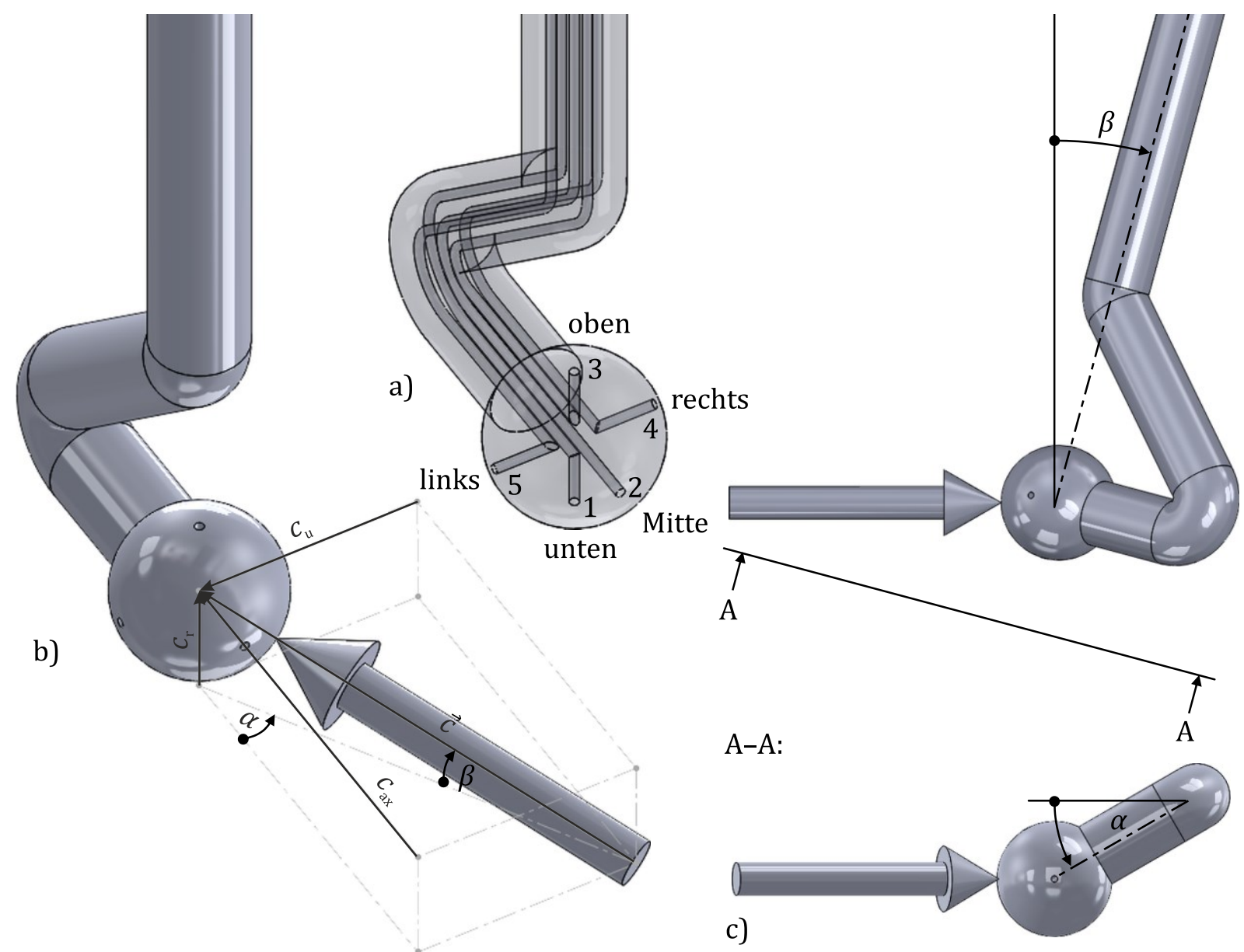

Abb. 5.3 Nomenklatur, Winkelkonventionen und Geschwindigkeitskomponenten an der kalibrierten Fünflochsonde. a) Bezeichnung der Druckbohrungen; b) Einbaulage im Versuchsstand; c) Einbaulage an der Kalibrierdüse. Im Bild ist $\beta=+15^{\circ}$ und $\alpha=+30^{\circ}$. 


\subsection{STRÖMUNGSFELD IM KONZENTRISCHEN RINGSPALTKANAL}

Die Gleichmäßigkeit der Anströmung zum Totraum spielt eine entscheidende Rolle für die Ausbildung des Wärmeübergangsprofils im Seitenraum. Ausgeprägte Ungleichverteilungen oder Nachlaufdellen können zu Abweichungen von einer idealen axialsymmetrischen WÜK-Verteilung führen und somit den generischen Modellcharakter des Versuches schmälern. Darüber hinaus würde die Berücksichtigung solcher Sekundäreffekte die Komplexität der Argumentations- und Rechenmodelle deutlich erhöhen, was in Anbetracht der ohnehin bereits komplizierten Strömungsstrukturen nicht wünschenswert wäre.

Zur Abklärung wurde in einem ersten Schritt das axiale Geschwindigkeitsprofil unmittelbar vor der Einströmöffnung für eine drallfreie Strömung mit Stützgitter bei $R e=110.000$ bestimmt

Abb. 5.4), und zwar einmal mittels Pitot-Rohr und einmal mit der Fünfloch-Pyramidensonde (vgl. Kap. 4.2.3). Auf Grund der endlichen Abmessungen der Sonde ist eine Traversierung bis zum Kanalrand nicht möglich. Dort fehlen entsprechend die Messwerte. Abb. 5.4 enthält jedoch nicht nur die aus dem dynamischen Druck berechnete Axialgeschwindigkeit $c_{\mathrm{ax}}$, sondern auch die querschnittsgemittelte integrale Geschwindigkeit $q_{\text {int }}$ über das gemessene Strömungsprofil

$$
q_{\text {int }}=\frac{1}{A} \int_{A} c_{\mathrm{ax}} d A=\frac{1}{\pi\left(r_{\mathrm{a}}^{2}-r_{\mathrm{i}}^{2}\right)} 2 \pi \int_{r_{\mathrm{i}}}^{r_{\mathrm{a}}} u(r) r d r
$$

sowie zum Vergleich die querschnittsgemittelte Geschwindigkeit $q$ im Achsnormalschnitt gemäß Gleichung 4.12. Außerdem sind die Ergebnisse einer CFD-Untersuchung für ein $90^{\circ}$-Sektor-Modell des Strömungskanals (ohne Totraum) unter Berücksichtigung der Streben von Einströmmodul und Stützgitter hinterlegt (siehe auch $>$ Kap. 6.4 und $>$ Abb. A.34).

Beide Sonden sowie das numerische Modell weisen ein ausgeprägtes Geschwindigkeitsprofil mit deutlich erkennbaren Grenzschichten am Innen- und Außenradius des Kanals und einer Maximalgeschwindigkeit zwischen 24,5 und 25,7 m/s auf. Unterschiede sind jedoch in der Form des Profils festzustellen: während das Geschwindigkeitsmaximum bei den beiden Sonden oberhalb der Kanalmitte (bei $r=45 \mathrm{~mm}$ bzw. $46 \mathrm{~mm}$ ) liegt, ist das simulierte Profil in Richtung Nabe (radial nach innen) verlagert (Maximum bei $r=42 \mathrm{~mm}$ ). Im Gegensatz zu den beiden anderen Profilen zeigt die mittels Pitotsonde bestimmte axiale Geschwindigkeitsverteilung bei einem Radius von $r=41 \mathrm{~mm}$ einen ausgeprägten Knick, der auf eine überlagerte Sekundärströmung an der Nabe hindeutet. Der Knick wurde sowohl im ein- als auch im ausfahrenden Traversiermodus reproduzierbar nachgewiesen. 


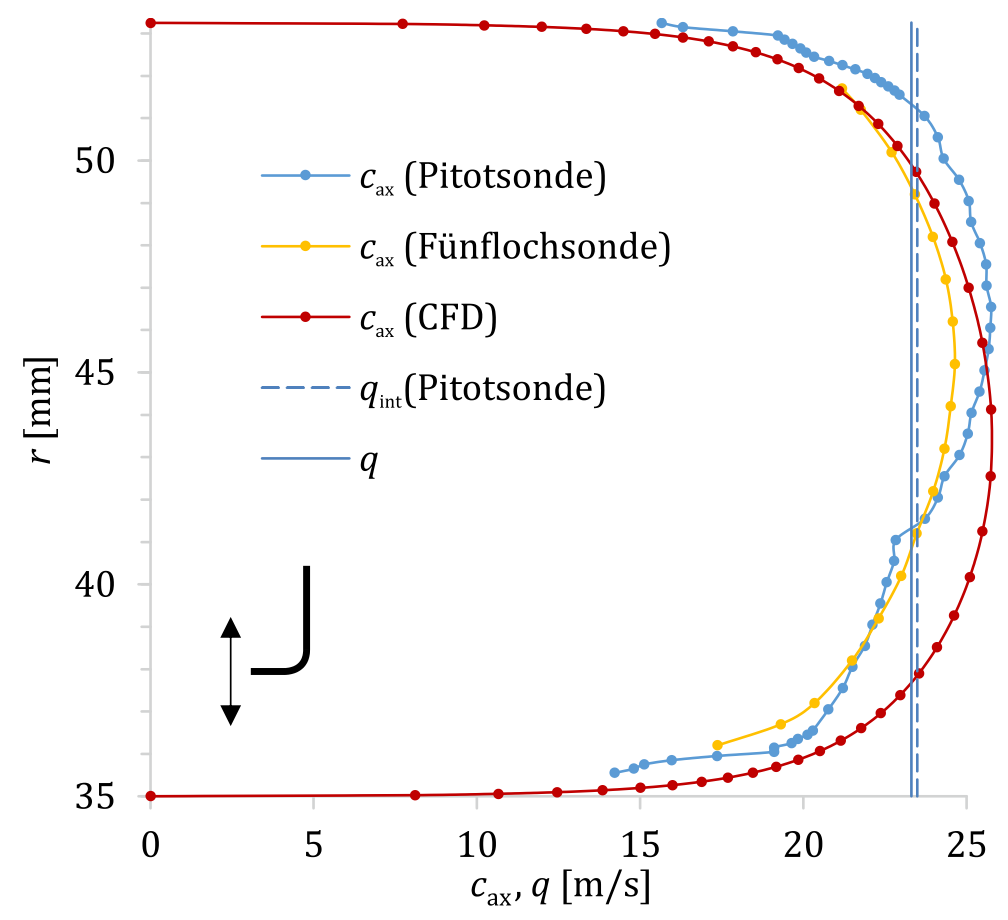

\section{Abb. 5.4 Axialgeschwindigkeitsprofil im kreisringspaltförmigen Hauptströmungskanal am Ein- lass in den Seitenraum ( $x=0 \mathrm{~mm})$, mit Stützgitter $\left(\gamma=0^{\circ}\right)$. $R e=110.000, p_{\mathrm{ref}}=(2,10 \ldots 2,12) \mathrm{bar}, \dot{m}_{\mathrm{VS}}=(0,284 \ldots 0,288) \mathrm{kg} / \mathrm{s}$.}

Die über $d A=2 \pi r d r$ gemittelte Geschwindigkeit $q_{\text {int }}$ des Pitotsonden-Profils ist wiederum quasi identisch mit der mittleren Kanalgeschwindigkeit $q$, was die Aussagekraft der Sondenmessungen unterstreicht. An dieser Stelle soll noch einmal auf die begrenzten Abmessungen der Sonden in Relation zur Kanalhöhe hingewiesen werden, bei denen die Pitot-Sonde deutlich besser abschneidet $\left(d_{\text {Sonde }} / \Delta r=5,5 \%\right)$ als die Fünflochsonde $\left(d_{\text {Sonde }} / \Delta r=13,2 \%\right)$.

Mit der Fünflochsonde wurden in einem zweiten Schritt die Strömungswinkel sowie die dreidimensionale Geschwindigkeitsverteilung im 4. Quadranten des Hauptströmungskanals für verschiedene Reynolds-Zahlen und Blechwinkel $\gamma$ am Drallgitter bestimmt. Dazu wurde durch Verdrehung der Außenwand die Umfangsposition der Sonde $\varphi$ zwischen 270 und $360^{\circ}$ (Abb. 4.16) in $5^{\circ}$-Schritten variiert und die Eintauchtiefe in 1-mm-Schritten mittels SVG traversiert.

Als Referenzfall wurde zunächst das einfache Stützgitter mit den vier geraden Stützblechen $\left(\gamma=0^{\circ}\right)$ bei Reynolds-Zahlen von $R e=40.000$ und $R e=110.000$ ( Abb. 5.5) untersucht. Ermittelt wurden jeweils der Drallwinkel $\alpha$, der Nickwinkel $\beta$, die Axialgeschwindigkeit $c_{\mathrm{ax}}$ und die aus der Umfangs- und der Radialkomponente zusammengesetzte resultierende Geschwindigkeit im Achsnormalschnitt $\vec{c}_{\text {res }}=\vec{c}_{\mathrm{u}}+\vec{c}_{\mathrm{r}}$ mit

$$
c_{\text {res }}=\left|\vec{c}_{\text {res }}\right|=\sqrt{c_{\mathrm{u}}^{2}+c_{\mathrm{r}}^{2}}
$$

In beiden Fällen liegen die gemessenen Drallwinkel über den gesamten Messbereich hinweg deutlich unter $\alpha=5^{\circ}$, bei $R e=40.000$ typischerweise zwischen 2,5 und $4^{\circ}$ und bei $R e=110.000$ 
zwischen 0 und 1,5 $5^{\circ}$, weshalb man von einer drallfreien Strömung sprechen kann. Es treten geringfügige lokale Unterschiede in der Drallwinkelverteilung auf. So stellt man für die kleinere der beiden Reynolds-Zahlen die geringsten Drallwinkel im Bereich der Kanalmitte fest, die zu den beiden Rändern hin anwachsen. Durchtrennt ist diese Verteilung im Bereich $\varphi=310^{\circ}$, wo lokal höhere Werte gemessen werden, dies deutet auf Nachlaufdellen des Stützbleches hin, welches sich stromauf bei $\varphi=315^{\circ}$ befindet (vgl. Abb. A.18 b). Eine solch stabile Schichtung lässt sich bei der größeren Reynolds-Zahl nicht mehr feststellen, wo die turbulente Vermischung offensichtlich größer ist. Minimale Drallwerte finden sich dort nabenseitig zwischen $\varphi=320^{\circ} \ldots 350^{\circ}$, die Verteilung wirkt ansonsten „regellos“. Der Nickwinkel $\beta$, der den Winkel zwischen Meridian- und Axialgeschwindigkeit beschreibt, liegt hier und in allen anderen untersuchten Fällen zwischen $\beta=-2^{\circ}$ am Innenradius des Kanals und $\beta=12^{\circ}$ am Außenradius und weist eine rein radiale Verteilung ohne lokale Extrema auf. Hierfür gibt es drei denkbare Erklärungsansätze: Entweder sind die Stromlinien so kurz vor der Einströmöffnung in den Totraum tatsächlich bereits radial nach außen gekrümmt, wobei die Krümmung nach außen hin ansteigt, oder der radiale Druckgradient im Ringspalt führt zu nennenswerten gemessenen Druckdifferenzen zwischen den in radialer Richtung gegenüberliegenden Druckbohrungen 1 und 3 der Fünflochsonde ( Abb. 5.3 a), sodass diese auf Grund Ihrer Abmessungen quasi auf zwei verschiedenen Stromlinien misst.

Die geringfügig negativen Werte direkt an der Innenwand könnten für eine Rezirkulation am Inliner sprechen, viel wahrscheinlicher sind jedoch sog. „Bodeneffekte“ im wandnahen Bereich, die die Messung des Nickwinkels beeinflussen könnten. Für letzteres spricht, dass sich die absoluten Werte für $\beta$ weder über der Reynolds-Zahl noch über den Drallwinkel (siehe unten) ändern. Sehr eindrucksvoll sind die oben bereits angesprochenen Nachlaufdellen des Stützgitters in den Konturplots der Axialgeschwindigkeit $c_{\mathrm{ax}}$ in Abb. $5.5 \mathrm{zu}$ erkennen, wo die Maximalwerte bei $\varphi=315^{\circ}$ einbrechen (vgl. Abb. A.18 b), jedoch in absoluten Werten nur um 1 bzw. $2 \mathrm{~m} / \mathrm{s}$, was als geringfügig eingeschätzt werden kann. Der Vektorplot für die resultierende Geschwindigkeit im Achsnormalschnitt zeigt für beide Fälle nur minimale Beträge in Umfangsrichtung an. Am Außendurchmesser überwiegt die Radialkomponente deutlich, $\vec{c}_{\text {res }}$ zeigt dort nahezu perfekt radial nach außen. 

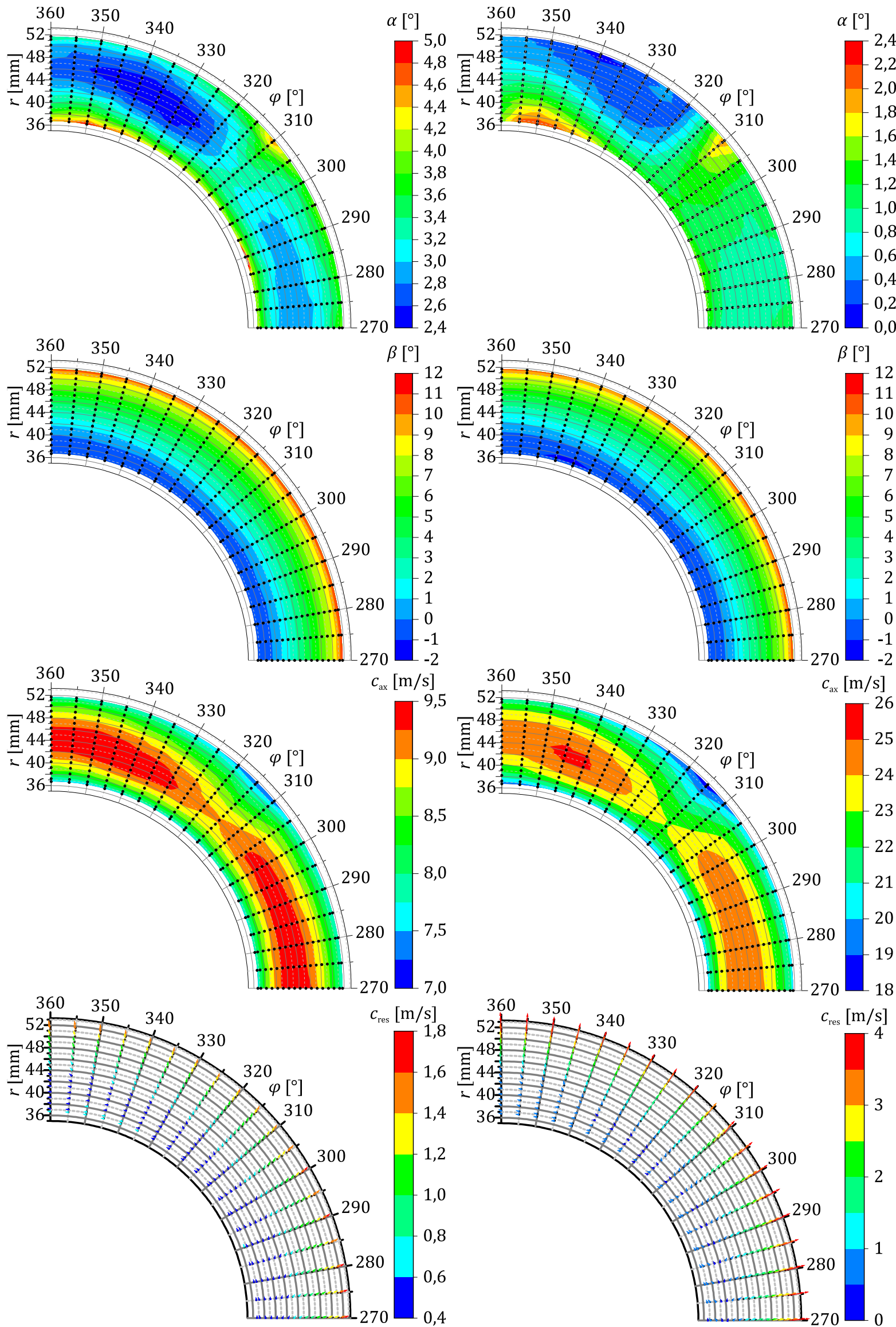

Abb. 5.5 Strömungswinkel und Geschwindigkeitsverteilung in der Hauptströmung am Einlass in den Seitenraum, mit Stützgitter. $\varphi=270^{\circ} \ldots 360^{\circ}$; links: $\operatorname{Re}=40.000$, rechts: $R e=110.000$ 
Nach einer entsprechenden Versuchsstandmodifikation wurden anschließend die Strömungsverhältnisse im Hauptströmungskanal mit Drallgitter bestimmt, und zwar für Blechwinkel $\gamma$ zwischen 0 und $30^{\circ}$ bei einer Reynolds-Zahl von jeweils $R e=110.000$.

Die Ergebnisse sind auf zwei Abbildungen verteilt, um Vergleichbarkeit zwischen den einzelnen Blechwinkeln herstellen zu können. So sind in Abb. 5.6 die beiden Strömungswinkel $\alpha$ und $\beta$ und in Abb. 5.7 die Geschwindigkeiten $c_{\mathrm{ax}}$ und $c_{\text {res }}$ dargestellt, und zwar jeweils in Spalten, bei denen der Blechwinkel über die Zeilen nach unten hin anwächst. In $>$ Abb. 5.8 sind jeweils die Umfangsmittelwerte aller Winkelgrößen und Geschwindigkeitskomponenten über dem Radius aufgetragen.

Vergleicht man jeweils die oberen Zeilen von Abb. 5.6 und Abb. 5.7 mit der rechten Spalte in - Abb. 5.5, so erkennt man nur geringe Unterschiede in den ermittelten Werten zwischen dem Stützgitter und dem Drallgitter ohne Anstellung $\left(\gamma=0^{\circ}\right)$. Die gemessenen Drallwinkel liegen beim Drallgitter noch tiefer. Offensichtlich haben die zwölf Drallbleche eine noch bessere Gleichrichterwirkung als die vier Stützbleche beim Stützgitter. Naturgemäß stellt man dadurch für das Drallgitter auch mehr Nachlaufdellen in der Axialgeschwindigkeit fest, und zwar wiederum exakt an den Winkelpositionen, an denen die Bleche angeordnet sind, und zwar bei $\varphi=285^{\circ}, 315^{\circ}$ und $345^{\circ}$. Nickwinkel und resultierendes Geschwindigkeitsfeld im Achsnormalschnitt sind gleich. Dementsprechend sollte es für die später zu akquirierenden WÜK-Ergebnisse egal sein, ob ein Stütz- oder ein Drallgitter eingebaut ist (mehr dazu siehe Kap. 5.7.2).

Wie beabsichtigt steigen die gemessenen Drallwinkel mit größer werdendem Blechwinkel an ( Abb. 5.6). Doch nicht nur der Mittelwert nimmt zu, sondern auch die Spreizung, also die Differenz zwischen dem höchsten und dem niedrigsten gemessenen Wert über die traversierte Kanalfläche. Diese beträgt für $\gamma=0^{\circ}$ lediglich $\Delta \alpha_{\max }=2,4^{\circ}$ und steigt auf etwa $5^{\circ}$ für $\gamma=30^{\circ}$ an. Deutlich zu erkennen in Abb. 5.6 ist auch die Minderumlenkung, die erwartungsgemäß mit steigendem Blechwinkel größer wird. Für den größten Blechwinkel $\gamma=30^{\circ}$ liegt der maximal gemessene Drallwinkel bei $27^{\circ}$, der mittlere sogar nur bei $25,1^{\circ}$ (siehe auch Abb. 5.8 a und Abb. 5.9), was einer Minderumlenkung $\Delta \gamma=\gamma-\bar{\alpha}$ von ca. $5^{\circ}$ entspricht.

Da es für weiterführende Untersuchungen zur Drallverteilung nicht immer möglich ist, aufwändig einen Viertelsektor des Strömungskanals zu traversieren, soll überprüft werden, ob und inwieweit der an einem einzelnen ausgewählten Referenzpunkt, hier: in der Kanalmitte bei $\varphi=360^{\circ}$ (12-Uhr-Position), gemessene Drall mit dem flächengemittelten Wert korreliert. Wie Abb. 5.9 zeigt, liegen die Ergebnisse über alle untersuchten Blechwinkel hinweg nah beieinander, genauso wie die beiden eingezeichneten quadratischen Regressionskurven, die sogar vorsichtig bis $\gamma=40^{\circ}$ extrapoliert werden können. Der punktuell bestimmte Drallwinkel ist damit repräsentativ für den 
gesamten Strömungskanal(sektor) und kann daher als Drallindikator verwendet werden, so zum Beispiel auch für die Drallstudie bei höheren Reynolds-Zahlen (Abb. 5.10). Wie zu vermuten war, nimmt die Umlenkung für höhere Reynolds-Zahlen ab, die Minderumlenkung $\Delta \gamma$ entsprechend zu, und zwar in etwa linear mit steigendem Blechwinkel ( Abb. 5.10 b). So beträgt die Minderumlenkung im Referenzpunkt für die maximale Reynolds-Zahl $R e=451.000$ und einen Blechwinkel von $\gamma=30^{\circ}$ immerhin $\Delta \gamma=10,3^{\circ}$. Um also einen tatsächlichen Drallwinkel von $\alpha=30^{\circ}$ an der Einströmung zum Totraum zu realisieren, muss der Blechwinkel des Drallgitters auf einen Wert deutlich über $30^{\circ}$ eingestellt werden. Auch wenn hierfür keine separaten Untersuchungen durchgeführt wurden, wird erwartet, dass die hier gewonnenen Erkenntnisse auch auf die Sondenmessungen während der WÜK-Versuche übertragbar sind, bei der die Sonde nicht mehr durch die Außenwand in den Kanal eingeführt werden kann, sondern über einen separaten Zugang stromaufwärts des Totraums von unten bis in die Kanalmitte geführt wird (Pos. 2 in Abb. 4.14). Nach diesem Exkurs sollen nun wieder die Ergebnisse der Sondentraversierung in Abb. 5.6 und Abb. 5.7 sowie die Umfangsmittelwerte in Abb. 5.8 betrachtet werden. Wie bereits erwähnt, ändert sich die Nickwinkelverteilung so gut wie gar nicht mit dem eingestellten Blechwinkel, selbst die umfangsgemittelten Werte zeigen nur marginale Abweichungen vom Innenradius bis hin zur Kanalmitte. Darüber hinaus sind die Verläufe quasi identisch, die Zuströmung zum Totraum in der Meridianebene also gleich.

Während für die Konfiguration ohne Drall noch ein ausgeprägtes Nachlaufprofil in der Axialgeschwindigkeitsverteilung zu erkennen ist, „verwaschen“ die $c_{\mathrm{ax}}$-Profile mit größer werdendem Blechwinkel durch die stärkere turbulente Vermischung zunehmend ( Abb. 5.7), wodurch auch die Spreizung der Werte sinkt. Dies ist auch in der umfangsgemittelten Darstellung in Abb. $5.8 \mathrm{~d}$ sichtbar. Dort sieht man, dass das Strömungsprofil flacher wird: während es für $\gamma=0^{\circ}$ und $10^{\circ}$ noch voll ausgebildet erscheint, weist es für $\gamma=30^{\circ}$ ein ausgeprägtes Plateau in der Kanalmitte auf, als wäre die Strömung noch in ihrem hydrodynamischen Anlauf begriffen. Ähnliches lässt sich auch in Abb. 5.8 c für die Absolutgeschwindigkeit $c=|\vec{c}|$ feststellen, die sich ja im Wesentlichen aus ihrer Haupteinflusskomponente $c_{\mathrm{ax}}$ speist.

Wie gewünscht bildet sich mit zunehmendem Drallwinkel eine ausgeprägte Strömung in Umfangsrichtung aus, deutlich zu erkennen an Betrag und Orientierung der $\vec{c}_{\text {res }}$-Vektoren in $>$ Abb. 5.7. Analog steigen die auch die umfangsgemittelten Umfangsgeschwindigkeiten in $\$$ Abb. 5.8 e an, wobei sich zunehmend ein charakteristisches $c_{\mathrm{u}}$-Profil ausbildet. Demgegenüber ändern sich Werte für die radiale Geschwindigkeitskomponente $c_{\mathrm{r}}$ - analog zum Nickwinkel $\beta$ - kaum mit dem Blechwinkel ( Abb. 5.8 f). 


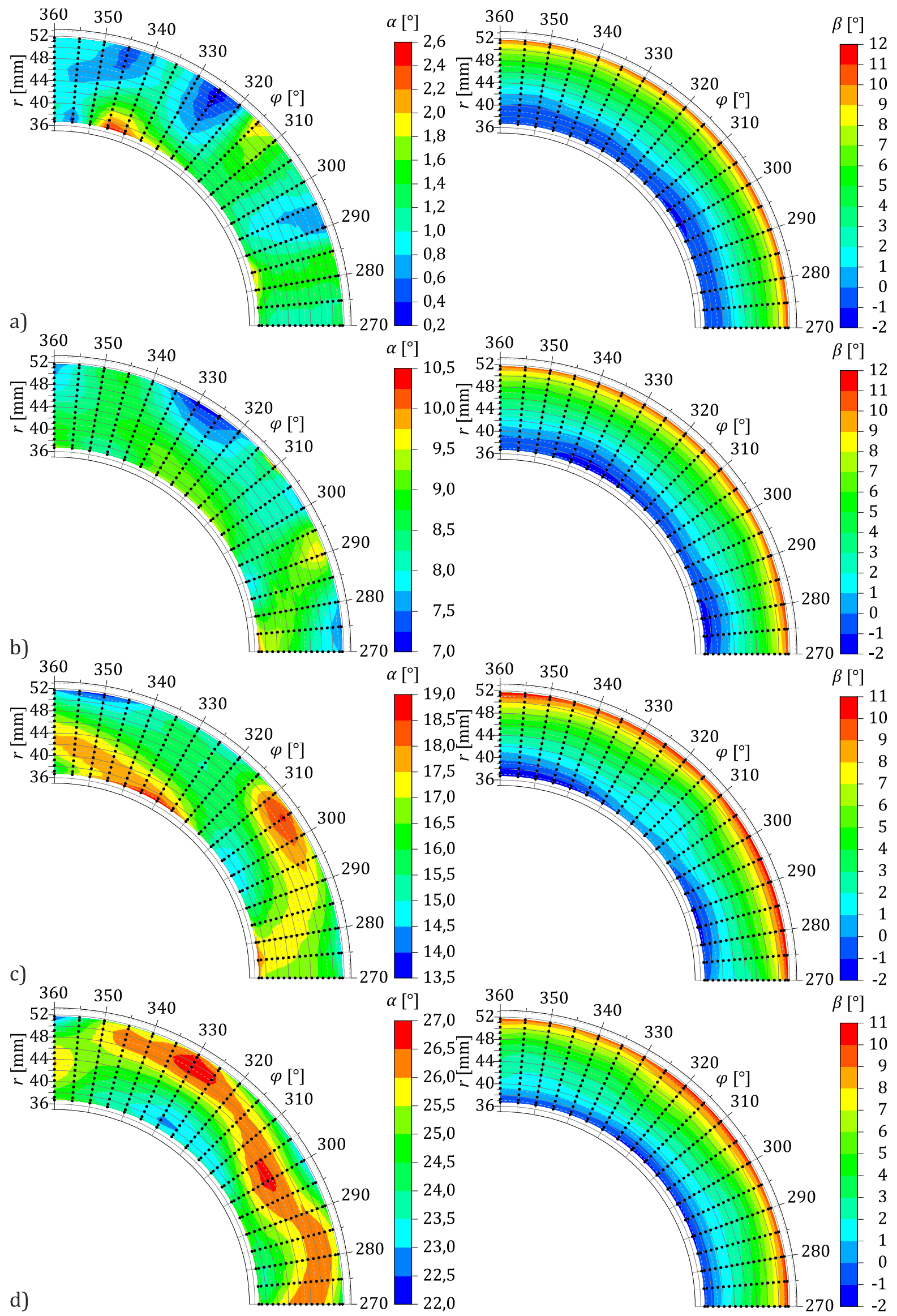

Abb. 5.6 Drallwinkel $\alpha$ und Nickwinkel $\beta$ in der Hauptströmung am Einlass in den Seitenraum. $R e=110.000, \varphi=270^{\circ} \ldots 360^{\circ}$ (4. Quadrant); a) $\gamma=0^{\circ}$, b) $\gamma=10^{\circ}$, c) $\gamma=20^{\circ}$, d) $\gamma=30^{\circ}$. 

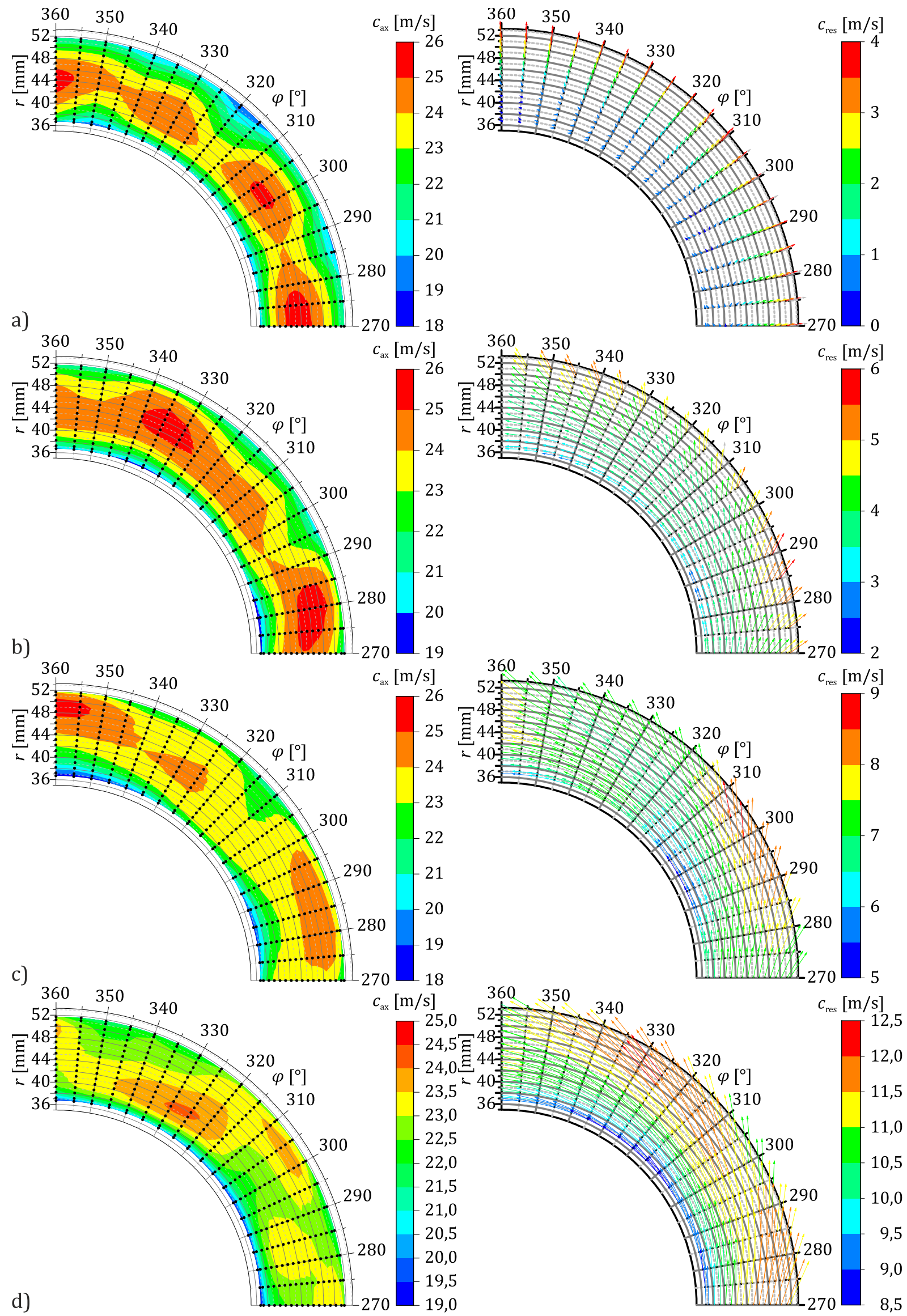

d)
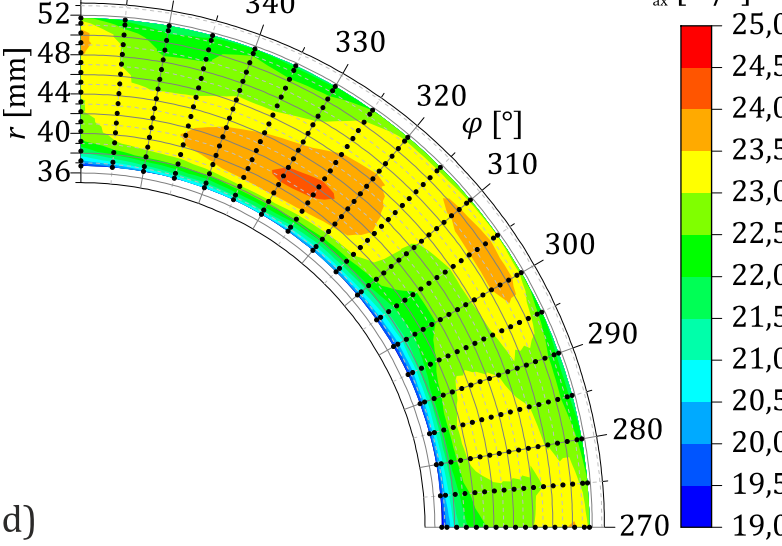

Abb. 5.7 Axial- und Achsnormalgeschwindigkeit $c_{\text {ax }}$ bzw. $c_{\text {res }}$ in der Hauptströmung am Einlass in den Seitenraum. $R e=110.000, \varphi=270^{\circ} \ldots 360^{\circ}$; a) $\gamma=0^{\circ}$, b) $\gamma=10^{\circ}$, c) $\gamma=20^{\circ}$, d) $\gamma=30^{\circ}$. 

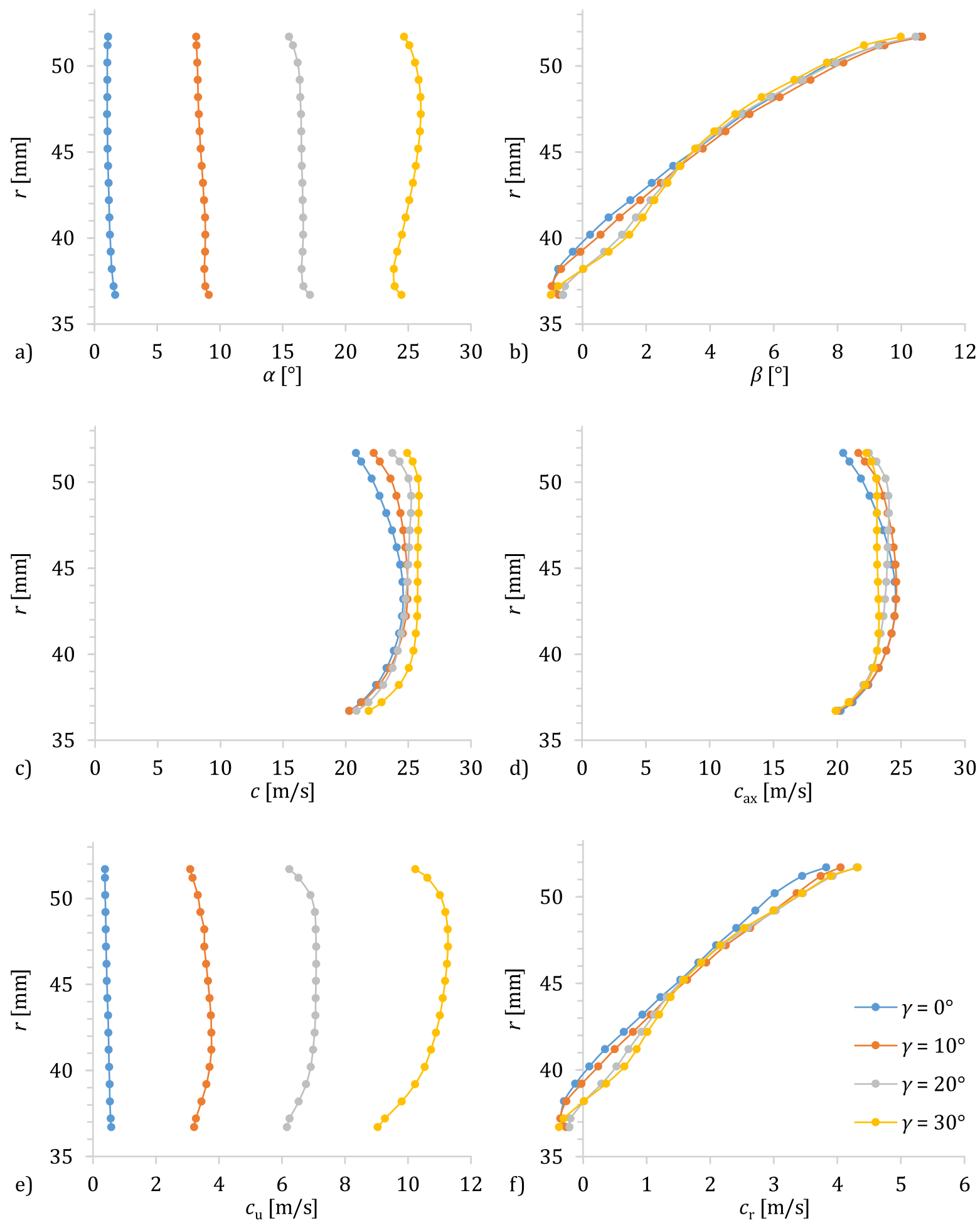

Abb. 5.8 Umfangsgemittelte Strömungswinkel und Geschwindigkeitskomponenten über der Kanalhöhe in der Hauptströmung am Einlass in den Seitenraum. $\varphi=270^{\circ} \ldots 360^{\circ} ; R e=110.000$;

a) Drallwinkel $\alpha$; b) Nickwinkel $\beta$; c) Absolutgeschwidnigkeit $c$; d) Axialgeschwindigkeit $c_{\text {ax }}$;

e) Umfangsgeschwindigkeit $c_{\mathrm{u}}$; f) Radialgeschwindigkeit $c_{\mathrm{r}}$. 


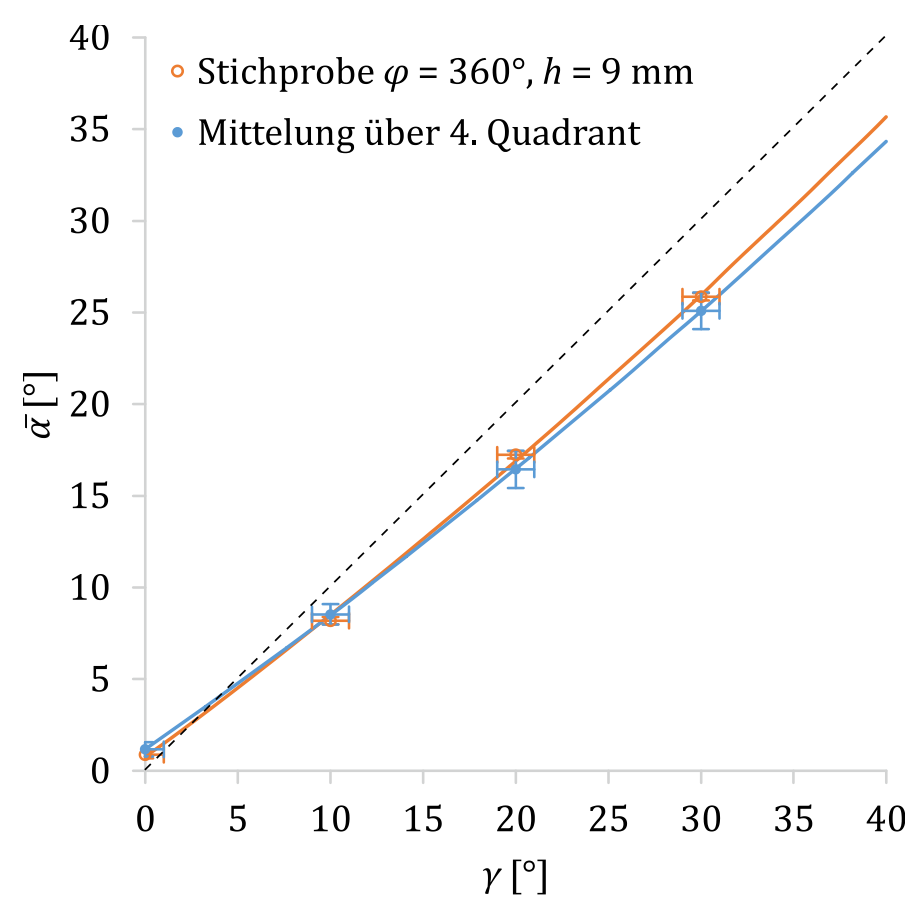

Abb. 5.9 Arithmetisch über den 4. Quadranten gemittelter und an einem Referenzpunkt gemessener Drallwinkel in der Strömung für verschiedene mittels Drallgitter eingestellte Blechwinkel, $R e=110.000$.
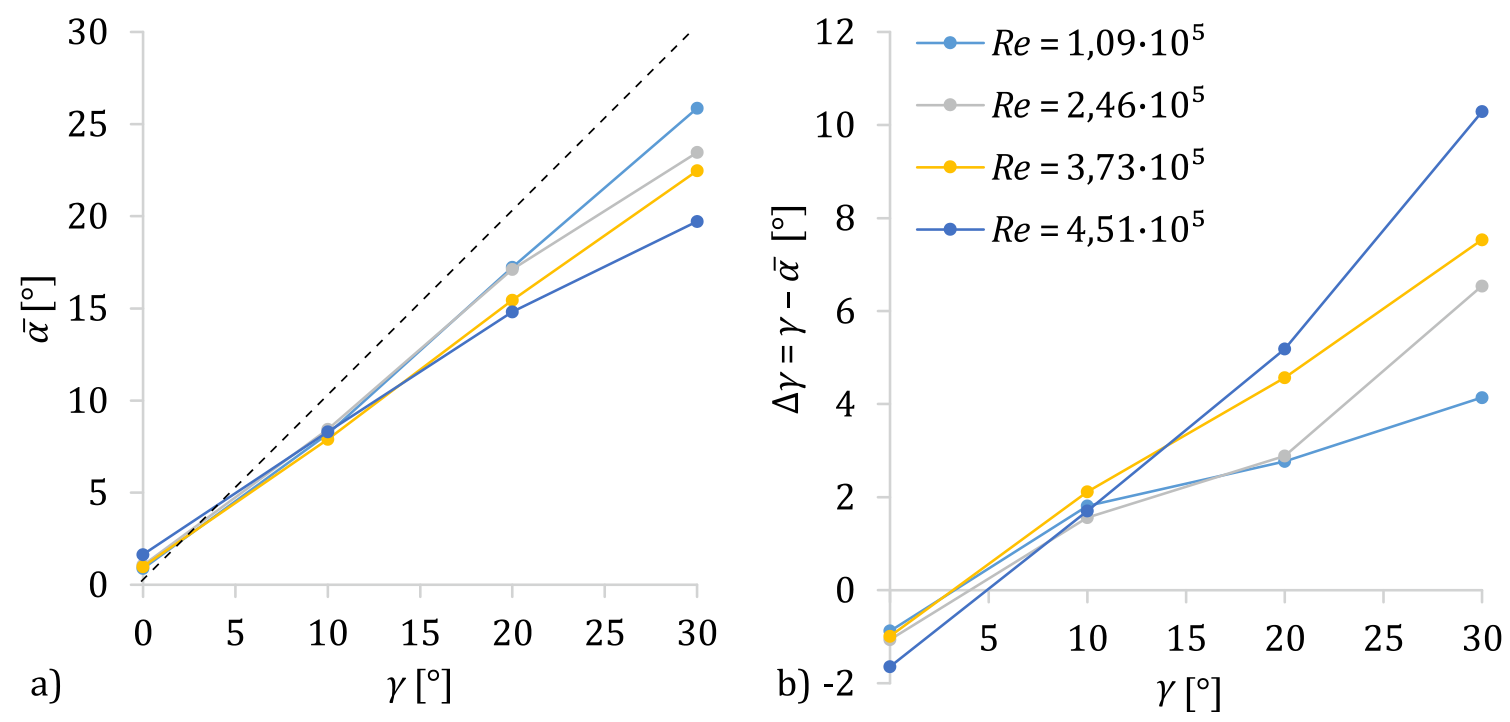

Abb. 5.10 Drallwinkel am Referenzpunkt für verschiedene mittels Drallgitter eingestellte Blechwinkel und erhöhte Reynolds-Zahlen. a) Absoluter Drallwinkel; b) Minderumlenkung. 


\subsection{DRUCKVERTEILUNG AN DER AUßENWANDINNENOBERFLÄCHE}

Mit Hilfe des in Kap. 4.2.2 beschriebenen alternativen Außenwandaufbaus wurde für zwei verschiedene Reynolds-Zahlen $R e=90.000$ und $R e=110.0000$ und maximale Seitenraumgeometrie die Druckverteilung entlang der Innenoberfläche der Außenwand über dem gesamten Umfang des Seitenraumes $\left(360^{\circ}\right.$-Traversierung) in $5^{\circ}$-Schritten bestimmt ( Abb. 5.11). Die vertikalen Linien bei $x=0$ und $x=45 \mathrm{~mm}$ kennzeichnen dabei die Lage der Einströmöffnung. Die Wanddrücke werden als Differenz bezüglich des Referenzdruckes $p_{\text {ref }}$ im Hauptströmungskanal bestimmt:

$$
\Delta p_{\mathrm{AW}, i}=p_{\mathrm{ref}}-p_{\mathrm{AW}, i}, i \in[1 ; 17]
$$

Dadurch kann auch bei geringeren Strömungsgeschwindigkeiten noch ein Messeffekt erzielt werden. Auf Grund des Druckverlustes zwischen der Hauptströmung und der Wirbelströmung im Seitenraum in Folge Reibung und Dissipation ist für alle Messungen $p_{\text {ref }}>p_{\mathrm{AW}, i}$, entsprechend $\Delta p_{\mathrm{AW}, i}>0$. Mit steigender Reynolds-Zahl nehmen auch die Druckverluste zu und $\Delta p_{\mathrm{AW}, i}$ steigt an. Während es sich bei dem Referenzdruck $p_{\text {ref }}$ um einen reinen statischen Druck handelt (Strömung parallel zur Kanalwand), misst man beim Wanddruck $p_{\mathrm{AW}, i}$ unterschiedliche Effekte, je nachdem, in welchem Winkel die Strömung an den Druckbohrungen zur Wand steht:

Strömung parallel zur Wand: $\quad$ statischer Druck, $r=0$;

Strömung senkrecht zur Wand: $\quad$ Staudruck (Totaldruck), $r=1$;

Strömung unter Winkel zur Wand: dynamischer Geschwindigkeitsanteil in gemessenem Druck enthalten, $0<r<1$.

Der Druckrückgewinnungsfaktor (Recovery-Faktor) $r$ bezieht dabei die Differenz zwischen dem gemessenen und dem tatsächlichen statischen Wanddruck $p$ auf den dynamischen Druck:

$$
r_{i}=\frac{p_{\mathrm{AW}, i}-p}{\frac{\rho}{2} u^{2}}
$$

Aus den obigen Überlegungen folgt, dass in den Bereichen der Außenwand, wo $\Delta p_{\mathrm{AW}, i}$ niedrig, also $p_{\mathrm{AW}, i}$ hoch ist, die Strömung steil auf die Wand zu bzw. von der Wand weg fließt oder nur eine geringe Tangentialgeschwindigkeit besitzt. Ersteres lässt sich in Abb. 5.11 sehr eindrucksvoll feststellen, und zwar zum einen im Bereich der Staulinie bei $x \approx 40 \mathrm{~mm}$, wo der Prallstrahl von der Einströmöffnung senkrecht auf die Außenwand trifft, und zum anderen bei $x \approx-18 \mathrm{~mm}$ und $x \approx 82 \mathrm{~mm}$, wo sich die Wirbel wieder von der Außenwand ablösen. Zwischen diesen drei Linien finden sich Bereiche mit großen Werten von $\Delta p_{\mathrm{AW}, i}$, also niedrigen Wanddrücken $p_{\mathrm{AW}, i}$. Dort strömt das Fluid schnell und in flachem Winkel über die Wand, was die Lage der sich ausbildenden Wirbelrollen des Primär- und Sekundärwirbels kennzeichnet. 

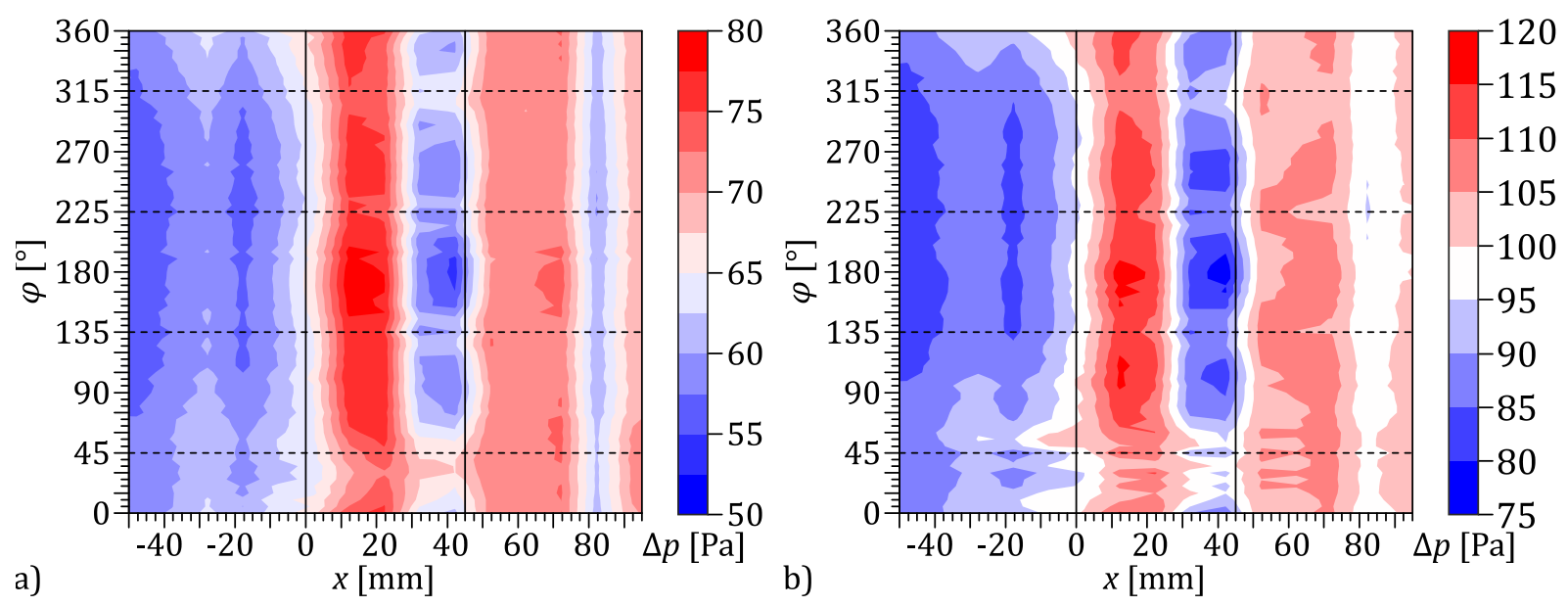

Abb. 5.11 Wanddruckverteilung entlang der Innenoberfläche der Außenwand für $360^{\circ}$-Umfangstraversierung, mit Stützgitter. $s=45 \mathrm{~mm}, b=145 \mathrm{~mm}, e=0$;

a) $R e=90.000$, b) $R e=110.000$.

Des Weiteren fällt auf, dass in beiden Versuchsfällen jeweils alle $90^{\circ}$ bei $\varphi=45^{\circ}, 135^{\circ}$ usw. (gekennzeichnet durch horizontale gestrichelte Linien im Diagramm; Winkeldefinition Abb. 4.16) Bereiche auftreten, in denen $\Delta p_{\mathrm{AW}, i}$ größer und demnach $p_{\mathrm{AW}, i}$ kleiner ist im Vergleich zu den übrigen Werten am Umfang. Dieser erhöhte Druckverlust wird durch die Nachlaufdellen des Stützgitters ( Abb. A.18 b) verursacht, welches zwar weit vor dem Eintritt in den Seitenraum am Beginn des Hauptströmungskanales angeordnet ist, aber dennoch bis in den Seitenraum hinein wirkt. Wie Abb. 5.12 a zeigt, ist dieser Effekt im Bereich der Staulinie mit einer maximalen Schwankungsbreite von $15 \mathrm{~Pa}$ am stärksten ausgeprägt und klingt axial zu den Rändern hin ab. Insgesamt wird der Nachlaufeffekt des Stützgitters jedoch auf Grund dieser doch relativ niedrigen Werte als gering eingeschätzt und keine Beeinflussung des Wärmeübergangs erwartet.

Mittelt man den Druck über dem Umfang und trägt ihn über der axialen Breite auf ( Abb. 5.12 b), so erhält man zusätzliche Validierungsdaten, die zum Beispiel auch für einen Vergleich mit CFDErgebnissen herangezogen werden können (Näheres hierzu siehe Kap. 6).

Weitere Versuche zur Wanddruckverteilung wurden zum Teil parallel mit den Strömungsmessungen in Kap. 5.3 durchgeführt, bei denen die Fünflochsonde durch den Seitenraum bis in den Hauptströmungskanal eingeführt war ( Abb. 4.16). Weil mit der Sonde nur Umfangspositionen zwischen $\varphi=270^{\circ}$ und $360^{\circ}$ traversiert wurden und die Wanddruckbohrungen gegenüber der Sonde um $90^{\circ} \mathrm{im}$ Uhrzeigersinn (mathematisch negativen Sinn) versetzt angeordnet sind, wurden im Folgenden nur Wanddruckplots zwischen $\varphi=180^{\circ} \ldots 270^{\circ}$ aufgenommen. Außerdem musste die Kavitätsbreite für die Sondentraversierung auf $b=120 \mathrm{~mm}$ reduziert werden ( Abb. 4.14). In einem ersten Schritt war zunächst zu untersuchen, ob und inwieweit die Sonde durch ihre Nachlaufdellen die Wandbohrungen verschattet und damit die Wanddruckverteilung verfälscht. 

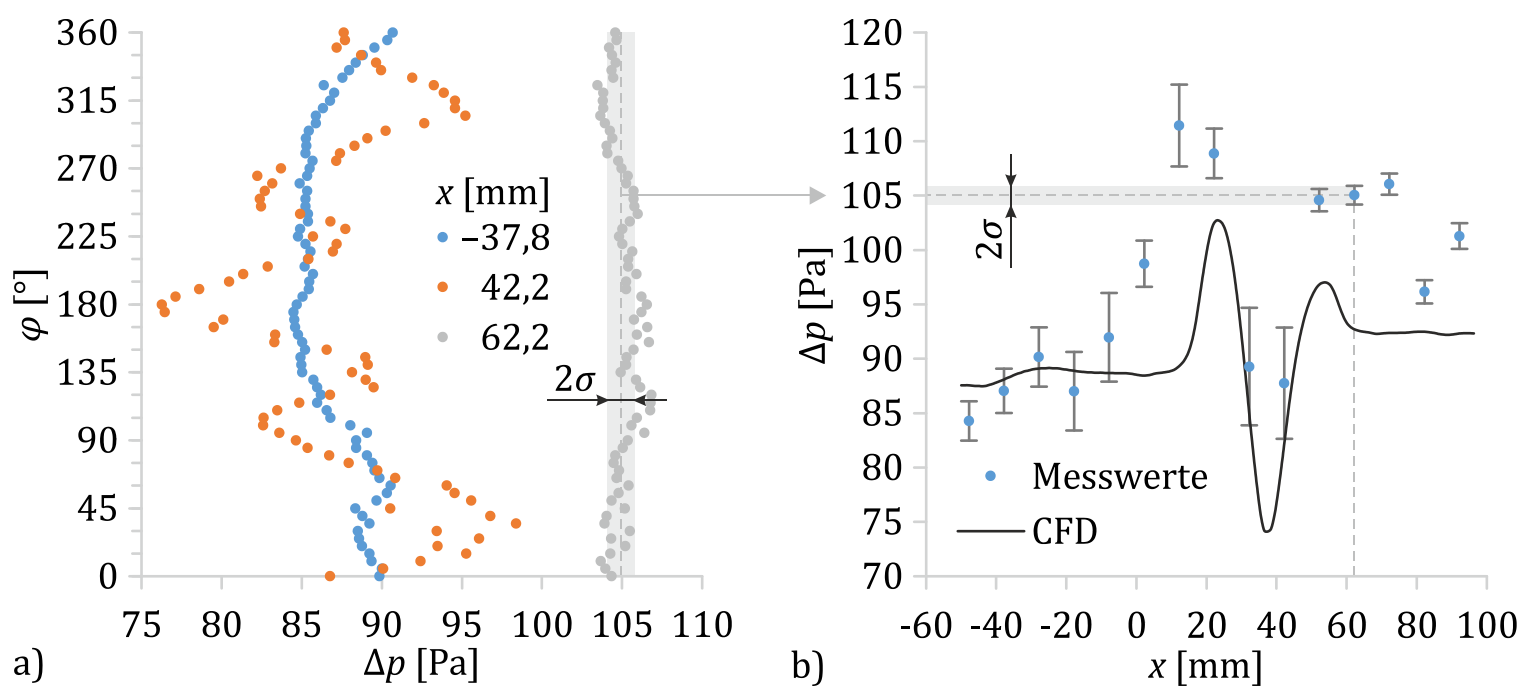

Abb. 5.12 Wanddruckverteilung am Umfang und entlang der Achse der Außenwand für $360^{\circ}$-Umfangstraversierung, mit Stützgitter. $s=45 \mathrm{~mm}, b=145 \mathrm{~mm}, e=0, R e=110.000$.

a) Umfangsverteilung für ausgewählte axiale Positionen,

b) Umfangsgemittelte axiale Druckverteilung und Vergleich mit CFD-Ergebnissen.

Wie der Vergleich in Abb. 5.13 zeigt, unterscheidet sich das Druckfeld mit und ohne Sonde kaum voneinander, was auch daran liegt, dass die Sonde gegenüber den Wandbohrungen in Drallrichtung (mathematisch positivem Sinn) versetzt ist. Die folgenden Ergebnisse für die Wanddruckverteilungen sind daher auch trotz Sonde als belastbar anzusehen.

Wie erwähnt, wurde zur Untersuchung von Dralleffekten auf die Strömung im Hauptströmungskanal ( Kap. 5.3) und später auf den Wärmeübergang im Seitenraum ( Kap. 5.7.7) das Stützgitter mit den vier im $90^{\circ}$-Winkel angeordneten Streben ( Abb. A.18 b) durch das verstellbare Drallgitter mit insgesamt 12 Leitblechen (Teilung $30^{\circ}$ ) ersetzt ( Abb. A.19).
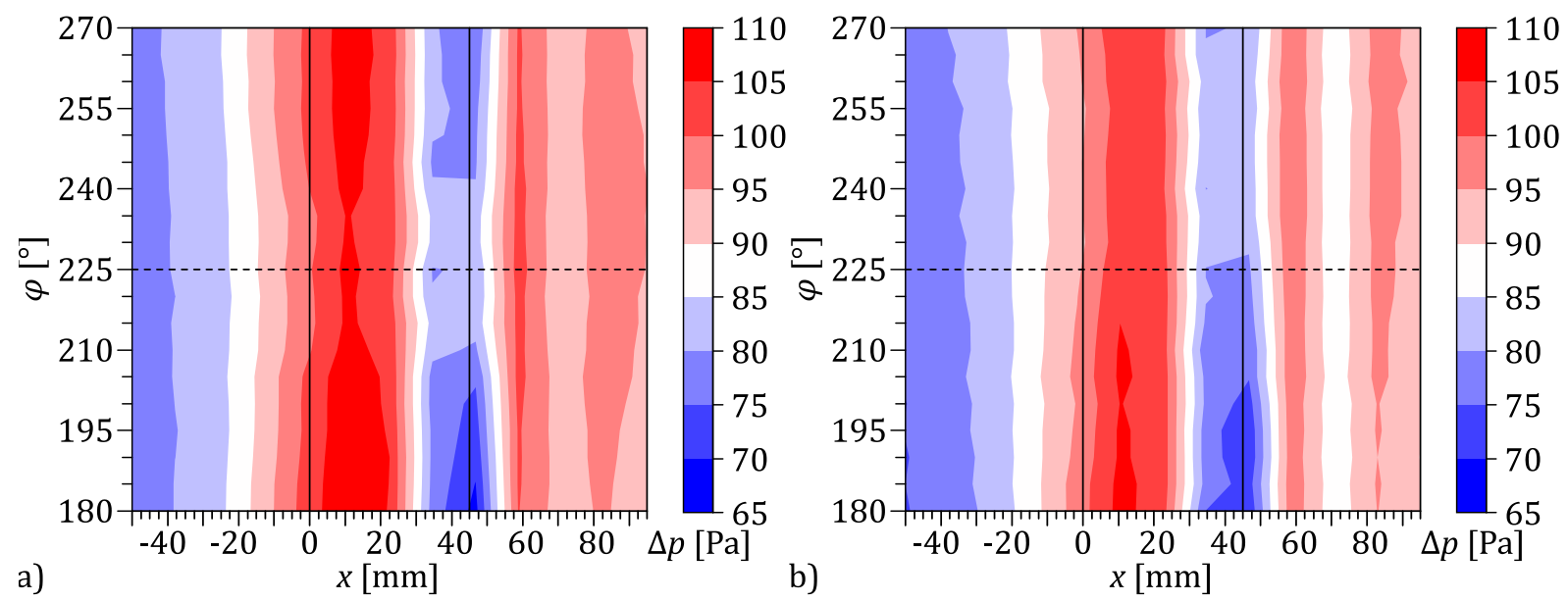

Abb. 5.13 Vergleich der gemessenen Wanddruckverteilung entlang der Innenoberfläche der Außenwand zur Untersuchung des Einflusses einer eventuellen Sondenverschattung; $s=45 \mathrm{~mm}, b=120 \mathrm{~mm}, e=0, R e=110.000$, mit Stützgitter; a) mit Sonde, b) ohne Sonde. 
Vergleicht man für ein und dieselbe Reynolds-Zahl die Wanddruckverteilung für das Stützgitter ( Abb. 5.13 a) und für das Drallgitter ohne Anstellung $\left(\gamma=0^{\circ}\right.$; Abb. 5.14 a), so erkennt man keine nennenswerten Unterschiede im untersuchten Umfangssektor. Damit sollten sich auch die WÜK-Ergebnisse zwischen beiden Fällen nicht unterscheiden, was in Kap. 5.7.2 näher beleuchtet wird.

Abb. 5.14 vermittelt ferner einen Eindruck darüber, wie stark sich die Wanddruck- und damit möglicherweise auch die WÜK-Verteilung im Seitenraum mit dem Drallwinkel in der Hauptströmung ändert, im konkreten Fall für eine Reynolds-Zahl von $R e=110.000$ und insgesamt vier Blechwinkel zwischen 0 und $40^{\circ}$. Während sich die Plots a bis c stark ähneln, treten erst für $\gamma=30^{\circ}$ ( Abb. 5.14 d) nennenswerte Unterschiede auf: die Wanddruckdifferenz $\Delta p_{\mathrm{AW}}$ liegt insgesamt tiefer, es treten also höhere Wanddrücke auf, was gemäß Gleichung 5.17 für einen erhöhten gemessenen dynamischen Druckanteil an den Bohrungen und damit einer stärkeren Durchwirbelung im Seitenraum spricht.
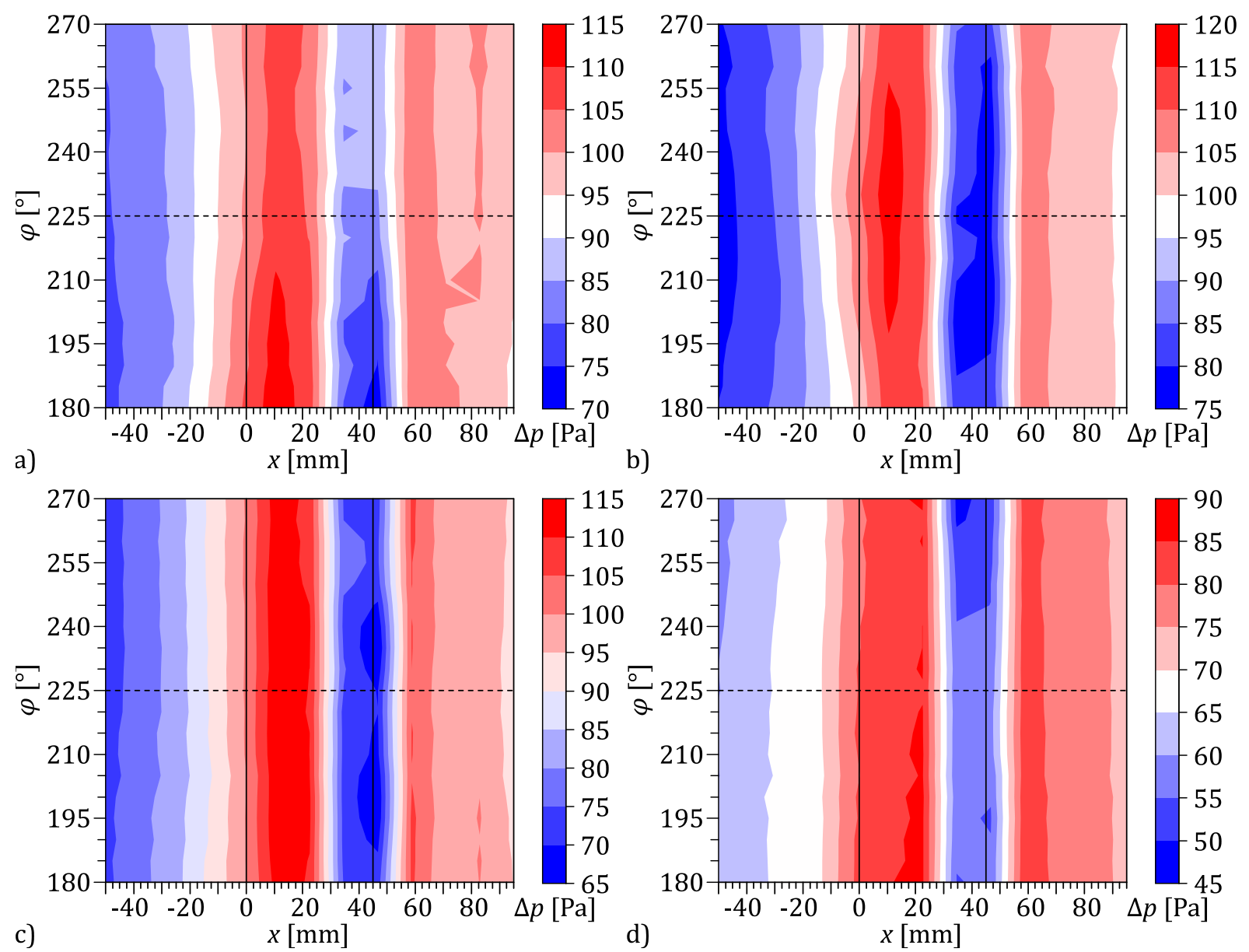

Abb. 5.14 Einfluss des Blechwinkels $\gamma$ in der Hauptströmung auf die Wanddruckverteilung entlang der Innenoberfläche der Außenwand; mit Drallgitter, a) $\gamma=0^{\circ}$, b) $\gamma=10^{\circ}$, c) $\gamma=20^{\circ}$, d) $\gamma=30^{\circ}$; $R e=110.000, s=45 \mathrm{~mm}, b=120 \mathrm{~mm}, e=0$. 
Noch deutlicher wird dies, wenn man die umfangsgemittelten Werte über den $90^{\circ}$-Sektor betrachtet (Abb. 5.15 a). Während die Druckwerte für $\gamma=0^{\circ} \ldots 20^{\circ}$ nur leicht abnehmen, liegt das Wanddruckprofil für $\gamma=30^{\circ}$ deutlich darunter, wobei die Form und die Spreizung des Profils faktisch erhalten bleibt und sich nur der Offset (Verschiebung hin zu niedrigeren Differenzdrücken) ändert. Noch stärker ausgeprägt ist dieser Effekt für $\gamma=40^{\circ}$ (siehe Abb. 5.15 b), wo der Mitteldruck noch einmal um einen Faktor 0,5 tiefer liegt als bei $\gamma=30^{\circ}$. Die in $>$ Abb. 5.15 b dargestellten Drücke sind im Vergleich zu Abb. 5.15 a keine Umfangsmittelwerte, sondern die Werte, die lediglich an der Umfangswinkelposition $\varphi=270^{\circ}$ gemessen worden sind. Wie sich zeigt, sind die Unterschiede zwischen gemittelten und nicht gemittelten Werten nur marginal. Es treten lediglich kleinere Abweichungen im Bereich des linken lokalen Maximums und des rechten lokalen Minimums auf. Die gemessenen Drücke an der $270^{\circ}$-Position sind somit repräsentativ für die gesamte Druckverteilung am Umfang und können im Folgenden auch für quantitative Aussagen herangezogen werden, ohne dass eine aufwändige Traversierung notwendig ist.

Zur Untersuchung des Einflusses des Drallwinkels bei höheren Reynolds-Zahlen ist der Betrieb des Versuchsstandes mit beiden Verdichtern notwendig. Auf Grund der höheren Durchsätze ergeben sich dadurch auch höhere Drücke im Versuchsstand gegenüber dem Solobetrieb des kleineren, drehzahlgeregelten Verdichters, welcher allein nur für Reynolds-Zahlen bis ca. $R e=110.000$ verwendet werden kann.
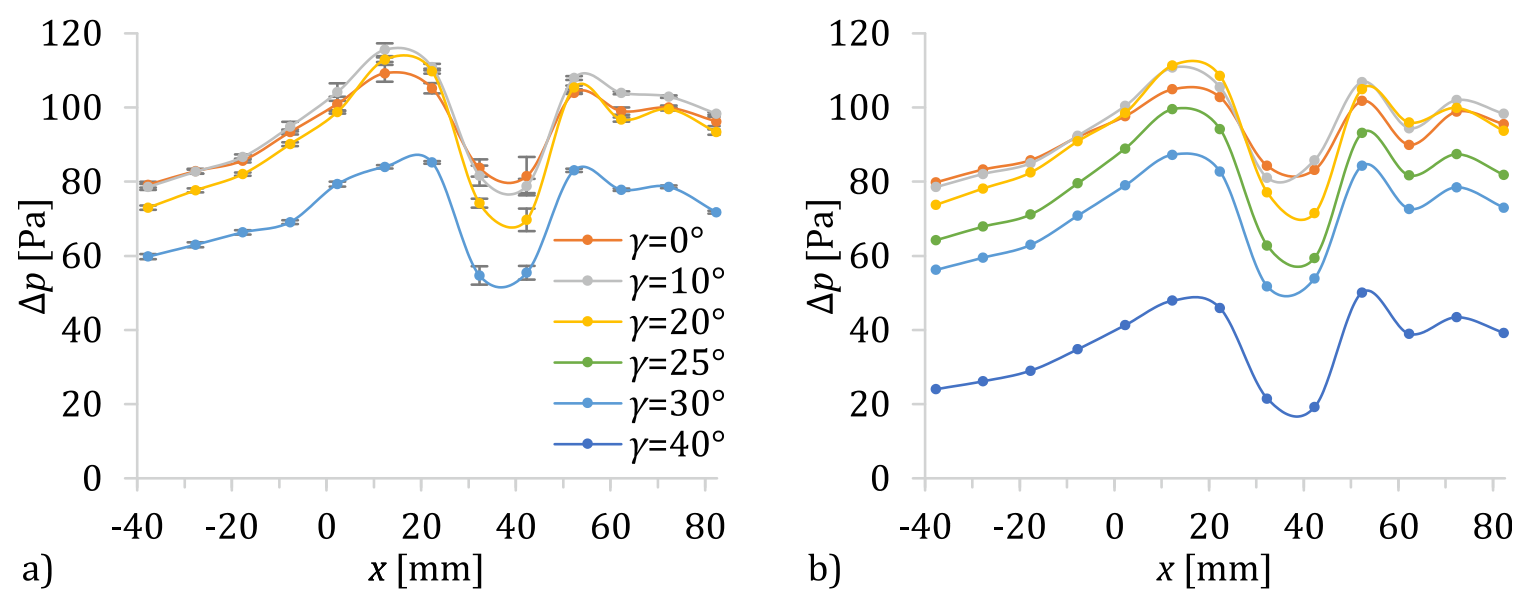

Abb. 5.15 Einfluss des am Drallgitter eingestellten Blechwinkels $\gamma$ auf die axiale Druckverteilung an der Innenoberfläche der Außenwand. $s=45 \mathrm{~mm}, b=120 \mathrm{~mm}, e=0$;

a) umfangsgemittelt im Bereich $180^{\circ} \leq \varphi \leq 270^{\circ}$ (3. Quadrant), $R e=109.000$;

b) bei Umfangswinkelposition $\varphi=270^{\circ}, R e=108.000$. 
Um den eventuellen Einfluss eines erhöhten Referenzdruckes auf die Wanddruckverteilung zu eruieren, wurde für diese Reynolds-Zahl die Wanddruckverteilung für verschiedene Blechwinkel einmal (wie bisher) im Solobetrieb bei ca. 2 bar bestimmt ( Abb. 5.16 a) und einmal im Parallelbetrieb der Kompressoren und mittels Bypass-Betrieb bei etwa 2,5 bar ( Abb. 5.16 b). Wie der Druck liegt auch die Dichte um einen Faktor 1,22 höher als im Solobetrieb, was mit niedrigeren querschnittgemittelten Geschwindigkeiten einhergeht. Wie sich zeigt, unterscheiden sich die Kurven über alle untersuchten Blechwinkel hinweg doch recht deutlich hinsichtlich Niveau und Form: so liegen die Werte bei $p_{\text {ref }}=2,54$ bar um einen Faktor drei höher als bei $p_{\text {ref }}=2,07$ bar. Das globale Minimum im Bereich des Prallstrahles fehlt gänzlich. Die Sensitivität der Werte gegenüber großen Blechwinkeln (z B. $\gamma=30^{\circ}$ ) ist ebenso geringer.

Für höhere Reynolds-Zahlen ( Abb. 5.17 b bis d) stellt sich wiederum das gewohnte Bild mit der bekannten Kurvenform und dem distinktiven Einfluss des Blechwinkels wie in Abb. 5.16 a dar. Wie aus Abb. 5.17 ferner zu entnehmen ist, nimmt der Einfluss des Blechwinkels mit steigender Reynolds-Zahl deutlich zu. So sind bei $R e=451.000$ schon für kleine Blechwinkel deutliche Unterschiede gegenüber der drallfreien Strömung erkennbar. Außerdem nimmt die lokale Verteilung eine deutlich ausgeprägtere Form an (größere Spreizung bezogen auf den Mittelwert) als bei kleineren Reynolds-Zahlen. Dies lässt auch auf erhöhte WÜK für diesen Fall schließen.
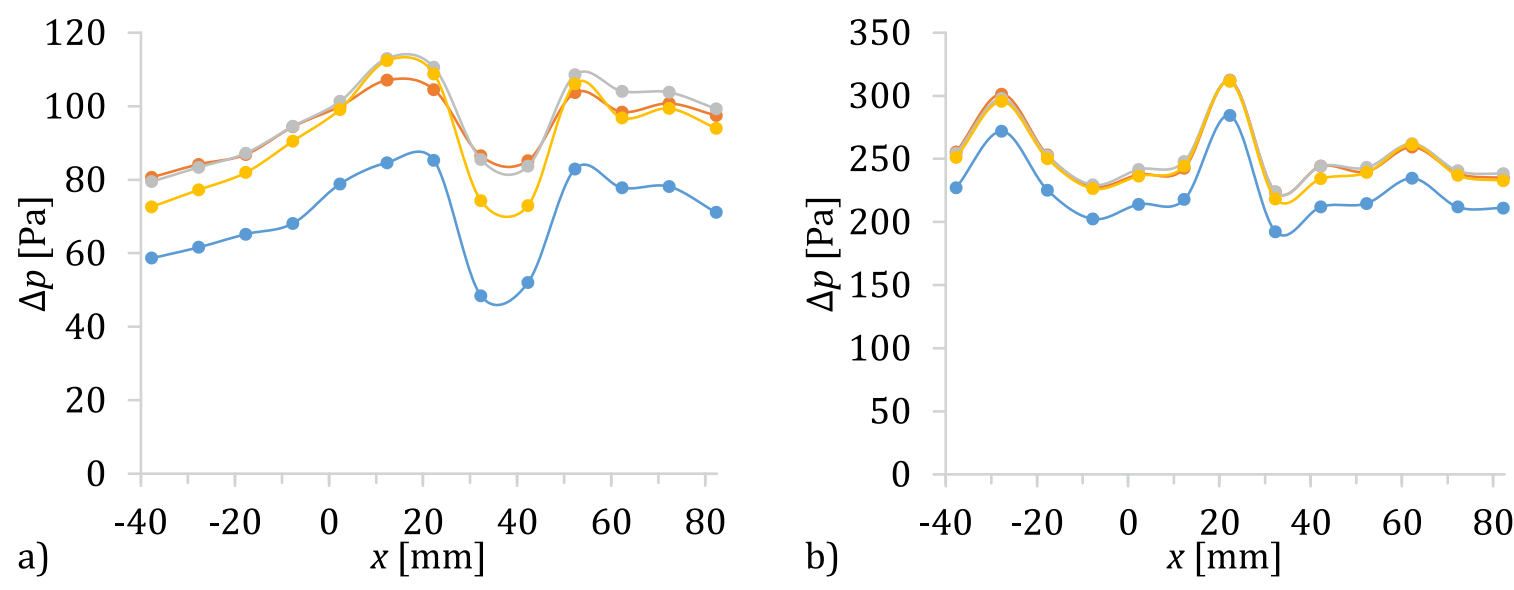

Abb. 5.16 Einfluss des Drucks bzw. der Geschwindigkeit in der Hauptströmung bei gleicher Reynolds-Zahl auf die Druckverteilung an der Innenoberfläche der Außenwand bei $\boldsymbol{\varphi}=\mathbf{0}^{\circ}$ für unterschiedliche Blechwinkel $\gamma . s=45 \mathrm{~mm}, b=120 \mathrm{~mm}, e=0$;

a) $R e=109.000, \boldsymbol{p}_{\text {ref }}=2,07$ bar, $q=22,8 \mathrm{~m} / \mathrm{s}, \dot{m}=0,277 \mathrm{~kg} / \mathrm{s}, T_{\text {ref }}=27,0{ }^{\circ} \mathrm{C}$, $\rho_{\text {ref }}=2,402 \mathrm{~kg} / \mathrm{m}^{3}$, Solobetrieb drehzahlgeregelter Verdichter, $y_{\mathrm{MSV}}=50 \%, y_{3 \mathrm{WV}}=100 \%$;

b) $R e=112.000, \boldsymbol{p}_{\text {ref }}=2,54$ bar, $q=19,4 \mathrm{~m} / \mathrm{s}, \dot{m}=0,288 \mathrm{~kg} / \mathrm{s}, T_{\text {ref }}=28,8^{\circ} \mathrm{C}$, $\rho_{\text {ref }}=2,93 \mathrm{~kg} / \mathrm{m}^{3}$, Parallelbetrieb beider Verdichter, $y_{\mathrm{MSV}}=100 \%, y_{3 \mathrm{WV}}=13,4 \%$. 

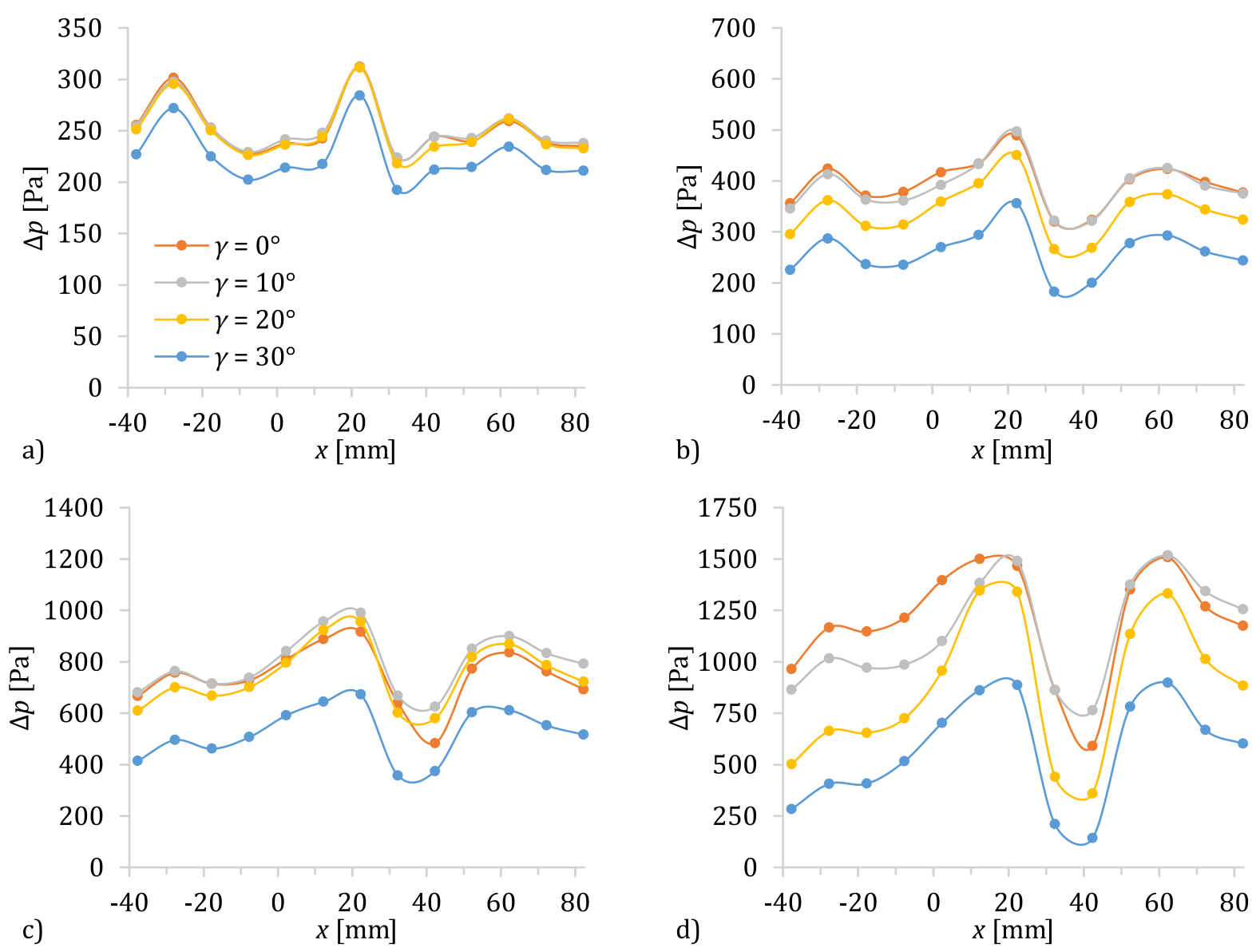

Abb. 5.17 Einfluss der Reynolds-Zahl in der Hauptströmung auf die Druckverteilung bei $\boldsymbol{\varphi}=\mathbf{0}^{\circ}$ für unterschiedliche Blechwinkel $\gamma$. a) $R e=113.000$, b) $R e=246.000$, c) $R e=373.000$, d) $R e=451.000 ; s=45 \mathrm{~mm}, b=120 \mathrm{~mm}, e=0$.

\subsection{THERMODYNAMISCHE STOFFWERTE DER WANDMATERIALIEN}

Wie in Kap. 4.4 ausgeführt, spielt die genaue Kenntnis der thermodynamischen Stoffwerte der Wandmaterialien, allen voran der Wärmeleitfähigkeit des verwendeten PTFE, welches sowohl für die Mittelringe, welche als Hilfswände agieren, als auch für die Flachdichtungen, die die axialen Wärmeverluste zwischen den Innenringen bestimmen, eine zentrale Rolle für die genaue Berechnung der Wärmeströme und damit der WÜK im Seitenraum.

Dazu wurden aus einer extrudierten und gewalzten PTFE-Platte mit einer Nennstärke von $20 \mathrm{~mm}$, wie sie auch für die Fertigung der Zwischenringe und Flachdichtungen genutzt wurde, insgesamt sechs Proben verschiedener geometrischer Form und Größe gefertigt. Form und Nennmaße der Proben sind in Tab. 5.1 aufgeführt, ihre Ausrichtung und Lage innerhalb der Platte ist in Abb. 5.18 dargestellt. 
Tab. 5.1 Form und Nennmaße der PTFE-Proben

\begin{tabular}{ccll}
\hline Pos. & Anzahl & Geometrie & Abmessungen \\
\hline 01 & 1 & Quader & $200 \times 120 \times 20$ \\
02 & 2 & Quader & $60 \times 60 \times 20$ \\
03 & 1 & Quader & $30 \times 30 \times 10$ \\
04 & 2 & Rund & $\emptyset 19 \times 4$ \\
\hline
\end{tabular}

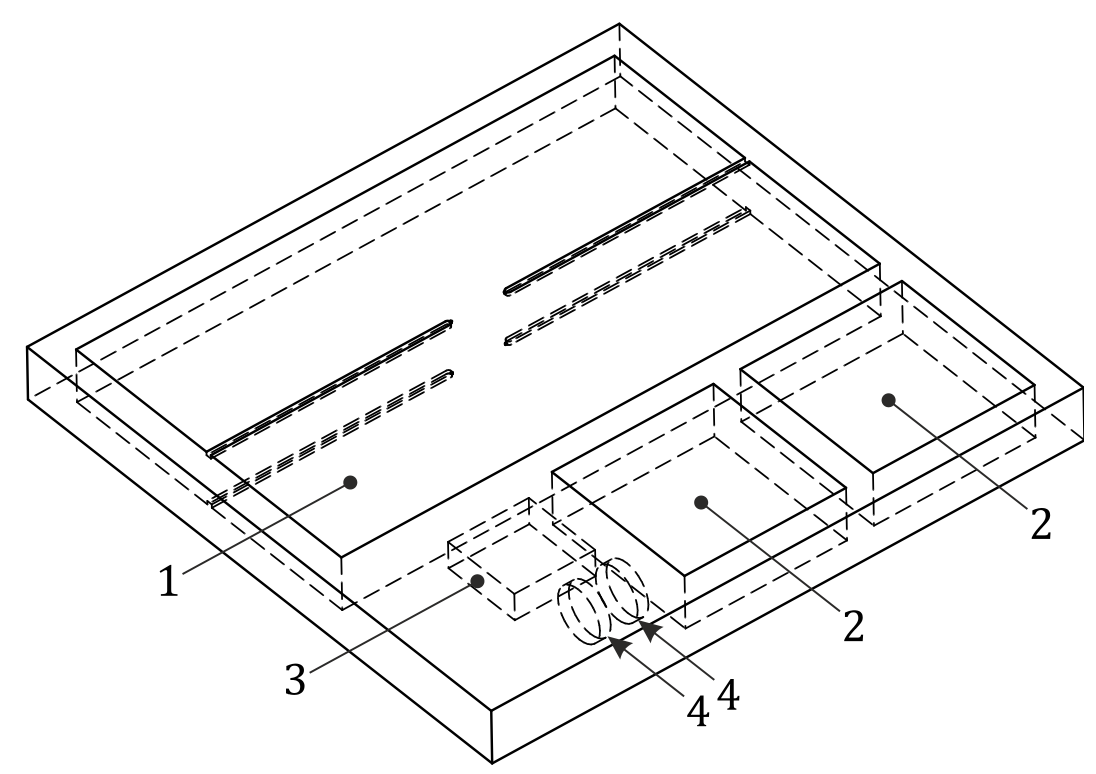

Abb. 5.18 Probennahme aus PTFE-Platte.

\subsubsection{Dichte}

Zur Bestimmung der Dichte der PTFE-Proben wurden die Probekörper zunächst mit einem Digitalmessschieber vermessen und anschließend mit einer Feinwaage gewogen ( Tab. 5.3). Die Mittelwerte und Messungenauigkeiten der Abmaße resultieren aus Mehrfachmessungen an verschiedenen Positionen und wurden „im Kopf gemittelt“. Die Abweichungen umfassen Maß-, Form- und Lagetoleranzen. Die Temperatur der Probekörper und der Umgebung betrug bei der Messung ca. $21^{\circ} \mathrm{C}$. Der Rauminhalt der Proben berechnet sich aus dem Volumen von Zylinder bzw. Quader, die relativen maximalen Abweichungen ergeben sich gemäß Gauß’scher Fehlerfortpflanzung ( Tab. 5.2).

Tab. 5.2 Berechnung der Mittelwerte und Abweichungen für die ermittelten Probenvolumina

\begin{tabular}{lcc}
\hline Statistische Kenngröße & Rundprobe & Quader \\
\hline Mittelwert & $V=\frac{\pi}{4} d^{2} h$ & $V=l \cdot b \cdot h$ \\
relative maximale Abweichung & $\delta V=2 \delta d+\delta h$ & $\delta V=\delta l+\delta b+\delta h$ \\
absolute maximale Abweichung & $\Delta V=V \cdot \delta V$ & $\Delta V=V \cdot \delta V$ \\
\hline
\end{tabular}


Für jeden Probekörper wurde genau eine Wägung durchgeführt. Sobald sich ein Gleichgewichtszustand eingestellt hat, wurde die Masse des Probekörpers notiert. Die absolute Standardunsicherheit des Messergebnisses kann dann nach [118] mit dem Zwei- bis Dreifachen der Wiederholbarkeit der Einzelmessung angegeben werden. Diese beträgt laut Datenblatt für die verwendete Waage $\Delta m= \pm 0,1 \mathrm{mg}$.

Die Dichte eines jeden Probenkörpers ( Tab. 5.3) berechnet sich durch einfache Quotientenbildung aus der jeweiligen Masse und dem zugehörigen Volumen:

$$
\rho=\frac{m}{V}
$$

Es handelt sich hierbei also um eine volumengemittelte Dichte, die bei Unterstellung von Isotropie auch den Wert für die lokale Dichte in jedem Stoffpunkt liefert:

$$
\rho(\vec{x})=\frac{d m}{d V} \equiv \bar{\rho}(V)=\frac{m}{V}=\frac{1}{V} \int_{V} \rho(V) d V .
$$

Auffällig ist, dass die Dichte mit steigendem Volumen der Probekörper scheinbar zunimmt. Dies könnte ein Indiz für eine Anisotropie sein, die auf eine Oberflächenverfestigung der gewalzten PTFE-Platte hindeuten könnte. Während die beiden Zylinderflachproben sowie der kleine Quader aus der Plattenmitte entnommen wurden, umfassen die beiden großen Quader die gesamte Plattendicke abzüglich eines Materialabtrages von ca. 0,5 mm von beiden Seiten durch das Überfräsen. Der Mittelwert (Erwartungswert) der Dichte von PTFE über alle Probekörper liegt bei

$$
\bar{\rho}=\mathrm{E}(\rho)=\frac{1}{N} \cdot \sum_{i=1}^{N} \rho_{i}=2,1463 \frac{\mathrm{g}}{\mathrm{cm}^{3}}
$$

Während der maximale Fehler der Einzelmessung

$$
\begin{gathered}
\delta \rho=\delta m+\delta V \\
\Delta \rho=\rho \cdot \delta \rho
\end{gathered}
$$

auf Grund der Unsicherheiten der zu Grunde liegenden physikalischen Größen bei bis zu $\pm 0,312 \%$ $\left( \pm 0,0067 \mathrm{~g} / \mathrm{cm}^{3}\right)$ liegen kann, beträgt die (Stichproben-) Standardabweichung nur

$$
\begin{aligned}
\Delta \rho=\sigma_{\rho}=\sqrt{\operatorname{Var}(\rho)}=\sqrt{\sigma_{\rho}^{2}} & =\sqrt{\frac{\sum_{i=1}^{N}\left(\rho_{i}-\bar{\rho}\right)^{2}}{N \cdot(N-1)}}= \pm 0,0029 \frac{\mathrm{g}}{\mathrm{cm}^{3}} \\
\frac{\Delta \rho}{\bar{\rho}} & = \pm 0,134 \%
\end{aligned}
$$

Auch die Spannweite der ermittelten Dichtewerte liegt mit 0,0156 g/ $\mathrm{cm}^{3}$ nur bei etwa $0,73 \%$ des Mittelwertes, weshalb der Mittelwert als verlässlich eingeschätzt wird und für alle weiteren Untersuchungen als Stoffparameter benutzt werden soll. 
Tab. 5.3 Abmessungen, Volumen, Masse und Dichte der PTFE-Proben

\begin{tabular}{|c|c|c|c|c|c|}
\hline Probe & $04-1$ & $04-2$ & 03 & $02-1$ & $02-2$ \\
\hline Geometrie & Rund & Rund & Quader & Quader & Quader \\
\hline Nenngröße & $\emptyset 19 \times 4$ & $\emptyset 19 \times 4$ & $30 \times 30 \times 10$ & $60 \times 60 \times 20$ & $60 \times 60 \times 20$ \\
\hline \multirow{2}{*}{$\varnothing d[\mathrm{~mm}]$} & 19,03 & 19,04 & \multirow{2}{*}{-} & \multirow{2}{*}{-} & \multirow{2}{*}{-} \\
\hline & $\pm 0,005$ & $\pm 0,01$ & & & \\
\hline \multirow{2}{*}{$l[\mathrm{~mm}]$} & \multirow{2}{*}{-} & \multirow{2}{*}{-} & 30,01 & 59,90 & 59,91 \\
\hline & & & $\pm 0,01$ & $\pm 0,03$ & $\pm 0,05$ \\
\hline \multirow{2}{*}{$b[\mathrm{~mm}]$} & \multirow{2}{*}{-} & \multirow{2}{*}{-} & 29,95 & 59,86 & 59,85 \\
\hline & & & $\pm 0,01$ & $\pm 0,03$ & $\pm 0,02$ \\
\hline \multirow{2}{*}{$h[\mathrm{~mm}]$} & 4,02 & 4,01 & 10,04 & 20,03 & 20,03 \\
\hline & $\pm 0,005$ & $\pm 0,005$ & $\pm 0,01$ & $\pm 0,015$ & $\pm 0,02$ \\
\hline \multirow{3}{*}{$V\left[\mathrm{~mm}^{3}\right]$} & 1143,39 & 1141,74 & 9023,95 & 71819,85 & 71819,84 \\
\hline & $\pm 0,177 \%$ & $\pm 0,300 \%$ & $\pm 0,166 \%$ & $\pm 0,175 \%$ & $\pm 0,217 \%$ \\
\hline & $\pm 2,023$ & $\pm 2,623$ & $\pm 15,01$ & $\pm 125,75$ & $\pm 155,65$ \\
\hline \multirow{3}{*}{$m[\mathrm{~g}]$} & 2,4432 & 2,4485 & 19,3633 & 154,5734 & 154,5868 \\
\hline & $\pm 0,0122 \%$ & $\pm 0,0123 \%$ & $\pm 0,00155 \%$ & $\pm 0,000194 \%$ & $\pm 0,000194 \%$ \\
\hline & $\pm 0,0003$ & $\pm 0,0003$ & $\pm 0,0003$ & $\pm 0,0003$ & $\pm 0,0003$ \\
\hline \multirow{3}{*}{$\rho\left[\frac{\mathrm{g}}{\mathrm{cm}^{3}}\right]$} & 2,1368 & 2,1445 & 2,1458 & 2,1522 & 2,1524 \\
\hline & $\pm 0,189 \%$ & $\pm 0,312 \%$ & $\pm 0,168 \%$ & $\pm 0,175 \%$ & $\pm 0,217 \%$ \\
\hline & $\pm 0,0040$ & $\pm 0,0067$ & $\pm 0,0036$ & $\pm 0,0038$ & $\pm 0,0047$ \\
\hline \multirow{3}{*}{$\bar{\rho}\left[\frac{\mathrm{g}}{\mathrm{cm}^{3}}\right]$} & \multicolumn{5}{|c|}{2,1463} \\
\hline & \multicolumn{5}{|c|}{ $\pm 0,134 \%$} \\
\hline & \multicolumn{5}{|c|}{ $\pm 0,0029$} \\
\hline
\end{tabular}

\subsubsection{Wärmeleitfähigkeit, Temperaturleitfähigkeit, spezifische Wärmekapazität}

\subsubsection{Ergebnisse bei Raumtemperatur}

In einem ersten Schritt wurde für drei PTFE-Proben die Wärmeleitfähigkeit bei Raumtemperatur durch das Fraunhofer-Institut für Fertigungstechnik und Angewandte Materialforschung (IFAM) in Dresden im Auftrag bestimmt. Dabei kamen zwei verschiedene Messverfahren zum Einsatz.

Beim transienten Flächenquellenverfahren (Hot-Disk-Verfahren) nach DIN EN ISO 22007-2 wird die Wärmeleitfähigkeit nicht direkt gemessen, sondern aus der Temperaturleitfähigkeit $a$, der spezifischen Wärmekapazität $c$ und der Dichte $\rho$ berechnet:

$$
\lambda=a \rho c
$$

Die Dichte wurde wie in Kap. 5.5.1 aus gewogener Masse und gemessenen Maßen der Probe ermittelt (Tab. 5.4). Die im Vergleich etwas höheren Dichtewerte resultieren aus kleineren gemessenen Abmaßen, die auf eine geringere Prüftemperatur der Probekörper schließen lassen. Die gewogenen Massen sind praktisch identisch. 
Tab. 5.4 Abmessungen, Masse und Dichte der PTFE-Proben [119]

\begin{tabular}{lccc}
\hline Probe & $04-1$ & $04-2$ & 03 \\
Geometrie & Rund & Rund & Quader \\
Nenngröße & $\emptyset 19 \times 4$ & $\emptyset 19 \times 4$ & $30 \times 30 \times 10$ \\
\hline$\varnothing d[\mathrm{~mm}]$ & 19,00 & 19,02 & - \\
$l[\mathrm{~mm}]$ & - & - & 29,96 \\
$b[\mathrm{~mm}]$ & - & - & 29,92 \\
$h[\mathrm{~mm}]$ & 3,99 & 3,98 & 9,99 \\
\hline$m[\mathrm{~g}]$ & 2,4433 & 2,4487 & 19,3638 \\
\hline$\rho\left[\frac{\mathrm{g}}{\mathrm{cm}^{3}}\right]$ & 2,160 & 2,165 & 2,162 \\
\hline $\bar{\rho}\left[\frac{\mathrm{g}}{\mathrm{cm}^{3}}\right]$ & & 2,162 \\
& & $\pm 0,003$ & \\
\hline & &
\end{tabular}

Für die Bestimmung der beiden anderen thermodynamischen Größen $a$ und $c_{p}$ kommen spezielle Sonden zum Einsatz ( Abb. 5.19). Mit Hilfe der Sonde in Abb. 5.19 a wird über eine dünne, beidseitig isolierte, bifilare Nickelspirale, welche zwischen zwei identischen Flachproben platziert wird, ein konstanter Wärmestrom appliziert, und der Temperatursprung in der Mitte der Probenoberfläche über einen in die Sonde integrierten Temperatursensor aufgezeichnet. Aus dem zeitlichen Verlauf kann unter Annahme der zweidimensionalen Wärmeleitung im finiten Zylinder durch Kurvenanpassung direkt die Temperaturleitfähigkeit bestimmt werden [119] ( Tab. 5.5).
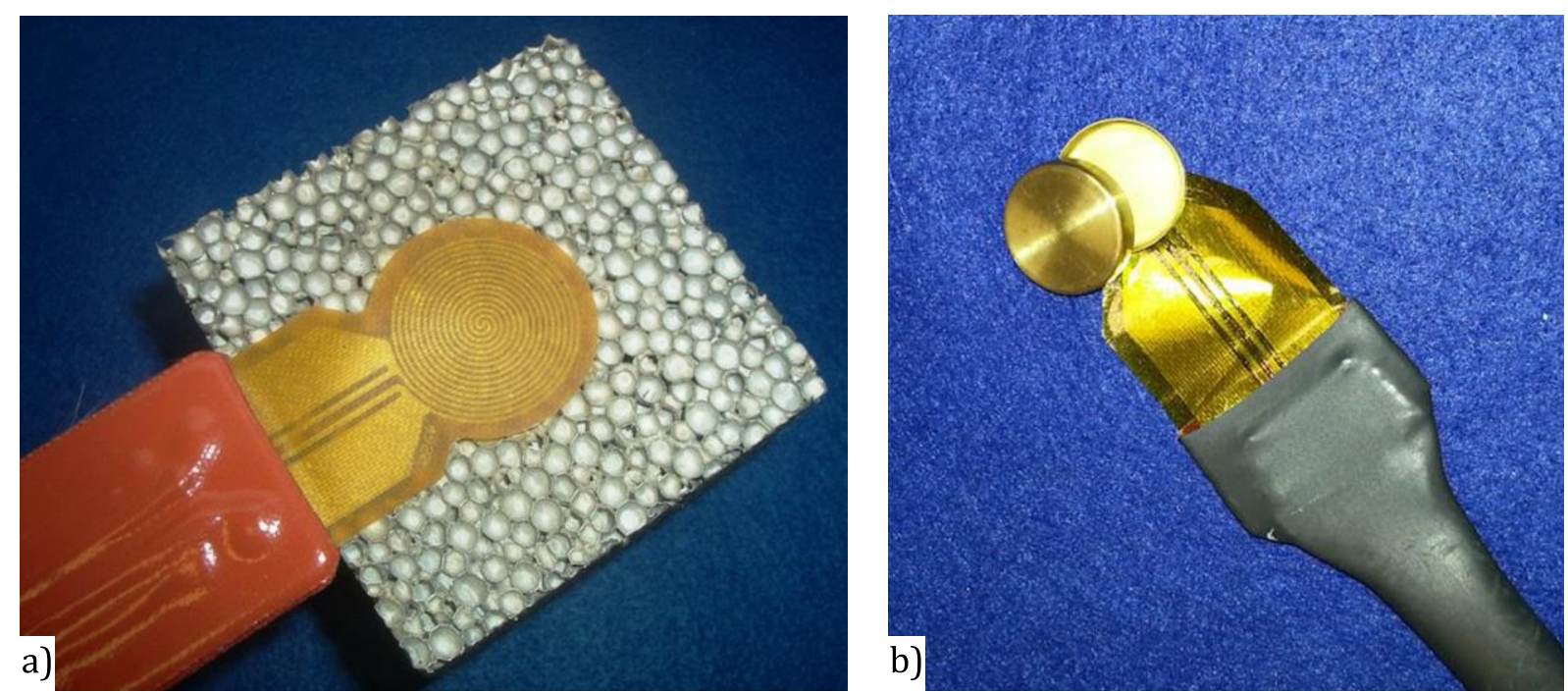

Abb. 5.19 Hot-Disk-Sonden: a) Temperaturleitfähigkeitssonde; b) Wärmekapazitätssonde. Bilder aus [119]. 
Eine abgewandelte Form der Sonde wird auch zur Bestimmung der spezifischen Wärmekapazität genutzt ( Abb. 5.19 b). Der Sensorkopf ist dabei einseitig in einen geteilten, verschließbaren, zylindrischen Behälter integriert, der thermisch isoliert ist und in den später der Probekörper eingelegt wird. In einer Referenzmessung wird zunächst bei einer definierten Heizleistung und -dauer die Aufheizung (Temperaturerhöhung) des leeren Behälters bestimmt. Anschließend wird bei identischen Umgebungsbedingungen und mit eingelegter Probe die Heizleistung so angepasst, dass die gemessene Temperaturerhöhung der des leeren Probenbehälters entspricht. Aus der notwendigen Leistungserhöhung und der zuvor ermittelten Probenmasse wird die spezifische Wärmekapazität berechnet ( Tab. 5.5).

Tab. 5.5 Mittels Hot-Disk-Verfahren bestimmte spezifische Wärmekapazität, Temperatur- und Wärmeleitfähigkeit der PTFE-Proben [119]

\begin{tabular}{cccccccc}
\hline Größe & Probe & Messung 1 & Messung 2 & Messung 3 & Messung 4 & $\boldsymbol{\mu}$ & $\sigma$ \\
\hline$c\left[\frac{\mathrm{J}}{\mathrm{kg} \mathrm{K}}\right]$ & $04-1 / 2$ & 921,4 & 971,7 & 941,1 & 914,1 & $\mathbf{9 3 7 , 2}$ & 25,8 \\
$a\left[\frac{\mathrm{mm}^{2}}{\mathrm{~s}}\right]$ & $04-1 / 2$ & 0,163 & 0,161 & 0,167 & 0,168 & $\mathbf{0 , 1 6 4}$ & 0,003 \\
$\lambda\left[\frac{\mathrm{W}}{\mathrm{m} \mathrm{K}}\right]$ & $04-1 / 2$ & 0,330 & 0,325 & 0,338 & 0,340 & $\mathbf{0 , 3 3 3}$ & 0,007 \\
\hline
\end{tabular}

Als zweites Verfahren kam das stationäre Referenz-Platten-Messverfahren zur Anwendung. In Anlehnung an DIN EN 12667, DIN EN 1946-2 bzw. ISO 8302 wird dort die Wärmeleitfähigkeit einer Flachprobe, in diesem Fall von Probekörper 03 ( Tab. 5.1), mittels eines speziellen Schichtaufbaus bestimmt ( Abb. 5.20), der auch eine Referenzprobe mit bekannter Wärmeleitfähigkeit umfasst. Über eine Heizfolie, welche auf eine Kupferplatte aufgeklebt ist, wird von oben ein flächiger Wärmestrom auf die Probe appliziert. Als Wärmesenke dient eine temperierte Aluminium-Kühlplatte, auf der der Versuchsaufbau angeordnet ist. Temperaturen werden sowohl an der Wärmequelle und -senke sowie zwischen den beiden Proben mittels Temperaturmessplatten gemessen. Zur Verringerung des thermischen Kontaktwiderstandes zwischen den einzelnen Schichten wird Wärmeleitfolie eingesetzt. Um Wärmeverluste an die Umgebung zu reduzieren, wird der Versuchsaufbau durch eine lose Schüttung von Styroporkugeln isoliert. Weiterhin werden die Temperaturen von Wärmequelle und -senke so eingestellt, dass die Temperatur zwischen den Proben der Raumtemperatur entspricht [119]. 

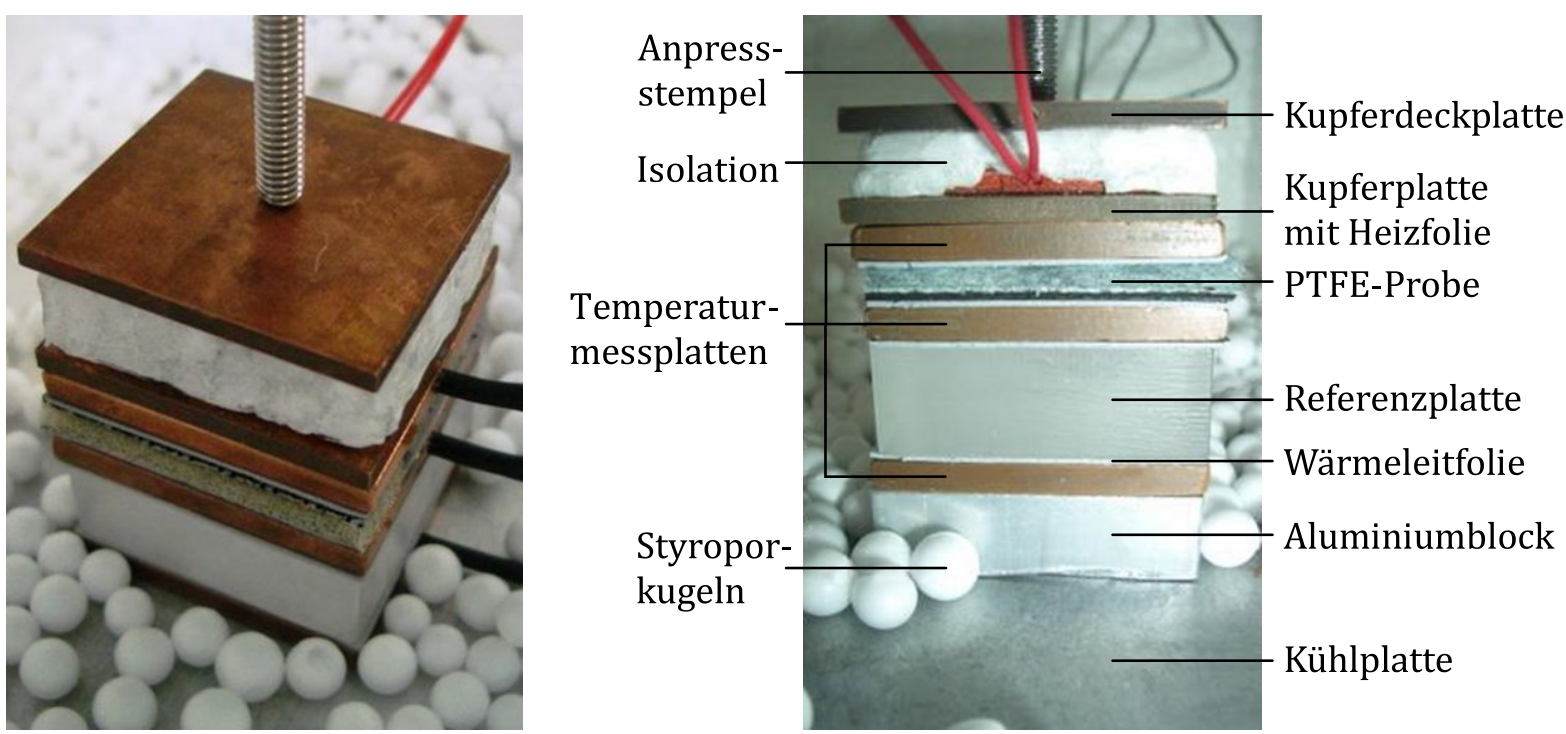

Abb. 5.20 Aufbau der Referenz-Platten-Apparatur [119].

Aus der Reihenschaltung der thermischen Widerstände der einzelnen Schichten und der Temperaturdifferenz über der Referenzprobe $\Delta T_{\text {ref }}$ sowie über der unbekannten Probe $\Delta T_{\operatorname{Pr}}$ im thermisch stationären Zustand ergibt sich unter Annahme eindimensionaler Wärmeleitung und Vernachlässigung von Wärmeverlusten nach außen:

$$
\dot{Q}=\frac{\Delta T_{\text {ref }}}{R_{\lambda, \text { ref }}+2 R_{\lambda, \text { Folie }}}=\frac{\Delta T_{\text {Pr }}}{R_{\lambda, \text { Pr }}+2 R_{\lambda, \text { Folie }}}
$$

Vernachlässigt man den Wärmeleitwiderstand der Folien, erhält man mit $R_{\lambda, \mathrm{i}}=s_{\mathrm{i}} /\left(\lambda_{\mathrm{i}} A\right)$ die Wärmeleitfähigkeit des zu untersuchenden Prüfkörpers:

$$
\lambda_{\mathrm{Pr}}=\lambda_{\text {ref }} \cdot \frac{S_{\mathrm{Pr}}}{S_{\mathrm{Ref}}} \cdot \frac{\Delta T_{\mathrm{ref}}}{\Delta T_{\mathrm{Pr}}}
$$

Auf Grund der asymmetrischen Einbaulage liegt das Temperaturniveau der Probe entweder oberhalb oder unterhalb der Raumtemperatur, was einen Sekundärwärmestrom zwischen Probe und Umgebung bedingt. Um die dadurch auftretenden Abweichungen bei der berechneten Wärmeleitfähigkeit auszugleichen, wird die Anordnung gedreht und die Probe in einer zweiten Messung unterhalb der Referenzplatte angeordnet. Durch Mittelwertbildung der Einzelmessungen erhält man dann ein aussagekräftiges Ergebnis ( Tab. 5.6), welches in derselben Größenordnung liegt wie der mittels Hot-Disk-Methode bestimmte Wert.

Tab. 5.6 Mittels Referenz-Platten-Apparatur bestimmte Wärmeleitfähigkeit der PTFE-Probe [119]

\begin{tabular}{rccccc}
\hline Größe & Probe & Messung 1 & Messung 2 & $\boldsymbol{\mu}$ & $\sigma$ \\
\hline$\lambda\left[\frac{\mathrm{W}}{\mathrm{m} \mathrm{K}}\right]$ & 03 & 0,293 & 0,341 & $\mathbf{0 , 3 1 7}$ & 0,034 \\
\hline
\end{tabular}




\subsubsection{Einfluss der Temperatur auf die Wärmeleitfähigkeit}

Um die Temperaturabhängigkeit der Wärmeleitfähigkeit feststellen zu können, wurden weiterführende Versuche an einer sehr ähnlichen Wärmestrommessplattenapparatur durchgeführt, welche freundlicherweise von der Professur für Technische Thermodynamik an der TU Dresden zur Verfügung gestellt wurde [120,121]. Bei dem in DIN 52616, DIN EN 1946-3 und DIN EN ISO 8301 genormten Verfahren wird eine quaderförmige Flachprobe des zu prüfenden Materials zwischen einer Heiz- und einer Kühlplatte angeordnet und aus der Messung des Wärmestroms sowie der Temperaturdifferenz über der Dicke der Probe die Wärmeleitfähigkeit bestimmt.

In der praktischen Umsetzung ( Abb. 5.21 a) wurden zwei wasserdurchströmte Aluminiumplatten als Heiz- bzw. Kühlplatte verwendet, deren Temperaturen über Umwälzthermostate unabhängig voneinander auf einen Vorgabewert ausgeregelt werden. Um Wärmeverluste an die Umgebung zu reduzieren und damit einen möglichst eindimensionalen Wärmestrom durch den Probekörper (Probe 01 in $\$ Tab. 5.1) zu realisieren, wird der Messaufbau mit Watte isoliert und in einer Klimaklammer [121] platziert (Abb. 5.21 b), deren Temperatur $T_{\mathrm{KK} \text {,soll }}$ auf den Mittelwert von Heiz- und Kühlplattensolltemperatur eingestellt wird:

$$
T_{\mathrm{KK}, \mathrm{soll}}=\frac{1}{2}\left(T_{\mathrm{H}, \mathrm{soll}}+T_{\mathrm{K}, \mathrm{soll}}\right)
$$

Die Wärmestromdichte, die durch den Prüfling strömt, wird mittels zweier Wärmestrommessplatten (Abb. 5.21 c) gemessen, die zwischen Probekörper und Heiz- bzw. Kühlplatte eingelegt und durch Beschweren des Versuchsaufbaus angepresst sind. Die tatsächlichen Temperaturen an den beiden Plattenoberflächen werden jeweils mittels zweier teflonisolierter NiCr-Ni-Thermoelemente vom Typ K mit einem Drahtdurchmesser von 0,2 mm bestimmt, welche in eingefrästen $\mathrm{Nu}$ ten von außen bis in die Mitte der Oberfläche geführt und eingeklebt worden sind (siehe Pos. 1 in

Abb. 5.18). Als Datenerfassungssystem diente ein ALMEMO-Universalmessgerät 2690A-710. Sowohl Wärmeflussplatten als auch Thermoelemente kamen werkskalibriert zum Einsatz.

Nach Vorgabe der Sollwerte für Heiz- und Kühltemperatur sowie der Mitteltemperatur benötigt das Messsystem ca. 1,5 h für den thermischen Ausgleich. Aus den zeitlich gemittelten stationären Gleichgewichtswerten wurden zunächst die mittleren Probenoberflächentemperaturen auf der Oberseite $\bar{T}_{\mathrm{o}}=\left(T_{\mathrm{o}, 1}+T_{\mathrm{o}, 2}\right) / 2$ und auf der Unterseite $\bar{T}_{\mathrm{u}}=\left(T_{\mathrm{u}, 1}+T_{\mathrm{u}, 2}\right) / 2$ sowie die mittlere Wärmestromdichte $\overline{\hat{q}}=\left(\hat{\dot{q}}_{\mathrm{o}}+\hat{\hat{q}}_{\mathrm{u}}\right) / 2$ bestimmt und daraus zusammen mit der Plattenstärke $s=20 \mathrm{~mm}$ die Wärmeleitfähigkeit der Probe berechnet:

$$
\bar{\lambda}\left(\bar{T}_{\mathrm{KK}}\right)=\frac{\overline{\hat{q}} \cdot s}{\bar{T}_{\mathrm{o}}-\bar{T}_{\mathrm{u}}}
$$



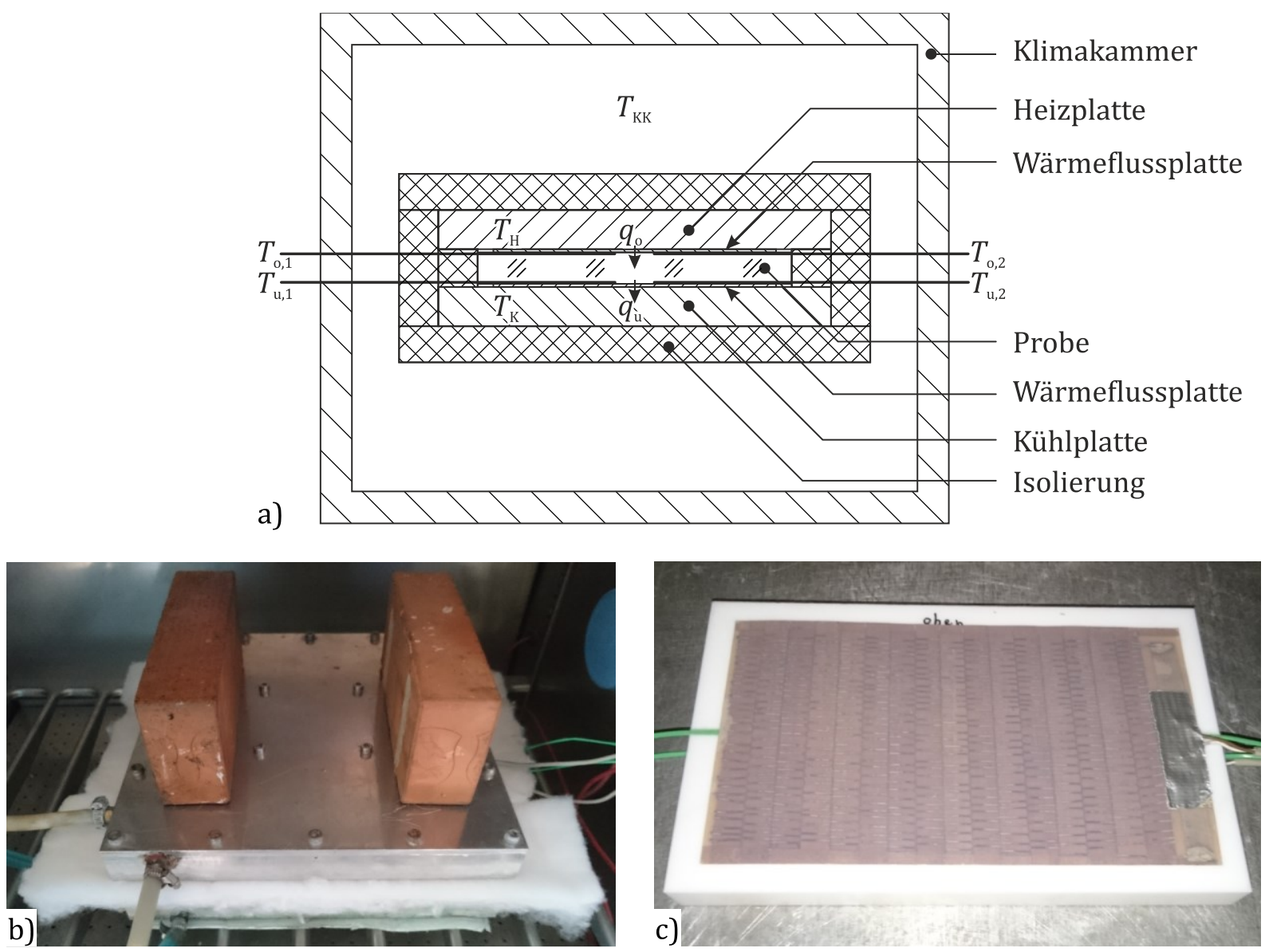

Abb. 5.21 Versuchsaufbau zur Bestimmung der Wärmeleitfähigkeit einer PTFE-Flachprobe mittels Wärmestrommessplattenapparatur.

a) Prinzipskizze und Messpunkte (nach [121]);

b) Versuchsaufbau mit Heiz- und Kühlkörper und Isolation in Klimakammer;

c) Probenkörper (weiß) mit Wärmestrommessplatte.

In mehreren Messreihen wurden unterschiedliche Mitteltemperaturen zwischen $T_{\mathrm{KK} \text {,soll }}=17,5^{\circ} \mathrm{C}$ und $45{ }^{\circ} \mathrm{C}$ eingestellt, wobei die Spreizung zwischen Heiz- und Kühlsolltemperatur stets $5 \mathrm{~K}$ betrug. Die Ergebnisse sind in Abb. 5.22 über der tatsächlichen Temperatur $\bar{T}_{\mathrm{KK}}$ der Klimakammer dargestellt. Die horizontalen Linien markieren die Spreizung zwischen den gemessenen Plattenoberflächentemperaturen $\bar{T}_{\mathrm{o}}$ und $\bar{T}_{\mathrm{u}}$ für jeden Versuch. Die vertikalen „Fehlerbalken“ kennzeichnen die Standardabweichung der Wärmeleitfähigkeiten im Zeitintervall, über dem gemittelt wurde, und enthalten damit die Unsicherheiten aller beteiligten Messgrößen.

Der arithmetische Mittelwert über den gesamten untersuchten Temperaturbereich liegt bei 0,304 W/(m K) ( Tab. 5.7) und damit sehr nah an den Werten, die mit den vorn beschriebenen Methoden bestimmt worden sind. Die Standardabweichung über alle Messpunkte beträgt 0,005 W/(m K) (entsprechend 1,7 \%), was für thermodynamische Stoffwerte als sehr klein eingeschätzt werden kann. Dargestellt ist dies durch den gefärbten „Vertrauensbereich“ in $>$ Abb. 5.22. 
Tab. 5.7 Mittels Wärmestrommessplattenapparatur bestimmte Wärmeleitfähigkeit der PTFE-Probe

\begin{tabular}{cccc}
\hline Größe & Probe & $\boldsymbol{\mu}$ & $\sigma$ \\
\hline$\lambda\left[\frac{\mathrm{W}}{\mathrm{m} \mathrm{K}}\right]$ & 01 & $\mathbf{0 , 3 0 4}$ & 0,005 \\
\hline
\end{tabular}

Es fällt auf, dass die gemessenen Wärmeleitfähigkeiten im Bereich von etwa $25^{\circ} \mathrm{C}$ um ca. $4 \%$ einbrechen. Ein ähnliches Verhalten wurde auch bereits bei $[122,123]$ festgestellt, allerdings bei einer etwas höheren Temperatur von ca. $31^{\circ} \mathrm{C}$, dafür jedoch deutlich ausgeprägter mit einer Abnahme der Wärmeleitfähigkeit um 17 \% von 0,315 W/(m K) auf 0,262 W/(m K). Ältere Arbeiten [124-127] berichten ebenso über diesen Effekt. Grund dafür ist, dass reines („virginales“) PTFE aufgrund seiner mizellären Struktur gleich zwei Phasenübergänge bei umgebungsnahen Temperaturen durchlebt: bei Temperaturerhöhung wandelt sich zwischen 19 und $35^{\circ} \mathrm{C}$ die vollkristalline Gitterstruktur von PTFE zunächst in ein teilkristallines Hexagonalgitter und anschließend in ein ungeordnetes amorphes Gitter um, verbunden mit einer sprunghaften Änderung der thermodynamischen Parametern mit einem großen Peak bei ca. $24^{\circ} \mathrm{C}$ und einem kleineren bei etwa $32{ }^{\circ} \mathrm{C}$ [128]. Da ein Großteil der Messungen im Versuchsstand oberhalb von $25^{\circ} \mathrm{C}$ stattfindet, kann eine signifikante Diskontinuität in den Stoffparametern und eine damit verbundene Beeinflussung der Ergebnisse weitgehend ausgeschlossen werden.

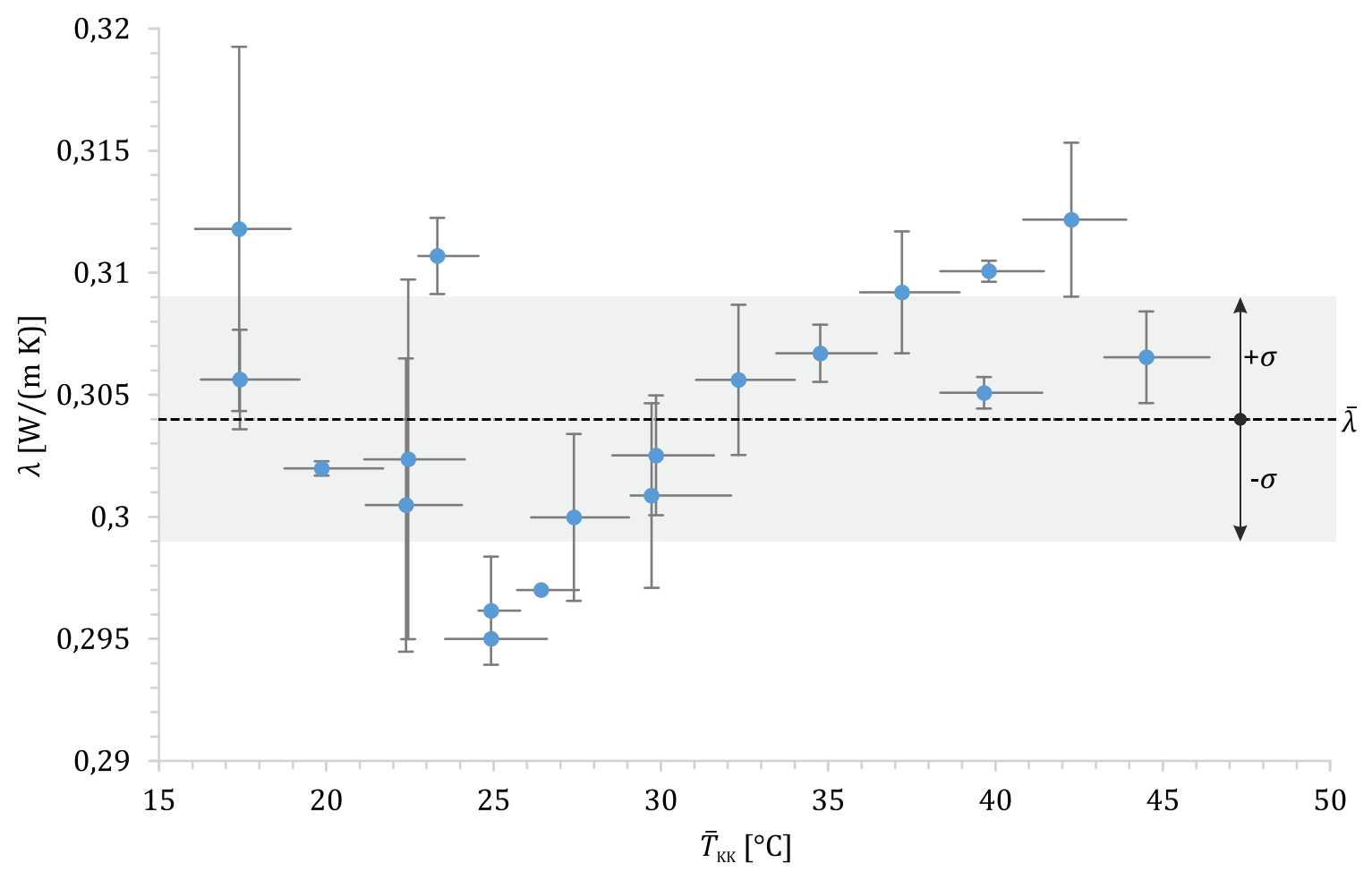

Abb. 5.22 Abhängigkeit der mittels Plattenapparatur gemessenen Wärmeleitfähigkeit der PTFEFlachprobe von der tatsächlichen Temperatur der Klimakammer $\bar{T}_{\mathrm{KK}}$. 


\subsubsection{Einfluss der Pressung auf die Wärmeleitfähigkeit}

Die Wärmeleitfähigkeit langkettiger Polymere wie PTFE weist auch eine Abhängigkeit hinsichtlich ihrer Pressung auf. Ursache hierfür ist die Stauchung der Moleküle in Kraftrichtung, die zu einer Verringerung der freien Weglänge der Phononen führt und damit mit einer Erhöhung der Wärmeleitfähigkeit einhergeht [128]. Die relative Änderung der Wärmeleitfähigkeit mit dem Druck bei konstanter Temperatur kann in linearisierter Form durch den Bridgman-Parameter $G$ beschrieben werden:

$$
G:=\left.\frac{1}{\lambda} \frac{d \lambda}{d p}\right|_{T} \approx \frac{1}{\lambda\left(p_{0}\right)} \frac{\lambda(p)-\lambda\left(p_{0}\right)}{p-p_{0}}
$$

welcher dem prozentualen Anstieg von $\lambda(p)$ gegenüber einem Referenzwert $\lambda_{0}=\lambda\left(p_{0}\right)$ beim Bezugsdruck $p_{0}$ entspricht. Die Einheit von $G$ wird üblicherweise mit $\mathrm{GPa}^{-1}$ oder $\mathrm{kbar}^{-1}$ angegeben. Für virginales PTFE sind in $>$ Tab. 5.8 die Literaturdaten verschiedener Autoren zusammengetragen (vgl. a. [129]), die teilweise noch in die richtige Einheit (Gl. 5.22) umgerechnet werden mussten. Im Mittel ergibt sich ein Wert von $G \approx 0,8 \mathrm{GPa}^{-1}=0,08 \% / \mathrm{MPa}$.

Tab. 5.8 Literaturdaten zum Bridgman-Parameter für virginales PTFE

\begin{tabular}{lcc}
\hline Autor, Jahr & $G=\frac{1}{\lambda_{0}} \frac{d \lambda}{d p}\left[\mathrm{GPa}^{-1}\right]$ & Ref. \\
\hline Barker, 1970 & 0,86 & {$[130]$} \\
Andersson, 1972 & 0,76 & {$[131]$} \\
Bohlin, 1974 & 0,73 & {$[132]$} \\
Dzhavadov, 1975 & 0,81 & {$[133]$} \\
Andersson, 1976 & 0,75 & {$[134]$} \\
\hline
\end{tabular}

Der PTFE-Zwischenring hat einen Innendurchmesser von $230 \mathrm{~mm}$ und einen Außendurchmesser von $240 \mathrm{~mm}$ und weist sowohl zum Innenring als auch zum Außenring eine Übermaßpassung mit einer Toleranz von jeweils $+0,5 /+0,4$ (wellenseitig) und $+0,1 / 0$ (nabenseitig) auf. Es ergibt sich somit ein maximales Übermaß von jeweils 0,5 mm am Innen- und am Außendurchmesser des Zwischenrings und damit eine maximale radiale Verschiebung von $u_{\mathrm{i}}=-u_{\mathrm{a}}=0,25 \mathrm{~mm}$.

Unter der Annahme, dass die Aufdehnung des Innenringes und die Stauchung des Außenringes auf Grund des viel größeren Elastizitätsmoduls der Aluminiumlegierung (70 GPa) gegenüber PTFE $(E=750 \mathrm{MPa}=0,75 \mathrm{GPa}$ ) vernachlässigbar sind, und unter Zugrundelegung eines zweiachsigen rotationssymmetrischen Spanungszustandes im PTFE-Zwischenring, d. h. freie Ausdehnung in axiale Richtung $\left(\sigma_{\mathrm{ax}}=0\right)$, ergeben sich folgende radiale Verläufe für die radiale Verschie- 
bung, die Radial-, Umfangs- sowie für die Vergleichsspannung nach der Hypothese der spezifischen elastischen Gestaltänderungsenergie (GEH):

$$
\begin{gathered}
u_{r}(r)=\frac{u_{\mathrm{i}} \frac{r_{\mathrm{i}}}{r_{\mathrm{a}}}-u_{\mathrm{a}}}{\frac{r_{\mathrm{a}}}{r}\left[\left(\frac{r_{\mathrm{i}}}{r_{\mathrm{a}}}\right)^{2}-1\right]}+\frac{u_{\mathrm{i}} \frac{r_{\mathrm{a}}}{r_{\mathrm{i}}}-u_{\mathrm{a}}}{\frac{r}{r_{\mathrm{a}}}\left[\left(\frac{r_{\mathrm{a}}}{r_{\mathrm{i}}}\right)^{2}-1\right]} \\
\sigma_{r}(r)=\frac{E}{1-v^{2}}\left(\frac{\partial u_{r}}{\partial r}+v \frac{u_{r}}{r}\right) \\
\sigma_{\varphi}(r)=\frac{E}{1-v^{2}}\left(\frac{u_{r}}{r}+v \frac{\partial u_{r}}{\partial r}\right) \\
\sigma_{\mathrm{V}, 3}(r)=\sqrt{\sigma_{r}^{2}-\sigma_{r} \sigma_{\varphi}+\sigma_{\varphi}^{2}}
\end{gathered}
$$

Mit einer Querkontraktionszahl von $v=0,4$ für PTFE nimmt $\sigma_{\mathrm{V}, 3}$ am Innendurchmesser einen maximalen Wert von $75 \mathrm{MPa}$ an („äquivalente Pressung“). Auf Grund des Einschrumpfens des PTFE-Ringes kann sich dessen (anisotrope) Wärmeleitfähigkeit also um bis zu

$$
\frac{\Delta \lambda}{\lambda_{0}} \approx 0,08 \frac{\%}{\mathrm{MPa}} \cdot 75 \mathrm{MPa}=6 \%
$$

erhöht haben, was als weitere systematische Unsicherheit von $\lambda_{\text {PTFE }}$ angesehen werden muss.

\subsubsection{Zusammenfassung der Wärmeleitfähigkeitsmessungen}

Tab. 5.9 liefert nochmals einen Überblick über die Wärmeleitfähigkeitswerte von PTFE, welche mittels der in den vorangegangen Teilkapiteln beschriebenen Messverfahren ermittelt wurden. Da dem mit Hilfe der Wärmestrommessplattenapparatur bestimmten Mittelwert mehr Einzelmessungen zu Grunde liegen, soll diesem bei der globalen Mittelwertbildung ein etwas höheres Gewicht eingeräumt werden. Für alle weiteren Betrachtungen wird daher ein mittlerer Wert von $\lambda_{\text {PTFE }}=0,31 \frac{\mathrm{W}}{\mathrm{m} \mathrm{K}}$ verwendet.

Tab. 5.9 Übersicht über Mittelwerte und Messunsicherheiten der mittels der verschiedenen Messverfahren ermittelten Wärmeleitfähigkeit von PTFE

\begin{tabular}{cclccc}
\hline Größe & Probe & \multicolumn{1}{c}{ Messverfahren } & Tabelle & $\boldsymbol{\mu}$ & $\sigma$ \\
\hline$\lambda\left[\frac{\mathrm{W}}{\mathrm{m} \mathrm{K}}\right]$ & $04-1 / 2$ & Hot-Disk-Verfahren & Tab. 5.5 & $\mathbf{0 , 3 3 3}$ & 0,007 \\
$\lambda\left[\frac{\mathrm{W}}{\mathrm{m} \mathrm{K}}\right]$ & 03 & Referenz-Platten-Apparatur & Tab. 5.6 & $\mathbf{0 , 3 1 7}$ & 0,034 \\
$\lambda\left[\frac{\mathrm{W}}{\mathrm{m} \mathrm{K}}\right]$ & 01 & Wärmestrommessplattenapparatur & Tab. 5.7 & $\mathbf{0 , 3 0 4}$ & 0,005 \\
\hline
\end{tabular}




\subsection{KALIBRIERUNG DES MESSAUFBAUS AN DER SAUGROHRSTRECKE}

Zur Kalibrierung der Wärmeübergangssensorik wurde der Messaufbau der späteren Totraumaußenwand vor dem Einbau in den Versuchsstand zunächst in eine Saugrohrstrecke $(L=8,5 \mathrm{~m}$, $D=200 \mathrm{~mm}$ ) der Professur für Magnetofluiddynamik, Mess- und Automatisierungstechnik eingesetzt ( Abb. 5.23) $[135,136]$. Ziel ist es, die mit beiden Methoden gemessenen lokalen WÜK mit bekannten Literaturwerten in einer Rohrströmung für verschiedene Reynolds-Zahlen zu vergleichen und ggf. zu korrigieren.

Mittels eines Gebläses (6) wird Luft über einen Einströmtrichter (1) aus der Umgebung angesaugt. Diesem kann optional noch ein mit Wasser temperierter Wärmeübertrager vorgeschaltet sein. Nach einer hydrodynamischen Anlaufstrecke (2) mit einer Länge von $L_{\mathrm{h}}=1,8 \mathrm{~m}\left(L_{\mathrm{h}} / D=9\right)$ durchströmt die Luft den Messringaufbau (3). Nach einer Auslaufstrecke (4) wird der Volumenstrom stromabwärts durch einen Drehkolbenzähler (5) ermittelt. Für die Durchflussmessung stehen dabei zwei verschiedene Apparaturen zur Verfügung, mit denen durch einfaches Auswechseln ein Reynolds-Zahl-Bereich von $R e \approx 17.000$ bis 500.000 untersucht werden kann. Bei einer konstanten Heizleistung je Ring zwischen 2 und 3 W werden mittels der in Kap. 4.4.3 beschriebenen Methode lokale Wärmeübergangskoeffizienten für Reynolds-Zahlen zwischen $R e=17.000$ und 280.000 bestimmt und über der thermischen Anlauflänge $x$ aufgetragen. Diese wird so definiert, dass sie am stromaufwärtigen Ende des ersten beheizten Messrings beginnt (Abb. 5.23 c).

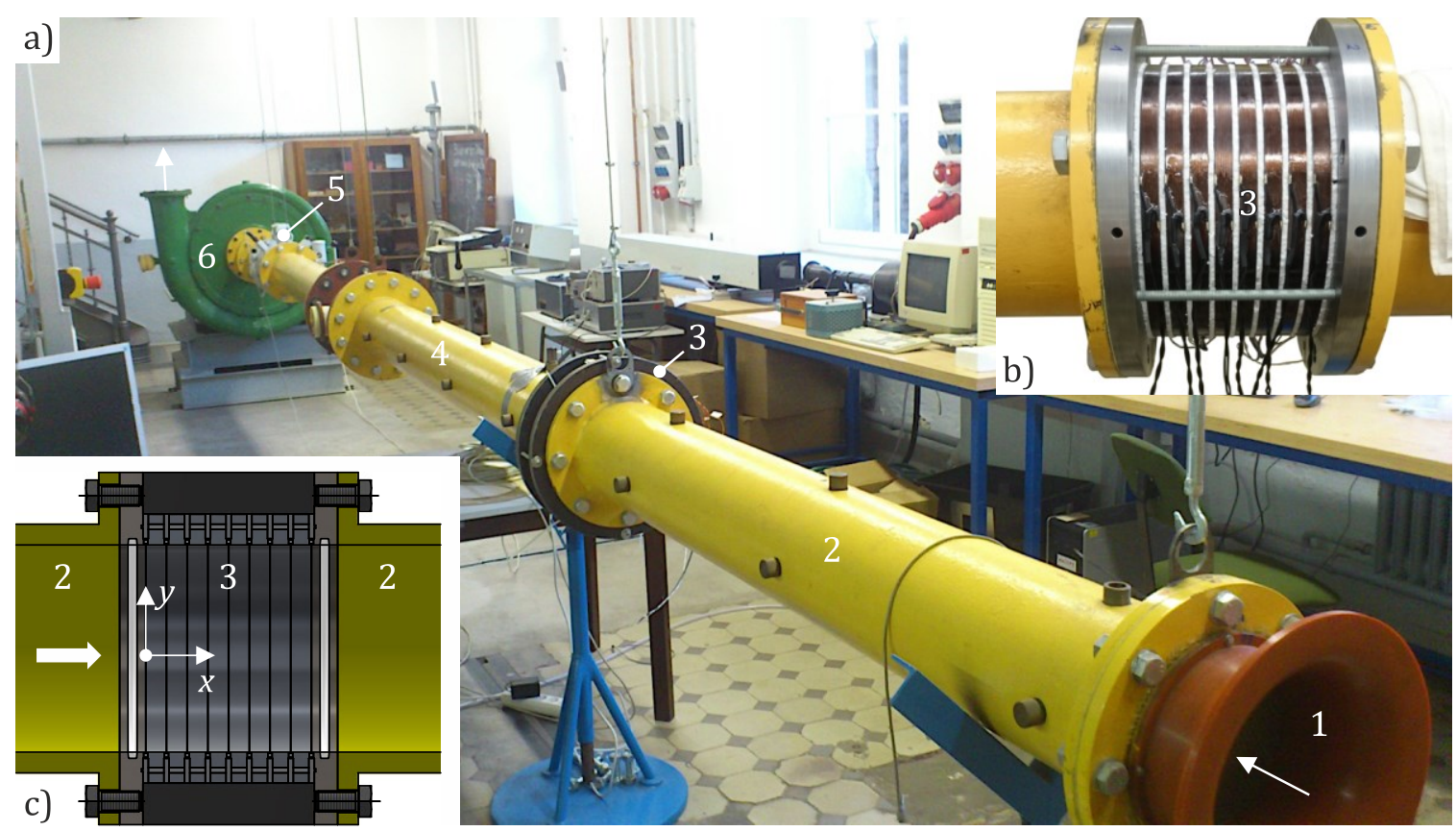

Abb. 5.23 Saugrohrstrecke zur Kalibrierung der Wärmeübergangssensorik in einer Rohrströmung: a) Versuchsaufbau; b) Einbau der Außenwand; c) Beginn der thermischen Anlaufstrecke $x$; 1 - Ansaugtrichter; 2 - Anlaufstrecke; 3 - Messringaufbau (spätere Außenwand des Versuchsstandseitenraumes); 4 - Auslaufstrecke; 5 - Volumenstrommessung; 6 - Sauggebläse. 
Die vergleichsweise einfache Geometrie des Strömungskanals gestattet den Vergleich mit Literaturdaten und analytischen Ansätzen. Einer der am häufigsten angewandten empirischen Ansätze ist die Nusselt-Korrelation für den lokalen Wärmeübergang bei erzwungener Konvektion in einer hydrodynamisch voll ausgebildeten, turbulenten, einphasigen Rohrströmung (z. B. nach VDI Wärmeatlas [90]):

$$
N u(x)=\frac{\frac{\xi}{8} \operatorname{Re} \operatorname{Pr}}{1+12,7 \sqrt{\frac{\xi}{8}}\left(\operatorname{Pr}^{\frac{2}{3}}-1\right)}\left[1+\frac{1}{3}\left(\frac{D}{x}\right)^{\frac{2}{3}}\right]
$$

mit $\xi=(1,8 \log R e-1,5)^{-2}, N u=\alpha D / \lambda, R e=q D / v, 10^{4} \leq R e \leq 10^{6}, 0,1 \leq \operatorname{Pr} \leq 1000$, die gleichermaßen für konstanten Wandwärmestrom ( $\dot{q}_{\mathrm{W}}=$ konst.) und konstante Wandtemperatur ( $T_{\mathrm{W}}=$ konst.) gilt. Formell besitzt die Korrelation allerdings nur Gültigkeit für thermische Lauflängen von $x / D \geq 1$. Diese liegt an der Saugrohrstrecke jedoch darunter $(x / D \leq 0,8)$. Außerdem ist die hydrodynamische Anlauflänge nicht ausreichend lang, um ein voll ausgebildetes turbulentes Strömungsprofil am Beginn der beheizten Oberfläche zu erhalten. Als Richtwert sind dazu

$$
\frac{L_{\mathrm{h}}}{D}=1,359 \operatorname{Re}^{\frac{1}{4}}
$$

notwendig. Das entspricht einer (Mindest-) Anlauflänge von $L_{\mathrm{h}} / D=15,5$ für $R e=17.000$ bzw. $L_{\mathrm{h}} / D=30,7$ für $R e=260.000$. Um den Einfluss der sich noch hydrodynamisch ausbildenden Strömung zu berücksichtigen, haben Haberland und Nitsche [139] einen auf der Reynolds-Analogie basierenden analytischen Korrekturansatz gebildet, der für $\operatorname{Pr}=1$ unter Zuhilfenahme eines vereinfachten Wandansatzes sowie empirischer Korrelationen für die Wandschubspannung und die turbulente Grenzschichtentwicklung die Nusselt-Zahlen, die bei einer sich gleichzeitig thermisch und hydraulisch entwickelnden Strömung auftreten würden, entsprechend korrigiert. Der Algorithmus hierzu ist in Anhang A4 festgehalten. Er enthält gegenüber der Originalquelle eine Berichtigung zu einer Formel, die dem Verlag zwar so gemeldet, bisher jedoch noch nicht korrigiert wurde.

Die benötigte Korrelation für den Wärmeübergang in einer Rohrströmung bei gleichzeitigem thermischem und hydraulischem Anlauf liefert zum Beispiel Deissler [140-142], der unter Anwendung dimensionsloser Gleichungen für Grenzschicht (Wandfunktionen) und Freiströmung und einem vereinfachten $k$ - $\varepsilon$-Turbulenzmodell auf Basis von Masse-, Impuls- und Energiebilanzen Integralgleichungen für die Entwicklung der Strömungs- und Temperaturgrenzschicht angibt, aus denen für jedes Wertepaar von $x / D$ und $R e$ explizit die lokale Nusselt-Zahl $N u$ berechnet werden kann. Für die vorliegende Arbeit wurden diese Gleichungen erstmals in finite Differenzen und Partialsummenfolgen umgewandelt und iterativ gelöst. Darauf aufbauend konnten auch Nusselt-Zahl-Verläufe für hydraulisch voll entwickelte Strömungen und sogar für Strömungen mit hydrodynamischem 
Vorlauf ermittelt werden, und zwar jeweils für konstante Wandtemperatur und konstanten Wandwärmestrom. Die genaue Vorgehensweise ist in Anhang A5 dokumentiert.

Die so ermittelten WÜK-Verläufe sind in Abb. 5.24 exemplarisch für eine Reynolds-Zahl von 270.000 dargestellt, gemeinsam mit den experimentellen Werten aus drei verschiedenen Messreihen (V1 bis V3) sowie den Ergebnissen einer CFD-Simulation, bei der ein Viertelmodell der Saugrohrstrecke mittels $k$ - $\omega$-SST-Modell in Ansys CFX 17.2 simuliert wurde, wobei für die beheizte Anlaufstrecke eine konstante Wandtemperatur angenommen und die berechneten WÜK umfangsgemittelt wurden ( Anhang A16).

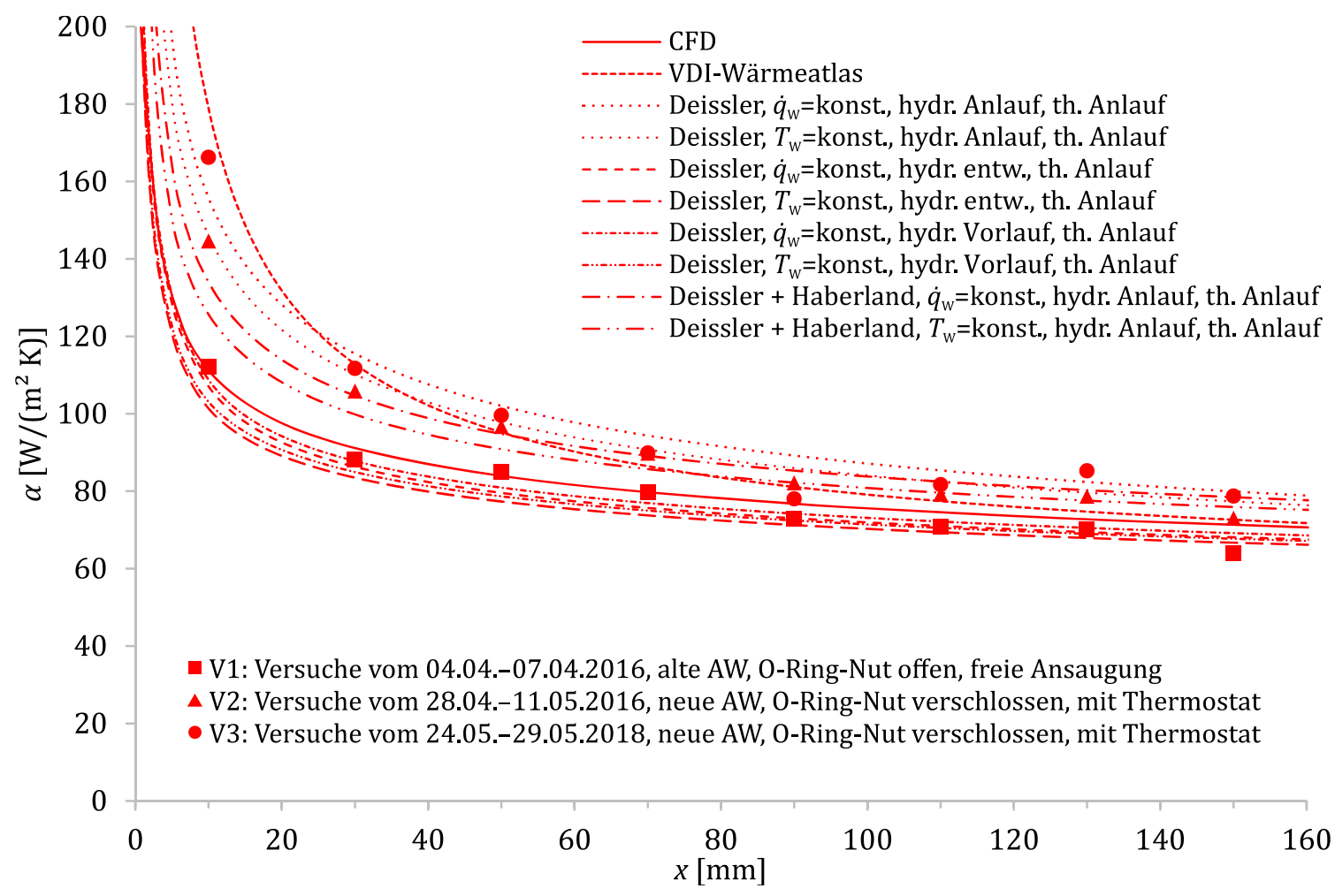

\section{Abb. 5.24 Berechnete WÜK-Verläufe für die Saugrohrströmung bei $R e=2,7 \cdot 10^{5}$ nach verschiede- nen Literaturansätzen $[90,139-142]$ im Vergleich mit Messwerten nach der stationären inversen Methode und mit Ergebnissen aus einer CFD-Analyse.}

Während im Bereich kleiner thermischer Lauflängen noch relativ große Abweichungen zwischen den verschiedenen Kurven sowie Streuungen bei den Messwerten auftreten, laufen für größere Werte von $x$ alle Ergebnisse auf nahezu denselben stationären Endwert zu. Dabei liegen die nach Deissler [140-142] berechneten Kurven für gleichzeitigen thermischen und hydrodynamischen Anlauf auf Grund der kleineren Strömungsgrenzschichten erwartungsgemäß am höchsten, die für vollausgebildete Strömung am niedrigsten, wobei die gemäß $>$ Anhang A5.8 berechneten WÜK-Verläufe für einen hydrodynamischen Vorlauf mit $L_{\mathrm{h}} / D=9$ nur unwesentlich darüber liegen und sich nahezu perfekt mit dem CFD-Resultat decken. Demgegenüber weisen die mittels des Ansatzes nach Haberland und Nitsche [139] korrigierten Verläufe deutlich höhere Werte auf. 
Die Unterschiede zwischen konstanter Wandtemperatur und konstantem Wandwärmestrom als Randbedingung für die beheizte Sektion sind dabei jeweils gering. Im Vergleich zu den übrigen Kurven fällt die Rohrströmungskorrelation nach VDI-Wärmeatlas etwas aus der Reihe [90], die vor allem für kleine $x / D$ ein anderes Anstiegsverhalten aufweist als alle anderen Kurven, formell aber auch wie angesprochen nicht für $x / D<1$ gültig ist und damit hier eigentlich nicht verwendet werden darf. Alle bis hierhin erläuterten Zusammenhänge gelten analog für den gesamten betrachteten Reynolds-Zahl-Bereich.

In Abb. 5.25 sind für alle untersuchten Reynolds-Zahlen die gemessenen Werte aus allen drei Versuchen V1 bis V3 und - jeweils im selben Farbton dazu - der Bereich zwischen der Kurve für hydrodynamischen Anlauf und $\dot{q}_{\mathrm{W}}=$ konst. und der Kurve für hydrodynamisch entwickelte Strömung und $T_{\mathrm{W}}=$ konst. nach Deissler unterlegt. Wie sich zeigt, liegen nahezu alle Messwerte innerhalb der dazugehörigen farbigen Bereiche. Einzig der Versuch V2 bei $R e=137.000$ liegt etwas darüber, die Werte für $R e=66.000$ systematisch etwas darunter, jedoch immer noch in unmittelbarer Nähe zu den Werten, die die Korrelationen voraussagen. Unterschiede zwischen den einzelnen Versuchen resultieren zum einen aus unterschiedlichen Zusammenbautoleranzen der beiden ansonsten identischen Außenwände und zum anderen aus leichten Modifikationen des Versuchsaufbaus an der Saugrohrstrecke, etwa ob die beiden 0-Ring-Nuten in den Spannscheiben für die Rohrströmungsuntersuchungen offen (V1) oder wandbündig verschlossen waren (V2 und V3, vgl. a. Abb. 5.23 c) oder ob an der Ansaugung noch ein zusätzlicher Wärmeübertrager zur Vergleichmäßigung der Ansaugtemperatur über der Zeit angeordnet war (V3). Im letzteren Fall existiert jedoch eine Unsicherheit darüber, ob die Rohrströmung auch gleichmäßig über dem Querschnitt temperiert werden konnte oder ob sie noch eine lokale Temperaturverteilung aufwies, was für die etwas höher liegenden WÜK in V3 gegenüber V1 und V2 sprechen würde. Daher sollen im Folgenden nur die Ergebnisse aus V1 und V2 mit freier Ansaugung weiter betrachtet werden. Da das CFD-Modell gegenüber den (halb-) analytischen und semi-empirischen Ansätzen einen geringeren Abstraktionsgrad aufweist, werden die numerischen Ergebnisse als realitätsnäher und damit verlässlicher bzw. aussagekräftiger angesehen. Aus diesem Grund sind in Abb. 5.26 zu Illustrationszwecken noch einmal abschließend Messwerte aus ausgewählten Versuchen gemeinsam mit den WÜK-Kurven aus den numerischen Strömungssimulationen zusammengestellt. Auf Grund der sehr guten Übereinstimmung vor allem im Bereich höherer thermischer Lauflängen, in denen die unterschiedlichen Berechnungsansätze immer weniger voneinander abweichen (siehe Abb. 5.24), können die mittels stationärer inverser Methode ermittelten WÜK unkorrigiert weiterverwendet werden - ganz im Gegensatz zur lokalen Übertemperaturmethode, wo die Messwerte noch durch eine mehr oder weniger komplexe Kalibrierfunktion umgewertet werden müssen $[73,135,136]$. 


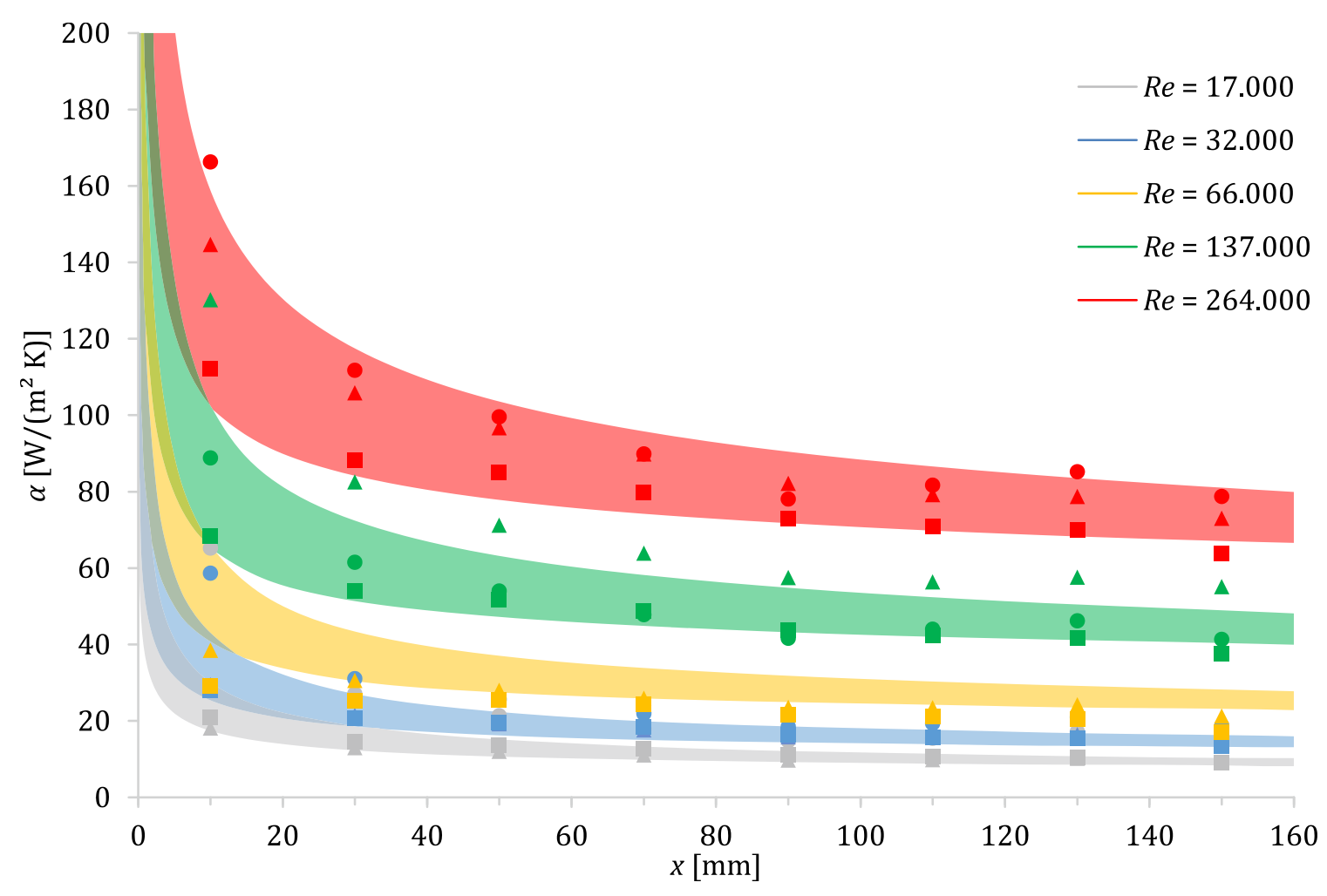

Abb. 5.25 In den verschiedenen Versuchsreihen mittels stationärer inverser Methode bestimmte lokale WÜK-Verläufe für die Saugrohrströmung bei unterschiedlichen Reynolds-Zahlen und jeweilige „Korrelationsbereiche“ nach Deissler [140-142].

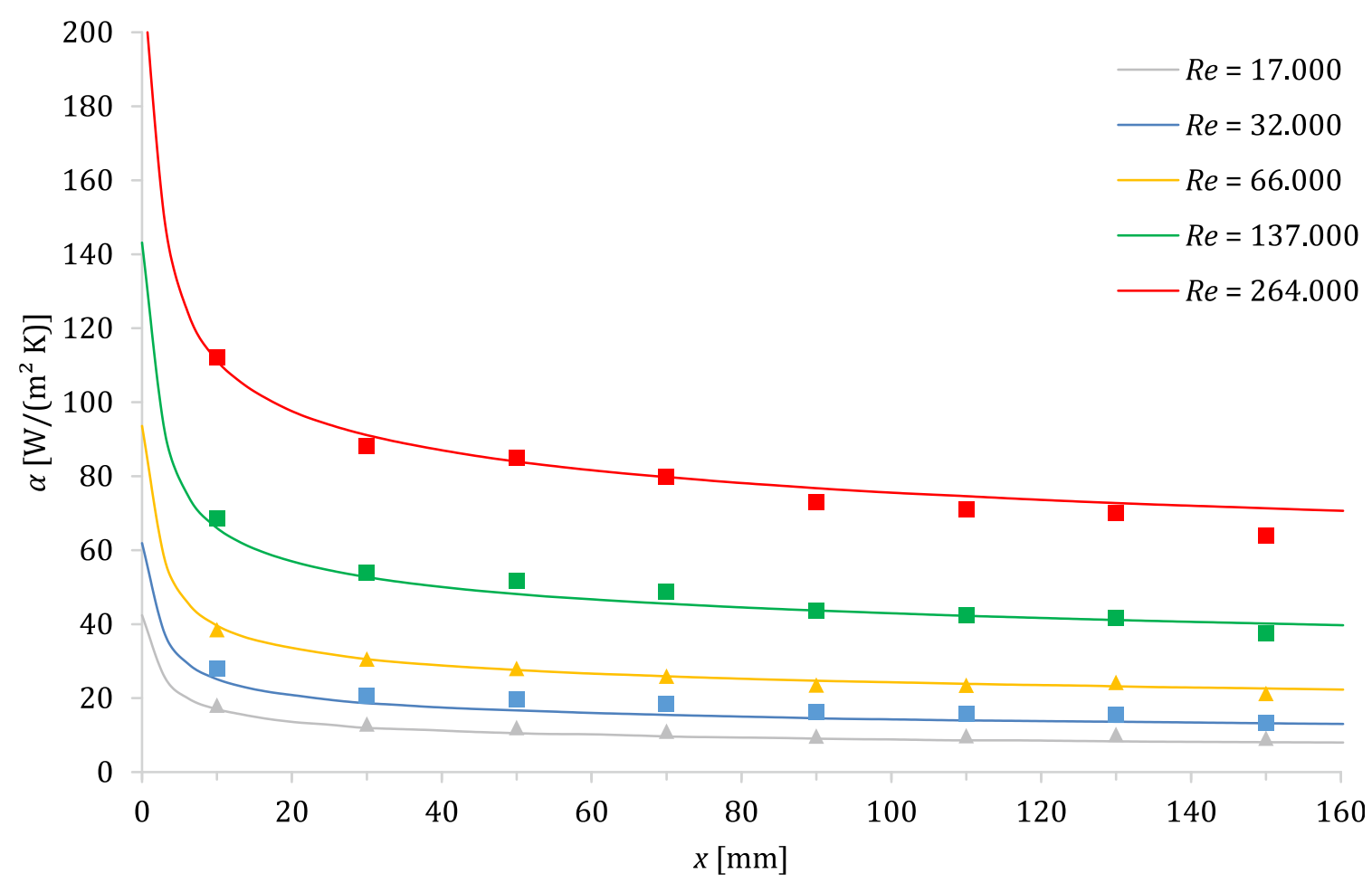

Abb. 5.26 Überblick über die mittels stationärer inverser Methode bestimmten lokalen WÜK-Verläufe für die Saugrohrströmung bei unterschiedlichen Reynolds-Zahlen im Vergleich mit Werten aus der CFD-Analyse. 


\subsection{MESSERGEBNISSE ZUM LOKALEN WÄRMEÜBERGANG IN GENERI- SCHEN SEITENRAUMGEOMETRIEN}

Die experimentellen Ergebnisse für den Wärmeübergang im Versuchsstandseitenraum werden im Folgenden als lokale WÜK $\alpha$ über der axialen Länge $x$ der Außenwandkontur dargestellt. Deren Nullpunkt befindet sich gemäß der vorher für den Seitenraum getroffenen Konvention (siehe

Abb. 4.4) an der Position der feststehenden (in Hauptströmungsrichtung gesehen) stromaufwärtigen Einströmseitenwand, wobei die Werte in gewohnter Weise in stromabwärtige Richtung aufgetragen sind. Zur Veranschaulichung sind in allen Diagrammen zusätzlich die Seitenraumkonturen in grau hinterlegt.

Ausgefüllte Punkte kennzeichnen die Intervallmittelwerte, die mittels der stationären inversen Methode auf Basis des gradientenbasierten Optimierungsverfahren ( Kap. 4.4.4) ermittelt wurden. Werte, die von der sensorbasierten lokalen Übertemperaturmethode stammen, sind durch Kreise ohne Füllung dargestellt. Sofern nicht anders angegeben, stehen die unterschiedlichen Farben der Symbole für die verschiedenen untersuchten Reynolds-Zahlen in der Hauptströmung, siehe Tab. 5.10.

Tab. 5.10 Symbolerklärung für Diagramme mit WÜK-Ergebnissen

\begin{tabular}{ll}
\hline Symbol & Definition \\
\hline$\bullet$ & Stationäre inverse Methode \\
$\circ$ & Lokale Übertemperaturmethode \\
$-\circ$ & $R e=110,000 \pm 3,300$ \\
$-\circ$ & $R e=90,000 \pm 1,700$ \\
$-\circ$ & $R e=61,900 \pm 1,200$ \\
$-\circ$ & $R e=41,800 \pm 900$ \\
\hline
\end{tabular}

\subsubsection{Vergleich der Messmethoden}

In einem ersten Schritt sollen die Ergebnisse der beiden Messmethoden miteinander verglichen werden. Dazu sind in Abb. 5.27 die lokalen WÜK für die maximal im Versuchsstand realisierbare Seitenraumgeometrie sowie für eine Konfiguration mit verringerter Einströmbreite jeweils bei der größten und bei der kleinsten untersuchten Reynolds-Zahl dargestellt. Wie sich zeigt, stimmen die Werte sehr gut miteinander überein, und das, obwohl sich die Strömungsverhältnisse im Seitenraum grundsätzlich von denen in einer geraden Rohrströmung unterscheiden. Dies bestätigt die Zulässigkeit der angewandten Kalibrierstrategie, bei der insbesondere die Werte der lokalen Übertemperaturmethode noch durch eine Kalibrierfunktion auf die entsprechende NusseltKorrelation für turbulente Rohrströmung korrigiert werden mussten (siehe Kap. 5.6). 

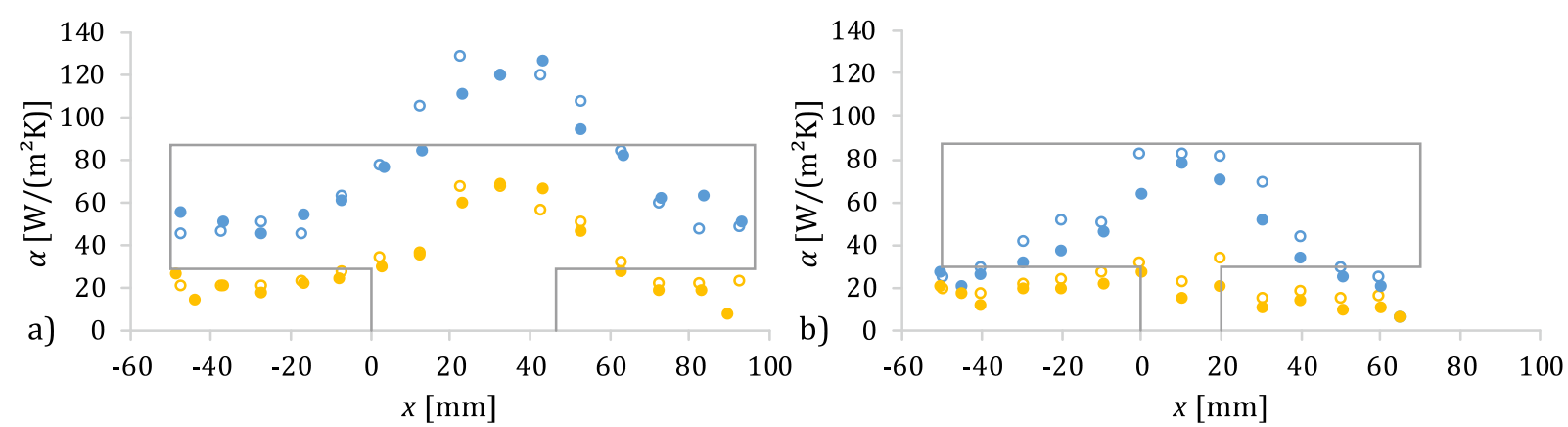

Abb. 5.27 Vergleich der mittels beider Messmethoden ermittelten lokalen WÜK-Verteilungen für je zwei verschiedene geometrische Konfigurationen und Reynolds-Zahlen:

a) $s=46,3 \mathrm{~mm}, b=146,3 \mathrm{~mm}, e=0$; b) $s=20 \mathrm{~mm}, b=120 \mathrm{~mm}, e=0$.

\subsubsection{Reproduzierbarkeit und Streuung der Messwerte}

Im nächsten Schritt soll abgeklärt werden, ob und inwieweit Variationen bei der Versuchsdurchführung die Reproduzierbarkeit und die Streuung der lokalen WÜK-Messwerte, welche mit der gradientenbasierten stationären inversen Methode gewonnen wurden, beeinflussen.

So wurde zum einen die pro Ring applizierte Heizleistung $P_{\text {el }}$ auf $3 \mathrm{~W}$ reduziert bzw. auf $5 \mathrm{~W}$ erhöht, um einen möglichen Einfluss der Übertemperatur der Wand gegenüber der Fluidtemperatur $T_{\mathrm{W}, \mathrm{i}}-T_{\mathrm{F}}$ abzuklären. Des Weiteren wurde für eine konstante Heizleistung von $4 \mathrm{~W}$ die Außenwand um die Versuchsstandlängsachse um den Winkel $\Delta \varphi$ verdreht. $\Delta \varphi$ wird, wie in Abb. 4.16 dargestellt, in mathematisch positiver Richtung (gegen den Uhrzeigersinn) mit Blick in Strömungsrichtung gezählt, ausgehend von der 12-Uhr-Position, wo sich bei $\Delta \varphi=0$ die Thermistorreihen $\mathrm{A}$ und $\mathrm{E}$ befinden.

In Abb. 5.28 sind beispielhaft für den symmetrischen Seitenraum mit der maximal untersuchten Geometrie $(s=46,3 \mathrm{~mm}, b=146,3 \mathrm{~mm}, e=0)$ und Reynolds-Zahl $\operatorname{Re}=(1,03 \ldots 1,11) \cdot 10^{5} \mathrm{die}$ lokalen WÜK für alle durchgeführten Variationsmessungen eingetragen. Die axiale Positionierung der Außenwand für die einzelnen Versuche ist wie in - Kap. 4.5.2 erläutert mit NP („Normalposition“) und ZP („Zwischenposition“) gekennzeichnet. Alle Messpunkte wurden zusätzlich durch eine Glockenkurve (vgl. Kap. 5.9) approximiert („angefittet“). Wie sich zeigt, liegen fast alle Messpunkte in einem Streuband von $\pm 15 \%$ um die jeweilige Glockenkurve. Dieser Wert kann als Wiederhol- bzw. Reproduktionsgenauigkeit interpretiert werden und deckt sich in etwa mit dem Wert für die lokale Übertemperaturmethode. Dort sind die Abweichungen mit $\pm 20 \%$ für Wiederholungsmessungen noch geringfügig größer [73]. Die „Ausreißer“ in den unmittelbaren Randbereichen der Kavität lassen auf größere Unsicherheiten der WÜK für teilbenetzte Messring schließen. Inwieweit dies der Fall ist, soll in $>$ Kap. 5.8.3 geklärt werden. 
Offensichtlich sind für die stationäre inverse Methode die ermittelten WÜK-Verläufe unabhängig von der applizierten Heizleistung $(P=3 \ldots 5 \mathrm{~W})$ sowie von der Verdrehung der Außenwand $\Delta \varphi$ und damit von der Positionierung der Messstellen am Umfang. Die WÜK-Werte an den Zwischenpositionen fügen sich gut zwischen den Werten für die Normalpositionen ein, und das obwohl die ZP-Werte häufig an anderen Messtagen aufgenommen wurden als die NP-Werte. Grund hierfür ist, dass die Reynolds-Zahl schneller und einfacher durch Auf- und Zufahren des Dreiwegeventils variiert werden kann als die Position der Außenwand. So gelingt es bei günstigem Versuchsverlauf, für eine geometrische Anordnung alle vier Reynolds-Zahlen an nur einem Versuchstag zu untersuchen und erst nach Abschalten der Verdichter die Außenwand (und/ oder die Seitenwände) zu verschieben.

Auf Grund von defekten und verlorenen WÜK-Sensoren wurde die Totraumaußenwand vor der Untersuchung des Dralleinflusses ( Kap. 5.7.7) aus dem Versuchsstand ausgebaut, demontiert und mit einem kompletten Satz neuer Sensortabletten instrumentiert. Die Materialtemperaturmessstellen blieben jedoch unberührt. Interessanterweise zeigen die mit der neuen Außenwand (AW) bestimmten WÜK systematisch etwas kleinere Werte, als mit dem Erstaufbau ermittelt wurde, siehe Abb. 5.28. Eine Vermutung, wonach die durch ein eventuelles stärkeres oder schwächeres Verspannen der Messringe zwischen den Spannscheiben veränderten Spalt- und Dichtmaße die berechneten WÜK beeinflussen, konnte durch eine Variantenrechnung mit Hilfe des thermischen Netzwerkmodelles - zumindest für eine gleichmäßige Spaltänderung über alle Ringe - entkräftet werden. Da die Werte jedoch gerade noch innerhalb des Streubandes liegen, werden diese systematischen Abweichungen als akzeptabel eingeschätzt.

Als größten Einfluss auf die Reproduzierbarkeit der Ergebnisse haben sich schnelle Änderungen der Verdichteransaug- und damit auch der Bezugstemperatur erwiesen, die sich auf Grund des Tagesganges der Temperatur ergeben. Solche kurzskaligen Temperaturänderungen im Minutenbereich erfordern streng genommen einen weiteren Ausgleichsprozess, bis auch die Wandtemperaturen im Versuchsstand erneut ein thermisches Gleichgewicht erreicht haben. Durch den Einbau des wassergekühlten Druckluftnachkühlers (vgl. Kap.4.1.4) wurde bereits eine deutlich verbesserte Temperaturstabilität erreicht. Eine neue Herausforderung besteht nunmehr darin, schwankende Kühlwasservorlauftemperaturen (vor allem im Sommer) durch Variation des Kühlwasserstromes auszugleichen. Die Regelung hierfür muss allerdings noch besser angepasst werden. 


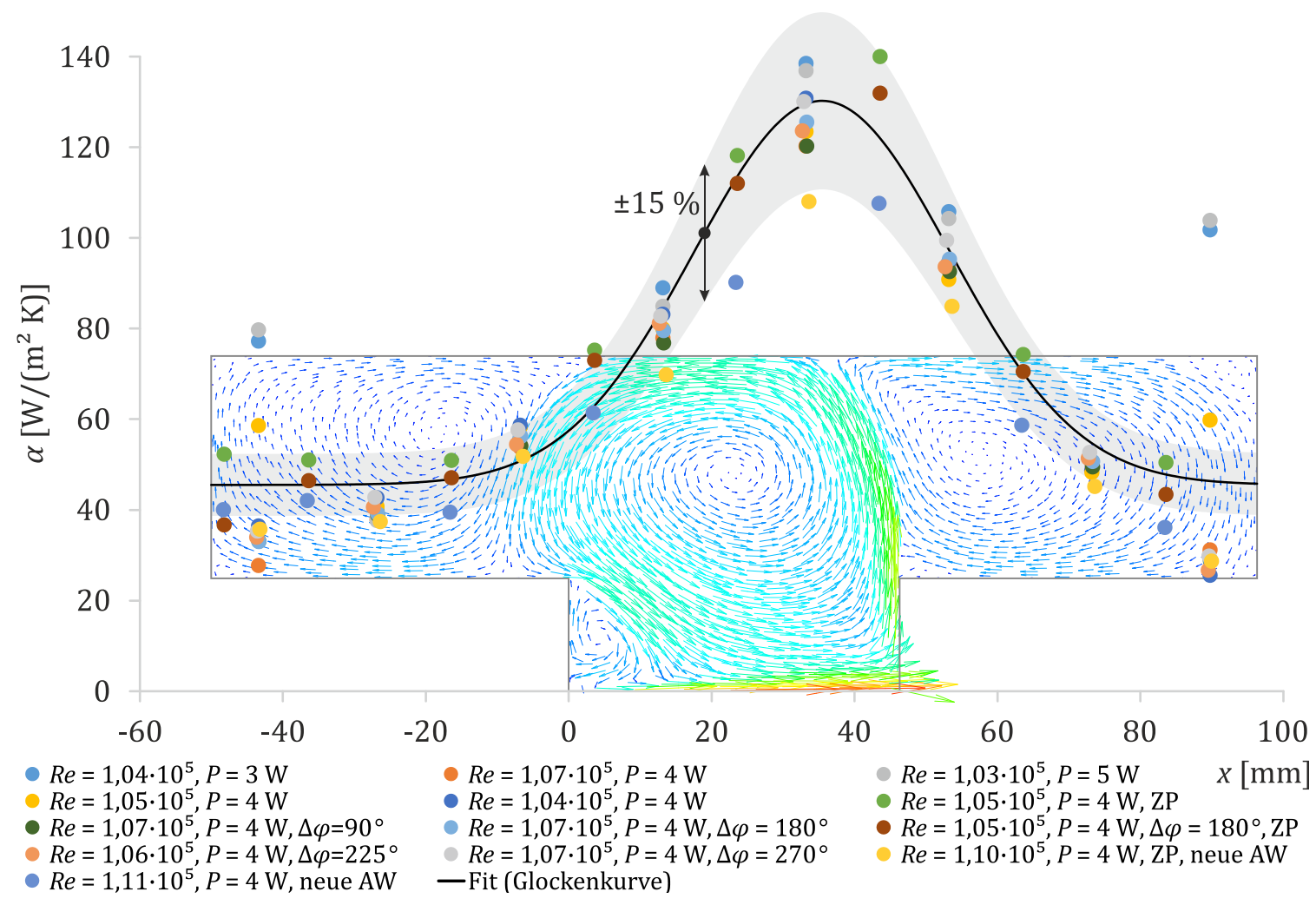

Abb. 5.28 Einfluss von Variationen bei der Versuchsdurchführung auf die Reproduzierbarkeit und Streuung der lokalen, umfangsgemittelten WÜK-Messwerte nach der stationären inversen Methode bei maximaler, symmetrischer Totraumgeometrie. $s=46,3 \mathrm{~mm}, b=146,3 \mathrm{~mm}, e=0, \operatorname{Re}=(1,03 \ldots 1,11) \cdot 10^{5}$.

\subsubsection{Einfluss der Reynolds-Zahl Re in der Hauptströmung}

Wie bereits in Kap. 4.5.2 erwähnt, wurde für alle in den folgenden vier Teilkapiteln diskutierten Geometrie- und Drallkonfigurationen immer auch die Reynolds-Zahl variiert, da von ihr ein wesentlicher (Quer-) Einfluss auf den Wärmeübergang im Totraum erwartet wird.

Aus sämtlichen anschließenden Bildern des Kap. 5.7 wird deutlich, dass höhere Reynolds-Zahlen in der Hauptströmung erwartungsgemäß zu größeren WÜK in der Kavität führen. Dabei skalieren die Verteilungen mit steigender Reynolds-Zahl ausschließlich entlang der Werteachse, während die Form der Verteilung und die Lage des Maximums weitgehend unverändert bleiben. Offensichtlich stellt die Reynolds-Zahl in der Hauptströmung (in der Formulierung von Gl. 4.3) ein gutes Maß für die Wirbelintensität im Seitenraum dar, die ihrerseits maßgeblich den erzwungenen Wärmeübergang entlang der Totraumaußenwand prägt. 


\subsubsection{Einfluss der Einströmbreite $s$}

Der Einfluss der Einströmbreite auf die lokale WÜK-Verteilung in einem symmetrischen Totraum ( $e=0$; Abb. 4.2) ist in Abb. 5.29 dargestellt. Die Einströmbreite wurde zwischen 10 und $46,3 \mathrm{~mm}$ variiert. In jedem Fall wurde die maximale Seitenraumbreite von $b=s+100 \mathrm{~mm}$ realisiert. Die gemessenen WÜK nehmen mit steigender Reynolds-Zahl in der Hauptströmung zu und weisen eine ausgeprägte lokale Verteilung auf. Die maximalen Werte ca. $5 \mathrm{~mm}$ stromauf der abströmseitigen Einströmseitenwand treten im Bereich des Auftreffens des Prallstrahls auf die Außenwand auf und nehmen mit kleiner werdender Einströmbreite ab. Für $s=20 \mathrm{~mm}$ und die kleinste untersuchte Reynolds-Zahl (Abb. 5.29 d) sowie für die kleinste Einströmbreite $s=$ 10 mm generell ( Abb. 5.29 e) brechen die WÜK gänzlich ein und weisen keine wahrnehmbare lokale Überhöhung mehr auf. In diesen Fällen ist die Einströmöffnung zu klein, als dass die Hauptströmung eine (signifikante) Wirbelbildung im Seitenraum induzieren könnte. Es wird vermutet, dass ein kleiner, schnell rotierender Wirbel in der Einströmöffnung die Strömung in den Seitenraum behindert. Dies deckt sich auch mit Beobachtungen an einem Flachwasserkanal, welche im Rahmen vorbetrachtender Untersuchungen durchgeführt wurden [76], und stellt eine wichtige Erkenntnis in Hinblick auf die Gestaltung von Seitenräumen in Turbinen dar.

\subsubsection{Einfluss der Breite der Kavität $b$}

Für einen symmetrischen Totraum mit der maximalen Einströmbreite von $s=46,3 \mathrm{~mm}$ wurde die Totraumbreite $b$ von 146,3 mm (maximal realisierbare Geometrie) bis 46,3 mm (quadratische Kavität) in Schritten von je $25 \mathrm{~mm}$ verringert. Wie in $>$ Abb. 5.30 zu sehen ist, zeigt die WÜKVerteilung über alle Reynolds-Zahlen hinweg nahezu keine Veränderungen mit abnehmender Einströmbreite. Vielmehr wird die Verteilung durch die beiden sich annähernden Seitenwände „abgeschnitten“. Die Streuung der berechneten WÜK (Unsicherheit) in den Randbereichen nimmt mit abnehmender Breite $b$ zu. Das wird vor allem am Beispiel der quadratischen Kavität deutlich ( Abb. 5.30 e). In diesem Fall befinden sich in Abhängigkeit von der axialen Position der Außenwand nur noch maximal zwei Messringe im Bereich der Strömung in der Kavität. Dem Finite-Elemente-Modell bleiben daher nur wenige Freiheitsgrade, um während der Optimierung die simulierten Temperaturen an die gemessenen Werte anzugleichen. Kleinere Abweichungen in den Messwerten haben dadurch einen noch größeren Einfluss auf die lokal berechneten WÜK. 


\subsubsection{Einfluss der Exzentrizität der Kavität e}

Die fünf Diagramme in Abb. 5.31 präsentieren die ermittelten WÜK-Verteilungen für eine Kavität mit einer Einströmbreite von $s=46,3 \mathrm{~mm}$, einer Totraumbreite von $b=96,3 \mathrm{~mm}$ und einer variablen Exzentrizität $e$ (Abb. 4.2) von $+25 \mathrm{~mm}$ (Kavität stromabwärts verlagert) bis $-25 \mathrm{~mm}$ (Kavität stromaufwärts verlagert). Das WÜK-Profil variiert deutlich mit der Exzentrizität und damit auch mit der Form des Totraumes. Die Kurven für $-12,5 \mathrm{~mm} \leq e \leq 12,5 \mathrm{~mm}(\mathrm{Abb} 5.31 \mathrm{~b}-$ d) weisen wie bisher für alle untersuchten Reynolds-Zahlen eine Glockenform auf, die jedoch in dieselbe Richtung schief ist, in die auch die Kavität zeigt. Für $e=25 \mathrm{~mm}$ ( Abb. 5.31 a) tritt ein zweites Maximum an der stromaufwärtigen Seitenwand auf, welches auf einen starken Sekundärwirbel in der linken oberen Ecke der Kavität hinweist. Im Gegensatz dazu steigen die WÜK-Kurven für $e=-25 \mathrm{~mm}$ (Abb. $5.31 \mathrm{e}$ ) streng monoton in stromabwärtige Richtung an und haben ihr globales Maximum an der stromabwärts befindlichen Seitenwand. Eine solche Form des Seitenraumes wird häufig für die Anzapfung von Dampfturbinen gewählt, um Sekundärwirbel zu vermeiden, die die Strömung des Dampfes durch die Kammer verringern. Die Kurvenmaxima bleiben über alle Werte von $e$ weitestgehend gleich, außer für den Fall mit $e=-25 \mathrm{~mm}$, wo der Primärwirbel einen stärkeren Wärmeübergang in der rechten oberen Ecke der Kavität bewirkt.

\subsubsection{Einfluss des Drallwinkels $\alpha$ in der Anströmung zum Seitenraum}

Zur Untersuchung des Einflusses von Drall in der Hauptströmung auf den Wärmeübergang wurde für einen symmetrischen Seitenraum mit maximalen Abmessungen ( $s=46,3 \mathrm{~mm}, b=146,3 \mathrm{~mm}$, $e=0$ ) bei jeweils vier verschiedenen Reynolds-Zahlen der Blechwinkel mittels des Drallgitters

( Abb. 5.9) zwischen $0^{\circ}$ (drallfreie Anströmung) und $40^{\circ}$ (starke Fehlanströmung) in $10^{\circ}$-Schritten variiert. Die Ergebnisse sind in Abb. 5.32 abgebildet und stellen sich wie folgt dar: Bei einer Steigerung des Blechwinkels von $\alpha=0^{\circ}$ auf $20^{\circ}$ steigen die WÜK im Totraum insgesamt an, wobei die Werte bei höheren Reynolds-Zahlen stärker vom Drall beeinflusst werden. Bei Blechwinkeln über $20^{\circ}$ hingegen bleiben die Maximalwerte im Staupunkt weitgehend konstant, es steigen nur noch die Werte am Rand der Verteilung, die Kurvenspreizung wird kleiner, und das für jede Reynolds-Zahl in etwa im selben Maß. Eine mögliche Erklärung lautet wie folgt: Durch die erhöhte Fliehkraft infolge des wachsenden Dralles wird sofort mehr Fluid in die Kavität gefördert, wodurch zunächst der Hauptwirbel stärker zirkuliert. Dessen Intensität steigt jedoch nur bis zu einem gewissen Drallwinkel an, darüber hinaus werden vor allem die Sekundärwirbel in den Totraumecken stärker angetrieben, wobei die Schleppverluste eine weitere Intensitätssteigerung des Hauptwirbels verhindern. 

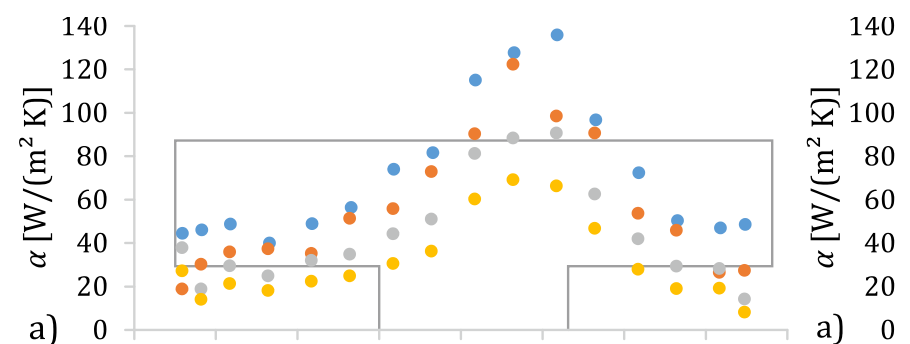

a) 0
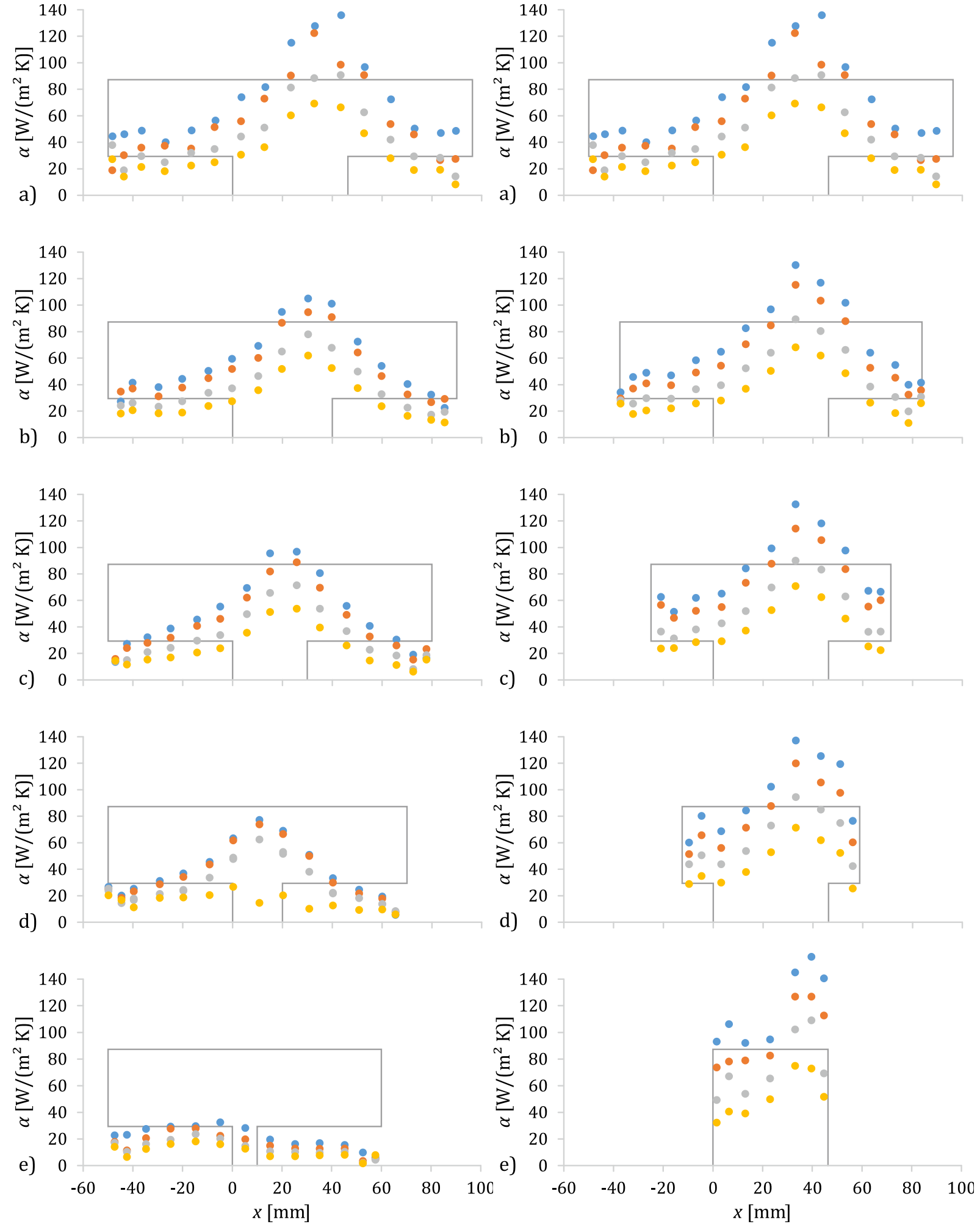

Abb. 5.29 Einfluss der Einströmbreite auf die WÜK-Verteilung im Seitenraum:
a) $s=46,3 \mathrm{~mm}$, b) $s=40 \mathrm{~mm}$,
c) $s=30 \mathrm{~mm}$, d) $s=20 \mathrm{~mm}$,
e) $s=10 \mathrm{~mm}$;
$b=s+100 \mathrm{~mm}, e=0$

Abb. 5.30 Einfluss der Breite der Kavität auf die WÜK-Verteilung im Seitenraum:

a) $b=146,3 \mathrm{~mm}$, b) $b=121,3 \mathrm{~mm}$,

c) $b=96,3 \mathrm{~mm}, \mathrm{~d}$ ) $b=71,3 \mathrm{~mm}$,

e) $b=46,3 \mathrm{~mm}$;

$s=46,3 \mathrm{~mm}, e=0$. 

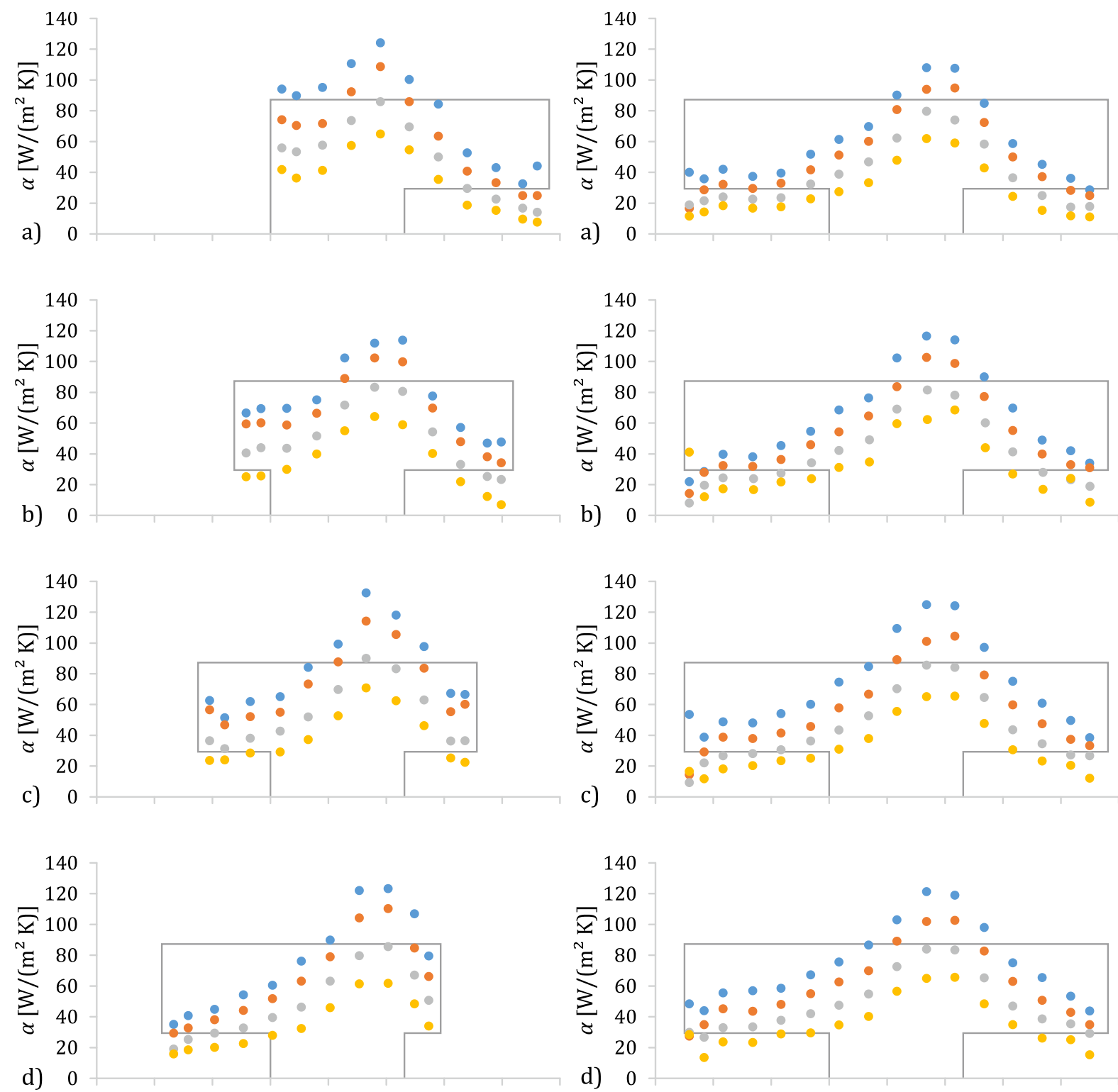

c) 0
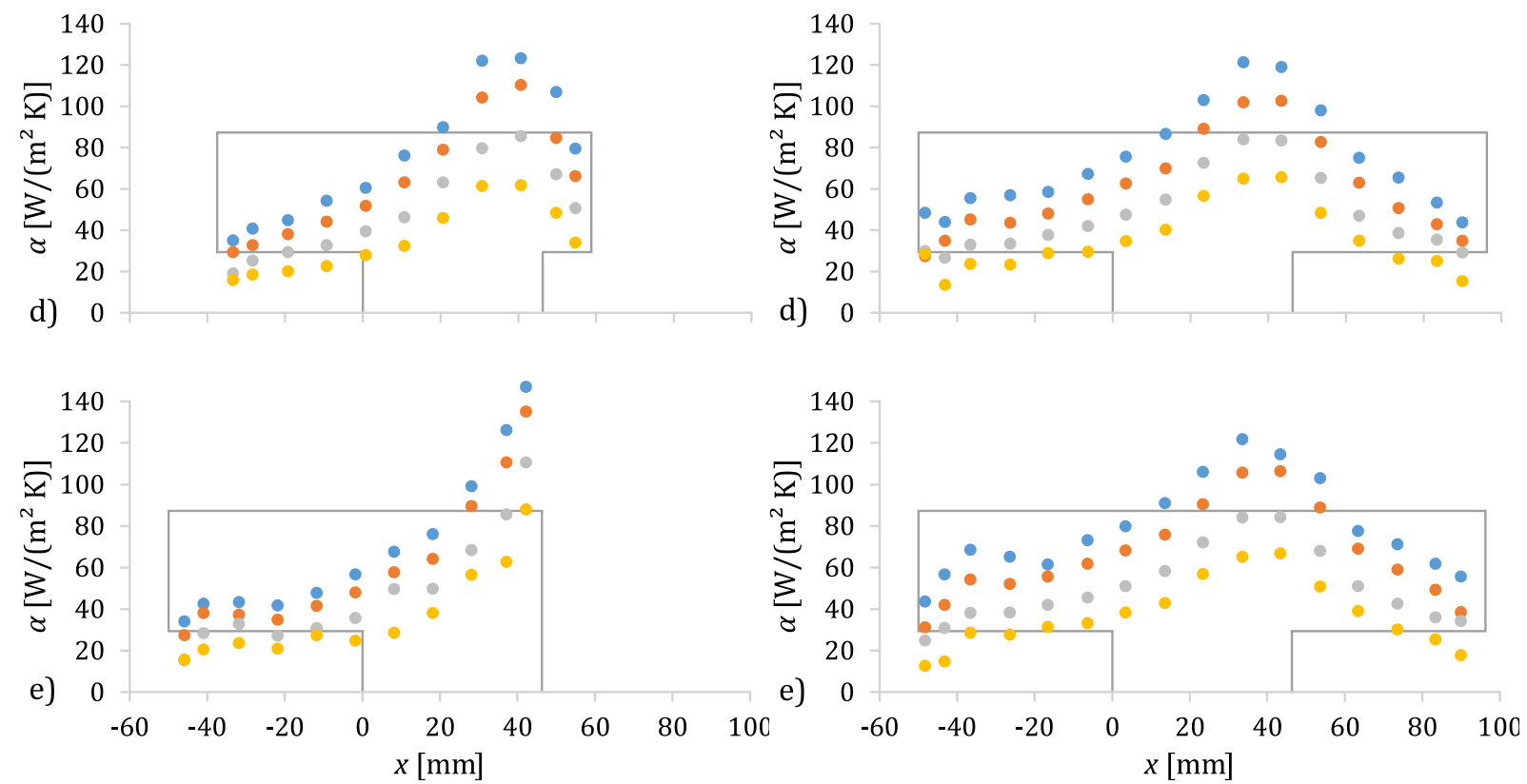

\section{Abb. 5.31 Einfluss der Exzentrizität der Kavität Abb. 5.32 Einfluss des Blechwinkels auf die} auf die WÜK-Verteilung im Seitenraum:

a) $e=25 \mathrm{~mm}$, b) $e=12,5 \mathrm{~mm}$, c) $e=0$, WÜK-Verteilung im Seitenraum:

d) $e=-12,5 \mathrm{~mm}, \mathrm{e}) e=-25 \mathrm{~mm}$; $s=46,3 \mathrm{~mm}, b=96,3 \mathrm{~mm}$.
a) $\gamma=0^{\circ}$, b) $\gamma=10^{\circ}$, c) $\gamma=20^{\circ}$,
d) $\gamma=30^{\circ}$, e) $\gamma=40^{\circ}$ $s=46,3 \mathrm{~mm}, b=146,3 \mathrm{~mm}, e=0 \mathrm{~mm}$ 


\subsection{ANALYSE UND ABSCHÄTZUNG VON MESSUNSICHERHEITEN}

\subsubsection{Unsicherheit der gemessenen Absolut- und Differenzdrücke}

Die Unsicherheit der gemessenen Absolut- und Differenzdrücke setzt sich zusammen aus der Unsicherheit des verwendeten Kalibrators, mit dem die Piezo-Sensoren kalibriert worden sind, der mittleren Abweichung des Fits von den kalibrierten Druckwerten sowie der Streuung der Messwerte während der Messung im Versuchsstand, die sich geometrisch zur Gesamtunsicherheit addieren:

$$
\Delta p_{\text {ges }}=\sqrt{\left[\Delta p_{\text {Kalib }}\right]^{2}+\left[\Delta p_{\text {Fit }}\right]^{2}+\left[\Delta p_{\text {Streu }}\right]^{2}}
$$

Für den verwendeten Präzisions-Druckkalibrator DPI 515 werden folgende Fehlergrenzen in Abhängigkeit von dem für die Kalibrierung verwendeten Messbereich des Kalibrators angegeben:

- $\quad$ 0,01 \% vom Endwert für Messbereiche von 700 mbar bis 210 bar;

- $\quad 0,03 \%$ vom Endwert für Messbereiche unterhalb 700 mbar.

Gemäß Herstellerangaben umfasst die Fehlergrenze die Nichtlinearität, Hysterese, Wiederholbarkeit und den Temperatureffekt zwischen $18^{\circ} \mathrm{C}$ und $28^{\circ} \mathrm{C}$, sowohl für Absolut- als auch für Überdruckkalibration. Voraussetzung ist der regelmäßige Nullabgleich sowie die turnusmäßige Nacheichung durch die Physikalisch-Technische Bundesanstalt (PTB).

Für die hochgenaue Messung der Differenzdrücke an Außenwand und Fünflochsonde wurde ein anderes, eigens dafür entwickeltes Messwerterfassungssystem G16.1 der Professur MFD eingesetzt, bei denen die integrierten Piezosensoren mit einem zweiten Druckregler (DPI 520) kalibriert worden sind. Dieser verfügt über zwei einsetzbare Druckbereiche: 0 bis 70 mbar und 0 bis 7 bar. Die Fehlergrenze beträgt dort:

- $0,05 \%$ vom Messwert von 20 bis $100 \%$ vom Messbereichsendwert;

- darunter 0,01\% vom Messbereichsendwert.

Die Approximationsgenauigkeit der Fits unterscheidet sich von Sensor zu Sensor und beträgt im Mittel etwa

- 24 Pa für die Absolutdrucksensoren mit Messbereich bis 8 bar (Betriebsmessstellen);

- 2 Pa für die Differenzdrucksensoren mit Messbereich bis 0,7 bar (u.a. Blendenwirkdruck);

- 0,6 bzw. 0,2 Pa für die Differenzdrucksensoren mit Messbereich bis 0,1 bar (hochaufgelöste Wanddruck- und Sondenmessungen). 
Die Streuung der Druckwerte umfasst sowohl niederfrequente Änderungen durch Betriebspunktfluktuationen als auch hochfrequentes Rauschen (zufälliger Fehler), etwa infolge von Störfeldern. Sie wird für jeden Sensor aus der Standardabweichung der Messwerte über den dazugehörigen Mittelungszeitraum ( Kap. 4.6) bestimmt und beträgt für ideal stationäre Betriebszustände

- 150 Pa für die Absolutdrucksensoren im Mitteldruckbereich bei 2 bar (Betriebsmessstellen);

- 2 bis 3 Pa für alle Differenzdrucksensoren.

In Tab. 5.11 sind noch einmal alle Teilunsicherheiten der Drucksensoren aufgeführt sowie die resultierende Gesamtunsicherheit für die einzelnen Sensorgruppen gemäß Gl. 5.29 angegeben.

Tab. 5.11 Teilunsicherheiten und Gesamtunsicherheit der eingesetzten Drucksensorgruppen

\begin{tabular}{|c|c|c|c|c|}
\hline Bezeichnung & $\begin{array}{l}\text { Betriebsdrücke/ } \\
\text { Blendenvordruck }\end{array}$ & $\begin{array}{l}\text { Betriebsüberdrücke/ } \\
\text { Blendenwirkdruck }\end{array}$ & $\begin{array}{c}\text { 5-Loch-Sonde } \\
\text { (Betriebsmessung/ } \\
\text { Kalibrierung) }\end{array}$ & $\begin{array}{l}\text { 5-Loch-Sonde } \\
\text { (Feinmessung)/ } \\
\text { Wanddrücke }\end{array}$ \\
\hline $\begin{array}{l}\text { Messbereich } \\
\text { Versuchsstand }\end{array}$ & $1-6$ bar (abs) & $\begin{array}{c} \pm 0,25 \text { bar (diff)/ } \\
+0,5 \text { bar (diff) }\end{array}$ & $\pm 0,25$ bar (diff) & $+0,01$ bar (diff) \\
\hline $\begin{array}{l}\text { Messbereich } \\
\text { Kalibrierung }\end{array}$ & 8 bar (abs) & 0,7 bar (rel) & 0,7 bar (rel) & 0,07 bar (rel) \\
\hline $\begin{array}{l}\text { Messwerter- } \\
\text { fassungsgerät }\end{array}$ & G14.1 & G14.1 & G14.2 & G16.1 \\
\hline Kalibrator & DPI 515 & DPI 515 & DPI 515 & DPI 520 \\
\hline$\overline{\Delta p_{\text {Kalib }}}$ & $80 \mathrm{~Pa}$ & $7 \mathrm{~Pa}$ & $7 \mathrm{~Pa}$ & $3,5 \mathrm{~Pa}$ \\
\hline$\Delta p_{\text {Fit }}$ & $24 \mathrm{~Pa}$ & $2 \mathrm{~Pa}$ & $2 \mathrm{~Pa}$ & $0,5 \mathrm{~Pa}$ \\
\hline$\Delta p_{\text {Streu }}$ & $150 \mathrm{~Pa}$ & $3 \mathrm{~Pa}$ & $2 \mathrm{~Pa}$ & $2 \mathrm{~Pa}$ \\
\hline$\overline{\Delta p_{\text {ges }}}$ & $172 \mathrm{~Pa}$ & $8 \mathrm{~Pa}$ & $8 \mathrm{~Pa}$ & $4 \mathrm{~Pa}$ \\
\hline
\end{tabular}

\subsubsection{Unsicherheit der gemessenen Temperaturen}

Die Betrachtung der Unsicherheiten der gemessenen Temperaturen erfolgt analog wie bei den Drucksensoren im vorangegangenen Teilkapitel. Für die Kalibrierung der Thermistoren für die Wandtemperaturmessungen sowie der Thermosonden wurde der Temperatur-Blockkalibrator CTD9300-165 der Firma WIKA verwendet. Die Kalibrierung und Auswertung erfolgte - wie schon bei den Drucksensoren - durch Herrn Gunter Eschmann von der Professur für Messtechnik. Für weitere Details sei daher auf [73] verwiesen. Der Metallblock-Kalibrator besitzt eine Genauigkeit (Messabweichung zwischen Mess- und Referenzwert) zwischen $0,1 \mathrm{~K}$ bei $-30{ }^{\circ} \mathrm{C}$ und $0,16 \mathrm{~K}$ bei $165^{\circ} \mathrm{C}$. Für eine Einsatztemperatur von $30^{\circ} \mathrm{C}$ kann daraus in erster Näherung ein Wert von $0,12 \mathrm{~K}$ linear interpoliert werden. 
Demgegenüber fallen die Fit-Genauigkeit der Kalibrierkurven sowie die Streuung der Temperaturmesswerte ( Tab. 5.12) nicht ins Gewicht. Interessant ist, dass die Gesamtabweichung von 0,12 K für die Thermistor-Messstellen nahezu identisch mit der maximal beobachteten Abweichung der Materialtemperaturen von $\pm 0,15 \mathrm{~K}$ innerhalb eines Aluminiumringes ist ( Kap. 4.6). Offensichtlich resultieren diese aus der begrenzten Genauigkeit der Temperaturkalibrierung. Für alle weiteren Betrachtungen zur Messunsicherheit der Thermistoren wird daher der konservativere Wert $\Delta T_{\text {ges }}=0,15 \mathrm{~K}$ verwendet.

Die Grenzabweichung für das unkalibrierte Thermoelement vom Typ K, welches zur Temperaturmessung an der Messblende eingesetzt wird, beträgt im Temperaturbereich zwischen -40 und $+375^{\circ} \mathrm{C}$ in der Toleranzklasse 1 nach DIN EN 60584-2 $\Delta T= \pm 1,5 \mathrm{~K}$.

Tab. 5.12 Teilunsicherheiten und Gesamtunsicherheit der eingesetzten Temperatursensoren

\begin{tabular}{lccc}
\hline Bezeichnung & $\begin{array}{c}\text { Materialtemperaturen } \\
\text { (Außenwand) }\end{array}$ & $\begin{array}{c}\text { Thermistor-Sonden } \\
\text { (Referenztemperatur) }\end{array}$ & $\begin{array}{c}\text { Thermoelement-Sonde } \\
\text { (Messblende) }\end{array}$ \\
\hline $\begin{array}{l}\text { Messbereich } \\
\text { Versuchsstand }\end{array}$ & $20-80^{\circ} \mathrm{C}$ & $20-80^{\circ} \mathrm{C}$ & $15-40^{\circ} \mathrm{C}$ \\
$\begin{array}{l}\text { Messbereich } \\
\text { Kalibrierung }\end{array}$ & $0-100^{\circ} \mathrm{C}$ & $0-100^{\circ} \mathrm{C}$ & $-40-375^{\circ} \mathrm{C}$ \\
\hline$\Delta T_{\text {Kalib }}$ & $0,12 \mathrm{~K}$ & $0,12 \mathrm{~K}$ & $1,5 \mathrm{~K}$ \\
$\Delta T_{\text {Fit }}$ & $0,005 \mathrm{~K}$ & $0,005 \mathrm{~K}$ & - \\
$\Delta T_{\text {Streu }}$ & $0,005 \mathrm{~K}$ & $0,008 \mathrm{~K}$ & $0,1 \mathrm{~K}$ \\
\hline$\Delta T_{\text {ges }}$ & $0,12 \mathrm{~K}$ & $0,12 \mathrm{~K}$ & $1,5 \mathrm{~K}$ \\
\hline
\end{tabular}

\subsubsection{Unsicherheit der berechneten Wärmeübergangskoeffizienten}

Wie in Kapitel in Kap. 4.4 beschrieben, zielen alle Modelle auf eine Umwertung der gemessenen Wandtemperaturen auf lokale WÜK ab. Aus diesen Grund haben die Abweichungen in den Materialtemperaturen auch den größten Einfluss auf die Unsicherheiten der berechneten WÜK. Demgegenüber nehmen Unsicherheiten in den übrigen Modellparametern (Stoffwerte, angenommene Randbedingungen etc.) nur eine untergeordnete Rolle ein und sollen im Folgenden - auch zur Vereinfachung - nicht weiter betrachtet werden.

\subsubsection{Unsicherheiten des thermischen Netzwerkmodells}

Gemäß Gleichung 4.15 berechnet sich der Wärmeübergangswiderstand $R_{\alpha, j}$ an jedem Ring $j$ aus den Temperaturen am jeweiligen Innen- $\left(T_{1, j}\right)$ und Außenring $\left(T_{3, j}\right)$, an den beiden benachbarten Innenringen $\left(T_{1, j-1}\right.$ und $\left.T_{1, j+1}\right)$ bzw. (für Endringe) am angrenzenden Flansch sowie der Fluidtemperatur $T_{\mathrm{F}}$ und den axialen $\left(R_{\lambda, \mathrm{s} 1, \mathrm{ges}}\right)$ und radialen Wärmeleitwiderständen $\left(R_{\lambda, 2}\right)$. Der WÜK 
ist gemäß Gleichung 4.16 dann der Kehrwert des Produktes aus WÜ-Widerstand und wärmeübertragender Innenringfläche $A_{\mathrm{i}}$.

Unter Anwendung des Gauß'schen Fehlerfortpflanzungsgesetzes und der Annahme, dass ausschließlich die gemessenen Materialtemperaturen $T_{i}$ Einfluss auf die Unsicherheit des Ergebnisses haben

$$
\Delta \alpha_{j}=\sqrt{\sum_{i}\left(\frac{\partial \alpha_{j}}{\partial T_{i}} \Delta T_{i}\right)^{2}}
$$

und alle mit der gleichen absoluten Abweichung von $\Delta T_{1, j}=\Delta T_{1, j-1}=\Delta T_{1, j+1}=\Delta T_{3, j}=\Delta T_{i} \equiv$ $\Delta T=0,15 \mathrm{~K}$ behaftet sind, folgt:

$$
\Delta \alpha_{j}=\sqrt{\left(\frac{\partial \alpha_{j}}{\partial T_{1, j}} \Delta T\right)^{2}+\left(\frac{\partial \alpha_{j}}{\partial T_{1, j-1}} \Delta T\right)^{2}+\left(\frac{\partial \alpha_{j}}{\partial T_{1, j+1}} \Delta T\right)^{2}+\left(\frac{\partial \alpha_{j}}{\partial T_{3, j}} \Delta T\right)^{2}}
$$

Setzt man Gleichung 4.15 in Gleichung 4.16 ein und bildet die partiellen Ableitungen, so erhält man

$$
\begin{aligned}
\Delta \alpha_{j}=\frac{\Delta T}{T_{1, j}-T_{\mathrm{F}}} \cdot \frac{1}{A_{i}}\left(\frac{1}{R_{\lambda, 2}^{2}}+\frac{2}{R_{\lambda, s 1, \mathrm{ges}}^{2}}+\frac{1}{R_{\lambda, 2}^{2} R_{\lambda, s 1, \mathrm{ges}}^{2}} \cdot\right. \\
\left.\left.\qquad \frac{T_{1, j-1}-T_{\mathrm{F}}}{T_{1, j}-T_{\mathrm{F}}} R_{\lambda, 2}+\frac{T_{1, j+1}-T_{\mathrm{F}}}{T_{1, j}-T_{\mathrm{F}}} R_{\lambda, 2}+\frac{T_{3, j}-T_{\mathrm{F}}}{T_{1, j}-T_{\mathrm{F}}} \mathrm{R}_{\lambda, \mathrm{s} 1, \mathrm{ges}}\right]^{2}\right)^{\frac{1}{2}}
\end{aligned}
$$

Die resultierenden relativen Abweichungen liegen im vorgestellten Anwendungsbeispiel für die äußeren Messringe bei 11,4\% bzw. 12,1\% und dazwischen bei 8,4\% bis 9,4\% Diese Genauigkeit kann als überaus zufriedenstellend für die schnelle Vorhersage der WÜK mit einem solchen vereinfachten Modell angesehen werden.

Tab. 5.13 Absolute und relative Abweichungen der WÜK aus dem Netzwerkmodell

\begin{tabular}{cccr}
\hline Modul $j$ & $\alpha_{j}\left[\frac{\mathrm{W}}{\mathrm{m}^{2} \mathrm{~K}}\right]$ & $\Delta \alpha_{j}\left[\frac{\mathrm{W}}{\mathrm{m}^{2} \mathrm{~K}}\right]$ & $\delta \alpha_{j}[-]$ \\
\hline 1 & 31,29 & 3,58 & $11,4 \%$ \\
2 & 35,87 & 3,37 & $9,4 \%$ \\
3 & 49,94 & 4,20 & $8,4 \%$ \\
4 & 71,62 & 6,03 & $8,4 \%$ \\
5 & 89,66 & 7,56 & $8,4 \%$ \\
6 & 56,81 & 4,88 & $8,6 \%$ \\
7 & 38,29 & 3,57 & $9,3 \%$ \\
8 & 29,92 & 3,62 & $12,1 \%$ \\
\hline
\end{tabular}




\subsubsection{Unsicherheiten der Rückwärtsrechnung mittels Optimierungsverfahren}

Das in Kap. 4.4.4 vorgestellte FE-Modell für die stationäre inverse Methode lässt auf Grund der Vielzahl seiner diskreten Elemente keine Abschätzung der Unsicherheiten für die berechneten WÜK auf analytischem Wege zu (zum Beispiel mittels Gauß'scher Fehlerfortpflanzung). Es eignet sich jedoch hervorragend für eine Probabilistikanalyse in Form einer Monte-Carlo-Simulation. Die gemessenen Materialtemperaturen werden dabei als Haupteinflussparameter betrachtet. Diese werden als normalverteilt mit einer Standardabweichung von 0,15 K modelliert ( Abb. 5.33), was in etwa der maximalen absoluten Abweichung zwischen den Temperaturmessstellen an den vier Umfangspositionen eines jeden Ringes entspricht. Die Umgebungs- und die Fluid- (Referenz) Temperatur weisen keine feststellbaren (Langzeit-) Schwankungen/ Fluktuationen auf und werden daher in der Analyse als konstant angenommen. Ebenfalls keine Unsicherheit wurde für die WÜK für die Konvektion von den Außenoberflächen sowie zur Hauptströmung angesetzt. Abweichungen in der (hohen) Wärmeleitfähigkeit der Metalle in Tab. 4.5 haben keinen Einfluss auf das simulierte Temperaturfeld, da diese Bereiche quasi-isotherm sind. Im Gegensatz dazu hat die Wärmeleitfähigkeit der Isolatoren, vor allem vom Styropor zwischen den Messringen und vom PTFE im Zwischenring, signifikanteren Einfluss. Während $\lambda_{\text {Sty }} \approx \lambda_{\text {Luft }}$ ist und auf Grund des hohen Luftanteils kaum variiert, wurde $\lambda_{\text {PTFE }}$ über dem relevanten Temperaturbereich präzise mittels dreier unabhängiger Messverfahren bestimmt und keine signifikante Abhängigkeit von der Temperatur festgestellt (vgl. \ Kap. 5.5). Thermische Kontaktwiderstände wurden ebenfalls nicht mit modelliert, da alle Teile eine geringe Oberflächenrauigkeit aufweisen und verschrumpft (Ringe) oder fest verbunden sind.

Insgesamt 100 unabhängige Datensätze (Shots) mit perfekt unkorrelierten Eingabeparametern wurden mittels Latin Hypercube Sampling in Verbindung mit einem Restriced-Pairing-Algorithmus erzeugt. Der maximale absolute Rangkorrelationskoeffizient nach Spearman für außerdiagonale Matrixelemente beträgt $|\tilde{r}|<0,011$ (siehe linke obere Submatrix in $>$ Tab. 5.14), der lineare Korrelationskoeffizient nach Pearson immerhin noch $|r|<0,083$. Für alle Simulationen wurde dieselbe Anzahl an Iterationen durchgeführt, damit auch die Abbruchfehler („Residuen“) in gleichem Maße in die Unsicherheiten mit einfließen können.

Die Rangkorrelationsmatrix in $\$ Tab. 5.14 zeigt, dass die lokalen WÜK an jedem Modul stark mit der Temperatur am dazugehörigen Innen- und Außenring korrelieren sowie - in etwas schwächerem Maße - mit den Temperaturen an den beiden benachbarten Innen- und Außenringen, erkennbar durch die zwei roten Diagonalen in der rechteckigen Submatrix in $\downarrow$ Tab. 5.14. Die resultierenden Unsicherheiten (relative Standardabweichungen) der iterierten WÜK bewegen sich zwischen 6 und 8,5 \% für die Ringe 2 bis 7 bzw. $56 \%$ und $46 \%$ für die Ringe 1 und 8 (Abb. 5.34, 
Tab. 5.15 und vertikale Fehlerbalken in $\$$ Abb. 4.23 d). Zum Vergleich: Für den zweiten, in Abb. 4.34 dargestellten Anwendungsfall liegen die Unsicherheiten bei 8 bis $10 \%$ (Ringe 2 bis 7) bzw. $24 \%$ und $29 \%$ (Ringe 1 und 8). Offensichtlich sind die ermittelten WÜK an den beiden äußeren Messringen umso weniger vertrauenswürdig, je kleiner der benetzte Anteil der Ringinnenoberfläche ist. Dieser beträgt im ersten Fall nur 29 \% und im zweiten Fall immerhin 64,5 \%.

Tab. 5.14 Rang-Korrelationsmatrix nach Spearman zwischen den Ein- und Ausgabeparametern der Probabilistik-Analyse

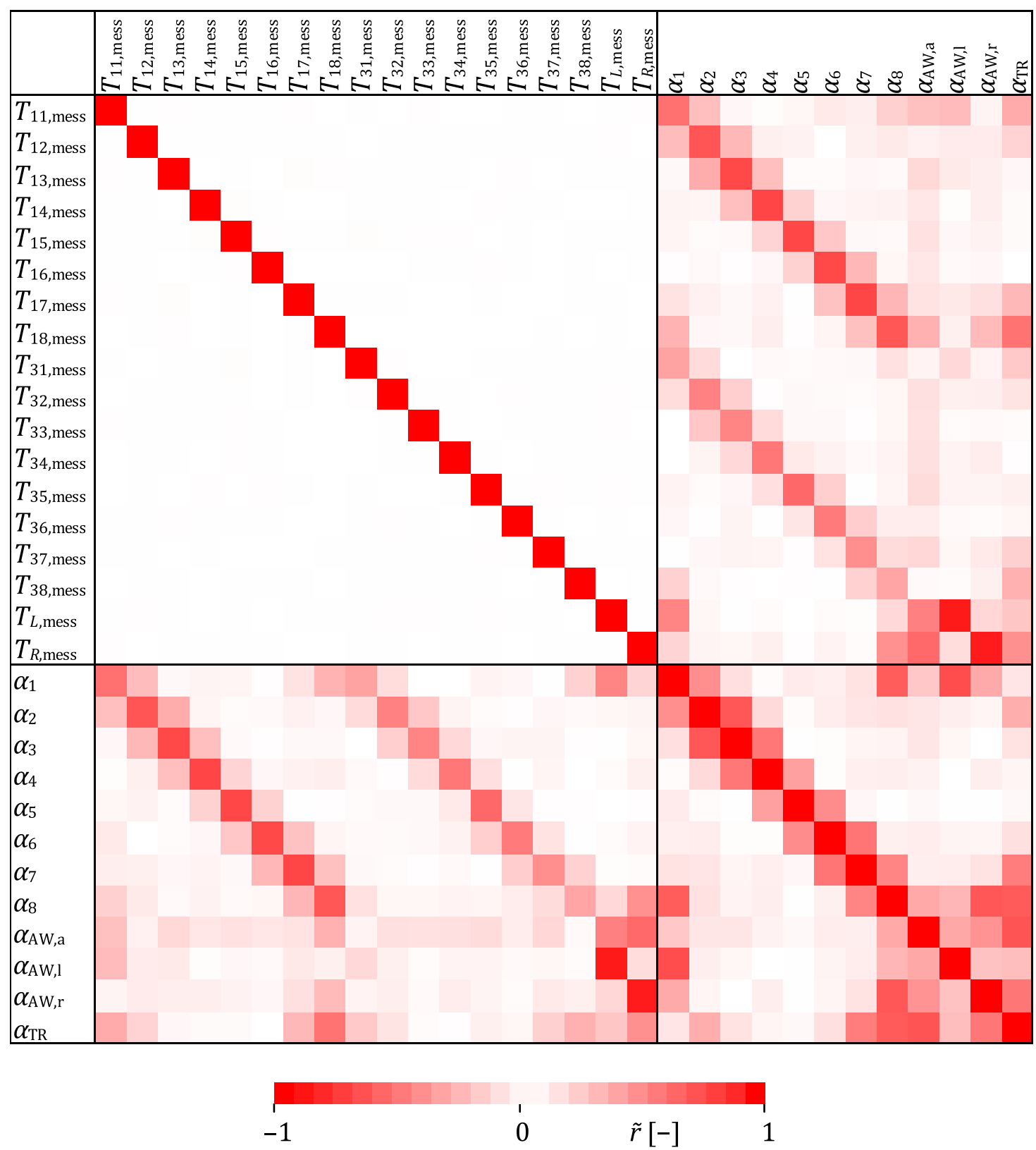




\begin{tabular}{|c|c|c|c|c|c|c|c|c|}
\hline Größe $x$ & $T_{11 \text {,mess }}$ & $T_{12, \text { mess }}$ & $T_{13 \text {,mess }}$ & $T_{14, \text { mess }}$ & $T_{15, \text { mess }}$ & $T_{16, \text { mess }}$ & $T_{17, \text { mess }}$ & $T_{18 \text {,mess }}$ \\
\hline$\mu\left[{ }^{\circ} \mathrm{C}\right]$ & 30,492 & 31,066 & 30,167 & 28,869 & 28,261 & 29,541 & 30,780 & 30,379 \\
\hline$\sigma[\mathrm{K}]$ & 0,150 & 0,152 & 0,152 & 0,150 & 0,151 & 0,151 & 0,150 & 0,151 \\
\hline$(x-\mu) / \sigma$ & \multicolumn{8}{|c|}{ Häufigkeitsverteilungen } \\
\hline$-3,4 \ldots-3$ & 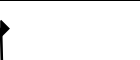 & & & & & & & \\
\hline$-3 \quad \ldots-2,6$ & 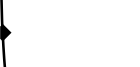 & & & & & & & \\
\hline$-2,6 \ldots-2,2$ & 5 & & & & & & & \\
\hline$-2,2 \ldots-1,8$ & & & & & & & & \\
\hline $\begin{array}{l}-1,8 \ldots-1,4 \\
-1,4 \ldots-1\end{array}$ & & & & & & & & \\
\hline$-1 \quad \ldots-0,6$ & & & & & & & & \\
\hline $\begin{array}{c}-0,6 \ldots-0,2 \\
-02\end{array}$ & & & & & & & & \\
\hline $0,2 \ldots 0,6$ & & & & & & & & \\
\hline $\begin{array}{lll}0,6 & \ldots & 1 \\
1 & \ldots & 1,4\end{array}$ & & & & & & & & \\
\hline $1,4 \ldots 1,8$ & & & & & & & & \\
\hline $1,8 \ldots 2,2$ & 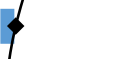 & & & 6 & & 6 & 6 & 1 \\
\hline $2,2 \ldots 2,6$ & 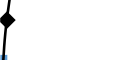 & & & 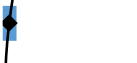 & & 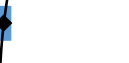 & 6 & \\
\hline $2,6 \ldots 3$ & & & & & & & & \\
\hline $3 \ldots 3,4$ & $b$ & 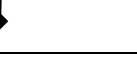 & & $b$ & & 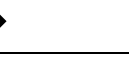 & & \\
\hline
\end{tabular}

\begin{tabular}{|c|c|c|c|c|c|c|c|c|}
\hline Größe $x$ & $T_{31 \text {,mess }}$ & $T_{32 \text {,mess }}$ & $T_{33 \text {,mess }}$ & $T_{34, \text { mess }}$ & $T_{35, \text { mess }}$ & $T_{36, \text { mess }}$ & $T_{37 \text {,mess }}$ & $T_{38 \text {,mess }}$ \\
\hline$\mu\left[{ }^{\circ} \mathrm{C}\right]$ & 35,086 & 36,073 & 35,639 & 34,526 & 33,931 & 34,960 & 35,959 & 34,861 \\
\hline$\sigma[\mathrm{K}]$ & 0,152 & 0,148 & 0,150 & 0,148 & 0,151 & 0,152 & 0,149 & 0,149 \\
\hline$(x-\mu) / \sigma$ & \multicolumn{8}{|c|}{ Häufigkeitsverteilungen } \\
\hline$-3,4 \ldots-3$ & $p$ & & & & & & & \\
\hline$-3 \quad \ldots-2,6$ & 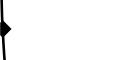 & & & & & & & \\
\hline$-2,6 \ldots-2,2$ & 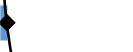 & & & & & & & \\
\hline$-2,2 \ldots-1,8$ & & & & & & & & \\
\hline $\begin{array}{l}-1,8 \ldots-1,4 \\
-1,4 \ldots-1\end{array}$ & & & & & & & & \\
\hline$-1 \quad \ldots-0,6$ & & & & & & & & \\
\hline $\begin{array}{lll}-0,0 & \ldots & -0,2\end{array}$ & & & & & & & & \\
\hline $\begin{array}{l}0,2 \ldots 0,6 \\
0,6 \ldots 1\end{array}$ & & & & & & & & \\
\hline $1 \quad \ldots 1,4$ & & & & & & & & \\
\hline $1,4 \ldots 1,8$ & & & & & & & & \\
\hline $1,8 \ldots 2,2$ & $\phi$ & & & & & & & 4 \\
\hline $2,2 \ldots 2,6$ & 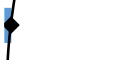 & & & 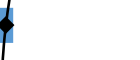 & & & 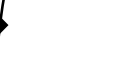 & \\
\hline $2,6 \ldots 3$ & 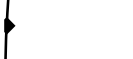 & & & & & & & \\
\hline $3 \ldots 3,4$ & 1 & 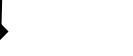 & & 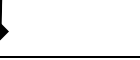 & & 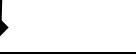 & & \\
\hline
\end{tabular}

Abb. 5.33 Häufigkeitsverteilung der Eingabeparameter der Monte-Carlo-Simulation (Temperaturen). 


\begin{tabular}{|c|c|c|c|c|c|c|c|c|}
\hline Größe $x$ & $\alpha_{1}$ & $\alpha_{2}$ & $\alpha_{3}$ & $\alpha_{4}$ & $\alpha_{5}$ & $\alpha_{6}$ & $\alpha_{7}$ & $\alpha_{8}$ \\
\hline$\mu\left[\mathrm{W} /\left(\mathrm{m}^{2} \mathrm{~K}\right)\right]$ & 14,004 & 32,321 & 45,526 & 69,813 & 96,654 & 55,867 & 30,485 & 16,391 \\
\hline$\sigma\left[\mathrm{W} /\left(\mathrm{m}^{2} \mathrm{~K}\right)\right]$ & 7,885 & 2,745 & 3,384 & 4,456 & 5,800 & 3,838 & 2,579 & 7,522 \\
\hline$(x-\mu) / \sigma$ & \multicolumn{8}{|c|}{ Häufigkeitsverteilungen } \\
\hline$-3,4 \ldots-3$ & ? & & & & & & & \\
\hline$-3 \quad \ldots-2,6$ & & & & & & & & \\
\hline$-2,6 \ldots-2,2$ & & & 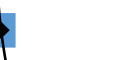 & & & & 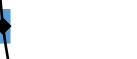 & \\
\hline$-2,2 \ldots-1,8$ & & & & & & & & \\
\hline$-1,8 \ldots-1,4$ & & & & & & & & \\
\hline $\begin{array}{l}-1,4 \ldots-1 \\
-1 \quad \ldots-0,6\end{array}$ & & & & & & & & \\
\hline $\begin{array}{c}-0,6 \ldots \\
-0\end{array}$ & & & & & & & & 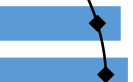 \\
\hline 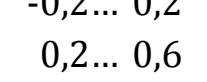 & & & & 4 & 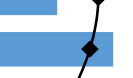 & 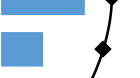 & 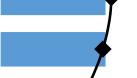 & 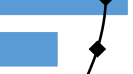 \\
\hline $\begin{array}{lll}0,6 \ldots & 1 \\
1 & \ldots & 1,4\end{array}$ & & & & & & & & \\
\hline $1,4 \ldots 1,8$ & & 4 & & 8 & & 1 & & 4 \\
\hline $1,8 \ldots 2,2$ & 8 & 4 & 4 & 6 & 6 & 4 & 6 & 4 \\
\hline $2,2 \ldots 2,6$ & & & & 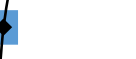 & & d & 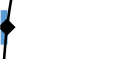 & \\
\hline $2,6 \ldots 3$ & $t$ & & & & & & & \\
\hline $3 \ldots 3,4$ & 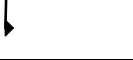 & & & 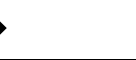 & & $b$ & & \\
\hline
\end{tabular}

Abb. 5.34 Häufigkeitsverteilung der Ausgabeparameter der Monte-Carlo-Simulation (WÜK).

Tab. 5.15 Absolute und relative Abweichungen der WÜK aus der Gradientenoptimierung

\begin{tabular}{cccr}
\hline Modul $j$ & $\alpha_{j}\left[\frac{\mathrm{W}}{\mathrm{m}^{2} \mathrm{~K}}\right]$ & $\Delta \alpha_{j}\left[\frac{\mathrm{W}}{\mathrm{m}^{2} \mathrm{~K}}\right]$ & \multicolumn{1}{c}{$\delta \alpha_{j}[-]$} \\
\hline 1 & 14,00 & 7,89 & $56,3 \%$ \\
2 & 32,32 & 2,74 & $8,5 \%$ \\
3 & 45,53 & 3,38 & $7,4 \%$ \\
4 & 69,81 & 4,46 & $6,4 \%$ \\
5 & 96,65 & 5,80 & $6,0 \%$ \\
6 & 55,87 & 3,84 & $6,9 \%$ \\
7 & 30,48 & 2,58 & $8,5 \%$ \\
8 & 16,39 & 7,52 & $45,9 \%$ \\
\hline
\end{tabular}




\subsubsection{Unsicherheiten der Rückwärtsrechnung mittels Trefftz-Funktionen}

Obwohl auch diese Methode auf einem FE-Modell basiert ( Kap. 0), erlaubt die besondere Form der Lösung als Linearkombination von Teilproblemen auf Basis von diskreten Knotentemperaturen (Gleichung 4.33) bzw. Wärmestromdichten (Gleichung 4.30) die Anwendung des Fehlerfortpflanzungsansatzes nach Gauß.

Basierend auf den Gleichungen im $>$ Anhang A7 wird der Lösungsvektor $\vec{c}=\left(c_{i}\right)$ für die Wärmestromdichten durch Multiplikation der inversen Matrix $\mathbf{A}^{-1}=\left(f_{i, j}\right)$ und der "rechten Seite“ $\vec{b}=\left(b_{i}\right)$ gebildet:

$$
\begin{gathered}
\vec{c}=\mathbf{A}^{-1} \vec{b} \\
c_{i}=\sum_{j=1}^{n} f_{i, j} b_{j}
\end{gathered}
$$

Die Matrixelemente $f_{i, j}$ der inversen Matrix $\boldsymbol{A}^{-1}$ hängen genau wie die Elemente $a_{i, j}$ der Koeffizientenmatrix $\boldsymbol{A}$ gemäß Gleichung A.50 nur vom Regularisierungsparameter $\beta$ und den Werten der partiellen Lösungen („Trefftz-Funktionen“) $\theta_{i, k}$ in den Knoten der Temperaturmessstellen ab, die sich nur aus Einheitswärmestrom-Randbedingungen zusammensetzen, jedoch nicht von den Solltemperaturen $T_{k}^{*}$ selbst, die ausschließlich im Vektor $\vec{b}$ enthalten sind (Gleichung A.51):

$$
c_{i}=-\sum_{j=1}^{n} f_{i, j} \sum_{k=1}^{m}\left(T_{0, k}-T_{k}^{*}\right) \theta_{j, k}, \quad f_{i, j} \neq f\left(T_{k}^{*}\right)
$$

Berücksichtigt man wiederum nur die Unsicherheiten in den Messwerten $T_{k}^{*}$, liefert die Anwendung des Gauß’schen Fortpflanzungsgesetzes

$$
\Delta c_{i}=\frac{\Delta \dot{q}_{i}}{\frac{\mathrm{W}}{\mathrm{m}^{2}}}=\sqrt{\sum_{k=1}^{m}\left(\frac{\partial c_{i}}{\partial T_{k}^{*}}\right)^{2}\left(\Delta T_{k}^{*}\right)^{2}}
$$

auf Gleichung 5.34 unter der erneuten Annahme $\Delta T_{k}^{*} \equiv \Delta T$ direkt die Abweichungen der Wärmestromdichte in den einzelnen Segmenten:

$$
\Delta \dot{q}_{i}=\Delta T \sqrt{\sum_{k=1}^{m}\left[\left(\sum_{j=1}^{n} f_{i, j} \theta_{j, k}\right)^{2}\right]} \frac{\mathrm{W}}{\mathrm{m}^{2}}
$$

Für Variante 6 ( Tab. 4.6), bei der die Segmentierung der Außenwandinnenoberfläche $\Gamma_{\text {inv }}$ der Ringteilung entspricht, ist die Wandtemperatur $\bar{T}_{\mathrm{W}, j}$ in jedem Segment $\Gamma_{\mathrm{inv}, j}$ auf Grund der hohen Wärmeleitfähigkeit von Aluminium praktisch konstant und entspricht der im jeweiligen Innenring berechneten Knotentemperatur $T_{1, j}$, die mit der Unsicherheit $\Delta T$ belegt ist. 
Gemäß Gleichung 4.46 berechnet sich die Unsicherheit des WÜK in jedem $\Gamma_{\text {inv,j }}$ (unter Berücksichtigung des aus Homogenitätsgründen in diesem Schritt gebotenen Basiswechsels $\dot{q}_{i} \rightarrow \dot{q}_{j}$ ) nach Gauß schließlich zu:

$$
\begin{gathered}
\Delta \bar{\alpha}_{j}=\sqrt{\left(\frac{\partial \bar{\alpha}_{j}}{\partial \dot{q}_{j}} \Delta \dot{q}_{j}\right)^{2}+\left(\frac{\partial \bar{\alpha}_{j}}{\partial T_{1, j}} \Delta T_{1, j}\right)^{2}} \\
\Delta \bar{\alpha}_{j}=\sqrt{\left[\frac{\Delta \dot{q}_{j}}{T_{1, j}-T_{\mathrm{B}}}\right]^{2}+\left[\frac{\dot{q}_{j} \Delta T}{\left(T_{1, j}-T_{\mathrm{B}}\right)^{2}}\right]^{2}}
\end{gathered}
$$

Die absoluten und relativen Abweichungen von Wärmestromdichte und WÜK sind in $>$ Tab. 5.16 angeführt. Mit Unsicherheiten von 5 bis 6,5\% für die inneren Messringe und immerhin noch 27,1 \% bzw. 28,2 \% für die beiden äußeren Ringe zeigt sich das Trefftz-Modell am unempfindlichsten gegenüber Abweichungen in den gemessenen Temperaturen im Vergleich zum gradientenbasierten inversen Berechnungsverfahren.

Tab. 5.16 Absolute und relative Abweichungen der WÜK aus der Rückwärtsrechnung mittels Trefftz-Funktionen

\begin{tabular}{crrrrcr}
\hline Modul $j$ & $\dot{q}_{j}\left[\frac{\mathrm{W}}{\mathrm{m}^{2}}\right]$ & $\Delta \dot{q}_{j}\left[\frac{\mathrm{W}}{\mathrm{m}^{2}}\right]$ & $\delta \dot{q}_{j}[-]$ & $\alpha_{j}\left[\frac{\mathrm{W}}{\mathrm{m}^{2} \mathrm{~K}}\right]$ & $\Delta \alpha_{j}\left[\frac{\mathrm{W}}{\mathrm{m}^{2} \mathrm{~K}}\right]$ & $\delta \alpha_{j}[-]$ \\
\hline 1 & 26,99 & 7,28 & $27,0 \%$ & 4,23 & 1,15 & $27,1 \%$ \\
2 & 219,04 & 12,32 & $5,6 \%$ & 31,93 & 1,93 & $6,0 \%$ \\
3 & 273,28 & 12,73 & $4,7 \%$ & 45,48 & 2,40 & $5,3 \%$ \\
4 & 330,73 & 12,75 & $3,9 \%$ & 70,05 & 3,50 & $5,0 \%$ \\
5 & 389,59 & 12,75 & $3,3 \%$ & 94,84 & 4,65 & $4,9 \%$ \\
6 & 297,32 & 12,73 & $4,3 \%$ & 55,67 & 2,85 & $5,1 \%$ \\
7 & 201,51 & 12,29 & $6,1 \%$ & 30,41 & 1,98 & $6,5 \%$ \\
8 & 18,14 & 5,10 & $28,1 \%$ & 2,93 & 0,83 & $28,2 \%$ \\
\hline
\end{tabular}




\subsubsection{Unsicherheiten der lokalen Übertemperaturmethode}

Die Unsicherheiten der lokalen Übertemperaturmethode können mittels Gauß'scher Fehlerfortpflanzung aus dem Wärmeübergangswiderstand berechnet werden:

$$
\alpha=\frac{1}{R_{\alpha} \cdot A_{\mathrm{Th}}}
$$

Um die relative Abweichung

$$
\frac{\Delta \alpha}{\alpha}=\sqrt{\left(\frac{\Delta R_{\alpha}}{R_{\alpha}}\right)^{2}+\left(\frac{\Delta A_{\mathrm{Th}}}{A_{\mathrm{Th}}}\right)^{2}}
$$

bestimmen zu können, werden sowohl die Unsicherheiten des Wärmeübergangswiderstandes $R_{\alpha}$ als auch der effektiven wärmeübertragenden Thermistorfläche $A_{\text {Th }}$ benötigt. Die mittlere relative Abweichung der Sensorfläche wurde auf Basis von hochauflösenden Fotografien eines vergleichbaren Sensors auf einen Wert von 2 \% geschätzt $[84,143]$. Die relative Abweichung des Wärmeübergangswiderstandes

$$
\frac{\Delta R_{\alpha}}{R_{\alpha}}=\sqrt{2 \cdot\left\{\left[\left(\Delta \operatorname{Dig}_{\mathrm{ADU}}+\Delta \operatorname{Dig}_{\mathrm{Fit}, T}\right) \cdot Z_{\alpha}\right]^{2}+\left(2 \frac{\Delta U}{U}\right)^{2}+\left(2 \frac{\Delta R}{R}\right)^{2}\right\}}
$$

setzt sich zusammen aus den Unsicherheiten des Thermistorwiderstandes $R$, seiner Messspannung $U$ sowie verschiedener Brückenspannungsverhältnisse [84,143], wobei alle Größen jeweils zwei Mal bestimmt werden müssen (Ruheversuch und Messung). Während die relativen Abweichungen für die Messung der elektrischen Spannung $U$ sowie des elektrischen Widerstandes $R$ durch den Hersteller des Digitalmultimeters vorgegeben sind, muss für die Verhältnisse der Brückenspannungen nur der Diskretisierungsfehler des Analog-Digital-Wandlers (ADU), die Approximationsgenauigkeit des Fits für die Übertemperatur sowie die Auflösung $Z$ der gesamten Analog-Digital-Messkette vom Ablesewert des ADU ( $A D C$ reading) in Dezimal-Ziffernschritten (sog. Digits) bis zum umgerechneten WÜK berücksichtigt werden. Der Diskretisierungsfehler im A/DWandler wird mit $\Delta \operatorname{Dig}_{\mathrm{ADU}}=4$ Digits abgeschätzt. Die Kalibriergenauigkeit des Temperaturfits berechnet sich aus der doppelten Standardabweichung zwischen dem Fit und den Messwerten sowie der maximalen Auflösung der Temperaturmessstellen $Z_{T}=340$ Digit/K [84,143]:

$$
\Delta \operatorname{Dig}_{\mathrm{Fit}, T}=2 \Delta T_{\mathrm{Fit}} \cdot Z_{T}
$$

Die Auflösung des Messwerterfassungssystems kennzeichnet die relative Sensitivität des aus der gesamten Messkette berechneten WÜK gegenüber dem abgelesenen Digit-Wert des Thermistors in stationären Endzustand in \%/Digit [82,83]:

$$
Z\left(\alpha, T_{0}\right)=\left.\frac{1}{\alpha} \cdot \frac{d \alpha}{d \operatorname{Dig}\left(T_{\infty}\right)}\right|_{\operatorname{Dig}\left(T_{0}\right)}
$$


Das Kennfeld $\alpha\left(\operatorname{Dig}\left(T_{\infty}\right), \operatorname{Dig}\left(T_{0}\right)\right)$ eines jeden Sensors setzt sich zusammen aus der Übertragungsfunktion des elektrischen Schaltkreises, aus welcher sich die Kalibrierkurve des Thermistors $\operatorname{Dig}(T)$ ergibt, sowie aus dem thermischen Ersatzmodell zur Berechnung des WÜK aus der Sprungantwort der Thermistoraufheizung. Dies kann z. B. ein thermisches Netzwerkmodell sein $[82,83]$.

Wie Abb. 5.35 zeigt, ist die Auflösung $Z\left(\alpha, T_{0}\right)$ eine Funktion des WÜK und der Starttemperatur des Thermistors zum Einschaltzeitpunkt (äquivalent zur Fluid- bzw. adiabaten Wandtemperatur) und ist begrenzt durch die minimal gerade noch zulässige Übertemperatur auf der einen und die maximal verfügbare Heizleistung auf der anderen Seite.

Im Ergebnis liegen die Unsicherheiten für die mittels der Sensoren ermittelten lokalen WÜK zwischen 2,53 und $2,64 \%[135,136]$.

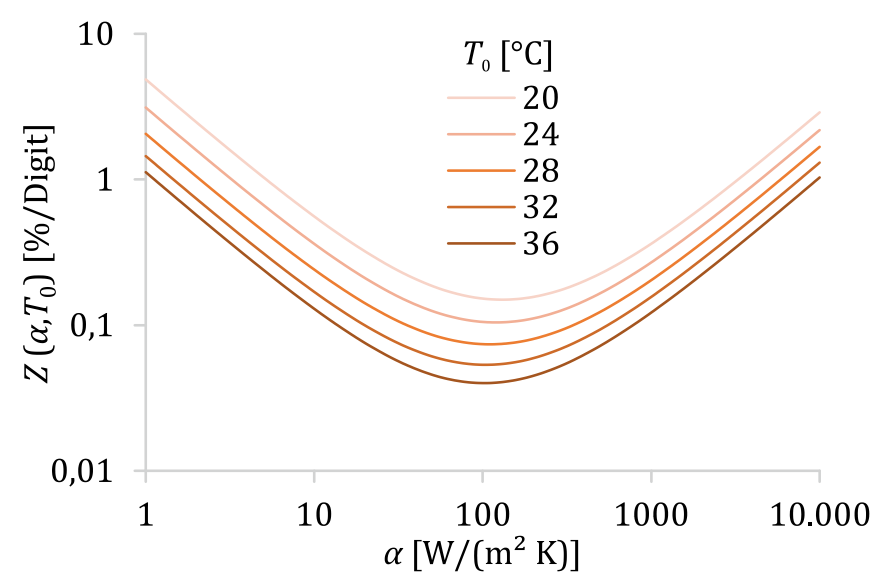

Abb. 5.35 Auflösung des Datenerfassungssystems als Funktion von WÜK und Fluidtemperatur.

\subsubsection{Unsicherheit der geometrischen Maße von Seitenraum und Strömungskanal}

Zur Bestimmung der Unsicherheiten der Ringspaltabmessungen, welche unmittelbar in die Berechnung der Reynolds-Zahl ( Kap. 5.8.6) eingehen, wurde mit Hilfe eines digitalen Messschiebers sowohl für den Innendurchmesser des Außenrohres $D_{\mathrm{VS}}$ als auch für den Außendurchmesser des Innenrohres $d_{\mathrm{VS}}$ jeweils der Minimal- und der Maximalwert einmal im Bereich der Einströmöffnung und einmal am gegenüberliegenden Rohrende bestimmt. Als die resultierenden Abweichungen für Innen- und Außendurchmesser werden dann die halben Spannweiten zwischen den jeweiligen globalen Extremwerten definiert (100-\%-Konfidenzintervall), zu denen noch die Längenmessabweichung des Messschiebers in Höhe von $\Delta L= \pm 0,02 \mathrm{~mm}$ hinzuaddiert werden müssen. Im Ergebnis sind

$$
\begin{aligned}
\Delta D & = \pm 0,4 \mathrm{~mm} \\
\Delta d & = \pm 0,3 \mathrm{~mm}
\end{aligned}
$$


Die Unsicherheit von Ringspalthöhe $\Delta(\Delta r)$ und hydraulischem Durchmesser $\Delta(\Delta d)$ beträgt dann:

$$
\begin{gathered}
\Delta(\Delta r)=\frac{1}{2}(\Delta D+\Delta d)= \pm 0,35 \mathrm{~mm} \\
\Delta(\Delta d)=\Delta D+\Delta d= \pm 0,7 \mathrm{~mm}
\end{gathered}
$$

Für die weiteren radialen und axialen Abmessungen der Kavität werden die Unsicherheiten aus den Maßtoleranzen und Passmaßen auf den Fertigungszeichnungen berechnet, und zwar durch getrennte Summierung der oberen Grenzmaße es und der unteren Grenzmaße ei („Toleranzketten"). Form- und Lagetoleranzen werden nicht berücksichtigt, da es sich bei den Totraumstrukturen ausschließlich um hochgenaue (maßhaltige) Dreh- oder Frästeile handelt. Ebenso wird die Unsicherheit durch die Längenänderung infolge thermischer Dehnung vernachlässigt, da sich die Betriebstemperaturen nicht signifikant von den Temperaturen während der Fertigung unterscheiden. Für die Totraumhöhen $h_{1}$ und $h_{2}$ ergibt sich damit:

$$
\begin{gathered}
h_{1}=(\emptyset 138 \mathrm{f} 7-\emptyset 106,5 \pm 0,4) \mathrm{mm} / 2=15_{, 75_{-0,242}^{+0,179} \mathrm{~mm}} \\
h_{2}=(\emptyset 200 \mathrm{D} 7-\emptyset 138 \mathrm{f} 7) \mathrm{mm} / 2=31_{+0,107}^{+0,150} \mathrm{~mm}
\end{gathered}
$$

Wie in Kap. 4.1.3 angesprochen, erfolgt die Einstellung der axialen Abmessungen der Kavität sowie der axialen Positionierung der Seitenwand indirekt durch die Abnahme und Kontrolle von äußeren Referenzmaßen ( Abb. 5.36). Die Nennwerte dieser Referenzmaße werden für jede Geometrievariante berechnet und mittels des Messschiebers auf $\pm 0,05 \mathrm{~mm}$ genau eingestellt. Die Unsicherheiten der Totraumabmessungen ergeben sich dann aus dieser Einstellgenauigkeit, dem Messfehler des Messschiebers sowie der Toleranzkette der beteiligten Bauteile und sind daher höher als die Abweichungen der radialen Abmessungen.

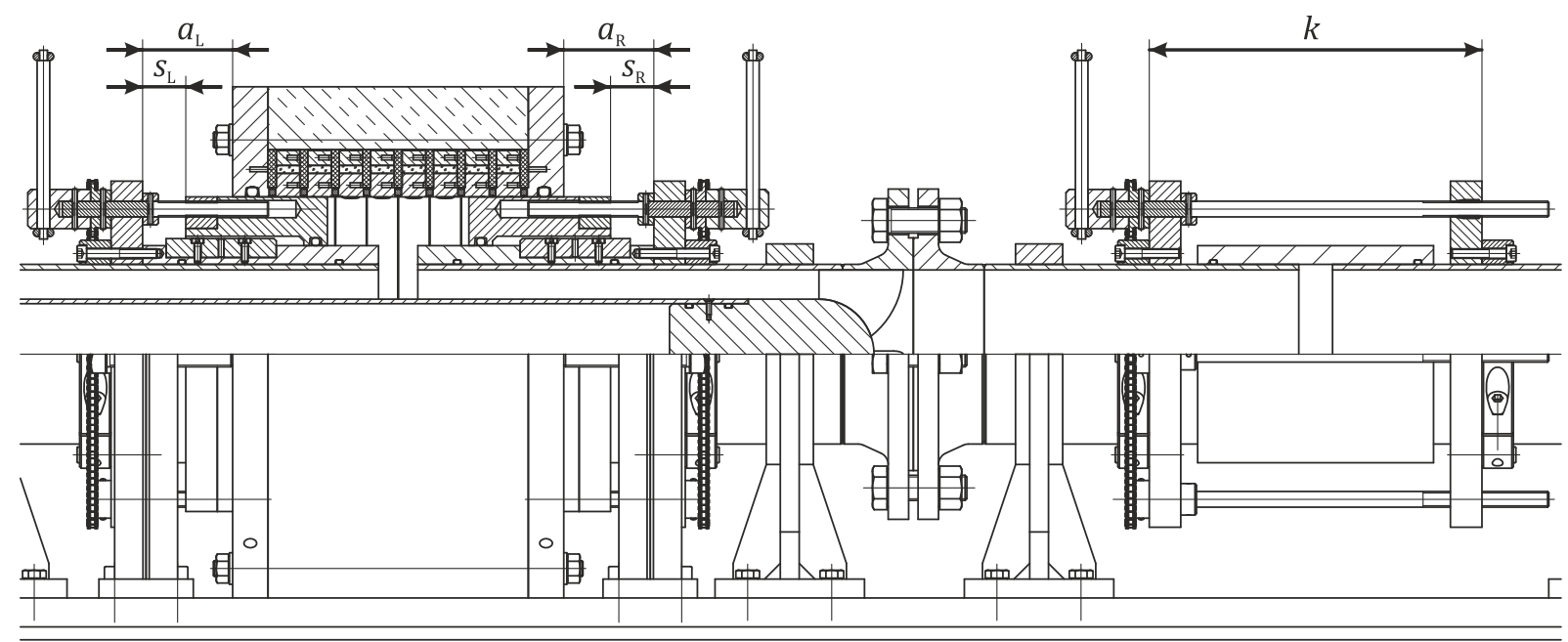

Abb. 5.36 Äußere axiale Referenzmaße zur Einstellung der lateralen Seitenraumabmessungen und der Außenwandposition. 
Die Einströmbreite $s$ wird durch Verschieben der stromabwärtigen Einströmseitenwand mit Hilfe des Kompensationsmoduls realisiert. Dazu wurde einmalig zur Inbetriebnahme des Versuchsstandes die Einströmöffnung vollständig zugefahren und der Abstand $k$ zwischen den beiden Spindellagern am Kompensationsmodul bestimmt. Durch Zufahren des Kompensationsmoduls auf den neuen Sollwert, der ebenfalls zu messen ist, nimmt die Einströmbreite im selben Maß zu. Für die Genauigkeit von $s$ ergibt sich damit:

$$
\Delta s= \pm 0,05 \mathrm{~mm}+2 \cdot \pm 0,02 \mathrm{~mm}= \pm 0,07 \mathrm{~mm}
$$

Die Position der linken und der rechten Totraumseitenwand $b_{\mathrm{R}}$ bzw. $b_{\mathrm{L}}$ (vgl. Abb. 4.4) wird wie in Abb. 5.36 dargestellt durch die Abstände $s_{\mathrm{L}}$ und $s_{\mathrm{R}}$ eingestellt. Aus den Allgemeintoleranzen der beteiligten Bauteile nach ISO $2768 \mathrm{mK}$ sowie der Einstell- und Messgenauigkeit für $s_{\mathrm{L}}$ und $s_{\mathrm{R}}$ ergibt sich dann

$$
\Delta b_{\mathrm{L}}=\Delta b_{\mathrm{R}}=( \pm 0,5 \mathrm{~mm} \pm 0,05 \mathrm{~mm} \pm 0,3 \mathrm{~mm}) \pm 0,05 \mathrm{~mm} \pm 0,02 \mathrm{~mm}= \pm 0,92 \mathrm{~mm}
$$

Die maximale Abweichung der Totraumbreite berechnet sich dann aus:

$$
\Delta b=\Delta s+\Delta b_{\mathrm{L}}+\Delta b_{\mathrm{R}}= \pm 1,91 \mathrm{~mm}
$$

und die der Exzentrizität äquivalent gemäß:

$$
\Delta e=\frac{1}{2}\left(\Delta b_{\mathrm{R}}+\Delta b_{\mathrm{L}}\right)= \pm 0,92 \mathrm{~mm}
$$

Die Genauigkeit für die axiale Positionierung der Außenwand, welche über das Maß $a_{\mathrm{L}}$ eingestellt und über das Maß $a_{\mathrm{R}}$ gegengeprüft wird, ergibt sich erneut aus der Einstell- und Messgenauigkeit von $a_{\mathrm{L}}$ sowie der Unsicherheit durch das ungleichmäßige Verpressen der PTFE-Dichtringe zwischen den Messringen, die aus der gleichmäßigen Aufteilung der gemessenen Gesamtstauchung der Außenwand auf alle neun Dichtstellen mit $\pm 0,2 \mathrm{~mm}$ angesetzt wurde:

$$
\Delta x= \pm 0,05 \mathrm{~mm} \pm 0,02 \mathrm{~mm} \pm 0,2 \mathrm{~mm}= \pm 0,27 \mathrm{~mm} \text { (WÜK) }
$$

Letztere entfällt bei der Dummy-Außenwand, die zur Untersuchung der Wanddruckverteilung sowie des Strömungsprofiles im Hauptströmungskanal eingesetzt wurde und aus einem geflanschten integralen Plastikzylinder besteht:

$$
\Delta x= \pm 0,05 \mathrm{~mm} \pm 0,02 \mathrm{~mm}= \pm 0,07 \mathrm{~mm} \text { (Druck) }
$$

Die Umfangsposition der Außenwand wird über eine auf den Flansch der Außenwand aufgeklebte Winkelskala und eine durch Ausloten auf der obersten Mantellinie der Totraumseitenwand aufgezeichnete Referenzmarkierung eingestellt. Die Einstellgenauigkeit entspricht dann der halben Skalenteilung:

$$
\Delta \varphi= \pm 0,5^{\circ}
$$




\subsubsection{Unsicherheit des Massenstromes an der Blendenmessstrecke}

Da es sich bei der Blendenmessstrecke um ein Messgerät handelt, welches unter Beachtung der normativen Anforderungen ohne Kalibrierung eingesetzt werden darf, muss die Unsicherheit des mit seiner Hilfe ermittelten Massenstromes unter Anwendung der Fehlerfortpflanzung abgeschätzt werden, für welche die Blendennorm DIN EN ISO 5167-1 folgende „Arbeitsgleichung“ angibt, die unmittelbar aus der Drosselgleichung 4.5 hervorgeht:

$$
\delta \dot{m}=\frac{\Delta \dot{m}}{\dot{m}}=\sqrt{\left(\frac{\Delta C}{C}\right)^{2}+\left(\frac{\Delta \varepsilon}{\varepsilon}\right)^{2}+\left(\frac{2 \beta^{4}}{1-\beta^{4}}\right)^{2}\left(\frac{\Delta D}{D}\right)^{2}+\left(\frac{2}{1-\beta^{4}}\right)^{2}\left(\frac{\Delta d}{d}\right)^{2}+\frac{1}{4}\left(\frac{\Delta(\Delta p)}{\Delta p}\right)^{2}+\frac{1}{4}\left(\frac{\Delta \rho_{1}}{\rho_{1}}\right)^{2}}
$$

Zur Beachtung: Hier und im Folgenden bezeichnet $d=71 \mathrm{~mm}$ der Blendenöffnungsdurchmesser und $D=107,5 \mathrm{~mm}$ den Rohrinnendurchmesser der Blendenmesstrecke.

Die wahrscheinliche relative Unsicherheit des Durchflusskoeffizienten setzt sich aus verschiedenen Termen zusammen, die die Maß-, Form und Lagetoleranzen der Blendenmessstrecke berücksichtigen:

$$
\delta C=\frac{\Delta C}{C}=\delta C_{0}+\delta C_{D}+\delta C_{R e}+\delta C_{L}+\delta C_{\Delta D}+\delta C_{\mathrm{e}}
$$

Die Grundabweichung $\delta C_{0}$ beträgt unter der Annahme, dass die Unsicherheiten von $\beta, D, R e_{D}$ und der spezifischen Oberflächenrauigkeit $R a / D$ hier vernachlässigbar sind:

$$
\delta C_{0}= \begin{cases}(0,7-\beta) \% & 0,1 \leq \beta<0,2 \\ 0,5 \% & 0,2 \leq \beta<0,6 \\ \left(\frac{5}{3} \beta-0,5\right) \% & 0,6 \leq \beta<0,75\end{cases}
$$

Für Blendenmessstrecken mit kleinem Innendurchmesser muss die Zusatzunsicherheit $\delta C_{D}$ aufgeschlagen werden:

$$
\delta C_{D}=0,9 \% \cdot(0,75-\beta) \cdot\left(2,8-\frac{D}{25,4 \mathrm{~mm}}\right) \text { falls } D<2,8 \text { in }
$$

Eine weitere Zusatzunsicherheit in Höhe von 0,5 \% wird fällig bei kleinen Reynolds-Zahlen und vergleichsweise großen Blendenverhältnissen:

$$
\delta C_{R e}=0,5 \% \text { falls } \beta>0,5 \text { und } R e_{D}<10000
$$

Falls nur verkürzte An-und Abströmstrecken gemäß Norm zur Verfügung stehen, muss eine weitere Zusatzunsicherheit von

$$
\delta C_{L}=0,5 \%
$$

hinzuaddiert werden. 
Bei zu hohen Durchmessersprüngen zwischen zwei Passstücken im Vorlauf kommen noch

$$
\delta C_{\Delta D}=0,2 \% \text { falls }\left\{\begin{array}{cc}
\frac{\Delta D}{D}>0,3 \%, & 2<\frac{L}{D}<10 \\
\frac{\Delta D}{D}>2 \%, & \frac{L}{D}>10
\end{array}\right\} \text { und } \frac{\Delta D}{D}<\min \left(0,002 \frac{\frac{S}{D}+0,4}{0,1+2,3 \beta^{4}} ; 0,05\right)
$$

hinzu. Sofern die Exzentrizität der Messblende gegenüber den Achsen von Ein- und Auslaufrohr in der Ebene der Druckentnahme zu groß wird, muss eine weitere Zusatzunsicherheit berücksichtigt werden:

$$
\delta C_{\mathrm{e}}=0,3 \% \text { falls } \frac{0,0025 D}{0,1+2,3 \beta^{4}}<e_{\mathrm{cl}} \leq \frac{0,005 D}{0,1+2,3 \beta^{4}}
$$

Für die hier vorliegende Hochpräzisions-Blendenmesstrecke sind keine dieser Zusatzunsicherheiten zu beachten. Die Unsicherheit des Durchflusskoeffizienten beträgt demnach für $\beta=0,663$

$$
\delta C=\delta C_{0}=0,605 \%
$$

Die Unsicherheit der Expansionszahl wird angegeben mit

$$
\delta \varepsilon=3,5 \% \cdot \frac{\Delta p_{\mathrm{W}}}{\kappa \cdot p_{1}}
$$

und ist abhängig vom Wirkdruck $\Delta p_{\mathrm{W}}$ und vom Vordruck $p_{1}$ und damit vom Massenstrom. Der Einfluss der Unsicherheiten von $\beta, \Delta p_{\mathrm{W}} / p_{1}$ und $\kappa$ wird hierbei explizit vernachlässigt.

Für die relativen Abweichungen von Blendenöffnungs- und Rohrinnendurchmesser sind (sofern nicht separat bestimmt, wie dies hier der Fall ist) die maximal zulässigen Werte gemäß DIN EN ISO 5167-2 anzusetzen: $\Delta D / D=0,3 \%$ und $\Delta d / d=0,05 \%$.

Die relative Abweichung der Dichte beträgt unter Anwendung der idealen Gasgleichung und unter Zugrundelegung der absoluten Abweichungen des Blendenwirkdrucks ( Tab. 5.11) und der Thermoelementsonde ( Tab. 5.12):

$$
\delta \rho_{1}=\frac{\Delta \rho_{1}}{\rho_{1}}=\sqrt{\left(\frac{\Delta p}{p}\right)^{2}+\left(\frac{\Delta T}{T}\right)^{2}}=0,51 \%
$$




\subsubsection{Unsicherheit der Reynolds-Zahl}

Die Unsicherheit der Reynolds-Zahl im Versuchsstand kann unter Anwendung des Gauß'schen Fehlerfortpflanzungsgesetzes wie folgt aus Gleichung 4.3 bestimmt werden:

$$
\delta R e=\frac{\Delta R e}{R e}=\sqrt{\left(\frac{\Delta \dot{m}}{\dot{m}}\right)^{2}+\left(\frac{\Delta \eta}{\eta}\right)^{2}+\left(\frac{\Delta D_{\mathrm{VS}}}{D_{\mathrm{VS}}+d_{\mathrm{VS}}}\right)^{2}+\left(\frac{\Delta d_{\mathrm{VS}}}{D_{\mathrm{VS}}+d_{\mathrm{VS}}}\right)^{2}}
$$

Hier kennzeichnen $\Delta d_{\mathrm{VS}}=\Delta d$ die Unsicherheit des Innen- und $\Delta D_{\mathrm{VS}}=\Delta D$ die Unsicherheit des Außendurchmessers des $\underline{\text { Ringspaltes }}$ aus Kap. 5.8.4. Eventuelle Abweichungen von der Rundheit oder von der Konzentrizität haben übrigens keinen Einfluss auf die Unsicherheit der Reynolds-Zahl, da der benetzte Umfang, auf dem Gl. 5.46 basiert, davon unberührt bleibt. Gemäß Gleichung 5.46 geht die Unsicherheit der Reynolds-Zahl im Wesentlichen mit der Unsicherheit des Massenstromes einher, welcher über die Blendenmessstrecke bestimmt wird (Kap. 5.8.5).

Die Temperaturabhängigkeit der dynamischen Viskosität von technischer Luft kann im Temperaturbereich zwischen 20 und $40{ }^{\circ} \mathrm{C}$ in exzellenter Näherung wie folgt linearisiert werden:

$$
\eta(T)=\left(4,656 \cdot 10^{-6}+4,572 \cdot 10^{-8} \frac{T}{\mathrm{~K}}\right) \mathrm{Pa} \mathrm{s}
$$

Die absolute Unsicherheit von $\eta$ ist dann mit $\Delta T=\Delta T_{\text {ref }}=0,15 \mathrm{~K}$ :

$$
\Delta \eta=4,572 \cdot 10^{-8} \frac{\mathrm{Pa} \mathrm{s}}{\mathrm{K}} \cdot \Delta T=6,858 \cdot 10^{-9} \mathrm{~Pa} \mathrm{~s}
$$

Unter Annahme einer einheitlichen Temperatur von $26^{\circ} \mathrm{C}$ und eines einheitlichen Druckes von 2 bar in Blendenmessstrecke und Versuchsstand sind in Abb. 5.37 für den Blendeneinsatz mit dem Öffnungsdurchmesser $d_{\mathrm{Bl}}=71 \mathrm{~mm}$ und verschiedene Reynolds-Zahlen $R e_{\mathrm{VS}}$ die relativen Teilunsicherheiten der dem Blendenmassenstrom zugrunde liegenden physikalischen Größen sowie die resultierende Gesamtunsicherheit der Reynolds-Zahl im Versuchsstand angegeben. Wie sich zeigt, liegt die Gesamtunsicherheit $\delta R e$ über einen weiten Reynolds-Zahl-Bereich unter $1 \%$. Einzig für kleine Reynolds-Zahlen wird der relative Fehler des Blendenwirkdruckes dominant, weshalb hierfür typischerweise ein geringerer Blendenöffnungsdurchmesser gewählt werden sollte, der eine höhere Wirkdruckdifferenz generiert.

Für die im Rahmen dieser Arbeiten untersuchten Reynolds-Zahlen von Re $>40.000$ liegt die Unsicherheit $\delta R e$ unter $1,5 \%$. 


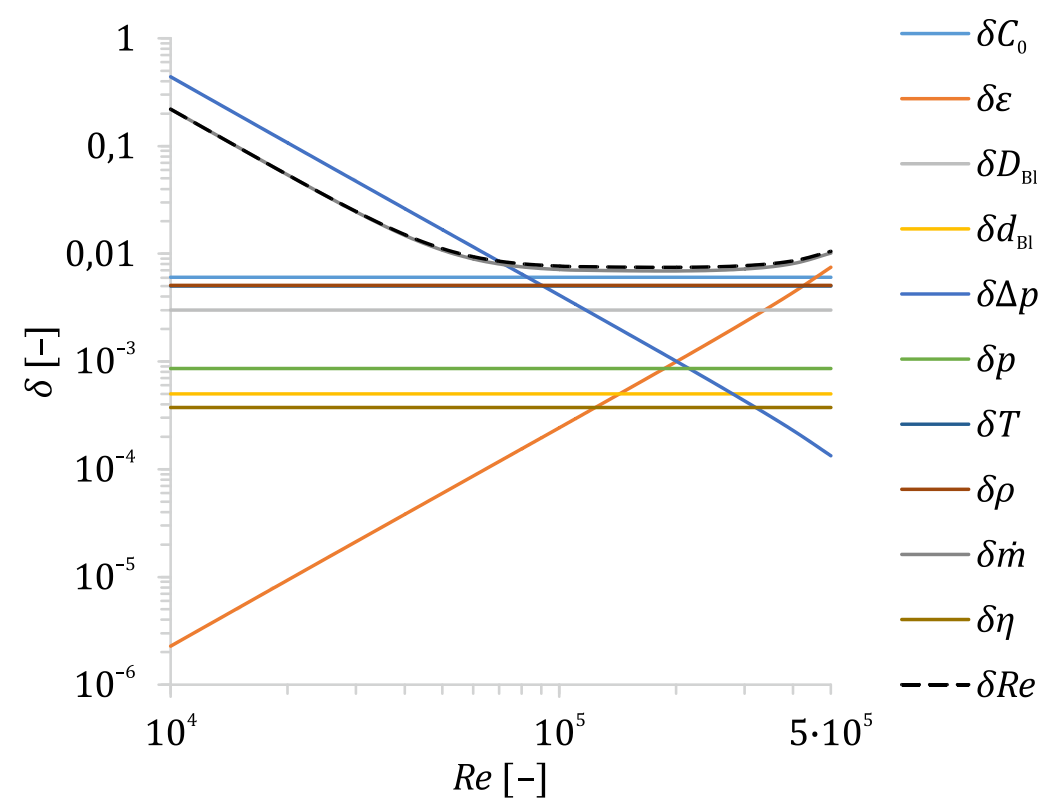

Abb. 5.37 Relative Gesamtunsicherheit der Reynolds-Zahl im Versuchsstand und Einzelunsicherheiten der zugrunde liegenden Messgrößen für den im Versuchsstand relevanten Reynolds-Zahl-Bereich. $p_{\mathrm{L}}=2 \mathrm{bar}, T_{\mathrm{L}}=26^{\circ} \mathrm{C}, D_{\mathrm{Bl}}=107,1 \mathrm{~mm}, d_{\mathrm{Bl}}=71 \mathrm{~mm}$.

\subsubsection{Unsicherheit der Nusselt-Zahl}

Gemäß Gl. 5.64 lässt sich die Unsicherheit der aus den WÜK berechneten Nusselt-Zahlen analog wie bei der Reynolds-Zahl (Gl. 5.46) abschätzen:

$$
\delta N u=\frac{\Delta N u}{N u}=\sqrt{\left(\frac{\Delta \alpha}{\alpha}\right)^{2}+\left(\frac{\Delta(\Delta d)}{\Delta d}\right)^{2}+\left(\frac{\Delta \lambda}{\lambda}\right)^{2}}
$$

Die Unsicherheit des WÜK $\delta \alpha$ kann aus Kap. 5.8.3 entnommen werden. Die relative Unsicherheit des hydraulischen Durchmessers beträgt unter Berücksichtigung von Gl. $5.43 \delta(\Delta d)=1,9 \%$ und muss noch geometrisch hinzuaddiert werden. Demgegenüber ist die Unsicherheit der Wärmeleitfähigkeit der Luft im Prüfstand vernachlässigbar. Sie kann wie schon bei der dynamischen Viskosität (Gl. 5.47) durch Linearisierung von $\lambda(T)$ im Temperaturbereich zwischen 20 und $40{ }^{\circ} \mathrm{C}$ und unter Annahme von $\Delta T_{\text {ref }}=0,15 \mathrm{~K}$ wie folgt abgeschätzt werden:

$$
\begin{gathered}
\lambda(T)=\left(6,396 \cdot 10^{-3}+6,793 \cdot 10^{-5} \frac{T}{\mathrm{~K}}\right) \frac{\mathrm{W}}{\mathrm{m} \mathrm{K}} \\
\Delta \lambda=6,793 \cdot 10^{-5} \frac{\mathrm{W}}{\mathrm{m} \mathrm{K}^{2}} \cdot \Delta T=1,019 \cdot 10^{-5} \frac{\mathrm{W}}{\mathrm{m} \mathrm{K}}
\end{gathered}
$$

Für $T=26^{\circ} \mathrm{C}$ beträgt $\delta \lambda=0,038 \%$. 


\subsubsection{Unsicherheit der Strömungswinkel und Geschwindigkeitskomponenten}

Wie in Kap. 5.2 erörtert, werden die Strömungswinkel und Geschwindigkeitskomponenten aus den gemessenen Druckdifferenzen an der Fünflochsonde berechnet. Entsprechend sind auch deren Unsicherheiten eng miteinander verknüpft. Ganz ähnlich wie bei Temperatur- und Druckmessungen setzen sich die Unsicherheiten auch hier aus den Teilbeträgen für die Kalibriergenauigkeit, für die Approximationsgüte des Fits und für die Unsicherheit während der Messung im Versuchsstand zusammen.

Die „Kalibriergenauigkeit“ umfasst die Ausricht- und die Einstellgenauigkeit der Sonde während der Kalibrierung. Die Ausrichtung der Sonde erfolgt manuell durch Sichtprüfung mit Hilfe der angebrachten Winkelskala ( $1^{\circ}$-Teilung) und einer Wasserwaage bzw. anhand einer horizontalen Sichtkante im Raum. Die Einstellgenauigkeit basiert auf der Güte der Umfangs- bzw. Sondenwinkeltraversierung mit Hilfe der eingesetzten Schrittmotoren. Sowohl für Einstell- als auch Ausrichtgenauigkeit wird ein Wert von $\pm 0,5^{\circ}$ angesetzt. Letzterer Wert gilt auch für die Ausrichtungs- bzw. Montagegenauigkeit der Sonde im Versuchsstand. Eine Winkeltraversierung erfolgt hier hingegen nicht:

$$
\begin{gathered}
\Delta \alpha_{\text {Ausricht,Kalib }}=\Delta \alpha_{\text {Einstell,Kalib }}=\Delta \alpha_{\text {Ausricht,Mess }}=0,5^{\circ} \\
\Delta \beta_{\text {Ausricht,Kalib }}=\Delta \beta_{\text {Einstell,Kalib }}=\Delta \beta_{\text {Ausricht,Mess }}=0,5^{\circ}
\end{gathered}
$$

Das dimensionslose Verhältnis $f_{Q}$ aus dem (nahezu konstanten) dynamischen Druck $\Delta p_{\text {dyn }} \operatorname{der}$ Düsenfreiströmung und dem Bezugsdifferenzdruck $\Delta p_{\mathrm{B}}$ an der Sonde wird zwar nicht aktiv eingestellt, variiert jedoch auf Grund der Drehung der Sonde im Freistrahl. Die Genauigkeit, mit der dieses Verhältnis bestimmt werden kann, folgt aus Gl. 5.3 gemäß Fehlerfortpflanzung zu:

$$
\delta f_{Q}=\sqrt{\left[\frac{\Delta(\Delta p)}{p_{\mathrm{K}}-p_{\mathrm{Umg}}}\right]^{2}+\left[\frac{4 \Delta(\Delta p)}{\Delta p_{\mathrm{B}}}\right]^{2}}, \quad \Delta f_{Q, \text { Einstell }}=\delta f_{Q} \cdot f_{Q}
$$

$\Delta f_{Q \text {,Einstell }}$ liegt über dem gesamten Kalibrierbereich zwischen 0,02 und 0,04. Weitergerechnet wird mit dem größeren der beiden Werte.

Für die Unsicherheiten der Fits, mit denen die an der Sonde in der Kalibrierströmung gemessenen Druckdifferenzen in die beiden Winkel bzw. den dynamischen Druckkoeffizienten umgewertet werden, werden die mittleren Standardabweichungen aus Kap. 5.2 verwendet:

$$
\begin{gathered}
\Delta \alpha_{\mathrm{Fit}}=\sigma_{\alpha}=0,29^{\circ} \\
\Delta \beta_{\mathrm{Fit}}=\sigma_{\beta}=0,24^{\circ} \\
\Delta f_{Q, \mathrm{Fit}}=\sigma_{f_{Q}}=0,097
\end{gathered}
$$


Als zusammengesetzte Größen erfolgt die Abschätzung der Unsicherheit des Gier- (Drall-) und des Nickwinkels während der Sondenmessung im Versuchsstand nicht auf Basis von deren stochastischen Schwankungen, sondern durch Fehlerfortpflanzung, die die Gesamtunsicherheit der gemessenen Druckdifferenzen $\Delta p_{i}:=p_{\mathrm{M}}-p_{i}$ mit $i \in\{0 ; \mathrm{U} ; \mathrm{L} ; \mathrm{R}\}$ berücksichtigt. Die Unsicherheiten der dimensionslosen Druckdifferenzen $x$ und $y$ aus den Gln. 5.1 und 5.2 berechnen sich demnach zu:

$$
\begin{aligned}
& \Delta x=\frac{\Delta(\Delta p)}{\left[\Delta p_{\mathrm{B}}\right]^{2}} \sqrt{\left[2 \Delta p_{\mathrm{O}}+\Delta p_{\mathrm{L}}+\Delta p_{\mathrm{R}}\right]^{2}+\left[2 \Delta p_{\mathrm{U}}+\Delta p_{\mathrm{L}}+\Delta p_{\mathrm{R}}\right]^{2}+2\left[\Delta p_{\mathrm{U}}-\Delta p_{\mathrm{O}}\right]^{2}} \\
& \Delta y=\frac{\Delta(\Delta p)}{\left[\Delta p_{\mathrm{B}}\right]^{2}} \sqrt{\left[2 \Delta p_{\mathrm{R}}+\Delta p_{\mathrm{U}}+\Delta p_{\mathrm{O}}\right]^{2}+\left[2 \Delta p_{\mathrm{L}}+\Delta p_{\mathrm{U}}+\Delta p_{\mathrm{O}}\right]^{2}+2\left[\Delta p_{\mathrm{L}}-\Delta p_{\mathrm{R}}\right]^{2}}
\end{aligned}
$$

Die Unsicherheit des daraus berechneten Gier-/Drallwinkels $\alpha$, des Nickwinkels $\beta$ sowie des dynamischen Druckkoeffizienten $f_{Q}$ (Gln. 5.6 bis 5.8) ergeben sich dann unter der gebräuchlichen Annahme, dass die Unsicherheit der Kalibrierkoeffizienten (Matrixelemente) $A_{i, k}, B_{i, k}, Q_{i, k}$ vernachlässigbar sind, wie folgt:

$$
\begin{aligned}
& \Delta \alpha_{\Delta p}=\sqrt{\left[\sum_{i=0}^{4} \sum_{k=0}^{5} A_{i, k} i x^{i-1} y^{k} \Delta x\right]^{2}+\left[\sum_{i=0}^{4} \sum_{k=0}^{5} A_{i, k} k x^{i} y^{k-1} \Delta y\right]^{2}} \\
& \Delta \beta_{\Delta p}=\sqrt{\left[\sum_{i=0}^{5} \sum_{k=0}^{4} B_{i, k} i x^{i-1} y^{k} \Delta x\right]^{2}+\left[\sum_{i=0}^{5} \sum_{k=0}^{4} B_{i, k} k x^{i} y^{k-1} \Delta y\right]^{2}} \\
& \Delta f_{Q, \Delta p}=\sqrt{\left[\sum_{i=0}^{4} \sum_{k=0}^{4} Q_{i, k} i x^{i-1} y^{k} \Delta x\right]^{2}+\left[\sum_{i=0}^{4} \sum_{k=0}^{4} Q_{i, k} k x^{i} y^{k-1} \Delta y\right]^{2}}
\end{aligned}
$$

Die resultierende Gesamtunsicherheit für Kalibrierung und Messung beträgt somit:

$$
\begin{gathered}
\Delta \alpha=\sqrt{3\left[\Delta \alpha_{\text {Einstell/Ausricht }}\right]^{2}+\left[\Delta \alpha_{\mathrm{Fit}}\right]^{2}+\left[\Delta \alpha_{\Delta p, \text { Mess }}\right]^{2}} \\
\Delta \beta=\sqrt{3\left[\Delta \beta_{\text {Einstell/Ausricht }}\right]^{2}+\left[\Delta \beta_{\mathrm{Fit}}\right]^{2}+\left[\Delta \beta_{\Delta p, \text { Mess }}\right]^{2}} \\
\Delta f_{Q}=\sqrt{\left[\Delta f_{Q, \text { Einstell }}\right]^{2}+\left[\Delta f_{Q, \mathrm{Fit}}\right]^{2}+\left[\Delta f_{Q, \Delta p, \text { Mess }}\right]^{2}}, \quad \delta f_{Q}=\frac{\Delta f_{Q}}{f_{Q}}
\end{gathered}
$$


Aus diesen Größen kann nun auch die Gesamtunsicherheit für die gemessenen Geschwindigkeitskomponenten (vgl. Gl. 5.10 bis Gl. 5.13 sowie Gl. 5.45) abgeleitet werden:

$$
\begin{gathered}
\delta c=\frac{1}{2} \sqrt{\left[\delta f_{Q}\right]^{2}+\left[\delta\left(\Delta p_{\mathrm{B}}\right)\right]^{2}+\left[\delta p_{\mathrm{ref}}\right]^{2}+\left[\delta T_{\mathrm{ref}}\right]^{2}}, \quad \Delta c=\delta c \cdot c \\
\Delta c_{\mathrm{r}}=\sqrt{[\Delta c \sin \beta]^{2}+[c \cos \beta \Delta \beta]^{2}} \\
\Delta c_{\mathrm{u}}=\sqrt{[\Delta c \cos \beta \sin \alpha]^{2}+[c \cos \beta \cos \alpha \Delta \alpha]^{2}+[c \sin \beta \sin \alpha \Delta \beta]^{2}} \\
\Delta c_{\mathrm{ax}}=\sqrt{[\Delta c \cos \beta \cos \alpha]^{2}+[c \cos \beta \sin \alpha \Delta \alpha]^{2}+[c \sin \beta \cos \alpha \Delta \beta]^{2}}
\end{gathered}
$$

Die mit obigen Gleichungen berechneten Abweichungen betragen beispielsweise für den in Abb. 5.6 d und Abb. 5.7 d dargestellten Fall $\left(R e=110.000, \gamma=30^{\circ}\right) \Delta \alpha=0,92^{\circ}, \Delta \beta=0,91^{\circ}$ und $\Delta c=1,14 \mathrm{~m} / \mathrm{s}$. Wie sich zeigt, tragen die Teilunsicherheiten infolge des Messfehlers der gemessenen Druckdifferenzen (Gln. 5.53 bis 5.55) nicht nennenswert zur Gesamtunsicherheit bei. Grund hierfür ist die sehr präzise Feinmessung der Differenzdrücke mit $\Delta(\Delta p)=4 \mathrm{~Pa}$. Zum Vergleich: für eine hypothetische, sehr stark verrauschte Druckmessung mit $\Delta(\Delta p)=30$ Pa betragen die Abweichung immer noch $\Delta \alpha=1,37^{\circ}, \Delta \beta=1,39^{\circ}$ und $\Delta c=1,47 \mathrm{~m} / \mathrm{s}$. 


\subsection{VERALLGEMEINERUNG DER ERGEBNISSE ALS NUSSELT-KORRE- LATION}

Wie in Kap. 5.7 beobachtet, zeigen sowohl die WÜK-Verläufe der stationären inversen Methode als auch die korrigierten Werte der lokalen Übertemperaturmethode ( Abb. 5.27) ein Maximum im Bereich des Staupunktes des Prallstrahls $\left(\alpha_{\max }\right)$, welcher von der stromabwärtigen Einströmseitenwand in den Totraum gelenkt wird (vgl. a. Abb. 5.28). In axialer Richtung fallen die WÜK zu den Totraumseitenwänden jeweils monoton mit annähernd demselben Anstieg ab und erreichen zu beiden Seiten hin in etwa gleiche Sättigungswerte $\alpha_{\text {min }}$. Für beide Methoden lässt sich dieser Verlauf daher sehr gut durch eine Glockenkurve ähnlich der Gauß'schen Normalverteilung annähern (vgl. Abb. 5.38):

$$
\alpha(x)=\alpha_{\min }+\Delta \alpha \cdot e^{-\frac{\left(x-x_{\mathrm{S}}\right)^{2}}{2 \sigma_{x}^{2}}}
$$

Die Wahl einer solchen Kurvenform, die sonst in der Wahrscheinlichkeitstheorie beheimatet ist, birgt gleich mehrere Vorteile. Alle ihre Parameter repräsentieren charakteristische Eigenschaften der Kurve. So entspricht $\alpha_{\text {min }}$ der Nullverschiebung (Offset) und damit dem Minimalwert der Kurve, $\Delta \alpha$ der Überhöhung im Maximalpunkt $\alpha_{\max }=\alpha_{\min }+\Delta \alpha$ und $x_{\mathrm{S}}$ der Abszisse des Maximalwertes und damit des Staupunktes (Erwartungswert). Die „Standardabweichung“ $\sigma_{x}$ kennzeichnet den Ordinatenabstand zwischen Maximum und den beiden Wendepunkten und ist somit ein Maß für die axiale Breite der Verteilung. Außerdem hat die Glockenkurve beispielsweise im Vergleich zu Polynomen nur genau ein globales Maximum und ist somit auch für $x$-Werte, die außerhalb des Definitionsbereiches liegen, extrapolierbar. Ein Charakteristikum der Funktion besteht hingegen auch darin, das sich die Werte im linken und rechten Ast der Kurve demselben Wert $\alpha_{\text {min }}$ annähern. Eine Unterscheidung separater Grenzwerte für $x \rightarrow \pm \infty$ ist so-
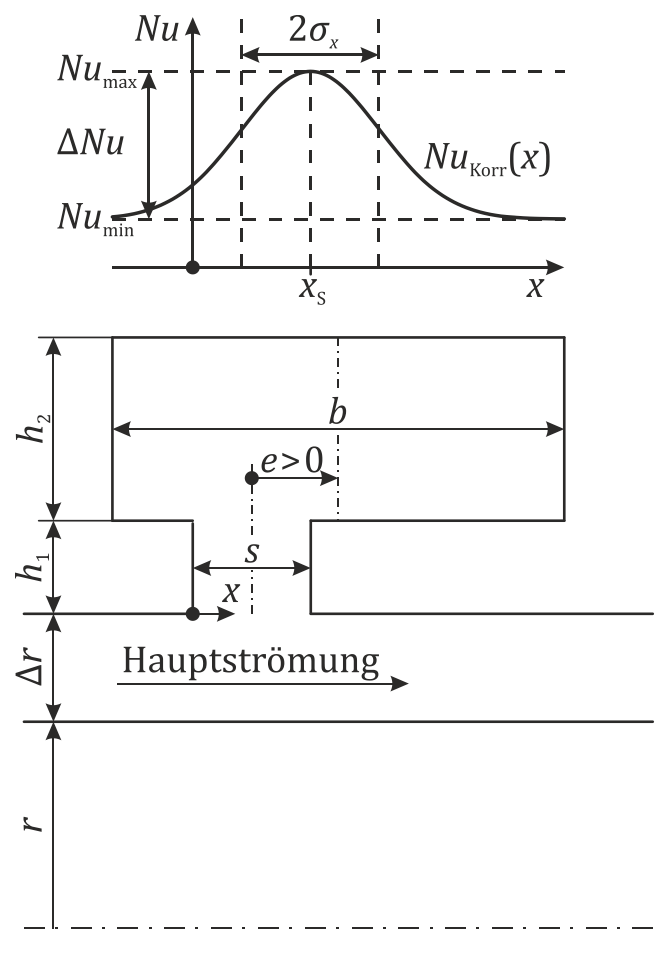

Abb. 5.38 Approximation des WÜK-Verlaufes mittels einer Gauß'schen Glockenkurve (entdimensionalisiert), charakteristische Kurvenparameter und zugehörige Seitenraumgeometrie. mit nicht möglich. 
In einem ersten Schritt wurden für jeden der in Abb. 5.29 bis Abb. 5.32 dargestellten Fälle separat die vier Parameter in Gleichung 5.63 so angepasst, dass die jeweilige Kurven nach der Methode der kleinsten Fehlerquadrate möglichst nahe an den dazugehörigen Messpunkten liegen (Least-square Fit; LSF). Bildet man nun über alle Konfigurationen hinweg die Korrelationskoeffizienten zwischen jeden dieser vier Werte und jeden der fünf verschiedenen geometrischen bzw. strömungstechnischen Konfigurationsparametern („Eingabewerten“), so erhält man die in $>$ Tab. 5.17 und Tab. 5.18 dargestellten Korrelationsmatrizen. Diese geben an, ob ein Ausgabeparameter stark $(|r|=0,5 \ldots 1)$ oder nur schwach mit dem jeweiligen Eingabeparameter korreliert, also linear (direkt oder indirekt proportional) von ihm abhängt. Da Messwerte naturgemäß Streuungen unterliegen und „Ausreißer" das Korrelationsverhalten verfälschen können, eignet sich der Spearman-Rangkorrelationskoeffizient, bei denen die Ein- und Ausgabewerte erst der Größe nach geordnet und dann gemäß ihrer „Platzierung“ (ihres Ranges) miteinander verglichen werden, etwas besser zur Abschätzung der Quereinflüsse. So korreliert $\alpha_{\min }$ beispielsweise sehr stark mit der Reynolds-Zahl Re und der Einströmbreite $s$, mit der Exzentrizität $e$ und dem Drallwinkel (Blechwinkel) $\gamma$ hingegen so gut wie gar nicht. Dies deckt sich auch mit den qualitativen Erkenntnissen aus Kap. 5.7.

Tab. 5.17 Matrix der linearen Korrelationskoeffizienten nach Pearson zwischen den Ein- und Ausgabeparametern der idealen Glockenkurven-Fits

\begin{tabular}{|c|rrrr|rrrrrr|}
\hline & $\alpha_{\min }$ & $\Delta \alpha$ & $x_{\mathrm{S}}$ & $\sigma_{x}$ & $N u_{\min }$ & $\Delta N u$ & $N u_{\max }$ & $\frac{\Delta N u}{N u_{\max }}$ & $\frac{x_{\mathrm{S}}}{s}$ & $\frac{\sigma_{x}}{b}$ \\
\hline$s$ & 0,51 & 0,08 & 0,30 & $-0,01$ & 0,51 & 0,08 & 0,08 & $-0,13$ & 0,39 & 0,05 \\
$b$ & $-0,41$ & $-0,11$ & $-0,23$ & $-0,15$ & $-0,41$ & $-0,11$ & $-0,11$ & 0,20 & $-0,20$ & $-0,38$ \\
$e$ & $-0,08$ & $-0,44$ & $-0,60$ & $-0,51$ & $-0,08$ & $-0,44$ & $-0,44$ & $-0,24$ & $-0,57$ & $-0,39$ \\
$\gamma$ & 0,00 & $-0,08$ & $-0,09$ & $-0,12$ & 0,00 & $-0,08$ & $-0,08$ & 0,01 & $-0,08$ & $-0,20$ \\
$R e$ & 0,55 & 0,13 & 0,08 & 0,14 & 0,55 & 0,13 & 0,13 & $-0,13$ & 0,09 & 0,16 \\
\hline
\end{tabular}

Tab. 5.18 Matrix der Rang-Korrelationskoeffizienten nach Spearman zwischen den Ein- und Ausgabeparametern der idealen Glockenkurven-Fits

\begin{tabular}{|c|rrrr|rrrrrr|}
\hline & $\alpha_{\min }$ & $\Delta \alpha$ & $x_{\mathrm{S}}$ & \multicolumn{1}{c}{$\sigma_{x}$} & $N u_{\min }$ & $\Delta N u$ & $N u_{\max }$ & $\frac{\Delta N u}{N u_{\max }}$ & $\frac{x_{\mathrm{S}}}{s}$ & $\frac{\sigma_{x}}{b}$ \\
\hline$s$ & 0,55 & 0,48 & 0,74 & $-0,24$ & 0,55 & 0,47 & 0,55 & $-0,27$ & 0,65 & $-0,11$ \\
$b$ & $-0,25$ & 0,14 & $-0,38$ & 0,21 & $-0,25$ & 0,14 & $-0,13$ & 0,20 & $-0,36$ & $-0,70$ \\
$e$ & $-0,08$ & $-0,19$ & $-0,26$ & $-0,09$ & $-0,08$ & $-0,19$ & $-0,24$ & $-0,06$ & $-0,31$ & $-0,09$ \\
$\gamma$ & 0,03 & 0,24 & $-0,03$ & 0,05 & 0,05 & 0,25 & 0,12 & 0,02 & $-0,08$ & $-0,55$ \\
$R e$ & 0,57 & 0,57 & 0,16 & 0,28 & 0,57 & 0,58 & 0,62 & $-0,17$ & 0,18 & 0,32 \\
\hline
\end{tabular}


Zur Verallgemeinerung der Ergebnisse in Form einer Korrelation wurde Gleichung 5.63 anschließend entdimensionalisiert, indem aus den WÜK zunächst Nusselt-Zahlen gemäß

$$
N u=\frac{\alpha \cdot l}{\lambda}=\frac{\alpha \cdot \Delta d}{\lambda}=\frac{\alpha \cdot\left(D_{\mathrm{VS}}-d_{\mathrm{VS}}\right)}{\lambda}
$$

gebildet sowie die geometrischen Größen als Abmessungsverhältnisse (sog. Ähnlichkeitssimplexe) formuliert wurden. Da die Spreizung $\Delta N u$ zum Teil sehr viel größer werden kann als $N u_{\text {min }}$ und beide Werte stark von der Reynolds-Zahl abhängen ( Tab. 5.18), hat es sich als günstig erwiesen, die Korrelation so umzuformulieren, dass stattdessen $N u_{\max }$ und das Verhältnis $\Delta N u / N u_{\max }$ als Parameter fungieren. Letzteres nimmt angenehmerweise immer Werte zwischen 0 und 1 an. Die dazugehörigen Korrelationskoeffizienten sind ebenfalls in $\$ Tab. 5.17 und $\$ Tab. 5.18 mit angegeben.

Es ergibt sich somit folgende Korrelation:

$$
N u_{\text {Korr }}(x)=N u_{\max } \cdot\left(1-\frac{\Delta N u}{N u_{\max }} \cdot\left[1-\exp \left\{-\left(\frac{s}{b}\right)^{2} \cdot \frac{\left(\frac{x}{s}-\frac{x_{\mathrm{S}}}{s}\right)^{2}}{2\left(\frac{\sigma_{x}}{b}\right)^{2}}\right\}\right]\right)
$$

Unter Berücksichtigung von Tab. 5.18 wurde anschließend für jeden der $i=4$ Kurvenparameter $y=\left\{N u_{\max } ; \Delta N u / N u_{\max } ; x_{\mathrm{S}} / s ; \sigma_{x} / b\right\}$ mit Hilfe von separaten Input-Output-Diagrammen der funktionelle Zusammenhang zu jeder der $j=5$ Einflussgrößen $x_{j}$ festgestellt, deren Rang-Korrelationskoeffizient zum jeweiligen Kurvenparameter betragsmäßig mindestens 0,2 beträgt. Bei der Wahl der elementaren Funktionsterme wurde in besonderem Maße darauf geachtet, dass die Grenzwerte von Gl. 5.65 plausibel sind, falls eine Einflussgröße Null wird. So darf für den Fall, dass die Kavität von der Hauptströmung abgeschnitten ist ( $s=0)$ oder keine Strömung fließt $(R e=0)$, kein (erzwungener) Wärmeübergang im Seitenraum vorhersagt werden: $N u_{\text {Korr }}(x)=N u_{\text {max }} \stackrel{!}{=} 0$. Gemäß der Ähnlichkeitstheorie eignet sich hierfür ein Potenzansatz hervorragend [75]:

$$
y_{i}=a_{i} \prod_{j} x_{j}^{c_{i, j}}
$$

Die Startwerte für die Funktionskoeffizienten $a_{i}$ und $c_{i, j}$ wurden - teils durch Fitting, teils durch Augenmaß - aus den Input-Output-Diagrammen ermittelt. In einem abschließenden Schritt wurden dann die Werte aller insgesamt 21 Koeffizienten (vgl. Gl. 5.69 bis 5.72) noch mittels eines „globalen“ LSF fein angepasst. Dazu wurden für alle Konfigurationen die Nusselt-Zahlen gemäß Gl. 5.65 berechnet und die quadratischen Abweichungen zwischen den korrelierten $\left(N u_{\text {Korr }}\right)$ und den gemessenen Nusselt-Zahlen $\left(N u_{\text {mess }}\right)$ berechnet. Da die gemessenen WÜK an den äußeren Messringen, die teilweise von der Wand überdeckt sind, eine höhere Unsicherheit aufweisen als 
an den voll benetzen Ringen (siehe Kap. 5.8.3), wurden die quadrierten Differenzen noch mit der benetzten Breite $\Delta x_{\mathrm{S}, i}$ des jeweiligen Ringes $i$ gewichtet, bevor deren Summe minimiert wird:

$$
d N u o p t=\sum_{s, b, e, R e, \gamma} \sum_{i} \Delta x_{\mathrm{S}, i} \cdot\left[N u_{\mathrm{Korr}}\left(x_{i}\right)-N u_{\mathrm{mess}}\left(x_{i}\right)\right]^{2} \stackrel{!}{\rightarrow} \min
$$

Die dazugehörige gewichtete mittlere quadratische Abweichung („,Standardabweichung“) ist dann

$$
\sigma_{N u}=\sqrt{\frac{d N u o p t}{\sum_{s, b, e, R e, \gamma} \sum_{i} \Delta x_{\mathrm{S}, i}}}
$$

Es ergeben sich die folgenden vier Größengleichungen („Subkorrelationen“), die Gl. 5.65 komplettieren:

$$
\begin{gathered}
N u_{\max }=0,071 \operatorname{Re}^{0,634} \operatorname{Pr}^{0,4} \cdot\left(\frac{s}{h_{1}}\right)^{0,738} \cdot\left(\frac{b}{h_{2}}\right)^{-0,181} \cdot e^{-0,0147 \varepsilon} \cdot(\cos \gamma)^{-0,196} \\
\frac{\Delta N u}{N u_{\max }}=\left(1,4 \cos \gamma+0,178 \varepsilon^{2}\right) \cdot R e^{-0,112} \cdot\left(\frac{s}{h_{1}}\right)^{-0,0427} \cdot\left(\frac{b}{h_{2}}\right)^{0,397} \\
\frac{x_{\mathrm{S}}}{s}=0,607 \operatorname{Re}^{-0,00878} \cdot\left(\frac{s}{h_{1}}\right)^{0,383} \cdot\left(\frac{b}{h_{2}}\right)^{-0,0491} \cdot e^{-0,0674 \varepsilon} \\
\frac{\sigma_{x}}{b}=0,0555 R e^{0,13} \cdot\left(\frac{b}{h_{2}}\right)^{-0,364} \cdot(\cos \gamma)^{0,083}
\end{gathered}
$$

mit der dimensionslosen Exzentrizität

$$
\varepsilon=\left\{\begin{array}{cl}
0 & e=0, b=s \\
\frac{2 e}{b-s} & b>s
\end{array}\right.
$$

welche für die untersuchten Seitenraumkonfigurationen Werte zwischen \pm 1 annimmt.

Für den Exponent der Prandtl-Zahl in Gleichung 5.69 wurde ein konstanter Wert von $n=0,4$ angenommen, der häufig in Nusselt-Korrelationen für turbulente erzwungene Wärmeübertragung vorkommt und perspektivisch in weiteren Versuchen mit anderen Arbeitsmedien (z. B. Dampf) noch abgeklärt werden muss. Interessanterweise ergibt sich unter dieser Annahme ein Exponent in der Reynolds-Zahl von $m \approx 2 / 3$ und damit ebenfalls einen Wert, der in anderen turbulenten Nusselt-Korrelationen dokumentiert ist.

Die Korrelation ist gültig für $40.000 \leq R e \leq 115.000, \operatorname{Pr} \approx 0,7$ und $\gamma \leq 40^{\circ}$, kann aber auf Grund der Anlage der Gleichung aus rein mathematischer Sicht auch vorsichtig auf andere Reynolds- und Prandtl-Zahlen extrapoliert werden, dann jedoch noch ohne Gewährleistung der Genauigkeit. 
In Abb. 5.41 bis $>$ Abb. 5.44 sind für alle Versuchskonfigurationen die mittels der vorgeschlagenen Korrelation berechneten WÜK-Verläufe im Vergleich mit den gemessenen Werten aus Kap. 5.7 dargestellt. Wie sich zeigt, treffen die berechneten Kurven die Messpunkte recht gut. Erwartungsgemäß treten kleinere Abweichungen in den Fällen auf, bei denen die WÜK im linken und im rechten Teil der Kavität deutlich unterschiedlich sind. Dies ist zum Beispiel für die beiden stromabwärts gekrümmten Seitenräume mit positiver Exzentrizität der Fall (vgl. Abb. 5.43 a,b). Entgegen den Feststellungen in Kap. 5.7.6 berechnet die Korrelation für den stromaufwärts zeigenden L-förmigen Seitenraum in Abb. 5.43 e interessanterweise ein lokales Maximum unmittelbar an der rechten Totraumseitenwand. Dies ergibt sich aus der Mindergewichtung der Randpunkte beim Anpassen der Fitparameter und ist insofern plausibler, als dass der Prallstrahl nicht bis in den hintersten Winkel der oberen rechten Ecke des Seitenraums strömen kann, da er zuvor nach links in die Kavität umgeleitet werden muss. Auch für den Fall der quadratischen Kavität, bei der nur wenige lokale Messpunkte vorlagen und nur maximal zwei Messringe gleichzeitig voll benetzt sind, bildet die Korrelation die Zielwerte gut ab. Die Kurven fügen sich sogar sehr gut in die in Abb. 2.13 dargestellten, aus der Literatur zusammengetragenen Verläufe ein, von denen eine Auswahl noch einmal nicht-logarithmisch in $>$ Abb. 5.39 aufgetragen ist. Sowohl die leicht gebogene Kurvenform als auch die Größenordnung stimmen überraschend gut überein, obwohl es sich bei den Literaturfällen um ebene quadratische Kavitäten handelt.

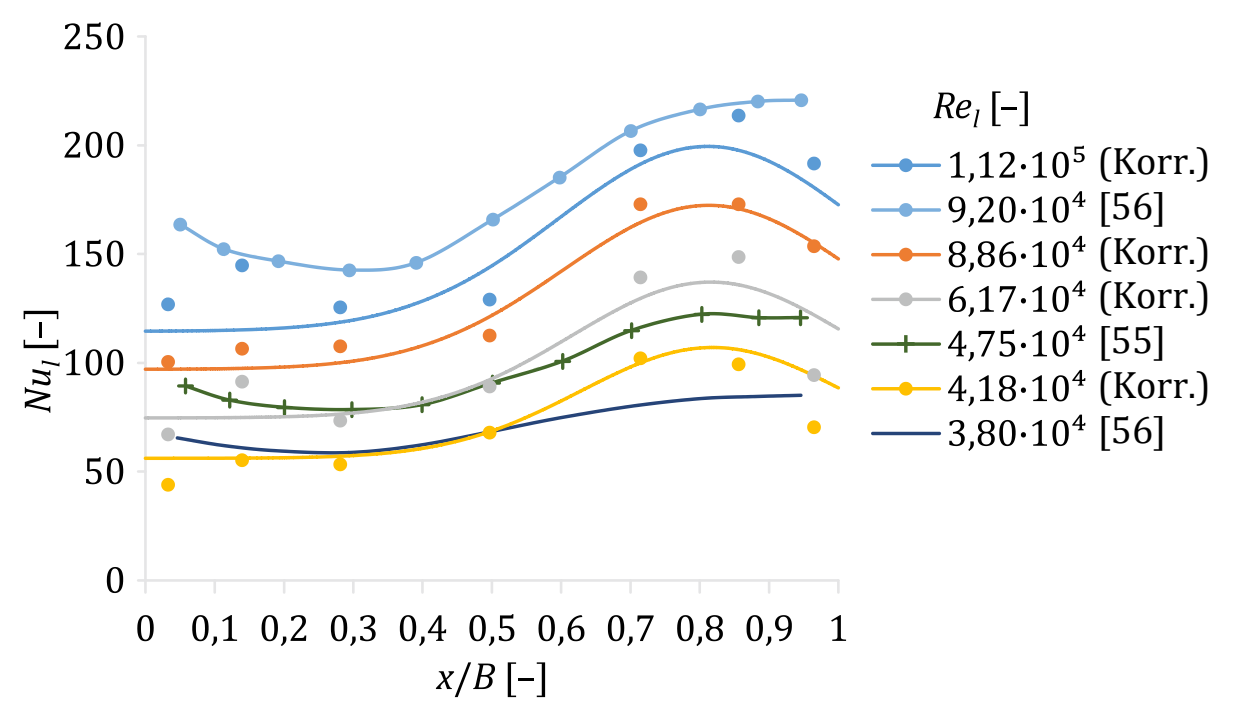

Abb. 5.39 Experimentell bestimmte Nusselt-Zahl-Verläufe im Seitenraum mit $s=b=46,3 \mathrm{~mm}$ im Vergleich zu Literaturwerten für ebene quadratische Kavitäten. 
Trägt man abschließend die mit der Korrelation berechneten über den gemessenen lokalen NusseltZahlen für alle Messpunkte in einem sog. Streudiagramm (Predicted-vs.-measured [scatter] plot) auf (Abb. 5.40), so zeigt sich, dass nahezu alle Punkte in einem Streuband von $\Delta N u_{\text {Korr }}= \pm 20$ um die Mitteldiagonale $N u_{\text {Korr }}=N u_{\text {mess }}$ streuen, was als relativ kleiner Fehler infolge der begrenzten Approximationsgenauigkeit der Korrelation gewertet werden kann. Die wenigen Ausreißer (Outlier) sind ausnahmslos die (weniger gewichteten) Randpunkte, für die etwas geringere Werte berechnet werden als tatsächlich (aus der Rückwärtsrechnung) ermittelt wurden, deren Genauigkeit dort jedoch wie mehrfach besprochen abfällt. Die gewichtete Standardabweichung zwischen korrelierten und gemessenen Werten für alle Messpunkte beträgt gemäß Gleichung $5.68 \sigma_{N u}=8,17$. Für die Versuche im Luftversuchsstand entspricht dies mit Gl. 5.64 einer mittleren Abweichung im WÜK von $\sigma_{\alpha}=5,99 \mathrm{~W} /\left(\mathrm{m}^{2} \mathrm{~K}\right)$.

Es soll an dieser Stelle noch einmal explizit darauf hingewiesen werden, dass die Nusselt-Korrelation (Gleichungen 5.65, 5.69-5.73) nur streng für die im Rahmen der vorliegenden Dissertation untersuchten Konfigurationen und die in $>$ Abb. 5.29 bis $>$ Abb. 5.32 gezeigten Versuche gültig ist. Das gilt insbesondere für die radialen Abmessungen von Hauptströmungskanal $(\Delta r)$ und Seitenraum $\left(h_{1}, h_{2}\right)$, die im Versuchsstand nicht variiert werden konnten. Sie wurden als konstante Werte in die Ähnlichkeitssimplexe mit eingerechnet, um mit dimensionslosen Verhältnissen arbeiten zu können. $\mathrm{Ob}$ und inwieweit die funktionalen Zusammenhänge auch für variierte Höhenmaße zutreffen, kann hier nicht geklärt werden und sollte ein zentraler Gegenstand weiterführender Arbeiten sein.

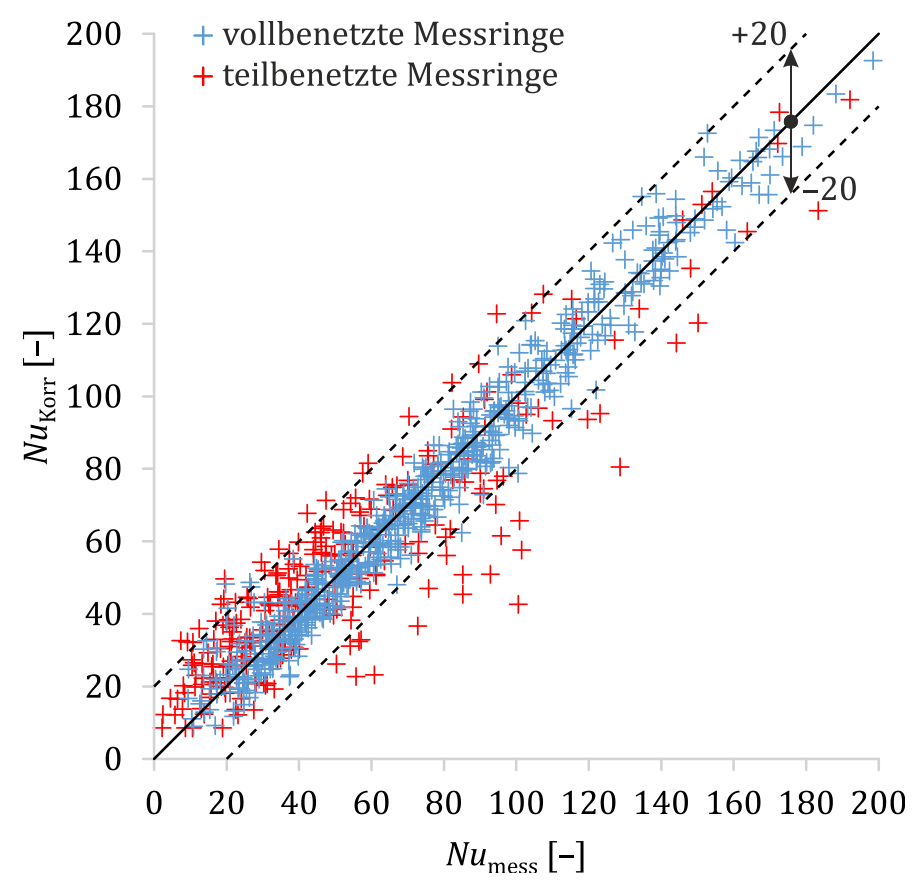

Abb. 5.40 Streudiagramm der mittels Korrelation für alle Messpunkte und Konfigurationen berechneten über den gemessenen lokalen Nusselt-Zahlen (predicted vs measured plot). 

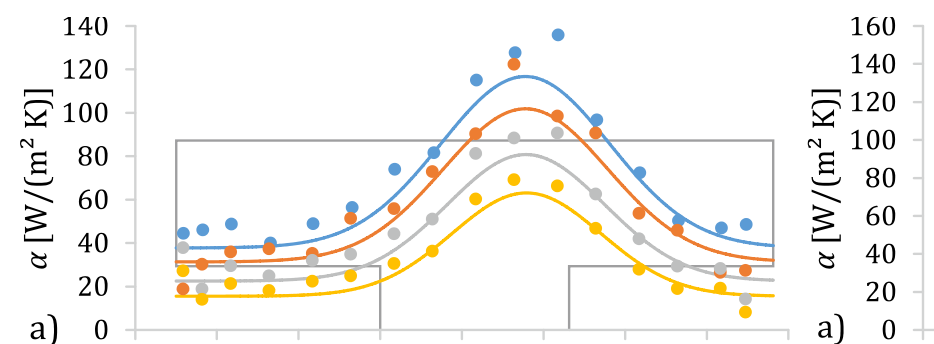

a) 0
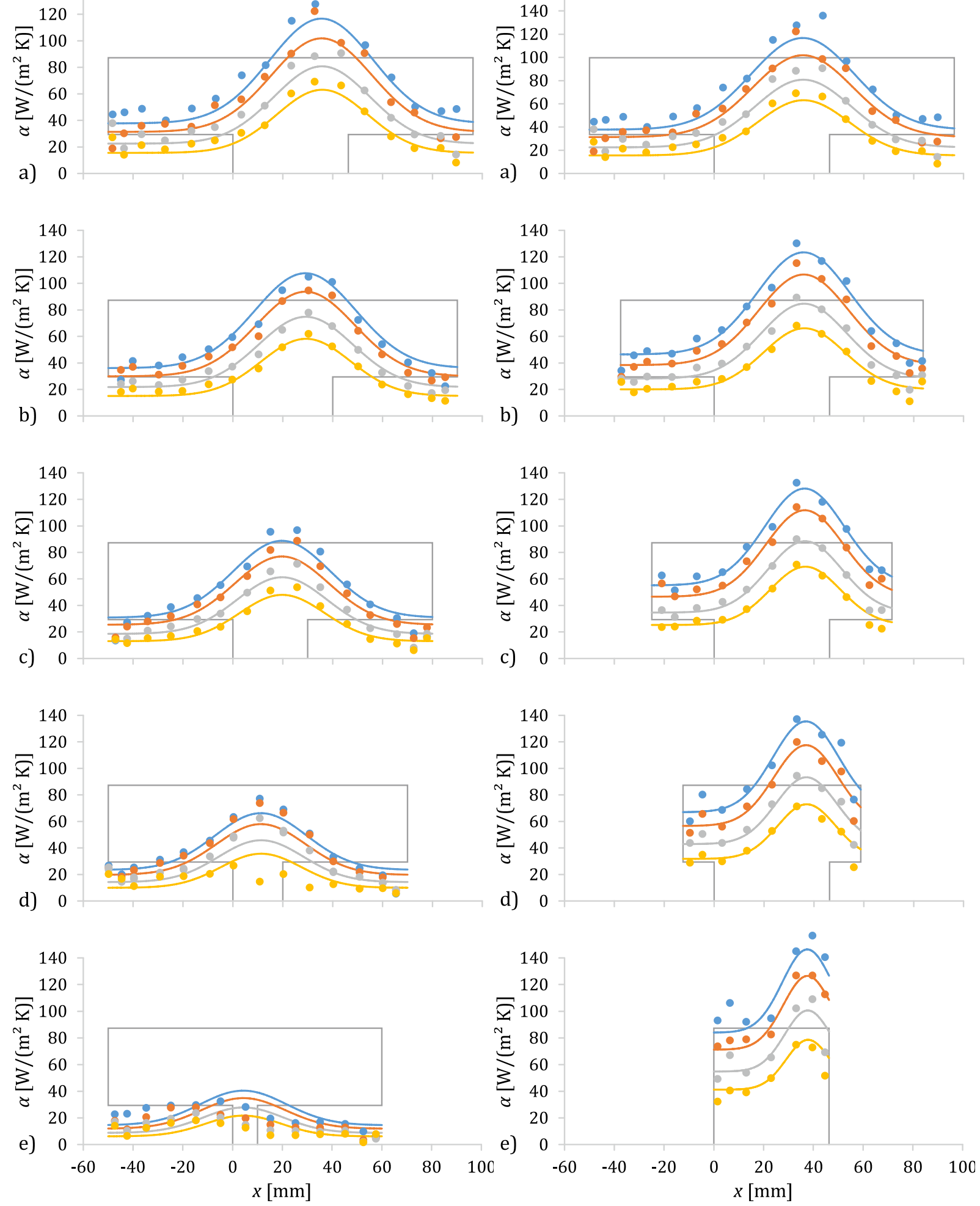

Abb. 5.41 Vergleich der Nusselt-Korrelation mit Abb. 5.42 Vergleich der Nusselt-Korrelation mit der gemessenen WÜK-Verteilung im Seitenraum für variierte Einströmbreite $s$. tenraum für variierte Totraumbreite $b$. 

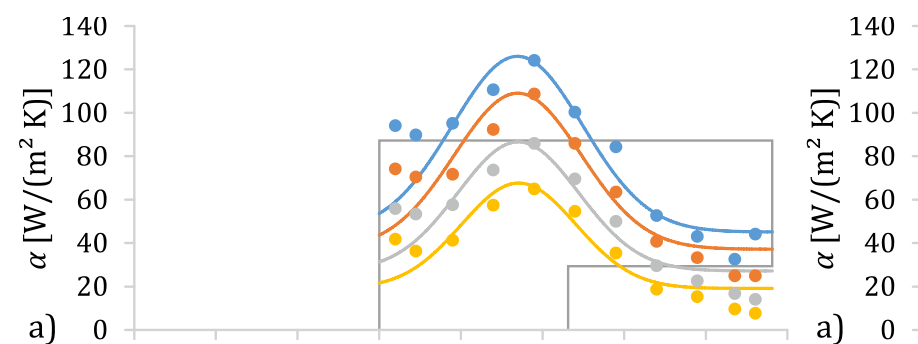

a) 0
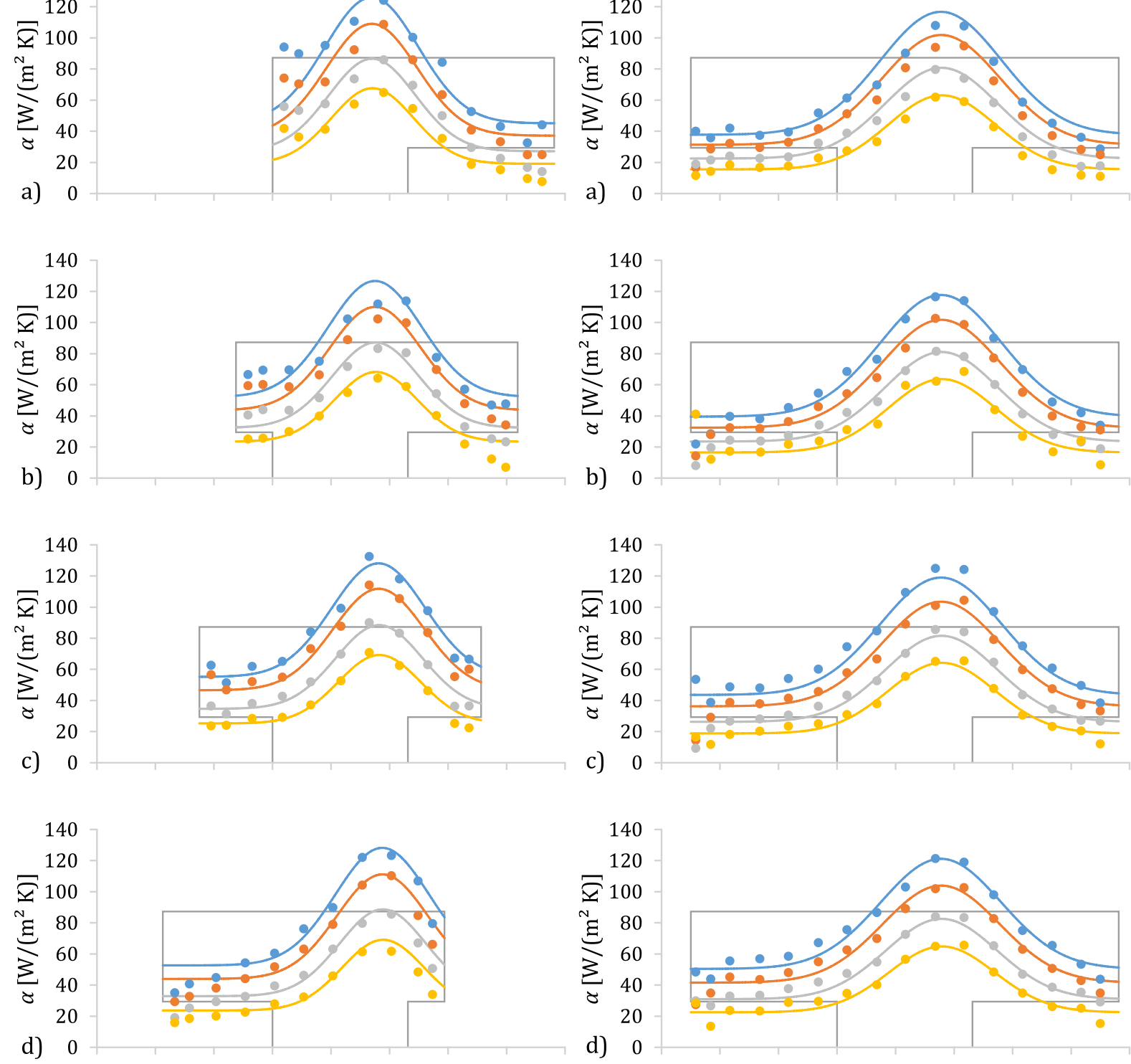

c) 0
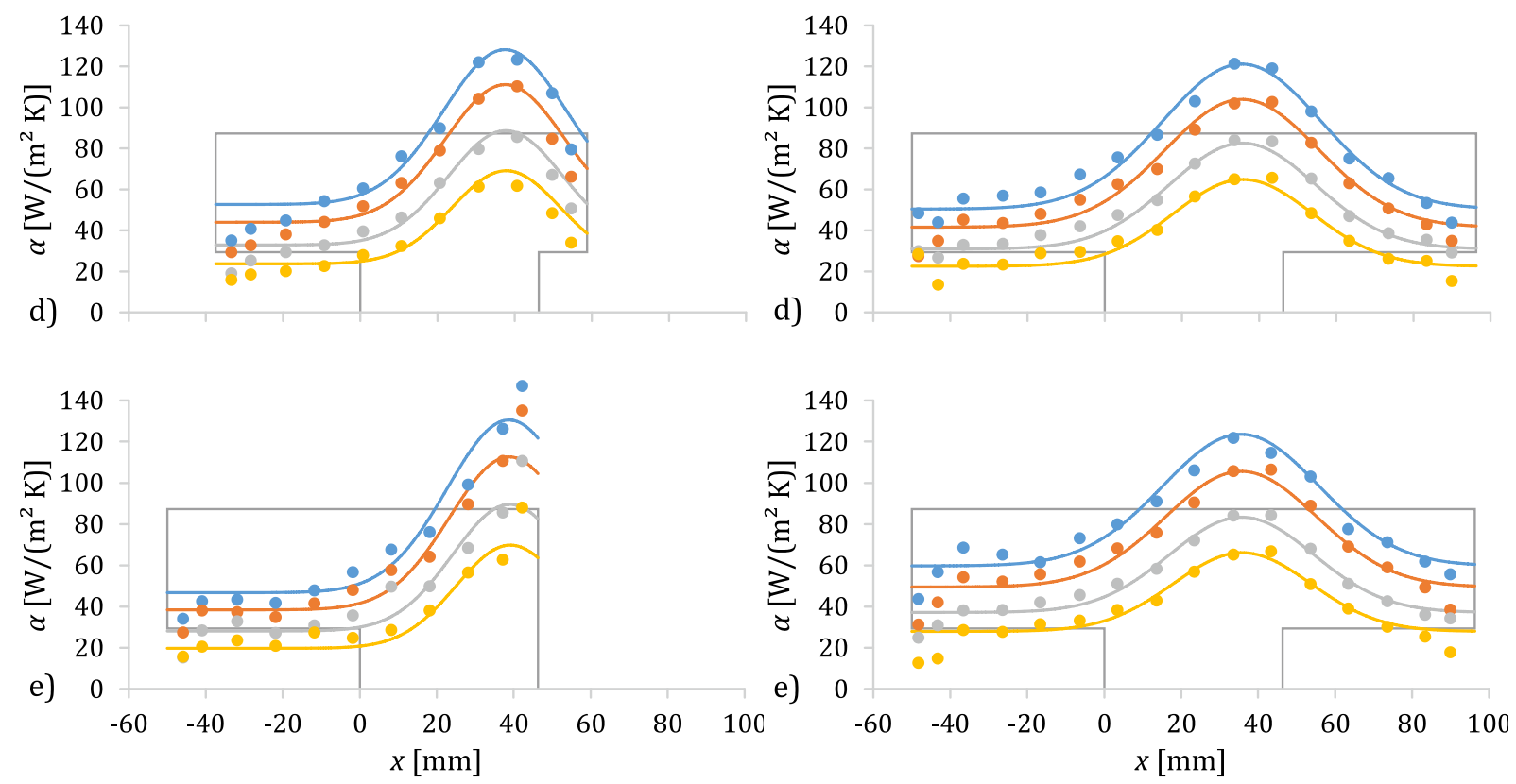

Abb. 5.43 Vergleich der Nusselt-Korrelation mit Abb. 5.44 Vergleich der Nusselt-Korrelation mit der gemessenen WÜK-Verteilung im der gemessenen WÜK-Verteilung im Seitenraum für variierte Exzentrizität $e$. 


\section{NUMERISCHE NACHRECHNUNG AUSGEWÄHLTER KONFIGURATIONEN MITTELS CFD-SIMULATION}

In diesem Kapitel soll nun überprüft werden, inwieweit die lokale WÜK-Verteilung in einzelnen Versuchskonfigurationen mittels numerischer Strömungsmechanik nachgebildet bzw. vorhergesagt werden kann und ob sich nicht experimentell untersuchte (Zwischen-) Konfigurationen zuverlässig nur noch auf numerischen Wege studieren lassen.

Die einzusetzenden (kommerziellen) CFD-Tools sollen dabei nur als Werkzeug dienen, d. h. es soll festgestellt werden, mit welchen vordefinierten Programmeinstellungen sich das bestmögliche Ergebnis erzielen lässt, um dem potenziellen Anwender einen Leitfaden mit einigen Best-PracticeRichtlinien an die Hand zu geben, mit dem er schnell und zielstrebig zum Ziel gelangt. Es kann und soll keine modelltheoretische Grundlagenuntersuchung stattfinden, weswegen an dieser Stelle explizit auf die Diskussion der theoretischen Grundlagen und Gleichungen verzichtet werden soll.

\subsection{CFD-BASISMODELL}

Alle folgenden CFD-Analysen wurden in der Simulationsumgebung Ansys Workbench 18.2 durchgeführt. Die Software gestattet einen strukturierten Workflow vom Aufbau eines vollparametrischen Geometriemodells ( Kap. 6.1.1), der automatischen Vernetzung mit immer gleichen Einstellungen ( Kap. 6.1.2), die ebenfalls parametrische Festlegung von Randbedingungen ( Kap. 6.1.3) und Simulationseinstellungen ( Kap. 6.1.4) bis hin zur Lösung des Problems ( Kap. 6.1.6) und der Auswertung der Ergebnisse ( Kap. 6.1.7).

\subsubsection{Geometrie}

Als Referenzgeometrie für das Basismodell dient ein symmetrischer Seitenraum mit T-förmigem Querschnitt und den maximal mit dem Versuchsstand realisierbaren Abmessungen $(s=46,3 \mathrm{~mm}, b=146,3 \mathrm{~mm}, e=0 \mathrm{~mm}$; andere Maße siehe Abb. 4.4).

Die Einlaufstrecke wurde in Richtung der Referenzdruckbohrung ( Abb. 6.1) verlängert und besitzt eine Länge von $L_{\text {ein }}=190 \mathrm{~mm}$. Der Auslauf erstreckt sich bis zum Ende des Innenrohrmoduls, kurz bevor die Strömung von dem ringspaltförmigen Hauptströmungskanal wieder in den Rohrquerschnitt übergeht. Die Länge der Nachlaufstrecke beträgt $L_{\text {aus }}=235 \mathrm{~mm}-s$.

Zum Einsatz kommen ein reines 2D-Modell (für Ansys Fluent), ein Sektormodell mit Umfangswinkeln zwischen 5 und $90^{\circ}$ sowie ein $360^{\circ}$-Vollmodell (für Ansys CFX).

Für die voll parametrisierte Geometrieerstellung dient das Programm Ansys DesignModeler 18.2. 


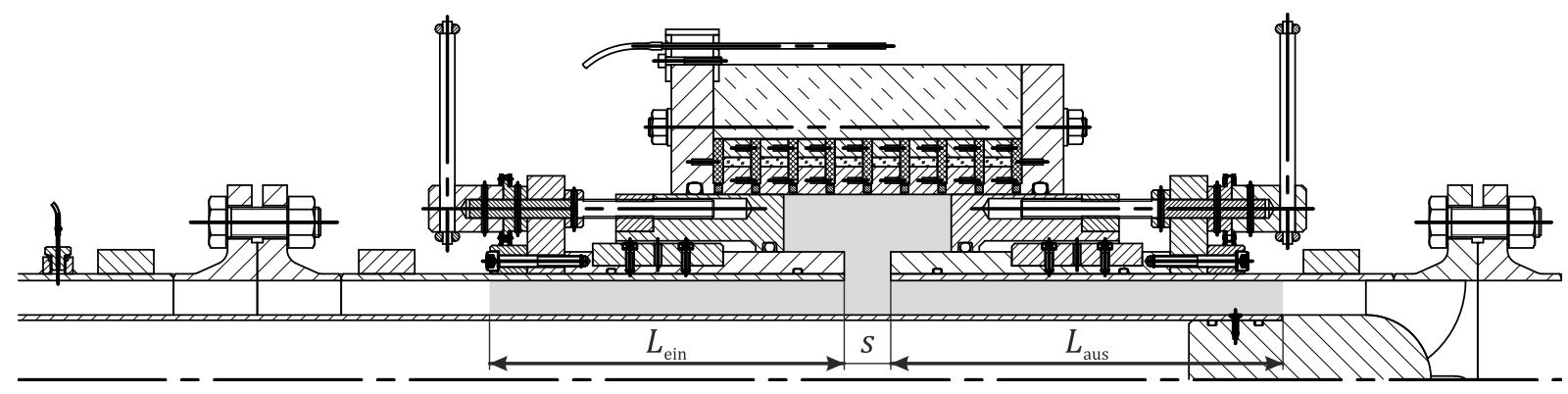

Abb. 6.1 Geometrie, Ein- und Auslaufstrecken des CFD-Basismodells (graue Schattierung) und Abbildung der Versuchsstandgeometrie. Im Bild dargestellt: $s=25 \mathrm{~mm}, b=90 \mathrm{~mm}, e=0$.

\subsubsection{Vernetzung}

Für alle Modelle wird ein strukturiertes Rechennetz in Ansys Meshing 18.2 generiert ( Abb. 6.2 a), welches ausschließlich aus Hexaederelementen (2D-Modelle: aus Viereckelementen, sog. Quads) mit Elementmittenknoten besteht. Für die Elementgröße des freien Netzes wurde ein Wert von $1 \mathrm{~mm}$ gewählt. Die wandnahen Bereiche wurden mittels Inflationsschicht verdichtet, wobei die Dicke der ersten („,wandnächsten“) Schicht, der geometrische Wachstumsfaktor sowie die Anzahl der Schichten festgelegt wurde, um in einer Netzunabhängigkeitsstudie gezielt den Einfluss der ersten Zelle auf den Wärmeübergang untersuchen zu können (siehe Kap. 6.3). Die wichtigsten Vernetzungseinstellungen sind in $>$ Tab. A.8 aufgelistet. Mit denselben Einstellungen wurde ein alternatives (blockstrukturiertes) Rechengitter in ICEM CFD 18.2 erstellt ( Abb. 6.2 b), bei dem die ersten zwölf Elemente dieselbe Höhe $(5 \mu \mathrm{m})$ in wandnormaler Richtung aufweisen wie die erste Zelle an der Wand, bevor die Dicke der nächsten 18 Schichten geometrisch mit einer Wachstumsrate von 1,31 anwächst ( Kap. 6.3).

Für das Basismodell ergeben sich die in $>$ Tab. A.9 aufgeführten Elementanzahlen.

a)
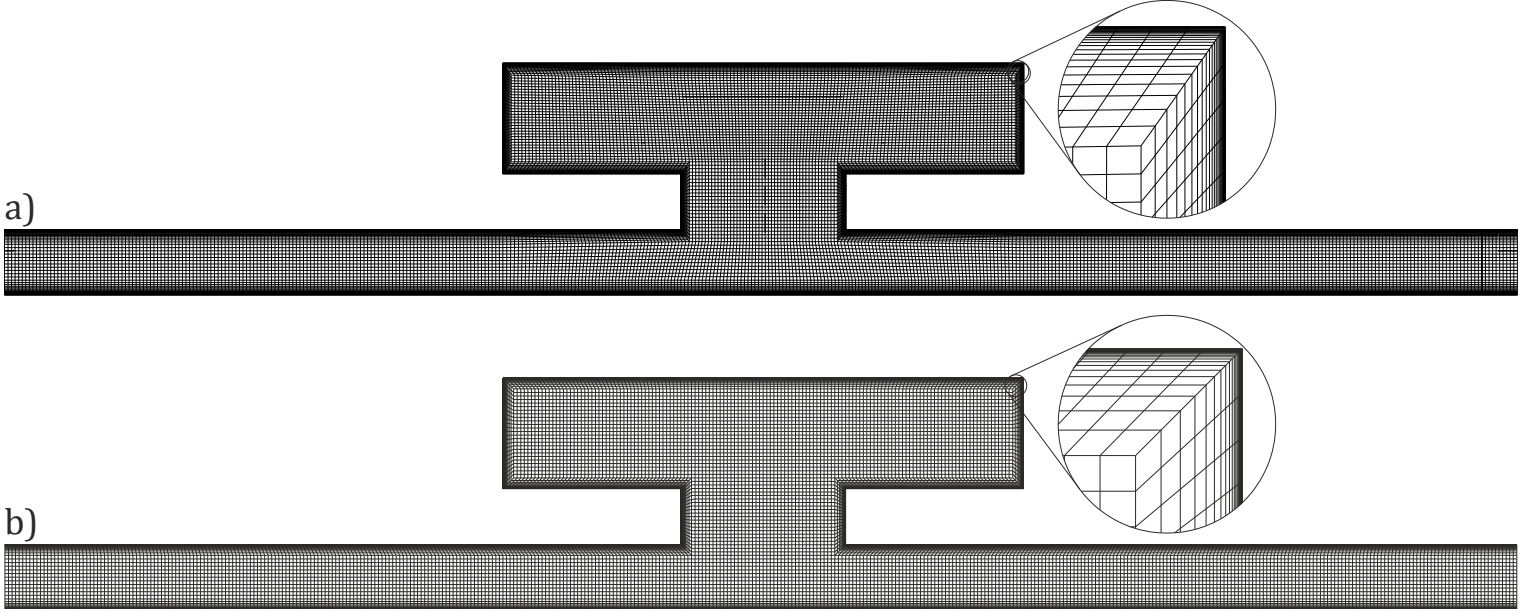

Abb. 6.2 Rechennetz des Basismodells. a) Vorder- und Seitenansicht des $5^{\circ}$-Modells aus Ansys Meshing; b) Vorderansicht des 2D-Modells aus ICEM CFD. 
Für eine konjugierte Simulation des Wärmeübergangs (conjugate heat transfer, CHT) mit FluidStruktur-Kopplung (fluid-solid interaction, FSI) wurde ein weiteres 2D-CFD-Modell mit zusätzlichen Wandstrukturen (solid domain) erstellt (Abb. 6.3). Das Fluidgebiet wurde dabei mit denselben Einstellungen wie in $>$ Tab. A.8 vernetzt und weist daher quasi ein identisches Netz auf wie in Abb. 6.2 b. Beim Solidgebiet besitzen die (Einström-) Seitenwände sowie die Außenwand die gleiche Geometrie wie das FE-Modell in $\$$ Kap. 4.4.4, nur deren Anordnung wurde an den vorliegenden Fall angepasst. Im Gegensatz zu $>$ Abb. 4.21 besteht das Solid-Netz (ähnlich wie das FreeFEM-Netz in Abb. 4.32) ausschließlich aus Tetraeder-Elementen (Elementgröße: $1 \mathrm{~mm}$ ), wobei sich die insgesamt acht verschiedenen (Ober-) Flächenkörper (einer je Werkstoff, siehe Tab. 4.5 und Abb. 4.21) an ihren gemeinsamen Trennlinien jeweils gemeinsam die Knoten teilen (conformal meshing). Das Netz der Wandstrukturen besteht aus 53.736 Elementen (108.897 Knoten). Zusammen mit dem Fluidnetz (hier: 46.646 Elemente, 141.261 Knoten) kommt das „CHT-Netz“ damit insgesamt auf 100.382 Elemente (250.158 Knoten).

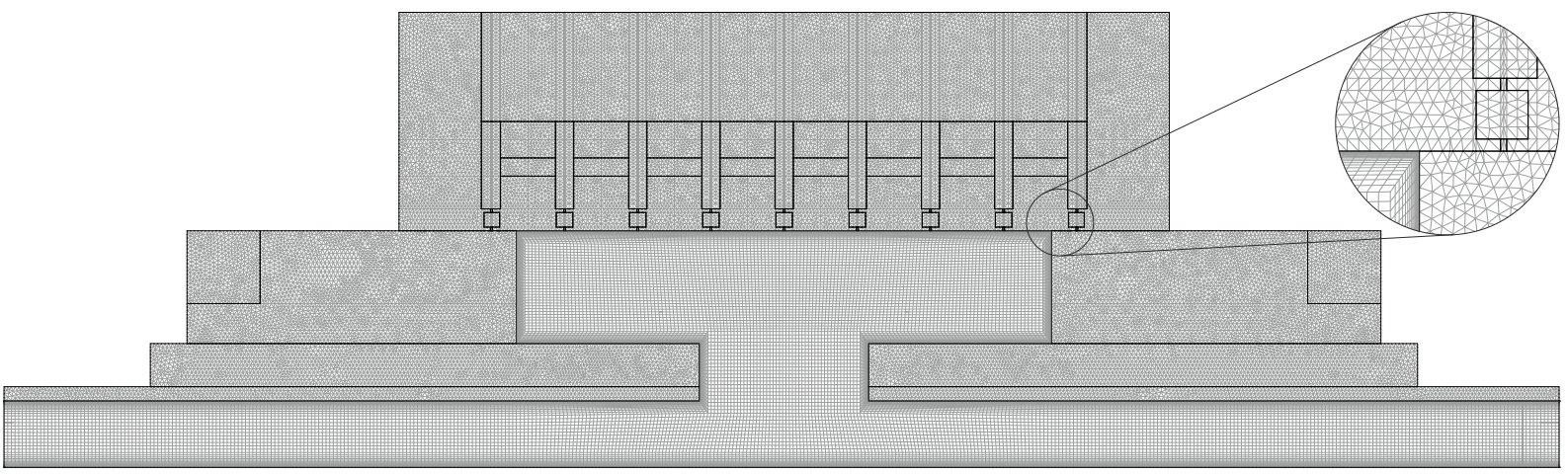

Abb. 6.3 Rechennetz des Modelles für die konjugierte WÜ-Simulation („CHT-Netz“).

\subsubsection{Randbedingungen}

Im Grundmodell wurde am Einlass ein über die gesamte Fläche als konstant angenommener Totaldruck vorgegeben, der dem an der dortigen Wandbohrung gemessenen Referenzdruck entspricht. Die Strömungsrichtung wird normal zur Fläche definiert, außer für CFD-Untersuchungen mit Drall (siehe unten). Ferner wurde die mit den beiden Temperaturmesssonden vor- und nach dem Versuchsstand ermittelte Referenztemperatur ( Kap. 4.4.2) als Totaltemperatur am Einlass gesetzt. Als Turbulenzrandbedingungen wurden ein isotroper Turbulenzgrad von Tu $=5 \%$ und eine Wirbellänge von $\lambda_{x}=2,84 \mathrm{~mm}$ festgesetzt. Diese Werte entsprechen den Bedingungen in einem Abstand von $x=25,2 \mathrm{~mm}$ hinter dem Turbulenzgitter („Sieb“, „Filtereinsatz“, „Gaze“; $>$ Abb. A.18 a) mit quadratischen Maschen und einem Drahtdurchmesser von $d=0,32 \mathrm{~mm}$, die nach den in der Literatur häufig verwendeten empirischen Korrelationen nach [144] wie folgt berechnet werden: 


$$
\begin{gathered}
T u=1,13\left(\frac{x}{d}\right)^{-\frac{5}{7}} \\
\frac{\lambda_{x}}{d}=0,2 \sqrt{\frac{x}{d}}
\end{gathered}
$$

Demnach sind Tu und $\lambda_{x}$ unabhängig von der Maschenweite und von der Reynolds-Zahl.

Am Auslass wurde der über die Blendenmessstrecke bestimmte Massenstrom gesetzt, der anteilig durch den Winkelsektor des CFD-Modells strömt:

$$
\dot{m}_{\mathrm{aus}}=\dot{m} \cdot \frac{\varphi}{360^{\circ}}
$$

für ein $90^{\circ}$-Modell beispielsweise ein Viertel des Gesamtmassenstroms.

Für die Untersuchungen mit Ansys Fluent sowie für die Nachrechung der Fälle mit Drallströmung generell hat sich eine Vertauschung der Ein- und Auslass-Impuls-Randbedingungen als zweckmäßig erwiesen: dort werden am Einlass der Massenstrom nach Gleichung 6.3 beziehungsweise die Geschwindigkeitskomponenten im Zylinderkoordinatensystem vorgegeben, wobei die axiale Geschwindigkeit $c_{\mathrm{ax}}$ mit der querschnittsgemittelten Geschwindigkeit $q$ aus Gleichung 4.12 gleichgesetzt und die Umfangskomponente $c_{\mathrm{u}}$ aus dem eingestellten Blechwinkel $\gamma$ des Drallgitters berechnet wird:

$$
\begin{gathered}
c_{\mathrm{ax}}=q \\
c_{\mathrm{r}}=0 \\
c_{\mathrm{u}}=q \tan \gamma
\end{gathered}
$$

Am Auslass wird ein mittlerer statischer Druck in Höhe des Referenzdruckes gesetzt sowie eine maximale Abweichung von diesem Mittelwert von $\delta p_{\max , A}= \pm 5 \%$ über der gesamten Fläche zugelassen, die jedoch in der Rechnung bei weitem nicht ausgeschöpft wird. Der Einfluss dieser Vertauschung der Randbedingungen ist in $>$ Kap. 6.4 beleuchtet.

Alle Wände werden mit einer Haftbedingung (no slip) und optional mit einer äquivalent Sandkornrauigkeit versehen, die nach [145] ziemlich genau der gemittelten Rautiefe („Zehnpunkthöhe") $R z$ entspricht

$$
k_{\mathrm{S}} \approx 0,978 \cdot R z
$$

und für die ein Richtwert von $16 \mu$ m für gedrehte Oberflächen angenommen wurde [146]. Für die den Seitenraum radial nach außen hin begrenzende, beheizte Außenwand wird entweder ein konstanter Wandwärmestrom von $\dot{Q}=4 \mathrm{~W}$ pro Ring, entsprechend 


$$
\overline{\hat{q}}_{\mathrm{W}}=\frac{\dot{Q}}{\pi D b}=318 \frac{\mathrm{W}}{\mathrm{m}^{2}}
$$

oder eine konstante Wandtemperatur in Höhe der über die benetzten Ringflächen gewichteten mittleren Temperatur der Innenringe angesetzt:

$$
\bar{T}_{\mathrm{W}}=\frac{1}{b} \sum_{i=1}^{8} T_{1, i} \cdot B_{i, \text { benetzt }}
$$

Alle übrigen Wände werden als adiabat betrachtet, da über sie auf Grund ihrer Isolationseigenschaften entweder nur ein vernachlässigbar kleiner Wärmestrom fließt (wie im Fall der übrigen Seitenraumbegrenzungen) oder der thermische Zustand der Flächen für den Wärmeübergang an der Außenwand keine Bedeutung hat.

Für die Sektormodelle werden die beiden Schnittflächen („hohe und niedrige Geometrie“) mit einer Rotationssymmetrie versehen, die für Dralluntersuchungen sogar eine notwendige Voraussetzung ist. Rotationssymmetrie hat sich jedoch auch bei drallfreien Fällen gegenüber den dort üblicherweise verwendeten Randbedingungen (Spiegelsymmetrie bzw. free slip-Wänden ohne Wandschlupf) als günstiger für die Konvergenz erwiesen, da Störungen durch die Trennflächen (Interfaces) durchlaufen können, was numerisch dämpfend wirkt. Außerdem weist die resultierende WÜK-Verteilung auf der Außenwand (vor allem bei kleinen Sektorwinkeln) dann keine unplausiblen Randeffekte auf. Alle Randbedingungen sind noch einmal in Tab. A.10 (Ansys CFX) bzw. Tab. A.11 (Ansys Fluent) zusammengefasst.

Für das CHT-Modell ( Abb. 6.3) wurden in Ansys Fluent die Wände in Tab. A.11 durch Kontaktflächen (fluid-solid interfaces) ersetzt ( Tab. A.12). Die Übergangsbedingungen (ÜB) zwischen den verschiedenen Werkstoff-Bereichen innerhalb der solid domain wurden durch Wände und gespiegelte „Schattenwände“ (shadow walls) realisiert, die im Prinzip genauso funktionieren wie Interfaces, auf Grund der deckungsgleichen Netze jedoch keine zusätzliche Nichtlinearität in das Problem einbringen. An den Netzverbindungsstellen zwischen Aluminium und Armaflex (vgl.

Abb. 4.21) wurde zusätzlich eine volumetrische Wärmequelle vorgesehen, die auf eine virtuelle Schichtdicke von $1 \mathrm{~mm}$ (nicht als Element modelliert sondern gerechnet) eine Wärmemenge von insgesamt $32 \mathrm{~W}(8 \times 4 \mathrm{~W})$ aufbringt $\left(\tilde{q}=326.429 \mathrm{~W} / \mathrm{m}^{3}\right)$. Als äußere Randbedingungen für das Solid-Gebiet wurde wiederum freie Konvektion an die Umgebung angenommen $\left(\alpha_{\mathrm{a}}=\alpha_{\mathrm{AW}}=\right.$ $10 \mathrm{~W} /\left(\mathrm{m}^{2} \mathrm{~K}\right)$; vgl. Abb. 4.22). 


\subsubsection{Medium/Stoffkennwerte}

Als Medium für die Simulationen wurde trockene technische Luft als Idealgas mit einer molaren Masse von $M=28,96 \mathrm{~kg} / \mathrm{kmol}$ angenommen. Die eventuelle Restfeuchte in der Versuchsluft ist auf Grund des Trockners in beiden Verdichtern gering ( Kap. 4.1.4) und wird daher vernachlässigt. Die Dichte der Luft berechnet sich in Abhängigkeit von statischem Druck und Temperatur gemäß der Idealgasgleichung zu

$$
\rho(p, T)=\frac{p}{R T}
$$

mit der spezifischen Gaskonstante

$$
R=\frac{\bar{R}}{M}
$$

Gegenüber dem in Ansys CFX verfügbaren Fluid Air Ideal Gas, welches nur für einen Normdruck von $101325 \mathrm{kPa}$ und eine Temperatur von $25^{\circ} \mathrm{C}$ Gültigkeit besitzt (eigentlich ein perfektes Gas)

$$
\begin{array}{cc}
M=28,96 \mathrm{~kg} / \mathrm{kmol} & p_{\text {ref }}=1 \mathrm{~atm}=101,325 \mathrm{kPa} \\
c_{p}=1004,4 \mathrm{~J} /(\mathrm{kg} \mathrm{K}) & h_{\mathrm{ref}}=0 \mathrm{~J} /(\mathrm{kg} \mathrm{K}) \\
\eta=1,831 \cdot 10^{-5} \mathrm{~Pa} \mathrm{~s} & s_{\mathrm{ref}}=0 \mathrm{~J} /(\mathrm{kg} \mathrm{K}) \\
\lambda=0,0261 \mathrm{~W} /(\mathrm{m} \mathrm{K}) & T_{\mathrm{ref}}=25^{\circ} \mathrm{C}
\end{array}
$$

bzw. dem in Ansys Fluent vorgefertigten Äquivalentes air

$$
\begin{gathered}
M=28,966 \mathrm{~kg} / \mathrm{kmol} \\
c_{p}=1006,43 \mathrm{~J} /(\mathrm{kg} \mathrm{K}) \\
\eta=1,7894 \cdot 10^{-5} \mathrm{~Pa} \mathrm{~s} \\
\lambda=0,0242 \mathrm{~W} /(\mathrm{m} \mathrm{K})
\end{gathered}
$$

$$
\begin{gathered}
p_{\text {ref }}=101,325 \mathrm{kPa} \\
h_{\text {ref }}=0 \mathrm{~J} /(\mathrm{kg} \mathrm{K}) \\
s_{\text {ref }}=194,336 \mathrm{~J} /(\mathrm{kg} \mathrm{K}) \\
T_{\text {ref }}=25^{\circ} \mathrm{C}
\end{gathered}
$$

wurde in einem ersten Schritt ein benutzerdefiniertes Material (CFX: Air SiSTer; Fluent: air-sister) erstellt, bei dem die Transportgrößen $\left(\eta, \lambda, c_{p}\right)$ mit Hilfe der Stoffdatenbank LibIDGAS der Hochschule Zittau-Görlitz für Ideale Gasgemische nach VDI 4670 aus den jeweiligen Mittelwerten für reine technische Luft bei (versuchsstandüblichen) Temperaturen zwischen $T=20 \ldots 35^{\circ} \mathrm{C}$ und einem Druck von $p=2$ bar gebildet worden sind:

$$
\begin{array}{cc}
M=28,96 \mathrm{~kg} / \mathrm{kmol} & p_{\mathrm{ref}}=101,325 \mathrm{kPa} \\
c_{p}=1004,97 \mathrm{~J} /(\mathrm{kg} \mathrm{K}) & h_{\mathrm{ref}}=0 \mathrm{~J} /(\mathrm{kg} \mathrm{K}) \\
\eta=1,8403 \cdot 10^{-5} \mathrm{~Pa} \mathrm{~s} & s_{\mathrm{ref}}=0 \mathrm{~J} /(\mathrm{kg} \mathrm{K}) \\
\lambda=0,02682 \mathrm{~W} /(\mathrm{m} \mathrm{K}) & T_{\mathrm{ref}}=0{ }^{\circ} \mathrm{C}
\end{array}
$$


Für weiterführende Untersuchungen wurde bei gleichen Referenzwerten wie oben ein Fluidmodell (CFX: Air; Fluent: air-temp) mit temperaturabhängigen Transportgrößen generiert, welches für Temperaturen von $T=0{ }^{\circ} \mathrm{C}$ bis $100{ }^{\circ} \mathrm{C}$ gültig ist. Dazu wurden in $0,5-\mathrm{K}-\mathrm{Sch}$ ritten die Stoffwerte für $c_{p}, \eta$ und $\lambda$ berechnet und daraus unter Anwendung der Methode der kleinsten Fehlerquadrate Polynome unterschiedlichen Grades gebildet:

$$
\begin{aligned}
\frac{c_{p}(T)}{\mathrm{kJ}}= & 6,97455 \cdot 10^{-13}\left(\frac{T}{\mathrm{~K}}\right)^{4}-4,55380 \cdot 10^{-10}\left(\frac{T}{\mathrm{~K}}\right)^{3}+3,36916 \cdot 10^{-7}\left(\frac{T}{\mathrm{~K}}\right)^{2} \\
& -1,03927 \cdot 10^{-4} \frac{T}{\mathrm{~K}}+1,01244 \\
\frac{\eta(T)}{\mathrm{Pa} \mathrm{s}}= & 4,36546 \cdot 10^{-14}\left(\frac{T}{\mathrm{~K}}\right)^{3}-7,42985 \cdot 10^{-11}\left(\frac{T}{\mathrm{~K}}\right)^{2}+7,87156 \cdot 10^{-8} \frac{T}{\mathrm{~K}}+2,66908 \cdot 10^{-7} \\
\frac{\lambda(T)}{\frac{\mathrm{W}}{\mathrm{m} \mathrm{K}}}= & -3,42663 \cdot 10^{-8}\left(\frac{T}{\mathrm{~K}}\right)^{2}+8,87149 \cdot 10^{-5} \frac{T}{\mathrm{~K}}+3,24267 \cdot 10^{-3}
\end{aligned}
$$

Die Gleichungen sind noch einmal im $>$ Anhang A13.3 im Ansys CCL-Format (CFX Command Language) hinterlegt.

Extrapolation der Stoffwerte wurde nicht gestattet, um unphysikalische Zwischenlösungen während des Iterationsprozesses zu vermeiden, die sonst im schlimmsten Fall zur völligen Divergenz des Problems führen können. Stattdessen werden die Werte bei der jeweiligen Maximal- bzw. Minimaltemperatur festgehalten („Clipping“), sollte bei einem Zwischenschritt (temporär) die jeweilige Temperatur über- bzw. unterschritten werden.

Je nachdem, ob $c_{p}$ temperaturabhängig ist oder nicht, ergeben sich spezifische Enthalpie und Entropie zu:

$$
\begin{gathered}
c_{p}=\text { konst. } \\
\downarrow \\
h-h_{\mathrm{ref}}=\int_{T_{\mathrm{ref}}}^{T} c_{p}(T) d T=c_{p}\left(T-T_{\mathrm{ref}}\right) \\
c_{p}=\text { konst. } \\
\downarrow-S_{\mathrm{ref}}=\int_{T_{\mathrm{ref}}}^{T} \frac{c_{p}(T)}{T} d T-R \ln \frac{p}{p_{\mathrm{ref}}}=c_{p} \ln \frac{T}{T_{\mathrm{ref}}}-R \ln \frac{p}{p_{\mathrm{ref}}}
\end{gathered}
$$




\subsubsection{Physikalische Modellierung/ Setup}

Für alle Simulationen wurde die Energiegleichung gelöst ohne Berücksichtigung von Temperatureffekten infolge lokal unterschiedlicher kinetischer Energie sowie ohne Energiedissipation infolge viskoser Reibung. Beide Effekte spielen bei subsonischen Strömungen, die weitgehend inkompressibel sind $(M a<0,3)$, eine sehr untergeordnete Rolle. In Ansys CFX steht hierfür das Thermal Energy-Modell zur Verfügung. Weiterhin wurden Auftrieb und Strahlung vernachlässigt, was auf Grund der beabsichtigt geringen Temperaturunterschiede zwischen Wand und Fluid zulässig ist und die Konvergenz der Lösung weiter verbessert.

Der Referenzdruck für die Simulation beträgt 0 bar (nicht zu verwechseln mit dem Referenzdruck der Stoffwerte, der z. B. für die Berechnung der spezifischen Entropie benötigt wird). Es wird also mit Absolutdrücken gerechnet, weshalb auch Absolutwerte für den Druck am Ein- bzw. Auslass gesetzt werden müssen. Besonders für Ansys Fluent hat sich das Setzen des Absolut- anstelle des Relativdruckes am Auslass sogar als notwendig erwiesen, da der Solver sonst keine Möglichkeit hat, das Druckprofil um den voreingestellten Wert (z. B. \pm 5 \%) zu variieren, sondern lokale Druckunterschiede durch Rückströmung auszugleichen versucht. In Ansys CFX wird dies zwar verhindert, indem temporär oder dauerhaft betroffene Elemente des Auslasses „verschlossen“, d. h. als Wand modelliert werden. Für die korrekte Abbildung des Strömungsfeldes sind jedoch beide Effekte nicht wünschenswert.

Zur korrekten, einheitlichen und vergleichbaren Berechnung der Wärmeübergangskoeffizienten muss in Ansys CFX der Expertenparameter „tbulkforhtc“ bzw. in Ansys Fluent die Referenztemperatur korrekt gesetzt werden. Als Wert wird für alle Simulationen jeweils dieselbe Temperatur wie am Einlass definiert, wie bereits in Kapitel 6.1.3 diskutiert wurde. Der Wand-WÜK (Wall bzw. Surface Heat Transfer Coefficient) wird dann von den Simulationsprogrammen automatisch zusammen mit dem Wandwärmestrom (Wall Heat Flux) und der Wandtemperatur (Wall Temperature) berechnet:

$$
\alpha_{\mathrm{W}}(\vec{x})=\frac{\hat{\dot{q}}_{\mathrm{W}}(\vec{x})}{T_{\mathrm{W}}(\vec{x})-T_{\text {ref }}}
$$

Bei konjugierten Simulationen findet ein iterativer Abgleich sowohl von $T_{\mathrm{W}}$ als auch von $\hat{\dot{q}}_{\mathrm{W}}$ an der Fluid-Solid-Interface statt, indem die Energiegleichung auch in der Solid-Domain gelöst wird. Bei reinen Strömungssimulationen hingegen muss je nach thermischer Randbedingung an der Außenwand entweder $\hat{\dot{q}}_{\mathrm{W}}$ oder $T_{\mathrm{W}}$ vorgegeben und die jeweils andere Größe über die Reynolds-Analogie zwischen Impuls- und Energietransport in der wandnächsten Zelle berechnet werden:

$$
\hat{\dot{q}}_{\mathrm{W}}=\frac{\rho c_{p} u^{*}}{T^{+}}\left(T_{\mathrm{W}}-T_{\mathrm{f}}\right)
$$


wobei $T_{\mathrm{f}}$ die Temperatur im wandnächsten Elementknoten (off-wall node) bezeichnet. Die dimensionslosen Größen $u^{*}$ und $T^{+}$werden gemäß der zu Grunde gelegten Wandmodellierungsmethode für die dimensionslose Geschwindigkeits- und Temperaturgrenzschicht berechnet. Für dissipations- $(\varepsilon-)$ basierte Turbulenzmodelle, die nicht bis zur Wand gelöst (,integriert“) werden können, da sie dort eine Singularität aufweisen, kommen skalierbare Wandfunktionen (Scalable Wall Functions) zum Einsatz, die den logarithmischen Grenzschichtbereich bis zur Wand extrapolieren. Wirbelfrequenz- $(\omega$-) basierte Turbulenzmodelle gestatten hingegen formell die Integration bis zur Wand (ohne Wandmodell), da sie Dämpfungsterme haben, die die Turbulenz in unterschiedliche Raumrichtungen unterschiedlich stark dämpfen können. Ansys Fluent bietet optional die Möglichkeit, die lineare Unterschicht unmittelbar an der Wand mit einem „Low-Re-Ansatz" als rein laminar zu rechnen. Ansys CFX verwendet für $\omega$-Modelle immer die sog. automatische Wandmodellierung (Automatic Wall Treatment), bei der in Abhängigkeit vom lokalen $y^{+}$Wert der wandnächsten Zelle automatisch zwischen Low-Re-Ansatz und Turbulenzmodell umgeschaltet (besser: überblendet) wird. Für detaillierte Ausführungen und Empfehlungen zur richtigen Wandmodellierung bei CFD-Simulationen siehe Anhang A14. Ein zentraler Gegenstand dieser Arbeit besteht neben Evaluation der verschiedenen Wandmodellierungsansätze daher vor allem in der Erprobung verschiedener Turbulenzmodelle. Voruntersuchungen im Rahmen studentischer Arbeiten [147] haben gezeigt, dass $\varepsilon$-basierte Modelle wie das $k$ - $\varepsilon$ - oder das RNG- $k$ - $\varepsilon$-Modell (Re-normalization group) für die Vorhersage des Wärmeübergangs im Seitenraum nicht geeignet sind, auch nicht mit verschiedenen Modellmodifikationen und -korrekturansätzen, wie Stromlinienkrümmungskorrektur oder Begrenzung der Turbulenzproduktion an der Wand (Production Delimiter). Daher sollen im Rahmen dieser Arbeit nur moderne, $\omega$-basierte (wandaufgelöste) Zweibzw. Fünfgleichungsturbulenzmodelle im Fokus stehen. Dazu gehören das für Standardindustrieanwendungen häufig genutzte $k$ - $\omega$-SST-Modell (Shear Stress Transport), bei dem mittels einer Blendfunktion zwischen dem $k-\omega$-Modell für wandnahe Bereiche und dem $k$ - $\varepsilon$-Modell für wandferne Bereiche übergeblendet wird, oder die komplexeren Reynolds-Stress-Modelle wie das $\omega$ RSM, welche im Stande sind, die Turbulenz anisotrop in alle Raumrichtungen zu modellieren. Transitionsmodelle, welche eigenständig zwischen laminarer Strömung und Turbulenzmodell umschalten können, für voll-turbulente Strömung jedoch nur im Wandbereich wirken, haben ebenfalls keine signifikanten Verbesserungen mit sich gebracht [147] und sollen im Folgenden ebenso vernachlässigt werden.

Sofern nicht anders angegeben, wurden für alle verwendeten physikalischen (Turbulenz-) Modelle die hinterlegten Standard-Modellparameter beibehalten. 


\subsubsection{Lösung/Konvergenz}

\subsubsection{Solver-Einstellungen/ Diskretisierungsschemen}

Für die Berechnungen in Ansys CFX wird der als einzige Option verfügbare, gekoppelte CFX-Solver (cfx5solve) verwendet. Sowohl für die Diskretisierung der Strömungsgleichungen (Advection Scheme) als auch der Turbulenzgleichung(en) wurde die Methode High Resolution gewählt, welche je nach örtlicher Konvergenz selbsttätig zwischen einem Verfahren erster Ordnung (Aufwind) und zweiter Ordnung überblendet ( Tab. A.14). In Ansys Fluent kommen der druckbasierte Solver SIMPLE (Semi-Implicit Method for Pressure Linked Equations) oder dessen verbesserte Version SIMPLEC (SIMPLE-consistent) zur Anwendung. Im Gegensatz zu den ebenfalls verfügbaren gekoppelten Methoden werden bei Segregrated Solvern das Geschwindigkeits- und das Druckfeld nicht gemeinsam gelöst, sondern durch Iteration einander angenähert (predictor-corrector-Prinzip). Auf Grund des strukturierten Netzes wird auf eine Korrektur der Elementschiefe (Skewness Correction) beim SIMLEC-Algorithmus verzichtet ( Tab. A.16). Fluent gestattet ferner die Festlegung der örtlichen Diskretisierungsschemen für jede einzelne zu lösende Gleichung. Hierbei wurden die in Tab. A.16 angegebenen Standardeinstellungen beibehalten.

\subsubsection{Zeitschrittweite/ Relaxationsfaktoren}

Ansys CFX verwendet auch für stationäre Berechnungen das Konzept einer sog. „falschen“ oder „Pseudo-Zeitschrittweite“ bzw. Zeitskala (Timescale) zur Unterrelaxation der zu lösenden (Erhaltungs-) Gleichungen [148], um die Lösung zu stabilisieren und örtliche Änderungen von einem Rechenschritt (fälschlicherweise als Zeitschritt bezeichnet) zum nächsten lokal zu dämpfen. Dabei sorgen große Zeitschritte für schnellere Konvergenz, verursachen jedoch unter Umständen Instabilitäten in den Residuen bis hin zu völligen Divergenz der Rechnung. Zur besseren manuellen Einstellung wird eine physikalische Zeitschrittweite gesetzt, deren initialer Wert in einer Größenordnung von $10^{-4}$ bis $10^{-5}$ s gewählt wird ( Tab. A.14) und zum Ende der Rechnung hin bei Bedarf noch manuell um ein bis zwei Größenordnungen angehoben werden kann, ohne dass deutliche Änderungen in der Lösung stattfinden.

Ansys Fluent verwendet für die Stabilisierung des Iterationsprozesses sog. explizite oder Unterrelaxationsfaktoren (URF) $\alpha$ zwischen 0 und 1, die ausdrücken, wie stark die Änderungen verschiedener Variablen $\phi$ zwischen zwei aufeinander folgenden Iterationsschritten $(n ; n-1)$ in die Lösung für den neuen Iterationsschritt eingehen:

$$
\phi_{n, \mathrm{korr}}=\phi_{n-1}+\alpha \cdot\left(\phi_{n}-\phi_{n-1}\right)=\alpha \phi_{n}+(\alpha-1) \phi_{n-1}
$$


$\alpha=1$ bedeutet demnach, dass die neue Lösung 1:1 für den nächsten Rechenschritt verwendet werden kann $\left(\phi_{n \text {,korr }}=\phi_{n}\right)$. Für Erhaltungsgleichungen besteht folgender Zusammenhang zwischen lokaler physikalischer Zeitschrittweite $\Delta t$ in CFX und dem (global angewendeten) URF in Fluent [149]:

$$
C F L=\frac{c \Delta t}{\Delta x}=\frac{\alpha}{1-\alpha}
$$

Für $\alpha=0,5$ ergibt sich eine Courant-Zahl von $C F L=1$, für $\alpha=0,75$ entsprechend $C F L=3$ und damit ein Wert über Eins, was bei stationären Simulationen mit impliziten Gleichungslösern jedoch kein Problem darstellt. Generell empfiehlt es sich, an den Standardeinstellungen für die URF ( Tab. A.16) keine Änderungen vorzunehmen, ganz besonders bei dem URF für die Energiegleichung nicht, der, sofern er nicht bei 1 gehalten wird, zu nicht plausiblen Ergebnissen führt, ohne dass sich dies in den Residuen erkennen lässt.

Für instationäre Lösungen (LES, URANS) wurde sowohl in Fluent als auch in CFX eine Zeitschrittweite von $2 \cdot 10^{-5} \mathrm{~s}=20 \mu$ s gewählt, was einer Courant-Zahl von $C F L<1$ entspricht und sich für die Stabilität/ Konvergenz der inneren Iterationen als günstig herausgestellt hat. Dies entspricht bei einer berechneten physikalischen Zeit von 2 s insgesamt 100.000 Zeitschritten, wobei für jede äußere Iteration maximal 15 bzw. 20 innere Iterationen zugelassen wurden. Bei vorzeitigem Erreichen der Konvergenzkriterien ( Kap. 6.1.6.4) ergeben sich entsprechend weniger innere Iterationen.

\subsubsection{Initialisierung/ Sonstige Einstellungen}

Für die Initialisierung des Lösungsprozesses wurden als Startwerte entweder die Ergebnisse einer vorangegangen Lösung gewählt, auch wenn diese ein anderes physikalisches Modell, einen anderen Lastschritt oder gar eine abweichende Geometrie aufwies, oder in dessen Ermangelung ganz zu Beginn mit dem Referenzdruck und der -temperatur, den dazugehörigen Stoffwerten und der zu erwartenden Axialgeschwindigkeit in der Hauptströmung gestartet (Domain Initialization). Für transiente Rechnungen (insbesondere die LES-Simulationen) wurde mit der auskonvergierten stationären Lösung einer vorher durchgeführten SST- oder RSM-Simulation des jeweiligen Anwendungsfalles oder - bei Fortsetzung der Lösung - mit einer vorher gesicherten Zwischenlösung weiter gearbeitet.

Die Einstellungen für die Partitionierung der Lösung für die lokale parallele oder distributive Rechnung auf dem Rechencluster (CFX: MeTiS k-way) sowie die Priorität und die Speicherallokation von Partitioner, Solver und Interpolator wurden nicht geändert. 


\subsubsection{Konvergenzkriterien/ -verlauf}

Als Hauptkonvergenzkriterien wurden die über alle Elemente im gesamten Rechengebiet quadratisch gemittelten, relativen/ „skalierten“ (RMS-) Residuen, also der quadratische Mittelwert der „Restglieder“ über alle Elemente bei der Lösung der Kontinuitätsgleichung (P-Mass), der Impulsgleichung in alle Raumrichtungen (U,V,W-mom), der Energiegleichung (Energy), der Turbulenzgleichungen (SST: k, epsilon, omega; RSM: uu, uv, vv, ww usw.) sowie bei konjugierten Rechnungen zusätzlich das Temperaturresiduum des Solids, gesetzt und in jedem Zeitschritt überwacht. Als Konvergenzziel wurde ein Wert von $10^{-5}$ festgelegt, bei stationären Berechnungen sogar noch ein bis zwei Größenordnungen darunter.

Ferner wurden (in CFX) auch die relativen Imbalancen (globale „Ungleichgewichte“), also die bilanzschließenden relativen Differenzen für alle Erhaltungsgrößen (Masse, Impuls in alle Raumrichtungen, Energie) über alle Ränder (Ein- und Auslässe), Quellen und -senken (bei CHT) berechnet. Das Konvergenzziel liegt für alle Imbalancen bei $10^{-4}=0,01 \%$ (transiente Berechnungen: $10^{-2}=1 \%$ ), siehe Tab. A.14 bzw. Tab. A.16.

Zusätzlich werden während des Lösungsprozesses bei jeder Iteration an verschiedenen charakteristischen Punkten innerhalb der Domain (sog. Monitorpunkten) verschiedene physikalische Größen ausgelesen und aufgezeichnet ( Tab. 6.1). Dies sind in erster Linie Drücke und WÜK in der $x$-y-Ebene entlang der Außenwand (in einem axialen Abstand von $15 \mathrm{~mm}$ ), sowie Geschwindigkeiten im Seitenraum und in der Einströmöffnung ( Abb. 6.4)

Tab. 6.1 Monitor-Punkte, deren Koordinaten und dort ausgewertete Größen

\begin{tabular}{ccrcc}
\hline Bezeichnung & Größen & $x[\mathrm{~mm}]$ & $y[\mathrm{~mm}]$ & $z[\mathrm{~mm}]$ \\
\hline P01 & $\alpha, p$ & -45 & 100 & 0 \\
P02 & $\alpha, p$ & -30 & 100 & 0 \\
P03 & $\alpha, p$ & -15 & 100 & 0 \\
P04 & $\alpha, p$ & 0 & 100 & 0 \\
P05 & $\alpha, p$ & 15 & 100 & 0 \\
P06 & $\alpha, p$ & 30 & 100 & 0 \\
P07 & $\alpha, p$ & 45 & 100 & 0 \\
P08 & $\alpha, p$ & 60 & 100 & 0 \\
P09 & $\alpha, p$ & 75 & 100 & 0 \\
P10 & $\alpha, p$ & 90 & 100 & 0 \\
\hline V01 & $c$ & 39 & 53,25 & 0 \\
V02 & $c$ & 45 & 85 & 0 \\
V03 & $c$ & 75 & 85 & 0 \\
V04 & $c$ & 0 & 85 & 0 \\
V05 & $c$ & -45 & 85 & 0 \\
\hline
\end{tabular}




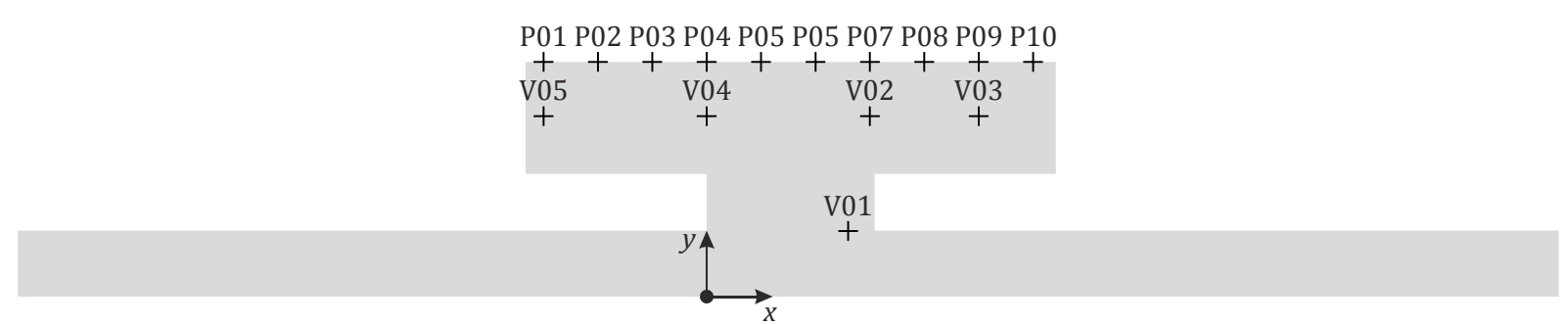

Abb. 6.4 Position der Monitorpunkte innerhalb der Domain.

Außerdem wird für jeden Rechenschritt $n$ der flächengewichtete arithmetische Mittelwert $\bar{\alpha}_{n}$, die zugehörige Standardabweichung $\sigma_{\alpha, n}$ sowie der Minimal- und Maximalwert $\alpha_{\min , n}$ bzw. $\alpha_{\max , n}$ der WÜK über alle Zellen $i$ der Außenwand mitgeschrieben:

$$
\begin{gathered}
\bar{\alpha}_{n}=\frac{1}{A} \sum_{i=1}^{N_{A}} \alpha_{i, n} \Delta A_{i} \\
\sigma_{\alpha, n}=\sqrt{\frac{1}{A} \sum_{i=1}^{N_{A}}\left(\alpha_{i, n}-\bar{\alpha}_{n}\right)^{2} \Delta A_{i}} \\
\alpha_{\min , n}=\min _{i} \alpha_{i, n} \\
\alpha_{\max , n}=\max _{i} \alpha_{i, n}
\end{gathered}
$$

wobei $N_{\mathrm{A}}$ die Anzahl aller Elemente der Außenwand bezeichnet. In Fluent wird zusätzlich die maximale relative Abweichung des aktuellen Flächenmittelwertes $\bar{\alpha}_{n}$ von allen Flächenmittelwerten $\operatorname{der} N=200$ vorhergehenden Iterationen

$$
\delta \alpha_{\max , n}=\max _{k \in[1 ; N]}\left|\frac{\bar{\alpha}_{n}-\bar{\alpha}_{n-k}}{\bar{\alpha}_{n}}\right|
$$

als zusätzliches benutzerdefiniertes Konvergenzkriterium aufgezeichnet und ein Wert von $10^{-4}$ als Ziel vorgegeben (,htc-opt“).

Abb. 6.5 zeigt einen typischen Konvergenzverlauf für die Residuen einer stationären zweidimensionalen SST-Rechnung mittels Ansys Fluent. Wie ersichtlich ist, konvergieren alle Residuen kontinuierlich zu Werten von $10^{-6}$ und zum Teil deutlich darunter. Dasselbe gilt auch für das WÜK-Konvergenzkriterium nach Gleichung 6.25. Die WÜK-Monitorpunkte in Abb. 6.6 haben schon nach gut 1000 Iterationen einen stationären Wert erreicht, weshalb die Rechnungen bereits zu diesem Zeitpunkt hätten abgebrochen werden können.

Die Simulationen mit $90^{\circ}$ - oder gar $360^{\circ}$-Netzen erreichen nicht ganz so niedrige Residuen. Es wird jedoch darauf geachtet, dass zumindest Werte von $10^{-5}$ erreicht werden und die Monitorpunkte stationär sind, was für alle stationären und instationären Rechnungen realisiert werden konnte. 


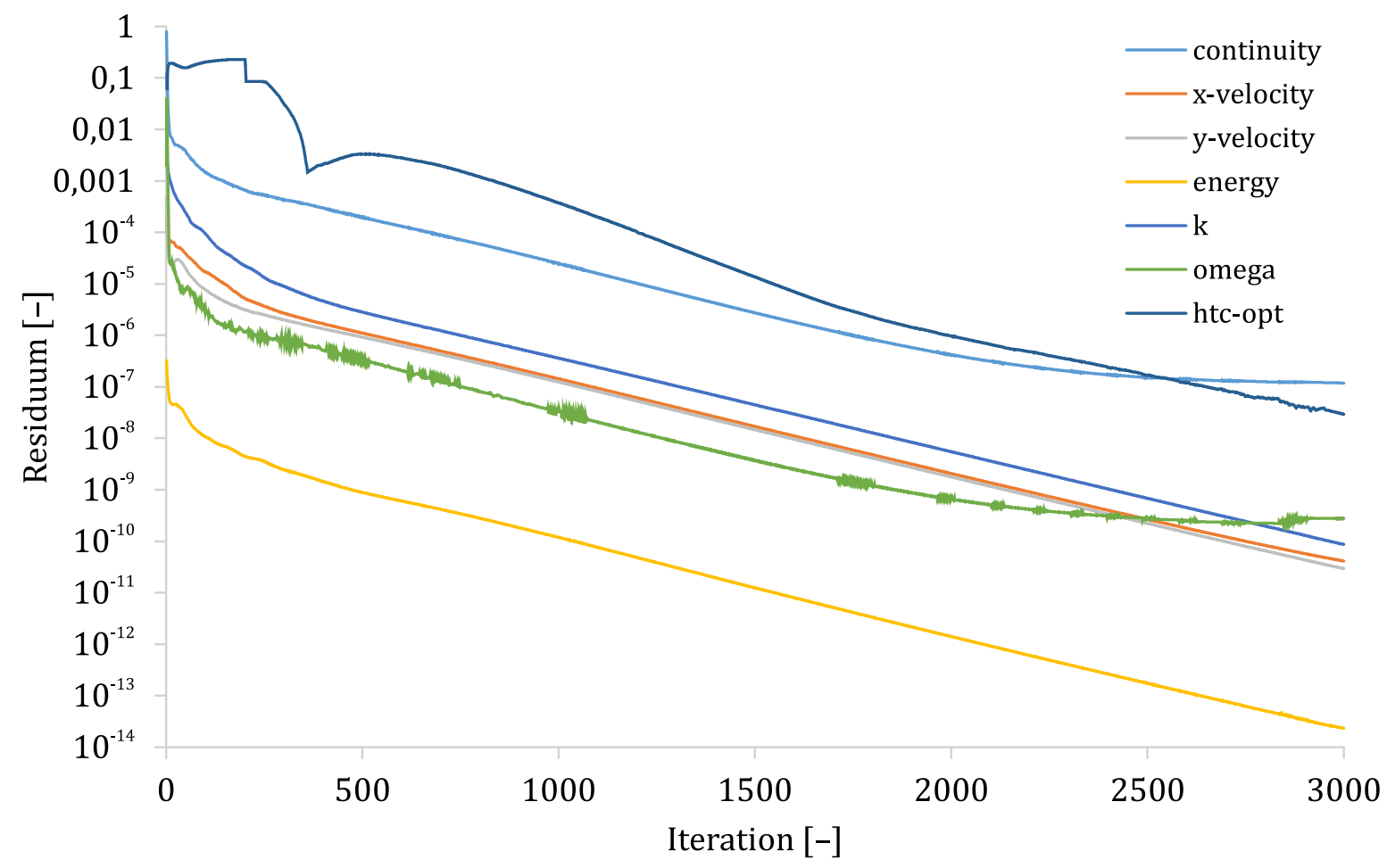

Abb. 6.5 Typischer Konvergenzverlauf der Residuen sowie des WÜK-Konvergenzkriteriums für ein 2D-SST-Modell in Ansys Fluent, berechnet mit dem Solver SIMPLEC.

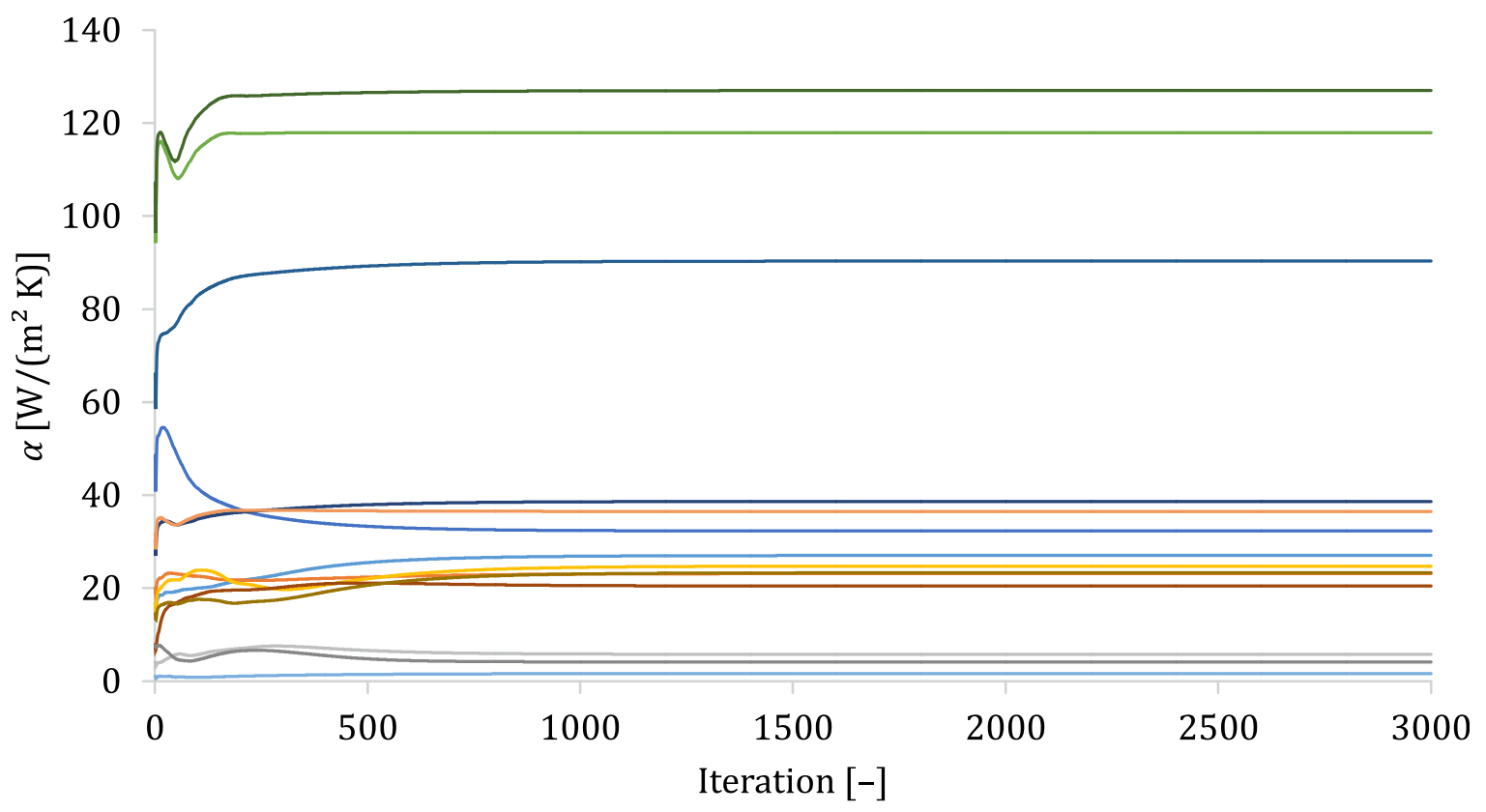

$\begin{array}{rrrr}- \text { htc-01 } & \text { htc-02 } & \text { htc-03 } & \text { htc-04 htc-05 }- \text { htc-06 }- \text { htc-07 } \\ \text { htc-08 } & \text { htc-09 } & \text { htc-10 } & \text { htc-avg }- \text { htc-max }- \text { htc-min }- \text { htc-rms }\end{array}$

Abb. 6.6 Typischer Konvergenzverlauf der WÜK-Monitorpunkte für ein 2D-SST-Modell in Ansys Fluent. 


\subsubsection{Rechenzeiten/ Hardware}

Um einen Eindruck zu gewinnen, welche Rechenressourcen gerade für die instationären Simulationen benötigt worden sind und wie lange die Rechnungen andauerten, sind in $\mathbf{T}$ Tab. A.17 einige Richtwerte angegeben. Während die zweidimensionalen RANS-Rechnungen in Ansys Fluent auf einem lokalen Arbeitsplatz-PC nur wenige Minuten dauern, beanspruchen gerade die skalenaufgelösten LES-Analysen, welche ausnahmslos auf dem Hochleistungsrechencluster (HPC) „Taurus“ des Zentrums für Informationsdienste und Hochleistungsrechnen (ZIH) an der TU Dresden durchgeführt wurden, enorme Ressourcen und Rechenzeit, weshalb diese nicht für systematische numerische Untersuchungen geeignet sind.

\subsubsection{Auswertung und Ergebnisse}

Zur Auswertung der transienten Simulationen ist es bereits während der Simulation erforderlich, einzelne Zwischenergebnisse in geeigneter Art und Weise herauszuschreiben, die später mittels (halb-) automatisierter Tools in Stapelverarbeitung ausgewertet werden können. Neben den in

Kap. 6.1.6.4 angegebenen Monitorpunkten, welche bei jeder (äußeren) Iteration ausgegeben werden, werden bei den LES-Analysen aller fünf Zeitschritte (entsprechend $100 \mu \mathrm{s}=0,1 \mathrm{~ms}$ ) folgende Skalar- und Vektorfelder gespeichert und als CGNS-Dateien (CFD General Notation System) herausgeschrieben:

- (Total-) Druck, (Total-) Temperatur, Geschwindigkeitskomponenten und -betrag auf der Symmetriefläche (2D-Simulationen: Domain);

- Temperatur, Wandtemperatur, Wandwärmestrom, Wandwärmeübergangskoeffizient, Druck an Außenwand.

Zur zeitlichen Auswertung der transienten Ergebnisse sowie zur zeitlichen Mittelwertbildung kommt das freie Datenanalyse- und -visualisierungsprogramm ParaView 5.4.1 zur Anwendung. Die örtliche Mittelung der zeitlichen Mittelwerte über dem Umfang der Außenwand erfolgt mittels PivotTabellen in Microsoft Excel 2013. Die Visualisierung der Ergebnisse erfolgt mit OriginPro 2017 oder Microsoft Excel. 


\subsection{MODELLDETAILLIERUNGSGRAD UND ABBRUCHFEHLER}

$\mathrm{Zu}$ Beginn der CFD-Untersuchungen stand die Frage, ob und inwieweit die exakt rotationssymmetrische Geometrie des Seitenraums durch ein Sektormodell abgebildet werden kann und wie groß der Kreissektorwinkel (Zentriwinkel $\theta$, Abb. 6.7) sein muss, um zum einen alle relevanten Strömungsstrukturen und die resultierenden Auswirkungen auf die berechnete WÜK-Verteilung zu erfassen, zum anderen aber auch den Rechenaufwand in einem begrenzten Rahmen zu halten.

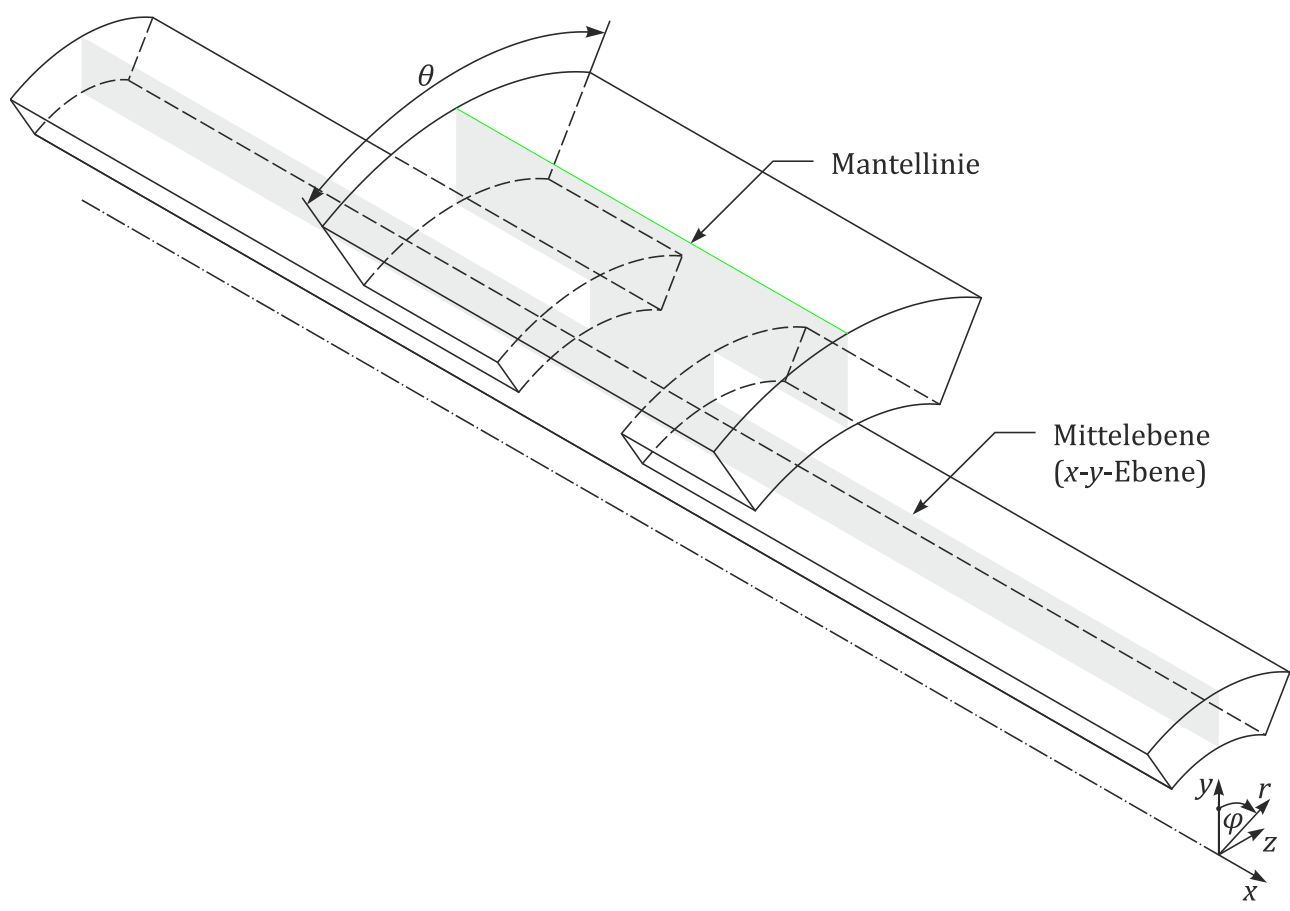

Abb. 6.7 Sektormodell, Mantellinie und Zentriwinkel (hier: $\theta=\mathbf{3 0}^{\circ}$ ).

Gleichzeitig war zu klären, ob die Auswertung der lokalen WÜK auf einer einzelnen Mantellinie der Außenwand repräsentativ ist, um die CFD-Ergebnisse mit den gemessenen Werten zu vergleichen ( Abb. 6.8 a), oder ob eine Umfangsmittelung der Werte notwendig ist (Abb. 6.8 b).

Wie aus Abb. 6.8 a ersichtlich wird, unterscheiden sich die WÜK-Verläufe zwischen den einzelnen Sektormodellen erheblich, wenn sie nur entlang der oberen Mantellinie der Außenwandinnenoberfläche ( $z=0, y=r=100 \mathrm{~mm}$, Abb. 6.7) abgetragen werden, obwohl diese in jedem CFD-Modell genau in der Mittelfläche der Außenwand liegt. Mittelt man hingegen die Werte über dem Umfang, liegen die Kurven deutlich näher beieinander ( Abb. 6.8 b). Während die „abgetragenen" und die gemittelten WÜK-Verläufe für $\theta=5^{\circ}$ und (naturgemäß) für das 2D-Modell identisch sind, liegen sie bei größeren Sektorwinkeln nur noch im mittleren Bereich sehr nah beieinander, wohingegen die Abweichungen im linken und rechten Bereich des Seitenraumes mit zunehmendem Sektorwinkel größer werden. 

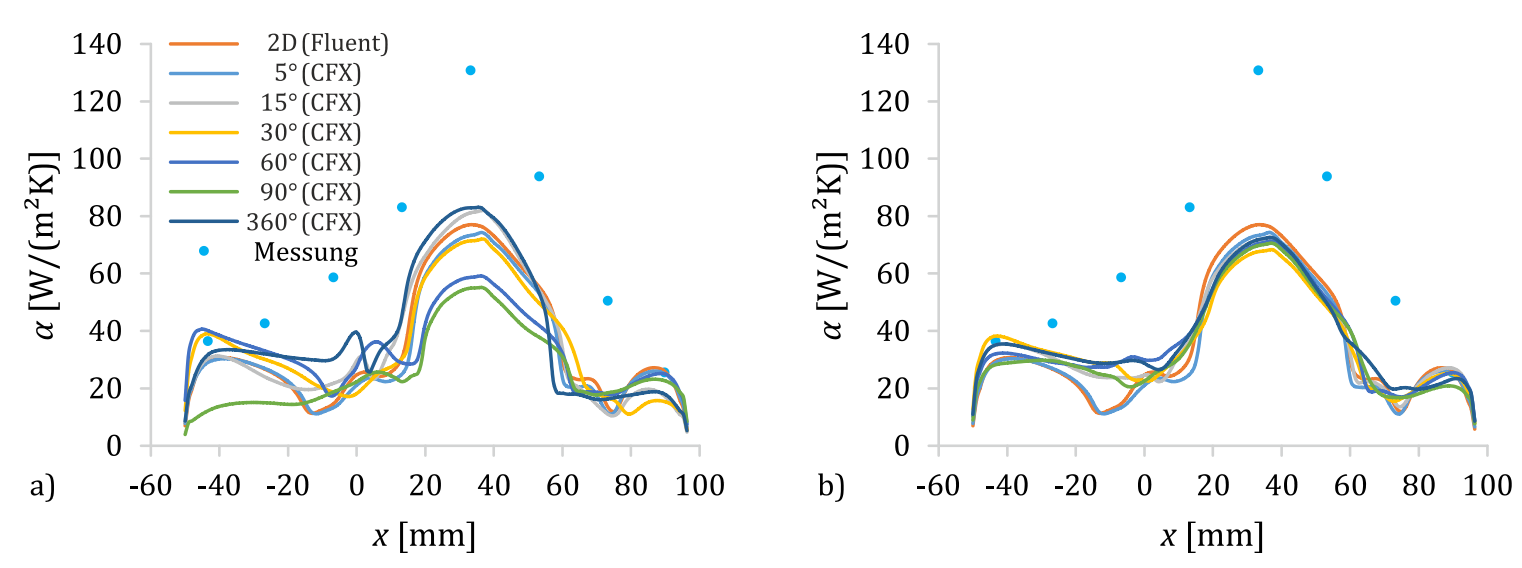

Abb. 6.8 Einfluss des modellierten Sektorwinkels auf die berechneten lokalen WÜK. $k$ - $\omega$-SST, $\hat{\dot{q}}_{\mathrm{W}}=$ konst. a) Auswertung auf der Mantellinie $(z=0, r=100 \mathrm{~mm})$ der Außenwand;

b) Umfangsgemittelte Werte.

Obwohl in der hier vorliegenden RANS-Rechnung ( $k-\omega$-SST) die Navier-Stokes-Gleichungen in stationärer Form (ohne Zeitterm) gelöst werden, führt die instationäre Natur der Seitenraumströmung offensichtlich dazu, dass auch in den berechneten WÜK-Verläufen Schwankungen auftreten. Bei kleinen Sektorwinkeln wird dieser Effekt durch die verhinderte Ausbreitung der Strömung in Umfangsrichtung offensichtlich noch unterdrückt. Um diese Vermutung zu untersuchen, wird für das $360^{\circ}$-Modell, bei dem die größten Schwankungen zu erwarten sind, eine nach ca. 5000 Iterationen konvergierte Rechnung für weitere 5000 Schritte weitergeführt und nach jedem Rechenschritt die WÜK auf der Außenwand herausgeschrieben. Mittels ParaView wurde der zeitliche Mittelwert (Index „Z“) der WÜK in jedem diskreten Punkt $(i, j)$ der Außenwand

$$
\bar{\alpha}_{\mathrm{Z}, i, j}=\frac{1}{N_{\mathrm{Z}}} \sum_{n=1}^{N_{\mathrm{Z}}} \alpha_{i, j, n}
$$

sowie die dazugehörige Standardabweichung (mittlere Streuung über der Zeit)

$$
\sigma_{\alpha, Z, i, j}=\sqrt{\frac{1}{N_{\mathrm{Z}}} \sum_{n=1}^{N_{\mathrm{Z}}}\left(\alpha_{i, j, n}-\bar{\alpha}_{\mathrm{Z}, i, j}\right)^{2}}
$$

ermittelt und in $>$ Abb. 6.9 dargestellt. Neben den „zeitlichen“ Schwankungen, die gemäß $>$ Abb. 6.9 b lokal zwischen 5 und 37 \% liegen, sind aus Abb. 6.9 a auch deutliche örtliche Schwankungen festzustellen, gerade über dem Umfang, wie besonders eindrücklich im Bereich der Staulinie ( $x \approx 45 \mathrm{~mm}$ ) anhand der Wirbelballen (4 Stück pro $90^{\circ}$-Sektor) zu sehen ist. Zur Visualisierung der Unsicherheiten sind - ähnlich wie in Abb. 6.8 b - in Abb. 6.10 zunächst die zeitlich $\underline{\text { und }}$ über dem Umfang (Index „ „U“) gemittelten WÜK der letzten $N_{\mathrm{Z}}=5000$ Iterationen über der axialen Koordinate aufgetragen: 


$$
\bar{\alpha}_{\mathrm{Z}, \mathrm{U}, i}=\frac{1}{N_{\mathrm{Z}} N_{\mathrm{U}}} \sum_{j=1}^{N_{\mathrm{U}}} \sum_{n=1}^{N_{\mathrm{Z}}} \alpha_{i, j, n}
$$

außerdem die über dem Umfang gemittelte zeitliche Streuung (Standardabweichung)

$$
\sigma_{\alpha, \mathrm{Z}, i}=\frac{1}{N_{\mathrm{U}}} \sum_{j=1}^{N_{\mathrm{U}}} \sigma_{\alpha, \mathrm{Z}, i, j}
$$

und die lokale Streuung (Standardabweichung) der zeitlichen Mittelwerte über dem Umfang an der jeweiligen axialen Position:

$$
\sigma_{\alpha, \mathrm{U}, i}=\sqrt{\frac{1}{N_{\mathrm{U}}} \sum_{j=1}^{N_{\mathrm{U}}}\left(\bar{\alpha}_{\mathrm{Z}, i, j}-\bar{\alpha}_{\mathrm{Z}, \mathrm{U}, i}\right)^{2}}
$$

Nach dem Gauß'schen Fehlerfortpflanzungsgesetz addieren sich diese beiden Werte geometrisch zur wahrscheinlichen Gesamtunsicherheit der mittels CFD ermittelten zeitlich und umfangsgemittelten WÜK an der Außenwand gemäß

$$
\sigma_{\alpha, \mathrm{ges}, i}=\sigma_{\alpha, \mathrm{Z}, \mathrm{U}, i}=\sqrt{\sigma_{\alpha, \mathrm{Z}, i}^{2}+\sigma_{\alpha, \mathrm{U}, i}^{2}}
$$

Zusätzlich sind auch der Minimal- und der Maximalwert über dem Umfang und alle Zeitschritte

$$
\begin{aligned}
& \alpha_{\text {max }, i}=\max _{j, n} \alpha_{i, j, n} \\
& \alpha_{\min , i}=\min _{j, n} \alpha_{i, j, n}
\end{aligned}
$$

sowie die in Abb. $6.8 \mathrm{~b}$ abgebildete „Momentanaufnahme“ nach 5000 Iterationen $(n=1)$ aufgetragen.

Unter der idealisierten Annahme sowohl in Umfangsrichtung als auch über der Zeit normalverteilter WÜK, ergibt sich gemäß der Gesamtstreuung in Gleichung 6.31 für den gemittelten WÜKVerlauf ein Konfidenzintervall, welches eine Breite von 9 bis $13 \mathrm{~W} /\left(\mathrm{m}^{2} \mathrm{~K}\right)$ im Mittelteil der Kavität und 4 bis $7 \mathrm{~W} /\left(\mathrm{m}^{2} \mathrm{~K}\right)$ im linken und rechten Teil besitzt und in dem 68,27 \% der berechneten Werte liegen (dunkelgrauer Bereich). Der Bereich zwischen den (in Zeit und Umfang) globalen Minima und Maxima („100-\%-Konfidenzintervall“, graue Fläche) ist noch deutlich größer.

Der exemplarische umfangsgemittelte WÜK-Verlauf nach 5000 Iterationen ( $n=1$; blaue Kurve) liegt innerhalb des 1- $\sigma$-Konfidenzintervalles und deckt sich gut mit dem zeitlich gemittelten Verlauf. Nennenswerte Abweichungen zwischen beiden Kurven treten treffenderweise in den Bereichen auf, in dem auch die relativen Standardabweichungen (vgl. Abb. 6.9 b) maximal sind. 1000 Iterationen später ( $n=1001)$ sieht die „Momentaufnahme“ des WÜK-Verlaufes an diesen Stellen bereits ganz 
anders aus, weshalb man den durch Gleichung 6.31 beschriebenen Vertrauensbereich auch als Maß für den zufälligen „Abbruchfehler“ der Simulation betrachten kann. Da dieser „Abbruchfehler“ bei kleinen Sektorwinkeln gegen Null geht und sich die Ergebnisse gemäß Abb. 6.8 b nur wenig zwischen den einzelnen Sektormodellen unterscheiden, werden für die nachfolgenden Untersuchungen, bei denen unter anderem die Sensitivität der Ergebnisse gegenüber dem verwendeten Rechennetz ( Kap. 6.3) sowie den angesetzten Randbedingungen ( Kap. 6.4) untersucht werden soll und daher ein Quereinfluss auf Grund eines möglichen „Abbruchfehlers“ kontraproduktiv wäre, das $5^{\circ}$-Modell (CFX) bzw. das 2D-Modell (Fluent) verwendet.
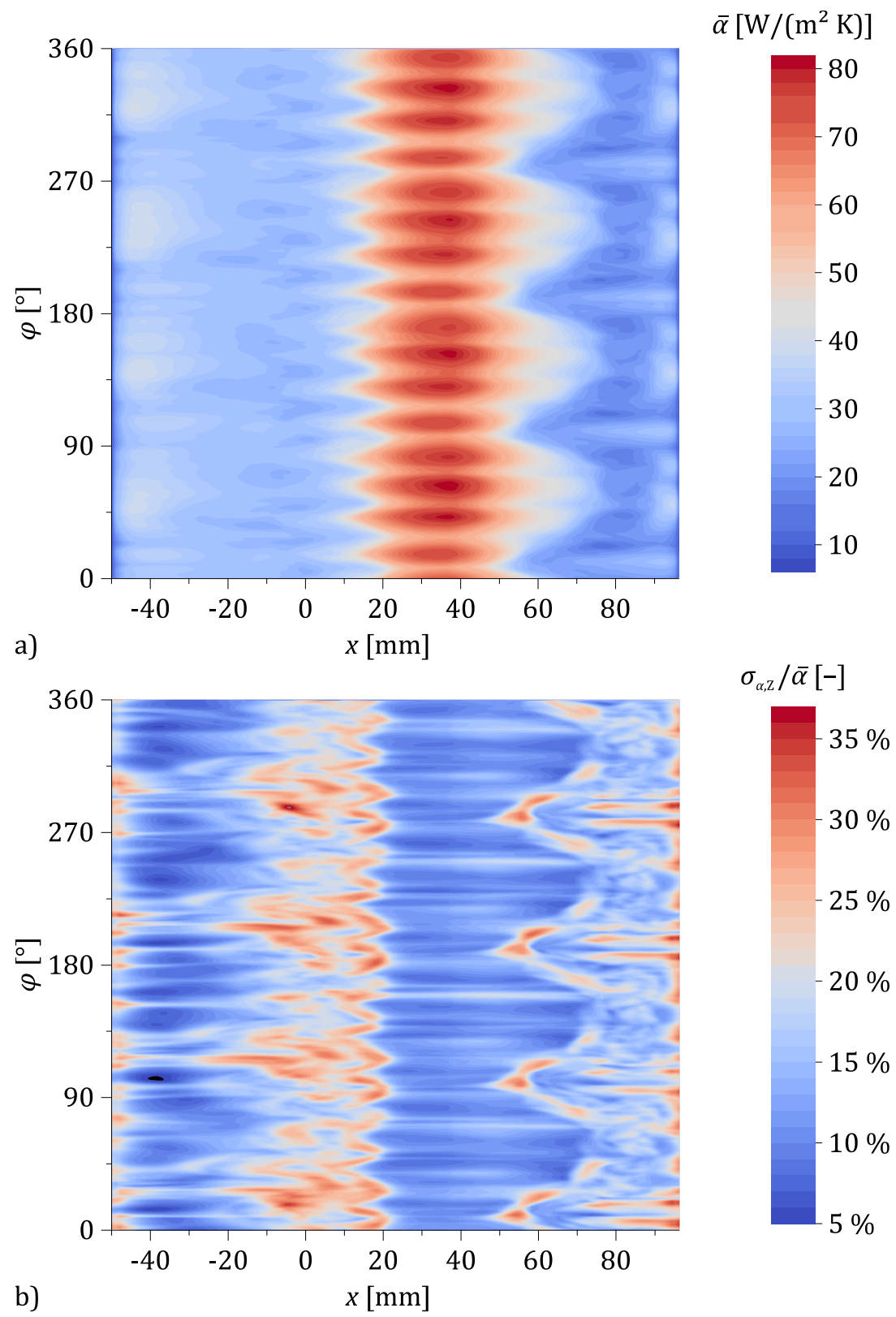

Abb. 6.9 Statistische Kenndaten der mittels CFD berechneten WÜK an der Außenwand eines $360^{\circ}$-Modelles über die letzten 5000 Iterationen. $k$ - $\omega$-SST, $\hat{\dot{q}}_{\mathrm{W}}=$ konst.

a) zeitlicher Mittelwert; b) relative Standardabweichung vom zeitlichen Mittelwert. 


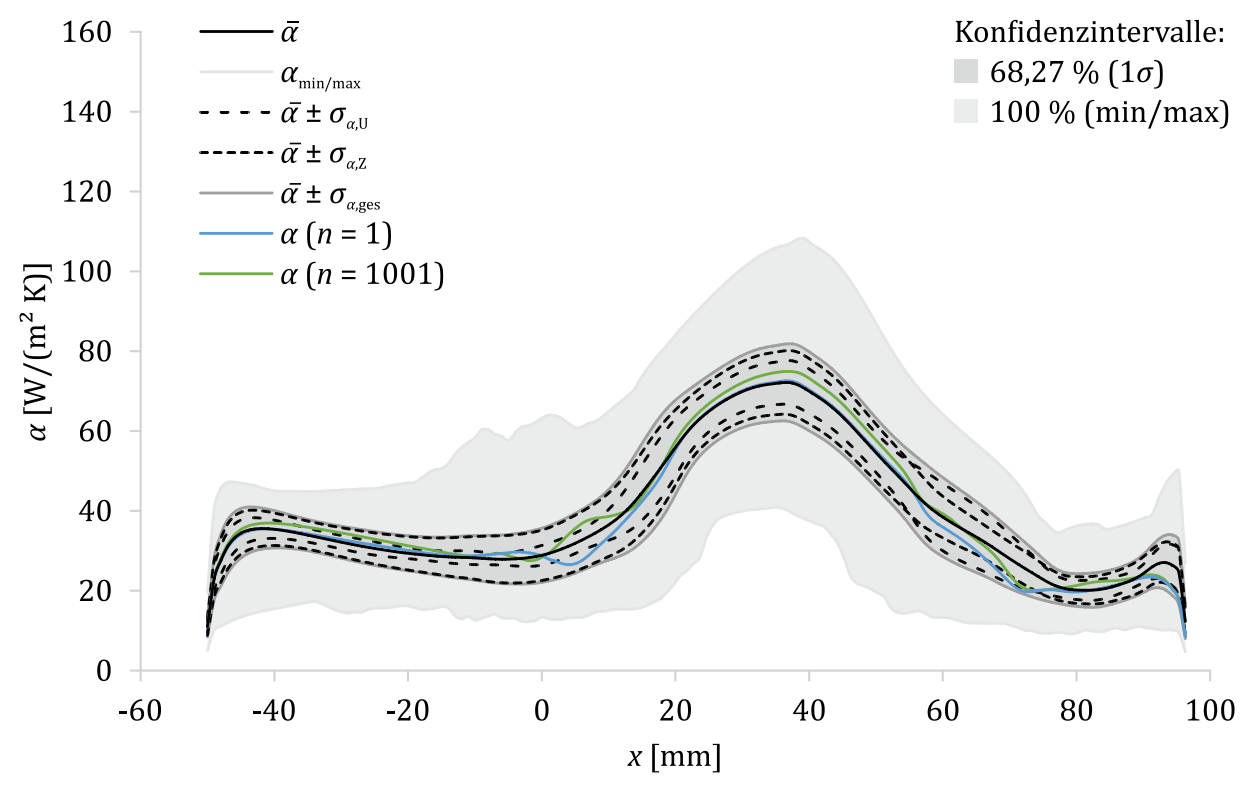

Abb. 6.10 Zeitlich und umfangsgemittelter WÜK-Verlauf, zugehörige Streuungen (Standardabweichungen) und Konfidenzintervalle der mittels CFD berechneten WÜK an der Außenwand eines $360^{\circ}$-Modelles über die letzten 5000 Iterationen. $k-\omega$-SST, $\hat{\dot{q}}_{\mathrm{W}}=$ konst.

\subsection{NETZUNABHÄNGIGKEITSSTUDIE}

Im nächsten Schritt soll sichergestellt werden, dass die örtliche Diskretisierung des verwendeten Rechennetzes („Netzfeinheit“/ „Netzgüte“) keinen oder nur einen vernachlässigbar geringen Einfluss auf das Simulationsergebnis, hier speziell auf die berechneten WÜK an der Außenwand, hat. Aufbauend auf dem „Basisnetz“ mit einer Elementgröße von $1 \mathrm{~mm}$ und einer Höhe der ersten Zelle an der Wand von $5 \mu \mathrm{m}$ (vgl. $>$ Tab. A.8) wurde in Abb. 6.11 a und b jeweils ein Netzparameter konstant gehalten und der andere einmal vergrößert und einmal verkleinert und die sich ergebenden WÜK-Verteilungen mit einander verglichen. Aus Abb. 6.11 a lässt sich erkennen, dass die Netze mit 0,5 und $1 \mathrm{~mm}$ Elementkantenlänge sehr ähnliche WÜK liefern, wohingegen das 2mm-Netz bereits zu grob erscheint, was sich vor allem in den großen Abweichungen im Staupunkt widerspiegelt. Im Sinne des Rechenaufwandes wird daher das Netz mit $1 \mathrm{~mm}$ Elementgröße als die wissenschaftlich und wirtschaftlich sinnvollste Variante angesehen. Demgegenüber ergeben sich aus Abb. 6.11 b für alle drei Wandschichtdicken nahezu identische WÜK-Werte über der gesamten Außenwand. Für die beiden kleinsten Schichtdicken liegt der dimensionslose Wandabstand an der Außenwand sowie an allen anderen Wänden der Domain unter dem empfohlenen Maximalwert von $y^{+}=1$, einer konservativen Annahme, die gewährleistet, dass auch bei geometrischem Wachstum der Schichten mindestens zwei bis vier Elemente innerhalb der viskosen Unterschicht liegen (Abb. A.29 in Anhang A14), sodass Gradienten an der Wand korrekt berechnet werden können. Dies ist gerade für die Untersuchung von Wärmeübertragungsphänomenen essentiell. Darauf aufbauend wurde in einer abschließenden Studie untersucht, ob die Anzahl der 
Zellen in der viskosen Unterschicht einen Einfluss auf den berechneten WÜ-Verlauf haben. Dazu wurde obiger Testfall in Ansys Fluent zum einen mit dem 2D-Basisnetz ( Abb. 6.2 a) sowie dem in ICEM CFD erstellten Netz nachgerechnet, bei dem die ersten zwölf Elemente an der Wand eine konstante Elementhöhe von $5 \mu$ m aufweisen ( Abb. 6.2 b) und bei einem $y^{+}$-Wert von unter Eins allesamt innerhalb der viskosen Unterschicht $\left(y^{+} \leq 11\right)$ liegen sollten ( Abb. A.29). Das Ergebnis ist in Abb. 6.11 c dargestellt. In Anbetracht der Tatsache, dass sich die Ergebnisse kaum voneinander unterscheiden, kann davon ausgegangen werden, dass beide Netze geeignet sind, Wandeffekte in geeignetem Maße aufzulösen. Dies wird auch dadurch bekräftigt, dass die wandnahen Geschwindigkeitsprofile, in Abb. A.30 exemplarisch für die rein axiale Hauptströmung im Bereich des Auslasses gezeigt, für beide Netze vollständig bis in die lineare Unterschicht aufgelöst werden. In Hinblick auf die späteren systematischen Analysen ( Kap. 6.4 und 6.6), bei denen die Netze für die verschiedenen Geometriekonfigurationen vollautomatisch generiert werden sollen, hat sich das Basisnetz, welches mit dem im Workflow von Ansys Workbench integrierten Vernetzungsprogramm Ansys Meshing erstellt wird und mit dem auch bereits alle bisherigen Grundlagenuntersuchungen durchgeführt worden sind, als beste Variante herausgestellt.
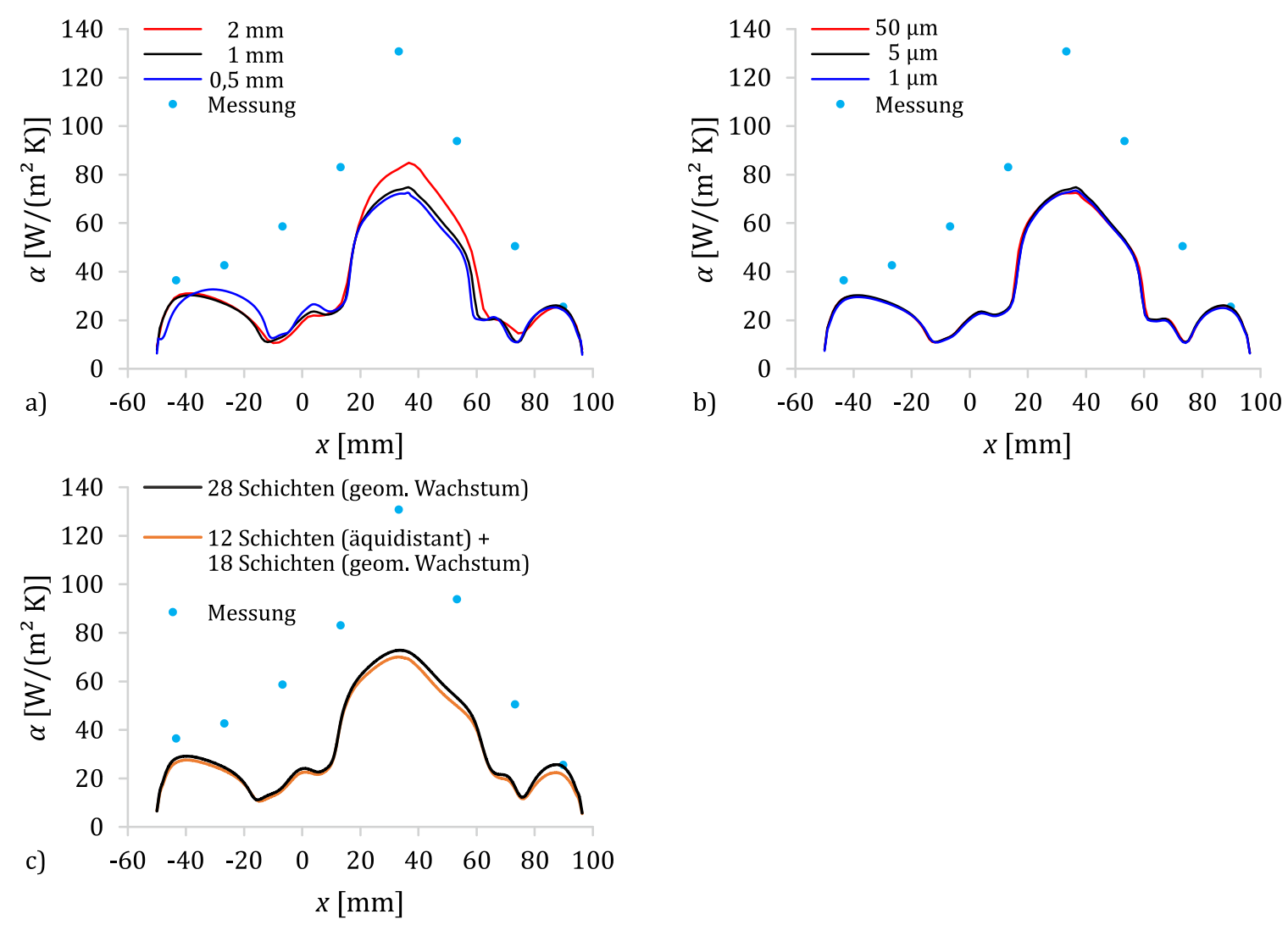

Abb. 6.11 Einfluss verschiedener Vernetzungsparameter auf die berechneten lokalen WÜK. $k-\omega$-SST, $\hat{\dot{q}}_{\mathrm{W}}=$ konst.

a) Elementgröße des freien Netzes; b) Schichtdicke der wandnächsten Zelle (beide $5^{\circ}$-Modell);

c) Auflösungsgrad der viskosen Unterschicht (2D-Modell, mit low-Re correction). 


\subsection{EINFLUSS DER RANDBEDINGUNGEN UND DER MODELLIERUNG}

Die richtige Auswahl geeigneter Randbedingungen sowie der zu Grunde gelegten physikalischen Modelle hat entscheidenden Einfluss auf die Abbildungsqualität und damit die Güte des Modells. Aus diesem Grund wurden in einer eigens durchgeführten Sensitivitätsstudie die wichtigsten physikalischen Eingabeparameter und Modelleinstellungen variiert und deren Einfluss auf das WÜErgebnis an der Außenwand beleuchtet. Als Basis wurde das in den Kap. 6.2 und 6.3 entwickelte, numerisch stabile, ideal konvergierende und netzunabhängige 2D-Modell in Ansys Fluent verwendet. Ausgehend von den Ausführungen in Kap. 6.1 sind in Tab. 6.2 noch einmal alle Daten des „Basisfalls“ zusammengefasst, die im Tab. 6.2 Randbedingungen und Modelleinstellungen Folgenden variiert werden sollen. des Fluent-Basismodells

In $\triangleright$ Abb. 6.12 bis Abb. 6.14 wird jeweils eine Einstellung verändert, alle sonstigen Randbedingungen bleiben unverändert und entsprechen, sofern nicht anders angegeben, den Angaben in Tab. 6.2. Der Basisfall ist jeweils als schwarze Kurve mit eingezeichnet.

\begin{tabular}{ll}
\hline Randbedingung & Wert/ Einstellung \\
\hline Einlass Impuls-RB & $\dot{m}_{\text {ein }}=0,271 \mathrm{~kg} / \mathrm{s}$ \\
Einlass Turbulenz-RB & $T u_{\text {ein }}=5 \%, \lambda_{\text {ein }}=2,84 \mathrm{~mm}$ \\
Einlass thermische RB & $T_{\text {ein }}=T_{\mathrm{B}}=33,55^{\circ} \mathrm{C}$ \\
Auslass Impuls-RB & $p_{\text {aus }}=2,06 \mathrm{bar}$ \\
Außenwand thermische RB & $T_{\mathrm{W}}=40^{\circ} \mathrm{C}$ \\
Außenwand Impuls-RB & hydr. glatt \\
sonst. Wände thermische RB & adiabat \\
sonst. Wände Impuls-RB & hydr. glatt \\
Turbulenzmodell & RSM-BSL \\
Medium & air (Idealgas) \\
\hline
\end{tabular}

Wie Abb. 6.12 a zeigt, stellt die Art der angesetzten thermischen Randbedingung an der beheizten Außenwand einen sehr wesentlichen Einflussparameter für die Berechnung der WÜK dar. Im Bereich des Staupunktes werden unter Annahme einer konstanten Wandtemperatur ( $T_{\mathrm{W}}=$ konst.; RB 1. Art) deutlich höhere Werte und ein „spitzerer“ Verlauf für die WÜK berechnet, die deutlich näher an den experimentell ermittelten Daten liegen, als wenn eine konstante Wandwärmestromdichte ( $\hat{\dot{q}}_{\mathrm{W}}=$ konst.; RB 2 . Art) vorgegeben wird, und zwar unabhängig vom verwendeten Turbulenzmodell (RSM-BSL, RSM- $\omega, k$ - $\omega$-SST und LES-WALE), vom modellierten Sektorwinkel, vom Simulationsprogramm (CFX, Fluent, OpenFOAM) sowie von allen sonstigen Randbedingungen. Ein ähnliches Verhalten wurde auch bereits bei der experimentellen Untersuchung des lokalen Wärmeübergangs von runden Prallstrahlen beobachtet [150]. Bei den in [151] durchgeführten numerischen Nachrechnungen, bei denen ebenfalls ein zweidimensionales axialsymmetrisches Modell des Prallstrahles in Ansys Fluent unter Einsatz des $k$ - $\omega$-SST-Turbulenzmodell zur Anwendung kam, wurde ebenso ein Unterschied in den berechneten, über den Radius gemittelten Nusselt-Zahl-Verläufen festgestellt, bei denen jedoch die Werte für $T_{\mathrm{W}}=$ konst. unter denen für $\hat{\dot{q}}_{\mathrm{W}}=$ konst. lagen. Dies deckt sich auch mit den Erfahrungen vom Wärmeübergang bei laminarer und turbulenter Rohrströmung. In beiden Veröffentlichungen waren im Gegensatz zu den aktuellen Beobachtungen die Nusselt-Zahlen unmittelbar im Staupunkt für $T_{\mathrm{W}}=$ konst. und 
$\hat{\dot{q}}_{\mathrm{W}}=$ konst. jeweils gleich. Leider liefert keiner der Autoren einen Erklärungsansatz für die randbedingten Unterschiede. In Anbetracht der Tatsache, dass im vorliegenden Fall die strömungstechnischen Effekte noch mannigfaltiger sind als in den beiden genannten Literaturfällen, sich nämlich zusammensetzt aus

- Ringspaltströmung,

- Ablösung an der stromaufwärts liegenden Einseitenströmwand,

- Scherströmung im Spalt, Einmischung („Austausch“) von Fluid aus Seitenraum,

- Wiederanlegen und -ablösen an der stromabwärts liegenden Einströmseitenwand,

- axialsymmetrischer Prallstrahl an der Außenwand mit erzwungenem Wärmeübergang,

- verzweigte Kavitätenströmung (confined impingement jet),

- Primär, Sekundär-, Tertiärwirbelbildung,

kann keine einfache Erklärung für die Unterschiede in den WÜK für die beiden Arten der thermischen Randbedingung gegeben werden. Da jedoch die Werte für konstante Wandtemperatur zumindest im Bereich des Prallstrahles näher an den mittels des FE-basierten Gradientenverfahren ( Kap. 4.4.4) aus den gemessenen Temperaturen bestimmten WÜK liegen, soll im Folgenden nur noch die RB 1. Art, $\left.T(x)\right|_{\mathrm{W}}=T_{\mathrm{W}}(x)$, verwendet werden. Dabei hat die Höhe der angenommenen Außenwandtemperatur keinen Einfluss auf das Ergebnis ( Abb. 6.12 b), selbst wenn man diese mit $\pm 5 \mathrm{~K}$ deutlich stärker variiert, als die Unsicherheiten in den Temperaturmessungen hergeben würden. Selbst wenn man einen abschnittsweise konstanten Temperaturverlauf aufprägt, der den diskreten Innenringtemperaturen entspricht, ändern sich die Werte kaum. Die kleineren Überschwinger an den Unstetigkeitsstellen können als numerische Artefakte in Folge der lokalen Nichtlinearität angesehen werden. Weiterhin spielt es keine Rolle, ob die sonstigen Wände als adiabat oder mit einer konstanten Wandtemperatur modelliert werden. Die WÜK-Verläufe decken sich außerdem sehr gut mit den Ergebnissen der konjugierten Rechnung (Abb. 6.3), bei der die Wandstrukturen mit modelliert wurden. Die Temperaturverteilung in der Gesamtdomain sowie speziell in der Außenwand ist in Abb. A.31 und die Verteilung der Wärmestromdichte entlang des Fluid-Solid-Interfaces sowie an weiteren Oberflächen des Modells in Abb. A.32 im -Anhang A15 dargestellt. Demgegenüber weichen die Graphen für konstante Wandwärmestromdichte deutlich vom CHT-Ergebnis ab ( Abb. 6.12 d). Die Sandkornrauigkeit an der Außenwand sowie an allen anderen Wänden hat ebenfalls keinen Einfluss auf das WÜK-Ergebnis (Abb. 6.12 c, nur für das $k$ - $\omega$-SST-Modell verfügbar).

Da die simulierten WÜK-Kurven vor allem in den Seitenbereichen des Seitenraumes nach wie vor nicht zufriedenstellend die gemessenen Werte treffen, soll im nächsten Schritt die Sensitivität der 
Ergebnisse hinsichtlich der Ein- und Auslassrandbedingungen untersucht werden ( Abb. 6.13). Wie erwartet beeinflusst die Wahl einer moderat anderen Einlasstemperatur, welche definitionsgemäß auch die Bezugstemperatur für die berechneten WÜK ist, den berechneten WÜK-Verlauf kaum ( Abb. 6.13 a). Verändert man Einlass- und Wandtemperatur jeweils um denselben Betrag (z. B. $\Delta T=5 \mathrm{~K})$, sind die Verläufe sogar identisch $($ Abb. 6.13 b). Auch der Turbulenzgrad am Einlass spielt keine führende Rolle, selbst wenn man ihn stark variiert ( Abb. 6.13 c). Während bisher auf Grund der gesetzten Massenstromrandbedingung die Geschwindigkeit am Einlass als gleichverteilt („Rechteckprofil“, uniform) modelliert wurde, soll abgeklärt werden, ob die Wahl eines realistischeren Einlassprofils einen Einfluss auf die das berechnete WÜK-Profil hat. Dazu wurde der gesamte Versuchsstand von der Stelle unmittelbar nach dem Strömungsumformer und dem Turbulenzsieb bis hin zum Ende des ringspaltförmigen Hauptströmungskanals ( Abb. A.16), jedoch ohne Totraumgeometrie, als Viertelmodell in Ansys CFX modelliert ( Abb. A.34 in Anhang A16) und für den betreffenden Betriebsfall mittels einer RANS-Simulation ( $k-\omega$-SST) das Strömungsprofil an der Interface bestimmt, die dem Einlass des Fluent-Modells entspricht.
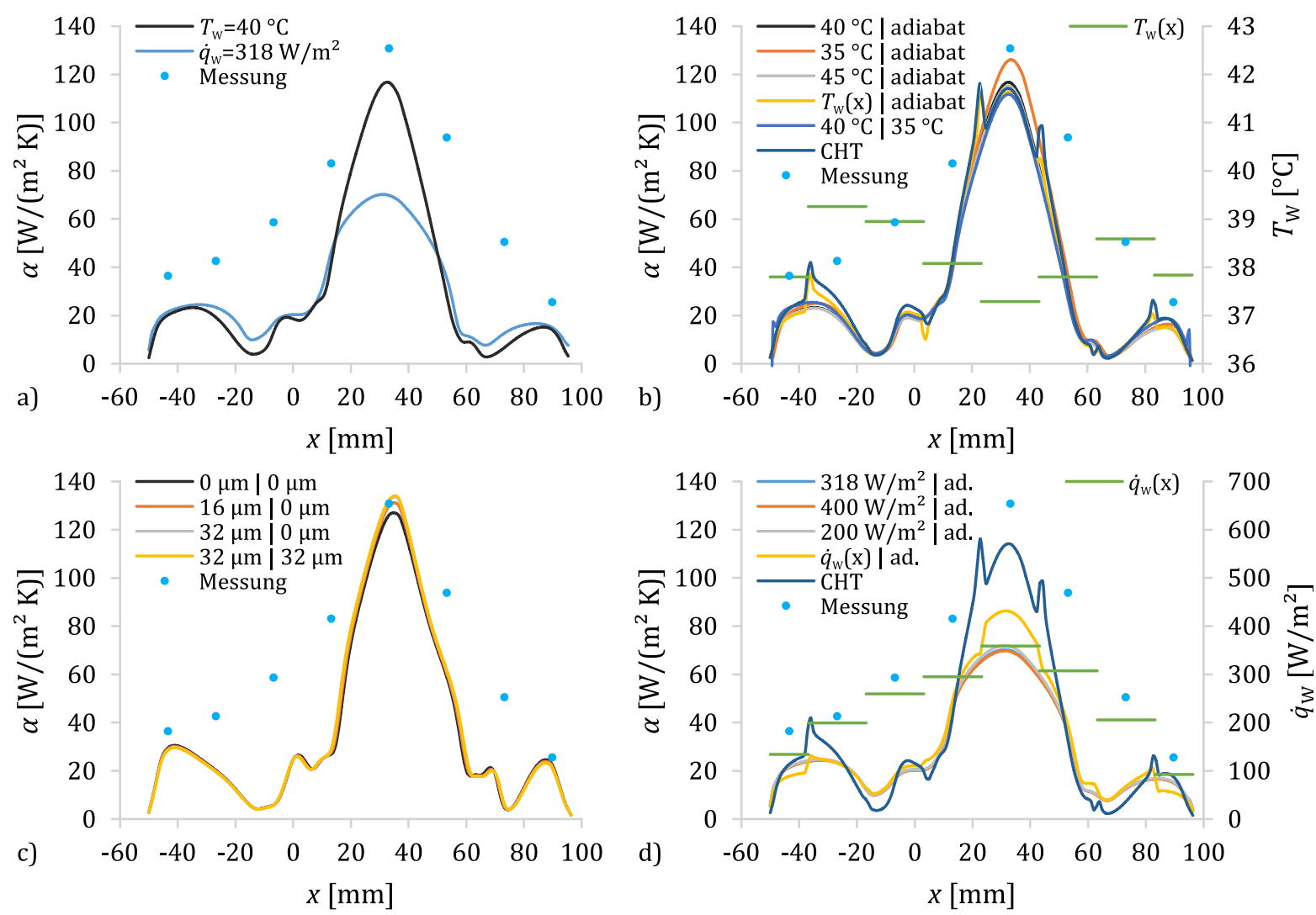

Abb. 6.12 Einfluss der Wandmodellierung auf den mit Ansys Fluent berechneten WÜK-Verlauf.

a) Art der thermischen Randbedingung an der Außenwand;

b) Temperatur $T_{\mathrm{W}}$ an der Außenwand I an den sonstigen Wänden;

c) Wandrauigkeit $k_{\mathrm{S}} \approx R z$ an der Außenwand | an den sonstigen Wänden ( $k-\omega$-SST);

d) Wärmestromdichte $\hat{\dot{q}}_{\mathrm{W}}$ an der Außenwand | an den sonstigen Wänden. 

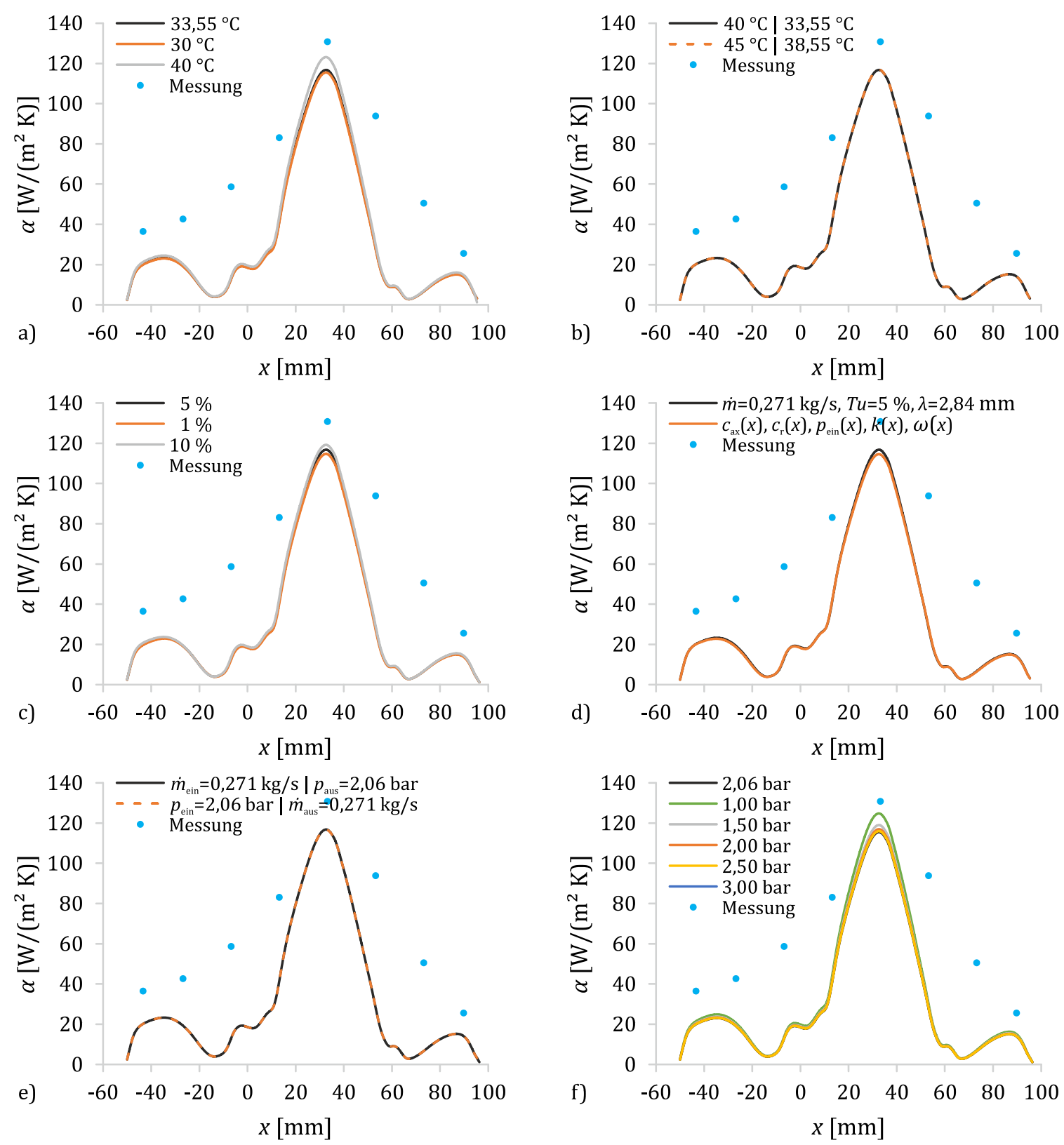

Abb. 6.13 Einfluss der Ein- und Auslassrandbedingungen auf den mit Ansys Fluent berechneten WÜK-Verlauf.

a) Einström-/ Bezugstemperatur $T_{\text {ein }}=T_{B}$;

b) Temperaturniveau (Wandtemperatur $T_{\mathrm{W}} \mid$ Einströmtemperatur $T_{\text {ein }}=T_{\mathrm{W}}-6,45 \mathrm{~K}$ );

c) Turbulenzgrad $T u_{\text {ein }}$ am Einlass; d) Einströmprofil;

e) Vertauschung der Ein- und Auslass-Impulsrandbedingung;

f) Druckniveau (Druck $p_{\text {aus }}$ am Auslass).

Die umfangsgemittelten Werte für die axiale und radiale Geschwindigkeitskomponente $c_{\mathrm{ax}}(r)$ bzw. $c_{r}(r)$, den statischen Druck $p(r)$, die turbulente kinetische Energie $k(r)$ sowie die turbulente Wirbelfrequenz $\omega(r)$ (in Fluent: „spezifische Dissipationsrate“) wurden anschließend als radiale Verteilungen auf den Einlass des 2D-Modells in Fluent übertragen ( Abb. A.35). Doch auch diese Maßnahme führte nicht zu einer besseren Abbildung der Messwerte ( Abb. 6.13 d). Dasselbe gilt auch, wenn man die Impulsrandbedingung an Ein- und Auslass vertauscht (Abb. 6.13 e). Selbst 
das Druckniveau im Versuchsstand, was über den Auslassdruck eingestellt wird und auf Grund der unterschiedlichen Dichte auch das Geschwindigkeitsniveau im Seitenraum bestimmt, hat keinerlei Einfluss auf den WÜK-Verlauf ( Abb. 6.13 e). Letzteres ist insoweit zufriedenstellend, als dass dies die in Gleichung 4.14 postulierte Unabhängigkeit der Reynolds-Zahl vom Druck auch für die WÜK bestätigt.

Im letzten Schritt sollen ausgewählte Modellierungsparameter hinsichtlich ihrer Wirkung auf den Wärmeübergang im Seitenraum studiert werden. So haben die Stoffwerte, egal ob die Transportgrößen $\left(\eta, \lambda, c_{p}\right)$ als konstant (air, air-sister) oder als Funktion der Temperatur (air-temp) angesetzt werden, keinen Einfluss auf das Ergebnis ( Abb. 6.14 a). Das ist auch so gewünscht und liegt an den beabsichtigt geringen Temperaturdifferenzen zwischen Wand und Fluid (siehe Kap. 4.1.3). Wichtig ist in jedem Falle die Berücksichtigung der Druck- und Temperaturabhängigkeit der Dichte über das Idealgasverhalten. Als einer der Haupteinflussfaktoren auf die berechneten WÜK wurde die Turbulenzmodellierung vermutet. Doch die (relativ weit entwickelten, dem Stand der Technik für RANS-Simulationen entsprechenden), $\omega$-basierten (wandaufgelösten) Zwei- bzw. Fünfgleichungsmodelle in Abb. 6.14 b zeigen keine signifikanten Unterschiede.
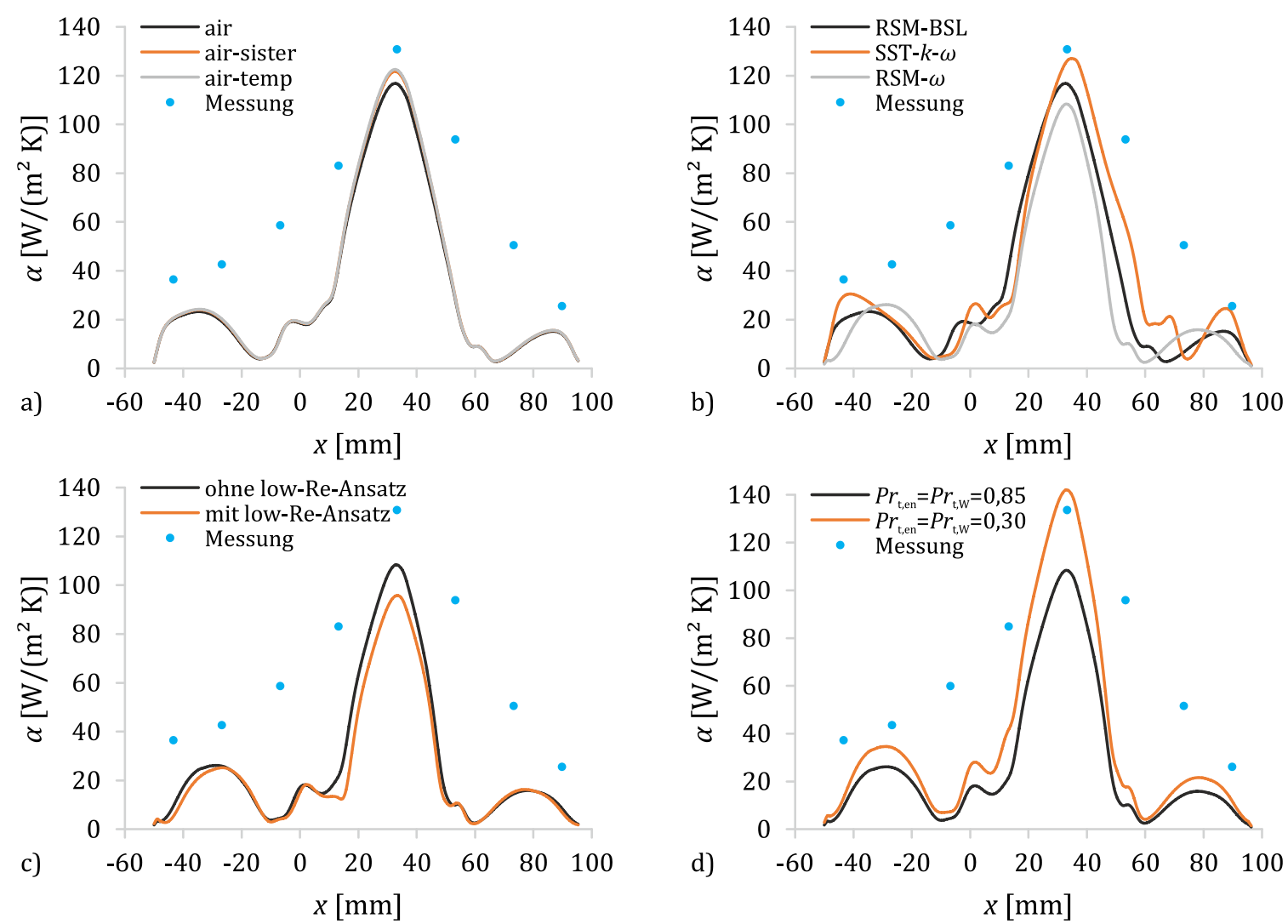

Abb. 6.14 Einfluss der Stoffwerte sowie der Turbulenzmodellierung auf den mit Ansys Fluent berechneten WÜK-Verlauf.

a) Stoffwerte (Nomenklatur Kap. 6.1.4); b) Turbulenzmodell; c) Wandmodellierung (RSM- $\omega$ );

d) Turbulente Prandtl-Zahl $P r_{\mathrm{t}}$ für Energiegleichung und Wandmodellierung (RSM- $\omega$ ). 
Für das SST- $k-\omega$ - und das RSM- $\omega$-Modell gibt es ferner die Möglichkeit, die lineare Unterschicht, in der die lokalen Zell-Reynolds-Zahlen sehr klein sind, durch entsprechende Modellanpassungen als rein laminar zu rechnen (sog. Low-Re-Ansatz; siehe Abb. 6.14 c). Es lässt sich ein minimaler Effekt im Bereich des Staupunktes feststellen. Die Werte in den Randbereichen der Kavität bleiben jedoch nach wie vor unverändert. Basierend auf einer numerischen Arbeit zum Wärmeübergang in Drallrohren [152] soll abschließend getestet werden, ob und inwieweit durch die Wahl einer anderen turbulenten Prandtl-Zahl $P r_{\mathrm{t}}$ für das RSM- $\omega$-Modell die lokalen WÜK noch feinangepasst werden können („Parameter Tuning“). Hintergrund ist, dass gerade bei den Turbulenzmodellen viele Modellkonstanten auf sehr einfache Standardversuchsfälle (ebene Platte, vollentwickelte Rohrströmung, backward-facing step etc.) kalibriert, für kompliziertere Strömungen aber nicht immer gültig sind. Einer dieser Konstanten ist $P r_{\mathrm{t}}$. Sie bestimmt maßgeblich die turbulente und damit auch die effektive Wärmeleitfähigkeit der Strömung:

$$
\lambda_{\text {eff }}=\lambda+\lambda_{\mathrm{t}}=\lambda+\frac{c_{p} \eta_{\mathrm{t}}}{P r_{\mathrm{t}}}
$$

In $>$ Abb. $6.14 \mathrm{~d}$ wurde die turbulente Prandtl-Zahl $P r_{\mathrm{t}}$ sowohl für die Energiegleichung als auch für die Wandmodellierung deutlich vom Standardwert abgeändert. Es zeigt sich, dass durch Verringerung von $P r_{\mathrm{t}}$ die WÜK im mittleren Bereich des Seitenraumes angehoben werden können. An den Rändern ist auf diese Art jedoch keine Wirkung zu erzielen.

\subsection{LARGE-EDDY-SIMULATION}

Nachdem alle bisherigen Versuche gescheitert sind, mit stationären Simulationen unter Verwendung von modernen $\omega$-basierten RANS- und Reynolds-Stress-Modellen die berechneten WÜK den Messwerten anzunähern, wurden abschließend skalenaufgelöste Großstruktursimulationen (Large-Eddy Simulationen; LES) durchgeführt. Zur Anwendung kam dabei das LES-Modell in der Formulierung LES-WALE (Wall-Adapting Local Eddy-Viscosity), welches mit Hilfe einer near-wall damping function die Konsistenz der Wirbelviskosität an der Wand gewährleistet (siehe auch - Anhang A14). Insgesamt wurden vier verschiedene Rechnungen mit unterschiedlichen Sektormodellen und thermischen Randbedingungen durchgeführt ( Tab. A.13), bei denen jeweils dasselbe Rechengitter wie in Kap. 6.1.2 verwendet wurde.

Um aus den transienten Daten ( Kap. 6.1.7) zum Schluss wieder belastbare Mittelwerte für die WÜK auf der Außenwand zu erhalten, stellt sich zunächst die Frage nach der korrekten zeitlichen (und örtlichen) Mittelung der Werte. Die richtige Vorgehensweise richtet sich dabei auch nach der Art der angesetzten thermischen Randbedingung an der Außenwand. Betrachten wir dazu exemp- 
larisch den in Abb. 6.15 blau dargestellten Verlauf des WÜK im Monitorpunkt P07 über alle gerechneten 100.000 Iterationen $(2 \mathrm{~s})$ des $90^{\circ}$-Modells mit konstanter Wandwärmestromdichte. P07 liegt in etwa im Bereich der Staulinie (siehe Abb. 6.4), weshalb dort mit den größten Werten, aber auch den größten Schwankungen im zeitlichen WÜK-Verlauf zu rechnen ist. Wie sich zeigt, „rauschen“ die Werte deutlich, wobei auf den ersten Blick kein Mittelwert zu erkennen ist. Immer wieder nehmen die WÜK extrem große Werte an (bis $25.000 \mathrm{~W} /\left(\mathrm{m}^{2} \mathrm{~K}\right)$; in $>$ Abb. 6.15 nur bis $200 \mathrm{~W} /\left(\mathrm{m}^{2} \mathrm{~K}\right)$ dargestellt). Das liegt an sporadisch auftreffenden, kälteren Wirbelballen (Cold Spots), die noch nicht an der Wärmeübertragung im Seitenraum teilgenommen haben und deren Temperatur nahe an der Bezugstemperatur (Einlasstemperatur) oder auf Grund des Joule-Thomsen-Effektes sogar darunter liegt. Diese Wirbelballen bewegen sich von der Staulinie ( Abb. A.36 a in - Anhang A19.1) aus axial in Richtung der Seitenwände und erzeugen in $x$-Richtung gestreckte, auseinanderlaufende Zonen hoher Wärmeübertragung: Während ihres Durchzuges rufen sie wegen $\hat{\dot{q}}_{\mathrm{W}}=$ konst. sehr hohe (oder sehr niedrige) rechnerische WÜK hervor, die sich in den zeitlichen Spitzen in Abb. 6.15 widerspiegeln („Artefakte“). Würde man die Werte nun arithmetisch über der Zeit mitteln, würden diese Peaks die Mittelwerte verfälschen und unplausible hohe und damit aussagelose Werte liefern ( Abb. A.36 b in Anhang A19.1). Als richtige Wahl erweist sich für $\dot{q}_{\mathrm{W}}=$ konst. daher die Anwendung des harmonischen zeitlichen Mittelwertes $\bar{\alpha}_{\text {harm }}$ (Beweis siehe Anhang A18.1):

$$
\frac{1}{\bar{\alpha}_{\mathrm{harm}}}=\frac{1}{n} \sum_{i=1}^{n} \frac{1}{\alpha_{i}}
$$

Dabei bezeichnet $\alpha_{i}$ den Wert des WÜK bei der Iteration $i$ und $n$ die Anzahl der Iterationen, über die gemittelt wird. Um herauszufinden, wie groß $n$ sein muss, damit der Mittelwert zum einen numerisch stabil wird, zum anderen aber Anfangseffekte nicht mit berücksichtigt werden, die aus der noch nicht vollständigen Abklingung der stationären Startlösung resultieren, wird ein „auflaufender" harmonischer Mittelwert unter Einbeziehung von immer mehr Einzelwerten gebildet, beginnend mit dem Wert der letzten Iteration $\alpha_{N}$, und in $>$ Abb. 6.4 von rechts nach links aufgetragen:

$$
\bar{\alpha}_{\text {harm }, \text { inv }, i}=\left[\frac{1}{N-i+1} \sum_{N}^{i} \frac{1}{\alpha_{i}}\right]^{-1}
$$

Wie sich im rechten Teil der schwarzen Kurve zeigt, reichen $n=N-i+1=10.000$ Iterationen noch nicht aus, um den Mittelwert zu stabilisieren. Wählt man allerdings eine zu hohe Anzahl an Iterationen (z. B. $n=70.000$ ), fällt die Mittelwertkurve nach links hin schon wieder ab - ein Zeichen für noch nicht abgeklungene Starteffekte. Als sehr guter Kompromiss hat sich eine Anzahl von $n=50.000$ Iterationen, also eine Mittelung über die letzte physikalische Sekunde, erwiesen. 
Dies bestätigen auch die in Abb. 6.16 dargestellten invers aufgetragenen auflaufenden Mittelwertkurven der übrigen Monitorpunkte. Trägt man nun den über $n=50.000$ Iterationen gleitenden harmonischen Mittelwert

$$
\bar{\alpha}_{\text {harm,gleit }, i}=\left[\frac{1}{n} \sum_{i-n+1}^{i} \frac{1}{\alpha_{i}}\right]^{-1} \quad \forall i \geq n
$$

der einzelnen Monitorpunkte vorwärts auf ( Abb. 6.17), so sieht man, dass wirklich erst die Mittelung über die letzten 50.000 Iterationen (zwischen $i=50.000$ und 100.000) einen „stationären" und damit vertrauenswürdigen Mittelwert liefert. Die sich für $i=N=100.000$ und $n=$ 50.000 ergebende, harmonisch gemittelte WÜK-Verteilung

$$
\bar{\alpha}_{\text {harm }, N, n}=\left[\frac{1}{n} \sum_{i=N-n+1}^{N} \frac{1}{\alpha_{i}}\right]^{-1}
$$

an der Außenwand für das $90^{\circ}$-Modell ( Abb. A.37) ist deutlich glatter und liegt naturgemäß systematisch etwas tiefer als die arithmetische gemittelte Verteilung in Abb. A.36 b. Die dazugehörige harmonische Standardabweichung der zeitlichen Mittelwerte berechnet sich nach [153] $\mathrm{zu}:$

$$
\sigma_{\alpha, \mathrm{harm}, N}=\bar{\alpha}_{\mathrm{harm}, N}^{2} \cdot \sqrt{\frac{1}{n} \sum_{i=N-n+1}^{N}\left(\frac{1}{\alpha_{i}}-\frac{1}{\bar{\alpha}_{\mathrm{harm}, N}}\right)^{2}}
$$

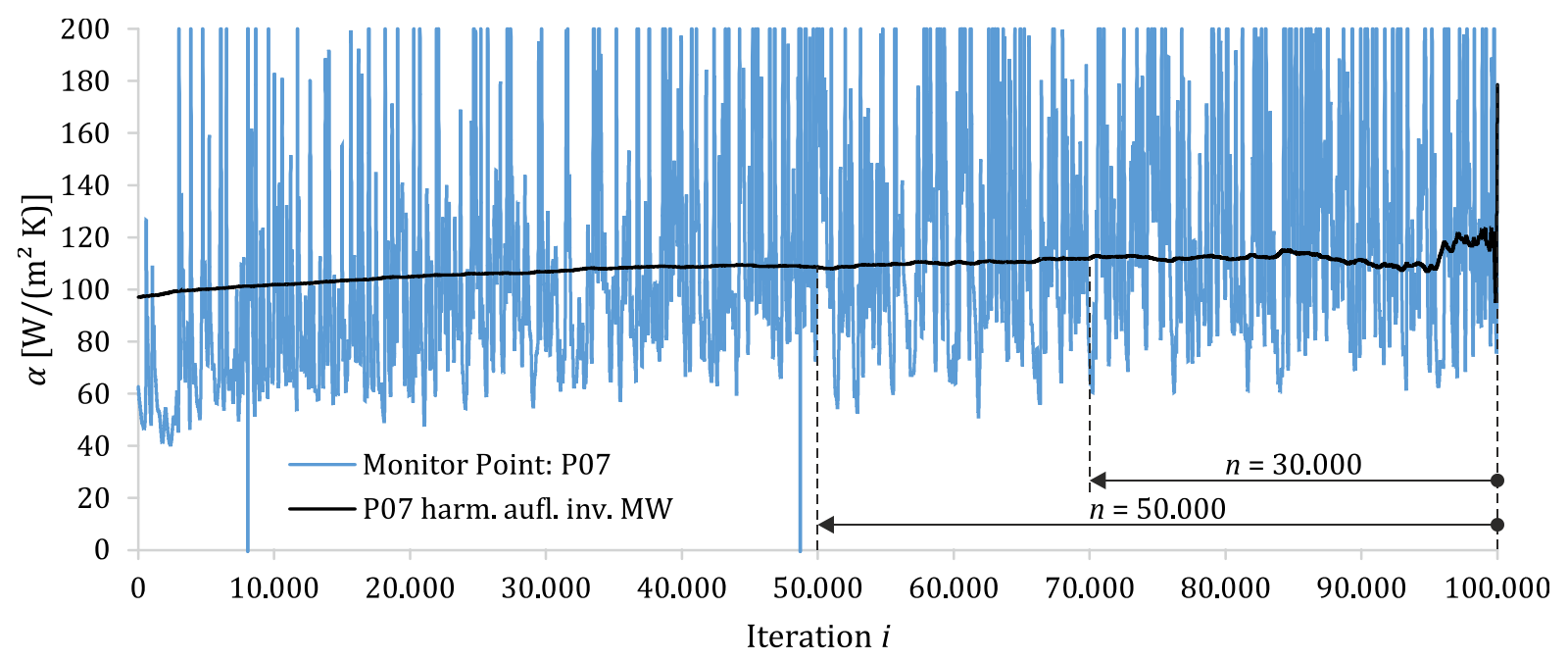

Abb. 6.15 Zeitlicher Verlauf und invers aufgetragener, auflaufender, harmonischer Mittelwert des WÜK im Monitorpunkt P07. $90^{\circ}$-Modell, $\hat{\hat{q}}_{\mathrm{W}}=$ konst., $t=0 \ldots 2 \mathrm{~s}$. 


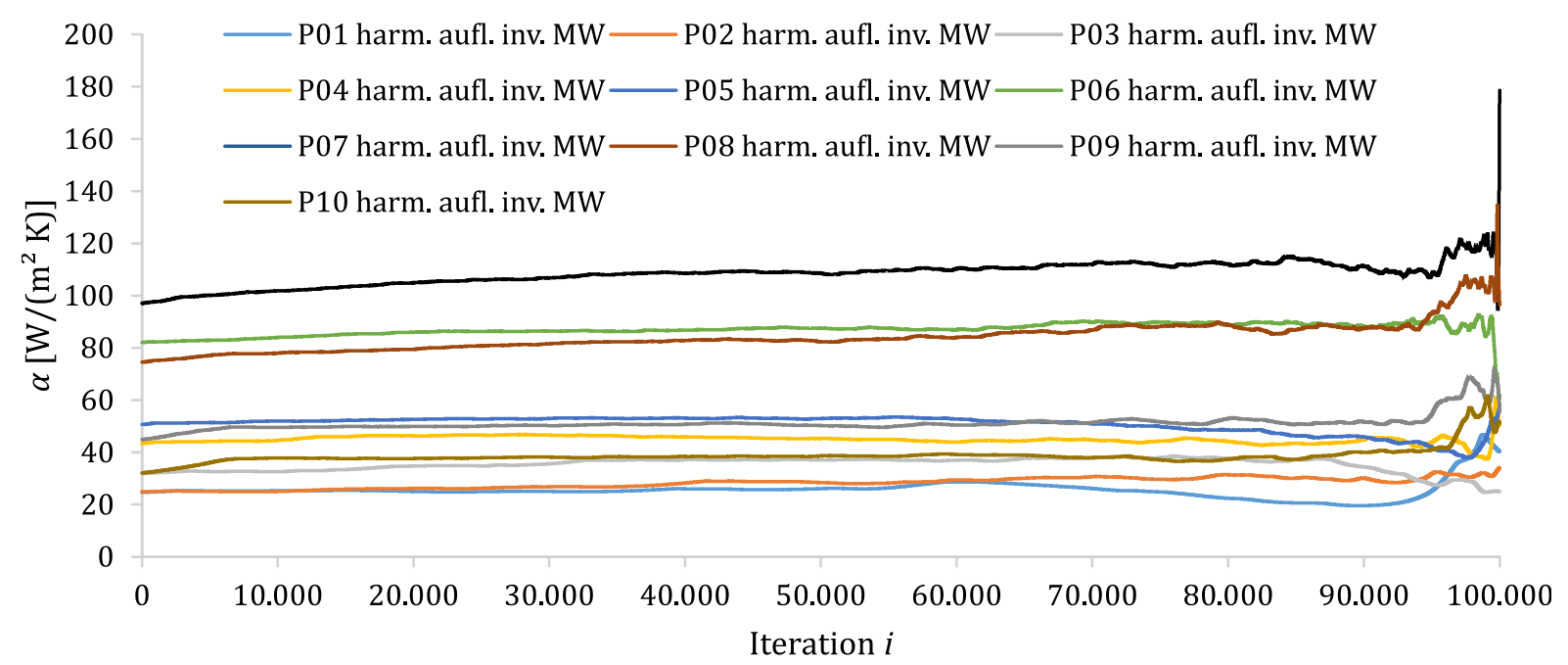

Abb. 6.16 Invers aufgetragene, auflaufende, harmonische Mittelwerte des WÜK in allen Monitorpunkten P01 bis P10. $90^{\circ}$-Modell, $\hat{q}_{\mathrm{W}}=$ konst., $t=0 \ldots 2 \mathrm{~s}$.

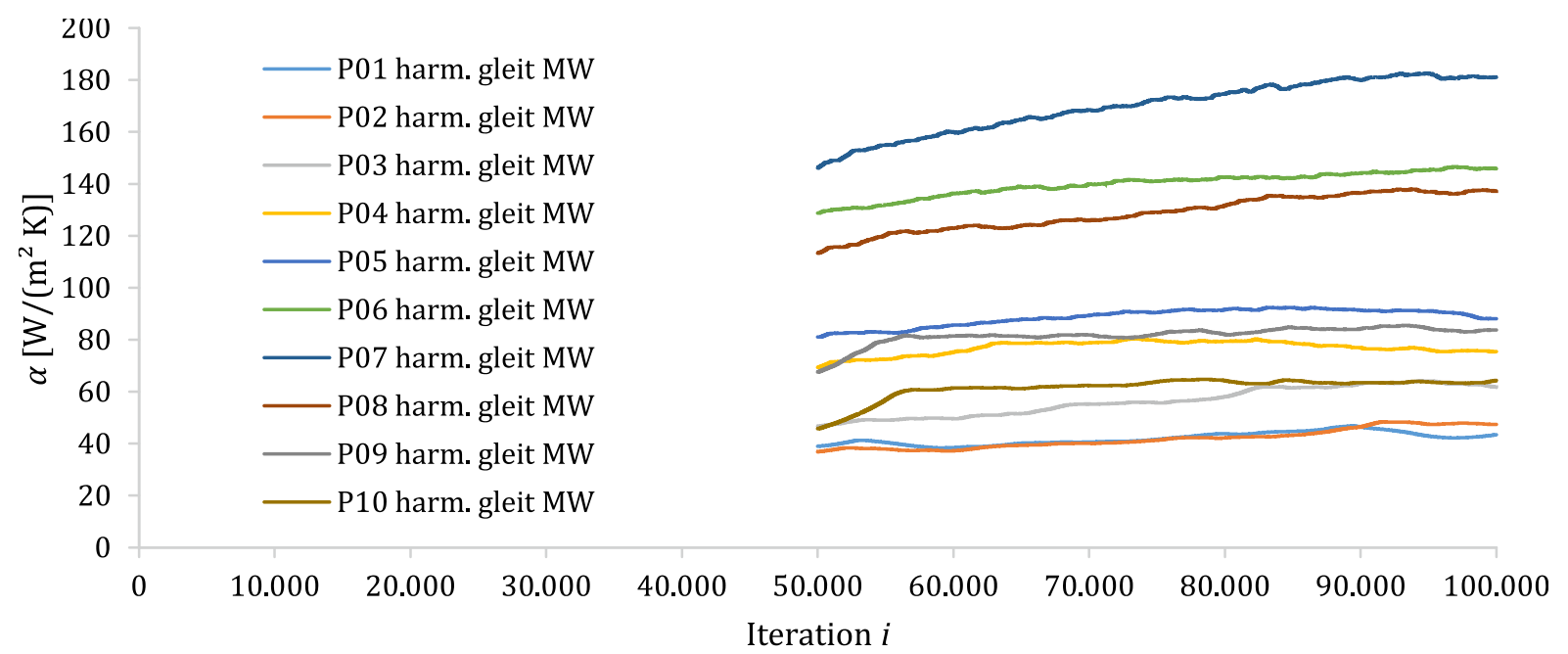

Abb. 6.17 Vorwärts aufgetragene, über $n=50.000$ Iterationen gleitende harmonische Mittelwerte des WÜK in allen Monitorpunkten P01 bis P10. $90^{\circ}$-Modell, $\hat{q}_{\mathrm{W}}=$ konst., $t=0 \ldots 2 \mathrm{~s}$.

Für die Fälle mit konstanter Wandtemperatur muss gemäß Anhang A18.2 der arithmetische Mittelwert (vgl. Gl. 6.26) und die dazugehörige Standardabweichung (Gl. 6.27) verwendet werden. Bildet man aus den zeitlich gemittelten WÜK ähnlich wie in Kap. 6.2 arithmetische Umfangsmittelwerte (Gleichung 6.29) und Standardabweichungen (Gleichungen 6.30, 6.31), so erhält man die zu Abb. 6.9 korrespondierende Abb. 6.18 für $\hat{\dot{q}}_{\mathrm{W}}=$ konst. Es zeigt im Vergleich höhere Werte im Staupunkt sowie einen monotoneren Verlauf im linken Bereich des Seitenraumes. Die relative Standardabweichung $\sigma_{\alpha \text {,ges }} / \bar{\alpha}$ liegt zwischen 25 und $35 \%$ und ist damit ebenfalls höher als in der stationären SST-Simulation. Noch höhere Mittelwerte und Schwankungen (50 bis 70 \%) bei ansonsten ähnlichem Kurvenverlauf weist die LES mit $T_{\mathrm{W}}=$ konst. auf ( Abb. 6.19). Im Vergleich zu den $90^{\circ}$-Modellen zeigen die in Abb. 6.20 für $\hat{\dot{q}}_{\mathrm{W}}=$ konst. und in Abb. 6.21 für $T_{\mathrm{W}}=$ konst. dargestellten Ergebnisse der 2D-Modelle ein deutlich differenziertes Verhalten. 


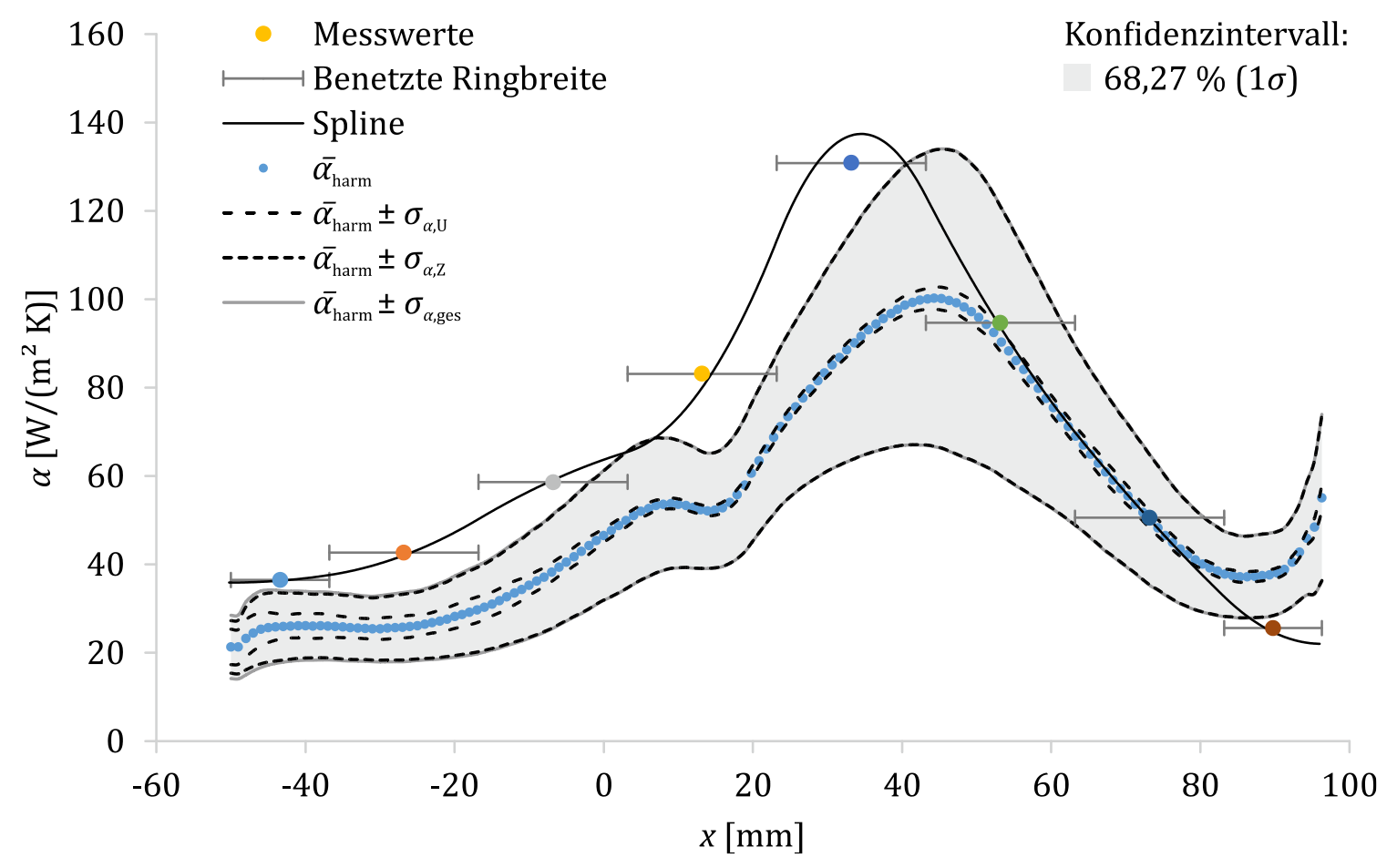

Abb. 6.18 Harmonischer Mittelwert der lokalen WÜK-Verteilung an der Außenwand des $90^{\circ}$-LES-

Modells über die letzten $\boldsymbol{n}=\mathbf{5 0 . 0 0 0}$ Iterationen. $\hat{\dot{q}}_{\mathrm{W}}=$ konst.

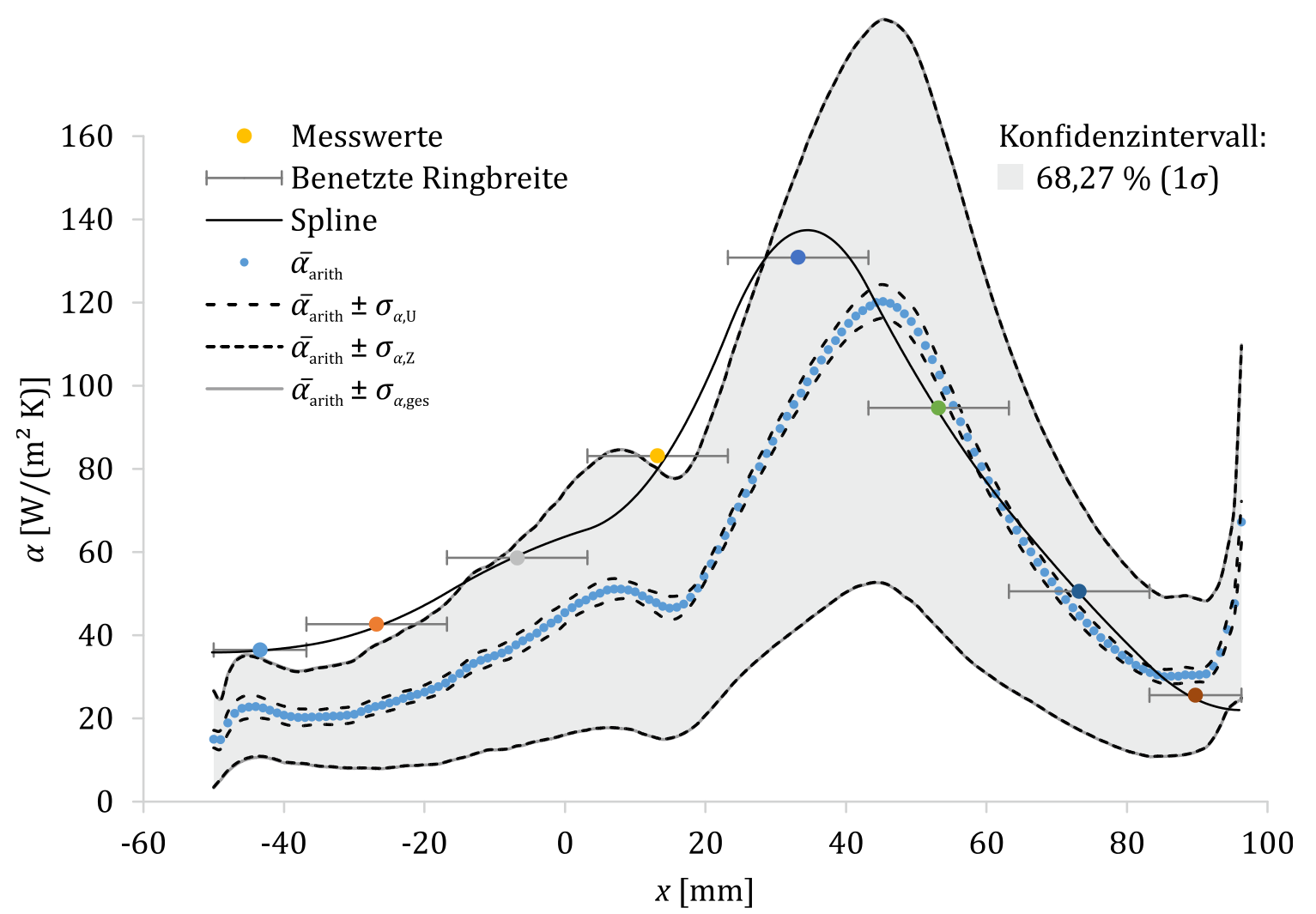

Abb. 6.19 Arithmetischer Mittelwert der lokalen WÜK-Verteilung an der Außenwand des $90^{\circ}$-LESModells über die letzten $\boldsymbol{n}=\mathbf{5 0 . 0 0 0}$ Iterationen. $T_{\mathrm{W}}=$ konst. 


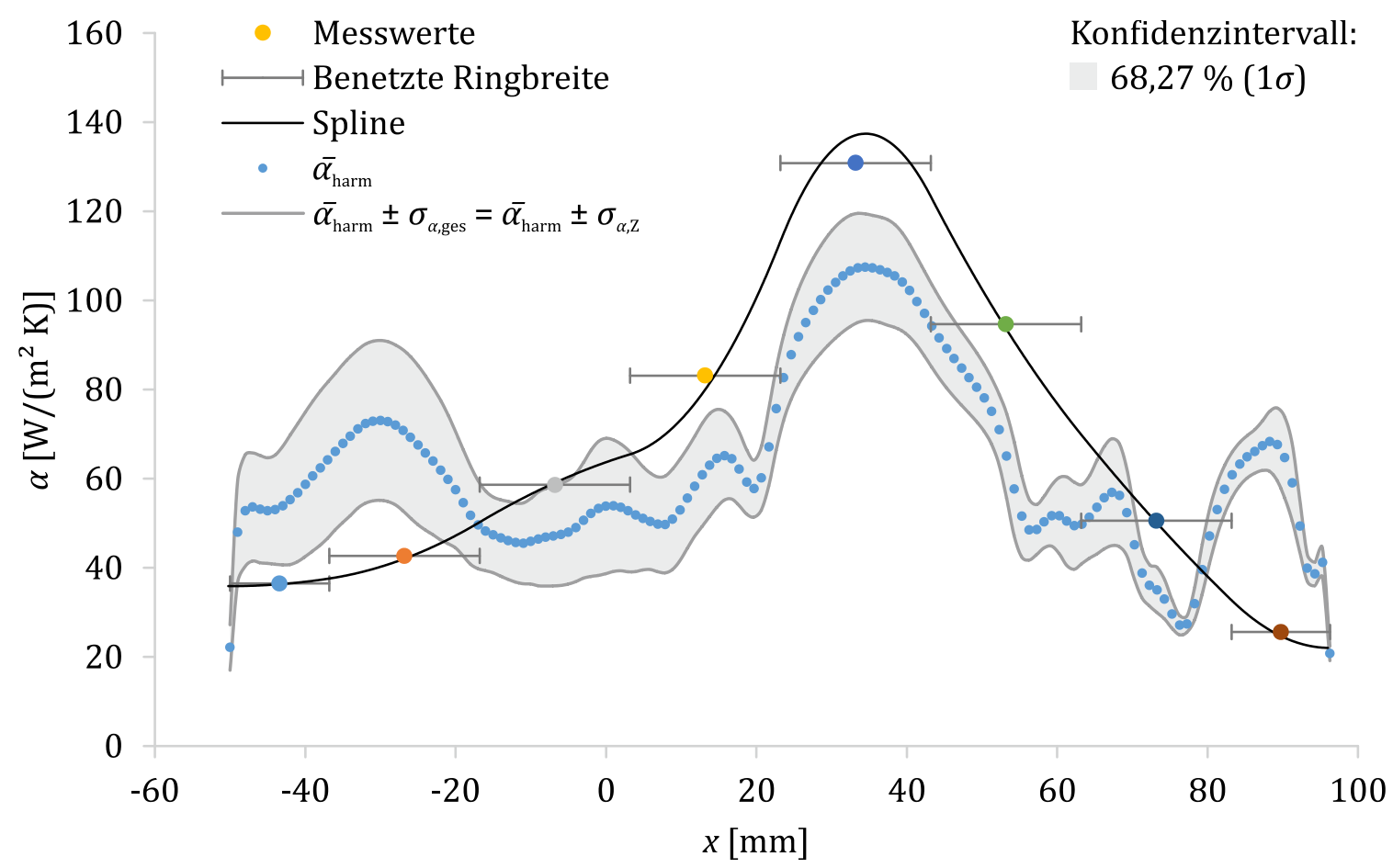

Abb. 6.20 Harmonischer Mittelwert der lokalen WÜK-Verteilung an der Außenwand des 2D-LESModells über die letzten $\boldsymbol{n}=\mathbf{5 0 . 0 0 0}$ Iterationen. $\hat{\dot{q}}_{\mathrm{W}}=$ konst.

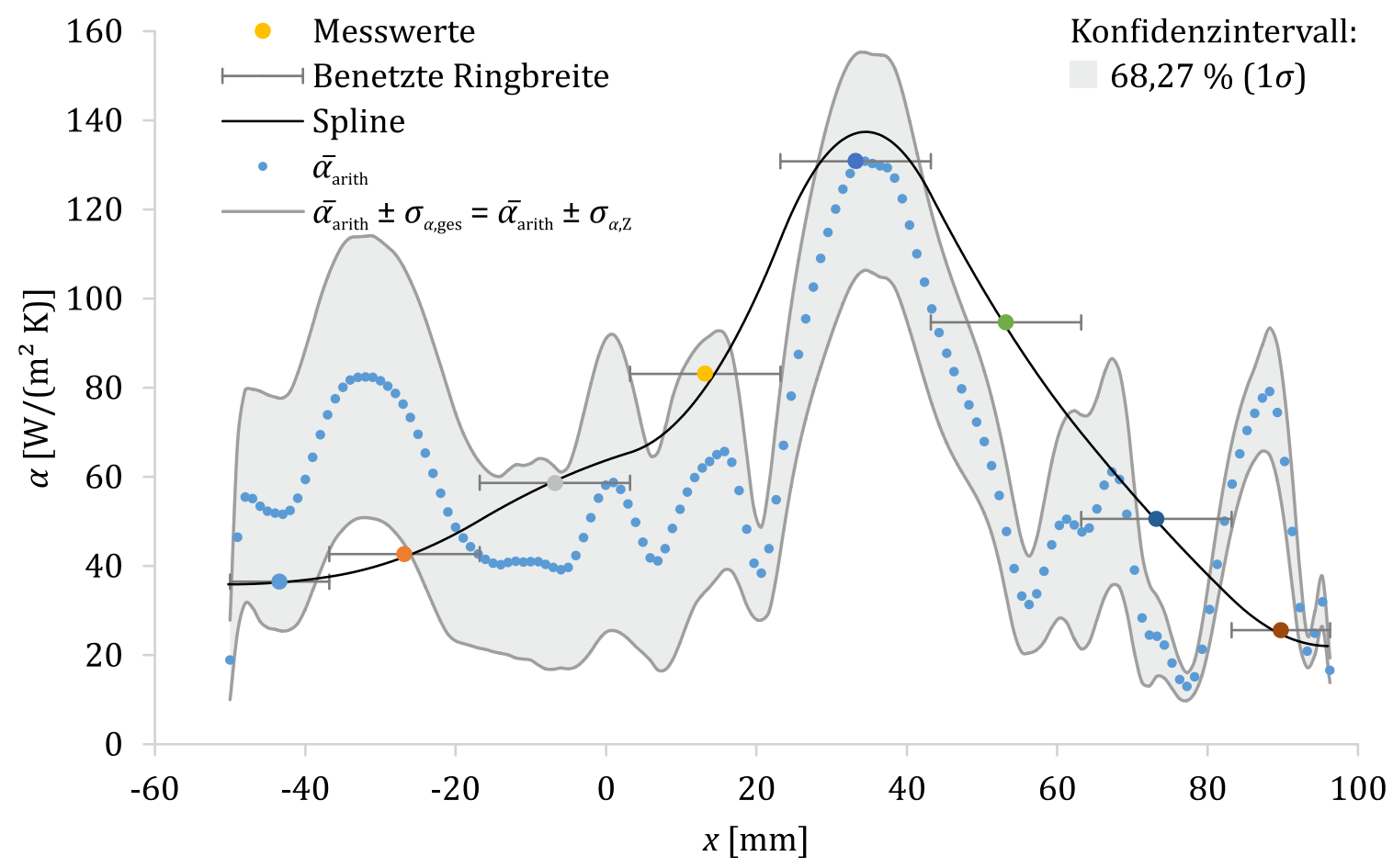

Abb. 6.21 Arithmetischer Mittelwert der lokalen WÜK-Verteilung an der Außenwand des 2D-LESModells über die letzten $\boldsymbol{n}=\mathbf{5 0 . 0 0 0}$ Iterationen. $T_{\mathrm{W}}=$ konst. 
Einen Aufschluss über die Ursachen kann ein Blick auf die (arithmetisch) gemittelten Druck-, Temperatur- und Geschwindigkeitsfelder

$$
\begin{aligned}
& \bar{p}_{\text {arith }}(x, y)=\frac{1}{n} \sum_{N-n+1}^{N} p_{i}(x, y) \\
& \bar{T}_{\text {arith }}(x, y)=\frac{1}{n} \sum_{N-n+1}^{N} T_{i}(x, y) \\
& \bar{c}_{\text {arith }}(x, y)=\frac{1}{n} \sum_{N-n+1}^{N} c_{i}(x, y)
\end{aligned}
$$

in Anhang A19 liefern. So weisen diese für die 2D-Modelle mehr lokale Maxima auf als für die $90^{\circ}$-Modelle, bedingt durch die größere Anzahl von vorausgesagten sekundären und tertiären Wirbeln im Seitenraum (vgl. z. B. Abb. A.46 c und Abb. A.52 c). Man ahnt, dass eine zweidimensionale Large-Eddy-Simulation nicht geeignet ist, um WÜK geeignet vorherzusagen, da die Wirbelausbreitung und Dissipation in Umfangsrichtung unterdrückt und offensichtlich nicht - wie bei den RANS-Modellen - zumindest modelliert wird. Die prozentualen Schwankungen gegenüber dem Mittelwert sind in der Folge höher als beim $90^{\circ}$-Modell und betragen zwischen $30 \%$ im linken und $5 \%$ im rechten Teil des Seitenraumes ( $\hat{\dot{q}}_{\mathrm{W}}=$ konst.; Abb. 6.20) bzw. zwischen 60 und $20 \%\left(T_{\mathrm{W}}=\right.$ konst.; Abb. 6.21).

\subsection{ERGEBNISSE DER SYSTEMATISCHEN NACHRECHNUNG}

Wie im voran gegangenen Kapitel beschrieben, liefern zweidimensionale Large-Eddy-Simulationen keine treffsichereren Ergebnisse in Bezug auf die richtige Vorhersage der gemessenen WÜKVerläufe und damit keinen Mehrwert gegenüber stationären 2D-Rechnungen mit modernen $\omega$ basierten Modellen. Ganz im Gegenteil: durch den fehlenden Ansatz für Turbulenzmodellierung in Umfangsrichtung erscheinen die Strömungsstrukturen und WÜK-Profile sogar eher unplausibel, weshalb 2D-LES vermutlich nach wie vor den Status eines Beta-Features in Ansys Fluent besitzt und vor der Verwendung erst freigeschaltet werden muss. Demgegenüber liegen gemessene und berechnete Werte bei den $90^{\circ}$-LES-Modellen näher beieinander. Der Rechenaufwand für ein Modell ist jedoch enorm (fast fünf Wochen für zwei physikalische Sekunden, siehe Tab. A.17). Daher sind solche Ansätze nicht für eine systematische Nachrechnung mehrerer Fälle geeignet. Obwohl auch die $\omega$-basierten Modelle die Messwerte nicht ganz getroffen haben, sollen mit ihrer Hilfe auf Grund des geringen Rechenaufwandes und des einfachen, automatisierbaren Workflows in Ansys Workbench alle untersuchten Seitenraumkonfigurationen (Kap. 5.7.4 bis Kap. 5.7.7) 
nachgerechnet werden. Es soll überprüft werden, ob auch für die anderen Versuchsfälle ähnliche Abweichungen vorausgesagt werden wie beim Basismodell.

Dazu werden für jede geometrische Seitenraumkonfiguration die Abmessungen $s, b$ und $e$ vorgegeben ( Abb. 4.2) und für jede untersuchte Reynolds-Zahl ( Tab. 5.10) und jeden Blechwinkel $\gamma$ arithmetische Mittelwerte für den Massenstrom $\dot{m}$ bzw. der querschnittsgemittelten Geschwindigkeit $q$, für Bezugstemperatur $T_{\text {ref }}$ und Bezugsdruck $p_{\text {ref }}$ sowie für die ringgemittelte Wandtemperatur $\bar{T}_{\mathrm{W}}$ über alle Repro-Messungen gebildet und gemäß Tab. A.11 als Randbedingungen auf das CFD-Modell aufgeprägt. Alle anderen Einstellungen entsprechen denen des Basisfalls (siehe ebenda) - mit Ausnahme des Turbulenzgrades: dieser beträgt hier abweichend $T u=10 \%$.

In Abb. 6.22 bis Abb. 6.25 sind die Ergebnisse für alle Konfigurationen dargestellt. Zu Vergleichszwecken wurden auch die jeweils gemessenen Werte aus den korrespondierenden Diagrammen in Abb. 5.29 bis Abb. 5.32 mit eingezeichnet. Die Farbkennzeichnung entspricht den in Tab. 5.10 angegebenen Reynolds-Zahlen. Beim Betrachten der vier Bilder wird auf den ersten Blick deutlich, dass - genau wie beim Referenzfall zuvor auch - die Lage und die WÜK im Bereich des Staupunktes sehr gut mit dem CFD-Modell vorhergesagt werden. Dabei skalieren die berechneten WÜK sehr schön mit der Reynolds-Zahl. In den weniger durchströmten Randbereichen der Kavität werden die Abweichungen zwischen berechneten und gemessenen WÜK größer. Bei den Seitenräumen mit geringer Einströmbreite $s$ (Abb. 6.22 d, e) versagt das CFD-Modell vollends. Hier werden keine nennenswerten bzw. gegenüber den Messwerten in Ausprägung und Lage völlig abweichende WÜK-Peaks vorhergesagt. Interessant ist, dass vor allem bei den drallfreien Strömungen in den Ecken des Seitenraumes stets ein kleineres lokales WÜK-Maximum berechnet wird, was in dieser Art auch bei den Messwerten zu erahnen ist (siehe z. B. Abb. 6.23 c, d oder Abb. $6.24 \mathrm{a}, \mathrm{c}$ ) und dort bisher auf numerische Instabilitäten in Folge reduzierter benetzter Ringoberfläche zurückgeführt wurde (vgl. Kap.4.4.4). Möglicherweise könnten dafür doch (auch) die kleineren Eckwirbel verantwortlich sein. Im Gegensatz zu den gemessenen Werten werden die berechneten WÜK-Verteilungen mit zunehmendem Drall in der Hauptströmung immer höher (Abb. 6.25 a-e). Über den Grund für diese Unterschiede kann nur spekuliert werden. Am wahrscheinlichsten ist, dass bei einem 2D-Modell zwar die Periodizität der Geschwindigkeit richtig berücksichtigt wird, nicht jedoch die Turbulenzdissipation in Umfangsrichtung. Diese hat gegenüber der drallfreien Strömung, bei denen sich die „Wirbelballen“ fast ausschließlich in axiale Richtungen entlang der Wand bewegen und dort der Modellfehler nicht allzu groß ist, doch einen nennenswerten Einfluss auf den Wärmeübergang, der mit steigendem Drallwinkel zunimmt. 

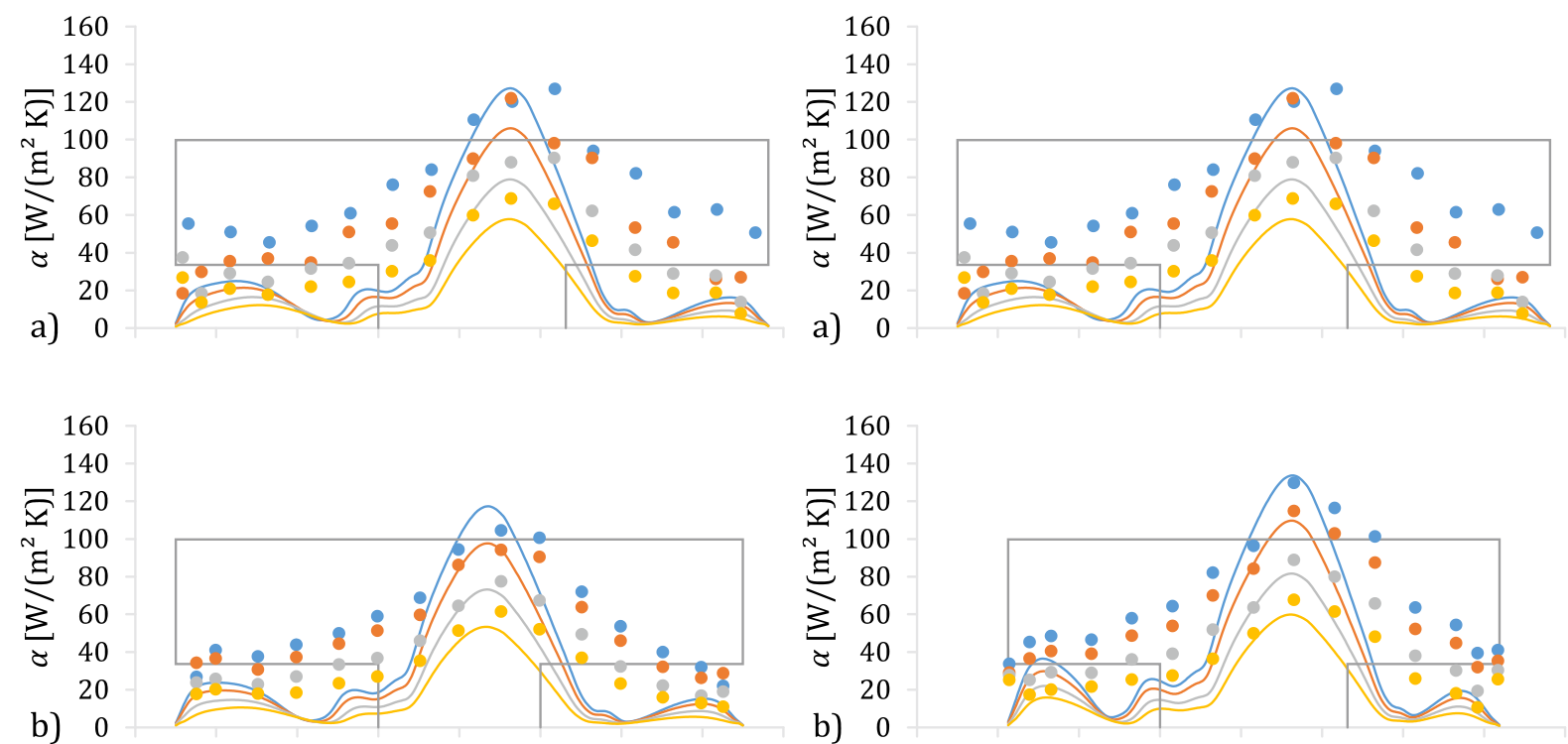

b) 0

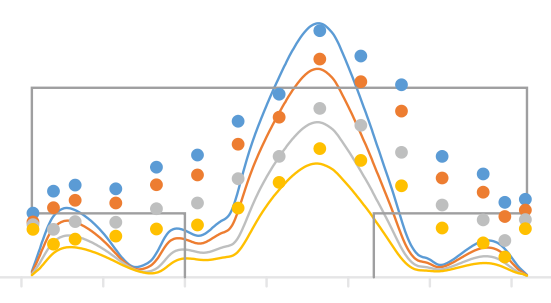

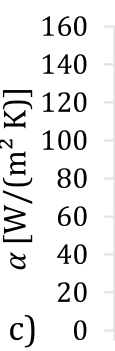

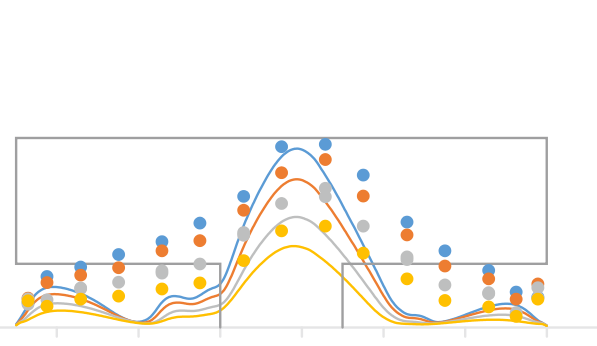

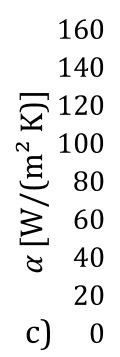

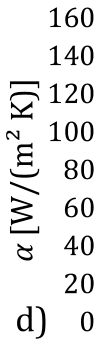
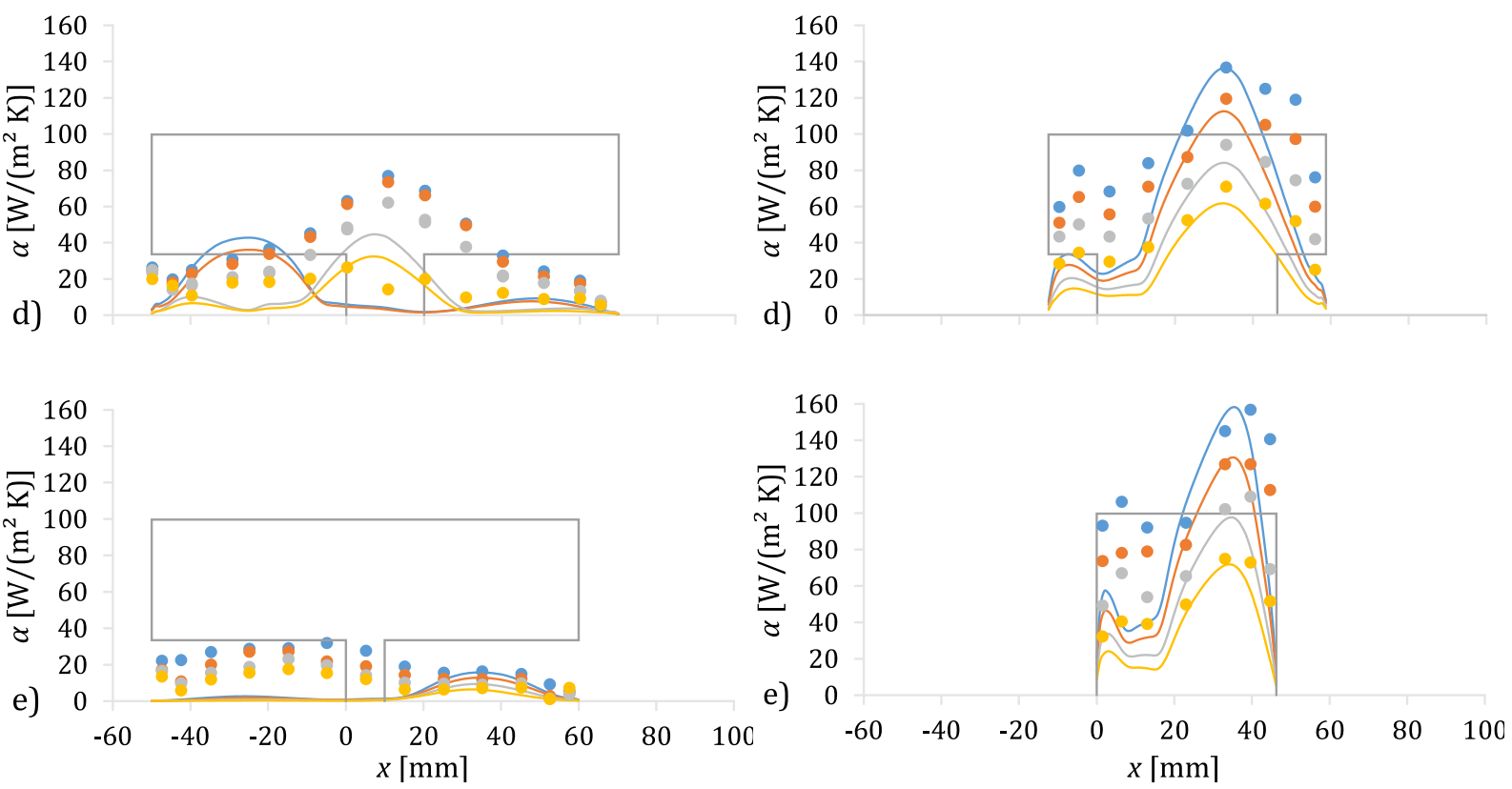

Abb. 6.22 CFD-Nachrechnung zum Einfluss der Einströmbreite auf die WÜK-Verteilung im Seitenraum:
a) $s=46,3 \mathrm{~mm}$, b) $s=40 \mathrm{~mm}$,
c) $s=30 \mathrm{~mm}$, d) $s=20 \mathrm{~mm}$,
e) $s=10 \mathrm{~mm} ; b=s+100 \mathrm{~mm}, e=0$.

Abb. 6.23 CFD-Nachrechnung zum Einfluss der Breite der Kavität auf die WÜK-Verteilung im Seitenraum:
a) $b=146,3 \mathrm{~mm}$, b) $b=121,3 \mathrm{~mm}$,
c) $b=96,3 \mathrm{~mm}, \mathrm{~d}$ ) $b=71,3 \mathrm{~mm}$,
e) $b=46,3 \mathrm{~mm} ; s=46,3 \mathrm{~mm}, e=0$. 

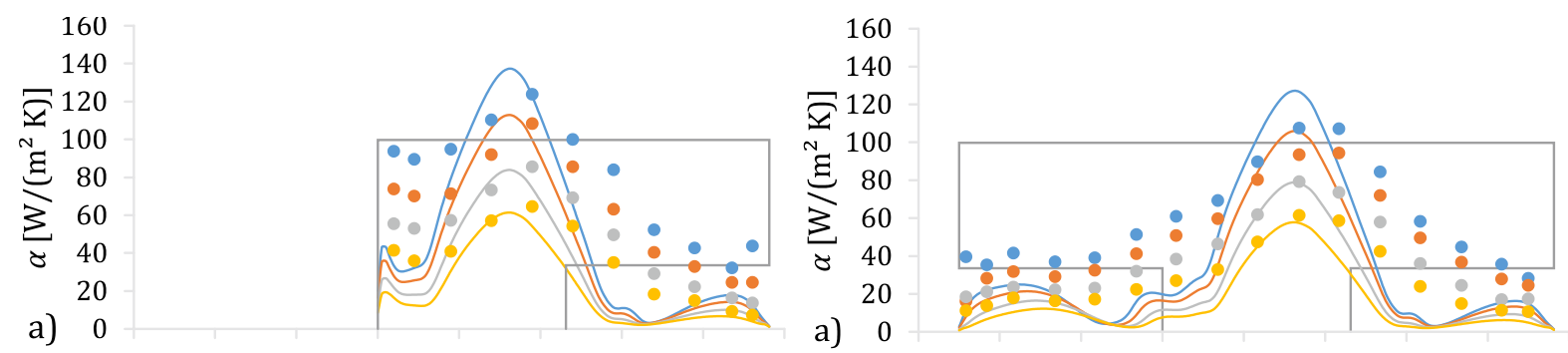

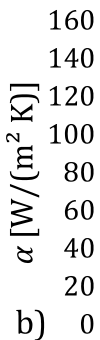

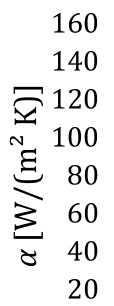

b) 0
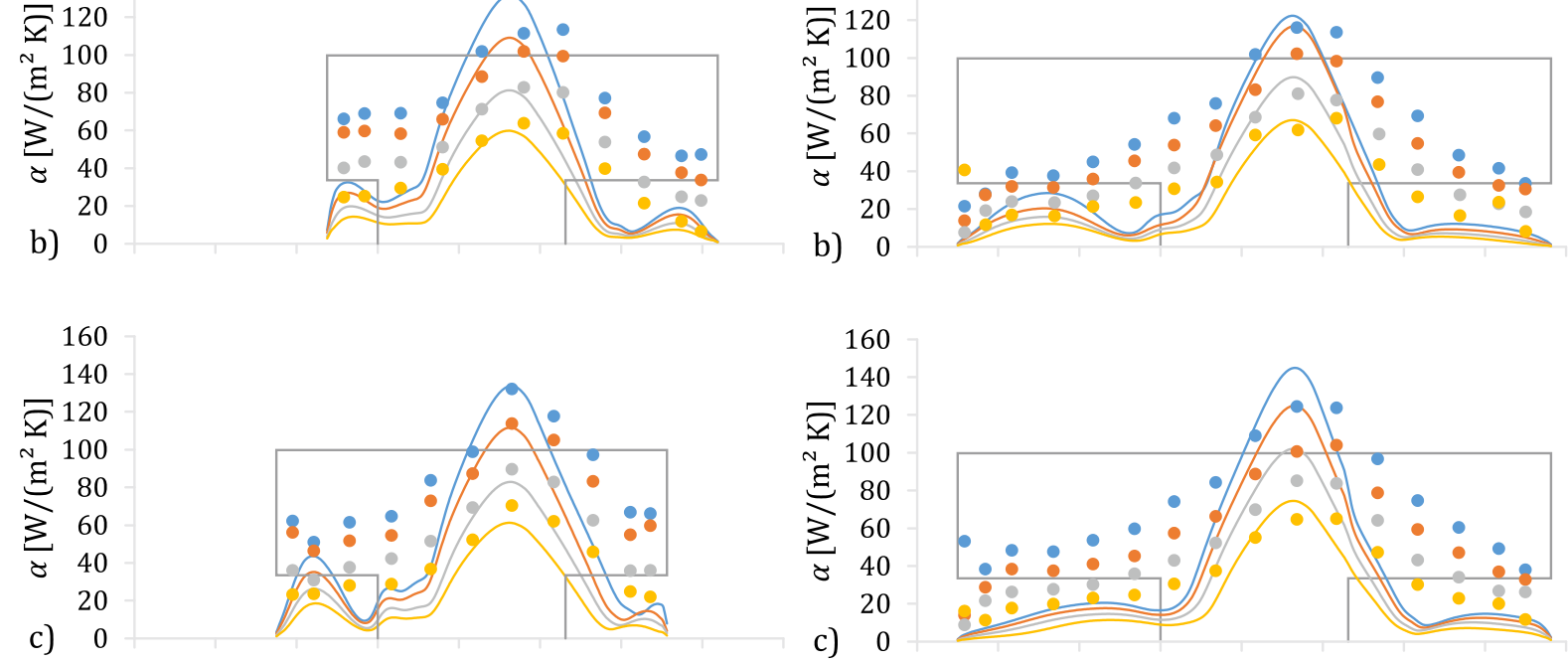

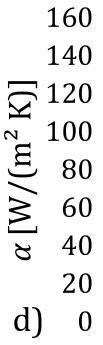
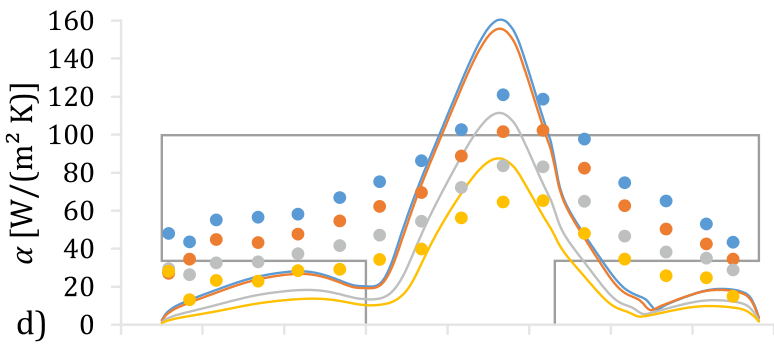

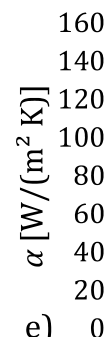

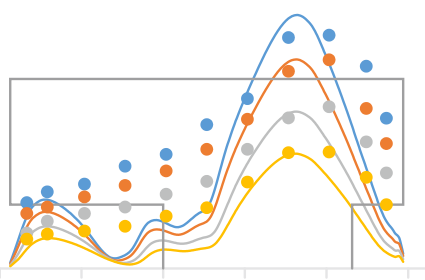

d)

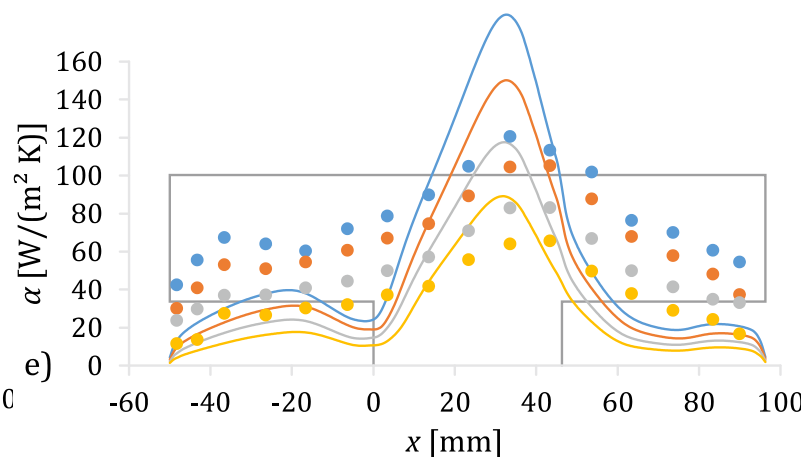

Abb. 6.24 CFD-Nachrechnung zum Einfluss der Exzentrizität der Kavität auf die WÜKVerteilung im Seitenraum:

a) $e=25 \mathrm{~mm}$, b) $e=12,5 \mathrm{~mm}$, c) $e=0$,

d) $e=-12,5 \mathrm{~mm}, \mathrm{e}) e=-25 \mathrm{~mm}$; $s=46,3 \mathrm{~mm}, b=96,3 \mathrm{~mm}$.

Abb. 6.25 CFD-Nachrechnung zum Einfluss des Drallwinkels auf die WÜK-Verteilung im Seitenraum:
a) $\alpha=0^{\circ}$, b) $\alpha=10^{\circ}$, c) $\alpha=20^{\circ}$,
d) $\alpha=30^{\circ}$, e) $\alpha=40^{\circ}$;
$s=46,3 \mathrm{~mm}, b=146,3 \mathrm{~mm}, e=0 \mathrm{~mm}$. 


\section{7 ÜBERTRAGUNG DER ERGEBNISSE AUF REALE TUR- BINENVERHÄLTNISSE}

Basierend auf der Ähnlichkeitstheorie dient die dimensionslose Nusselt-Zahl

$$
N u=\frac{\alpha \cdot l}{\lambda}
$$

dazu, die Intensität des Wärmeübergangs zwischen zwei verschiedenen Systemen zu vergleichen. Um die WÜK-Ergebnisse vom Modell („M“) auf die reale Turbine („, $\left.\mathrm{T}^{\prime}\right)$ zu übertragen, müssen die Nusselt-Zahlen für beide Systeme übereinstimmen, sodass die Wärmeübergangsmechanismen ähnlich zueinander sind:

$$
N u_{\mathrm{T}}=N u_{\mathrm{M}}
$$

Für reine erzwungene Konvektion ist dies der Fall, wenn die charakteristischen Reynolds-Zahlen für beide Systeme gleich sind, d. h. wenn die Strömungen ähnlich sind (Reynolds-Ähnlichkeit):

$$
R e_{\mathrm{T}}=R e_{\mathrm{M}}
$$

Verschiedene Korrelationen berücksichtigen auch den Einfluss der Prandtl-Zahl des Fluides, welche das Verhältnis zwischen Strömungs- und Temperaturgrenzschichtdicke charakterisiert:

$$
N u=k \cdot R e^{m} \cdot \operatorname{Pr}^{n}
$$

Unter der Voraussetzung identischer Reynolds-Zahlen (Gleichung 7.3) kann die Nusselt-Zahl im Modell um das Verhältnis der Prandtl-Zahlen korrigiert werden, um die Nusselt-Zahl für die Turbine zu erhalten:

$$
N u_{\mathrm{T}}=N u_{\mathrm{M}} \cdot\left(\frac{P r_{\mathrm{T}}}{P r_{\mathrm{M}}}\right)^{n}
$$

Durch Einsetzen von Gleichung 7.1 in Gleichung 7.5 ergibt sich der WÜK für die Turbine zu

$$
\alpha_{\mathrm{T}}=\alpha_{\mathrm{M}} \cdot \Lambda \cdot \frac{\lambda_{\mathrm{T}}}{\lambda_{\mathrm{M}}} \cdot\left(\frac{P r_{\mathrm{T}}}{P r_{\mathrm{M}}}\right)^{n}
$$

wobei $\Lambda=l_{\mathrm{M}} / l_{\mathrm{T}}$ der vom Maschinentyp abhängige Maßstab zwischen Modell und Turbine ist, z. B. $\Lambda=1: 4$.

Für voll turbulente Strömung ist sogar ein Extrapolieren der Werte auch für noch höhere Reynolds-Zahlen, die nicht gemessenen worden sind, denkbar. 
In diesem Fall erweitert sich Gleichung 7.6 gemäß Gleichung 7.4 zu:

$$
\alpha_{\mathrm{T}}=\alpha_{\mathrm{M}} \cdot \Lambda \cdot \frac{\lambda_{\mathrm{T}}}{\lambda_{\mathrm{M}}} \cdot\left(\frac{R e_{\mathrm{T}}}{R e_{\mathrm{M}}}\right)^{m} \cdot\left(\frac{P r_{\mathrm{T}}}{P r_{\mathrm{M}}}\right)^{n}
$$

bzw. für lokal unterschiedliche WÜK:

$$
\alpha_{\mathrm{T}}(x)=\alpha_{\mathrm{M}}(\Lambda x) \cdot \Lambda \cdot \frac{\lambda_{\mathrm{T}}}{\lambda_{\mathrm{M}}} \cdot\left(\frac{R e_{\mathrm{T}}}{R e_{\mathrm{M}}}\right)^{m} \cdot\left(\frac{P r_{\mathrm{T}}}{P r_{\mathrm{M}}}\right)^{n}
$$

Der genaue Wert des Parameters $n$, welcher in Kap. 5.9 zunächst mit 0,4 angenommen wurde, muss noch in weiterführenden Versuchen mit anderen Arbeitsfluiden (z. B. mit feuchter Luft oder Dampf) ermittelt werden. $\alpha_{\mathrm{M}}(x)$ kann entweder aus den Diagrammen mit den gemessenen WÜKVerläufen in Kap. 5.6 oder den Ergebnissen der CFD-Analyse ( Kap. 6.6) entnommen werden. Alternativ kann auch direkt auf die in Kap. 5.9 vorgeschlagene Nusselt-Korrelation zurückgegriffen und aus ihr gemäß Gl. 7.1 die anzusetzenden lokalen WÜK errechnet werden. Mittels dieses Ansatzes wurden in [154] erstmals lokale WÜK-Verläufe für einen Gehäuseseitenraum mit abgeschalteter Dampfzuführung in einer ausgeführten Dampfturbine ( Abb. 7.1) bei drei verschiedenen Lastfällen (Volllast, Teillast, Schwachlast) berechnet und als thermische Randbedingung 3. Art für die FE-Berechnung der thermomechanischen Gehäusebeanspruchung verwendet.

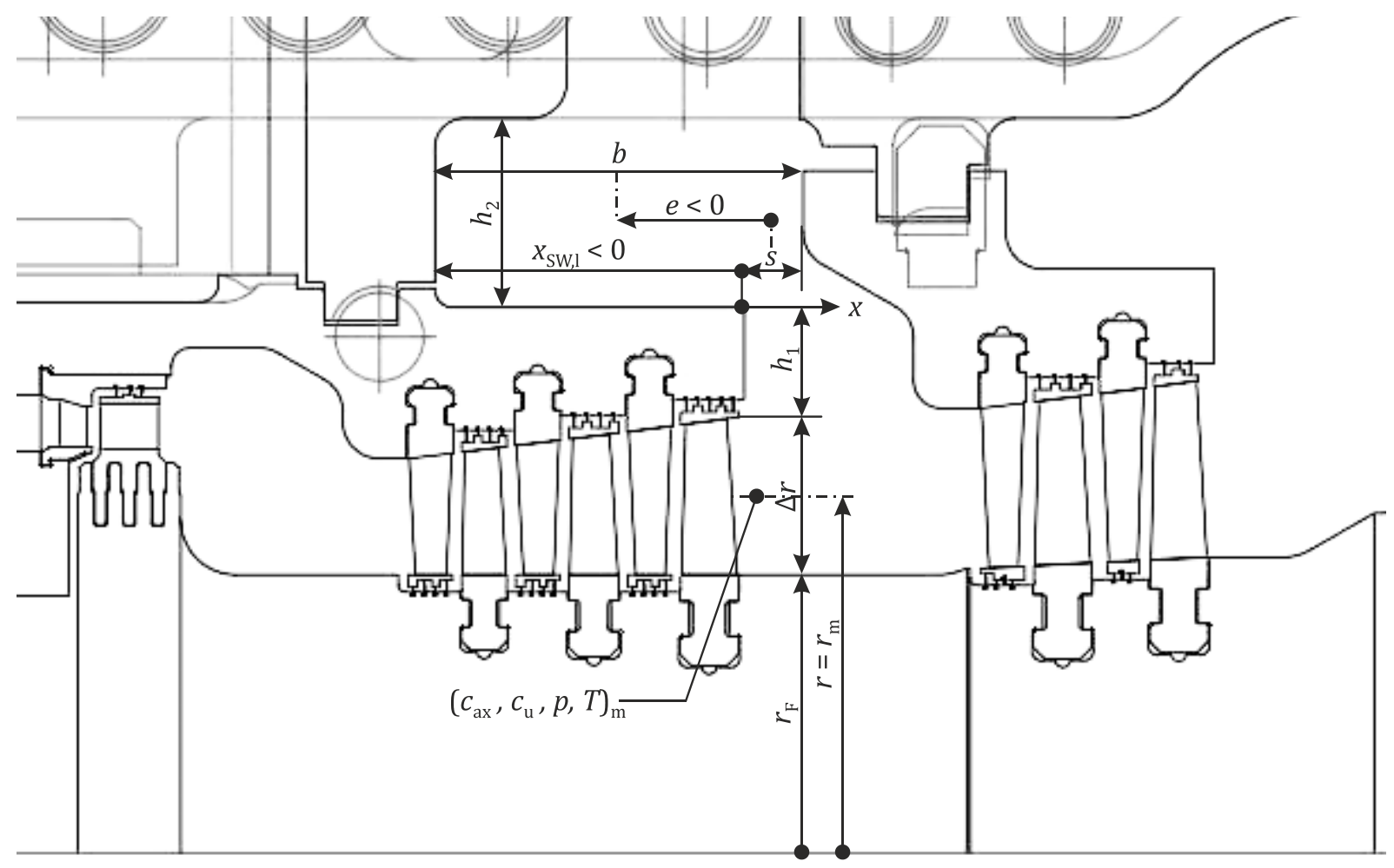

Abb. 7.1 Charakteristische thermodynamische Größen und Abmessungen am Längsschnitt eines Seitenraumes in einer ausgeführten Dampfturbine zur Berechnng lokaler Nusselt-ZahlVerläufe mittels der vorgeschlagenen Korrelation 5.65. 


\section{ZUSAMMENFASSUNG UND SCHLUSSFOLGERUNGEN}

Wie die Literaturrecherche zu Beginn dieser Arbeit gezeigt hat, besteht nach wie vor der Bedarf an strukturiertem Wissen zum lokalen Wärmeübergang in Gehäuseseitenräumen von Dampfturbinen. Trotz umfangreicher theoretischer und experimenteller Forschungstätigkeiten vor allem im russischsprachigen Raum bis ca. 1990 existieren bislang noch keine verallgemeinerungsfähigen Ergebnisse, die sich auf beliebige Seitenräume und Betriebsbedingungen anwenden lassen.

Um einen ersten Teil dieser Wissenslücke zu schließen, wurde im Rahmen dieser Arbeit ein neuartiger, mit Druckluft betriebener Seitenraumversuchsstand konzipiert und errichtet. Hierzu wurde die bestehende Druckluftinfrastruktur des Technikumgebäudes um einen weiteren Versuchsstrang erweitert. Es konnte nachgewiesen werden, dass mit dem implementierten EMSRSchema Druck, Temperatur und Durchsatz in der Versuchssektion unabhängig voneinander eingestellt und zuverlässig messtechnisch erfasst werden können.

Der Versuchsstand bildet den kreisringspaltförmigen Hauptströmungskanal einer repräsentativen Dampfturbine mit einem axialsymmetrischen Seitenraum ab. Der Dimensionierung der Versuchsstandgeometrie liegt dabei Reynolds-Ähnlichkeit zu Grunde. Die Basis hierfür bildeten Parameterstudien zu ausgeführten Dampfturbinen des Industriepartners. Mittels eines speziell entwickelten verstellbaren Chevron-Drallerzeugers kann der Anströmung zum Seitenraum zusätzlich ein variabler Drallwinkel aufgeprägt werden. In umfangreichen Vorversuchen konnte die Gleichmäßigkeit der Strömung durch Sondentraversierversuche mittels einer aufwändig kalibrierten Fünflochsonde unmittelbar vor dem Seitenraum sowie durch die Bestimmung der Wanddruckverteilung im Seitenraum festgestellt werden. Bei der Umsetzung des generischen Modellseitenraumes wurde auf größtmögliche Variabilität der axialen Abmessungen und somit der Querschnittsform geachtet, wobei spezielle Kettenantriebe zur Anwendung kamen. Der modulare Aufbau gestattet ferner eine einfache Auf-, Um- und Nachrüstbarkeit des Prüfstandes.

Die Bestimmung des Wärmeübergangs im Seitenraum erfolgt mittels zweier rückwirkungsarmer Messverfahren. Für die stationäre inverse Methode, welche im Mittelpunkt der vorliegenden Arbeit steht, wurde die Außenwand des Totraumes aus acht identischen, eigens hierfür hergestellten und instrumentierten Messringen zusammengesetzt. Jeder verfügt über eine Heizwicklung sowie kalibrierte Thermistoren zur Messung der Wandtemperaturen an verschiedenen Stellen am Umfang und über der Ringdicke. Für die Rückwärtsrechnung der lokalen WÜK aus den gemessenen stationären Materialtemperaturen wurden vier verschiedene Verfahren entwickelt und vorgestellt, welche sich hinsichtlich ihrer Komplexität und ihrer Rechenzeit unterscheiden. Während 
das thermische Netzwerkmodell die Berechnung der WÜK quasi in Echtzeit noch während der Messung ermöglicht, benötigen die beiden gradientenbasierten bzw. gradientenfreien Optimierungsverfahren ein Finite-Elemente-Modell und mehrere Iterationen. Demgegenüber ermöglicht die inverse Berechnung mittels Trefftz-Funktionen und Regularisierung einen Weg zur iterationsfreien analytischen Vorwärtsrechnung. Mit Ausnahme des gradientenfreien Verfahrens, welches auf einem Evolutionären Algorithmus basiert, nach mehreren Tausend Iterationen jedoch noch kein globales Optimum gefunden hat, liefern alle Methoden praktisch identische Werte. Selbiges gilt auch für den Vergleich mit den Ergebnissen der zweiten Messmethode, einer Anwendung der lokalen Übertemperaturmethode auf Basis miniaturisierter Sensoren.

Mit dem vorgestellten Versuchsstand sind im Rahmen dieser Dissertation erstmalig systematische experimentelle Untersuchungen zum Wärmeübergang in Seitenräumen durchgeführt worden, jedenfalls soweit dies die bisherige Literaturrecherche hergibt. Es konnte gezeigt werden, dass neben der Reynolds-Zahl in der Hauptströmung auch der Drall sowie die axialen Abmessungen des Seitenraumes einen nennenswerten, jedoch unterschiedlich stark ausgeprägten Einfluss auf die lokale WÜK-Verteilung entlang der Innenoberfläche der Totraumaußenwand haben, welche in guter Näherung durch eine Glockenkurve abgebildet werden kann. Als geometrischer Haupteinflussparameter konnte hierbei die Breite der Einströmöffnung zum Seitenraum identifiziert werden. Die Breite des Seitenraumes selbst sowie dessen Position gegenüber dem Einströmspalt spielen demgegenüber eine etwas untergeordnete Rolle.

Zur Verallgemeinerung der umfangreichen Versuchsergebnisse wurden die WÜK-Verläufe für sämtliche untersuchten Versuchskonfigurationen entdimensionalisiert und in Form einer einzigen Nusselt-Korrelation zusammengefasst. Die Korrelationsbildung gestaltete sich schwierig, da es hierbei keine standardisierte Vorgehensweise gibt. Vielmehr mussten die funktionellen Abhängigkeiten in einem Trial-and-error-Prinzip ermittelt werden. Die Korrelation ist im Stande, alle Messpunkte mit zufriedenstellender Genauigkeit abzubilden, und liefert darüber hinaus eine Berechnungsvorschrift, mit der lokale Wärmeübergangskoeffizienten in geometrisch unterschiedlichen Seitenräumen über einen für Turbinen repräsentativen Reynolds-Zahl-Bereich hinweg und unter Berücksichtigung des Drallwinkels in der Hauptströmung einfach und schnell auf analytischem Wege berechnet werden können.

In einer breit angelegten Unsicherheitsanalyse wurde die zu erwartende Genauigkeit aller relevanten gemessenen und von ihnen abgeleiteten Größen abgeschätzt. So konnte unter anderem gezeigt werden, dass die WÜK im Mittelbereich der Kavität bei allen inversen Methoden mit hoher Genauigkeit bestimmt werden können. Lediglich in den unmittelbaren axialen Randbereichen des 
Seitenraumes nimmt die Unsicherheit auf Grund der nur teilweisen Überdeckung durch die dortigen Messringe zu.

Begleitend zu den experimentellen Untersuchungen wurde parallel ein vollparametrisches CFDModell erstellt, welches als Werkzeug für die Nachrechnung beliebiger Versuchskonfigurationen zur Verfügung steht. Trotz umfangreicher Parameter- und Modellvariationen ist es bisher noch nicht gelungen, die experimentell ermittelten WÜK im Seitenraum vollständig mittels numerischer Simulation vorherzusagen. Vor allem in den Randbereichen der Kavität außerhalb des Staupunktes sind die Abweichungen noch deutlich zu groß. Weder die Anpassung der einzelnen Randbedingungen, die Wahl verschiedenster (verfügbarer) Turbulenzmodelle noch die Durchführungen konjugierter Analysen oder skalenaufgelöster transienter Simulationen konnten hier signifikante Verbesserungen bewirken. Dies rechtfertigt auf der einen Seite die Notwendigkeit der groß angelegten experimentellen Versuchskampagnen, verdeutlicht aber auch den Bedarf an weiteren Untersuchungen hinsichtlich der geeigneten numerischen Nachrechnung der Versuchsfälle. Die hier dokumentierten Studien können dabei als Einstieg dienen.

Insgesamt konnten im Rahmen der durchgeführten und dokumentierten Arbeiten wichtige Erkenntnisse, Ansätze und Hilfsmittel generiert werden, welche zum besseren Verständnis des Wärmeübergangs in Seitenräumen bei reiner erzwungener Konvektion und einphasiger, vollentwickelter turbulenter Strömung beitragen und nach geeigneter Implementierung die Vorhersagegenauigkeit der thermischen Gehäuseverformungen von Industriedampfturbinen auch für den lastflexiblen Betrieb steigern können. 



\section{AUSBLICK}

In den beiden laufenden Projekten ECOFLEX-turbo 4.3.4 A und C wird aktuell ein neuartiger Dampfversuchsstand inklusive erweiterter Dampfversuchsanlage entwickelt und im Zentrum für Energietechnik der TU Dresden aufgebaut ( Abb. A.53). Der Seitenraumversuchsstand „SiSTeR2“ ermöglicht es, den Wärmeübergang in generischen Dampfturbinenseitenräumen bei noch realitätsnäheren Bedingungen zu erforschen und erstmalig auch einen eventuellen Einfluss der veränderten Prandtl-Zahl festzustellen. Im Vergleich zu seiner luftbetriebenen Schwester wird die Dampfversuchsstrecke zunächst keinen axialsymmetrischen sondern einen ebenen Versuchsaufbau aufweisen. Dieser gestattet es, die lateralen Abmessungen des Hauptströmungskanals (Kanalhöhe $h$ ) sowie des Seitenraumes (Höhe des Eintrittsspaltes $h_{1}$, Höhe der Kavität $h_{2}$ ) sowie den Anstellwinkel des Seitenraumes gegenüber dem Strömungskanal - z. B. durch „Einlegen“ von Distanzstücken - zu variieren und deren Auswirkungen auf den WÜK-Verlauf zu bestimmen. Dabei kann die instrumentierte Außenwand einfach wiederverwendet werden. Dies ist bisher mit dem rotationssymmetrischen Druckluftaufbau nicht ohne weiteres möglich. Hier müssten alle Bauteile, bei denen sich Radien und/ oder Winkel verändern würden, ausgetauscht und neu gefertigt werden - einschließlich der Außenwand.

In einem ebenen Versuchsaufbau sind auch Sondentraversierversuche einfacher möglich, indem die Sonde von den seitlichen Stirnflächen aus eingeführt wird, ohne dass der komplette Versuchsaufbau gewechselt werden muss. Optische Messungen (PIV, LDA) wären ebenso denkbar. Die ebene Außenwand gestattet ferner auch die parallele Messung von Wanddrücken simultan zu den WÜK-Messungen. Beides ermöglicht noch grundlegendere Aussagen zu den Strömungs- und Wärmeübergangsmechanismen im Seitenraum, ganz nach dem Vorbild der russischen Forschungsarbeiten, die in Kap. 2.1 dargelegt wurden.

Zur Bestimmung der lokalen WÜK an der Außenwand sollen sogar drei verschiedene Verfahren zur Anwendung kommen: für die stationäre inverse Methode soll diesmal kein Scheiben- bzw. Segmentaufbau mehr sondern eine Messplatte aus einem homogenen, vergleichsweise schlecht thermisch leitendem Metall mit äußerer elektrischer Beheizung eingesetzt werden, bei der kalibrierte Eindraht-Thermoelemente (zweiter Pol ist Grundplatte) in einem gleichmäßigen Raster über der Länge, Breite und Dicke angeordnet sind. Zur Umsetzung der lokalen Übertemperaturmethode soll auf speziell für Dampfatmosphäre angepasste WÜK-„Messtaschensensoren“ zurückgegriffen werden, welche die Professur MFD bereits erfolgreich an der MAN-Versuchsdampfturbine in Oberhausen erprobt hat. Dort wurden auch Wärmestromlanzen getestet, die durch das 
Gehäuse geführt werden. Bei diesen wird über separate Heizwiderstände ein definierter Wärmestrom punktuell an das Fluid abgegeben, der über mehrere, über die Lanzenlänge angeordnete Thermoelemente bestimmt wird (lokal angewendete Wärmestrom-Temperaturdifferenz-Methode). Ob solche Lanzen in adaptierter Form auch im Dampfversuchsstand eingesetzt werden können, wird noch eruiert.

In der ersten Ausbaustufe soll im Dampfversuchsstand zunächst eine reine Rechteckkanalströmung umgesetzt werden, bei der die vollinstrumentierte Außenwand in die obere Kanalwand eingesetzt wird. Dort kann die Messsensorik zunächst erprobt und deren Messwerte mit den bekannten (Nusselt-) Korrelationen für Kanal-, Spalt- bzw. ebene Plattenüberströmung verglichen und gegebenenfalls kalibriert werden. Als zusätzliches viertes Messprinzip wäre es hierbei auch denkbar, die WÜK-Sensoren im Wandschubspannungsmessmodus zu betreiben. Aus dem axialen Druckgradient, welcher parallel über eine Reihe von Wanddruckbohrungen in der Messplatte bestimmbar ist, können die Wandschubspannungen und daraus unter Zugrundelegung der Reynolds-Analogie direkt lokale WÜK berechnet werden [85]:

$$
\tau_{\mathrm{W}}=\frac{h}{2} \frac{d p}{d x}=k \cdot N u^{\frac{1}{n}}, \quad n \approx \frac{1}{3}
$$

Damit die vorgesehene Versuchssektion mit ihren aus spannungstechnischer Sicht ungünstigen ebenen Wänden den hohen Temperaturen und dem Innendruck standhält, soll diese in einem Druckbehälter (Abb. A.53) untergebracht werden, der durch Druckausgleichsbohrungen mit dem Strömungskanal verbunden ist und diesen in jedem Betriebspunkt kraftfrei hält. Zum schnelleren Durchwärmen kann der Behälter optional mit Dampf vorgespült werden. Damit (im ausgekühlten Zustand) eine einfache und schnelle Zugänglichkeit an die Messstrecke möglich ist, soll das Druckgehäuse einen Schnellverschluss besitzen, wie er im Autoklavenbau üblich ist, bei dem über zwei halbkreisförmige Schalen die Flansche von Behälter und Deckel formschlüssig miteinander verbunden werden („Klammerverschluss“).

Um einen möglichst großen Druck- und Reynolds-Zahl-Bereich im Versuchsstand realisieren zu können, verfügt die Versuchsanlage über mehrere unabhängige Regelventile (vgl. A Abb. A.53 und

Abb. A.54). Während RV 1 den Druck im nachgeschalteten System regelt, teilen RV 2a und b den Massenstrom auf, der durch den Versuchsstand bzw. über den Bypass vorbei strömt. Eine Besonderheit der Anlage stellt der Dampfstrahlverdichter (besser: -ventilator) dar. Bei Bedarf kann über den Treibstrahl im engsten Querschnitt ein Großteil des Dampfmassenstroms, der durch den Versuchsstand fließt, wieder angesaugt und über eine Rezirkulationsleitung im Kreis gefördert werden. Auf diese Weise lässt sich ein Vielfaches der tatsächlich anlagenseitig zur Verfügung stehenden Dampfmenge rezirkulieren, allerdings unter Inkaufnahme einer moderaten antreibenden 
Druckdifferenz zwischen Treib- und Saugseite. Der „Verstärkungsfaktor“ hängt ganz Wesentlich vom Druckverlust in der Versuchsschleife ab. Bei maximal 0,5 bar kann im Auslegungspunkt bis zum 4,5-fachen des Treibdampfmassenstroms umgewälzt werden, wodurch der Reynolds-ZahlBereich im Versuchsstand deutlich erweitert werden kann. Die Feineinstellung erfolgt durch Vergrößern und Verkleinern der kritischen Querschnittsfläche über einen pneumatisch verstellbaren Ventilkegel im Dampfstrahlverdichter. Der Gemischmassenstrom wird quasi druckverlustfrei über zwei in Reihe geschaltete Ultraschall-Durchflussmessgerät gemessen, welchen eine lange gerade Anströmstrecke vorausgeht.

Ein wesentlicher Vorteil der geplanten Versuchsinfrastruktur besteht in der Möglichkeit der Durchführung von Langzeituntersuchungen durch den Brennerbetrieb des Dampfkessels, bei dem die Versuchsgasturbine nicht in Betrieb sein muss. Durch Austausch der Versuchsstrecke oder des gesamten Behälters steht die erweiterte Anlage prinzipiell auch für andere Anwendungen und Forschungstätigkeiten zur Verfügung. So ist im Rahmen des AG-Turbo-Anschlussvorhabens OptiSysKom 1.1b („Thermofluiddynamik in Gehäuseseitenräumen mit Dampfzufuhr und -entnahme im lastflexiblen Betrieb von Industriedampfturbinen“; FKZ 03EE5035E, Laufzeit 01.03.2020 bis 28.02.2023) vorgesehen - nach erfolgreicher Messung des eindimensionalen lokalen Wärmeübergangs für ausgewählte, ebene Seitenraumkonfigurationen - die Versuchstrecke mit einer geometrisch fixen, skalierten charakteristischen 3D-Turbinenseitenraumgeometrie und einer variablen Dampfzuführung und -entnahme zu ersetzen. Im Fokus steht hierbei die Evaluierung der zweidimensionalen Fluidtemperaturverteilung im Seitenraum bei verschiedenen $\mathrm{Zu}$ und Abdampfbedingungen (Menge, Temperatur, Stutzenanordnung) sowie Strömungsparametern in der Hauptströmung (Reynolds-Zahl, Druck, Vordrall). Eine geeignete Apparatur zur Drallerzeugung im Hauptströmungskanal stromauf des Seitenraumes ist entsprechend vorzusehen. Lokale WÜK sollen an ausgewählten Stellen im Seitenraum mittels WÜK-Sensoren gemessen werden, wenn auch nur begleitend.

Auch der druckluftbetriebene Seitenraumversuchsstand „SiSTeR“ soll parallel im Nachfolgeprojekt weitergenutzt werden. Er soll ebenfalls um eine kombinierte Fluidzufuhr und -entnahme erweitert werden, bei der die Menge des ab- bzw. zugeführten Fluides sowie die Temperatur der Zuströmung einstellbar ausgeführt sein sollen, ebenso wie die Position, Größe und Neigungswinkel des Stutzens an der Seitenraumaußenwand. Dies erfordert eine komplette Neuauslegung, Konstruktion, Fertigung, Instrumentierung und Kalibrierung der den Seitenraum radial nach außen hin begrenzenden Außenwand. Nach Inbetriebnahme und Erprobung der neuen Versuchsanordnung soll eine für die industrielle Praxis relevante Seitenraumkonfiguration mit Stutzen sowie 
eine weitere noch abzustimmende Anordnung, bei der Größe, Umfangsposition und Neigungswinkel sowie der Massenstrom und Strömungsrichtung des zu- bzw. abgeführten Fluides (in/ gegen Drallrichtung der Hauptströmung) zu spezifizieren sind, untersucht und der Einfluss auf den lokalen Wärmeübergang an der Innenoberfläche der Außenwand bestimmt werden.

Es ist zu erwarten, dass die Entnahme bzw. die Injektion von Fluid die Charakteristik der Temperaturverteilung und des Wärmeüberganges im Seitenraum und damit das thermomechanische Verhalten des Dampfturbinengehäuses signifikant verändert. Die geplanten Arbeiten an beiden Versuchseinrichtungen können hier wertvolle Beiträge für das bessere Verständnis liefern.

Perspektivisch ergibt sich ein großes Potenzial für die weitere Untersuchung des WÜK in Dampfanwendungen („Nachnutzung“), auch im Rahmen weiterer Nachfolgeprojekte. Während zunächst weiterhin die Studie reiner erzwungener Konvektion in überhitztem Dampf im Fokus steht, ist in Zukunft zum Beispiel auch eine Erweiterung des Versuchsstandes für die Untersuchung von Kondensations-, Verdampfungs- und Auftriebsphänomenen denkbar.

Dabei sollten die Kompetenzen der bis jetzt in den drei unterschiedlichen Teilprojekten organisierten Forschungsstellen (TU Dresden: Professur TEA, Professur MFD; Universität Duisburg-Essen) und Industriepartner (Siemens Görlitz und Mühlheim, MAN Oberhausen) weiter gebündelt werden. Die AG Turbo bietet dafür nach wie vor die ideale Plattform auf nationaler Ebene.

Eine noch engere wissenschaftliche Zusammenarbeit und Vernetzung sollte auch mit internationalen Forscherteams wie den ukrainischen Kollegen um Frau Prof. Dr. Svitlana Alyokhina von der Nationalen Akademie der Wissenschaften in Kharkiv angestrebt werden, an deren Vorgängerinstitution früher auch ein Teil der sowjetischen Forschung zum Wärmeübergang in Seitenräumen von Großdampfturbinen stattgefunden hat und wo heute noch immer an ähnlichen Themenstellungen geforscht wird [21]. Generell wird eindringlich empfohlen, auch weiterhin die (wenn auch schwer verfügbare aber wertvolle) russisch-sprachige Literatur der Vergangenheit und Gegenwart zur Recherche heranzuziehen und aufzuarbeiten und diese auf Grund der Sprachbarrieren nicht endgültig in der Grauen Literatur versinken zu lassen.

Der Erfahrungsaustausch sollte auch mit den Kollegen forciert werden, welche auf dem Gebiet der numerischen Strömungssimulation von Seitenräumen und ähnlichen Kavitäten arbeiten. Stellvertretend seien hier zum Beispiel die polnischen Partner um Prof. Dr.-Ing. Romuald Rządkowski und Dr.-Ing. habil. Piotr Lampart vom Institut für Strömungsmaschinen der Polnischen Akademie der Wissenschaft in Danzig genannt, die sich unter anderem mit der konjugierten Large-Eddy-Simulation von Kavitätenströmungen beschäftigen. Nur durch die begleitende Verbesserung der bestehenden CFD-Modelle können die Strömungs- und WÜ-Vorgänge noch besser verstanden und interpretiert werden. 


\section{LITERATUR}

[1] J. F. Clarke: An almost unknown great man. Charles Parsons and the significance of the patents of 1884, Occasional papers in the history of science and technology, Vol. 4, Newcastle upon Tyne Polytechnic, Newcastle upon Tyne, 1984. ISBN: 978-0-906721-18-6.

[2] C. A. Parsons: Improvements in rotary motors actuated by elastic fluid pressure and applicable also as pumps, Patent No. 6735, 23.04.1884.

[3] Siemens AG: Siemens steam turbine portfolio. Steam turbines from $10 \mathrm{~kW}$ to 1,900 MW, 2019, https://assets.new.siemens.com/siemens/assets/public.1560517188.c3192f5e-0979-4c719028-45f1913a80f2.steam-turbine-overview-2019.pdf, abgerufen 20.09.2019.

[4] T.-U. Kern, M. Wechsung: Dampfturbinen in der künftigen Energieversorgung, Siemens Day, 2015.

[5] Siemens AG: The Enhanced Platform. The Next Generation of Industrial Steam Turbines, 2014, https://assets.new.siemens.com/siemens/asets/api/uuid:2f615669d938f0d9ad97d384cb 53cdcf65b96c26/version:1527680067/brochure-ep-small-140714.pdf, abgerufen 20.09.2019.

[6] Siemens AG: 3D-Zeichnung einer Industriedampfturbine des Typs SST-600. EOG20041001-02, 2004, https://www.siemens.com/press/pool/de/pressebilder/2009/fossil_power_generation/300dpi/EFPG20041001-01_300dpi.jpg, abgerufen 20.09.2019.

[7] C. Heße: Entwicklung eines wissensbasierten modularen Verfahrens zur Beurteilung der thermischen Verkrümmung von Industriedampfturbinengehäusen, Dissertation, Technische Universität Dresden, Professur für Thermische Energiemaschinen und -anlagen, 2011, http://nbn-resolving.de/urn:nbn:de:bsz:14-qucosa-66668.

[8] В. А. Маляренко, В. Н. Голощапов, В. А. Барсуков, О. В. Котульская, О. Ю. Черноусенко: Теплообмен и газодинамика в камерах отбора паровых турбин, Наукова думка, Киев, 1991. ISBN: 978-5-12-002104-3.

[9] E. R. Plotkin, A. S. Leizerovich, I. V. Muratova: Investigation of Heat-Transfer Conditions in the K200-130 Steam Turbine, Thermal Engineering, Vol. 18, Nr. 5, 1971, S. 41-45.

[10] A. S. Leizerovich, E. R. Plotkin: Correlating the results of experimental investigations into heat transfer coefficients of steam turbine stator elements, Thermal Engineering, Vol. 38, Nr. 10, 1991, S. 550-553.

[11] A. I. Aleshin, A. S. Leizerovich, E. R. Plotkin: Conditions of Heat Transfer and Gasdynamics in Steam Turbine Extraction Chambers, Thermal Engineering, Vol. 23, Nr. 8, 1976, S. 37-42.

[12] В. А. Барсуков: Исследование газодинамики и теплообмена в камерах отбора паровых турбин, дис. канд. техн. наук., Институт проблем машиностроения, Харьков, 1980. 
[13] В. А. Маляренко, В. Н. Голощапов, В. А. Барсуков: Распределение газодинамических параметров потока в модели камеры регенеративного отбора паровой турбины, Энергетическое машиностроение, Nr. 25, 1978, S. 52-60.

[14] Ю. М. Мацевитый, Е.А.Барсуков, В.Н.Голощапов, О. В. Котульская: Гидравлический расчет камеры отбора паровой турбины, Теплоэнергетика, Vol. 30, Nr. 11, 1983, S. 61-62.

[15] Y. M. Matsevityi, V. A. Barsukov, V. N. Goloshchapov, V. A. Malyarenko: Estimation of the heattransfer conditions in the neighborhood of the stagnation point during impingement of a jet on an obstacle, Journal of Engineering Physics, Vol. 37, Nr. 2, 1979, S. 899-903. DOI: $10.1007 / \mathrm{BF} 00861440$.

[16] В. А. Маляренко: Исследование теплообмена в камерах отбора турбин типа К-300-240 ПОТ ХТЗ в пусковых режимах, Энергетическое машиностроение, Nr. 34, 1982, S. 68-77.

[17] В. А. Маляренко, В. А. Барсуков: Обобщенная методика расчета коэффициентов теплоотдачи в камерах регенеративного отбора паровых турбин, Энергетическое машиностроение, Nr. 30, 1980, S. 74-83.

[18] Ю. Е. Маховко, Е. М. Верников: Определение локальных коэффициентов теплоотдачи в корпусах паровых турбин, Энергомашиностроение, Vol. 19, Nr. 8, 1973, S. 10-12.

[19] Ю. Е. Маховко, А. Н. Коваленко, Е. М. Верников: Условия теплообмена стенке камеры регенеративного отбора турбины при включенном отборе пара, Энергомашиностроение, Vol. 21, Nr. 9, 1975, S. 37-38.

[20] В. Н. Голощапов, О. В. Котульская, М. П. Позигун: Теплообмен на поверхности камеры регенеративного отбора паровой турбины, Энергетика и электрификация, Nr. 6, 2000, S. 7-11.

[21] Ю. М. Мацевитый, С.В. Алехина, В. Н. Голощапов, О. В. Котульская: Теплообмен в элементах конструкций паровых турбин, Харьков, 2012. ISBN: 978-966-02-6322-2.

[22] Л. М. Зысина-Моложен, Л. В. Зысин, М. П. Поляк: Теплообмен в турбомашинах, Машиностроение, Ленинград, 1974.

[23] Л. М. Зысина-Моложен: Расчет теплового пограничного слоя в потоке сжимаемого газа, Инженерно-физический журнал, Vol. 5, Nr. 6, 1962, S. 21-26.

[24] Д. Чэнь: Моделирование течений в трактах отбора для определения их сопротивления и влияния на структуру потока в околоотборных ступенях паровых турбин, дис. канд. техн. наук., Государственный Технический Университет, Санкт-Петербург, 2000.

[25] B. Rosic, C. Maria Mazzoni, Z. Bignell: Aerodynamic Analysis of Steam Turbine Feed-Heating Steam Extractions, Journal of Engineering for Gas Turbines and Power, Vol. 136, Nr. 11, 2014, S. 197. DOI: $10.1115 / 1.4027569$. 
[26] R. Rządkowski, P. Lampart, L. Kwapisz, M. Szymaniak, M. Drewczyński: Transient Thermodynamic, Thermal and Structure Analysis of a Steam Turbine During Its Start-Up, ASME Turbo Expo 2010, Power for Land, Sea, and Air Volume 2: Combustion, Fuels and Emissions, Parts A and B, Glasgow, UK, 14.-18.06.2010, ASME, 2010, S. 1103-1112. ISBN: 978-0-7918-4399-4. DOI: $10.1115 /$ GT2010-22813.

[27] R. Rządkowski, P. Lampart, M. Szymaniak, M. Drewczyński: Transient Thermodynamic and Thermoelastic Analysis of an Improved Heat-up Procedure for a Large Power Steam Turbine, Journal of Vibration Engineering \& Technologies, Vol. 2, Nr. 2, 2014, S. 73-88.

[28] M. Topel: Steam turbine thermal modeling for improved transient operation, Lizenziatsarbeit, KTH Stockholm, Industrial Engineering and Management, Department of Energy Technology, Heat and Power Division, 2014, http://kth.diva-portal.org/smash/record.jsf?pid=diva2\%3A765657\&dswid=6539.

[29] M. Topel, M. Jöcker, S. Paul, B. Laumert: Differential Expansion Sensitivity Studies During Steam Turbine Start-Up, Proceedings of the ASME Turbo Expo, Turbine Technical Conference and Exposition 2015, Montreal, Quebec, Canada, 15.-19.06.2015, ASME, New York, N.Y., 2015, V008T26A006. ISBN: 978-0-7918-5679-6. DOI: 10.1115/GT2015-42214.

[30] M. Schinnerl, W. Beer, R. Willinger: Interpretation of Unexpected Aggregation of Condensate in Shrouded HP-Stages of an Industrial Steam Turbine, Proceedings of the ASME Turbo Expo, ASME 2014 Turbo Expo: Turbine Technical Conference and Exposition, Düsseldorf, 16.-20.06.2014, ASME, New York, N.Y., 2014. ISBN: 978-0-7918-4558-5. DOI: 10.1115/GT2014-26083.

[31] A. S. Leizerovich: Steam turbines for modern fossil-fuel power plants, Fairmont Press, Lilburn, Boca Raton, 2008. ISBN: 978-0-88173-549-9.

[32] A. Aroussi, S. A. Ferris: An Experimental and Computational Study of Captive Eddies in Rectangular Cavities, International Congress on Instrumentation in Aerospace Simulation Facilities, Williamsburg, VA, USA, IEEE, 1987, S. 22-32.

[33] A. Aroussi, S. A. Ferris: Recirculating flow in double cavity geometries prediction of LDA measurements, 4th International Symposium on Applications of Laser Anemometry to Fluid Mechanics, Lissabon, Portugal, 11.-14.07.1988, Instituto Superior Tecnico, Lisbon, 1988, Nr. 5.3.

[34] A. Aroussi: Measurement and prediction of recirculating flows in a 'T' shaped cavity, Proceedings of the International Symposium on Computational Fluid Dynamics, Sydney, Australia, 23.27.08.1987, Amsterdam, North-Holland, 1988, S. 229-237.

[35] A. Aroussi: Flow in multiple cavity geometries, International Congress on Instrumentation in Aerospace Simulation Facilities, Forschungszentrum Göttingen, 18.-21.09.1989, 1989, S. 245-253. DOI: 10.1109/ICIASF.1989.77678. 
[36] A. Aroussi, I. Grant, Y.-Y. Yan: Forced convection in joined rectangular grooves numerical simulation of PIV and LDA measurements, International Congress on Instrumentation in Aerospace Simulation Facilities, Rockville, MD, 27.-31.10.1991, 1991, S. 94-102. DOI: 10.1109/ICIASF.1991.186230.

[37] A. Aroussi, I. Grant, Y.-Y. Yan, E. Owen: A comparison between LDA and PIV measurements and numerical predictions of the flow in an L-shaped cavity, Laser Anemometry - Advances and Applications, Cleveland, OH, 05.-09.08.1991, 1991, S. 267-272.

[38] A. Aroussi, Y.-Y. Yan, I. Grant: Experimental verification of numerical simulation of turbulent flow past enclosures in offshore structures, Optics and Lasers in Engineering, Vol. 16, Nr. 4-5, 1992, S. 391-409. DOI: 10.1016/0143-8166(92)90099-S.

[39] M. K. Chyu, R. J. Goldstein: Local mass transfer in rectangular cavities with separated turbulent flow, Proceedings of the Eighth International Heat Transfer Conference, San Francisco, CA, Aug. 17-22, 1986, Hemisphere Publishing Corp., Washington, DC, 1986, S. 1065-1070.

[40] V. V. Degtyareva, V. A. Mukhin, V. E. Nakoryakov: Experimental study of mass exchange in axisymmetric cavities, Journal of Engineering Physics, Vol. 43, Nr. 2, 1982, S. 829-833. DOI: $10.1007 / \mathrm{BF} 00825005$.

[41] H. Inaba, T. Fukuda, M. Sugawara: Mixed, forced and natural convective heat transfers in cavities heated from bottom surface, Bulletin of the JSME, Vol. 29, Nr. 249, 1986, S. 862-866.

[42] D. H. Lee, J. S. Lee, H. J. Park, M. K. Kim: Experimental and numerical study of heat transfer downstream of an axisymmetric abrupt expansion and in a cavity of a circular tube, Journal of Mechanical Science and Technology, Vol. 25, Nr. 2, 2011, S. 395-401. DOI: 10.1007/s12206-0101222-6.

[43] V. I. Terekhov, N. I. Yarygina: Forced-Convection Heat Transfer from the Bottom of Trenches with Rectangular or Inclined Walls, Experimental Heat Transfer, Vol. 9, Nr. 2, 1996, S. 133-148. DOI: $10.1080 / 08916159608946518$.

[44] H. Togun, S. N. Kazi, A. Badarudin: A Review of Experimental Study of Turbulent Heat Transfer in Separated Flow, Australian Journal of Basic and Applied Sciences, Vol. 5, Nr. 10, 2011, S. 489-505.

[45] A. F. Charwat, C. F. Dewey Jr., J. N. Roos, J. A. Hitz: An Investigation of Separated Flows. Part II: Flow in the Cavity and Heat Transfer, Journal of the Aerospace Sciences, Vol. 28, Nr. 7, 1961, S. 513527. DOI: $10.2514 / 8.9099$.

[46] J. Fox: Heat transfer and air flow in a transverse rectangular notch, International Journal of Heat and Mass Transfer, Vol. 8, Nr. 2, 1965, S. 269-279. DOI: 10.1016/0017-9310(65)90114-6.

[47] R. L. Haugen, A. M. Dhanak: Heat Transfer in Turbulent Boundary-Layer Separation Over a Surface Cavity, Journal of Heat Transfer, Vol. 89, Nr. 4, 1967, S. 335-340. DOI: 10.1115/1.3614394. 
[48] M. Hiwada, I. Mabuchi, M. Kumada: Three-dimensional flow and heat transfer in a rectangular cavity, Heat transfer - Japanese research, Vol. 14, Nr. 1, 1985, S. 75-96.

[49] D. E. Metzger, R. S. Bunker, M. K. Chyu: Cavity Heat Transfer on a Transverse Grooved Wall in a Narrow Flow Channel, Journal of Heat Transfer, Vol. 111, Nr. 1, 1989, S. 73-79. DOI: $10.1115 / 1.3250661$.

[50] R. F. Richards, M. F. Young, J. C. Haiad: Turbulent forced convection heat transfer from a bottom heated open surface cavity, International Journal of Heat and Mass Transfer, Vol. 30, Nr. 11, 1987, S. 2281-2287. DOI: 10.1016/0017-9310(87)90221-3.

[51] A. Saima, T. Aiba: Heat transfer in a separated flow generated by a transverse rectangular notch, Journal of the Research Institute of Science and Technology, Nihon University, Vol. 3, Nr. 40, 1971, S. 539-545.

[52] R. A. Seban: Heat transfer and flow in a shallow rectangular cavity with subsonic turbulent air flow, International Journal of Heat and Mass Transfer, Vol. 8, Nr. 11, 1965, S. 1353-1368. DOI: $10.1016 / 0017-9310(65) 90127-4$.

[53] R. A. Seban, J. Fox: Heat transfer to the air flow in a surface cavity, International developments in heat transfer, Proceedings of the 1961-62 Heat Transfer Conference, continued discussions, University of Colorado Boulder, Colorado, USA; Central Hall Lecture Theatre, Westminster, London, England, August 28 - September 1, 1961; January 8-12, 1962, The American Society of Mechanical Engineers, United Engineering Center, New York, 1963, S. 426-431.

[54] I. M. Varfolomeyev, Y. F. Gortyshev, V. K. Shchukin: Heat transfer and dynamics of supersonic air flow past cavities, Heat Transfer - Soviet Research, Vol. 13, Nr. 4, 1981, S. 86-91.

[55] H. Yamamoto, N. Seki, S. Fukusako: Forced convection heat transfer on a heated bottom surface of cavity with different wall-height, Wärme- und Stoffübertragung, Vol. 17, Nr. 2, 1983, S. 73-83. DOI: $10.1007 / \mathrm{BF} 01007221$.

[56] H. Yamamoto, N. Seki, S. Fukusako: Forced Convection Heat Transfer on Heated Bottom Surface of a Cavity, Journal of Heat Transfer, Vol. 101, Nr. 3, 1979, S. 475-479. DOI: 10.1115/1.3451012.

[57] M. Yoshiwara: Convective Heat Transfer from the Wall Surface of a Cavity to the External Stream. Effects of Temperature Nonuniformity on the Wall Surface for Correlations of Heat Transfer Model, JSME International Journal, Series B, Vol. 44, Nr. 2, 2001, S. 262-273. DOI: 10.1299/jsmeb.44.262.

[58] M. Yoshiwara, K. Hiraoka, T. Hara: Heat Transfer from an Open Cavity, Transactions of the Japan Society of Mechanical Engineers, Vol. 44, Nr. 378, 1978, S. 659-660.

DOI: 10.1299/kikai1938.44.659.

[59] M. Yoshiwara, Y. Katto: Convective Heat Transfer from Wall Surface of Cavity Array to External Stream. Correlations by Heat Transfer Model, Vol. 57, Research Institute of Industrial Technology, Nihon University, 1999. 
[60] A. Y. D'yachenko, V. I. Terekhov, N. I. Yarygina: Vortex formation and heat transfer in turbulent flow past a transverse cavity with inclined frontal and rear walls, International Journal of Heat and Mass Transfer, Vol. 51, 13-14, 2008, S. 3275-3286. DOI: 10.1016/j.ijheatmasstransfer.2007.11.039.

[61] P. G. Tret'yakov: Calculating the heating of turbine casings, Thermal Engineering, Vol. 11, Nr. 4, 1964, S. 79-82.

[62] P. G. Tret'yakov: Determination of the coefficient of heat transfer in turbines from measurement data, Thermal Engineering, Vol. 14, Nr. 4, 1967, S. 64-69.

[63] R. Strozewski: Temperaturfeldberechnung einer Industriedampfturbine, Diplomarbeit, Hochschule Zittau/Görlitz, 2013.

[64] C. Hesse, S. Schoene, U. Gampe, J. Kober, H. Fogt: Semi-Analytical Procedure for Prediction of Radial Clearances in Industrial Steam Turbines, ASME Turbo Expo 2010: Power for Land, Sea, and Air, Glasgow, UK, 14.-18.06.2010, 14.-18.06.2010, S. 2119-2126. DOI: 10.1115/GT2010-22183.

[65] F. F. Lapp, S. Schuster, S. Hecker, D. Brillert: The Multi-Phase Flow Test Facility "EMMA" to Investigate Local Heat Transfer Coefficients and Liquid Water Films at Wet Steam Conditions, Proceedings of ASME Turbo Expo, 21.-25.09.2020, ASME, New York, N.Y., 2020. DOI: 10.1115/GT202016307.

[66] F. F. Lapp, S. Schuster, S. Hecker, D. Brillert: Experimental validation of an analytical condensation model for the improvement of steam turbine design regarding flexibility requirements, Proceedings of 14th European Conference on Turbomachinery Fluid Dynamics \& Thermodynamics, Danzig, Polen, 12.-16.04.2021, 2021.

[67] T. Diurno, M. Poggiali, L. Mazzei, A. Andreini, B. Facchini, G. Girezzi: Development of a numerical correlation for heat transfer coefficients in steam turbines inner chambers, International Conference on Material Science, Smart Strucures and Applications, Erode, Indien, 21.-22.11.2019, AIP Publishing, 2019, Nr. 020067. DOI: 10.1063/1.5138800.

[68] M. Ciałkowski, G. Buchheim: Calculating the heat transfer coefficient by solving the inverse heat conduction problem, Turbomachinery - fluid dynamic and thermodynamic aspects, Erlangen, 01.03.03.1995, VDI Verlag, Düsseldorf, 1995, S. 245-258. ISBN: 978-3-18-091186-1.

[69] M. Ciałkowski, U. Gampe, C. Heße: Numeric-analytical method for solution of the inverse problem of heat conduction, Conference on Modern Technologies and Power, Krakau, 2007.

[70] M. Ciałkowski, U. Gampe, J. Kołodziej, C. Hesse: Investigation of thermal loading by solution of inverse problem, Proceedings of IFToMM International Symposium on Dynamics of Steam and Gas Turbines, Gdańsk, 01.-03.12.2009, IFFM Publ, 01.-03.12.2009, S. 91-106. ISBN: 978-83-88237$51-5$. 
[71] M. Ciałkowski, J. Kołodziej, U. Gampe, C. Hesse, L. Semklo: Solution of Nonlinear Transient Heat Conduction Inverse Problem, 7th International Conference on Computational Heat and Mass Transfer, Istanbul, Türkei, 18.-22.07.2011, 2011, Paper No. 167.

[72] M. Ciałkowski, A. Maćkiewicz, J. Kołodziej, U. Gampe, A. Frąckowiak: Analytical-numerical solution of the inverse problem for the heat conduction equation, Journal of Mechanics of Materials and Structures, Vol. 7, Nr. 3, 2012, S. 239-253. DOI: 10.2140/jomms.2012.7.239.

[73] G. Eschmann: Experimentelle Untersuchungen zum Einsatz spezieller Messtechnik für die Bestimmung des Wärmeübergangs in Seitenräumen von Dampfturbinengehäusen, Dissertation, Technische Universität Dresden, Professur für Magnetofluiddynamik, Mess- und Automatisierungstechnik, 2020.

[74] M. Putzke: Entwurf einer Versuchseinrichtung für Untersuchungen zum Wärmeübergang in Toträumen von Dampfturbinengehäusen, Großer Beleg, Technische Universität Dresden, Professur für Thermische Energiemaschinen und -anlagen, 2011.

[75] D. Spura: Voruntersuchungen zur experimentellen Modellierung des Wärmeübergangs in Seitenräumen von Dampfturbinengehäusen, Diplomarbeit, Technische Universität Dresden, Professur für Thermische Energiemaschinen und -anlagen, 2013.

[76] D. Spura, J. Lueckert, S. Schoene, U. Gampe: Concept development for the experimental investigation of forced convection heat transfer in circumferential cavities with variable geometry, International Journal of Thermal Sciences, Vol. 96, 2015, S. 277-289. DOI: 10.1016/j.ijthermalsci.2014.08.018.

[77] S. Clauss, J. P. Schnitzler, B. Barabas, P. S. Nagabhushan, F. K. Benra, H. J. Dohmen: Test Rig Design to Explore Water Droplet Behavior in a Four Stage Axial Compressor, ASME 2013 International Mechanical Engineering Congress and Exposition, Volume 8B: Heat Transfer and Thermal Engineering, San Diego, California, USA, 15.-21.11.2013, 2013. ISBN: 978-0-7918-5635-2. DOI: 10.1115/IMECE2013-63429.

[78] W. Uffrecht, A. Günther, V. Caspary: Kleine Thermistoren zur Messung von Wärmeübergangskoeffizienten, Technisches Messen, Vol. 79, Nr. 12, 2012, S. 549-558. D0I: 10.1524/teme.2012.0230.

[79] W. Uffrecht, A. Günther, V. Caspary: Electro-Thermal Measurement of Heat Transfer Coefficients, Proceedings of ASME Turbo Expo, Kopenhagen, Dänemark, 11.-15.06.2012, ASME, New York, N.Y., 2012. ISBN: 978-0-7918-4467-0. DOI: 10.1115/GT2012-68144.

[80] G. Eschmann, A. Kuntze, W. Uffrecht, E. Kaiser, S. Odenbach: Experimental and numerical investigation of heat transfer coefficients in gaseous impinging jets. First test of a recent sensor concept for steady and unsteady flow, International Journal of Thermal Sciences, Vol. 96, 2015, S. 290-304. DOI: 10.1016/j.ijthermalsci.2015.03.016. 
[81] W. Uffrecht, B. Heinschke, A. Günther, V. Caspary, S. Odenbach: Measurement of heat transfer coefficients at up to $25,500 \mathrm{~g}-A$ sensor test at a rotating free disk with complex telemetric instrumentation, International Journal of Thermal Sciences, Vol. 96, 2015, S. 331-344.

DOI: 10.1016/j.ijthermalsci.2015.03.006.

[82] B. Heinschke, W. Uffrecht, A. Günther, S. Odenbach, V. Caspary: Telemetric Measurement of Heat Transfer Coefficients in Gaseous Flow: First Test of a Recent Sensor Concept in a Rotating Application, Proceedings of ASME Turbo Expo, Düsseldorf, Germany, 16.-20.06.2014, ASME, New York, N.Y., 2014. ISBN: 978-0-7918-4575-2. DOI: 10.1115/GT2014-26239.

[83] B. Heinschke, W. Uffrecht, S. Odenbach, V. Caspary: Telemetric Heat Transfer Coefficient Measurements in an Open Rotor Stator System Air Gap at up to 8500 rpm, Proceedings of ASME Turbo Expo, Oslo, Norwegen, 11.-15.06.2018, ASME, New York, N.Y., 2018. DOI: 10.1115/GT201875060.

[84] B. Heinschke: Experimentelle und theoretische Untersuchung zu Strömung und lokalem Wärmeübergang im geschlossenen Rotor-Stator-System, Dissertation, Technische Universität Dresden, Professur für Magnetofluiddynamik, Mess- und Automatisierungstechnik, 2020.

[85] A. Kuntze, S. Odenbach, W. Uffrecht: Experimental investigation of moment coefficients in open rotor-stator disc systems, 13th European Conference on Turbomachinery Fluid dynamics \& Thermodynamics, Lausanne, 08.-12.04.2019, 2019.

[86] M. Heymann, K. Rühling: Pilotanwendung des Kennzeichensystems RDS-PP an der komplexen Anlagentechnik des Technikums „Zentrum für Energietechnik“ (ZET) der TU Dresden, 28.08.2012.

[87] R. J. Moffat: Gas Temperature Measurement, in: A. I. Dahl, C. M. Herzfeld (Hrsg.), Temperature, Its Measurement and Control in Science and Industry, Reinhold Pub. Corp., New York, 1962, S. 553571.

[88] A. Frąckowiak, D. Spura, U. Gampe, M. Ciałkowski: Determination of heat transfer coefficient in Tshaped cavity by means of solving the inverse heat conduction problem, Proceedings of XI International Conference on Computational Heat, Mass and Momentum Transfer, Krakau, Polen, 21.24.05.2018, 2018.

[89] A. Frąckowiak, D. Spura, U. Gampe, M. Ciałkowski: Determination of heat transfer coefficient in a T-shaped cavity by means of solving the inverse heat conduction problem, International Journal of Numerical Methods for Heat \& Fluid Flow, Vol. 30, Nr. 4, 2019, 1725-1742. DOI: 10.1108/HFF09-2018-0484.

[90] Verein Deutscher Ingenieure (Hrsg.): VDI-Wärmeatlas. Berechnungsunterlagen für Druckverlust, Wärme- und Stoffübertragung, 10. Ausg., Springer, Berlin, Heidelberg, 2006. ISBN: 978-3-54025504-8. 
[91] D. Spura, G. Eschmann, W. Uffrecht, U. Gampe: Experimental Investigation of Heat Transfer in Cavities of Steam Turbine Casings under Generic Test Rig Conditions, Proceedings of ASME Turbo Expo, Oslo, Norwegen, 11.-15.06.2018, ASME, New York, N.Y., 2018. DOI: 10.1115/GT2018-75463.

[92] D. Spura, G. Eschmann, W. Uffrecht, U. Gampe: Experimental Investigation of Heat Transfer in Cavities of Steam Turbine Casings Under Generic Test Rig Conditions, Journal of Engineering for Gas Turbines and Power, Vol. 141, Nr. 5, 2019, S. 51021. DOI: 10.1115/1.4041452.

[93] J. V. Beck, A. M. Osman: Nonlinear inverse problem for the estimation of time-and-space-dependent heat-transfer coefficients, Journal of Thermophysics and Heat Transfer, Vol. 3, Nr. 2, 1989, S. 146152. DOI: $10.2514 / 3.141$.

[94] W.-L. Chen, Y.-C. Yang, H.-L. Lee: Inverse problem in determining convection heat transfer coefficient of an annular fin, Energy Conversion and Management, Vol. 48, Nr. 4, 2007, S. 1081-1088. DOI: 10.1016/j.enconman.2006.10.016.

[95] K. Grysa, A. Maciag, A. Pawinska: Solving nonlinear direct and inverse problems of stationary heat transfer by using Trefftz functions, International Journal of Heat and Mass Transfer, Vol. 55, Nr. 23-24, 2012, S. 7336-7340. DOI: 10.1016/j.ijheatmasstransfer.2012.07.072.

[96] D. N. Hào, P. X. Thanh, D. Lesnic: Determination of the heat transfer coefficients in transient heat conduction, Inverse Problems, Vol. 29, Nr. 9, 2013, S. 095020 ff. DOI: 10.1088/0266$5611 / 29 / 9 / 095020$.

[97] K. Kalidasan, R. Velkennedy, J. Taler, D. Taler, P. Oclon, P. Rajesh Kanna: Numerical study of air convection in a rectangular enclosure with two isothermal blocks and oscillating bottom wall temperature, International Journal of Numerical Methods for Heat \& Fluid Flow, Vol. 28, Nr. 1, 2018, S. 103-117. DOI: 10.1108/HFF-03-2017-0125.

[98] A. Maciag, M. J. Al - Khatib: Stability of solutions of the overdetermined inverse heat conduction problems when discretized with respect to time, International Journal of Numerical Methods for Heat \& Fluid Flow, Vol. 10, Nr. 2, 2000, S. 228-245. DOI: 10.1108/09615530010312554.

[99] B. Maciejewska, K. Strąk, M. Piasecka: The Solution of a Two-dimensional Inverse Heat Transfer Problem Using the Trefftz Method, Procedia Engineering, Vol. 157, 2016, S. 82-88.

DOI: 10.1016/j.proeng.2016.08.341.

[100] B. Maciejewska: The application of the Beck's method combined with the FEM and Trefftz functions to determine the heat transfer coefficient in minichannel, Journal of Theoretical and Applied Mechanics, 2017, S. 103-116. DOI: 10.15632/jtam-pl.55.1.103.

[101] B. Maciejewska, M. Piasecka: An application of the non-continuous Trefftz method to the determination of heat transfer coefficient for flow boiling in a minichannel, Heat and Mass Transfer, Vol. 53, Nr. 4, 2017, S. 1211-1224. DOI: 10.1007/s00231-016-1895-1. 
[102] B. Maciejewska, K. Strak, M. Piasecka: The solution of a two-dimensional inverse heat transfer problem using two methods, International Journal of Numerical Methods for Heat \& Fluid Flow, Vol. 28, Nr. 1, 2018, S. 206-219. DOI: 10.1108/HFF-10-2016-0414.

[103] P. Ocłoń, P. Cisek, M. Rerak, D. Taler, R. V. Rao, A. Vallati, M. Pilarczyk: Thermal performance optimization of the underground power cable system by using a modified Jaya algorithm, International Journal of Thermal Sciences, Vol. 123, 2018, S. 162-180. DOI: 10.1016/j.ijthermalsci.2017.09.015.

[104] M. Piasecka, B. Maciejewska: The study of boiling heat transfer in vertically and horizontally oriented rectangular minichannels and the solution to the inverse heat transfer problem with the use of the Beck method and Trefftz functions, Experimental Thermal and Fluid Science, Vol. 38, 2012, S. 19-32. DOI: 10.1016/j.expthermflusci.2011.11.002.

[105] J. Taler: Determination of local heat transfer coefficient from the solution of the inverse heat conduction problem, Forschung im Ingenieurwesen, Vol. 71, Nr. 2, 2007, S. 69-78. DOI: $10.1007 / \mathrm{s} 10010-006-0044-2$.

[106] J. Taler, D. Taler, P. Ludowski: Measurements of local heat flux to membrane water walls of combustion chambers, Fuel, Vol. 115, 2014, S. 70-83. DOI: 10.1016/j.fuel.2013.06.033.

[107] Zmywaczyk, J. Koniorczyk, P. Preiskorn, M. Machowski, B.: An Inverse Approach to Estimate Heat Transfer Coefficients of 122 mm Medium-Range Missile During Correction Engine Operation, Problems of Mechatronics Armament, Aviation, Safety Engineering, Vol. 5, Nr. 1 (15), 2014, S. 25-39.

[108] L. M. Jiji: Heat Conduction, 2. Ausg., Springer, Berlin, 2009. ISBN: 978-3-642-01267-9.

[109] D. W. Mackowski: Conduction Heat Transfer. Notes for MECH 7210, 2011, http://www.eng.auburn.edu/ dmckwski/mech7210/condbook.pdf, abgerufen 19.03.2020.

[110] J. Taler: Superposition Method for Multidimensional Heat Conduction Problems, in: R. B. Hetnarski (Hrsg.), Encyclopedia of thermal stresses, SpringerReference, Dordrecht, 2014, S. 4708-4718. ISBN: 978-94-007-2738-0. DOI: 10.1007/978-94-007-2739-7_410.

[111] J. Hadamard: Sur les problèmes aux dérivées partielles et leur signification physique, Princeton University Bulletin, 1902, S. 49-52.

[112] A. N. Tichonov, V. J. Arsenin: Solutions of ill-posed problems, Scripta series in mathematics, Winston and Sons, Washington, D.C., New York, 1977. ISBN: 978-0-470-99124-4.

[113] F. Hecht: New development in FreeFem++, Journal of Numerical Mathematics, Vol. 20, 3-4, 2012, S. 251-265. DOI: 10.1515/jnum-2012-0013.

[114] A. Frąckowiak, D. Spura, U. Gampe, M. Ciałkowski: Solution of inverse heat conduction problem with using the Trefftz function, Contemporary Issues of Heat and Mass Transfer, Kołobrzeg, Polska, 16.-19.09.2019, Publishing House of the Koszalin University of Technology, Koszalin, 2019, S. 163-178. ISBN: 978-83-7365-513-3. 
[115] D. Spura: Bedienungsanleitung Totraumversuchsstand SiSTeR, 163/20, Professur für Thermische Energiemaschinen und -anlagen, Dresden, 2020.

[116] R. G. Dominy, H. P. Hodson: An Investigation of Factors Influencing the Calibration of Five-Hole Probes for Three-Dimensional Flow Measurements, Journal of Turbomachinery, Vol. 115, Nr. 3 1993, S. 513-519. DOI: 10.1115/1.2929281.

[117] J. Frey: Skript Strömungsmesstechnik, Institut für Luft- und Raumfahrt, Arbeitsgruppe Experimentelle Aerodynamik (EAD), 2018.

[118] Mettler-Toledo AG: Wägefibel, 2008, http://lab.mt.com/gwp/waegefibel/W_gefibel-d720905.pdf, abgerufen 19.02.2020.

[119] A. Schlott, T. Klemm: Messtechnische Bestimmung der spezifischen Wärmekapazität und der Wärmeleitfähigkeit von Polytetrafluorethylen (PTFE), Fraunhofer Institut für Fertigungstechnik und Angewandte Materialforschung, Dresden, 27.05.2015.

[120] S. Pinnau: Wärmeleitfähigkeitsmessung nach DIN 52616, 2016, https://tu-dresden.de/ing/maschinenwesen/iet/tt/die-professur/technische-ausstattung/versuchsanlagen/waermeleitfaehigkeitsmessung, abgerufen 24.10.2018.

[121] S. Pinnau: Klimakammer, 2018, https://tu-dresden.de/ing/maschinenwesen/iet/tt/die-professur/technische-ausstattung/versuchsanlagen/klimakammer, abgerufen 24.10.2018.

[122] J. Blumm, A. Lindemann, M. Meyer, C. Strasser: Characterization of PTFE Using Advanced Thermal Analysis Techniques, International Journal of Thermophysics, Vol. 31, Nr. 10, 2010, S. 1919-1927. DOI: $10.1007 / \mathrm{s} 10765-008-0512-\mathrm{z}$

[123] J. Blumm: Thermal Conductivity of Engineering Materials, in: M. Kutz (Hrsg.), Handbook of Measurement in Science and Engineering, John Wiley \& Sons, Hoboken, N.J., 2013, S. 1151-1188. ISBN: 978-1-118-38464-0.

[124] K. Eiermann, K.-H. Hellwege: Thermal Conductivity of High Polymers from $-180^{\circ} \mathrm{C}$ to $90{ }^{\circ} \mathrm{C}$, Journal of Polymer Science, Vol. 57, Nr. 165, 1962, S. 99-106. DOI: 10.1002/pol.1962.1205716508.

[125] K. Eiermann, K.-H. Hellwege, W. Knappe: Quasistationäre Messung der Wärmeleitfähigkeit von Kunststoffen im Temperaturbereich von $-180{ }^{\circ} \mathrm{C}$ bis $+90^{\circ} \mathrm{C}$, Kolloid-Zeitschrift, Vol. 174, Nr. 2, 1961, S. 134-142. DOI: 10.1007/BF01559375.

[126] R. C. Steere: Detection of Polymer Transitions by Measurement of Thermal Properties, Journal of Applied Polymer Science, Vol. 10, Nr. 11, 1966, S. 1673-1685.

DOI: 10.1002/app.1966.070101107.

[127] К. Б. Тлебаев, А. И. Купчишин: Радиационные эффекты в тепловых свойствах линейного полимера и композитов, Proceedings of 9th International Conference on Interaction of Radiation with Solids, Minsk, Weißrussland, 20.-22.09.2011, 2011, S. 177-179. 
[128] M. Kutz (Hrsg.): Handbook of Measurement in Science and Engineering, John Wiley \& Sons, Hoboken, N.J., 2013. ISBN: 978-1-118-38464-0.

[129] R. G. Ross, P. Andersson, B. Sundqvist, G. Backstrom: Thermal conductivity of solids and liquids under pressure, Reports on Progress in Physics, Vol. 47, Nr. 10, 1984, S. 1347-1402. DOI: $10.1088 / 0034-4885 / 47 / 10 / 002$.

[130] R. E. Barker, R. Y. S. Chen: Grüneisen Parameter from Thermal Conductivity Measurements under Pressure, The Journal of Chemical Physics, Vol. 53, Nr. 7, 1970, S. 2616-2620. DOI: $10.1063 / 1.1674380$.

[131] P. Andersson, G. Bäckström: Specific heat of solids at high pressures from simultaneous measurements of thermal conductivity and diffusivity, High temperatures - High pressures, Vol. 4, 1972, S. 101-109.

[132] L. Bohlin, P. Andersson: A comment on the thermal conductivity of insulating solids at high pressures, Solid State Communications, Vol. 14, Nr. 8, 1974, S. 711-714. DOI: 10.1016/00381098(74)90869-2.

[133] L. N. Dzhavadov: Measurement of thermophysical properties of dielectrics under pressure, High temperatures, high pressures, Nr. 7, 1975, S. 49-54.

[134] P. Andersson, G. Bäckström: Thermal conductivity of solids under pressure by the transient hot wire method, Review of Scientific Instruments, Vol. 47, Nr. 2, 1976, S. 205-209. DOI: $10.1063 / 1.1134581$.

[135] D. Spura, G. Eschmann, W. Uffrecht, U. Gampe, S. Odenbach: COOREFLEX 4.3.6: Thermisches und mechanisches Verhalten von Turbinengehäusen, Statusbericht, Tagungsband zum 15. Statusseminar der AG Turbo, Bergisch-Gladbach, 12.-13.12.2016, Technische Informationsbibliothek Universitätsbibliothek, Hannover, 2017. DOI: 10.2314/GBV:880233656.

[136] D. Spura, G. Eschmann, W. Uffrecht, U. Gampe, S. Odenbach: Verbundprojekt: COOREFLEX-turbo, Vorhaben-Nr.: 4.3.6, Thermisches und mechanisches Verhalten von Turbinengehäusen, AG Turbo, Abschlussbericht, Zeitraum: 01.12.2013-31.11.2016, TU Dresden, Fakultät Maschinenwesen, Institut für Energietechnik, Professur für Thermische Energiemaschinen und -anlagen, Dresden, 2017. DOI: $10.2314 / G B V: 886938767$.

[137] M. S. Bhatti, R. K. Shah: Turbulent and Transition Flow Convective Heat Transfer in Ducts, in: S. Kakac, R. K. Shah, W. Aung (Hrsg.), Handbook of single-phase convective heat transfer, Wiley, New York, 1987. ISBN: 978-0-471-81702-4.

[138] W. Zhi-qing: Study on correction coefficients of liminar and turbulent entrance region effect in round pipe, Applied Mathematics and Mechanics, Vol. 3, Nr. 3, 1982, S. 433-446. DOI: $10.1007 / B F 01897224$. 
[139] C. Haberland, W. Nitsche: Ein einfaches analytisches Verfahren zur Berechnung des turbulenten Wärmeübergangs hinter einem hydrodynamischen Vorlauf, Wärme- und Stoffübertragung, Vol. 12, Nr. 1, 1979, S. 45-54. DOI: 10.1007/BF01672440.

[140] R. G. Deissler: Analysis of turbulent heat transfer and flow in the entrance regions of smooth passages, NACA Technical Note No. 3016, National Advisory Committee for Aeronautics, Lewis Flight Propulsion Lab, Cleveland, OH, USA, 01.10.1953.

[141] R. G. Deissler: Analytical investigation of turbulent flow in smooth tubes with heat transfer with variable fluid properties for Prandtl number of 1, NACA Technical Note No. 2242, National Advisory Committee for Aeronautics, Lewis Flight Propulsion Lab, Cleveland, OH, USA, 01.12.1950.

[142] R. G. Deissler: Analytical and experimental investigation of fully developed turbulent flow of air in a smooth tube with heat transfer with variable fluid properties, NACA Technical Note No. 2629, National Advisory Committee for Aeronautics, Lewis Flight Propulsion Lab, Cleveland, OH, USA, 01.02.1952.

[143] B. Heinschke: Messung von Wärmeübergangskoeffizienten an einer schnell rotierenden Scheibe mit variierter Strömungssituation, Diplomarbeit, TU Dresden, Professur für Magnetofluiddynamik, Mess- und Automatisierungstechnik, 2014.

[144] P. E. Roach: The generation of nearly isotropic turbulence by means of grids, International Journal of Heat and Fluid Flow, Vol. 8, Nr. 2, 1987, S. 82-92. DOI: 10.1016/0142-727X(87)90001-4.

[145] T. Adams, C. Grant, H. Watson: A Simple Algorithm to Relate Measured Surface Roughness to Equivalent Sand-grain Roughness, International Journal of Mechanical Engineering and Mechatronics, 2012. DOI: 10.11159/ijmem.2012.008.

[146] H. Haberhauer, F. Bodenstein: Maschinenelemente. Gestaltung, Berechnung, Anwendung, 14., bearb. Aufl., Springer-Lehrbuch, Springer, Berlin, 2007. ISBN: 978-3-540-34463-6.

[147] C. Hauptmann: Numerische Untersuchungen zu Strömung und Wärmeübergang in generischen Versuchsstandmodellen von Seitenräumen in Dampfturbinengehäusen, Großer Beleg, TU Dresden, Professur für Thermische Energiemaschinen und -anlagen, 2017.

[148] Solver setting for CFD simulations. Type of Solvers and Solution Control Parameters, http://www.cfdyna.com/CFDHT/SolverSetting.html, abgerufen 23.02.2020.

[149] Fluent Theory Guide 18.2, 15.07.2017.

[150] R. J. Goldstein, A. I. Behbahani, K.K. Heppelmann: Streamwise distribution of the recovery factor and the local heat transfer coefficient to an impinging circular air jet, International Journal of Heat and Mass Transfer, Vol. 29, Nr. 8, 1986, S. 1227-1235. DOI: 10.1016/0017-9310(86)90155-9.

[151] B. Sagot, G. Antonini, A. Christgen, F. Buron: Jet impingement heat transfer on a flat plate at a constant wall temperature, International Journal of Thermal Sciences, Vol. 47, Nr. 12, 2008, S. 1610-1619. DOI: 10.1016/j.ijthermalsci.2007.10.020. 
[152] S. M. Nelly, W. Nieratschker, M. Nadler, K. Einsle, A. Delgado: Calibration of the Reynolds Stress Model for the Simulation of Gas Flows in Corrugated Tubes, Heat Transfer Engineering, Vol. 39, 1314, 2017, S. 1235-1242. DOI: 10.1080/01457632.2017.1363632.

[153] N. Norris: The Standard Errors of the Geometric and Harmonic Means and Their Application to Index Numbers, The Annals of Mathematical Statistics, Vol. 11, Nr. 4, 1940, S. 445-448. DOI: $10.1214 / \mathrm{aoms} / 1177731830$.

[154] D. Spura, G. Eschmann, W. Uffrecht, U. Gampe, S. Odenbach: Verbundprojekt: ECOFlex-turbo, Vorhaben-Nr.: 4.3.4 A, Untersuchung von Wärmeübergang in Dampfturbinenkomponenten - Generisch variable Geometrien von Turbinenseitenräumen, AG Turbo, Abschlussbericht, Zeitraum: 01.08.2017-31.10.2020, TU Dresden, Fakultät Maschinenwesen, Institut für Energietechnik, Professur für Thermische Energiemaschinen und -anlagen, Dresden, 2020.

[155] L. Schiller: Die Entwicklung der laminaren Geschwindigkeitsverteilung und ihre Bedeutung für Zähigkeitsmessungen. Mit einem Anhang über den Druckverlust turbulenter Strömung beim Eintritt in ein Rohr, Zeitschrift für Angewandte Mathematik und Mechanik, Vol. 2, Nr. 2, 1922, S. 96-106. DOI: $10.1002 /$ zamm.19220020203.

[156] R. K. Shah: A Correlation for Laminar Hydrodynamic Entry Length Solutions for Circular and Noncircular Ducts, Journal of Fluids Engineering, Vol. 100, Nr. 2, 1978, S. 177-179. DOI: $10.1115 / 1.3448626$.

[157] J. A. Fay: Introduction to fluid mechanics, 2. Aufl., MIT Press, Cambridge, MA, USA, 1998. ISBN: 978-0-262-06165-0.

[158] H. L. Langhaar: Steady Flow in the Transition Length of a Straight Tube, Journal of Applied Mechanics, Nr. 9, 1942, S. 55-58.

[159] D. Spura: Wandmodellierung in CFD, IET-Wiki, 2019, https://wiki.iet.mw.tu-dresden.de/IETWiki/Wandmodellierung_in_CFD, abgerufen 28.02.2020.

[160] P. Bradshaw (Hrsg.): Turbulence, Springer Berlin Heidelberg; Imprint; Springer, Berlin, Heidelberg, 1976. ISBN: 978-3-662-22568-4. 


\section{EIDESSTATTLICHE ERKLÄRUNG}

Hiermit erkläre ich an Eides statt, dass ich die vorliegende Dissertation selbständig und ohne fremde Hilfe verfasst, andere als die angegebenen Quellen und Hilfsmittel nicht benutzt und die den benutzten Quellen wörtlich oder inhaltlich entnommenen Stellen als solche kenntlich gemacht habe. Die Arbeit wurde bisher in gleicher oder ähnlicher Form keiner anderen Prüfungskommission vorgelegt und auch nicht veröffentlicht.

David Spura

Dresden, 17. Dezember 2020 



\section{ANHANG}

A1 Korrelationen nach Malyarenko et al. ...................................................................................... A-3

A2 Korrelationen nach Goloshchapov et al................................................................................... A-6

A3 Korrelationen nach Malyarenko (Wirbelansatz) ……………………….............................. A-7

A4 Korrelationen nach Haberland und Nitsche ................................................................................ A-9

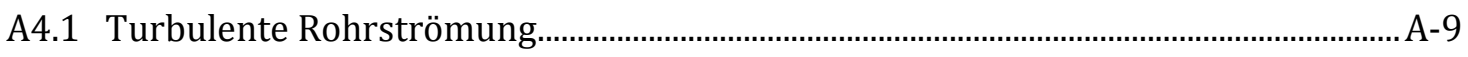

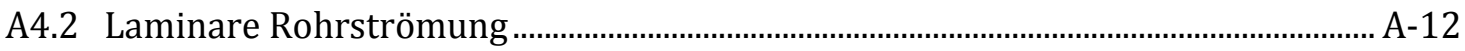

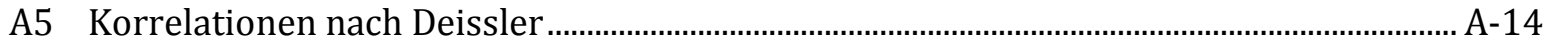

A5.1 Definition von Reynolds-Zahl und Nusselt-Zahl ...................................................... A-14

A5.2 Konvention für Summationsindizes ………................................................................ A-14

A5.3 Geschwindigkeits-/ Temperaturprofil ......................................................................... A-15

A5.4 Entwicklung der Temperaturgrenzschicht................................................................... A-17

A5.5 Bestimmung der Kernstrom- („Bulk“-) Temperatur und -geschwindigkeit........... A-19

A5.6 Reynolds-Zahl und Nusselt-Zahl ............................................................................. A-20

A5.7 Entwicklung der Strömungsgrenzschicht ……………….............................................. A-21

A5.8 Berücksichtigung des nicht voll ausgebildeten hydrodynamischen Vorlaufs....... A-22

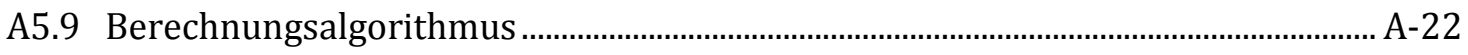

A5.10 Mittlere (lauflängengemittelte) Nusselt-Zahl ............................................................. A-25

A6 Konstruktive Details von Versuchsstand und -anlage ....................................................... A-28

A7 Liste der aufgezeichneten Messstellen ……........................................................................... A-38

A8 Superpositionsprinzip für die Lösung der Fourier-Gleichung ................................................ A-46

A9 Lösungsschema für die inverse Berechnung von WÜK mittels Trefftz-Funktionen....... A-48

A10 Einstellungen für den evolutionären Algorithmus...................................................................... A-49

A11 Lösungsschema zur Berechnung der Kalibrierkoeffizienten für die Fünflochsonden... A-50

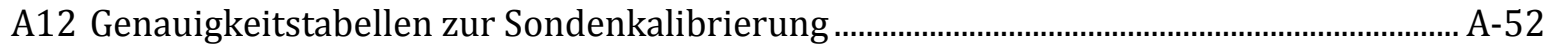

A13 Detaileinstellungen für die CFD-Modelle ................................................................................. A-55

A13.1 Vernetzungseinstellungen und -statistik .................................................................. A-55

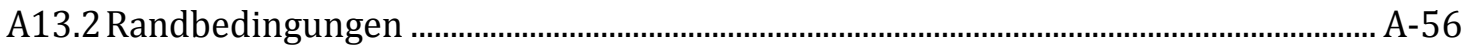

A13.3Stoffwertdefinitionen Ansys CCL ………........................................................................ A-59

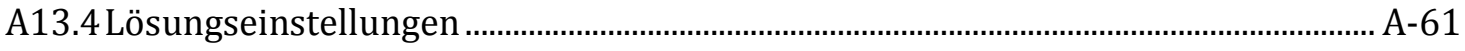


A14 Richtlinien zur Wandmodellierung für CFD-Analysen ........................................................... A-64

A15 Ergebnisse der konjugierten Simulation mit Fluid-Struktur-Kopplung ............................ A-68

A16 Viertelmodell der Saugrohrstrecke ....................................................................................... A-70

A17 Viertelmodell des Versuchsstandes ohne Totraum ...................................................................... A-71

A18 Herleitung der zeitlichen Mittelung transienter WÜK ……................................................. A-72

A18.1 Konstante Wandwärmestrom dichte ......................................................................... A-72

A18.2 Konstante Wandtemperatur ....................................................................................... A-73

A18.3 Konjugierte Rechnung .................................................................................................. A-74

A19 Weitere Ergebnisse der Large-Eddy-Simulationen ................................................................... A-75

A19.190-Modell, konstante Wandwärmestromdichte (Ansys CFX).................................. A-75

A19.290-Modell, konstante Wandtemperatur (Ansys CFX) ……………………................ A-80

A19.32D-Modell, konstante Wandwärmestromdichte (Ansys Fluent) ................................ A-84

A19.42D-Modell, konstante Wandtemperatur (Ansys Fluent).............................................. A-86

A20 Ausblick auf den Dampfversuchsstand „SiSTeR2“ ................................................................... A-88 


\section{A1 KORRELATIONEN NACH MALYARENKO ET AL.}

$$
\begin{aligned}
& N u(x, \varphi)=N u_{0}(\varphi) \cdot f\left(\bar{x}^{*}\right)-1,53 \varphi \\
& N u_{0}(\varphi)=N u_{0}^{\prime} \cdot(\cos \gamma)^{-0,6}-2,84 \cdot 10^{-4} \cdot R e \cdot \gamma \cdot\left(\varphi+\frac{\pi}{2}\right) \\
& 0 \leq \gamma \leq \frac{\pi}{4} \\
& N u_{0}^{\prime}=N u_{0}^{\prime \prime} \cdot g\left(\bar{x}_{0}^{*}\right) \\
& N u_{0}^{\prime \prime}=1,91 \cdot \operatorname{Re}^{0,5} \cdot \operatorname{Pr}^{0,4} \cdot \bar{h}^{-0,3} \\
& 5 \cdot 10^{3} \leq R e \leq 5 \cdot 10^{4} \\
& 6 \leq \bar{h} \leq 37 \\
& g\left(\bar{x}_{0}^{*}\right)=\left\{\begin{array}{cl}
0,5+0,14 \bar{x}_{0}^{*} & 1 \leq \bar{x}_{0}^{*} \leq 3,57 \\
1 & \bar{x}_{0}^{*}>3,57
\end{array}\right. \\
& f\left(\bar{x}^{*}\right)= \begin{cases}1-0,07 \bar{x}^{*}-0,04 \bar{x}^{* 2} & 0 \leq \bar{x}^{*}<1,25 \\
0,95 e^{-0,11 \bar{x}^{*}} & \bar{x}^{*} \geq 1,25\end{cases} \\
& \bar{x}^{*}=\frac{x}{x^{*}} \\
& \bar{x}_{0}^{*}=\frac{x_{0}}{x^{*}} \\
& x^{*}=0,58 \cdot b_{0} \cdot \bar{h}^{0,7} \\
& \bar{h}=\frac{h}{b_{0}} \\
& R e=\frac{u_{\mathrm{m}} \cdot b_{0}}{v} \\
& N u=\frac{\alpha \cdot b_{0}}{\lambda}
\end{aligned}
$$

$N u_{0}^{\prime \prime} \quad$ Nusselt-Zahl im Staupunkt des Prallstrahls an der Wand (ohne Drall, ohne Anzapfung, ohne Seitenwände)

$N u_{0}^{\prime} \quad$ Nusselt-Zahl im Staupunkt des Prallstrahls an der Wand (ohne Drall, ohne Anzapfung, mit Seitenwänden)

$N u_{0}(\varphi)$ Nusselt-Zahl entlang der Staulinie am Umfang der Kammer (mit Drall, Anzapfung und Seitenwänden) 
$N u(x, \varphi)$ lokale Nusselt-Zahl-Verteilung axial und über dem Umfang der Kammer (mit Drall, Anzapfung und Seitenwänden)

$g\left(\bar{x}_{0}^{*}\right) \quad$ Korrekturfaktor für den Einfluss der am nächsten zur Strahlachse gelegenen Seitenwand

$f\left(\bar{x}^{*}\right) \quad$ axiale Verteilung der Nusselt-Zahlen

$x_{0} \quad$ Abstand der Strahlachse von der nächsten Seitenwand

$x^{*} \quad$ axiale Halbbreite des Strahls vor dem Auftreffen an der Wand

$x \quad$ axiale Koordinate (beginnend von der Strahlachse)

$\gamma$ [rad] Drallwinkel des Strahls am Austritt des Spaltes zur Kammer gegenüber Radialenrichtung

$\varphi[\mathrm{rad}]$ Winkelkoordinate in der Mittelschnittebene; positive Werte für die Winkelkoordinate $\varphi$ werden vom Meridianschnitt gegenüber der Anzapfung aus entgegengesetzt zur Drallrichtung aufgetragen.

$\bar{h} \quad$ „Skalierungsmaßstab“ der Strömung [8,17]

$h \quad$ Höhe der Kammer (Abstand zwischen Außenwand und Ende des Einströmspaltes $\triangleq$ Beginn des Prallstrahles)

Re Reynolds-Zahl des Strahles am kammerseitigen Ende des Einströmspaltes

$u_{\mathrm{m}} \quad$ mittlere Geschwindigkeit des Strahles am kammerseitigen Ende des Einströmspaltes

$b_{0} \quad$ axiale Breite des Strahles am kammerseitigen Ende des Einströmspaltes

Die noch unbekannten Parameter $u_{\mathrm{m}}$ und $b_{0}$ können für eine Anzapfkammer gemäß $[8,17]$ aus dem Massenstrom in der Hauptströmung und der Anzapfung sowie den Abmessungen des Anzapfspaltes unter Zuhilfenahme von Abb. A.1 ermittelt werden. Hierbei sind:

$q_{\mathrm{Sp}} \quad$ querschnittsgemittelte Geschwindigkeit am kammerseitigen Ende des Einströmspaltes; berechenbar aus dem Anzapfmassenstrom $\dot{m}_{\mathrm{Z}}$ :

$$
q_{\mathrm{Sp}}=\dot{m}_{\mathrm{Z}} /\left(\rho \cdot A_{\mathrm{Sp}}\right) \text { mit } A_{\mathrm{Sp}}=\pi D_{\mathrm{Sp}} S
$$

$q_{\mathrm{HS}} \quad$ querschnittsgemittelte Geschwindigkeit in der Hauptströmung; berechenbar aus dem Massenstrom $\dot{m}_{\mathrm{HS}}$ :

$$
q_{\mathrm{HS}}=\dot{m}_{\mathrm{HS}} /\left(\rho \cdot A_{\mathrm{HS}}\right) \operatorname{mit} A_{\mathrm{HS}}=\frac{\pi}{4}\left(d_{\mathrm{a}}^{2}-d_{\mathrm{i}}^{2}\right)
$$

$\bar{H} \quad$ Verhältnis zwischen Spalthöhe $H$ und Spaltbreite $S ; \bar{H}=H / S$

$\psi \quad$ Füllgrad des Spaltes mit Strahl; $\psi=b_{0} / S$

$\bar{K} \quad$ Strahlparameter; $\bar{K}=u_{\mathrm{m}} / q_{\mathrm{HS}}$

Ein Ansatz für reine Toträume $\left(q_{\mathrm{Sp}}=0\right)$ ist jedoch nicht bekannt. 

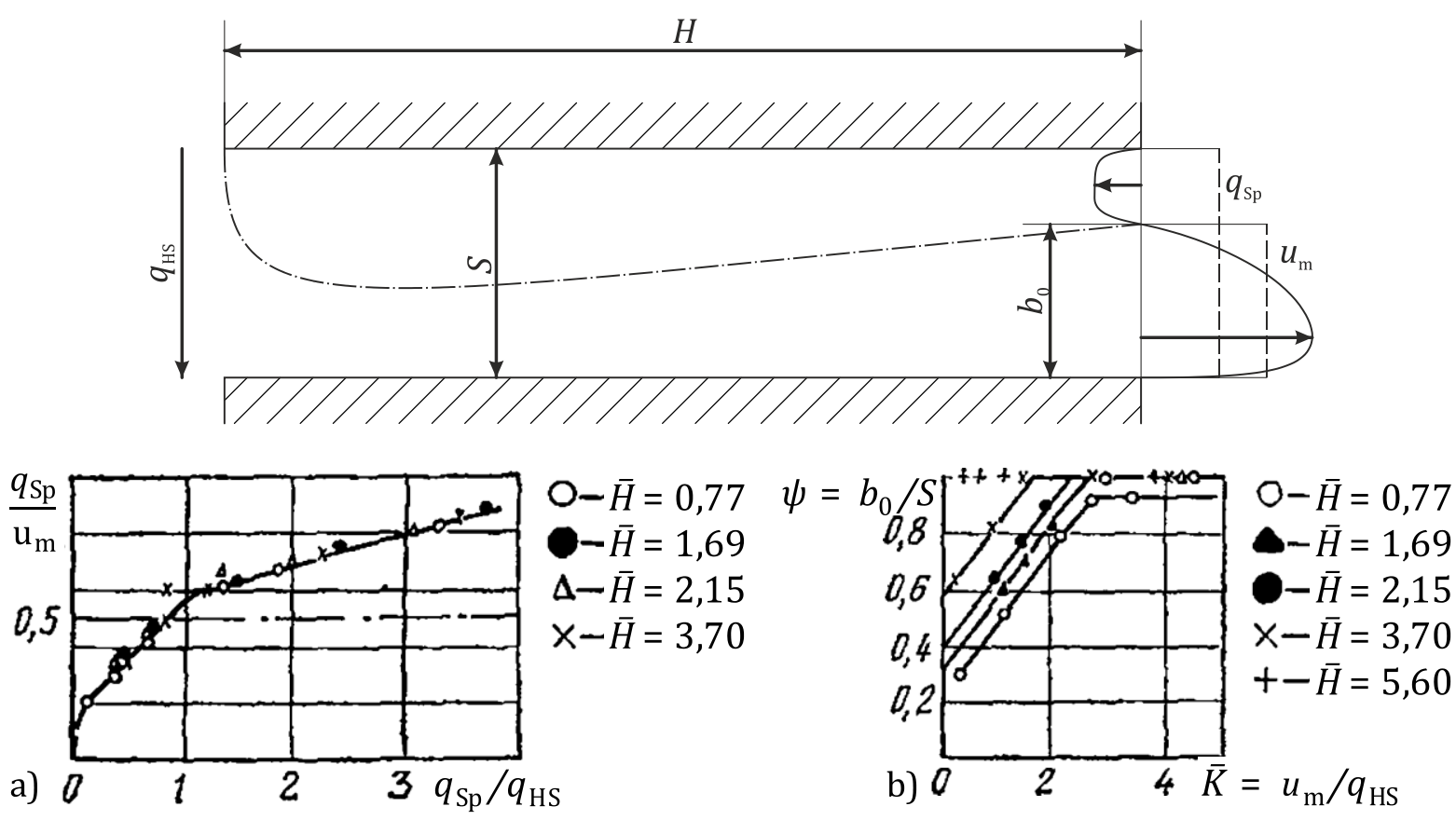

Abb. A.1 Diagramme zur Ermittlung der Breite $b_{0}$ sowie der maximalen Geschwindigkeit $u_{\mathrm{m}}$ des Strahles am kammerseitigen Ende des Einströmspaltes in Abhängigkeit der querschnittsgemittelten Geschwindigkeit in der Hauptströmung $q_{\mathrm{HS}}$ sowie in der Anzapfung $q_{\mathrm{Sp}}[8,17]$

Alternativ zu Abb. A.1 b liefert [20] einen Algorithmus zur iterativen Berechnung des Strahlparameters $K$, des Füllgrades $\psi=b / S=\psi(\bar{H}, \delta, K)$ sowie der daraus resultierenden querschnittsgemittelten Geschwindigkeit $u_{\mathrm{m}}$ des Freistrahls am kammerseitigen Ende des Einströmspaltes. Dieser berücksichtigt auch den Einfluss des Neigungswinkels $\delta$ der Einströmöffnung gegenüber der Axialenrichtung sowie den Drallwinkel $\alpha_{2_{\mathrm{R}}}$ in der Hauptströmung. Als Startwert für die Berechnung wird $\psi=1$ empfohlen.

$$
\begin{aligned}
& u_{\mathrm{m}}(\psi)=\frac{\dot{m}_{\mathrm{Z}}}{\psi \rho \pi D_{\mathrm{Sp}} S}=\frac{q_{\mathrm{Sp}}}{\psi} \\
& K(\psi)=\frac{u_{\mathrm{m}}(\psi)}{q_{\mathrm{HS}}} \\
& \bar{H}_{\delta}=\frac{\bar{H}}{\sin ^{2} \delta} \\
& K_{\text {krit }}=1,8 \sin \delta \cdot e^{-0,2 \bar{H}_{\delta}} \\
& \psi_{0}=\left\{\begin{array}{cc}
0,63+0,185 \bar{H}_{\delta} & \bar{H}_{\delta}<2,0 \\
1 & \bar{H}_{\delta} \geq 2,0
\end{array}\right. \\
& \psi_{90^{\circ}}= \begin{cases}\psi_{0}-0,30\left(K_{\text {krit }}-K\right) & K<K_{\text {krit }} \\
\psi_{0} & K \geq K_{\text {krit }}\end{cases} \\
& \psi=\psi_{90^{\circ}}\left(1+0,16 \sqrt{1-\sin \alpha_{2_{\mathrm{R}}}}\right)
\end{aligned}
$$


$\delta \quad$ Neigungswinkel der Einströmöffnung gegenüber der Hauptströmung $\left(\delta \leq 90^{\circ}\right)$

$\bar{H}_{\delta} \quad$ relative Spalthöhe bei geneigter Einströmöffnung

$K_{\text {krit }} \quad$ kritischer Wert des Strahlparameters, unterhalb dessen der Einfluss auf den Füllgrad groß ist

$\psi_{0} \quad$ Hilfsgröße zur Berechnung des Füllgrades aus der relativen Einströmspalthöhe

$\psi_{90^{\circ}} \quad$ Füllgrad bei rein axialer Abströmung aus dem Laufrad vor der Kammer $\left(\alpha_{2_{\mathrm{R}}}=90^{\circ}\right)$

$\psi \quad$ Füllgrad bei einem Laufradabströmwinkel $\alpha_{2_{\mathrm{R}}} \gtrless 90^{\circ}$

\section{A2 KORRELATIONEN NACH GOLOSHCHAPOV ET AL.}

$$
\begin{gathered}
\bar{\alpha}(L)=\frac{1}{L} \int_{0}^{L} \alpha(x) d x \\
N u_{0}= \begin{cases}0,032 \operatorname{Re}^{0,765} \operatorname{Pr}^{0,43} \bar{h}^{1,13} e^{-0,155 \bar{h}} & \bar{h} \leq 10 \\
0,129 \operatorname{Re}^{0,765} \operatorname{Pr}^{0,43} e^{-0,05 \bar{h}} & \bar{h}>10\end{cases} \\
\bar{L}_{G}=\frac{L_{G}}{b}
\end{gathered}
$$




$$
\begin{aligned}
& K_{\varphi}= \begin{cases}0,0138 & R e \leq 1,371 \cdot 10^{4} \\
0,052 & R e>1,371 \cdot 10^{4}\end{cases} \\
& \overline{N u}_{\mathrm{SR}}= \begin{cases}0,978 \overline{N u} & R e \leq 1,371 \cdot 10^{4} \\
0,920 \overline{N u} & R e>1,371 \cdot 10^{4}\end{cases} \\
& \overline{N u}_{\mathrm{Fl}}= \begin{cases}\overline{N u}(0,730+0,016 \bar{L}) & 0 \leq \bar{L} \leq 12,5 \\
\overline{N u}[0,930+0,00933(\bar{L}-12,5)] & 12,5 \leq \bar{L} \leq 21 \\
\overline{N u} & \bar{L}>21\end{cases} \\
& \overline{N u}_{\mathrm{SR}, \mathrm{Fl}}= \begin{cases}0,975 \overline{N u} & R e \leq 1,371 \cdot 10^{4} \\
0,917 \overline{N u} & R e>1,371 \cdot 10^{4}\end{cases} \\
& \overline{N u}_{\mathrm{SR}, \text { Drall }}= \begin{cases}\overline{N u}_{\mathrm{SR}}\left[1-1,156\left(\tan \delta_{U}\right)^{0,2}\right] & 0 \leq \delta_{U} \leq 15^{\circ} \\
0,88 \overline{N u}_{\mathrm{SR}} & 15^{\circ}<\delta_{U} \leq 32^{\circ}\end{cases} \\
& \tan \delta_{U}=\frac{C_{U_{C}}}{C_{\mathrm{SR}}} \\
& C_{U_{\mathrm{C}}}=C_{2 U_{R}} \frac{2 R_{\mathrm{B}}}{D}
\end{aligned}
$$

\section{A3 KORRELATIONEN NACH MALYARENKO (WIRBELANSATZ)}

$$
\begin{aligned}
& u_{\mathrm{p}, \mathrm{s}_{\max }}=u_{*} \frac{X_{m_{0}}}{X_{m_{i}}} \\
& \bar{X}_{m_{0}}=\frac{X_{m_{0}}}{b_{0}} \approx 0,46 \bar{h}
\end{aligned}
$$

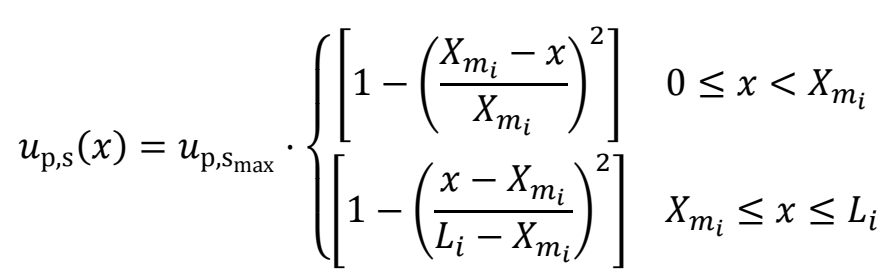

$$
\begin{aligned}
& N u_{x}(x)=\left\{\begin{array}{lc}
c \cdot \operatorname{Re}_{x}\left(\int_{0}^{x} \frac{u_{\mathrm{p}, \mathrm{s}}(x)}{v} d x\right)^{-\frac{1}{2}} & x \leq x_{k} \text { (laminar) } \\
d \cdot \operatorname{Re}_{x}\left(\int_{x_{k}}^{x} \frac{u_{\mathrm{p}, \mathrm{s}}(x)}{v} d x+0,72\left(2915 \frac{N u_{x_{k}}}{R e_{x_{k}}}\right)^{9}\right)^{-\frac{1}{5}} & x>x_{k} \text { (turbulent) }
\end{array}\right. \\
& R e_{x}=\frac{u_{\mathrm{p}, \mathrm{s}}(x) \cdot x}{v}
\end{aligned}
$$




$$
\begin{gathered}
N u_{x}=\frac{\alpha(x) \cdot x}{\lambda} \\
R e_{x_{k}}=2,8 \cdot 10^{6} r_{\varepsilon} r_{\mathrm{M}} r_{x} r_{\mathrm{f}}=5,6 \cdot 10^{4} \\
N u_{x_{k}}=N u_{x}\left(x_{k}\right) \\
r_{\varepsilon}=0,05, r_{\mathrm{M}}=1, r_{x}=0,4, r_{\mathrm{f}}=1 \\
c=0,55, d=0,0471
\end{gathered}
$$

$u_{\mathrm{p}, \mathrm{s}} \quad$ Geschwindigkeitsverlauf auf der Grenzschicht in der jeweiligen Strömungsregion

$u_{\mathrm{p}, \mathrm{s}_{\max }} \quad$ Maximalwert der Geschwindigkeit auf der Grenzschicht in der jeweiligen Strömungsregion

$u_{*} \quad$ Maximalgeschwindigkeit an der Außenwand bei unbehinderter Prallströmung (ohne Seitenwände)

$X_{m_{0}} \quad$ Abstand zwischen Wirbelzentrum und Außenwand

$X_{m_{i}} \quad$ Abstand zwischen Wirbelzentrum und der jeweils betrachteten Wand

$X_{m_{i}}=X_{m_{0}}$ für Seitenwände

$X_{m_{i}}=X_{m_{1}}$ für Innenoberflächen

$R e_{x_{k}} \quad$ kritische Reynolds-Zahl bei der Transition der Grenzschicht vom laminaren ins turbulente Strömungsregime

$N u_{x_{k}} \quad$ kritische Nusselt-Zahl bei der Transition der Grenzschicht vom laminaren ins turbulente Strömungsregime

$x \quad$ aus der Ecke der Kammer in Strömungsrichtung entlang der entsprechenden Fläche gemessene Koordinate

$L_{i} \quad$ Gesamtlänge der entsprechenden Fläche 


\section{A4 KORRELATIONEN NACH HABERLAND UND NITSCHE}

$$
\begin{aligned}
& \left.N u_{D}(x)\right|_{L}=\frac{\left.N u_{D}(x)\right|_{L}}{N u_{D}(x)} \cdot N u_{D}(x)=k_{N u} \cdot N u_{D}(x) \\
& k_{N u}=\frac{\left.N u_{D}(x)\right|_{L}}{N u_{D}(x)}=\frac{\left.\tau_{W}(x)\right|_{L}}{\tau_{W}(x)} \cdot \frac{u_{\delta_{T}}(x)}{\left.u_{\delta_{T}}(x)\right|_{L}}=k_{\tau} \cdot k_{u_{\delta}}
\end{aligned}
$$

$\left.(\cdot)\right|_{L} \quad$ hydrodynamischer Vorlauf vorhanden

(x) Lauflänge (bei hydrodynamischem Vorlauf: $x=0$ am Beginn des thermischen Anlaufs)

\section{A4.1 Turbulente Rohrströmung}

$$
\begin{aligned}
& u(x, y)=u_{\mathrm{m}}(x) \cdot\left[\frac{y}{\delta_{U}(x)}\right]^{\frac{1}{n}}, \quad n=3,6 \cdot R e_{\mathrm{E}}^{0,0537} \\
& u_{\delta_{T}}(x)=u_{\mathrm{m}}(x) \\
& \left.u_{\delta_{T}}(x)\right|_{L}=\left.u_{\mathrm{m}}(x)\right|_{L} \cdot\left[\frac{\delta_{T}(x)}{\left.\delta_{U}(x)\right|_{L}}\right]^{\frac{1}{n}} \\
& k_{u_{\delta}}=\frac{u_{\delta_{T}}(x)}{\left.u_{\delta_{T}}(x)\right|_{L}}=\frac{u_{\mathrm{m}}(x)}{\left.u_{\mathrm{m}}(x)\right|_{L}} \cdot\left[\frac{\left.\delta_{U}(x)\right|_{L}}{\delta_{T}(x)}\right]^{\frac{1}{n}}=k_{u} \cdot k_{\delta}^{\frac{1}{n}} \\
& \frac{\tau_{W}(x)}{\tau_{W, \infty}}=0,714 \cdot R e_{\mathrm{E}}^{0,05} \cdot\left(\frac{x}{D}\right)^{-0,12} \\
& \frac{\left.\tau_{W}(x)\right|_{L}}{\tau_{W, \infty}}=\frac{\tau_{W}(x+L)}{\tau_{W, \infty}}=0,714 \cdot R e_{\mathrm{E}}^{0,05} \cdot\left(\frac{x+L}{D}\right)^{-0,12} \\
& k_{\tau}=\frac{\left.\tau_{W}(x)\right|_{L}}{\tau_{W}(x)}=\left(\frac{x+L}{x}\right)^{-0,12} \\
& \frac{\delta(x)}{R}=\min \left(1,6 R e^{-0,2}\left(\frac{x}{D}\right)^{0,54} ; 1\right) \\
& \frac{\delta_{T}(x)}{R}=\frac{\delta(x)}{R}=1,6 R e^{-0,2}\left(\frac{x}{D}\right)^{0,54} \\
& \frac{\left.\delta_{U}(x)\right|_{L}}{R}=\frac{\delta(x+L)}{R}=1,6 R e^{-0,2}\left(\frac{x+L}{D}\right)^{0,54} \\
& k_{\delta}=\frac{\left.\delta_{U}(x)\right|_{L}}{\delta_{T}(x)}=\left(\frac{x+L}{x}\right)^{0,54}
\end{aligned}
$$




$$
\begin{gathered}
\frac{u_{\mathrm{m}}(x)}{u_{\mathrm{E}}}=\frac{1}{1-\frac{2}{n+1} \cdot \frac{\delta(x)}{R}+\frac{1}{2 n+1} \cdot\left[\frac{\delta(x)}{R}\right]^{2}} \\
\frac{\left.u_{\mathrm{m}}(x)\right|_{L}}{u_{\mathrm{E}}}=\frac{u_{\mathrm{m}}(x+L)}{u_{\mathrm{E}}} \\
k_{u}=\frac{u_{\mathrm{m}}(x)}{\left.u_{\mathrm{m}}(x)\right|_{L}}=\frac{u_{\mathrm{m}}(x)}{u_{\mathrm{m}}(x+L)}
\end{gathered}
$$

$u_{\mathrm{E}} \ldots$ Einströmgeschwindigkeit (entspricht der querschnittsgemittelten Geschwindigkeit) $u_{\mathrm{m}}$...Kerngeschwindigkeit der Strömung (Geschwindigkeit am Ende der Strömungsgrenzschicht) Herleitung von Formel A.47

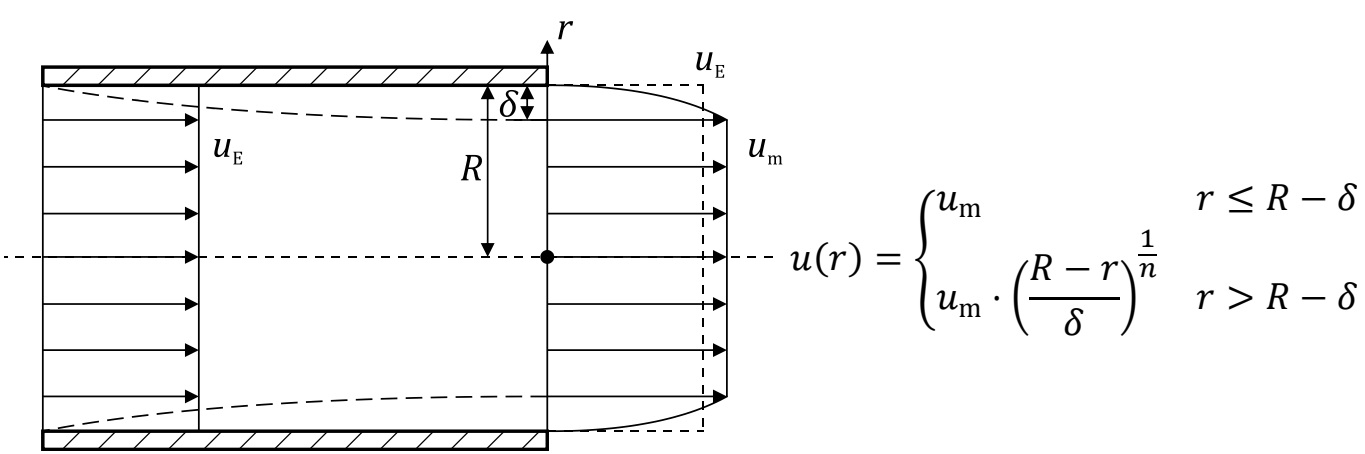

$$
\begin{aligned}
\dot{V}=u_{\mathrm{E}} \cdot \pi R^{2} & =2 \pi \int_{0}^{R} u(r) r d r \\
& =2 \pi \cdot\left[\int_{0}^{R-\delta} u_{\mathrm{m}} r d r+\int_{R-\delta}^{R} u_{\mathrm{m}} \cdot\left(\frac{R-r}{\delta}\right)^{\frac{1}{n}} r d r\right] \\
& =2 \pi \cdot\left\{u_{\mathrm{m}} \frac{(R-\delta)^{2}}{2}+u_{\mathrm{m}} \cdot \delta^{-\frac{1}{n}} \cdot\left[\frac{(R-r)^{\frac{1}{n}+2}}{\frac{1}{n}+2}-\frac{R \cdot(R-r)^{\frac{1}{n}+1}}{\frac{1}{n}+1}\right]_{R-\delta}^{R}\right\} \\
u_{\mathrm{E}} \cdot \pi R^{2} & =2 \pi \cdot\left\{u_{\mathrm{m}} \frac{(R-\delta)^{2}}{2}+u_{\mathrm{m}} \cdot \delta^{-\frac{1}{n}} \cdot\left[-\frac{\delta^{\frac{1}{n}+2}}{\frac{1}{n}+2}+\frac{R \cdot \delta^{\frac{1}{n}+1}}{\frac{1}{n}+1}\right]\right\} \\
u_{\mathrm{E}} R^{2} & \left.=u_{\mathrm{m}}(R-\delta)^{2}+2 u_{\mathrm{m}} \cdot\left[-\frac{\delta^{2}}{\frac{1}{n}+2}+\frac{R \delta}{\frac{1}{n}+1}\right]_{\mathrm{m}}^{2}\right) \\
\frac{u_{\mathrm{E}}}{u_{\mathrm{m}}} & =\left(1-\frac{\delta}{R}\right)^{2}-\frac{2 n}{2 n+1}\left(\frac{\delta}{R}\right)^{2}+\frac{2 n}{n+1} \frac{\delta}{R} \\
& =1+\left(\frac{2 n}{n+1}-2\right) \frac{\delta}{R}+\left(1-\frac{2 n}{2 n+1}\right)\left(\frac{\delta}{R}\right)^{2} \\
\frac{u_{\mathrm{E}}}{u_{\mathrm{m}}} & =1-\frac{2}{n+1} \frac{\delta}{R}+\frac{1}{2 n+1}\left(\frac{\delta}{R}\right)^{2}
\end{aligned}
$$


In diesem Zusammenhang wurde ein Fehler in der Originalveröffentlichung von Haberland und Nitsche [139] festgestellt. Dort steht auf Seite 50 in Gleichung (21):

$$
\frac{u_{\mathrm{m}}}{u_{\mathrm{E}}}=\frac{1}{1-(1-c)\left[\frac{2 \delta}{R}-\left(\frac{\delta}{R}\right)^{2}\right]}
$$

Richtig ist jedoch:

$$
\frac{u_{\mathrm{m}}}{u_{\mathrm{E}}}=\frac{1}{1-(1+c)\left[\frac{2 \delta}{R}-\left(\frac{\delta}{R}\right)^{2}\right]}
$$

Nur so wird der im dortigen Bild 6 dargestellte Kurvenverlauf erreicht und kommt man durch Einsetzen auf die richtige Formel A.47:

$$
\begin{aligned}
\frac{u_{\mathrm{E}}}{u_{\mathrm{m}}} & =1-(1+c)\left[\frac{2 \delta}{R}-\left(\frac{\delta}{R}\right)^{2}\right] \quad c=\frac{2}{2-\frac{\delta}{R}} \cdot\left[\frac{n}{2 n+1} \cdot\left(\frac{\delta}{R}-\frac{1+2 n}{n+1}\right)\right] \\
& =1-\left[1+\frac{2}{2-\frac{\delta}{R}} \cdot \frac{n}{2 n+1} \cdot\left(\frac{\delta}{R}-\frac{1+2 n}{n+1}\right)\right]\left[\frac{2 \delta}{R}-\left(\frac{\delta}{R}\right)^{2}\right] \\
& =1-\left[1+\frac{2}{2-\frac{\delta}{R}} \cdot\left(\frac{n}{2 n+1} \cdot \frac{\delta}{R}-\frac{n}{n+1}\right)\right] \cdot \frac{\delta}{R}\left(2-\frac{\delta}{R}\right) \\
& =1-2 \frac{\delta}{R}+\left(\frac{\delta}{R}\right)^{2}-\frac{2 n}{2 n+1}\left(\frac{\delta}{R}\right)^{2}+\frac{2 n}{n+1} \frac{\delta}{R} \\
& =1-\left(2-\frac{2 n}{n+1}\right) \frac{\delta}{R}+\left(1-\frac{2 n}{2 n+1}\right)\left(\frac{\delta}{R}\right)^{2} \\
\frac{u_{\mathrm{E}}}{u_{\mathrm{m}}} & =1-\frac{2}{n+1} \frac{\delta}{R}+\frac{1}{2 n+1}\left(\frac{\delta}{R}\right)^{2} \text { q.e.d. }
\end{aligned}
$$

Dieser Fehler wurde dem Springer-Verlag am 16.10.2014 in einer E-Mail an Herrn Michael Voigt so mitgeteilt. Er ist jedoch bisher noch nicht korrigiert worden. 


\section{A4.2 Laminare Rohrströmung}

Das von Haberland und Nitsche vorgestellte Konzept kann auch auf laminare Rohrströmung übertragen werden:

$$
\begin{aligned}
& u(x, y)=u_{\mathrm{m}}(x) \cdot\left\{2 \frac{y}{\delta_{U}(x)}-\left[\frac{y}{\delta_{U}(x)}\right]^{2}\right\} \\
& u_{\delta_{T}}(x)=u_{\mathrm{m}}(x) \\
& \left.u_{\delta_{T}}(x)\right|_{L}=\left.u_{\mathrm{m}}(x)\right|_{L} \cdot\left\{2 \frac{\delta_{T}(x)}{\left.\delta_{U}(x)\right|_{L}}-\left[\frac{\delta_{T}(x)}{\left.\delta_{U}(x)\right|_{L}}\right]^{2}\right\} \\
& k_{u_{\delta}}=\frac{u_{\delta_{T}}(x)}{\left.u_{\delta_{T}}(x)\right|_{L}}=\frac{u_{\mathrm{m}}(x)}{\left.u_{\mathrm{m}}(x)\right|_{L}} \cdot \frac{1}{2 \frac{\delta_{T}(x)}{\left.\delta_{U}(x)\right|_{L}}-\left[\frac{\delta_{T}(x)}{\left.\delta_{U}(x)\right|_{L}}\right]^{2}}=\frac{k_{u}}{2 k_{\delta}-k_{\delta}{ }^{2}} \\
& c_{\mathrm{f}}=\frac{3,44}{\sqrt{R e_{x}}} \\
& \Rightarrow \tau_{W}(x) \sim x^{-\frac{1}{2}} \\
& k_{\tau}=\frac{\left.\tau_{W}(x)\right|_{L}}{\tau_{W}(x)}=\left(\frac{x+L}{x}\right)^{-0,5} \\
& \delta(x) \sim \frac{x}{\sqrt{R e_{x}}} \\
& R e_{x}=\frac{u x}{v}=\frac{u D}{v} \cdot \frac{x}{D}=R e \cdot \frac{x}{D}=R e \cdot \frac{x}{2 R} \\
& \delta(x)=C \cdot \frac{\sqrt{x}}{\sqrt{R e}} \sqrt{D}=C \frac{\sqrt{x}}{\sqrt{R e}} \frac{2 R}{\sqrt{D}} \\
& L_{\mathrm{entw}}=0,0575 \cdot D \cdot R e
\end{aligned}
$$

mit

$$
\delta\left(L_{\mathrm{entw}}\right)=R
$$

$$
\begin{gathered}
\Rightarrow \frac{\delta(x)}{R}=\min \left(4,17 R e^{-0,5}\left(\frac{x}{D}\right)^{0,5} ; 1\right) \\
k_{\delta}=\frac{\delta_{T}(x)}{\left.\delta_{U}(x)\right|_{L}}=\left(\frac{x}{x+L}\right)^{0,5}
\end{gathered}
$$




$$
\begin{gathered}
\frac{u_{\mathrm{m}}(x)}{u_{\mathrm{E}}}=\frac{1}{1-\frac{2}{3} \cdot \frac{\delta(x)}{R}+\frac{1}{6} \cdot\left[\frac{\delta(x)}{R}\right]^{2}} \\
\frac{\left.u_{\mathrm{m}}(x)\right|_{L}}{u_{\mathrm{E}}}=\frac{u_{\mathrm{m}}(x+L)}{u_{\mathrm{E}}} \\
k_{u}=\frac{u_{\mathrm{m}}(x)}{\left.u_{\mathrm{m}}(x)\right|_{L}}=\frac{u_{\mathrm{m}}(x)}{u_{\mathrm{m}}(x+L)}
\end{gathered}
$$

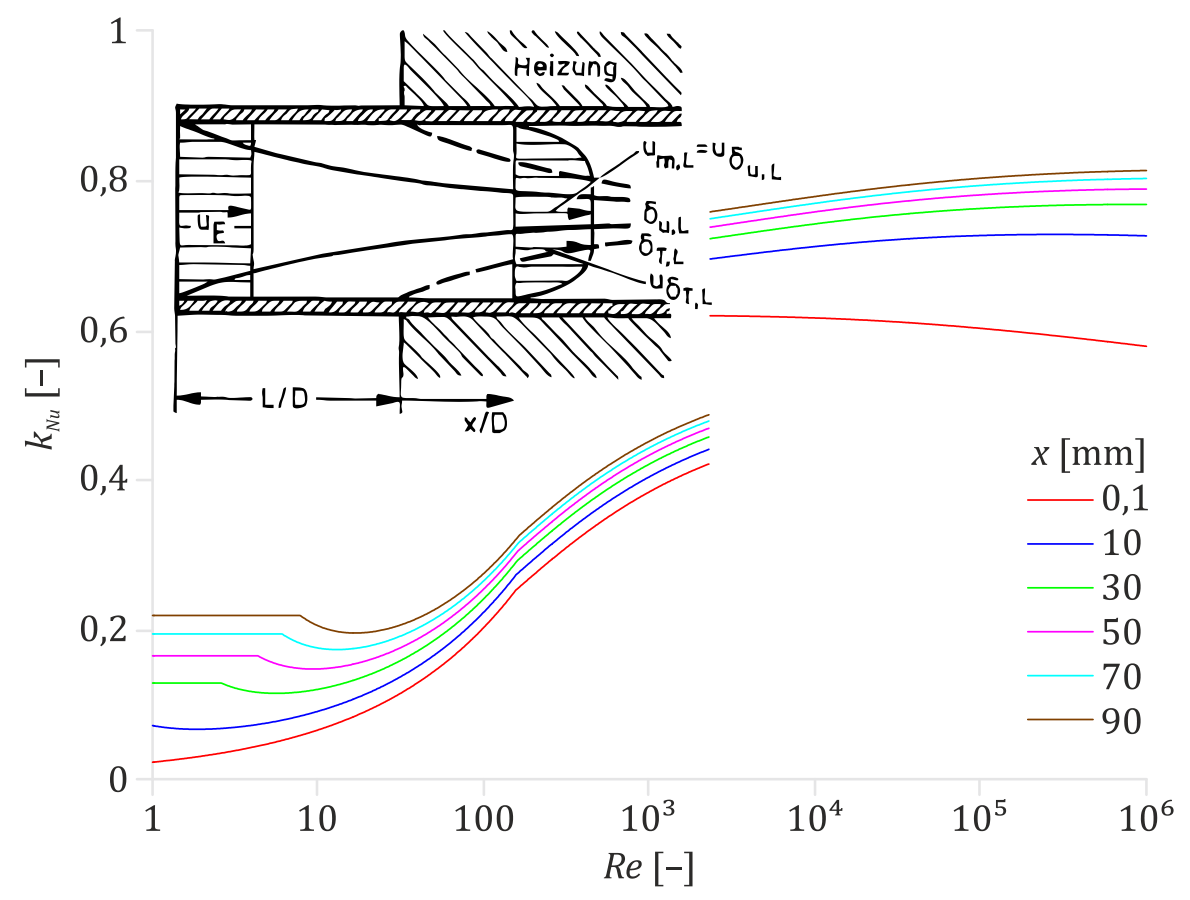

Abb. A.2 Korrekturfaktor für die Nusselt-Zahlen an unterschiedlichen axialen Positionen $x$ für laminare und turbulente Rohrströmung bei hydrodynamischem Vorlauf in Abhängigkeit von der Reynolds-Zahl (nach [139]); $L_{\mathrm{h}}=1,8 \mathrm{~m}, D=200 \mathrm{~mm}$. 


\section{A5 KORRELATIONEN NACH DEISSLER}

A5.1 Definition von Reynolds-Zahl und Nusselt-Zahl

$$
\begin{aligned}
& R e_{0} \equiv \frac{\rho_{0} u_{\mathrm{b}} D}{\mu_{0}} \\
& N u_{0} \equiv \frac{h D}{k_{0}} \\
& h \equiv \frac{q_{0}}{T_{0}-T_{\mathrm{b}}} \\
& u_{\mathrm{b}} \equiv \frac{\int_{0}^{r_{0}} u\left(r_{0}-y\right) d y}{\int_{0}^{r_{0}}\left(r_{0}-y\right) d y} \\
& T_{\mathrm{b}} \equiv \frac{\int_{0}^{r_{0}} T \rho u\left(r_{0}-y\right) d y}{\int_{0}^{r_{0}} \rho u\left(r_{0}-y\right) d y}
\end{aligned}
$$

$(\cdot)_{0} \ldots$ Größen an der Wand

$T_{\mathrm{b}} \ldots$ massenstromgemittelte (Bulk-) Temperatur

$u_{\mathrm{b}} \ldots$ querschnittsgemittelte (Bulk-) Geschwindigkeit

\section{A5.2 Konvention für Summationsindizes}

$(\cdot)_{i} \ldots$ Summation über $y^{+}$

$(\cdot)_{j} \ldots$ Summation über $\frac{x}{D}$ 


\section{A5.3 Geschwindigkeits-/ Temperaturprofil}

Strömung im wandfernen Bereich $\left(\mathrm{y}^{+}>26\right)$ :

$$
u^{+}=\int_{0}^{y^{+}} \frac{d y^{+}}{\left(1-\beta t^{+}\right)^{d}+\frac{n^{2} u^{+} y^{+}}{1-\beta t^{+}}} \quad[142] \quad t^{+}=\int_{0}^{y^{+}} \frac{d y^{+}}{\frac{\left(1-\beta t^{+}\right)^{d}}{P r}+\frac{n^{2} u^{+} y^{+}}{1-\beta t^{+}}}
$$

mit den experimentellen Konstanten $n=0,109$ und $\kappa=0,36$ [142].

Für $\beta=0$ (keine nennenswerte Aufheizung/ Abkühlung des Fluides durch Wärmeübergang):

$$
u^{+}=\int_{0}^{y^{+}} \frac{1}{1+n^{2} u^{+} y^{+}} d y^{+}
$$

$$
T^{+}=\int_{0}^{y^{+}} \frac{1}{\frac{1}{P r}+n^{2} u^{+} y^{+}} d y^{+}
$$

bzw.

$$
\frac{d u^{+}}{d y^{+}}=\frac{1}{1+n^{2} u^{+} y^{+}}
$$

$$
\frac{d T^{+}}{d y^{+}}=\frac{1}{\frac{1}{P r}+n^{2} u^{+} y^{+}}
$$

Diskretisierung in Radienrichtung (Index $i$ ):

$$
\frac{u_{i+1}^{+}-u_{i}^{+}}{y_{i+1}^{+}-y_{i}^{+}}=\frac{1}{1+n^{2} u_{i}^{+} y_{i}^{+}} \quad \frac{t_{i+1}^{+}-t_{i}^{+}}{y_{i+1}^{+}-y_{i}^{+}}=\frac{1}{\frac{1}{\operatorname{Pr}}+n^{2} u_{i}^{+} y_{i}^{+}}
$$

folgt:

$$
u_{i+1}^{+}=u_{i}^{+}+\frac{y_{i+1}^{+}-y_{i}^{+}}{1+n^{2} u_{i}^{+} y_{i}^{+}} \quad t_{i+1}^{+}=t_{i}^{+}+\frac{y_{i+1}^{+}-y_{i}^{+}}{\frac{1}{P r}+n^{2} u_{i}^{+} y_{i}^{+}}
$$

mit den Werten an der Wand

$$
u_{i=0}^{+}=0
$$

$$
t_{i=0}^{+}=0
$$


Strömung im wandnahen Bereich $\left(y^{+}<26\right)$ :

Für $\beta=0$ :

$$
u^{+}=\frac{1}{\kappa} \ln y^{+}+C
$$

$$
T^{+}=\frac{1}{\kappa} \ln y^{+}+D
$$

mit

$$
C=u^{+}\left(y^{+}=26\right)-\frac{1}{\kappa} \ln 26
$$

$$
D=T\left(y^{+}=26\right)-\frac{1}{\kappa} \ln 26
$$

und $u^{+}, T^{+}\left(y^{+}=26\right)$ aus den Gleichungen A.48

Diskretisierung:

$$
u_{i}^{+}=\frac{1}{\kappa} \ln y_{i}^{+}+C \quad T_{i}^{+}=\frac{1}{\kappa} \ln y_{i}^{+}+D
$$

liefert für vollentwickelte Profile:

$$
u_{i}^{+}=\left\{\begin{array}{cc}
u_{i-1}^{+}+\frac{y_{i}^{+}-y_{i-1}^{+}}{1+n^{2} u_{i-1}^{+} y_{i}^{+}} & y^{+} \leq 26 \\
\frac{1}{\kappa} \ln y_{i}^{+}+C & y^{+}>26
\end{array} \quad T_{i}^{+}=\left\{\begin{array}{cc}
T_{i-1}^{+}+\frac{y_{i}^{+}-y_{i-1}^{+}}{\frac{1}{P r}+n^{2} u_{i-1}^{+} y_{i}^{+}} & y^{+} \leq 26 \\
\frac{1}{\kappa} \ln y_{i}^{+}+D & y^{+}>26
\end{array}\right.\right.
$$

Und für sich ausbildende Profile:

$$
u_{\mathrm{a}, i}^{+}=\left\{\begin{array}{cl}
u_{i}^{+} & y^{+} \leq \delta^{+} \\
u_{\mathrm{a}, i-1}^{+} & y^{+}>\delta^{+}
\end{array} \quad T_{\mathrm{a}, i}^{+}=\left\{\begin{array}{cc}
T_{i}^{+} & y^{+} \leq \delta_{h}^{+} \\
T_{\mathrm{a}, i-1}^{+} & y^{+}>\delta_{h}^{+}
\end{array}\right.\right.
$$




\section{A5.4 Entwicklung der Temperaturgrenzschicht}

Konstanter Wandwärmestrom, variable Fluideigenschaften (Gase):

$$
\frac{x}{D}=\frac{1}{2 r_{0}^{+2}} \int_{0}^{\delta_{h}^{+}}\left(t_{\delta}^{+}-t^{+}\right) \frac{\rho}{\rho_{0}} u^{+}\left(r_{0}^{+}-y^{+}\right) d y^{+}
$$

Mit $\rho=\rho_{0}$ folgt:

$$
\frac{x}{D}=\frac{1}{2 r_{0}^{+2}} \int_{0}^{\delta_{h}^{+}}\left(t_{\delta_{(h)}^{+}}^{+}-t^{+}\right) \cdot u^{+} \cdot\left(r_{0}^{+}-y^{+}\right) d y^{+}
$$

Dabei ist $t_{\delta_{(h)}}^{+}=t^{+}\left(\delta_{h}^{+}\right)$(voll entwickeltes Profil)!

Diskretisierung für auflaufende Werte:

$$
\begin{gathered}
\left(\frac{x}{D}\right)_{i}=\frac{1}{2 r_{0}^{+2}} \underbrace{\sum_{i} \underbrace{\left(t_{\delta_{h}}^{+}-t_{i}^{+}\right) \cdot u_{i}^{+} \cdot\left(r_{0}^{+}-y_{i}^{+}\right) \Delta y_{i}^{+}}_{1, i}}_{s_{1, i}} \\
2 r_{0}^{+^{2}}\left(\frac{x}{D}\right)_{i}=s_{1, i}=\sum f_{1, i} \\
2 r_{0}^{+^{2}}\left(\frac{x}{D}\right)_{i+1}=s_{1, i}+f_{1, i+1} \\
\left(\frac{x}{D}\right)_{i+1}=\frac{1}{2 r_{0}^{+2}}\left[2 r_{0}^{+2}\left(\frac{x}{D}\right)_{i}+f_{1, i+1}\right] \\
\left(\frac{x}{D}\right)_{i}=\frac{1}{2 r_{0}^{+2}}\left[2 r_{0}^{+2}\left(\frac{x}{D}\right)_{i-1}+f_{1, i}\right] \\
\left(\frac{x}{D}\right)_{i}=\frac{1}{2 r_{0}^{+2}\left[2 r_{0}^{+2}\left(\frac{x}{D}\right)_{i-1}+\left(t_{\delta_{h}}^{+}-t_{i}^{+}\right) \cdot u_{i}^{+} \cdot\left(r_{0}^{+}-y_{i}^{+}\right) \Delta y_{i}^{+}\right]} \\
\left(\frac{x}{D}\right)_{i=0}=0
\end{gathered}
$$


$\underline{\text { Konstante Wandtemperatur, konstante Fluideigenschaften: }}$

$$
\frac{X}{D}=\frac{1}{2} \int_{0}^{[]} \frac{t_{\delta}^{+}}{r_{0}^{+}} d\left[\frac{1}{r_{0}^{+}} \int_{0}^{\delta_{h}^{+}} \frac{t_{\delta}^{+}-t^{+}}{t_{\delta}^{+}} u^{+}\left(r_{0}^{+}-y^{+}\right) d y^{+}\right]
$$

Ein Integralzeichen mit leeren Klammern für die obere Grenze $\int^{[~] ~ h e i ß t, ~ d a s s ~ d i e ~ I n t e g r a t i o n s v a-~}$ riable als obere Grenze verwendet werden soll.

Substituiert man die Obergrenze und die Integrationsvariable mit $a$, so folgt für die dimensionslose Anlauflänge:

$$
\frac{X}{D}=\left[\frac{x}{D}\right]_{\frac{x}{D}=0}^{\frac{x}{D}=\frac{X}{D}}=\frac{1}{2} \int_{a\left(\frac{x}{D}=0\right)}^{a\left(\frac{x}{D}=\frac{X}{D}\right)} \frac{t_{\delta}^{+}}{r_{0}^{+}} d a
$$

Diskretisierung für auflaufende Werte von $x / D$ :

$$
\begin{gathered}
\left(\frac{X}{D}\right)_{j}=\frac{1}{2} \sum_{j} \frac{t_{\delta, j}^{+}}{r_{0, j}^{+}}\left(a_{j}-a_{j-1}\right) \\
\left(\frac{X}{D}\right)_{j-1}=\left(\frac{X}{D}\right)_{j}+\frac{1}{2} \frac{t_{\delta, j}^{+}}{r_{0, j}^{+}}\left(a_{j}-a_{j-1}\right)
\end{gathered}
$$

mit

$$
\left(\frac{X}{D}\right)_{j=0}=0
$$

und

$$
\begin{gathered}
a_{i, j}=\frac{1}{r_{0, j}^{+}} \sum_{i} \frac{t_{\delta, j}^{+}-t_{a, i, j}^{+}}{t_{\delta, j}^{+}} \cdot u_{a, i, j}^{+} \cdot\left(r_{0, j}^{+}-y_{i}^{+}\right) \Delta y_{i}^{+} \\
a_{i, j}=a_{i-1, j}+\frac{1}{r_{0, j}^{+}} \frac{t_{\delta, j}^{+}-t_{a, i, j}^{+}}{t_{\delta, j}^{+}} \cdot u_{a, i, j}^{+} \cdot\left(r_{0, j}^{+}-y_{i}^{+}\right) \Delta y_{i}^{+}
\end{gathered}
$$

wobei

$$
\begin{aligned}
& a_{i=0, j}=0 \\
& a_{i, j=0}=0
\end{aligned}
$$


A5.5 Bestimmung der Kernstrom- („Bulk“-) Temperatur und -geschwindigkeit

$$
t_{b}^{+}=\frac{\int_{0}^{r_{0}^{+}} \frac{t^{+} u^{+}\left(r_{0}^{+}-y^{+}\right)}{1-\beta t^{+}} d y^{+}}{\int_{0}^{r_{0}^{+}} \frac{u^{+}\left(r_{0}^{+}-y^{+}\right)}{1-\beta t^{+}} d y^{+}}
$$

Dabei stehen positive Werte von $\beta$ für Wärmezufuhr an das Gas, negative Werte für Wärmeabfuhr. Für die Fälle, bei denen die Fluidparameter als konstant angenommen werden, sind die Kurven für $\beta=0$ zu nutzen [140].

Mit $\beta=0$ (konstante Fluideigenschaften):

$$
t_{b}^{+}=\frac{\int_{0}^{r_{0}^{+}} t^{+} \cdot u^{+} \cdot\left(r_{0}^{+}-y^{+}\right) d y^{+}}{\int_{0}^{r_{0}^{+}} u^{+} \cdot\left(r_{0}^{+}-y^{+}\right) d y^{+}}
$$

Diskretisierung:

$$
t_{b, i}^{+}=\frac{s_{2, i}}{s_{3, i}}
$$

mit

$$
\begin{gathered}
s_{2, i}=\sum_{i} t_{a, i}^{+} \cdot u_{a, i}^{+} \cdot\left(r_{0}^{+}-y_{i}^{+}\right) \Delta y_{i}^{+} \\
s_{2, i}=s_{2, i-1}+t_{a, i}^{+} \cdot u_{a, i}^{+} \cdot\left(r_{0}^{+}-y_{i}^{+}\right) \Delta y_{i}^{+} \\
s_{2, i=0}=0
\end{gathered}
$$

und

$$
\begin{gathered}
s_{3, i}=\sum_{i} u_{a, i}^{+} \cdot\left(r_{0}^{+}-y_{i}^{+}\right) \Delta y_{i}^{+} \\
s_{3, i}=s_{3, i-1}+u_{a, i}^{+} \cdot\left(r_{0}^{+}-y_{i}^{+}\right) \Delta y_{i}^{+} \\
s_{3, i=0}=0 \\
u_{b}^{+}=\frac{2}{r_{0}^{+2}} \int_{0}^{r_{0}^{+}} u^{+}\left(r_{0}^{+}-y^{+}\right) d y^{+}
\end{gathered}
$$

Diskretisierung:

$$
u_{b, i}^{+}=\frac{2}{r_{0}^{+2}} s_{3, i}
$$




\section{A5.6 Reynolds-Zahl und Nusselt-Zahl}

$$
\begin{aligned}
& N u_{0}=\frac{2 r_{0}^{+} P r}{t_{b}^{+}} \\
& R e_{0}=2 u_{b}^{+} r_{0}^{+}
\end{aligned}
$$

Nusselt- und Reynolds-Zahlen, bei denen sich die Stoffdaten auf eine andere Temperatur als die Wandtemperatur beziehen, können mit Hilfe der folgenden Verhältnisse gebildet werden [140]:

$$
\begin{gathered}
\frac{t_{b}}{t_{0}}=1-\beta t_{b}^{+} \\
\frac{t_{i}}{t_{0}}=1-\beta t_{\delta}^{+} \\
\frac{\rho_{b}}{\rho_{0}}=\frac{1}{1-\beta t_{b}^{+}} \\
\frac{\mu_{b}}{\mu_{0}}=\frac{k_{b}}{k_{0}}=\left(1-\beta t_{b}^{+}\right)^{d}
\end{gathered}
$$

mithin:

$$
\begin{array}{rlrl}
R e_{b} & =R e_{0} \frac{1}{\left(\frac{t_{b}}{t_{0}}\right)^{d+1}} & N u_{b}=\frac{N u_{0}}{\left(\frac{t_{b}}{t_{0}}\right)^{d}} \\
R e_{i}=\operatorname{Re}_{b} \frac{\left(\frac{t_{b}}{t_{0}}\right)^{d}}{\left(\frac{t_{i}}{t_{0}}\right)^{d}} & N u_{i}=\frac{N u_{b}}{\left(\frac{t_{i}}{t_{b}}\right)^{d}}
\end{array}
$$

Mit $\beta=0$ (konstante Fluideigenschaften) ist

$$
\begin{gathered}
R e_{i}=R e_{b}=R e_{0} \\
N u_{i}=N u_{b}=N u_{0}
\end{gathered}
$$




\section{A5.7 Entwicklung der Strömungsgrenzschicht}

Dimensionslose Form der Impulsgleichungen für konstante Stoffwerte:

$$
\begin{gathered}
\frac{X}{D}=\int_{\frac{R e}{2}}^{u_{\delta}^{+} r_{0}^{+}}\left[\frac{1}{4}\left(\frac{\delta^{+}}{r_{0}^{+}}\right) \frac{1}{r_{0}^{+}}\left(2-\frac{\delta^{+}}{r_{0}^{+}}\right) u_{\delta}^{+}-\frac{1}{2 r_{0}^{+3}} \int_{0}^{\delta^{+}} u^{+}\left(r_{0}^{+}-y^{+}\right) d y^{+}\right] d\left(r_{0}^{+} u_{\delta}^{+}\right)+ \\
\int_{0}^{[} \frac{1}{2 r_{0}^{+2}} d\left[\int_{0}^{\delta^{+}}\left(u_{\delta}^{+}-u^{+}\right) u^{+}\left(r_{0}^{+}-y^{+}\right) d y^{+}\right]
\end{gathered}
$$

Substitution einiger Terme:

$$
\frac{X}{D}=\left[\frac{x}{D}\right]_{\frac{x}{D}=0}^{\frac{x}{D}=\frac{X}{D}}=\int_{u_{\delta}^{+} r_{0}^{+}\left(\frac{x}{D}=0\right)}^{u_{\delta}^{+} r_{0}^{+}\left(\frac{x}{D}=\frac{X}{D}\right)}\left[\frac{1}{4} \cdot \frac{\delta^{+}}{r_{0}^{+^{2}}} \cdot\left(2-\frac{\delta^{+}}{r_{0}^{+}}\right) \cdot u_{\delta}^{+}-A\right] d\left(u_{\delta}^{+} r_{0}^{+}\right)+\int_{B\left(\frac{x}{D}=0\right)}^{B\left(\frac{x}{D}=\frac{X}{D}\right)} \frac{1}{2{r_{0}^{+2}}^{2}} d B
$$

Diskretisierung für auflaufende Werte von $x / D$ :

$$
\begin{aligned}
& \left(\frac{X}{D}\right)_{j}=\sum_{j}\left\{\left[\frac{1}{4} \cdot \frac{\delta_{j}^{+}}{r_{0, j}^{+2}} \cdot\left(2-\frac{\delta_{j}^{+}}{r_{0, j}^{+}}\right) \cdot u_{\delta, j}^{+}-A_{j}\right]\left(u_{\delta, j}^{+} r_{0, j}^{+}-u_{\delta, j-1}^{+} r_{0, j-1}^{+}\right)+\frac{1}{2 r_{0, j}^{+}{ }^{2}}\left(B_{j}-B_{j-1}\right)\right\} \\
& \left(\frac{X}{D}\right)_{j}=\left(\frac{X}{D}\right)_{j-1}+\left[\frac{1}{4} \cdot \frac{\delta_{j}^{+}}{{r_{0, j}^{+}}^{2}} \cdot\left(2-\frac{\delta_{j}^{+}}{r_{0, j}^{+}}\right) \cdot u_{\delta, j}^{+}-A_{j}\right]\left(u_{\delta, j}^{+} r_{0, j}^{+}-u_{\delta, j-1}^{+} r_{0, j-1}^{+}\right)+\frac{1}{2{r_{0, j}^{+}}^{2}}\left(B_{j}-B_{j-1}\right) \\
& \left(\frac{X}{D}\right)_{j=0}=0 \\
& \left(u_{\delta}^{+} r_{0}^{+}\right)_{j=0}=u_{b}^{+} r_{0, j=0}^{+} \equiv \frac{R e_{0}}{2} \\
& A_{i, j} \cdot 2{r_{0, j}^{+}}^{3}=\sum_{i} u_{a, i, j}{ }^{+} \cdot\left(r_{0, j}^{+}-y_{i}^{+}\right) \Delta y_{i}^{+} \equiv s_{3, i} \\
& A_{i, j}=\frac{1}{2 r_{0, j}^{+3}} \cdot\left(2 r_{0, j}^{+3} \cdot A_{i-1, j}+u_{a, i, j}{ }^{+} \cdot\left(r_{0, j}^{+}-y_{i}^{+}\right) \Delta y_{i}^{+}\right) \\
& A_{i=0, j}=0 \\
& B_{i, j}=\sum_{i}\left(u_{\delta, j}^{+}-u_{a, i, j}^{+}\right) \cdot u_{a, i, j}^{+} \cdot\left(r_{0, j}^{+}-y_{i}^{+}\right) \Delta y_{i}^{+} \\
& B_{i, j}=B_{i-1, j}+\left(u_{\delta, j}^{+}-u_{a, i, j}^{+}\right) \cdot u_{a, i, j}^{+} \cdot\left(r_{0, j}^{+}-y_{i}^{+}\right) \Delta y_{i}^{+} \\
& B_{i=0, j}=0 \\
& B_{i, j=0}=0
\end{aligned}
$$




\section{A5.8 Berücksichtigung des nicht voll ausgebildeten hydrodynamischen Vorlaufs}

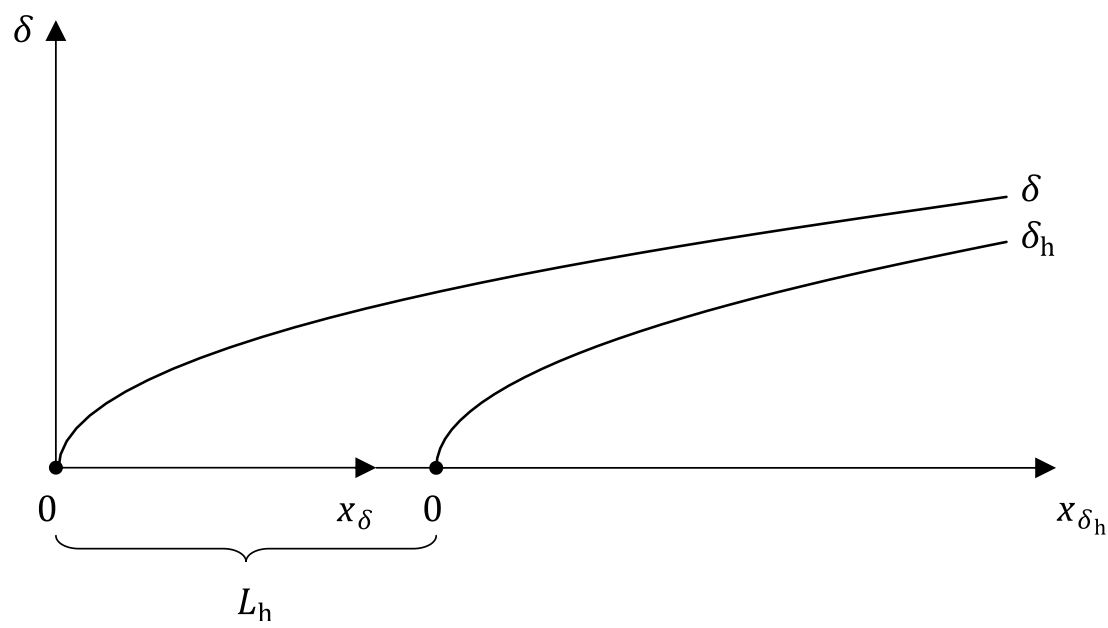

$$
\begin{gathered}
x_{\delta_{h}}=x_{\delta}-L_{\mathrm{h}} \\
x_{\delta}=x_{\delta_{h}}+L_{\mathrm{h}} \\
\left(\frac{x}{D}\right)_{\delta}=\left(\frac{x}{D}\right)_{\delta_{h}}+\frac{L_{\mathrm{h}}}{D}>\left(\frac{x}{D}\right)_{\delta_{h}} \geq 0
\end{gathered}
$$

Vorgabe separater Sollwerte für $x / D$ :

$$
\left(\frac{x}{D}\right)_{\delta, \text { Soll }}=\left(\frac{x}{D}\right)_{\delta_{h}, \text { Soll }}+\frac{L_{\mathrm{h}}}{D}
$$

\section{A5.9 Berechnungsalgorithmus}

Zur Berechnung des lokalen Verlaufes der Nusselt-Zahl $N u$ über der aufsteigenden Lauflänge $x / D$ bei einer gewünschten Reynolds-Zahl $R e_{\text {Soll }}$ werden die Größen $r_{0}^{+}, \delta_{h}^{+}$und $\delta^{+}$ausgehend von geeigneten Startwerten solange variiert, bis die kalkulierten Werte von $R e,\left.\frac{x}{D}\right|_{\delta^{+}}$und $\left.\frac{x}{D}\right|_{\delta_{h}^{+}}$mit den angegebenen Sollwerten von $\operatorname{Re}_{\text {Soll }}$ bzw. $(x / D)_{\text {Soll }}$ übereinstimmen. Danach werden die Werte von $(x / D)_{j-1},\left(u_{\delta}^{+} r_{0}^{+}\right)_{j-1}, B_{j-1}$ und $a_{j-1}$ zwischengespeichert und mit dem nächst größeren Wert von $(x / D)_{j-1}$ fortgefahren.

Die nachgerechneten Nusselt-Zahl-Verläufe sowohl für eine hydrodynamisch voll ausgebildete Strömung sowie für gleichzeitigen thermischen und hydrodynamischen Anlauf jeweils bei konstanter Wandtemperatur bzw. konstantem Wärmestrom sind für verschiedene Reynolds-Zahlen zusammen mit den unterlegten Diagrammen aus [140] in den Bildern A.3 bis A.6 dargestellt. Wie sich zeigt, stimmen die Werte für alle Fälle sehr gut überein, was das oben angegebene Diskretisierungs- und Berechnungsschema prinzipiell bestätigt. 


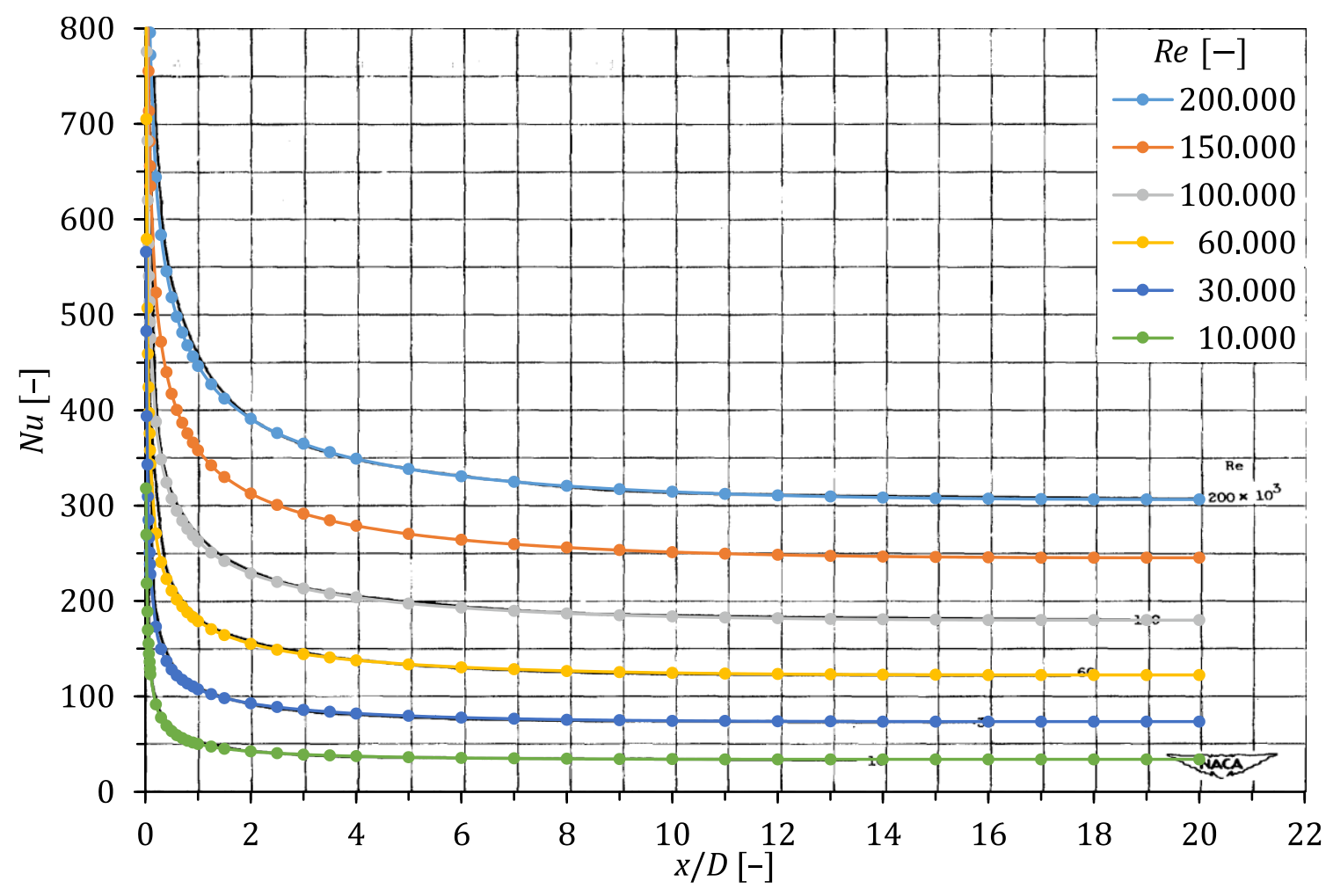

Abb. A.3 Variation der Nusselt-Zahl über der Anströmlänge für unterschiedliche Reynolds-Zahlen bei gleichzeitigem thermischen und hydrodynamischen Anlauf, konstantem Wandwärmestrom, einheitlicher Einströmtemperatur- und -geschwindigkeitsverteilung, konstanten Stoffwerten und $\operatorname{Pr}=$ 0,73 (Fig. 19 in [140]) sowie nachgerechnete Werte.

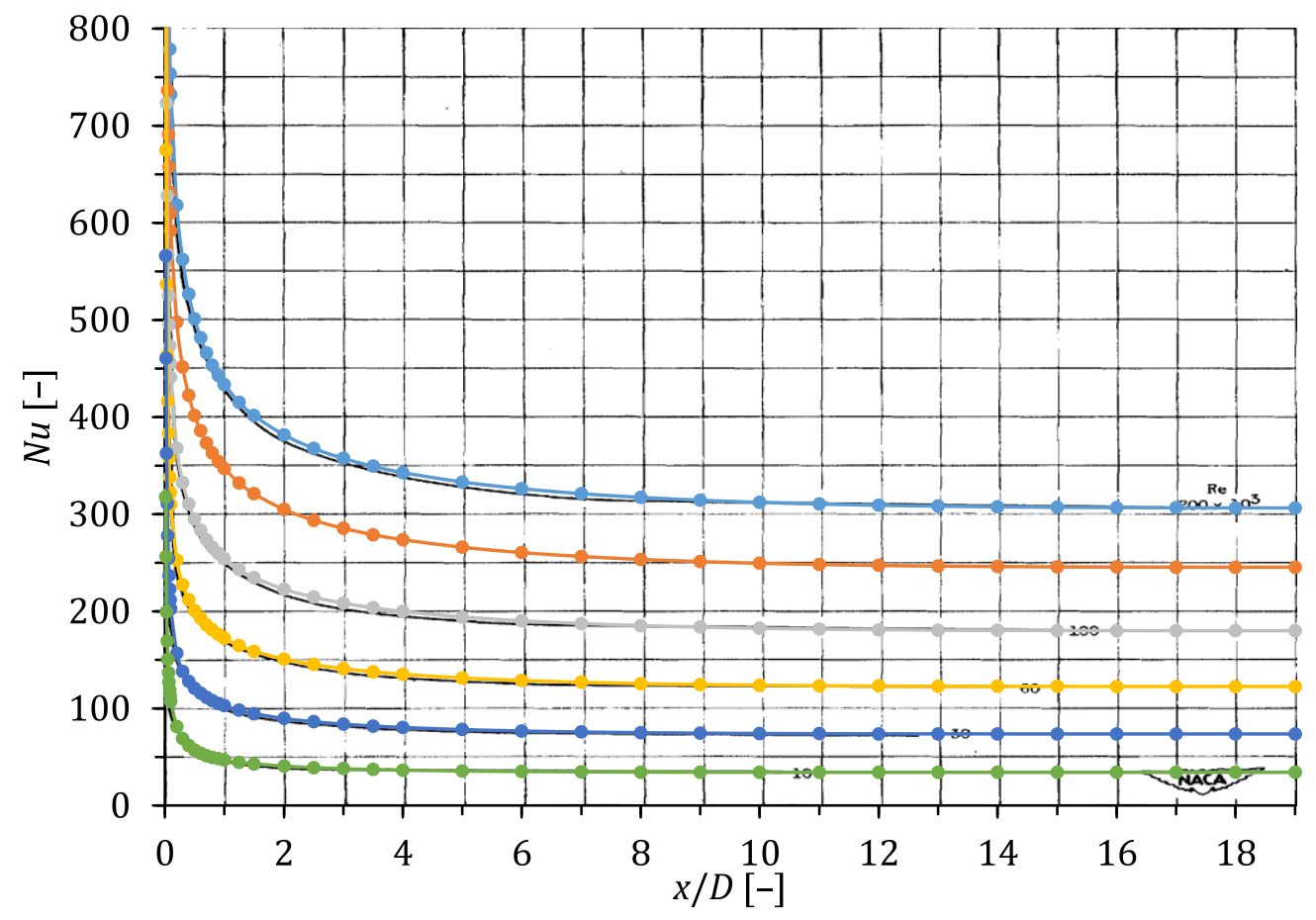

Abb. A.4 Variation der Nusselt-Zahl über der Anströmlänge für unterschiedliche Reynolds-Zahlen bei gleichzeitigem thermischen und hydrodynamischen Anlauf, konstanter Wandtemperatur, einheitlicher Einströmtemperatur- und -geschwindigkeitsverteilung, konstanten Stoffwerten und $P r=0,73$ (Fig. 22 in [140]) sowie nachgerechnete Werte. 


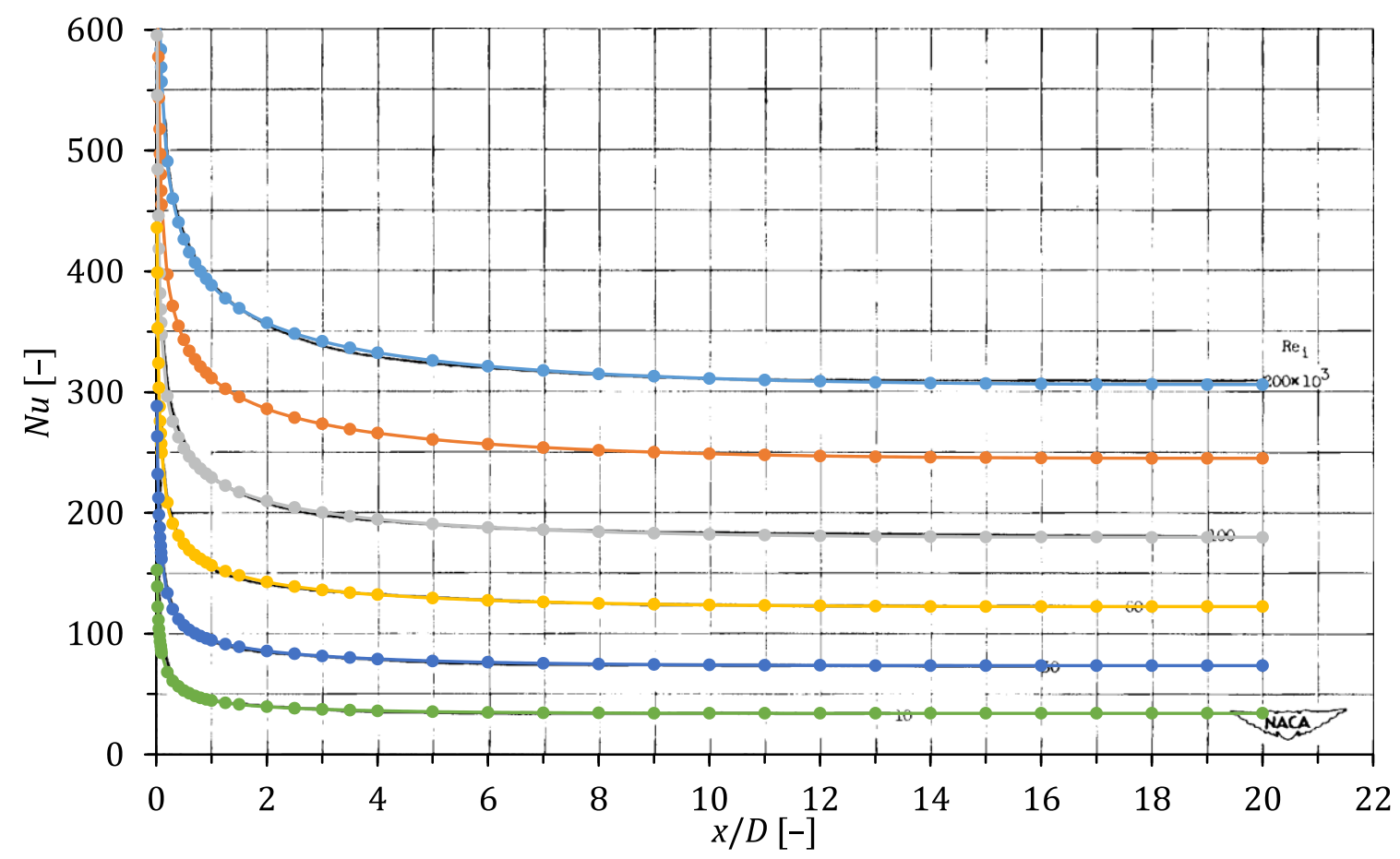

Abb. A.5 Variation der Nusselt-Zahl über der Anströmlänge für unterschiedliche Reynolds-Zahlen bei hydrodynamisch voll ausgebildeter Strömung und thermischen Anlauf, konstantem Wandwärmestrom, einheitlicher Einströmtemperaturverteilung, voll entwickelter Geschwindigkeitsverteilung, konstanten Stoffwerten und $P r=0,73$ (Fig. 5 a in [140]) sowie nachgerechnete Werte.

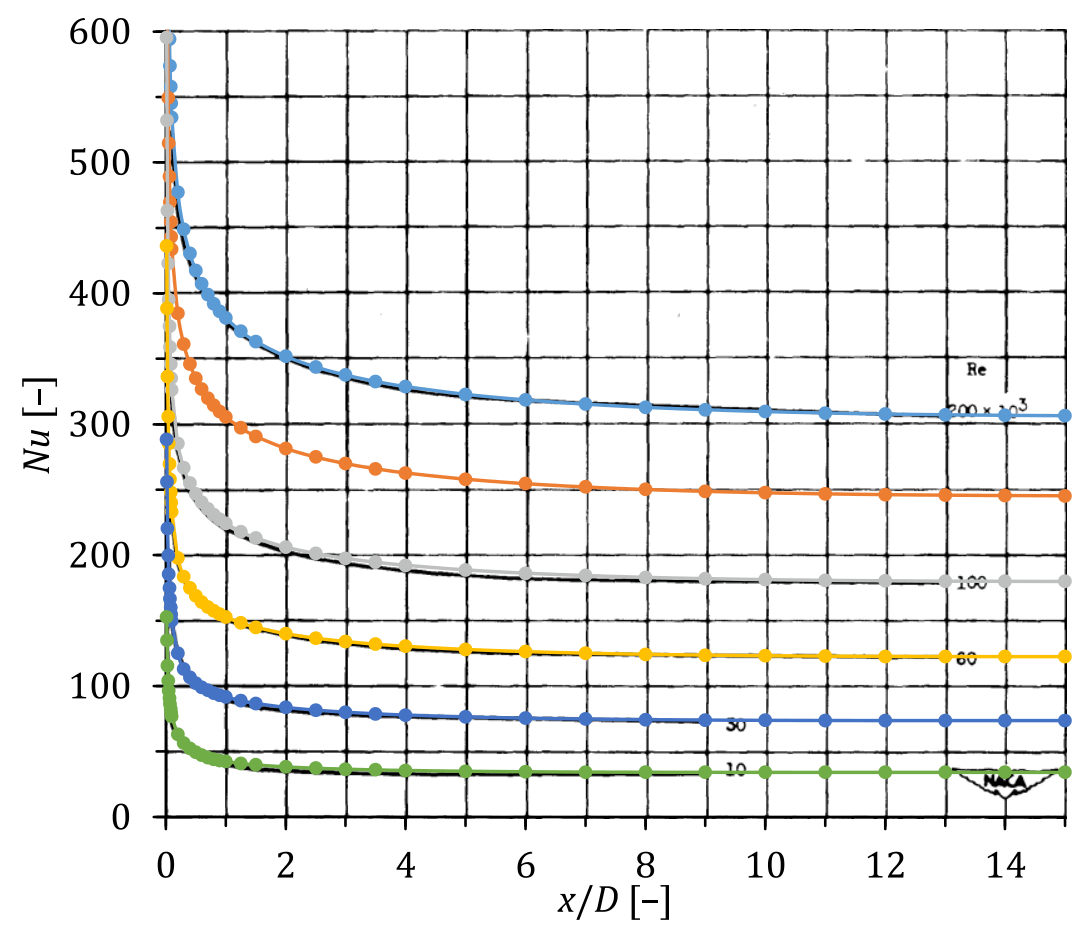

Abb. A.6 Variation der Nusselt-Zahl über der Anströmlänge für unterschiedliche Reynolds-Zahlen bei hydrodynamisch voll ausgebildeter Strömung und thermischen Anlauf, konstanter Wandtemperatur, einheitlicher Einströmtemperaturverteilung, voll entwickelter Geschwindigkeitsverteilung, konstanten Stoffwerten und $P r=0,73$ (Fig. 18 in [140]) sowie nachgerechnete Werte. 


\section{A5.10 Mittlere (lauflängengemittelte) Nusselt-Zahl}

Die mittlere Nusselt-Zahl für ein gegebenes $X / D$ wird wie folgt berechnet [140]:

$$
N u_{i, \mathrm{av}}=\frac{X / D}{\int_{0}^{X / D} \frac{d(X / D)}{N u_{i}}}
$$

Diskretisierung für auflaufende Werte (Wechsel des Indizes von $i \rightarrow j$, da über $x / D$ summiert wird:

$$
\begin{aligned}
& \overline{N u}_{j}=N u_{j, \mathrm{av}}=\underbrace{\frac{\left(\frac{x}{D}\right)_{j}}{\sum_{j} \underbrace{\left\{\frac{1}{2} \cdot\left(\frac{1}{N u_{j}}-\frac{1}{N u_{j-1}}\right) \cdot\left[\left(\frac{x}{D}\right)_{j}-\left(\frac{x}{D}\right)_{j-1}\right]\right\}}_{f_{j}}}}_{s_{j}} \\
& \overline{N u}_{j+1}=\underbrace{\frac{\left(\frac{x}{D}\right)_{j+1}}{\sum_{j} \underbrace{\left\{\frac{1}{2} \cdot\left(\frac{1}{N u_{j+1}}-\frac{1}{N u_{j}}\right) \cdot\left[\left(\frac{x}{D}\right)_{j+1}-\left(\frac{x}{D}\right)_{j}\right]\right\}}_{f_{j+1}}}}_{s_{j+1}} \\
& \frac{\left(\frac{x}{D}\right)_{j}}{\bar{N}_{j}}=s_{j}=\sum f_{j} \\
& \frac{\left(\frac{x}{D}\right)_{j+1}}{\overline{N u}_{j+1}}=s_{j}+f_{j+1} \\
& \frac{\left(\frac{x}{D}\right)_{j+1}}{\overline{N u}_{j+1}}=\frac{\left(\frac{x}{D}\right)_{j}}{\overline{N u}_{j}}+f_{j+1}
\end{aligned}
$$

Bestimmung von $\overline{N u}_{j=0}$ :

Da sowohl $\overline{N u}_{j=0} \rightarrow \infty$ als auch $N u_{0} \rightarrow \infty$, muss für einen sehr klein zu wählenden Wert von $(x / D)_{j=0}$ ein (endlich großer) Wert für $\overline{N u}_{j=0}$ gefunden werden. Dieser kann in Anlehnung an den Korrekturfaktor $k_{L}$ für die Anlauflänge bei einer voll entwickelten turbulenten Rohrströmung nach VDI-Wärmeatlas bestimmt werden:

Mit

$$
\begin{aligned}
& N u_{x}=N u_{\infty} \cdot\left[1+\frac{1}{3}\left(\frac{D}{x}\right)^{\frac{2}{3}}\right] \\
& \overline{N u}=N u_{\infty} \cdot\left[1+\left(\frac{D}{x}\right)^{\frac{2}{3}}\right]
\end{aligned}
$$

folgt:

$$
\overline{N u}=3 \cdot N u_{x}-2 \cdot N u_{\infty}
$$


Tatsächlich zeigt sich, dass die Wahl von $\overline{N u}_{j=0}$ kaum Auswirkung auf den Verlauf von $\overline{N u}(x / D)$ hat, selbst wenn ein Wert angenommen wird, der um Größenordnungen größer ist.

Um die Wahl eines Wertes für $\overline{N u}_{0}$ zu umgehen, ist es auch möglich, von der „anderen Seite“, d. h. von $x / D \rightarrow \infty$ her zu integrieren. Dabei gilt analog:

mit

$$
\overline{N u}_{j+1}=\frac{\left(\frac{x}{D}\right)_{j+1}}{\frac{\left(\frac{x}{D}\right)_{j}}{\overline{N u}_{j}}+\frac{1}{2} \cdot\left(\frac{1}{N u_{j+1}}-\frac{1}{N u_{j}}\right) \cdot\left[\left(\frac{x}{D}\right)_{j+1}-\left(\frac{x}{D}\right)_{j}\right]}
$$

$$
\overline{N u}_{\infty}=\lim _{\frac{x}{D} \rightarrow \infty} \frac{\frac{x}{D}}{\int_{0}^{\frac{x}{D}} \frac{d\left(\frac{x}{D}\right)}{N u}}=N u_{\infty}
$$

Hierbei zeigt sich jedoch, dass die „Wahl“ von $\overline{N u}_{\infty}$ deutlichen Einfluss auf die Steigung der Kurve hat, vor allem im Bereich kleiner $x / D$. So ist es zwar möglich, durch geschicktes Anpassen die Kurven von Deissler nachzubilden. Für die so erzeugten Kurven ergibt sich eine Asymptote, für die die Werte von $\bar{N} \bar{u}_{\infty} \rightarrow \infty$ streben, bei der die Polstelle jedoch bei einem Wert $x / D>0$ liegt. Dies ist jedoch unphysikalisch. Die Asymptote muss immer bei $x / D=0$ liegen:

$$
\lim _{\bar{x} \rightarrow+0} \overline{N u}=" \infty "
$$

Im doppelt logarithmischen Diagramm müssten sich dann die Kurven von $\overline{N u}$ für $x / D \rightarrow+0$ einer Geraden annähern, dürfen also weder links- noch rechtsgekrümmt sein:

$$
\begin{aligned}
& \lim _{\bar{x} \rightarrow+0} \frac{d \ln \overline{N u}}{d(\ln x)}=-c \\
& \lim _{\bar{x} \rightarrow+0} \frac{d^{2} \ln \overline{N u}}{[d(\ln x)]^{2}}=0
\end{aligned}
$$

Dieses Problem hat Deissler in seiner Veröffentlichung offensichtlich auch, weshalb zu vermuten ist, dass auch er die Werte für $\overline{N u}$ über eine Rückwärtsrechnung ermittelt und er deshalb nur die Werte bis $x / D=0,5$ dargestellt hat.

Dass die Rückwärtsrechnung numerisch instabil ist, sieht man auch daran, dass, wenn man einen Wert für $\overline{N u}_{\infty}$ wählt, der kleiner ist, zwar keine Asymptote mehr auftritt, die Werte von $\overline{N u}$ jedoch unterhalb eines gewissen Wertes von $x / D$ wieder kleiner und null werden. Auch das ist unphysikalisch. Für die Rückwärtsrechnung sind die verwendete Trapezregel sowie das Euler-vorwärtsVerfahren zur Auflösung des Integrals nicht geeignet. Entweder man wählt hier eine Diskretisierungsmethode höherer Ordnung (z. B. die Simpson-Regel) oder man verwendet die Werte aus der Vorwärtsrechnung, die bereits in der vorliegenden Form numerisch stabil sind. 

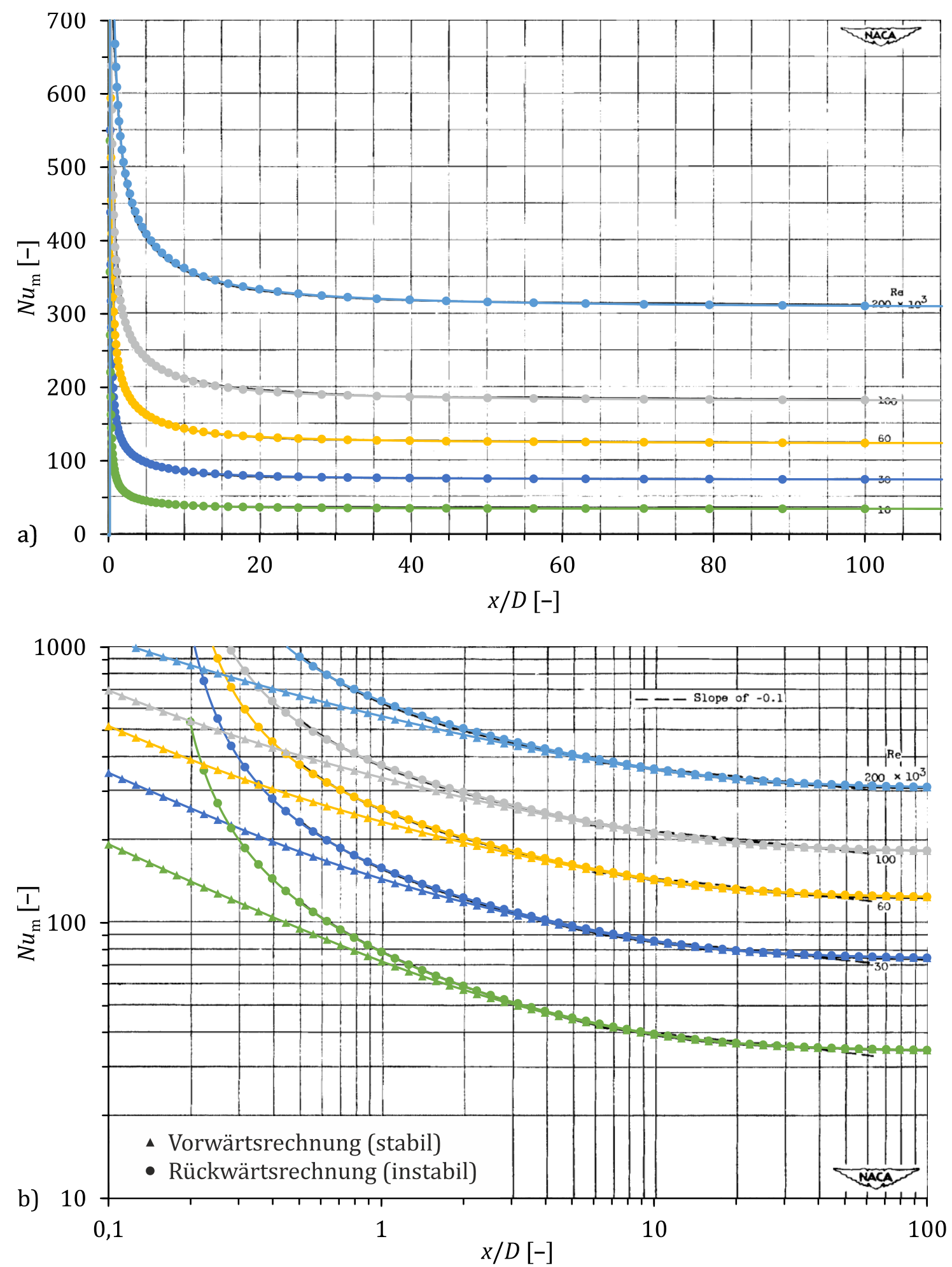

Abb. A.7 Variation der über der Anströmlänge gemittelten Nusselt-Zahl für unterschiedliche Reynolds-Zahlen bei gleichzeitigem thermischen und hydrodynamischen Anlauf, konstantem Wandwärmestrom, einheitlicher Einströmtemperatur- und -geschwindigkeitsverteilung, konstanten Stoffwerten und $P r=0,73$ (Fig. 20 in [140]) sowie nachgerechnete Werte.

a) lineare Achsteilung; b) doppelt-logarithmische Achsteilung. 


\section{A6 KONSTRUKTIVE DETAILS VON VERSUCHSSTAND UND -ANLAGE}

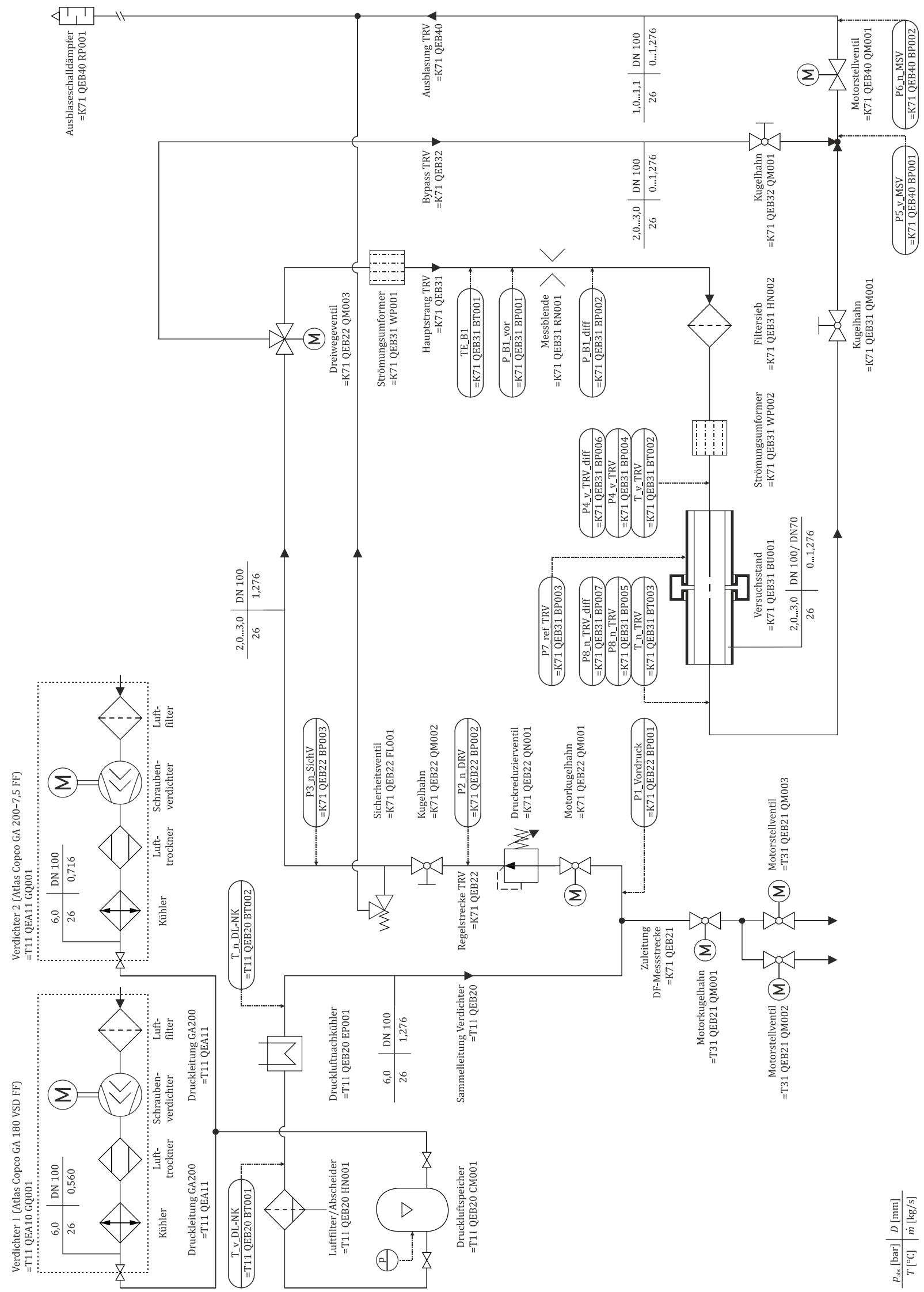

Abb. A.8 Rohrleitungs- und Instrumentenfließschema der erweiterten Druckluftversuchsanlage. 


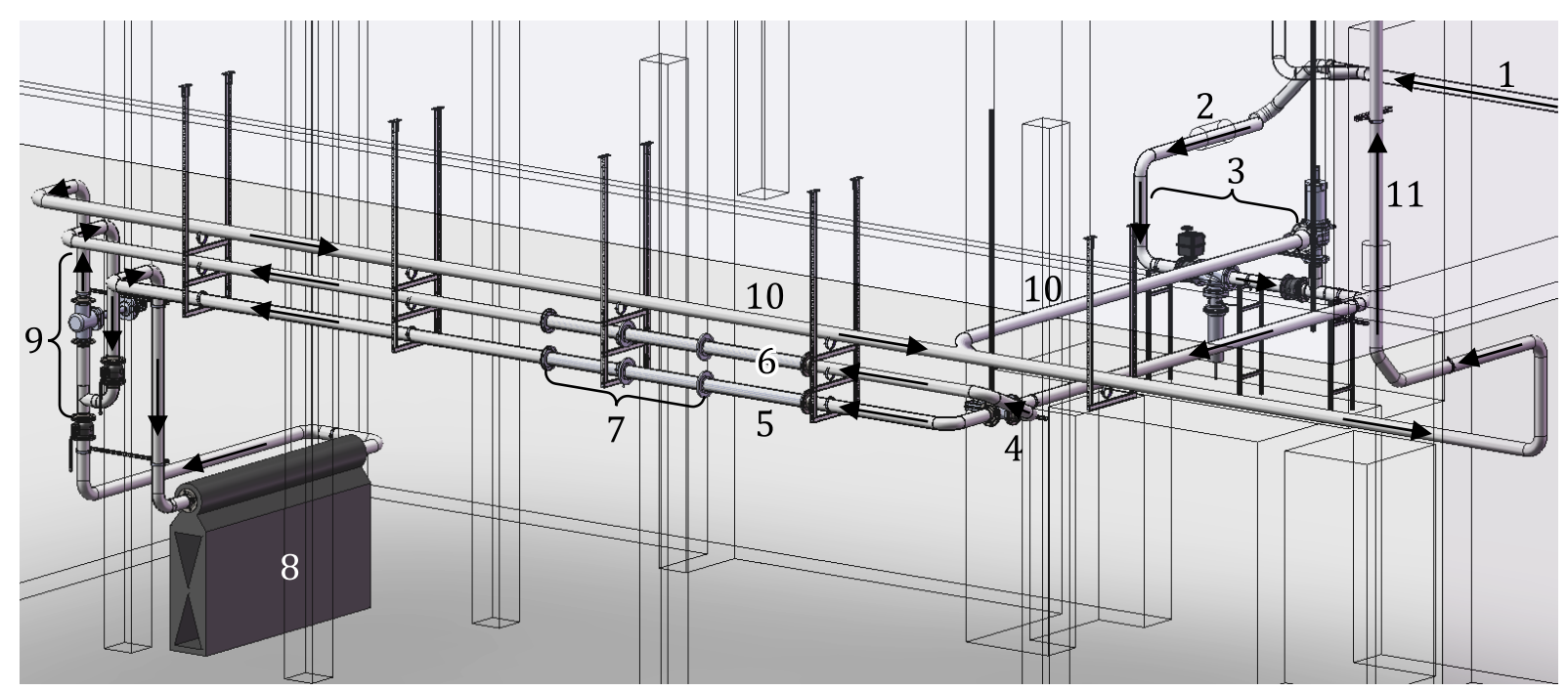

Abb. A.9 Installationsplan der erweiterten Druckluftversuchsanlage:

1 - Vorlauf von Verdichtern ( Abb. A.10); 2 - Wanddurchbruch; 3 - Regelgruppe 1 ( Abb. A.11 a);

4 - Dreiwegeventil; 5 - Hauptstrang; 6 - Bypass; 7 - Blendenmessstrecke ( Abb. A.13);

8 - Versuchsstand ( Abb. A.14); 9 - Regelgruppe 2 ( Abb. A.11 b); 10 - Ausblasleitung; 11 - Ausblasung über Dach.

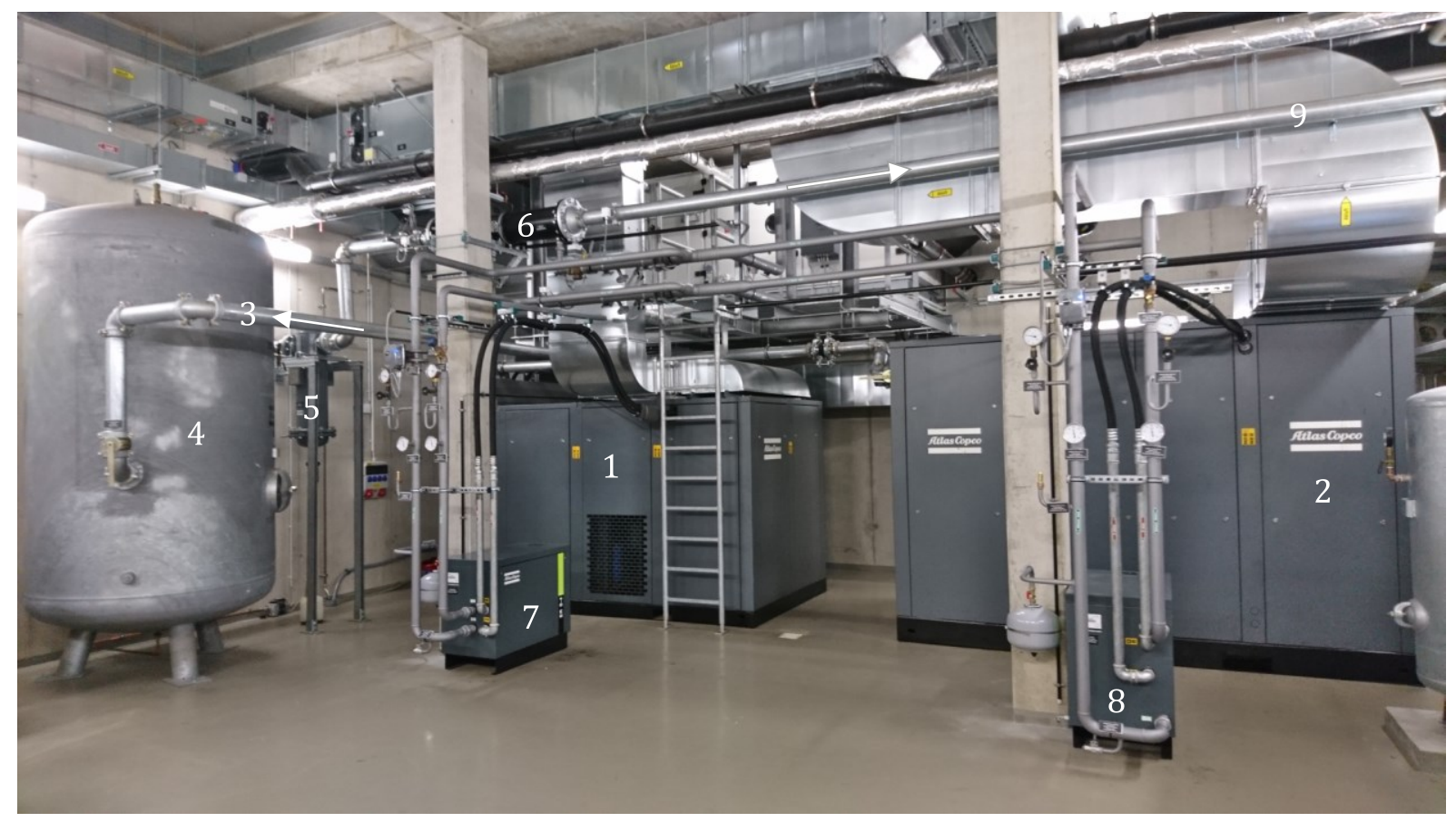

Abb. A.10 Druckluftbereitstellung durch die beiden Schraubenverdichter mit nachgeschaltetem Windkessel im ZET, Raum K106:

1 - Atlas Copco GA 180 VSD FF; 2 - Atlas Copco GA 200-7,5 FF; 3 - Sammeldruckleitung;

4 - Druckluftspeicher; 5 - Luftfilter; 6 - Druckluftnachkühler; 7 - Ölkühler ER-S4;

8 - Ölkühler ER-S5; 9 - Vorlauf zu den Versuchsständen. 

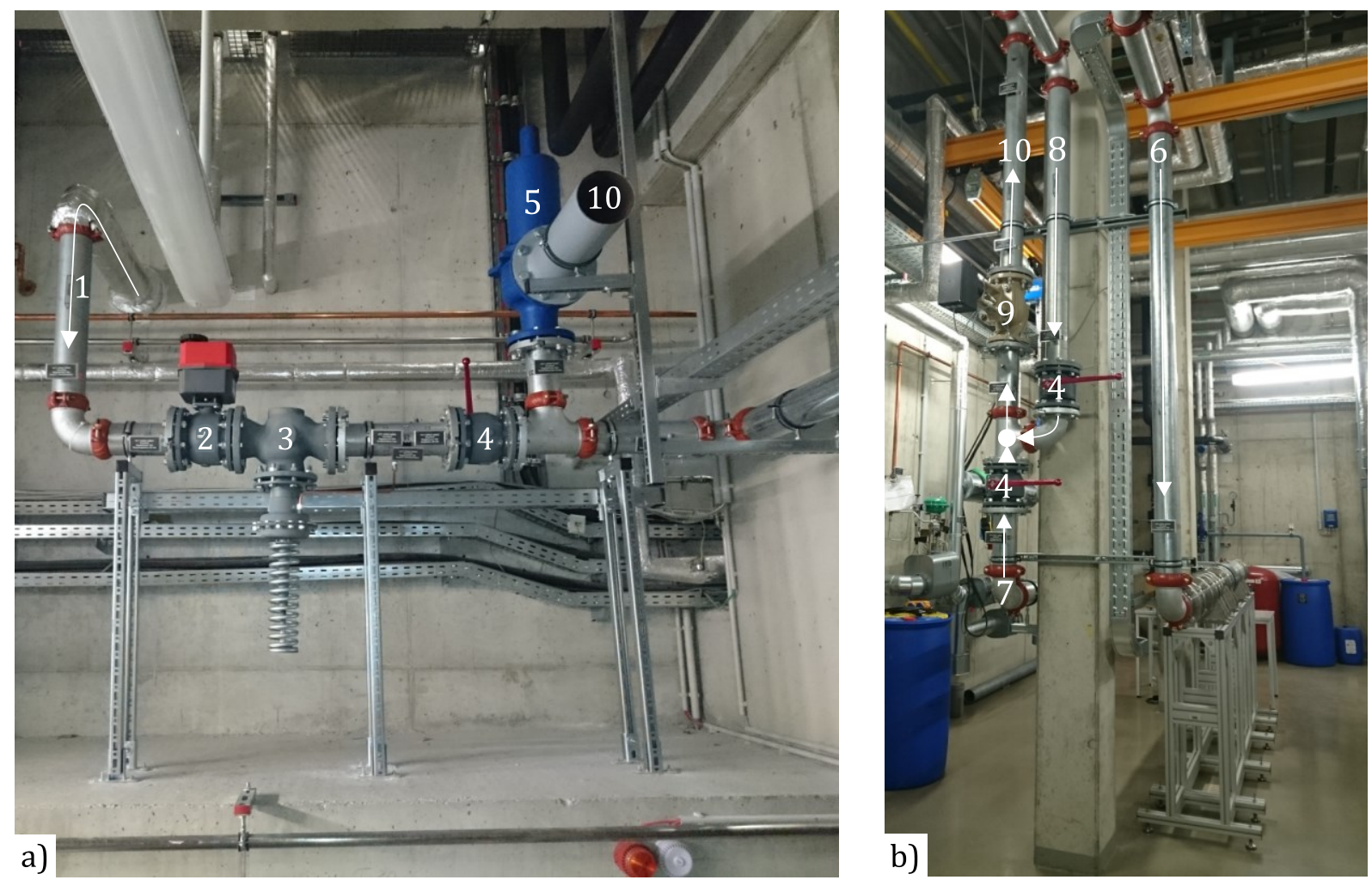

Abb. A.11 Regelgruppen für die Druckregelung in der Versuchsanlage:

a) Einstellung des Druckniveaus im nachgeschalteten System mittels Druckminderer;

b) Luftführung und Wiedereinbindung des Bypass, Nachdrosselung mit Motorstellventil; 1 - Vorlauf von den Verdichtern; 2 - Motorkugelhahn; 3 - Druckreduzierventil;

4 - Handkugelhahn; 5 - Sicherheitsventil; 6 - Hauptstrang (Vorlauf);

7 - Hauptstrang (Rücklauf); 8 - Bypass; 9 - Motorstellventil; 10 - Ausblaseleitung.

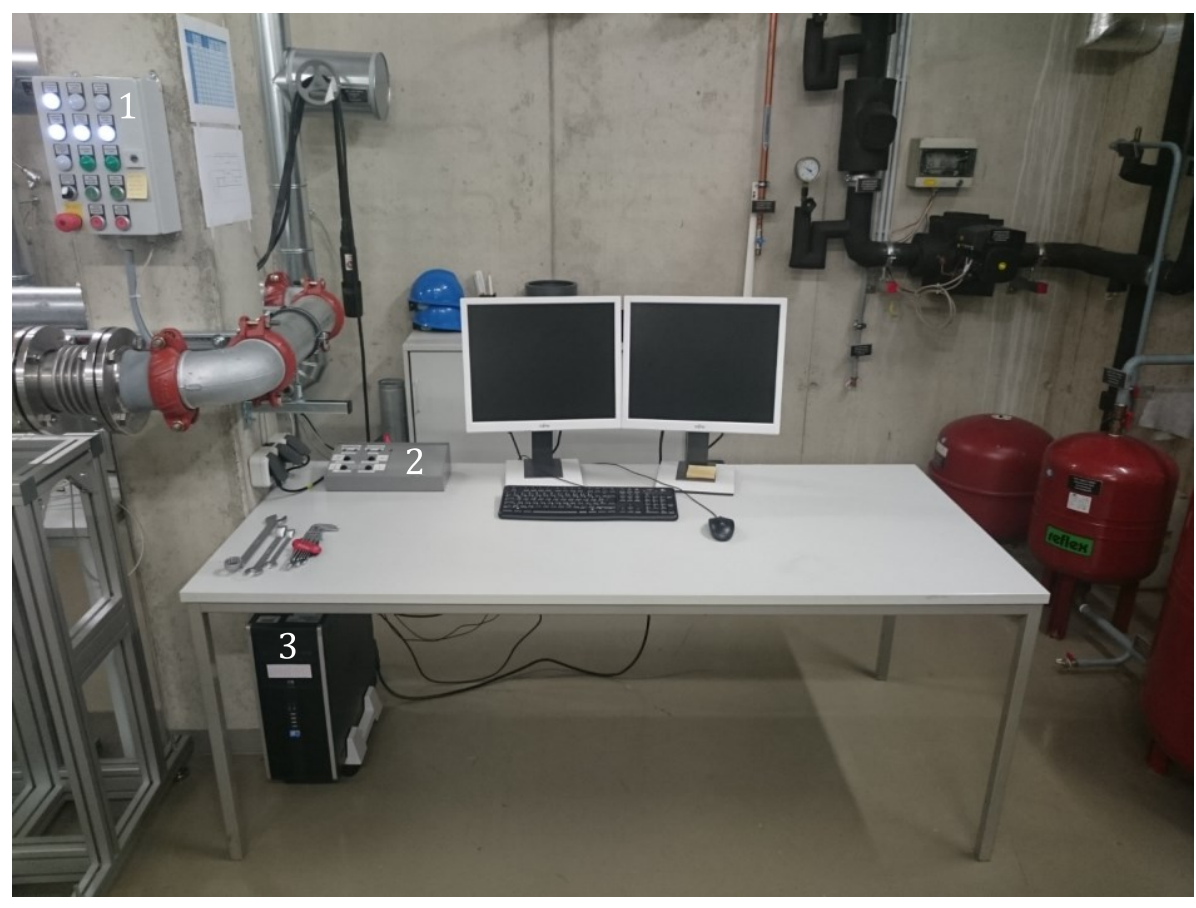

Abb. A.12 PC-Arbeitsplatz mit Schalteinrichtungen:

1 - Betriebspanel für Verdichter und Absperrkugelhähne;

2 - Betriebspanel für Stellventile (MSV/ 3WV); 3 -Messrechner 


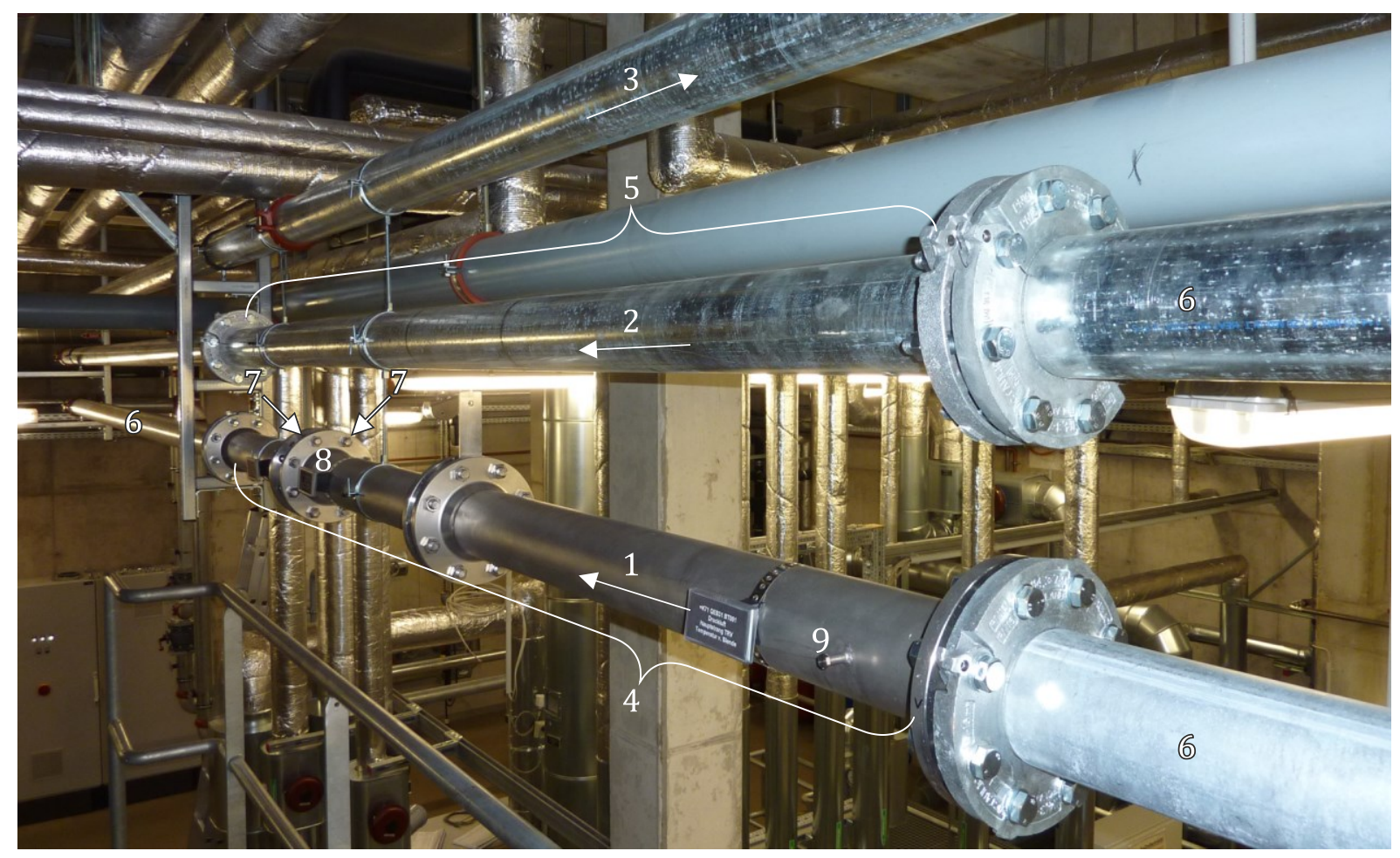

\section{Abb. A.13 Blendenmessstrecke:}

1 - Hauptstrang mit Versuchsstand; 2 - Bypass; 3 - Ausblasung; 4 - Blendenmessstrecke im Hauptstrang; 5 - Passstück für Blendenmessstrecke im Bypass (nachrüstbar);

6 -An- bzw. Abströmstrecken; 7 - Eckdruckentnahmen; 8 - Messblende $(\varnothing d=71 \mathrm{~mm}$; auswechselbar); 9 - Anschluss für Thermoelement.

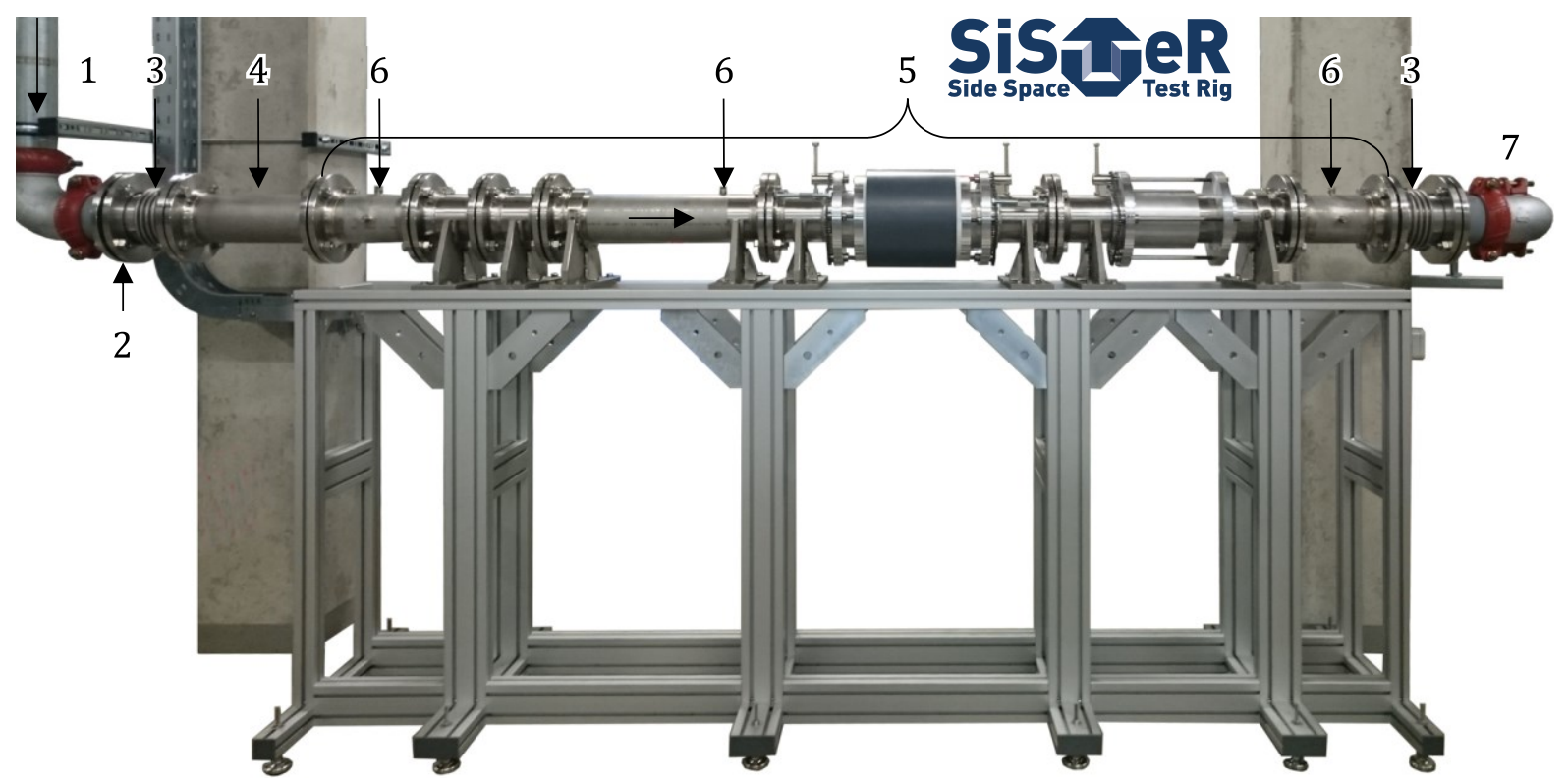

\section{Abb. A.14 Einbindung des Versuchsstandes in das Druckluftsystem:}

1 - Zuströmung; 2 - Zwischenflanschströmungsgleichrichter und -turbulenzsieb;

3 - Kompensator; 4 - Passstück; 5 - Versuchsstand; 6 - Anschlüsse für Druck-, Temperaturund Geschwindigkeitsmessung $\left(270^{\circ}, 0^{\circ}\right.$ bzw. $\left.90^{\circ}\right) ; 7$ - Abströmung (nach hinten). 


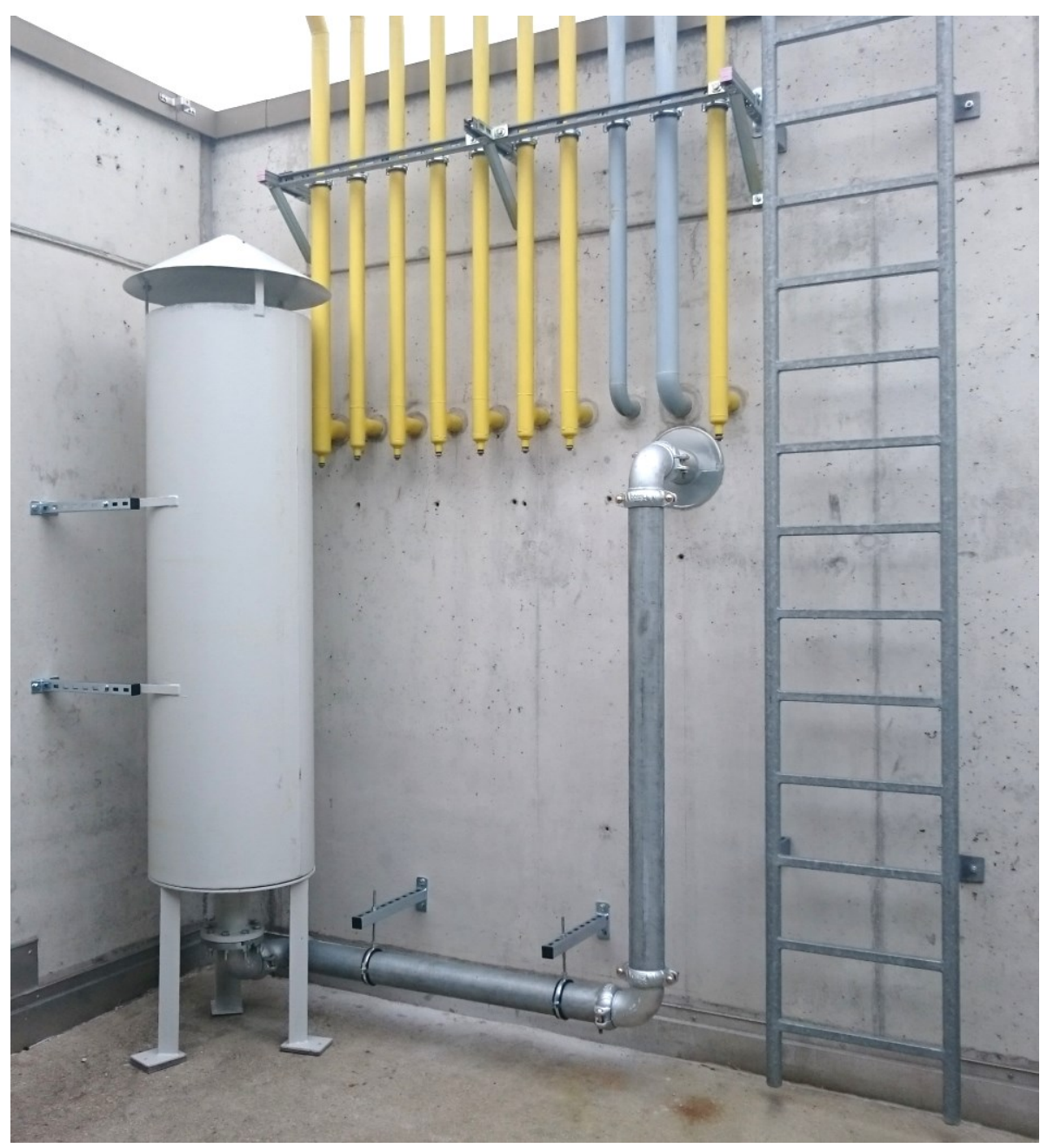

Abb. A.15 Ausblasung der Versuchsluft über einen Schalldämpfer in die Umgebung.

Tab. A.1 Einstellungen für die Steuerungen der beiden Schraubenverdichter

\begin{tabular}{llll}
\hline \multicolumn{1}{c}{ Atlas Copco GA180 VSD FF } & \multicolumn{2}{c}{ Atlas Copco GA 200 FF } \\
\hline Sollwert & 5,0 bar(Ü) & Belastungsdruck & 4,5 bar(Ü) \\
Indirekter Stopp & $+0,2 \mathrm{bar}$ & Entlastungsdruck & 5,5 bar(Ü) \\
Direkter Stopp & $+0,3 \mathrm{bar}$ & & \\
Proportionalbereich & $10,0 \%$ & Y-Zeit & $10 \mathrm{~s}$ \\
Integrationszeit & $10,0 \mathrm{~s}$ & Belastungsverzögerung & $1 \mathrm{~s}$ \\
Mindestdrehzahl & $600 \mathrm{~min}^{-1}$ & Anzahl Starts/h & 3 \\
Maximaldrehzahl & $70 \mathrm{bzw.100 \%}$ & Anzahl Starts/d & 3 \\
Minimum Stopzeit & $5 \mathrm{~s}$ & Minimum Stop Zeit & $20 \mathrm{~s}$ \\
Zulässige Startzeit & $0 \mathrm{~s}$ & Erforderliche Stopzeit & 5 \\
\hline
\end{tabular}




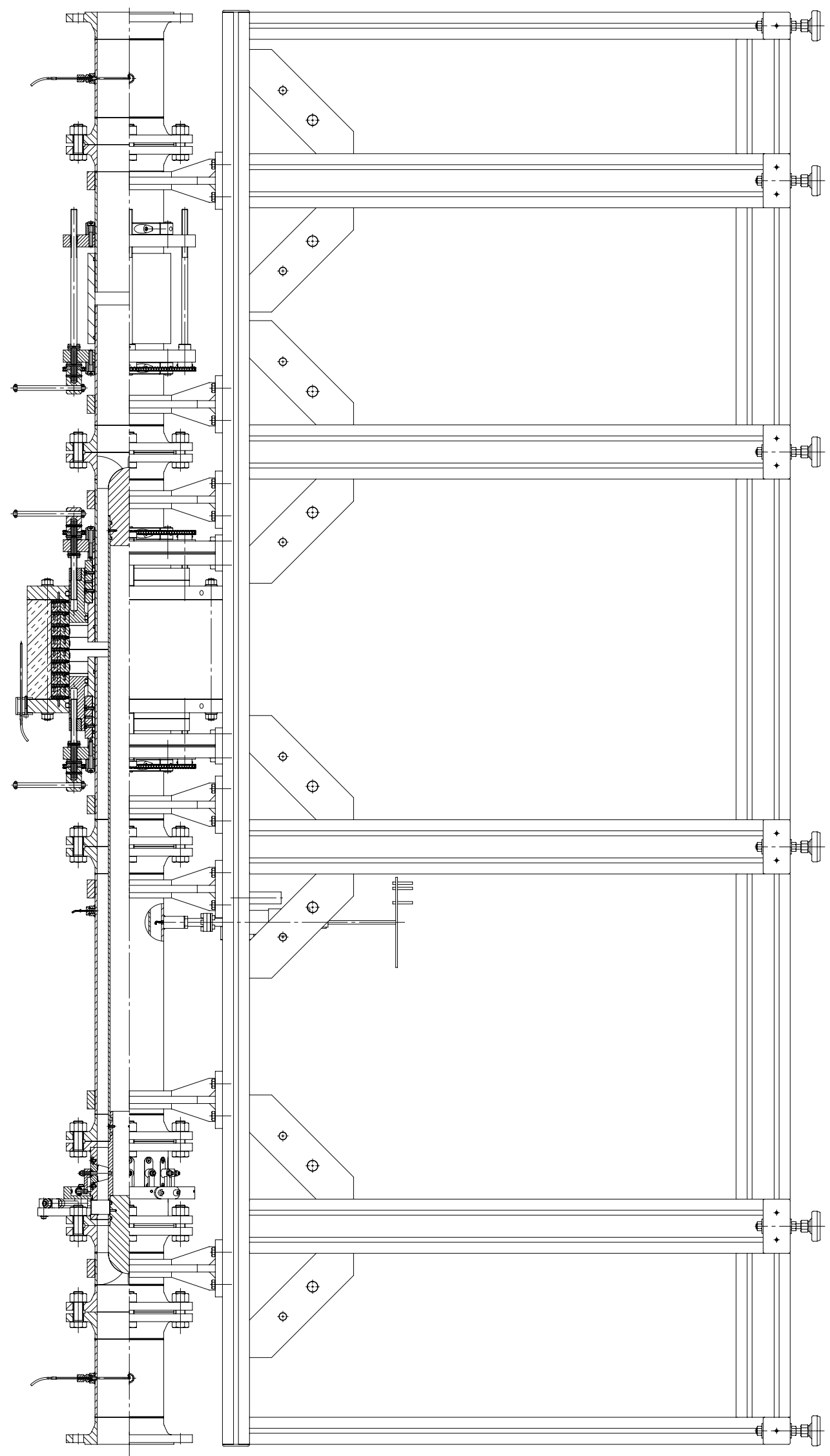

Abb. A.16 Experimenteller Aufbau des Versuchsstandes (Viertelschnittdarstellung).

Alle Flansche sowie Drallgitter, Ein- und Abströmmodul wurden zu Veranschaulichungszwecken um $45^{\circ}$ in die Ansichtsebene gedreht, um im Schnitt alle Bauteile zu sehen (vgl. Abb. A.18 b). 


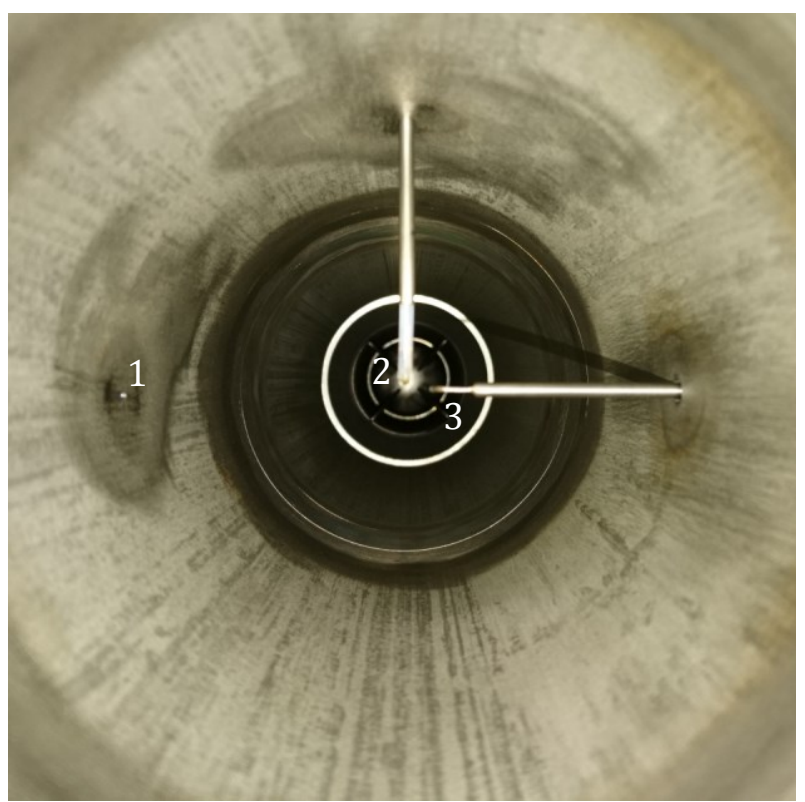

Abb. A.17 Druck-, Temperatur- und Geschwindigkeitsmessung in der Rohrströmung (hier: im Demontagemodul hinter dem Versuchsstand; Blick entgegen der Strömungsrichtung).

1 - Wandanbohrung für statische Druckmessung; 2 - Thermistorsonde für Temperaturmessung; 3 - Pitotsonde für Geschwindigkeitsmessung.
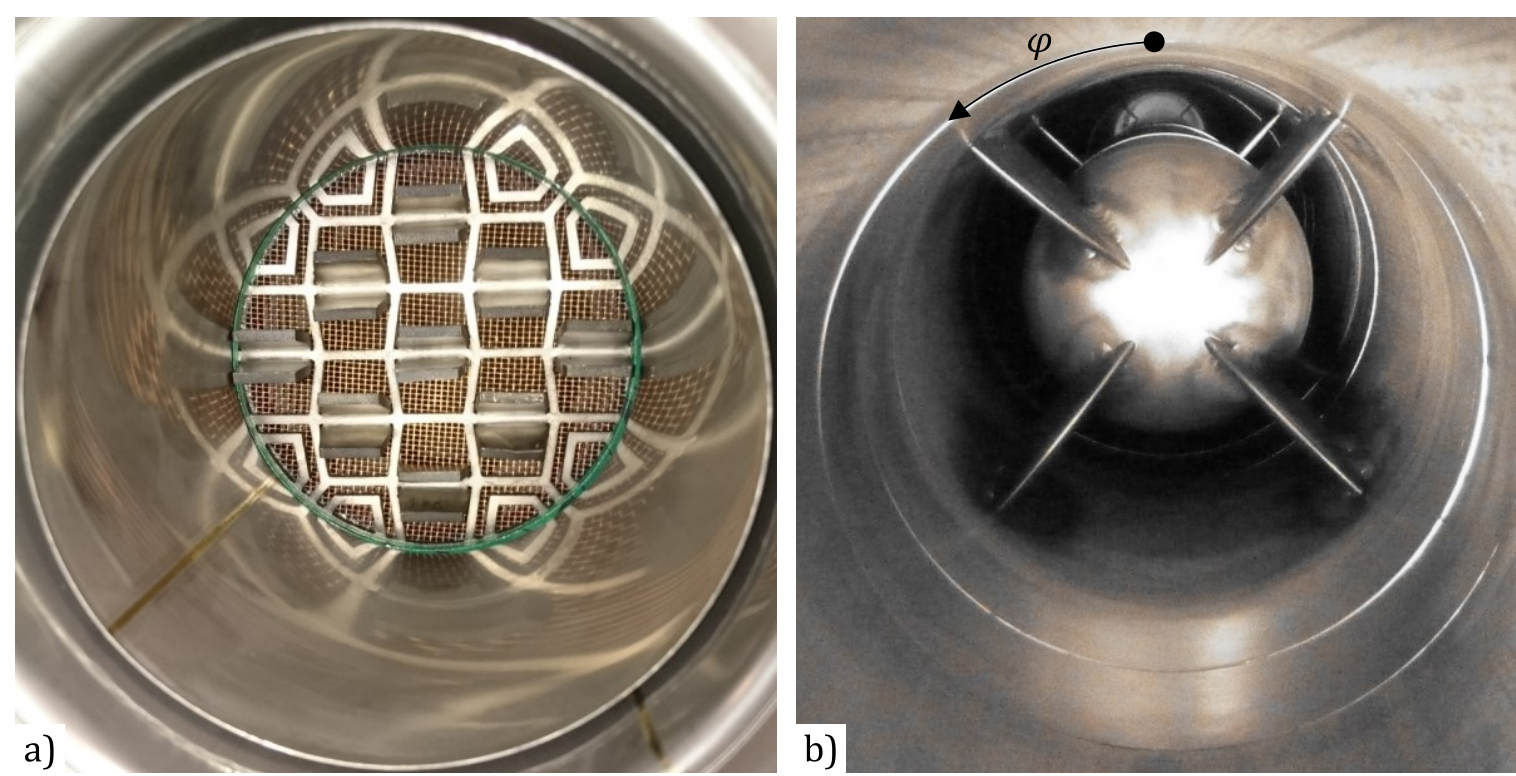

Abb. A.18 Innenansicht des Strömungskanals auf der Versuchsstandsachse:

a) Strömungsgleichrichter, Turbulenzgitter und Kompensatorleitrohr vor Versuchsstand;

b) Kreisringspaltförmiger Strömungskanal mit Verdrängungskörper und Innenrohrlagerungen. 

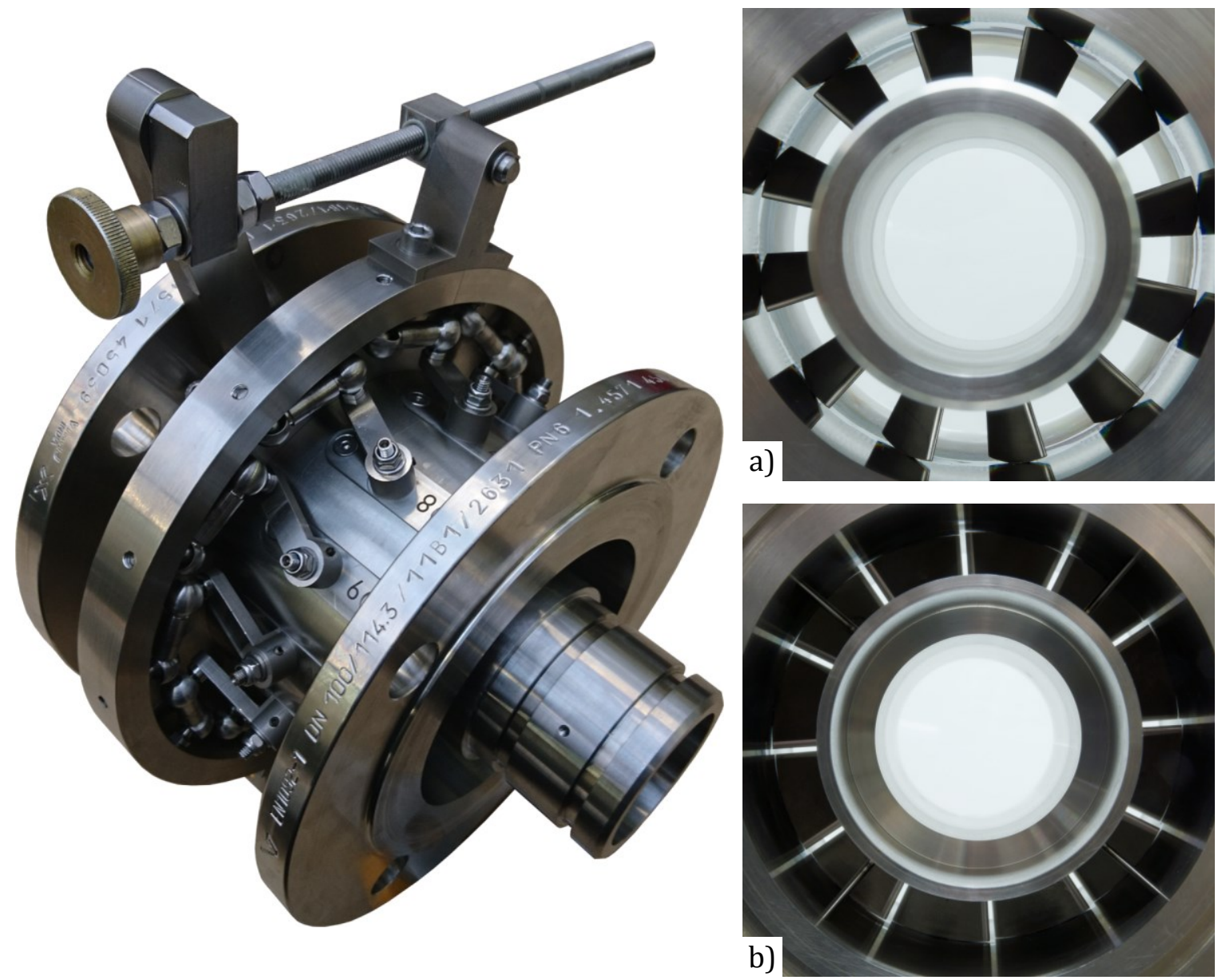

Abb. A.19 Variables Drallgitter (VGV) mit Drallblech-Verstellmechanismus: a) $\gamma \approx 50^{\circ}$; b) $\gamma \approx 85^{\circ}$.

Bei den beiden Detailabbildungen handelt es sich um "gestackte“ Bilder, siehe Abb. 4.13.
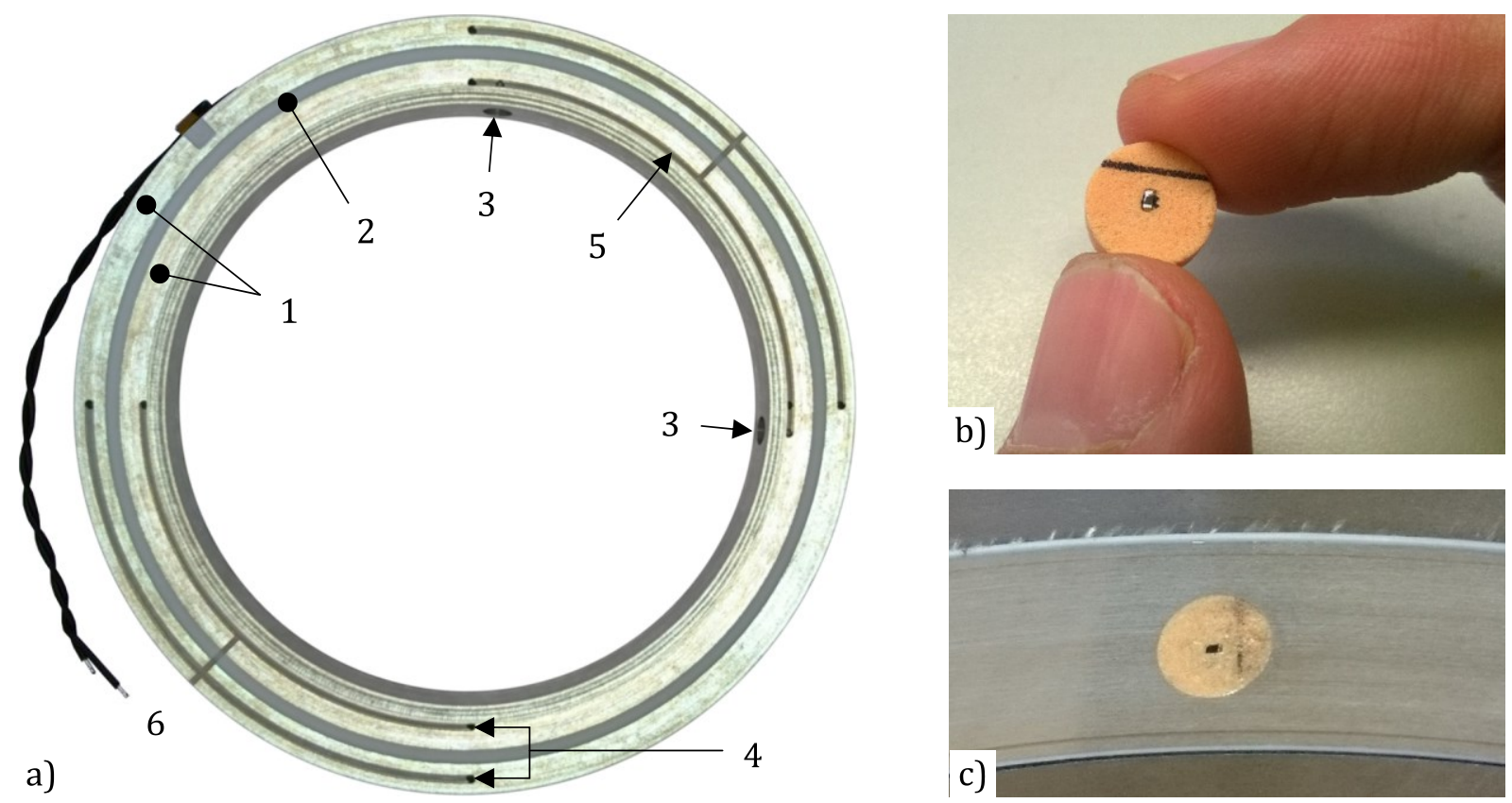

Abb. A.20 Instrumentierung eines einzelnen Messrings.

a) Vorderansicht, b) WÜK-Sensor im Vergleich und c) im eingebauten Zustand;

1 - Aluminium-Ring; 2 - PTFE-Ring; 3 - WÜK-Sensor; 4 - Materialtemperaturmessstelle;

5 - Umfangsnut; 6 - Anschluss für Heizwicklung. 

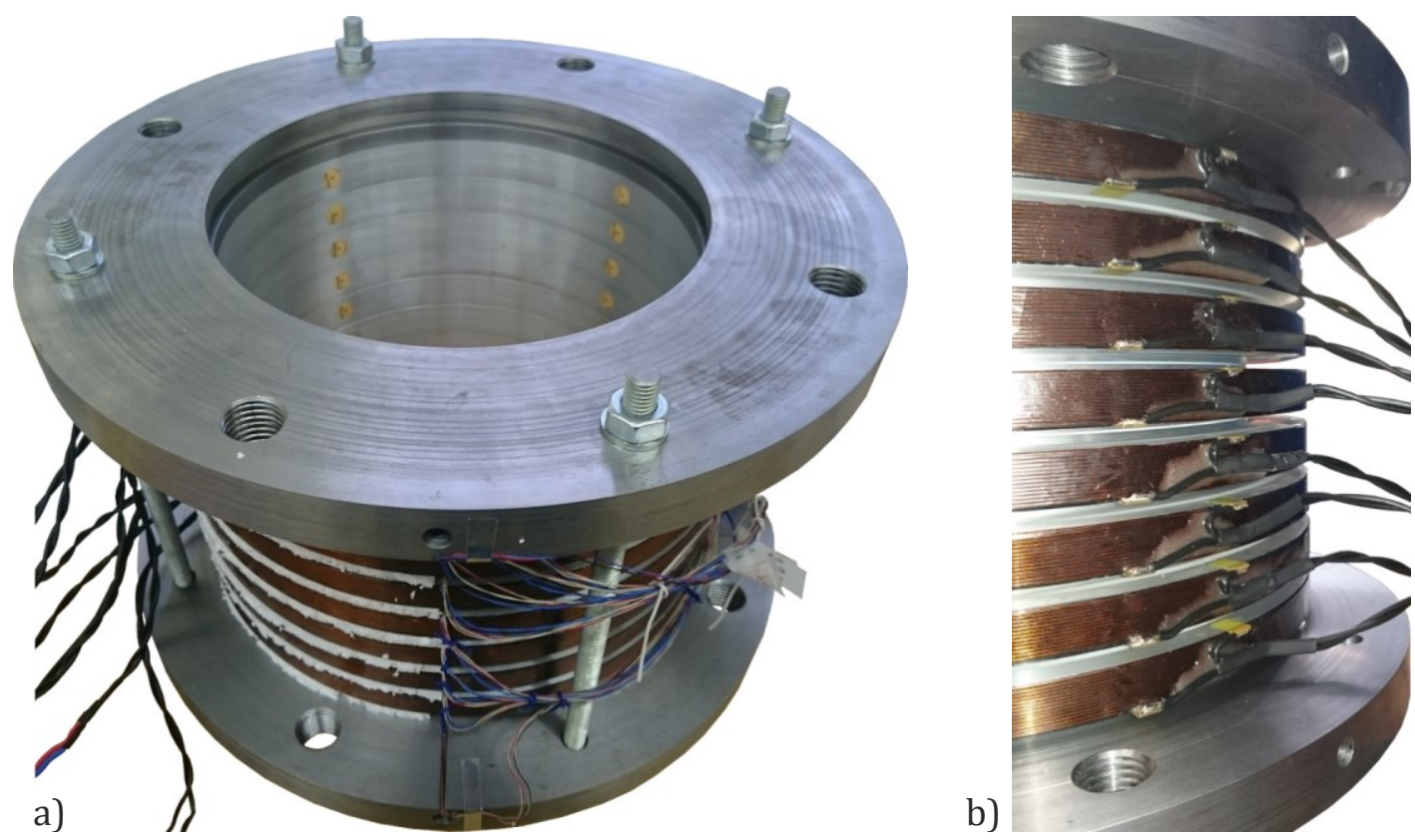

Abb. A.21 Vollinstrumentierte, montierte Außenwand in Scheibenbauweise.

a) teilisoliert mit Polystyrol-Halbschalen und Durchführung der Thermistordrähte;

b) Verlötung der Kabel für die Heizwicklungen.

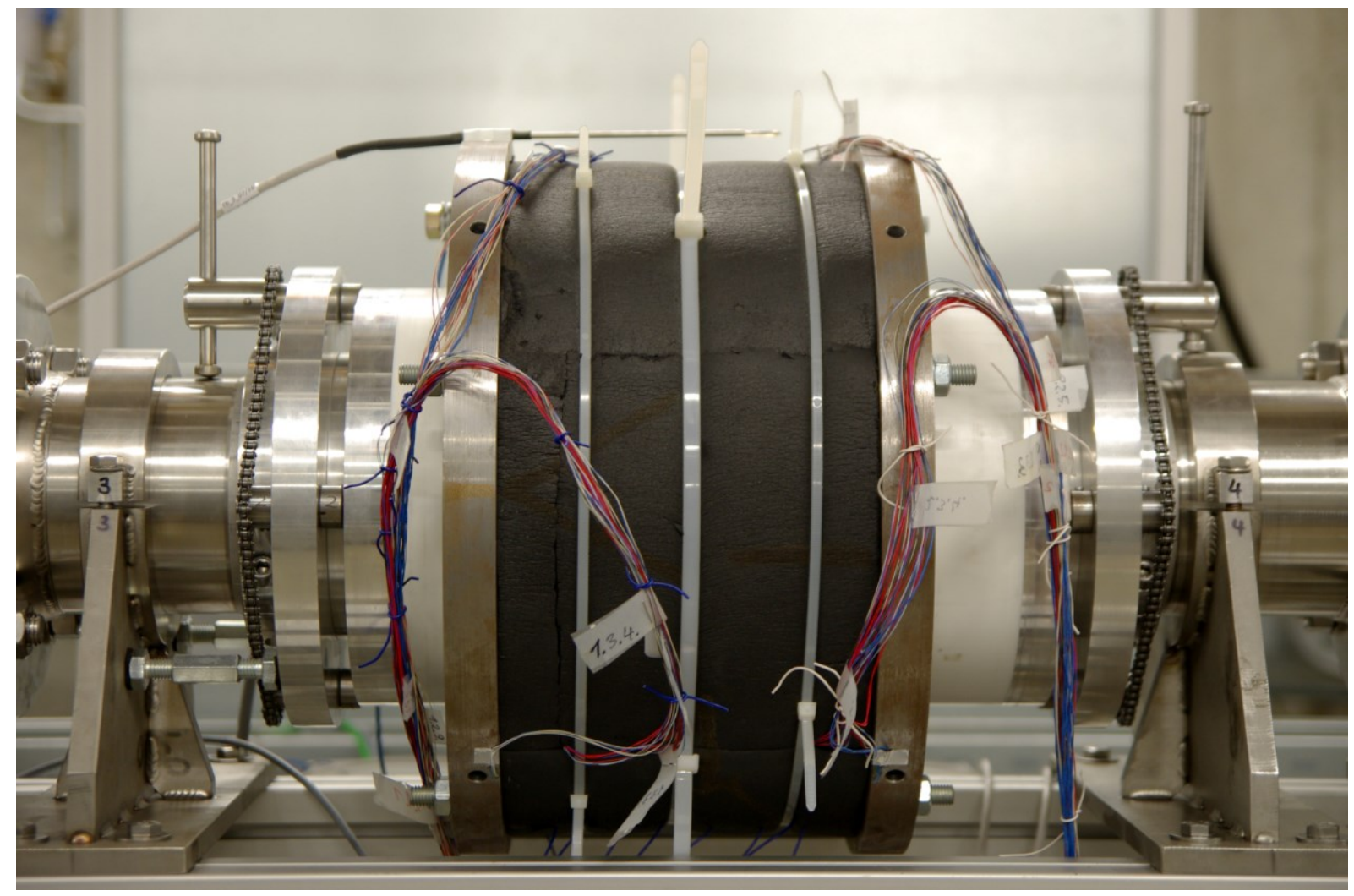

Abb. A.22 Totraummodul mit Seitenwandverstellmechanismus und Totraumaußenwand. 


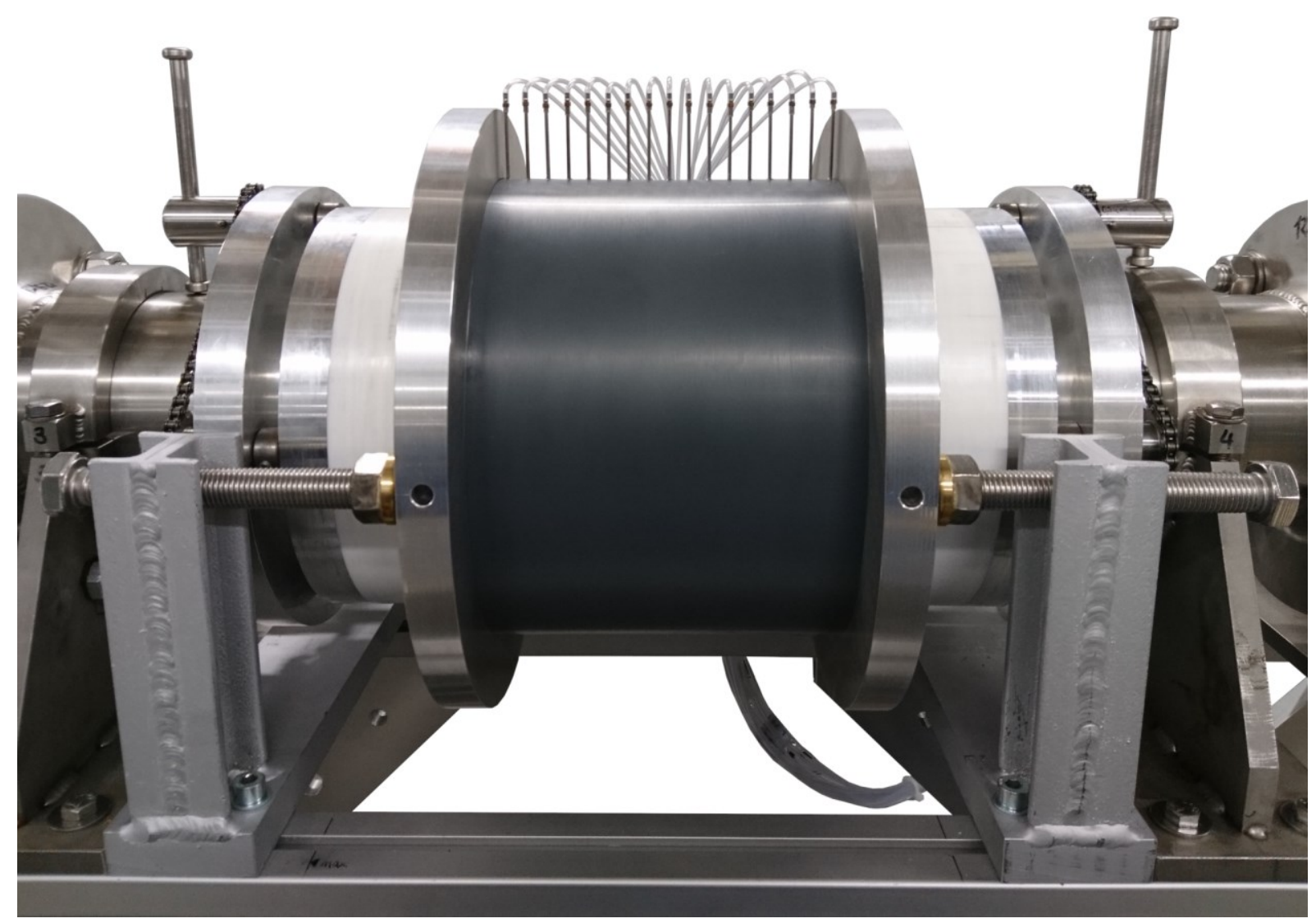

Abb. A.23 Alternative Totraumaußenwand zur Messung der Wanddruckverteilung an der äußeren Mantelfläche der Kavität. 


\section{A7 LISTE DER AUFGEZEICHNETEN MESSSTELLEN}

Tab. A.2 Übersicht über aufgezeichnete Messstellen an Versuchsanlage und Versuchsstand

\begin{tabular}{|c|c|c|c|c|}
\hline Bezeichnung & Beschreibung & Kal.-bereich & Messbereich & Gerät_Anschluss \\
\hline P1_Vordruck & Druck v. Druckminderer & 7 bar abs & $0,15-7$ bar abs & G14_1_1p16_0 \\
\hline P2_n_DRV & Druck n. Druckminderer & 5 bar abs & $0,15-7$ bar abs & G14_1_1p16_1 \\
\hline P3_n_SichV & Druck n. Sicherheitsventil & 5 bar abs & $0,15-7$ bar abs & G14_1_1p16_2 \\
\hline P4_V_TRV & Druck v. TR-Versuchsstand & 3 bar abs & $0,15-7$ bar abs & $\begin{array}{l}\text { G14_1_1p16_3; } \\
\text { G14_1_2p16_6L }\end{array}$ \\
\hline P5_V_MSV & Druck v. Motorstellventil & 3 bar abs & $0,15-7$ bar abs & G14_1_1p16_4 \\
\hline P6_n_MSV & Druck n. Motorstellventil & 3 bar abs & $0,15-7$ bar abs & G14_1_1p16_5 \\
\hline P7_ref_TRV & Referenzdruck Versuchsstand & 3 bar abs & $0,15-7$ bar abs & $\begin{array}{l}\text { G14_1_1p16_6; } \\
\text { G14_2_1p16_0L }\end{array}$ \\
\hline P8_n_TRV & Druck n. TR-Versuchsstand & 4 bar abs & $0,15-7$ bar abs & $\begin{array}{l}\text { G14_1_1p16_7; } \\
\text { G14_1_2p16_7L }\end{array}$ \\
\hline p_B1_vor & Druck v. Blende & 5 bar abs & $0,15-7$ bar abs & $\begin{array}{l}\text { G14_1_2p16_0; } \\
\text { G14_1_2p16_4H }\end{array}$ \\
\hline p_B2_vor & Druck v. Blende (Bypass) & 5 bar abs & $0,15-7$ bar abs & G14_1_2p16_1 \\
\hline p_Baro & Barometer (Atmosphäre) & 1 bar abs & $0,15-1,15$ bar abs & G14_1_2p16_2 \\
\hline p_Sonde_diff & Differenzdruck Sonde & $\pm 0,2$ bar diff & $\pm 0,25$ bar diff. & G14_1_2p16_3 \\
\hline p_B1_diff & Differenzdruck ü. Blende & 0,5 diff & 0,5 bar diff. & G14_1_2p16_4L \\
\hline p_B2_diff & Differenzdruck ü. Blende (Bypass) & 0,5 diff & 0,5 bar diff. & G14_1_2p16_5L \\
\hline P4_v_TRV_diff & Differenzdruck v. TR-Versuchsstand & $\pm 0,2$ bar diff & $\pm 0,25$ bar diff. & G14_1_2p16_6H \\
\hline P8_n_TRV_diff & Differenzdruck n. TR-Versuchsstand & $\pm 0,2$ bar diff & $\pm 0,25$ bar diff. & G14_1_2p16_7H \\
\hline $\mathrm{pM}$ & Diff.-druck 5-Loch-Sonde Mitte & $\pm 0,2$ bar diff & $\pm 0,25$ bar diff. & G14_2_1p16_0-4H \\
\hline pU & Diff.-druck 5-Loch-Sonde unten & $\pm 0,2$ bar diff & $\pm 0,25$ bar diff. & G14_2_1p16_1L \\
\hline pO & Diff.-druck 5-Loch-Sonde oben & $\pm 0,2$ bar diff & $\pm 0,25$ bar diff. & G14_2_1p16_2L \\
\hline $\mathrm{pR}$ & Diff.-druck 5-Loch-Sonde rechts & $\pm 0,2$ bar diff & $\pm 0,25$ bar diff. & G14_2_1p16_3L \\
\hline $\mathrm{pL}$ & Diff.-druck 5-Loch-Sonde links & $\pm 0,2$ bar diff & $\pm 0,25$ bar diff. & G14_2_1p16_4L \\
\hline TE_B1 & Temperatur vor Blende & $15-40^{\circ} \mathrm{C}$ & $0-1023,75^{\circ} \mathrm{C}$ & G14_1_SPI_00 \\
\hline TE_B2 & Temperatur vor Blende (Bypass) & $15-40^{\circ} \mathrm{C}$ & $0-1023,75^{\circ} \mathrm{C}$ & G14_1_SPI_01 \\
\hline $3 \mathrm{WV}$ & Hub Dreiwegeventil & $0-100 \%$ & $0-1 \mathrm{k} \Omega$ & G14_1_SPI_02 \\
\hline MSV & Hub Motorstellventil & $0-100 \%$ & $0-1 \mathrm{k} \Omega$ & G14_1_SPI_03 \\
\hline R1TG & Außenringtemperatur Ring 1, Reihe G (3 Uhr) & $20-80^{\circ} \mathrm{C}$ & $0-100^{\circ} \mathrm{C}$ & G14_1_AN0_0 \\
\hline R1TH & Außenringtemperatur Ring 1, Reihe H (12 Uhr) & $20-80^{\circ} \mathrm{C}$ & $0-100^{\circ} \mathrm{C}$ & G14_1_AN0_1 \\
\hline R2TG & Außenringtemperatur Ring 2, Reihe G (3 Uhr) & $20-80^{\circ} \mathrm{C}$ & $0-100^{\circ} \mathrm{C}$ & G14_1_AN0_2 \\
\hline R2TH & Außenringtemperatur Ring 2, Reihe H (12 Uhr) & $20-80^{\circ} \mathrm{C}$ & $0-100^{\circ} \mathrm{C}$ & G14_1_AN0_3 \\
\hline R3TG & Außenringtemperatur Ring 3, Reihe G (3 Uhr) & $20-80^{\circ} \mathrm{C}$ & $0-100^{\circ} \mathrm{C}$ & G14_1_AN0_4 \\
\hline R3TH & Außenringtemperatur Ring 3, Reihe H (12 Uhr) & $20-80^{\circ} \mathrm{C}$ & $0-100^{\circ} \mathrm{C}$ & G14_1_AN0_5 \\
\hline R4TG & Außenringtemperatur Ring 4, Reihe G (3 Uhr) & $20-80^{\circ} \mathrm{C}$ & $0-100^{\circ} \mathrm{C}$ & G14_1_AN0_6 \\
\hline R4TH & Außenringtemperatur Ring 4, Reihe H (12 Uhr) & $20-80^{\circ} \mathrm{C}$ & $0-100^{\circ} \mathrm{C}$ & G14_1_AN0_7 \\
\hline R1TE & Außenringtemperatur Ring 1, Reihe E (9 Uhr) & $20-80^{\circ} \mathrm{C}$ & $0-100^{\circ} \mathrm{C}$ & G14_1_AN1_0 \\
\hline R1TF & Außenringtemperatur Ring 1, Reihe F (6 Uhr) & $20-80^{\circ} \mathrm{C}$ & $0-100^{\circ} \mathrm{C}$ & G14_1_AN1_1 \\
\hline R2TE & Außenringtemperatur Ring 2, Reihe E (9 Uhr) & $20-80^{\circ} \mathrm{C}$ & $0-100^{\circ} \mathrm{C}$ & G14_1_AN1_2 \\
\hline R2TF & Außenringtemperatur Ring 2, Reihe F (6 Uhr) & $20-80^{\circ} \mathrm{C}$ & $0-100^{\circ} \mathrm{C}$ & G14_1_AN1_3 \\
\hline R3TE & Außenringtemperatur Ring 3, Reihe E (9 Uhr) & $20-80^{\circ} \mathrm{C}$ & $0-100^{\circ} \mathrm{C}$ & G14_1_AN1_4 \\
\hline R3TF & Außenringtemperatur Ring 3, Reihe F (6 Uhr) & $20-80^{\circ} \mathrm{C}$ & $0-100^{\circ} \mathrm{C}$ & G14_1_AN1_5 \\
\hline R4TE & Außenringtemperatur Ring 4, Reihe E (9 Uhr) & $20-80^{\circ} \mathrm{C}$ & $0-100^{\circ} \mathrm{C}$ & G14_1_AN1_6 \\
\hline R4TF & Außenringtemperatur Ring 4, Reihe F (6 Uhr) & $20-80^{\circ} \mathrm{C}$ & $0-100^{\circ} \mathrm{C}$ & G14_1_AN1_7 \\
\hline R1TC & Innenringtemperatur Ring 1, Reihe C (3 Uhr) & $20-80^{\circ} \mathrm{C}$ & $0-100^{\circ} \mathrm{C}$ & G14_1_AN2_0 \\
\hline R1TD & Innenringtemperatur Ring 1, Reihe D (12 Uhr) & $20-80^{\circ} \mathrm{C}$ & $0-100^{\circ} \mathrm{C}$ & G14_1_AN2_1 \\
\hline R2TC & Innenringtemperatur Ring 2, Reihe C (3 Uhr) & $20-80^{\circ} \mathrm{C}$ & $0-100^{\circ} \mathrm{C}$ & G14_1_AN2_2 \\
\hline R2TD & Innenringtemperatur Ring 2, Reihe D (12 Uhr) & $20-80^{\circ} \mathrm{C}$ & $0-100^{\circ} \mathrm{C}$ & G14_1_AN2_3 \\
\hline R3TC & Innenringtemperatur Ring 3, Reihe C (3 Uhr) & $20-80^{\circ} \mathrm{C}$ & $0-100^{\circ} \mathrm{C}$ & G14_1_AN2_4 \\
\hline
\end{tabular}




\begin{tabular}{|c|c|c|c|c|}
\hline Bezeichnung & Beschreibung & Kal.-bereich & Messbereich & Gerät_Anschluss \\
\hline R3TD & Innenringtemperatur Ring 3, Reihe D (12 Uhr) & $20-80^{\circ} \mathrm{C}$ & $0-100^{\circ} \mathrm{C}$ & G14_1_AN2_5 \\
\hline R4TC & Innenringtemperatur Ring 4, Reihe C (3 Uhr) & $20-80^{\circ} \mathrm{C}$ & $0-100^{\circ} \mathrm{C}$ & G14_1_AN2_6 \\
\hline R4TD & Innenringtemperatur Ring 4, Reihe D (12 Uhr) & $20-80^{\circ} \mathrm{C}$ & $0-100^{\circ} \mathrm{C}$ & G14_1_AN2_7 \\
\hline T_V_TRV & Temperatur vor Versuchsstand & $20-80^{\circ} \mathrm{C}$ & $0-100^{\circ} \mathrm{C}$ & G14_1_AN3_0 \\
\hline T_n_TRV & Temperatur nach Versuchsstand & $20-80^{\circ} \mathrm{C}$ & $0-100^{\circ} \mathrm{C}$ & G14_1_AN3_1 \\
\hline T_Umgeb & Umgebungstemperatur am Versuchsstand & $20-80^{\circ} \mathrm{C}$ & $0-100^{\circ} \mathrm{C}$ & G14_1_AN3_2 \\
\hline T_14_1 & Gerätetemperatur G14.1 & $20-80^{\circ} \mathrm{C}$ & $0-100^{\circ} \mathrm{C}$ & G14_1_AN3_3 \\
\hline F1TA & Flanschtemperatur Ring 1, Reihe A (9 Uhr) & $20-80^{\circ} \mathrm{C}$ & $0-100^{\circ} \mathrm{C}$ & G14_1_AN3_4 \\
\hline F1TB & Flanschtemperatur Ring 1, Reihe B (6 Uhr) & $20-80^{\circ} \mathrm{C}$ & $0-100^{\circ} \mathrm{C}$ & G14_1_AN3_5 \\
\hline F1TC & Flanschtemperatur Ring 1, Reihe C (3 Uhr) & $20-80^{\circ} \mathrm{C}$ & $0-100^{\circ} \mathrm{C}$ & G14_1_AN3_6 \\
\hline F1TD & Flanschtemperatur Ring 1, Reihe D (12 Uhr) & $20-80^{\circ} \mathrm{C}$ & $0-100^{\circ} \mathrm{C}$ & G14_1_AN3_7 \\
\hline R1TA & Innenringtemperatur Ring 1, Reihe A (9 Uhr) & $20-80^{\circ} \mathrm{C}$ & $0-100^{\circ} \mathrm{C}$ & G14_1_AN4_0 \\
\hline R1TB & Innenringtemperatur Ring 1, Reihe B (6 Uhr) & $20-80^{\circ} \mathrm{C}$ & $0-100^{\circ} \mathrm{C}$ & G14_1_AN4_1 \\
\hline R2TA & Innenringtemperatur Ring 2, Reihe A (9 Uhr) & $20-80^{\circ} \mathrm{C}$ & $0-100^{\circ} \mathrm{C}$ & G14_1_AN4_2 \\
\hline R2TB & Innenringtemperatur Ring 2, Reihe B (6 Uhr) & $20-80^{\circ} \mathrm{C}$ & $0-100^{\circ} \mathrm{C}$ & G14_1_AN4_3 \\
\hline R3TA & Innenringtemperatur Ring 3, Reihe A (9 Uhr) & $20-80^{\circ} \mathrm{C}$ & $0-100^{\circ} \mathrm{C}$ & G14_1_AN4_4 \\
\hline R3TB & Innenringtemperatur Ring 3, Reihe B (6 Uhr) & $20-80^{\circ} \mathrm{C}$ & $0-100^{\circ} \mathrm{C}$ & G14_1_AN4_5 \\
\hline R4TA & Innenringtemperatur Ring 4, Reihe A (9 Uhr) & $20-80^{\circ} \mathrm{C}$ & $0-100^{\circ} \mathrm{C}$ & G14_1_AN4_6 \\
\hline R4TB & Innenringtemperatur Ring 4, Reihe B (6 Uhr) & $20-80^{\circ} \mathrm{C}$ & $0-100^{\circ} \mathrm{C}$ & G14_1_AN4_7 \\
\hline R1WA & WÜK-Sensor Ring 1, Reihe A (9 Uhr) & $20-80^{\circ} \mathrm{C}$ & $0-100^{\circ} \mathrm{C}$ & G14_1_AN5_0 \\
\hline R1WB & WÜK-Sensor Ring 1, Reihe B (6 Uhr) & $20-80^{\circ} \mathrm{C}$ & $0-100^{\circ} \mathrm{C}$ & G14_1_AN5_1 \\
\hline R2WA & WÜK-Sensor Ring 2, Reihe A (9 Uhr) & $20-80^{\circ} \mathrm{C}$ & $0-100^{\circ} \mathrm{C}$ & G14_1_AN5_2 \\
\hline R2WB & WÜK-Sensor Ring 2, Reihe B (6 Uhr) & $20-80^{\circ} \mathrm{C}$ & $0-100^{\circ} \mathrm{C}$ & G14_1_AN5_3 \\
\hline R3WA & WÜK-Sensor Ring 3, Reihe A (9 Uhr) & $20-80^{\circ} \mathrm{C}$ & $0-100^{\circ} \mathrm{C}$ & G14_1_AN5_4 \\
\hline R3WB & WÜK-Sensor Ring 3, Reihe B (6 Uhr) & $20-80^{\circ} \mathrm{C}$ & $0-100^{\circ} \mathrm{C}$ & G14_1_AN5_5 \\
\hline R4WA & WÜK-Sensor Ring 4, Reihe A (9 Uhr) & $20-80^{\circ} \mathrm{C}$ & $0-100^{\circ} \mathrm{C}$ & G14_1_AN5_6 \\
\hline R4WB & WÜK-Sensor Ring 4, Reihe B (6 Uhr) & $20-80^{\circ} \mathrm{C}$ & $0-100^{\circ} \mathrm{C}$ & G14_1_AN5_7 \\
\hline R5TG & Außenringtemperatur Ring 5, Reihe G (3 Uhr) & $20-80^{\circ} \mathrm{C}$ & $0-100^{\circ} \mathrm{C}$ & G14_2_AN0_0 \\
\hline R5TH & Außenringtemperatur Ring 5, Reihe H (12 Uhr) & $20-80^{\circ} \mathrm{C}$ & $0-100^{\circ} \mathrm{C}$ & G14_2_AN0_1 \\
\hline R6TG & Außenringtemperatur Ring 6, Reihe G (3 Uhr) & $20-80^{\circ} \mathrm{C}$ & $0-100^{\circ} \mathrm{C}$ & G14_2_AN0_2 \\
\hline R6TH & Außenringtemperatur Ring 6, Reihe H (12 Uhr) & $20-80^{\circ} \mathrm{C}$ & $0-100^{\circ} \mathrm{C}$ & G14_2_AN0_3 \\
\hline R7TG & Außenringtemperatur Ring 7, Reihe G (3 Uhr) & $20-80^{\circ} \mathrm{C}$ & $0-100^{\circ} \mathrm{C}$ & G14_2_AN0_4 \\
\hline R7TH & Außenringtemperatur Ring 7, Reihe H (12 Uhr) & $20-80^{\circ} \mathrm{C}$ & $0-100^{\circ} \mathrm{C}$ & G14_2_AN0_5 \\
\hline R8TG & Außenringtemperatur Ring 8, Reihe G (3 Uhr) & $20-80^{\circ} \mathrm{C}$ & $0-100^{\circ} \mathrm{C}$ & G14_2_AN0_6 \\
\hline R8TH & Außenringtemperatur Ring 8, Reihe H (12 Uhr) & $20-80^{\circ} \mathrm{C}$ & $0-100^{\circ} \mathrm{C}$ & G14_2_AN0_7 \\
\hline R5TE & Außenringtemperatur Ring 5, Reihe E (9 Uhr) & $20-80^{\circ} \mathrm{C}$ & $0-100^{\circ} \mathrm{C}$ & G14_2_AN1_0 \\
\hline R5TF & Außenringtemperatur Ring 5, Reihe F (6 Uhr) & $20-80^{\circ} \mathrm{C}$ & $0-100^{\circ} \mathrm{C}$ & G14_2_AN1_1 \\
\hline R6TE & Außenringtemperatur Ring 6, Reihe E (9 Uhr) & $20-80^{\circ} \mathrm{C}$ & $0-100^{\circ} \mathrm{C}$ & G14_2_AN1_2 \\
\hline R6TF & Außenringtemperatur Ring 6, Reihe F (6 Uhr) & $20-80^{\circ} \mathrm{C}$ & $0-100^{\circ} \mathrm{C}$ & G14_2_AN1_3 \\
\hline R7TE & Außenringtemperatur Ring 7, Reihe E (9 Uhr) & $20-80^{\circ} \mathrm{C}$ & $0-100^{\circ} \mathrm{C}$ & G14_2_AN1_4 \\
\hline R7TF & Außenringtemperatur Ring 7, Reihe F (6 Uhr) & $20-80^{\circ} \mathrm{C}$ & $0-100^{\circ} \mathrm{C}$ & G14_2_AN1_5 \\
\hline R8TE & Außenringtemperatur Ring 8, Reihe E (9 Uhr) & $20-80^{\circ} \mathrm{C}$ & $0-100^{\circ} \mathrm{C}$ & G14_2_AN1_6 \\
\hline R8TF & Außenringtemperatur Ring 8, Reihe F (6 Uhr) & $20-80^{\circ} \mathrm{C}$ & $0-100^{\circ} \mathrm{C}$ & G14_2_AN1_7 \\
\hline R5TC & Innenringtemperatur Ring 5, Reihe C (3 Uhr) & $20-80^{\circ} \mathrm{C}$ & $0-100^{\circ} \mathrm{C}$ & G14_2_AN2_0 \\
\hline R5TD & Innenringtemperatur Ring 5, Reihe D (12 Uhr) & $20-80^{\circ} \mathrm{C}$ & $0-100^{\circ} \mathrm{C}$ & G14_2_AN2_1 \\
\hline R6TC & Innenringtemperatur Ring 6, Reihe C (3 Uhr) & $20-80^{\circ} \mathrm{C}$ & $0-100^{\circ} \mathrm{C}$ & G14_2_AN2_2 \\
\hline R6TD & Innenringtemperatur Ring 6, Reihe D (12 Uhr) & $20-80^{\circ} \mathrm{C}$ & $0-100^{\circ} \mathrm{C}$ & G14_2_AN2_3 \\
\hline R7TC & Innenringtemperatur Ring 7, Reihe C (3 Uhr) & $20-80^{\circ} \mathrm{C}$ & $0-100^{\circ} \mathrm{C}$ & G14_2_AN2_4 \\
\hline R7TD & Innenringtemperatur Ring 7, Reihe D (12 Uhr) & $20-80^{\circ} \mathrm{C}$ & $0-100^{\circ} \mathrm{C}$ & G14_2_AN2_5 \\
\hline R8TC & Innenringtemperatur Ring 8, Reihe C (3 Uhr) & $20-80^{\circ} \mathrm{C}$ & $0-100^{\circ} \mathrm{C}$ & G14_2_AN2_6 \\
\hline R8TD & Innenringtemperatur Ring 8, Reihe D (12 Uhr) & $20-80^{\circ} \mathrm{C}$ & $0-100^{\circ} \mathrm{C}$ & G14_2_AN2_7 \\
\hline- & - & $20-80^{\circ} \mathrm{C}$ & $0-100^{\circ} \mathrm{C}$ & G14_2_AN3_0 \\
\hline- & - & $20-80^{\circ} \mathrm{C}$ & $0-100^{\circ} \mathrm{C}$ & G14_2_AN3_1 \\
\hline
\end{tabular}




\begin{tabular}{|c|c|c|c|c|}
\hline Bezeichnung & Beschreibung & Kal.-bereich & Messbereich & Gerät_Anschluss \\
\hline- & - & $20-80^{\circ} \mathrm{C}$ & $0-100^{\circ} \mathrm{C}$ & G14_2_AN3_2 \\
\hline T_14_2 & Gerätetemperatur G14.2 & $20-80^{\circ} \mathrm{C}$ & $0-100^{\circ} \mathrm{C}$ & G14_2_AN3_3 \\
\hline F2TA & Flanschtemperatur Ring 2, Reihe A (9 Uhr) & $20-80^{\circ} \mathrm{C}$ & $0-100^{\circ} \mathrm{C}$ & G14_2_AN3_4 \\
\hline F2TB & Flanschtemperatur Ring 2, Reihe B (6 Uhr) & $20-80^{\circ} \mathrm{C}$ & $0-100^{\circ} \mathrm{C}$ & G14_2_AN3_5 \\
\hline F2TC & Flanschtemperatur Ring 2, Reihe C (3 Uhr) & $20-80^{\circ} \mathrm{C}$ & $0-100^{\circ} \mathrm{C}$ & G14_2_AN3_6 \\
\hline F2TD & Flanschtemperatur Ring 2, Reihe D (12 Uhr) & $20-80^{\circ} \mathrm{C}$ & $0-100^{\circ} \mathrm{C}$ & G14_2_AN3_7 \\
\hline R5TA & Innenringtemperatur Ring 5, Reihe A (9 Uhr) & $20-80^{\circ} \mathrm{C}$ & $0-100^{\circ} \mathrm{C}$ & G14_2_AN4_0 \\
\hline R5TB & Innenringtemperatur Ring 5, Reihe B (6 Uhr) & $20-80^{\circ} \mathrm{C}$ & $0-100^{\circ} \mathrm{C}$ & G14_2_AN4_1 \\
\hline R6TA & Innenringtemperatur Ring 6, Reihe A (9 Uhr) & $20-80^{\circ} \mathrm{C}$ & $0-100^{\circ} \mathrm{C}$ & G14_2_AN4_2 \\
\hline R6TB & Innenringtemperatur Ring 6, Reihe B (6 Uhr) & $20-80^{\circ} \mathrm{C}$ & $0-100^{\circ} \mathrm{C}$ & G14_2_AN4_3 \\
\hline R7TA & Innenringtemperatur Ring 7, Reihe A (9 Uhr) & $20-80^{\circ} \mathrm{C}$ & $0-100^{\circ} \mathrm{C}$ & G14_2_AN4_4 \\
\hline R7TB & Innenringtemperatur Ring 7, Reihe B (6 Uhr) & $20-80^{\circ} \mathrm{C}$ & $0-100^{\circ} \mathrm{C}$ & G14_2_AN4_5 \\
\hline R8TA & Innenringtemperatur Ring 8, Reihe A (9 Uhr) & $20-80^{\circ} \mathrm{C}$ & $0-100^{\circ} \mathrm{C}$ & G14_2_AN4_6 \\
\hline R8TB & Innenringtemperatur Ring 8, Reihe B (6 Uhr) & $20-80^{\circ} \mathrm{C}$ & $0-100^{\circ} \mathrm{C}$ & G14_2_AN4_7 \\
\hline R5WA & WÜK-Sensor Ring 5, Reihe A (9 Uhr) & $20-80^{\circ} \mathrm{C}$ & $0-100^{\circ} \mathrm{C}$ & G14_2_AN5_0 \\
\hline R5WB & WÜK-Sensor Ring 5, Reihe B (6 Uhr) & $20-80^{\circ} \mathrm{C}$ & $0-100^{\circ} \mathrm{C}$ & G14_2_AN5_1 \\
\hline R6WA & WÜK-Sensor Ring 6, Reihe A (9 Uhr) & $20-80^{\circ} \mathrm{C}$ & $0-100^{\circ} \mathrm{C}$ & G14_2_AN5_2 \\
\hline R6WB & WÜK-Sensor Ring 6, Reihe B (6 Uhr) & $20-80^{\circ} \mathrm{C}$ & $0-100^{\circ} \mathrm{C}$ & G14_2_AN5_3 \\
\hline R7WA & WÜK-Sensor Ring 7, Reihe A (9 Uhr) & $20-80^{\circ} \mathrm{C}$ & $0-100^{\circ} \mathrm{C}$ & G14_2_AN5_4 \\
\hline R7WB & WÜK-Sensor Ring 7, Reihe B (6 Uhr) & $20-80^{\circ} \mathrm{C}$ & $0-100^{\circ} \mathrm{C}$ & G14_2_AN5_5 \\
\hline R8WA & WÜK-Sensor Ring 8, Reihe A (9 Uhr) & $20-80^{\circ} \mathrm{C}$ & $0-100^{\circ} \mathrm{C}$ & G14_2_AN5_6 \\
\hline R8WB & WÜK-Sensor Ring 8, Reihe B (6 Uhr) & $20-80^{\circ} \mathrm{C}$ & $0-100^{\circ} \mathrm{C}$ & G14_2_AN5_7 \\
\hline p_AW_01_diff & Differenzdruck Außenwand Pos. 1 & $+0,07$ bar & $+0,1$ bar diff. & G16_1_PC0_0_L \\
\hline p_AW_02_diff & Differenzdruck Außenwand Pos. 2 & $+0,07$ bar & $+0,1$ bar diff. & G16_1_PC0_1_L \\
\hline p_AW_03_diff & Differenzdruck Außenwand Pos. 3 & $+0,07$ bar & $+0,1$ bar diff. & G16_1_PC0_2_L \\
\hline p_AW_04_diff & Differenzdruck Außenwand Pos. 4 & $+0,07$ bar & $+0,1$ bar diff. & G16_1_PC0_3_L \\
\hline p_AW_05_diff & Differenzdruck Außenwand Pos. 5 & $+0,07$ bar & $+0,1$ bar diff. & G16_1_PC0_4_L \\
\hline p_AW_06_diff & Differenzdruck Außenwand Pos. 6 & $+0,07$ bar & $+0,1$ bar diff. & G16_1_PC0_5_L \\
\hline p_AW_07_diff & Differenzdruck Außenwand Pos. 7 & $+0,07$ bar & $+0,1$ bar diff. & G16_1_PC0_6_L \\
\hline p_AW_08_diff & Differenzdruck Außenwand Pos. 8 & $+0,07$ bar & $+0,1$ bar diff. & G16_1_PC0_7_L \\
\hline p_AW_09_diff & Differenzdruck Außenwand Pos. 9 & $+0,07$ bar & $+0,1$ bar diff. & G16_1_PC1_0_L \\
\hline p_AW_10_diff & Differenzdruck Außenwand Pos. 10 & $+0,07$ bar & $+0,1$ bar diff. & G16_1_PC1_1_L \\
\hline p_AW_11_diff & Differenzdruck Außenwand Pos. 11 & $+0,07$ bar & $+0,1$ bar diff. & G16_1_PC1_2_L \\
\hline p_AW_12_diff & Differenzdruck Außenwand Pos. 12 & $+0,07$ bar & $+0,1$ bar diff. & G16_1_PC1_3_L \\
\hline p_AW_13_diff & Differenzdruck Außenwand Pos. 13 & $+0,07$ bar & $+0,1$ bar diff. & G16_1_PC1_4_L \\
\hline p_AW_14_diff & Differenzdruck Außenwand Pos. 14 & $+0,07$ bar & $+0,1$ bar diff. & G16_1_PC1_5_L \\
\hline p_AW_15_diff & Differenzdruck Außenwand Pos. 15 & $+0,07$ bar & $+0,1$ bar diff. & G16_1_PC1_6_L \\
\hline p_AW_16_diff & Differenzdruck Außenwand Pos. 16 & $+0,07$ bar & $+0,1$ bar diff. & G16_1_PC1_7_L \\
\hline p_AW_17_diff & Differenzdruck Außenwand Pos. 17 & $+0,07$ bar & $+0,1$ bar diff. & G16_1_PC2_0_L \\
\hline
\end{tabular}


Tab. A.3 Armaturen und Messstellen an der Versuchsanlage und deren Bezeichnung gemäß Kraftwerkskennzeichnungssystem RDS-PP

\begin{tabular}{llll}
\hline Bezeichnung 1 & Bezeichnung 2 & Bezeichnung 3 & RDS-PP \\
\hline Motorkugelhahn DN 100 & Druckluft & Regelstrecke TRV & $=$ K71 QEB22 QM001 \\
Druckminderer 3 bar & Druckluft & Regelstrecke TRV & $=$ K71 QEB22 QN001 \\
Kugelhahn DN 100 & Druckluft & Regelstrecke TRV & $=$ K71 QEB22 QM002 \\
Sicherheitsventil 3,5 bar & Druckluft & Regelstrecke TRV & $=$ K71 QEB22 FL001 \\
Druck v. Druckminderer & Druckluft & Regelstrecke TRV & $=$ K71 QEB22 BP001 \\
Druck n. Druckminderer & Druckluft & Regelstrecke TRV & $=$ K71 QEB22 BP002 \\
Druck n. Sicherheitsventil & Druckluft & Regelstrecke TRV & $=$ K71 QEB22 BP003 \\
Dreiwegestellventil DN 100 Bypass/ Hauptstr. & Druckluft & Regelstrecke TRV & $=$ K71 QEB22 QM003 \\
Strömungsumformer & Druckluft & Hauptstrang TRV & $=$ K71 QEB31 WP001 \\
Messblende & Druckluft & Hauptstrang TRV & $=$ K71 QEB31 RN001 \\
Strömungsumformer & Druckluft & Hauptstrang TRV & $=$ K71 QEB31 BU001 \\
Totraumversuchsstand SiSTeR & Druckluft & Hauptstrang TRV & $=$ K71 QEB31 WP002 \\
Kugelhahn DN 100 Absp. Hauptstrang & Druckluft & Hauptstrang TRV & $=$ K71 QEB31 QM001 \\
Temperatur v. Blende & Druckluft & Hauptstrang TRV & $=$ K71 QEB31 BT001 \\
Druck v. Blende & Druckluft & Hauptstrang TRV & $=$ K71 QEB31 BP001 \\
Differenzdruck ü. Blende & Druckluft & Hauptstrang TRV & $=$ K71 QEB31 BP002 \\
Kugelhahn DN 100 Absp. Bypass & Druckluft & Bypass TRV & $=$ K71 QEB32 QM001 \\
Motorstellventil DN 100 Druckniveau TRV & Druckluft & Ausblasung TRV & $=$ K71 QEB40 QN001 \\
Druck v. Motorstellventil & Druckluft & Ausblasung TRV & $=$ K71 QEB40 BP001 \\
Druck n. Motorstellventil & Druckluft & Ausblasung TRV & $=$ K71 QEB40 BP002 \\
Ausblaseschalldämpfer & Druckluft & Ausblasung TRV & $=$ K71 QEB40 RP001 \\
\hline
\end{tabular}

Tab. A.4 Sonstige Armaturen und Messstellen an der Druckluftversorgungsanlage und deren Bezeichnung gemäß Kraftwerkskennzeichnungssystem RDS-PP

\begin{tabular}{llll}
\hline Bezeichnung 1 & Bezeichnung 2 & Bezeichnung 3 & RDS-PP \\
\hline Absperrklappe DN 80 & Kühlwasser & RK2 Bereich T VL =T11/ =T33 =W30 PCB22 QM011 \\
Absperrklappe DN 80 & Kühlwasser & RK2 Bereich T RL =T11/ =T33 =W30 PCB22 QM021 \\
Absperrklappe DN 80 & Kühlwasser & RK2 Bereich T VL =T33 & $=$ W30 PCB22 QM016 \\
Absperrklappe DN 80 & Kühlwasser & RK2 Bereich T RL =T33 & $=$ W30 PCB22 QM026 \\
Kugelhahn DN 15 Entlüftung & Kühlwasser & Wasserkühlung Verdichter VL =W30 PCB22 QM017 \\
Kugelhahn DN 15 Entlüftung & Kühlwasser & Wasserkühlung Verdichter RL =W30 PCB22 QM027 \\
Temperatur Kühlwasser VL & Kühlwasser & Wasserkühlung Verdichter VL =W30 PCB22 BT101 \\
Druckluftnachkühler 44 kW & Druckluft & Sammelleitung Verdichter & $=$ T11 QEB20 EP001 \\
Temperatur v. DL-Nachkühler & Druckluft & Sammelleitung Verdichter & $=$ T11 QEB20 BT001 \\
Temperatur n. DL-Nachkühler & Druckluft & Sammelleitung Verdichter & $=$ T11 QEB20 BT002 \\
Strömungswächter & Druckluft & Sammelleitung Verdichter & $=$ T11 QEB20 BF001 \\
Absperrklappe DN 50 & Kühlwasser & Druckluftnachkühler VL & $=$ W30 PCB22 QM111 \\
Absperrklappe DN 50 & Kühlwasser & Druckluftnachkühler RL & $=$ W30 PCB22 QM115 \\
Regulierventil DN 40 & Kühlwasser & Druckluftnachkühler VL & $=$ W30 PCB22 QN111 \\
Temperatur Kühlwasser VL & Kühlwasser & Druckluftnachkühler VL & $=$ W30 PCB22 BT111 \\
Temperatur Kühlwasser RL & Kühlwasser & Druckluftnachkühler RL & $=$ W30 PCB22 BT114 \\
Temperatur Kühlwasser RL & Kühlwasser & Druckluftnachkühler RL & $=$ W30 PCB22 BT115 \\
Druck Kühlwasser VL & Kühlwasser & Druckluftnachkühler VL & $=$ W30 PCB22 BP111 \\
Druck Kühlwasser RL & Kühlwasser & Druckluftnachkühler RL & $=$ W30 PCB22 BP115 \\
Kugelhahn DN 15 Entleerung & Kühlwasser & Druckluftnachkühler VL & $=$ W30 PCB22 QM112
\end{tabular}




\begin{tabular}{|c|c|c|c|}
\hline Bezeichnung 1 & Bezeichnung 2 & Bezeichnung 3 & RDS-PP \\
\hline Kugelhahn DN 15 Entlüftung & Kühlwasser & Druckluftnachkühler RL & =W30 PCB22 QM116 \\
\hline Ölkühler ER-S4 135 kW & Verd.-ölkreisl. & Atlas Copco GA 180 VSD FF & $=\mathrm{T} 11 \mathrm{QES} 10 \mathrm{EP} 001$ \\
\hline Motorabsperrklappe DN 50 & Kühlwasser & Ölkühler ER-S4 VL & =W30 PCB22 QM121 \\
\hline Regulierventil DN 32 & Kühlwasser & Ölkühler ER-S4 RL & $=\mathrm{W} 30$ PCB22 QN125 \\
\hline Sicherheitsventil 10 bar & Kühlwasser & Ölkühler ER-S4 VL & =W30 PCB22 FL121 \\
\hline Temperatur Kühlwasser VL & Kühlwasser & Ölkühler ER-S4 VL & =W30 PCB22 BT121 \\
\hline Temperatur Kühlwasser RL & Kühlwasser & Ölkühler ER-S4 RL & =W30 PCB22 BT125 \\
\hline Druck Kühlwasser VL & Kühlwasser & Ölkühler ER-S4 VL & =W30 PCB22 BP121 \\
\hline Druck Kühlwasser RL & Kühlwasser & Ölkühler ER-S4 RL & =W30 PCB22 BP125 \\
\hline Membran-Ausdehnungsgefäß 2,2 bar & Kühlwasser & Ölkühler ER-S4 VL & =W30 PCB22 CM121 \\
\hline Kugelhahn DN 15 Entleerung & Kühlwasser & Ölkühler ER-S4 VL & =W30 PCB22 QM122 \\
\hline Ölkühler ER-S5 150 kW & Verd.-ölkreisl. & Atlas Copco GA 200-7,5 FF & $=\mathrm{T} 11 \mathrm{QES} 11 \mathrm{EP} 001$ \\
\hline Motorabsperrklappe DN 50 & Kühlwasser & Ölkühler ER-S5 VL & =W30 PCB22 QM131 \\
\hline Regulierventil DN 32 & Kühlwasser & Ölkühler ER-S5 RL & =W30 PCB22 QN135 \\
\hline Sicherheitsventil 10 bar & Kühlwasser & Ölkühler ER-S5 VL & =W30 PCB22 FL131 \\
\hline Temperatur Kühlwasser VL & Kühlwasser & Ölkühler ER-S5 VL & =W30 PCB22 BT131 \\
\hline Temperatur Kühlwasser RL & Kühlwasser & Ölkühler ER-S5 RL & =W30 PCB22 BT135 \\
\hline Druck Kühlwasser VL & Kühlwasser & Ölkühler ER-S5 VL & =W30 PCB22 BP131 \\
\hline Druck Kühlwasser RL & Kühlwasser & Ölkühler ER-S5 RL & =W30 PCB22 BP135 \\
\hline Membran-Ausdehnungsgefäß 2,2 bar & Kühlwasser & Ölkühler ER-S5 VL & $=\mathrm{W} 30$ PCB22 CM131 \\
\hline Kugelhahn DN 15 Entleerung & Kühlwasser & Ölkühler ER-S5 VL & =W30 PCB22 QM132 \\
\hline Schraubenverdichter GA 180 VSD FF & Druckluft & Zentr. Versorgung & =T11 QEA10 GQ001 \\
\hline Schraubenverdichter GA 200-7,5 FF & Druckluft & Zentr. Versorgung & =T11 QEA11 GQ001 \\
\hline Klappe DN 80 & Druckluft & Druckleitung GA 180 & =T11 QEB10 QM001 \\
\hline Klappe DN 100 & Druckluft & Druckleitung GA 200 & =T11 QEB11 QM001 \\
\hline Druckluftspeicher & Druckluft & Sammelleitung Verdichter & =T11 QEB20 CM001 \\
\hline Druck Druckluftspeicher & Druckluft & Sammelleitung Verdichter & =T11 QEB20 BP001 \\
\hline Klappe DN 100 & Druckluft & Speicher VL & =T11 QEB20 QM001 \\
\hline Klappe DN 100 & Druckluft & Speicher RL & =T11 QEB20 QM002 \\
\hline Filter m. Abscheider & Druckluft & Sammelleitung Verdichter & =T11 QEB20 HN001 \\
\hline Motorkugelhahn DN 100 & Druckluft & Zuleitung DF-Messstrecke & =T31 QEB21 QM001 \\
\hline
\end{tabular}


Tab. A.5 Nicht aufgezeichnete, versuchsrelevante Betriebsmessstellen in der Versuchsanlage, deren Ort und Ablesung

\begin{tabular}{|c|c|c|}
\hline Messort & Betriebsmessstellen & Ablesung \\
\hline $\begin{array}{l}\text { Schraubenverdichter: } \\
\text { Atlas Copco GA 180 VSD FF } \\
\text { (ZET, Raum K106) }\end{array}$ & $\begin{array}{l}\text { Druck Kompressorauslass } \\
\text { Temperatur Kompressorauslass } \\
\text { Taupunkt Trockner } \\
\text { Drehzahl } \\
\text { Relativer Volumenstrom }\end{array}$ & $\begin{array}{l}\text { vor Ort am Betriebspanel } \\
\text { und Online-Zugriff der } \\
\text { Elektronikon } ® \text {-Steuerung }\end{array}$ \\
\hline $\begin{array}{l}\text { Schraubenverdichter: } \\
\text { Atlas Copco GA 200-7,5 FF } \\
\text { (ZET, Raum K106) }\end{array}$ & $\begin{array}{l}\text { Druck Kompressorauslass } \\
\text { Temperatur Kompressorauslass } \\
\text { Taupunkt Trockner }\end{array}$ & vor Ort am Betriebspanel \\
\hline $\begin{array}{l}\text { Filter mit Abscheider } \\
\text { Atlas Copco PD1200F }\end{array}$ & Differenzdruck Filter & örtliche Anzeige \\
\hline $\begin{array}{l}\text { Druckluftspeicher } \\
\text { Lohenner ZU } 47 \\
\text { (ZET, Raum K106) }\end{array}$ & Druck Speicher & örtliche Anzeige \\
\hline \multirow{3}{*}{$\begin{array}{l}\text { Druckluftnachkühler } \\
\text { Parker Hiross WFN } 090 \\
\text { (ZET, Raum K106) }\end{array}$} & $\begin{array}{l}\text { Temperatur DL-Eintritt } \\
\text { Temperatur DL-Austritt } \\
\text { Solltemperatur DL-Austritt } \\
\text { Ventilhub Regelventil KW-VL }\end{array}$ & PLS \\
\hline & Temperatur KW-RL & PLS und örtliche Anzeige \\
\hline & $\begin{array}{l}\text { Temperatur KW-VL } \\
\text { Druck KW-VL } \\
\text { Druck KW-RL }\end{array}$ & örtliche Anzeige \\
\hline $\begin{array}{l}\text { Ölkühler/ Wärmerückgewin- } \\
\text { nungsmodule } \\
\text { Atlas Copco ER-S4/ S5 } \\
\text { (ZET, Raum K106) }\end{array}$ & $\begin{array}{l}\text { Temperatur KW-VL } \\
\text { Temperatur KW-RL } \\
\text { Druck KW-VL } \\
\text { Druck KW-RL } \\
\end{array}$ & örtliche Anzeige \\
\hline $\begin{array}{l}\text { Raumlufttechnik } \\
\text { (ZET, Raum K107) }\end{array}$ & $\begin{array}{l}\text { Temperatur Zuluft } \\
\text { Istwert Raumtemperatur } \\
\text { Sollwert Raumtemperatur } \\
\text { Drehzahl Lüfter Klimagerät } \\
\text { Ventilhub HW-VL Klimagerät }\end{array}$ & PLS \\
\hline
\end{tabular}



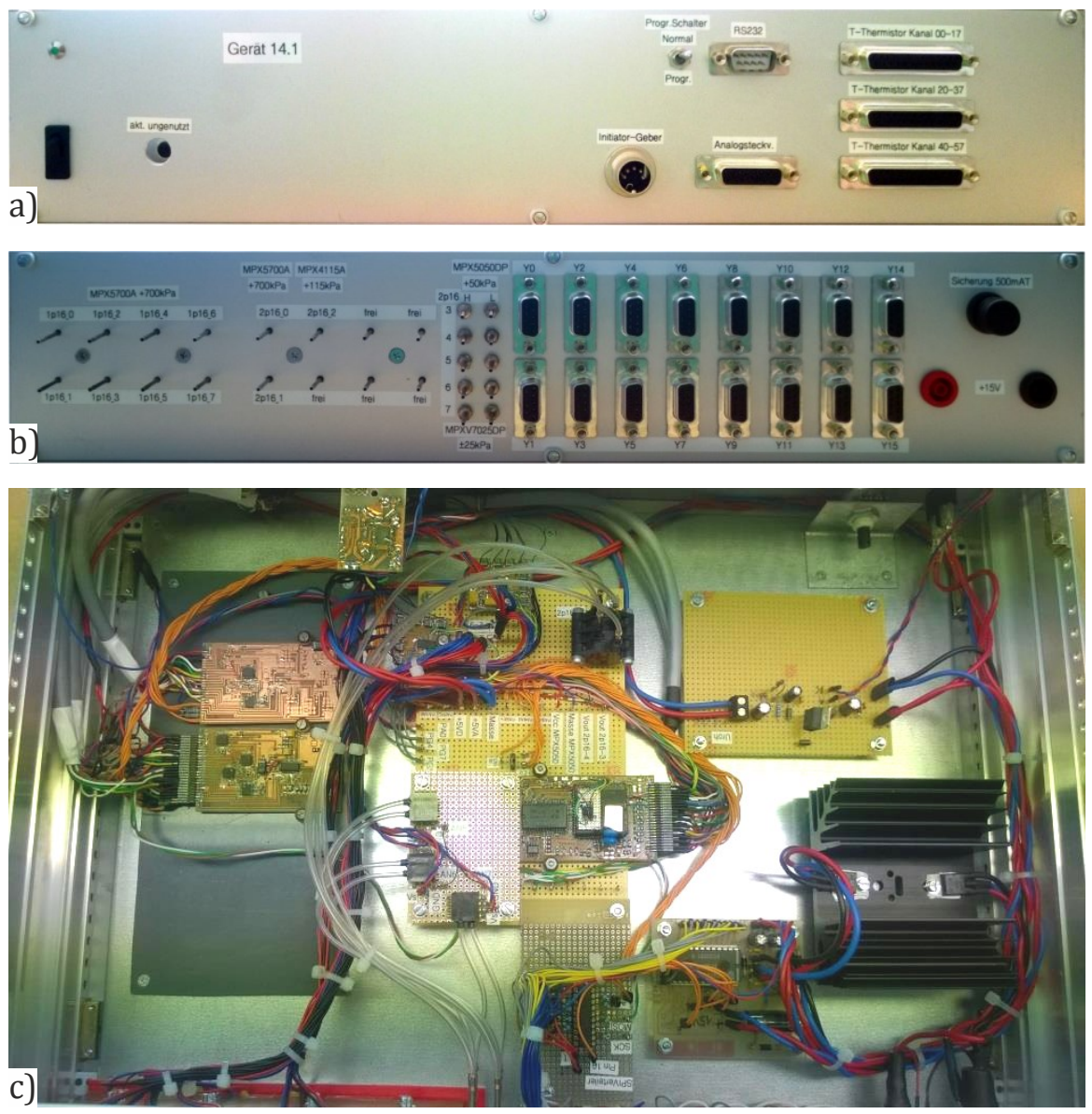

Abb. A.24 Messwerterfassungsgerät G14.1 (Fotos: Gunter Eschmann):

a) Vorder-; b) Rück-; c) Innenansicht. 


\begin{tabular}{|c|c|c|c|c|c|c|c|c|}
\hline \multicolumn{7}{|c|}{ ]0: MeBwerte } & \multicolumn{2}{|r|}{ - $\square x$} \\
\hline \multicolumn{7}{|c|}{ Datensatz $062 \mathrm{~A}, 5,63 \vee 5,03 \vee 25,45^{\circ} \mathrm{C}$} & \multicolumn{2}{|c|}{ Soeicherm $=0$ atrel } \\
\hline & Adresse 0 & Adresse 1 & Adresse 2 & Adresse 3 & Adresse 4 & Adresse 5 & Adresse 6 & Adresse 7 \\
\hline PE & 120 & 123 & 108 & 92 & 120 & 116 & 104 & 95 \\
\hline p16 & 11987 & 11907 & 12037 & 12129 & 11898 & 11871 & 11976 & 11902 \\
\hline AN & 0 & 0 & 0 & 0 & 0 & 0 & 28190 & 40365 \\
\hline ANO & 0 & 0 & 0 & 0 & 0 & 0 & 0 & 0 \\
\hline AN1 & 0 & 0 & 0 & 0 & 0 & 0 & 0 & 0 \\
\hline AN2 & 0 & 0 & 0 & 0 & 0 & 0 & 0 & 0 \\
\hline AN3 & 0 & 0 & 0 & 5798 & 0 & 0 & 0 & 0 \\
\hline AN4 & 0 & 0 & 0 & 0 & 0 & 0 & 0 & 0 \\
\hline AN5 & 0 & 0 & 0 & 0 & 0 & 0 & 0 & 0 \\
\hline $2 \mathrm{p} 16$ & 12084 & 12020 & 58078 & 2200 & 2464 & 36094 & 36151 & 36177 \\
\hline SPI & 828 & 0 & 3418 & 3664 & 0 & 0 & 0 & 0 \\
\hline SPI & 0 & 0 & 0 & 0 & 0 & 0 & 0 & 0 \\
\hline $\begin{array}{l}\text { TNT } \\
\text { a) }\end{array}$ & 11050 & 47934 & 65535 & 65535 & 65535 & 65535 & 20192 & 0 \\
\hline
\end{tabular}
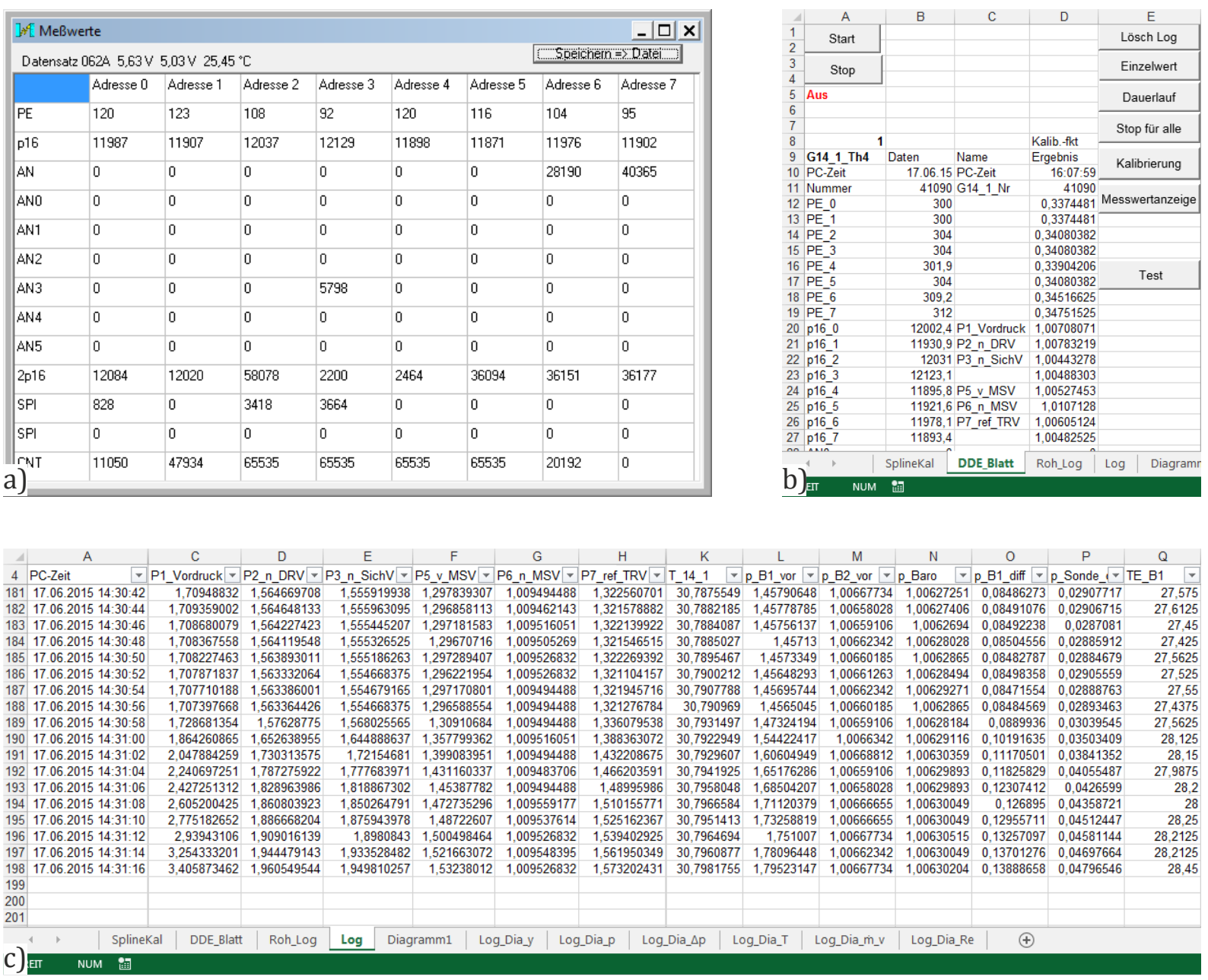

Abb. A.25 Datenerfassung und -verarbeitung - von den Rohdaten zum Messschrieb (Daten-Log):

a) Telemetrieprogramm mit den digitalen Werten (Digits) der einzelnen SPI-Kanäle;

b) Microsoft-Excel-Arbeitsblatt mit den eingetragenen Rohdaten und den mit den „Kalibrierfunktionen" umgerechneten physikalischen Messgrößen

c) Datentabelle mit den fortgeschriebenen, synchronisierten Messwerten („Messschrieb“) 


\section{A8 SUPERPOSITIONSPRINZIP FÜR DIE LÖSUNG DER FOURIER-GLEI- CHUNG}

Linearzerlegung des Temperaturfeldes als Lösung der Fourier-Gleichung:

$$
T(x, y)=T_{0}(x, y)+\sum_{j=1}^{n} c_{j} \theta_{j}(x, y)=T_{0}+c_{1} \theta_{1}+c_{2} \theta_{2}+\cdots
$$

Fourier-Gleichung (lineare PDGL): $\quad \nabla \cdot(\lambda \nabla T)=\tilde{q}$

$$
\begin{gathered}
\nabla \cdot\left(\lambda \nabla T_{0}\right)+c_{1} \nabla \cdot\left(\lambda \nabla \theta_{1}\right)+c_{2} \nabla \cdot\left(\lambda \nabla \theta_{2}\right)+\cdots=\tilde{q} \\
\nabla \cdot\left(\lambda \nabla T_{0}\right)=\tilde{q} \\
\epsilon_{1} \nabla \cdot\left(\lambda \nabla \theta_{1}\right)=0 \\
\epsilon_{2} \nabla \cdot\left(\lambda \nabla \theta_{2}\right)=0
\end{gathered}
$$

RB 1. Art (Dirichlet-RB):

$$
\begin{gathered}
\left.T(x)\right|_{\mathrm{W}}=T_{\mathrm{W}}(x), \quad x \in \Gamma \\
T_{0, \mathrm{~W}}+c_{1} \theta_{1, \mathrm{~W}}+c_{2} \theta_{2, \mathrm{~W}}+\cdots=T_{\mathrm{W}}(x) \\
T_{0}=T_{\mathrm{W}}(x) \\
\epsilon_{\mathrm{T}} \theta_{1, \mathrm{~W}}=0 \\
\epsilon_{2} \theta_{2, \mathrm{~W}}=0
\end{gathered}
$$

RB 2. Art (Neumann-RB): $\left.\quad \lambda(\nabla T \cdot \vec{n})\right|_{\mathrm{W}}=\dot{q}_{\mathrm{W}}(x), \quad x \in \Gamma$

$$
\begin{gathered}
\left.\lambda\left[\left(\nabla T_{0}+c_{1} \nabla \theta_{1}+c_{2} \nabla \theta_{2}+\cdots\right) \cdot \vec{n}\right]\right|_{\mathrm{W}}=\dot{q}_{\mathrm{W}}(x) \\
\left.\lambda\left(\nabla T_{0} \cdot \vec{n}\right)\right|_{\mathrm{W}}=\dot{q}_{\mathrm{W}}(x) \\
\left.\epsilon_{1} \lambda\left(\nabla \theta_{1} \cdot \vec{n}\right)\right|_{\mathrm{W}}=0 \\
\left.\epsilon_{2}\left(\nabla \theta_{2} \cdot \vec{n}\right)\right|_{\mathrm{W}}=0
\end{gathered}
$$

RB 3. Art (Robin-RB): $\quad \lambda \nabla T \cdot \vec{n}=-\alpha(x)\left(\left.T(x)\right|_{\mathrm{W}}-T_{\infty}\right), \quad x \in \Gamma$

$$
\begin{gathered}
\left.\lambda\left[\left(\nabla T_{0}+c_{1} \nabla \theta_{1}+c_{2} \nabla \theta_{2}+\cdots\right) \cdot \vec{n}\right]\right|_{\mathrm{W}}=-\alpha(x)\left(T_{0, \mathrm{~W}}+c_{1} \theta_{1, \mathrm{~W}}+c_{2} \theta_{2, \mathrm{~W}}+\cdots-T_{\infty}\right) \\
\left.\lambda\left(\nabla T_{0} \cdot \vec{n}\right)\right|_{\mathrm{W}}=-\alpha(x)\left(T_{0, \mathrm{~W}}-T_{\infty}\right) \\
\left.\epsilon_{\mathrm{T}} \lambda\left(\nabla \theta_{1} \cdot \vec{n}\right)\right|_{\mathrm{W}}=-\alpha(x) \epsilon_{\mathrm{T}} \theta_{1, \mathrm{~W}} \\
\left.\epsilon_{2}\left(\nabla \theta_{2} \cdot \vec{n}\right)\right|_{\mathrm{W}}=-\alpha(x) \epsilon_{2} \theta_{2, \mathrm{~W}}
\end{gathered}
$$

„Kunstgriff“ RB 2. Art:

$$
\begin{gathered}
\lambda \nabla T \cdot \vec{n}=c_{1} \dot{q}_{\mathbb{1}, 1}, \quad x \in \Gamma_{\mathrm{inv}, 1} \\
\lambda \nabla T_{0} \cdot \vec{n}=0 \\
\epsilon_{1} \lambda \nabla \theta_{1} \cdot \vec{n}=\epsilon_{1} \dot{q}_{\mathbb{1}, 1}=1 \\
\epsilon_{2} \lambda \nabla \theta_{2} \cdot \vec{n}=0
\end{gathered}
$$


Tab. A.6 Zu lösende Differenzialgleichungen und zugehörige Randbedingungen für alle Teilprobleme

\begin{tabular}{cccccc}
\hline Glied & \multicolumn{1}{c}{$T_{0}$} & $\theta_{1}$ & $\theta_{2}$ & $\cdots$ & $\theta_{n}$ \\
\hline PDGL & $\nabla \cdot\left(\lambda \nabla T_{0}\right)=\tilde{q}$ & $\nabla \cdot\left(\lambda \nabla \theta_{1}\right)=0$ & $\nabla \cdot\left(\lambda \nabla \theta_{2}\right)=0$ & $\cdots$ & $\nabla \cdot\left(\lambda \nabla \theta_{n}\right)=0$ \\
\hline$\Gamma_{\mathrm{i}}$ & $-\lambda \frac{\partial T_{0}}{\partial n}=\alpha_{\mathrm{i}}\left(T-T_{\mathrm{F}}\right)$ & $-\lambda \frac{\partial \theta_{1}}{\partial n}=\alpha_{\mathrm{i}} \theta_{1}$ & $-\lambda \frac{\partial \theta_{2}}{\partial n}=\alpha_{\mathrm{i}} \theta_{2}$ & $\cdots$ & $-\lambda \frac{\partial \theta_{n}}{\partial n}=\alpha_{\mathrm{i}} \theta_{n}$ \\
$\Gamma_{\mathrm{v}}$ & $-\lambda \frac{\partial T_{0}}{\partial n}=\alpha_{\mathrm{v}}\left(T-T_{\mathrm{U}}\right)$ & $-\lambda \frac{\partial \theta_{1}}{\partial n}=\alpha_{\mathrm{v}} \theta_{1}$ & $-\lambda \frac{\partial \theta_{2}}{\partial n}=\alpha_{\mathrm{v}} \theta_{2}$ & $\cdots$ & $-\lambda \frac{\partial \theta_{n}}{\partial n}=\alpha_{\mathrm{v}} \theta_{n}$ \\
$\Gamma_{\mathrm{h}}$ & $-\lambda \frac{\partial T_{0}}{\partial n}=\alpha_{\mathrm{h}}\left(T-T_{\mathrm{U}}\right)$ & $-\lambda \frac{\partial \theta_{1}}{\partial n}=\alpha_{\mathrm{h}} \theta_{1}$ & $-\lambda \frac{\partial \theta_{2}}{\partial n}=\alpha_{\mathrm{h}} \theta_{2}$ & $\cdots$ & $-\lambda \frac{\partial \theta_{n}}{\partial n}=\alpha_{\mathrm{h}} \theta_{n}$ \\
$\Gamma_{\text {inv }, 1}$ & $-\lambda \frac{\partial T_{0}}{\partial n}=0$ & $-\lambda \frac{\partial \theta_{1}}{\partial n}=1$ & $-\lambda \frac{\partial \theta_{2}}{\partial n}=0$ & $\cdots$ & $-\lambda \frac{\partial \theta_{n}}{\partial n}=0$ \\
$\Gamma_{\text {inv }, 2}$ & $-\lambda \frac{\partial T_{0}}{\partial n}=0$ & $-\lambda \frac{\partial \theta_{1}}{\partial n}=0$ & $-\lambda \frac{\partial \theta_{2}}{\partial n}=1$ & $\cdots$ & $-\lambda \frac{\partial \theta_{n}}{\partial n}=0$ \\
$\vdots$ & $\vdots$ & $\vdots$ & $\vdots$ & $\ddots$ & $\vdots$ \\
$\Gamma_{\text {inv }, n}$ & $-\lambda \frac{\partial T_{0}}{\partial n}=0$ & $-\lambda \frac{\partial \theta_{1}}{\partial n}=0$ & $-\lambda \frac{\partial \theta_{2}}{\partial n}=0$ & $\cdots$ & $-\lambda \frac{\partial \theta_{n}}{\partial n}=1$ \\
\hline
\end{tabular}


A9 LÖSUNGSSCHEMA FÜR DIE INVERSE BERECHNUNG VON WÜK MITTELS TREFFTZ-FUNKTIONEN

$$
\begin{gathered}
\sum_{k=1}^{m}\left(\theta_{j, k} \sum_{j=1}^{n} c_{j} \theta_{j, k}\right)+\beta c_{j}=-\sum_{k=1}^{m}\left(T_{0, k}-T_{k}^{*}\right) \theta_{j, k} \\
\sum_{j=1}^{n}\left\{\left[\sum_{k=1}^{m}\left(\theta_{i, k} \theta_{j, k}\right)+\delta_{i, j} \beta\right] c_{j}\right\}=-\sum_{k=1}^{m}\left(T_{0, k}-T_{k}^{*}\right) \theta_{j, k} \\
\mathbf{A} \vec{c}=\vec{b} \\
\mathbf{A}=\left(a_{i, j}\right) \\
a_{i, j}=\sum_{k=1}^{m} \theta_{i, k} \theta_{j, k}+\delta_{i, j} \beta
\end{gathered}
$$

mit dem Kronecker-Symbol $\delta_{i, j}$ :

$$
\delta_{i, j}=\left\{\begin{array}{lll}
1 & \forall & i=j \\
0 & \forall & i \neq j
\end{array}\right.
$$

$\beta$ wird nur zur Matrixdiagonale hinzuaddiert (daher „regularisiert“).

$$
\begin{gathered}
\vec{c}=\left(c_{i}\right) \\
\vec{b}=\left(b_{i}\right) \\
b_{i}=-\sum_{k=1}^{m}\left(T_{0, k}-T_{k}^{*}\right) \theta_{i, k}
\end{gathered}
$$

Für den Lösungsvektor $\vec{c}$ folgt dann:

$$
\vec{c}=\mathbf{A}^{-1} \vec{b}
$$




\section{A10 EINSTELLUNGEN FÜR DEN EVOLUTIONÄREN ALGORITHMUS}

Tab. A.7 Einstellungen für den evolutionären Algorithmus in Dakota

\begin{tabular}{ll}
\hline Parameter & Wert \\
\hline method & coliny_ea \\
seed & 11011011 \\
population_size & 50 \\
fitness_type & merit_function \\
mutation_type & offset_normal \\
mutation_rate & 1.0 (bis 10.000 Iterationen); 0.5 (danach) \\
crossover_type & two_point \\
crossover_rate & 0.0 \\
replacement_type & chc \\
replacement_size & 10 \\
max_iterations & 20000 \\
max_function_evaluations & 20000 \\
\hline
\end{tabular}




\section{A11 LÖSUNGSSCHEMA ZUR BERECHNUNG DER KALIBRIERKOEFFI- ZIENTEN FÜR DIE FÜNFLOCHSONDEN}

$$
\begin{gathered}
\nabla^{2}=\sum_{m=0}^{N_{m}}\left\{\left[\sum_{i=0}^{N_{i}-1} \sum_{k=0}^{N_{k}-1}\left(A_{i, k} x_{m}^{i} y_{m}^{k}\right)-\alpha_{\text {Soll }, m}\right]^{2}\right\} \rightarrow \min \\
\frac{\partial \nabla^{2}}{\partial A_{i, k}}=2 \sum_{m=0}^{N_{m}}\left\{x_{m}^{i} y_{m}^{k}\left[\sum_{i=0}^{N_{i}-1} \sum_{k=0}^{N_{k}-1}\left(A_{i, k} x_{m}^{i} y_{m}^{k}\right)-\alpha_{\text {Soll }, m}\right]\right\}=0 \forall i, k \\
\underbrace{\sum_{m=0}^{N_{m}}\left\{x_{m}^{i} y_{m}^{k} \sum_{i=0}^{N_{i}-1} \sum_{k=0}^{N_{k}-1}\left(A_{i, k} x_{m}^{i} y_{m}^{k}\right)\right\}}_{(*)}=\sum_{m=0}^{N_{m}}\left\{x_{m}^{i} y_{m}^{k} \alpha_{\text {Soll }, m}\right\}
\end{gathered}
$$

Zur Unterscheidung zwischen aufzusummierenden und nicht aufzusummierenden Größen in (*) wird ein Basiswechsel für die Doppelsumme vorgenommen: $i \rightarrow a, k \rightarrow b$

$$
(*)=\sum_{m=0}^{N_{m}}\left\{x_{m}^{i} y_{m}^{k} \sum_{a=0}^{N_{i}-1} \sum_{b=0}^{N_{k}-1}\left(A_{a, b} x_{m}^{a} y_{m}^{b}\right)\right\}
$$

Anwendung des Assoziativ- und Kommutativgesetzes liefert

$$
(*)=\sum_{m=0}^{N_{m}} \sum_{a=0}^{N_{i}-1} \sum_{b=0}^{N_{k}-1} x_{m}^{i} y_{m}^{k} x_{m}^{a} y_{m}^{b} A_{a, b}=\sum_{a=0}^{N_{i}-1} \sum_{b=0}^{N_{k}-1} A_{a, b} \sum_{m=0}^{N_{m}} x_{m}^{i+a} y_{m}^{k+b}
$$

Demnach kann Gleichung A.52 als lineares Gleichungssystem mit $N_{i} \cdot N_{k}$ Unbekannten formuliert werden.

$$
\mathbf{B} \vec{c}=\vec{d}
$$

Dazu müssen die Terme so sortiert werden, dass diese in eine Matrix mit $N_{i} N_{k} \times N_{i} N_{k}$ Elementen bzw. in Spaltenvektoren mit $N_{i} N_{k}$ Elementen einsortiert werden können. Dies passiert wie folgt:

$$
\begin{gathered}
\mathbf{B}=\left(b_{e, f}\right) \in \mathbb{R}\left\{N_{i} N_{k} \times N_{i} N_{k}\right\} \\
\vec{c}=\left(c_{e}\right) \in \mathbb{R}\left\{N_{i} N_{k}\right\} \\
\vec{d}=\left(d_{e}\right) \in \mathbb{R}\left\{N_{i} N_{k}\right\} \\
b_{e, f}=\sum_{m=0}^{N_{m}} x_{m}^{i(e)+p(f)} y_{m}^{k(e)+q(f)} \forall e, f \in\left[0 ; N_{i} N_{k}-1\right] \\
c_{e}=A_{i(e), k(e)} \forall e \in\left[0 ; N_{i} N_{k}-1\right] \\
d_{e}=\sum_{m=0}^{N_{m}} x_{m}^{i(e)} y_{m}^{k(e)} \alpha_{\text {Soll }, m} \forall e \in\left[0 ; N_{i} N_{k}-1\right]
\end{gathered}
$$


mit

$$
\begin{gathered}
i(e)=\operatorname{Int} \frac{e}{N_{k}}=\left\lfloor\frac{e}{N_{k}}\right\rfloor \\
k(e)=e \bmod N_{k}=e \backslash N_{k} \\
p(f)=\operatorname{Int} \frac{f}{N_{k}}=\left\lfloor\frac{f}{N_{k}}\right\rfloor \\
q(f)=f \bmod N_{k}=f \backslash N_{k}
\end{gathered}
$$

Int bezeichnet Ganzzahldivision, Mod deren Rest (modulo).

Die Lösung des Gleichungssystems erfolgt mit

$$
\vec{c}=\mathbf{B}^{-1} \vec{d}
$$

Die Koeffizienten $A_{i, k}$ ergeben sich schließlich aus

$$
A_{i(e), k(e)}=c_{e} \forall e \in\left[0 ; N_{i} N_{k}-1\right]
$$




\section{A12 GENAUIGKEITSTABELLEN ZUR SONDENKALIBRIERUNG}

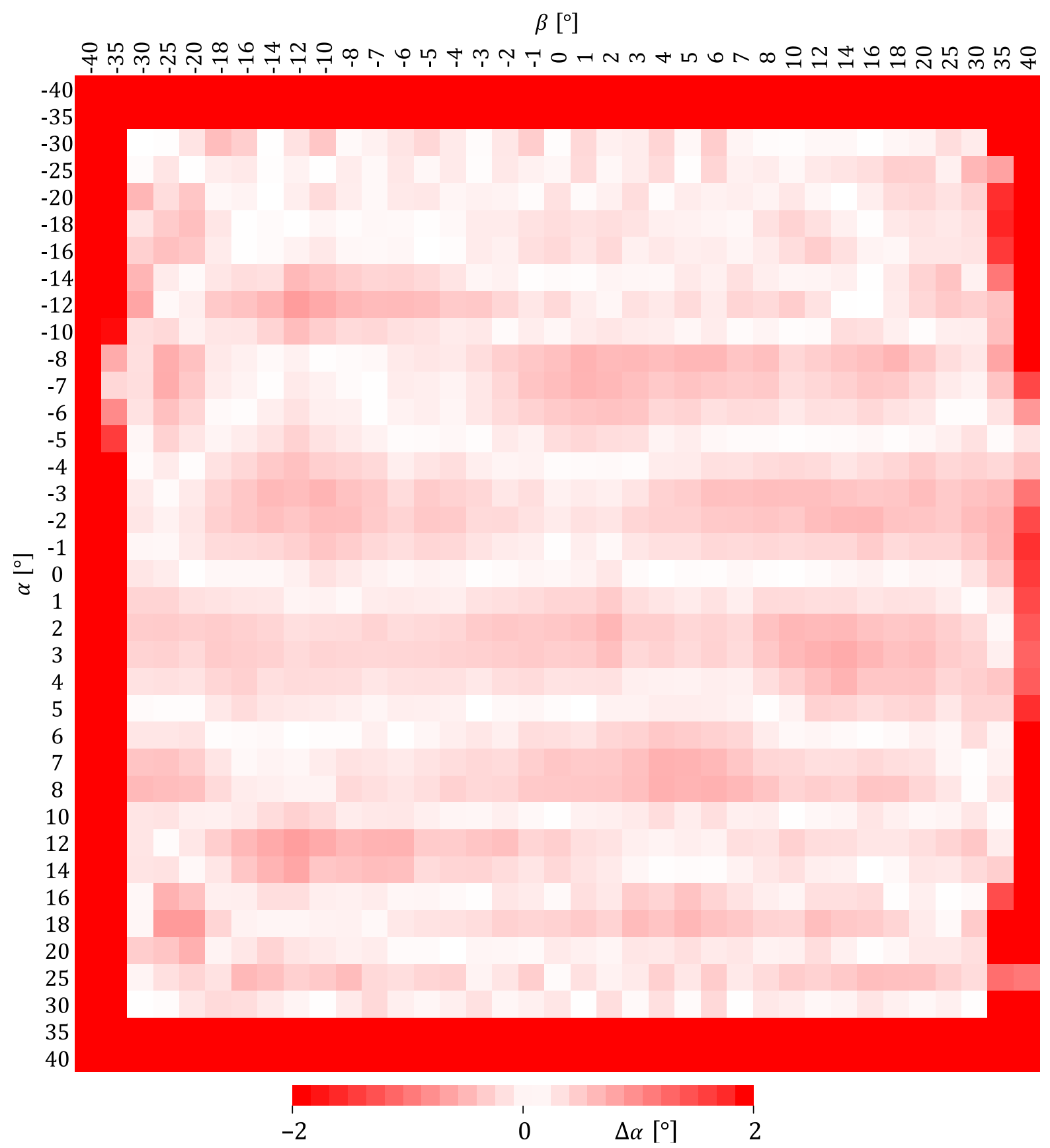

Abb. A.26 Approximationsgenauigkeit des Kalibrierpolynoms zur Berechnung des Drallwinkels für die Fünflochsonde. 


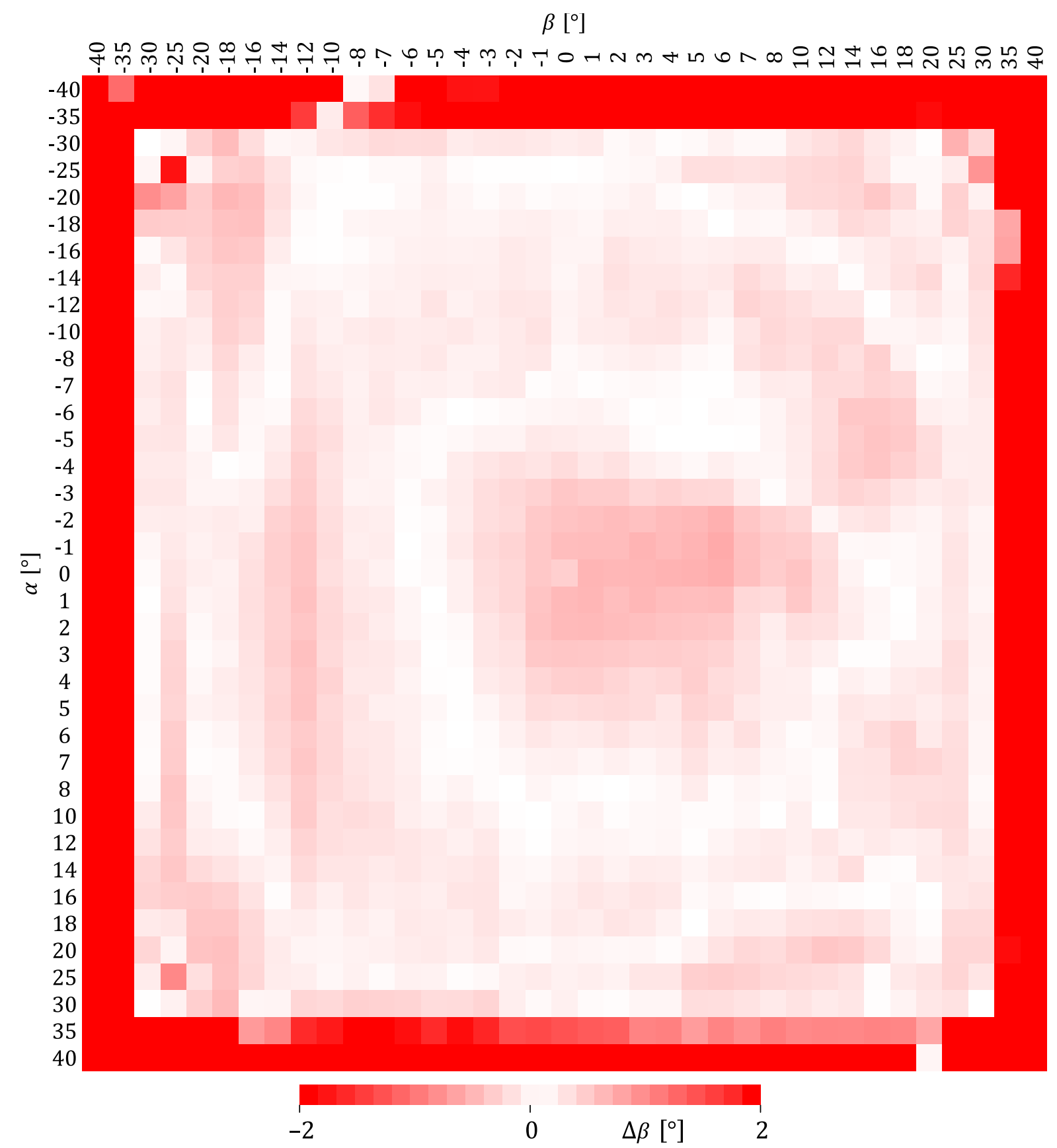

Abb. A.27 Approximationsgenauigkeit des Kalibrierpolynoms zur Berechnung des Nickwinkels für die Fünflochsonde. 


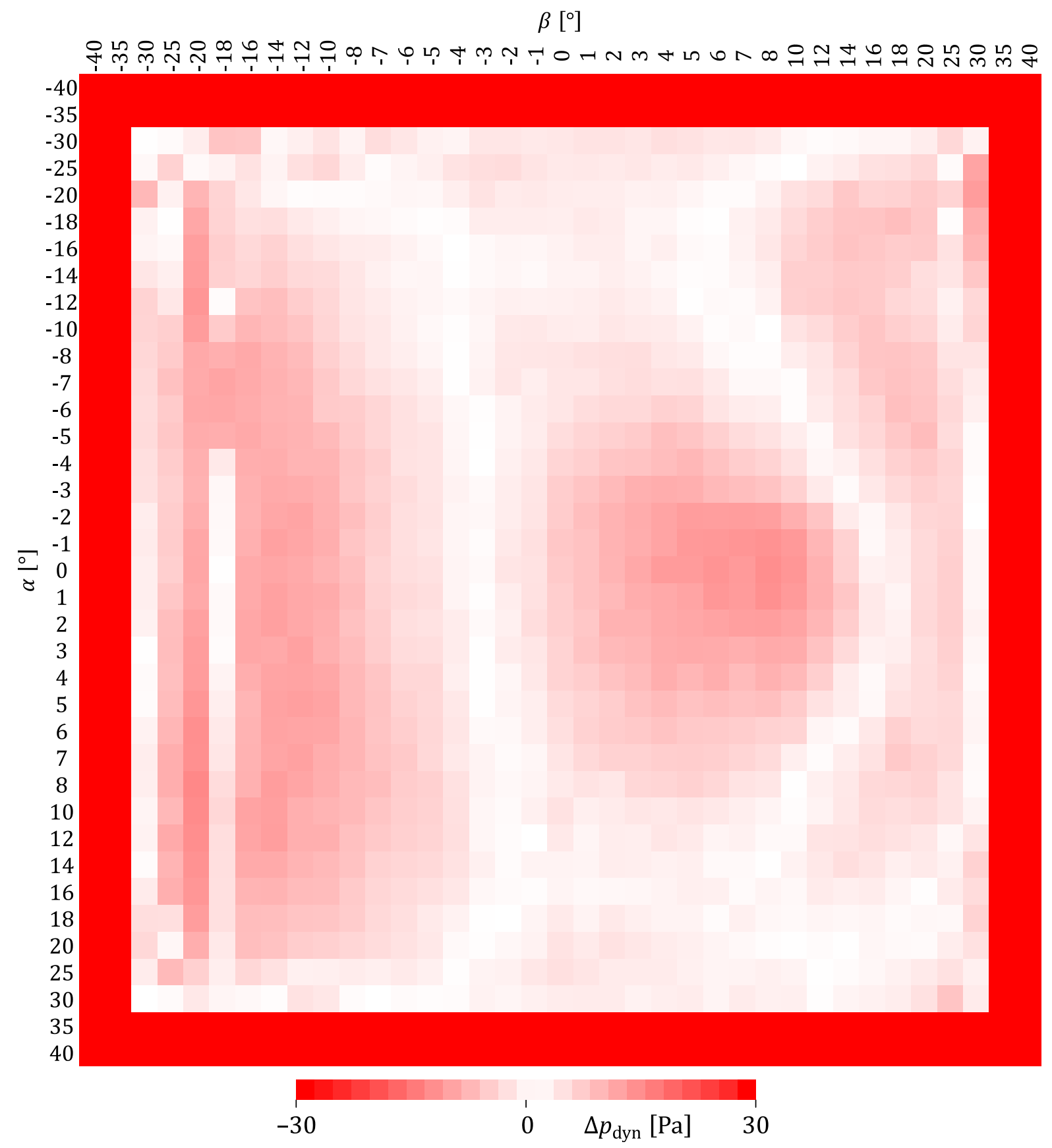

Abb. A.28 Approximationsgenauigkeit des Kalibrierpolynoms zur Berechnung des dynamischen Druckes für die Fünflochsonde. 


\section{A13 DETAILEINSTELLUNGEN FÜR DIE CFD-MODELLE}

\section{A13.1 Vernetzungseinstellungen und -statistik}

Tab. A.8 Vernetzungseinstellungen zur Erstellung einheitlicher Rechengitter mittels Ansys Meshing 18.2

\begin{tabular}{lll}
\hline Feature & Eigenschaft & Einstellung \\
\hline Methode & Methode & Multizone \\
& Strukturiertes Netz & Hexaeder \\
& Schalenvernetzungsmethode & Programmgesteuert \\
& Freies Netz & Nicht zulässig \\
\hline Methode (2D) & Methode & Multizone Vierecke/ Dreiecke \\
& Schalenvernetzungsmethode & Programmgesteuert \\
& Freies Netz & Nur Quads \\
& Elementansatzfunktion & Globale Einstellung verwenden \\
\hline Prismenschichten & Option zur Erzeugung & Dicke der ersten Schicht \\
(Inflation) & Höhe der ersten Schicht & $5 \mu$ m \\
& Max. Anzahl der Schichten & 28 \\
& Wachstumsrate & 1,2 \\
\hline Elementgröße & Typ & Elementgröße \\
& Elementgröße & 1 mm \\
& Größenfunktion & Gleichmäßig \\
& Verhalten & Flexibel \\
\hline Flächenvernetzung (2D) & Strukturiertes Netz & Ja \\
& Methode & Trapezoide \\
\hline
\end{tabular}

Tab. A.9 Elementanzahl der verschiedenen Rechennetze für das Basismodell

\begin{tabular}{lllrr}
\hline $\begin{array}{l}\text { Geometrie- } \\
\text { modell }\end{array}$ & $\begin{array}{l}\text { Vernetzungspro- } \\
\text { gramm }\end{array}$ & $\begin{array}{l}\text { Simulations- } \\
\text { programm }\end{array}$ & $\begin{array}{r}\text { Anzahl } \\
\text { Elemente }\end{array}$ & $\begin{array}{r}\text { Anzahl } \\
\text { Knoten }\end{array}$ \\
\hline $2 \mathrm{D}$ & Ansys Meshing & Ansys Fluent & 43.535 & 44.189 \\
$2 \mathrm{D}$ & ICEM CFD & Ansys Fluent & 43.728 & 44.372 \\
$5^{\circ}$ & Ansys Meshing & Ansys CFX & 391.815 & 441.890 \\
$15^{\circ}$ & Ansys Meshing & Ansys CFX & 1.131 .910 & 1.193 .103 \\
$30^{\circ}$ & Ansys Meshing & Ansys CFX & 2.263 .820 & 2.342 .017 \\
\hline $60^{\circ}$ & Ansys Meshing & Ansys CFX & 4.513 .496 & 4.625 .355 \\
$90^{\circ}$ & Ansys Meshing & Ansys CFX & 6.719 .232 & 6.864 .668 \\
$360^{\circ}$ & Ansys Meshing & Ansys CFX & 26.428 .272 & 26.833 .248 \\
\hline
\end{tabular}




\section{A13.2 Randbedingungen}

Tab. A.10 Randbedingungen für das Ansys CFX-Modell

\begin{tabular}{|c|c|c|c|}
\hline Rand & RB-Typ & Randbedingung & Einstellung \\
\hline \multirow[t]{9}{*}{ Einlass } & Inlet & Flow Regime & Subsonic \\
\hline & & Mass and Momentum & Total Pressure (stable) \\
\hline & & Relative Pressure & $p_{\mathrm{T}, \mathrm{ein}}=p_{\text {ref }}$ \\
\hline & & Flow Direction & Normal to Boundary Condition \\
\hline & & Turbulence & Intensity and Length Scale \\
\hline & & Fractional Intensity & $T u_{\text {ein }}=5 \%$ \\
\hline & & Eddy Length Scale & $\lambda_{\text {ein }}=2,84 \mathrm{~mm}$ \\
\hline & & Heat Transfer & Total Temperature \\
\hline & & Total Temperature & $T_{\mathrm{T}, \mathrm{ein}}=T_{\mathrm{ref}}$ \\
\hline \multirow[t]{3}{*}{ Auslass } & Outlet & Mass and Momentum & Mass Flow Rate \\
\hline & & Mass Flow Rate & $\dot{m}_{\mathrm{aus}}=\dot{m} \cdot \varphi / 360^{\circ}$ \\
\hline & & Mass Flow Rate Area & As specified \\
\hline Beheizte & Wall & Mass and Momentum & No Slip Wall \\
\hline \multirow[t]{5}{*}{ Wand } & & Wall Roughness & Smooth Wall - oder - Rough Wall \\
\hline & & Sand Grain Roughness & $k_{\mathrm{S}}=R z$ \\
\hline & & Heat Transfer & Heat Flux - oder - Temperature \\
\hline & & Heat Flux in - bzw. - & $\hat{\dot{q}}_{\mathrm{W}}=318 \mathrm{~W} / \mathrm{m}^{2}$ \\
\hline & & Fixed Temperature & $T_{\mathrm{W}}=40^{\circ} \mathrm{C}$ \\
\hline Unbeheizte & Wall & \multicolumn{2}{|c|}{ wie Beheizte Wand, jedoch } \\
\hline Wände & & Heat Transfer & Adiabatic \\
\hline \multirow[t]{5}{*}{ Symmetrie } & Interface & Interface Type & Fluid Fluid \\
\hline & & Interface Models & Rotational Periodicity \\
\hline & & Axis Definition & Coordinate Axis \\
\hline & & Rotation Axis & Global X \\
\hline & & Mesh Connection & GGI \\
\hline Symmetrie & Interface & Mass and Momentum & Conservative Interface Flux \\
\hline \multirow[t]{2}{*}{ Seite $1 / 2$} & & Turbulence & Conservative Interface Flux \\
\hline & & Heat Transfer & Conservative Interface Flux \\
\hline
\end{tabular}


Tab. A.11 Randbedingungen für das Ansys Fluent-Modell

\begin{tabular}{|c|c|c|c|}
\hline Rand & RB-Typ & Randbedingung & Einstellung \\
\hline \multirow[t]{9}{*}{ Einlass } & \multirow[t]{9}{*}{ mass-flow-inlet } & Reference Frame & Absolute \\
\hline & & Mass Flow Spec. Method & Mass Flow Rate \\
\hline & & Mass Flow Rate & $\dot{m}_{\mathrm{ein}}=\dot{m} \cdot \varphi / 360^{\circ}$ \\
\hline & & Direction Spec. Method & Normal To Boundary \\
\hline & & Turbulence Spec. Method & Intensity and Length Scale \\
\hline & & Turb. Intensity (fraction) & $T u_{\text {ein }}=5 \%$ \\
\hline & & Turbulent Length Scale & $\lambda_{\text {ein }}=2,84 \mathrm{~mm}$ \\
\hline & & Reynolds-Stress Spec. Meth. & $\mathrm{k}$ or Turbulent Intensity \\
\hline & & Total Temperature & $T_{\mathrm{T}, \mathrm{ein}}=T_{\mathrm{ref}}$ \\
\hline \multirow{7}{*}{$\begin{array}{l}\text { Einlass } \\
\text { (alt.) }\end{array}$} & \multirow[t]{7}{*}{ velocity-inlet } & wie mass-flow-inlet, jedoch & \\
\hline & & Velocity Spec. Method & Components \\
\hline & & Coordinate System & Cylindrical \\
\hline & & Axial Velocity & $c_{\mathrm{ax}}=q$ \\
\hline & & Radial Velocity & $c_{\mathrm{r}}=0$ \\
\hline & & Swirl Velocity & $c_{\mathrm{u}}=q \tan \alpha$ \\
\hline & & Swirl Angular Velocity & $\omega=0 \mathrm{rad} / \mathrm{s}$ \\
\hline \multirow[t]{7}{*}{ Auslass } & \multirow[t]{7}{*}{ pressure-outlet } & Backflow Reference Frame & Absolute \\
\hline & & Gauge Pressure & $p_{\text {aus }}=p_{\text {ref }}$ \\
\hline & & Pressure Profile Multiplier & 1 \\
\hline & & $\begin{array}{l}\text { Backflow Direction Spec. } \\
\text { Meth. }\end{array}$ & Normal To Boundary \\
\hline & & Backflow Pressure Spec. & Total Pressure \\
\hline & & Average Pressure Spec. & Yes \\
\hline & & \multicolumn{2}{|c|}{ Turbulenzeinstellungen wie Einlass } \\
\hline Beheizte & wall & Wall Motion & Stationary Wall \\
\hline \multirow[t]{6}{*}{ Wand } & & Shear Condition & No Slip \\
\hline & & Roughness Height (nur $k-\omega$ ) & $k_{\mathrm{S}}=R z$ \\
\hline & & Roughness Constant & 0,5 \\
\hline & & Thermal Conditions & Heat Flux - oder - Temperature \\
\hline & & Heat Flux - bzw. - & $\dot{q}_{\mathrm{W}}=318 \mathrm{~W} / \mathrm{m}^{2}$ oder $\overline{\dot{q}}_{\mathrm{W}}$ \\
\hline & & Temperature & $T_{\mathrm{W}}=40^{\circ} \mathrm{C}$ oder $\bar{T}_{\mathrm{W}}$ \\
\hline Unbeheizte & wall & wie Beheizte Wand, jedoch & \\
\hline Wände & & Heat Flux & 0 \\
\hline Domain & interior & - & - \\
\hline
\end{tabular}


Tab. A.12 Zusätzliche Randbedingungen für das CHT-Modell in Ansys Fluent

\begin{tabular}{llll}
\hline Rand & RB-Typ & Randbedingung & Einstellung \\
\hline Kontakt & Interface & Coupled Wall & Yes \\
& & Matching & No \\
& & Mapped & Yes \\
& & Static & No \\
& & Enable Local Tolerance & $0,0001 \mathrm{~m}$ \\
\hline Kontakt Seite 1/2 & Wall & Thermal Conditions & via Mapped Interface \\
(source/ target) & & Wall Thickness & $0 \mathrm{~m}$ \\
& & Heat Generation Rate & $0 \mathrm{~W} / \mathrm{m}^{3}$ \\
\hline Wand/ Schattenwand & Wall & Thermal Conditions & Coupled \\
(solid) & & Material Name & Aluminium \\
& & Wall Thickness & $0,001 \mathrm{~m}$ (Alu-Armaflex) \\
& & & $0 \mathrm{~m}($ sonstige) \\
& & Heat Generation Rate & $326.429 \mathrm{~W} / \mathrm{m}^{3}$ (Alu-Armaflex) \\
& & & $0 \mathrm{~W} / \mathrm{m}^{3}$ (sonstige) \\
\hline Außenoberflächen & Wall & Thermal Conditions & Convection \\
(solid) & & HTC & $10 \mathrm{~W} /\left(\mathrm{m}^{2} \mathrm{~K}\right)$ \\
& & Free Stream Temp. & $30,47^{\circ} \mathrm{C}$ \\
& & Wall Thickness & $0 \mathrm{~m}$ \\
& & Heat Generation Rate & $0 \mathrm{~W} / \mathrm{m}^{3}$ \\
\hline
\end{tabular}

Tab. A.13 Randbedingungen und Modellparameter der durchgeführten Large-Eddy-Simulationen

\begin{tabular}{llllll}
\hline Modellname & Programm & Sektorwinkel $\begin{array}{l}\text { thermische RB } \\
\text { Außenwand }\end{array}$ & $\begin{array}{l}\text { Physikalische } \\
\text { Zeit }\end{array}$ & $\begin{array}{l}\text { Letzte } \\
\text { Rechnung }\end{array}$ \\
\hline LES_TRV_90 & CFX & $90^{\circ}$ & $\dot{q}_{\mathrm{W}}=318 \mathrm{~W} / \mathrm{m}^{2}$ & $0 \ldots 2 \mathrm{~s}$ & 12.09 .2018 \\
LES_TRV_90_TW & CFX & $90^{\circ}$ & $T_{\mathrm{W}}=40^{\circ} \mathrm{C}$ & $0 \ldots 2 \mathrm{~s}$ & 28.09 .2019 \\
LES_TRV_2D_qW & Fluent & 2D & $\dot{q}_{\mathrm{W}}=318 \mathrm{~W} / \mathrm{m}^{2}$ & $0 \ldots 2 \mathrm{~s}$ & 06.04 .2019 \\
LES_TRV_2D & Fluent & 2D & $T_{\mathrm{W}}=40^{\circ} \mathrm{C}$ & $0 \ldots 2 \mathrm{~s}$ & 06.02 .2019 \\
\hline
\end{tabular}




\section{A13.3 Stoffwertdefinitionen Ansys CCL}

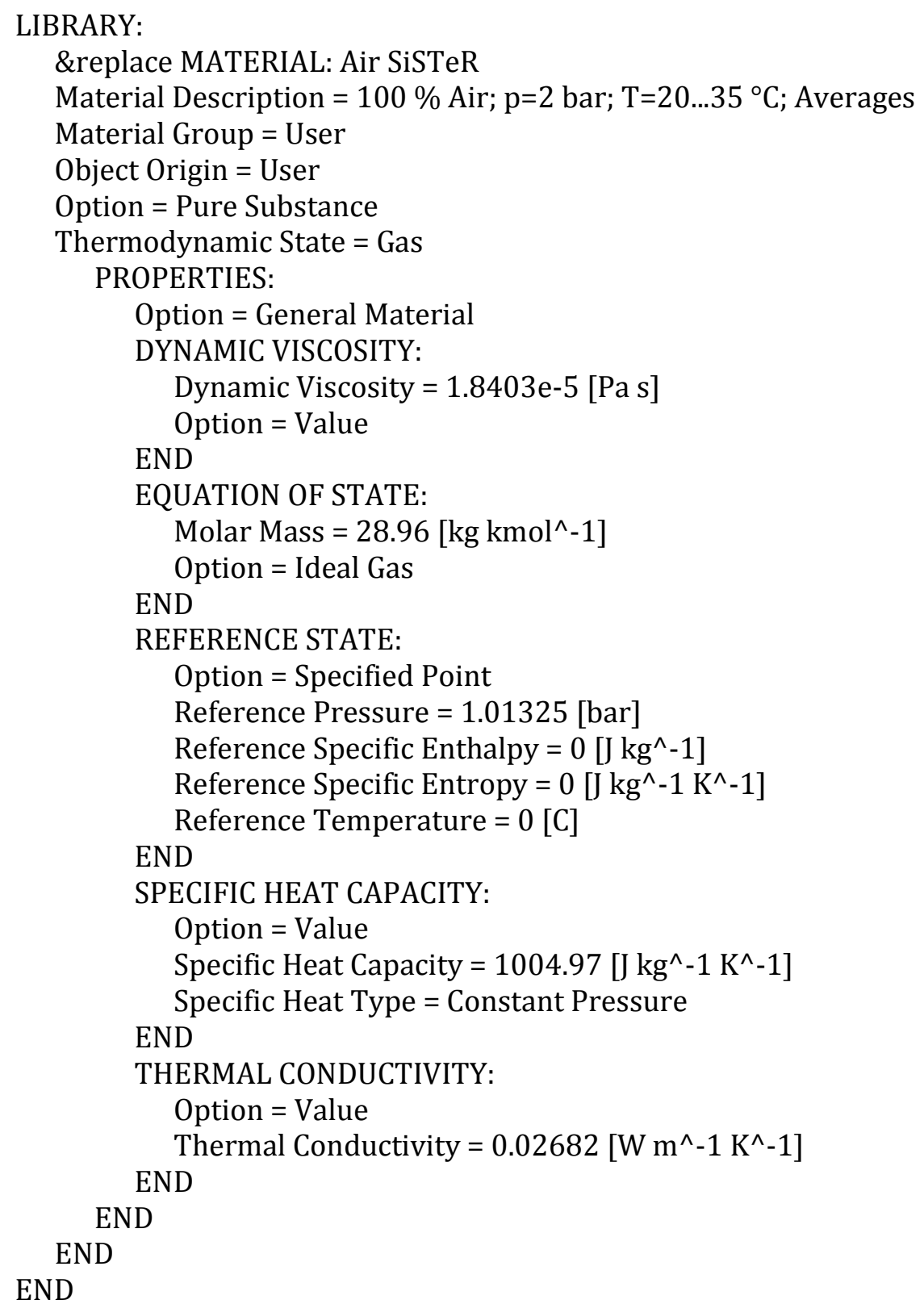




\section{LIBRARY:}

\&replace MATERIAL: Air

Material Description $=100 \%$ Air; $p=2$ bar; $\mathrm{T}=0 . .100^{\circ} \mathrm{C}$

Material Group = User

Object Origin $=$ User

Option $=$ Pure Substance

Thermodynamic State $=$ Gas

PROPERTIES:

Option $=$ General Material

DYNAMIC VISCOSITY:

Dynamic Viscosity $=\left(4.36546 \mathrm{e}-14\left[\mathrm{~K}^{\wedge}-3\right]^{*}\right.$ Temperature^3-7.42985e-11 $\left[\mathrm{K}^{\wedge}\right.$ -

2] ${ }^{*}$ Temperature ${ }^{\wedge} 2+7.87156 \mathrm{e}-8\left[\mathrm{~K}^{\wedge}-1\right]^{*}$ Tempera-

Option $=$ Value ture+2.66908e-7) [Pa s]

END

EQUATION OF STATE:

Molar Mass $=28.96\left[\mathrm{~kg} \mathrm{kmol}^{\wedge}-1\right]$

Option = Ideal Gas

END

REFERENCE STATE:

Option $=$ Specified Point

Reference Pressure $=1.01325$ [bar]

Reference Specific Enthalpy $=0$ [J kg^-1]

Reference Specific Entropy $=0$ [J kg^ $\left.-1 \mathrm{~K}^{\wedge}-1\right]$

END

Reference Temperature $=0[\mathrm{C}]$

SPECIFIC HEAT CAPACITY:

Option $=$ Value

Specific Heat Capacity $=\left(6.97455 \mathrm{e}-13\left[\mathrm{~K}^{\wedge}-4\right]^{*}\right.$ Temperature ${ }^{\wedge} 4-4.55380 \mathrm{e}-10\left[\mathrm{~K}^{\wedge}-\right.$ $3]^{*}$ Temperature ${ }^{\wedge} 3+3.36916 \mathrm{e}-7\left[\mathrm{~K}^{\wedge}-2\right]^{*}$ Temperature $^{\wedge} 2$ $1.03927 \mathrm{e}-4\left[\mathrm{~K}^{\wedge}-1\right]^{*}$ Temperature+1.01244e+0) $\left[\mathrm{kJ} \mathrm{kg}{ }^{\wedge}-1\right.$ $\left.\mathrm{K}^{\wedge}-1\right]$

Specific Heat Type $=$ Constant Pressure

\section{END}

TABLE GENERATION:

Error Tolerance $=0.001$

Maximum Points $=1000$

Maximum Temperature $=100[\mathrm{C}]$

Minimum Temperature $=0[\mathrm{C}]$

Temperature Extrapolation $=$ Off

END

THERMAL CONDUCTIVITY:

Option $=$ Value

Thermal Conductivity $=\left(-3.42663 \mathrm{e}-8\left[\mathrm{~K}^{\wedge}-2\right]^{*}\right.$ Temperature ${ }^{\wedge} 2+8.87149 \mathrm{e}-5\left[\mathrm{~K}^{\wedge}-\right.$ 1]*Temperature+3.24267e-3) [W $\left.\mathrm{m}^{\wedge}-1 \mathrm{~K}^{\wedge}-1\right]$

END

END

END

END 


\section{A13.4 Lösungseinstellungen}

Tab. A.14 Solver-Einstellungen für stationäre Berechnungen in Ansys CFX

\begin{tabular}{lll}
\hline Feature & Eigenschaft & Einstellung \\
\hline Analysis Type & Option & Steady State \\
\hline Advection Scheme & Option & High Resolution \\
\hline Turbulence Numerics & Option & High Resolution \\
\hline Convergence Control & Min. Iterations & 1 \\
& Max. Iterations & 5000 \\
\hline Fluid Timescale Control & Physical Timescale & $10^{-4} \mathrm{~s}\left(\right.$ Energy: $\left.10^{-2} \mathrm{~s}\right)$ \\
\hline Convergence Criteria & Residual Type & $\mathrm{RMS}$ \\
& Residual Target & $10^{-5}$ \\
\hline Elapsed Wall Clock Time & Conservation Target & $10^{-4}=0,01 \%$ \\
\hline Interrupt Control & Logical Expression & $8 \mathrm{~h}$ \\
\hline Run Settings & Precision & None \\
\hline
\end{tabular}

Tab. A.15 Solver-Einstellungen für transiente Berechnungen in Ansys CFX

\begin{tabular}{lll}
\hline Feature & Eigenschaft & Einstellung \\
\hline Analysis Type & Option & Transient \\
& Total Time & $2 \mathrm{~s}$ \\
& Time Steps & $2 \cdot 10^{-5} \mathrm{~s}\left(90^{\circ}\right) \mathrm{bzw} .5 \cdot 10^{-5} \mathrm{~s}\left(5^{\circ}\right)$ \\
& Initial Time & Automatic \\
\hline Advection Scheme & Option & Central Difference \\
& Bounded CDS & No \\
\hline Transient Scheme & Option & Second Order Backward Euler \\
& Timestep Initialization & Automatic \\
\hline Convergence Control & Min. Coeff. Loops & 1 \\
& Max. Coeff. Loops & 20 \\
& Fluid Timescale Control & Coefficient Loops \\
\hline Convergence Criteria & Residual Type & RMS \\
& Residual Target & $10^{-5}$ \\
& Conservation Target & $0,01=1 \%$ \\
\hline Run Settings & Precision & Double Precision \\
\hline
\end{tabular}


Tab. A.16 Solver-Einstellungen in Ansys Fluent

\begin{tabular}{|c|c|c|}
\hline Feature & Eigenschaft & Einstellung \\
\hline \multirow[t]{2}{*}{ Pressure-Velocity Couling } & Scheme & $\underline{\text { SIMPLE oder SIMPLEC }}$ \\
\hline & Skewness Correction & 0 \\
\hline \multirow[t]{10}{*}{ Spatial Discretization } & Gradient & Least Squares Cell Based \\
\hline & Pressure & Second Order \\
\hline & Density & Second Order Upwind \\
\hline & Momentum & Second Order Upwind \\
\hline & Momentum (transient) & Bounded Central Differencing \\
\hline & Swirl Velocity & Second Order Upwind \\
\hline & Turbulent Kinetic Energy & First Order Upwind \\
\hline & Specific Dissipation Rate & First Order Upwind \\
\hline & Reynolds Stresses & First Order Upwind \\
\hline & Energy & Second Order Upwind \\
\hline \multirow[t]{5}{*}{ Transient Formulation } & Time Discretization & Bounded Second Order Implicit \\
\hline & Non-Iterative Time Adv. & No \\
\hline & Frozen Flux Formulation & No \\
\hline & Warped-Face Gradient Corr. & No \\
\hline & High Order Term Relaxation & No \\
\hline \multirow[t]{10}{*}{ Under-Relaxation Factors } & Pressure & 0,3 \\
\hline & Density & 1 \\
\hline & Body Forces & 1 \\
\hline & Momentum & 0,7 \\
\hline & Swirl Velocity & 0,9 \\
\hline & Turbulent Kinetic Energy & 0,8 \\
\hline & Specific Dissipation Rate & 0,8 \\
\hline & Turbulent Viscosity & 1 \\
\hline & Reynolds Stresses & 0,5 \\
\hline & Energy & $1(!)$ \\
\hline \multirow[t]{10}{*}{ Residual } & Continuity & $10^{-5}$ \\
\hline & $\mathrm{x} / \mathrm{y}$-Velocity & $10^{-5}$ \\
\hline & Energy & $10^{-5}$ \\
\hline & Omega & $10^{-5}$ \\
\hline & uu, vv, ww, uv-stress & $10^{-5}$ \\
\hline & Normalize & No \\
\hline & Scale & Yes \\
\hline & Compute Local Scale & No \\
\hline & Convergence Criterion & Absolute \\
\hline & Convergence Conditions & All conditions are met \\
\hline Run Calculation (Stat.) & Number of Iterations & $\min .3000$ \\
\hline & Time Stepping Method & Fixed \\
\hline \multirow[t]{4}{*}{ (Transiente Rechnungen) } & Time Step Size & $2 \cdot 10^{-5} \mathrm{~s}$ \\
\hline & Number of Timesteps & 50.000 \\
\hline & Extrapolate Variables & No \\
\hline & Max Iterations / Time Step & 15 \\
\hline
\end{tabular}


Tab. A.17 Beispiele für Rechenzeiten und verwendete Ressourcen für die durchgeführten CFD-Simulationen

\begin{tabular}{lcccccc}
\hline Modell & \multicolumn{2}{c}{$\begin{array}{l}\text { Rechen- } \\
\text { umgebung }\end{array}$} & $\begin{array}{c}\text { Rechenknoten CPU-Kerne } \\
\text { („Maschinen“) }\end{array}$ & $\begin{array}{c}\text { (Äußere) } \\
\text { pro Knoten }\end{array}$ & $\begin{array}{c}\text { Berechnete } \\
\text { Iterationen }\end{array}$ & Rechenzeit \\
phys. Zeit & \\
\hline SST-2D (Fluent) & lokal & 1 & 3 & 3.000 & stationär & $15 \mathrm{~min}$ \\
CHT-2D (Fluent) & lokal & 1 & 3 & 6.000 & stationär & $14 \mathrm{~h}$ \\
SST-90 $^{\circ}$ (CFX) & Taurus & 4 & 48 & 1.000 & stationär & $8 \mathrm{~h}$ \\
SST-360 $^{\circ}$ (CFX) & Taurus & 8 & 96 & 5.000 & stationär & $47 \mathrm{~h}(\approx 2 \mathrm{~d})$ \\
RSM-360 $^{\circ}$ (CFX) & Taurus & 8 & 96 & 6.700 & stationär & $47 \mathrm{~h}(\approx 2 \mathrm{~d})$ \\
LES-2D $\left(\right.$ Fluent) $^{\circ}$ & Taurus & 1 & 12 & 100.000 & $2 \mathrm{~s}$ & $88 \mathrm{~h}(\approx 4 \mathrm{~d})$ \\
LES-5 $^{\circ}$ (CFX) & Taurus & 2 & 12 & 50.000 & $1 \mathrm{~s}$ & $230 \mathrm{~h}(\approx 9,5 \mathrm{~d})$ \\
LES-90 $^{\circ}$ (CFX) & Taurus & 20 & 12 & 100.000 & $2 \mathrm{~s}$ & $800 \mathrm{~h}(\approx 33 \mathrm{~d})$ \\
\hline
\end{tabular}




\section{A14 RICHTLINIEN ZUR WANDMODELLIERUNG FÜR CFD-ANALYSEN}

Folgende Erwägungen sollten bei der Wandmodellierung (near-wall treatment) in CFD-Simulationen getroffen werden:

1. Bei CFD-Simulationen können verschiedene Mechanismen im Fokus stehen: so kann es in manchen Fällen nur von Interesse sein, die „freie Strömung“ möglichst genau vorherzusagen (z. B. Umströmung von Körpern, Durchströmung komplexer Geometrien), wobei Wandeffekte uninteressant sind. Bei anderen Untersuchungen wiederum stehen Wandeffekte im Mittelpunkt (Reibung, Wandschubspannung, WÜK etc.).

2. Die Haftbedingung an Wänden sollte in CFD-Simulationen mitmodelliert werden, da sie eine wesentliche Haupteinflussgröße auf die Ausbildung des Strömungsfeldes ist.

3. Bei turbulenten Strömungen sind die Turbulenzgrößen an der Wand gänzlich anders als in der freien Strömung (Anisotropie, viskose Dämpfung etc.).

4. Deshalb kommt der richtigen Modellierung (oder besser: der richtigen Behandlung) von Wänden eine wichtige Bedeutung zu (near-wall treatment).

5. Mit den ursprünglichen $k-\varepsilon$-Modellen, die bereits in den 1960er/ 1970er Jahren entwickelt worden sind, konnten zwar Wirbelstrukturen besser vorhergesagt werden als mit allen anderen Modellen, die es bis zu diesem Zeitpunkt gab (Potenzialströmung ohne Reibung, einfache Wirbelviskositätsmodelle). An der Wand versagen $k$ - $\varepsilon$-Modelle aber, da sie dort eine Singularität aufweisen. Sie können daher nicht bis zur Wand integriert werden. Dasselbe gilt übrigens auch per se für RSM- und LES-Modelle.

6. Um nun aber doch Wände richtig modellieren zu können, wurden entsprechende Wandfunktionen eingeführt.

7. Mit ihnen konnten weitgehend vernünftige Werte (Geschwindigkeiten, Gradienten) am Ende der ersten („wandnächsten“) Zelle gefunden werden, mit denen dann ins Innere der Strömung weiter gerechnet werden kann.

8. Da die Rechenleistung zu damaligen Zeit noch beschränkt war, hatten die Rechennetze noch eine geringe Anzahl an Zellen. Das Verfeinern an der Wand war rechentechnisch noch nicht möglich, weshalb übliche Netze damals häufig noch $y^{+}$-Werte größer als 30 hatten.

9. In diesem Bereich befindet sich der logarithmische Bereich (vgl. 1/7-Wandgesetz), der in dimensionsloser Schreibweise bei geschickter Normierung beteiligten Größen immer die gleiche Funktion hat (siehe Abb. A.29; $\kappa=0,41, C=5,25$ ) und mit dem eine eindeutige Zuordnung des $y^{+}$-Wertes der ersten Zelle zum $u^{+}$-Wert möglich ist. 
10. Auch die Größen an der Wand ( $\tau_{W}$ etc.) können über $u^{+}=y^{+}$berechnet werden.

11. Für $y^{+}<30$ sollten Wandansätze nicht verwendet werden, da die erste Zelle dann schon im Buffer-Layer liegt und dort das logarithmische Gesetz nicht mehr gilt und die Turbulenz bereits anisotrop wird. Die Ergebnisse werden in der Folge falsch.

12. Semiempirische Ansätze wie die Wandfunktionen gelten allerdings nur unter bestimmten Modellbedingungen (Grenzschichten im Gleichgewicht, sog. equilibrium boundary layers; weitgehend wandparallele Strömungen). Daher ist es wünschenswert, die Wand mit aufzulösen, sprich mit dem (komplexeren) Turbulenzmodell (möglichst weit) bis an die Wand zu rechnen, da dies raumaufgelöste Effekte besser beschreiben kann als ein eindimensionaler Wandansatz.

13. Da der $k$ - $\varepsilon$-Ansatz wie oben beschrieben dazu mathematisch nicht imstande ist, wurden $\omega$ basierte Modelle entwickelt, bei dem geschickt eine neue abgeleitete Größe $\omega$ (CFX: Wirbelfrequenz; Fluent: spezifische Dissipationsrate) aus der turbulenten kinetischen Energie $k$ und der Wirbeldissipationsrate $\varepsilon$ berechnet wird.

14. Diese Modelle können bis an die Wand rechnen, da sie Dämpfungsterme haben, die die Turbulenz in unterschiedliche Raumrichtungen unterschiedlich dämpfen können.

15. Voraussetzung hierfür ist, dass das Netz zur Wand hin verfeinert werden muss.

16. Als Richtwert hat sich ein $y^{+}$-Wert der wandnächsten Zelle von 1 bewährt, um die lineare Unterschicht $\left(y^{+} \leq 5\right)$ mit mindestens zwei, besser vier Zellen aufzulösen und so Gradienten an der Wand sauber abbilden zu können. Bei einer voreingestellten Wachstumsrate von $q=1,2$ liegen dann knapp vier Zellen in der laminaren Unterschicht:

$$
\sum_{i=1}^{4} y^{+} \cdot q^{i-1}=1+1,2+1,44+1,728=5,368
$$

17. Auch ein Wert von $y^{+}=2$ kann noch geeignet sein, wenn die Wachstumsrate der Inflationsschicht entsprechend klein ist oder die wandnächsten Zellen äquidistant sind.

18. Bei Strömungen mit großer Reynolds-Zahl ist die viskose Unterschicht sehr klein gegenüber der Hauptströmung, sie beeinflusst sie so gut wie nicht (kleine Impulsverlustdicke).

19. Bei kleineren Reynolds-Zahlen und in Fällen, bei denen ein besonderes Interesse an der richtigen Abbildung der Werte unmittelbar an der Wand besteht, sollte die lineare/ laminare Unterschicht aber nicht turbulent gerechnet werden, da dies unter Umständen dazu führen kann, dass Effekte, die für turbulente Strömungen charakteristisch sind (z. B. spontane Strömungsablösung), fälschlicherweise auch für die laminare Unterströmung vorhergesagt werden. 
20. Ganz allgemein sind Turbulenzmodelle für Reynolds-Zahlen größer 10.000 kalibriert und können nicht für laminare Strömungen extrapoliert werden (dafür sind Transitionsmodelle notwendig, das sei aber nur am Rande erwähnt).

21. Sie würden also die Impulsverlustdicken falsch berechnen (bis zu 25\%).

22. Daher gibt es bei $\omega$-basierten Modellen (SST- $k-\omega$, RSM- $\omega$, RSM-BSL) die Option der sog. „LowRe-Korrektur".

23. Für Zellen mit kleiner Zellen-Reynolds-Zahl wird dann der $\omega$-Ansatz korrigiert, sodass die betroffenen Zellen de facto laminar gerechnet werden.

24. Für welche wandnächsten Zellen das zutrifft, das entscheidet der Solver bei jedem Iterationsschritt wieder aufs Neue.

25. In Ansys CFX kann ist für $\omega$-basierte Modelle nur das „Automatic Wall Treatment“ verfügbar. Anhand des $y^{+}$-Wertes der wandnächsten Zellen entscheidet CFX selbst, ob ein Low-Re-Ansatz gewählt werden kann oder ob mit dem unkorrigierten $\omega$-Turbulenzmodell gerechnet wird. Man ist also immer „auf der sicheren Seite“.

26. Das LES-WALE-Modell (Wall-Adaptive Local Eddy-Viscosity) beinhaltet eine ganz ähnlich funktionierende near-wall damping function, die für Konsistenz der Wirbelviskosität an der Wand sorgt. Sie ist daher die LES-Methode der Wahl. Das Smagorinski-LES-Modell kann das nicht, es ist daher nur für Strömung zu verwenden, bei denen es auf die Wirbelstrukturen selbst und nicht auf die Gegebenheiten an der Wand ankommt.

27. Das LES-WMLES (Wall-Modelled LES) ist ein Hybridmodell, was an der Wand eine RANS-Gleichung löst (siehe oben) und in der freien Strömung eine LES durchführt.

28. Für CFD-basierte Wärmeübergangsuntersuchungen eignen sich daher $\omega$-basierte Modelle mit einem Low-Re-Ansatz am besten, bei denen der dimensionslose Abstand aller Zellen an den (interessierenden) Wänden mindestens einen Wert von $y^{+} \leqslant 1$ aufweist.

Diese Anleitung wurde auch im Wiki des Instituts für Energietechnik an der TU Dresden veröffentlicht [159]. 


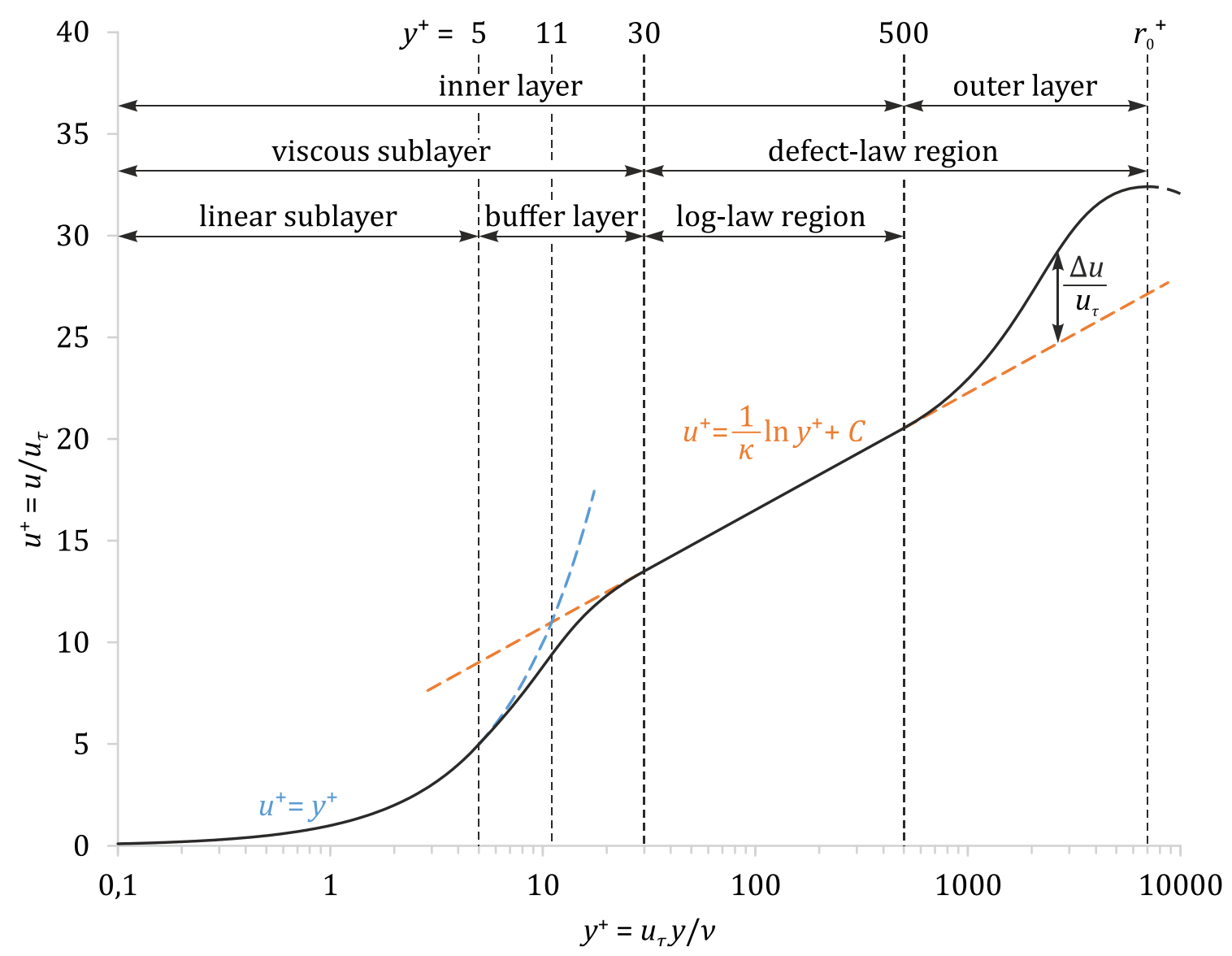

Abb. A.29 Dimensionsloses Geschwindigkeitsprofil und Schichten einer turbulenten Strömung in Wandnähe, hier für eine vollentwickelte Kanalströmung (dimensionsloser Rohrradius $\boldsymbol{r}_{0}^{+}$) (nach [160]).

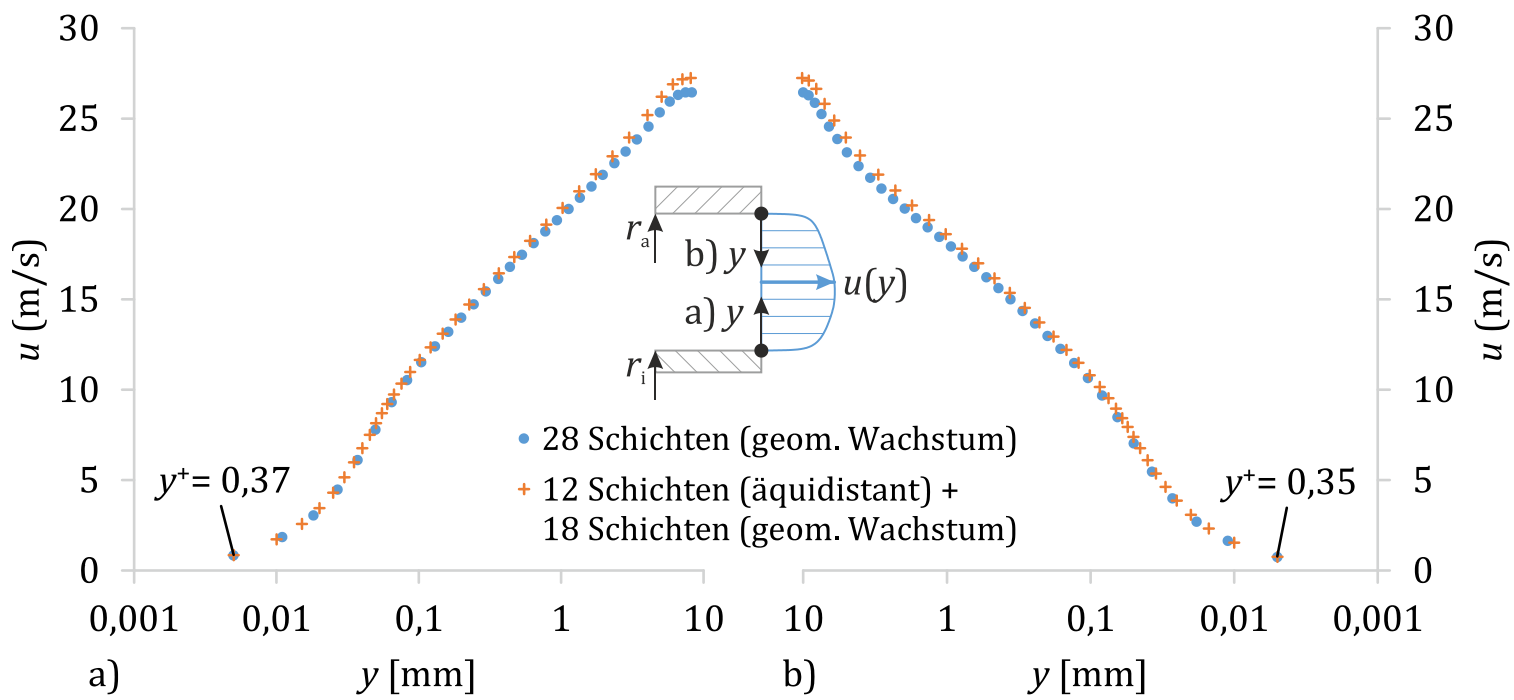

Abb. A.30 Einfluss des Auflösungsgrades der viskosen Unterschicht auf das wandnahe Geschwindigkeitsprofil in der Hauptströmung am Auslass. 2D-Modell, Elementgröße: $1 \mathrm{~mm}$, Höhe der ersten Zelle: $5 \mu \mathrm{m}, k$ - $\omega$-SST, Low-Re-Korrektur, $\hat{\dot{q}}_{\mathrm{W}}=$ konst.

a) Grenzschichtprofil an der Innenoberfläche des Hauptströmungskanals, $y=r-r_{\mathrm{i}}$;

b) Grenzschichtprofil an der Außenoberfläche des Hauptströmungskanals, $y=r_{\mathrm{a}}-r$. 


\section{A15 ERGEBNISSE DER KONJUGIERTEN SIMULATION MIT FLUID- STRUKTUR-KOPPLUNG}

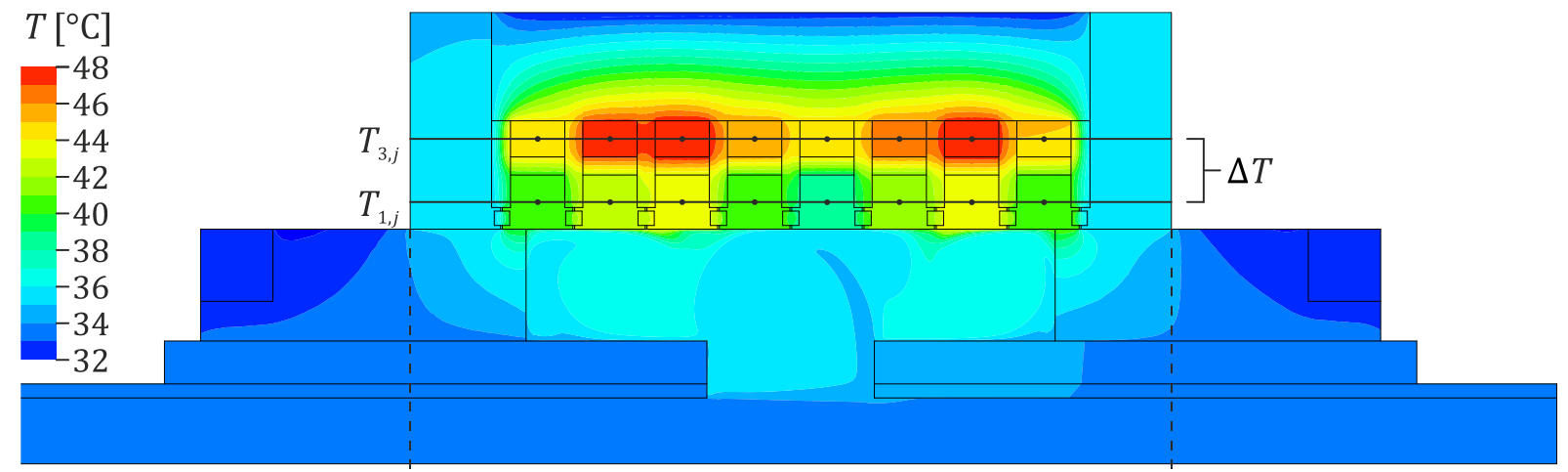

a)

b)

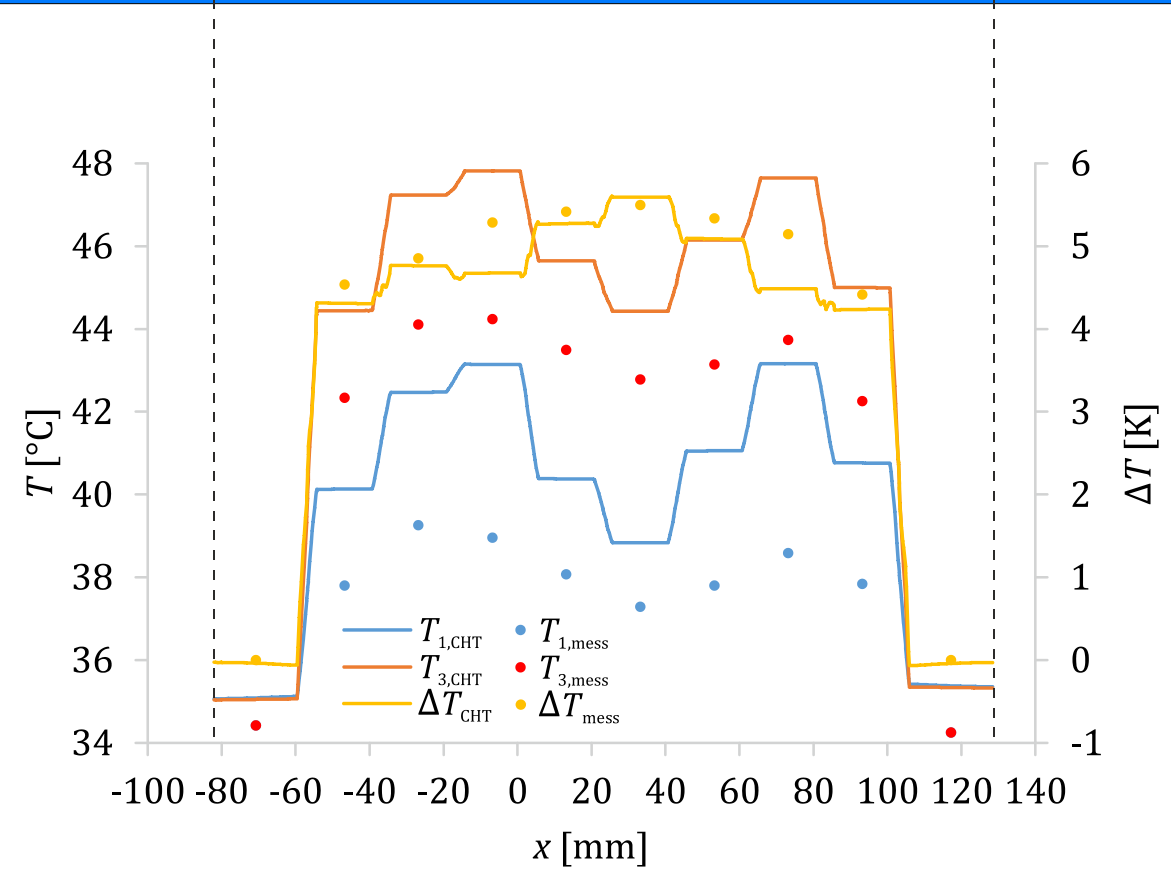

Abb. A.31 Temperaturverteilung im konjugierten Modell;

a) im gesamten Fluid und Solid; b) in der Außenwand im Vergleich mit den gemessenen Ringtemperaturen in den Innenringen $\left(T_{1}\right)$ und in den Außenringen $\left(T_{3}\right)$. 


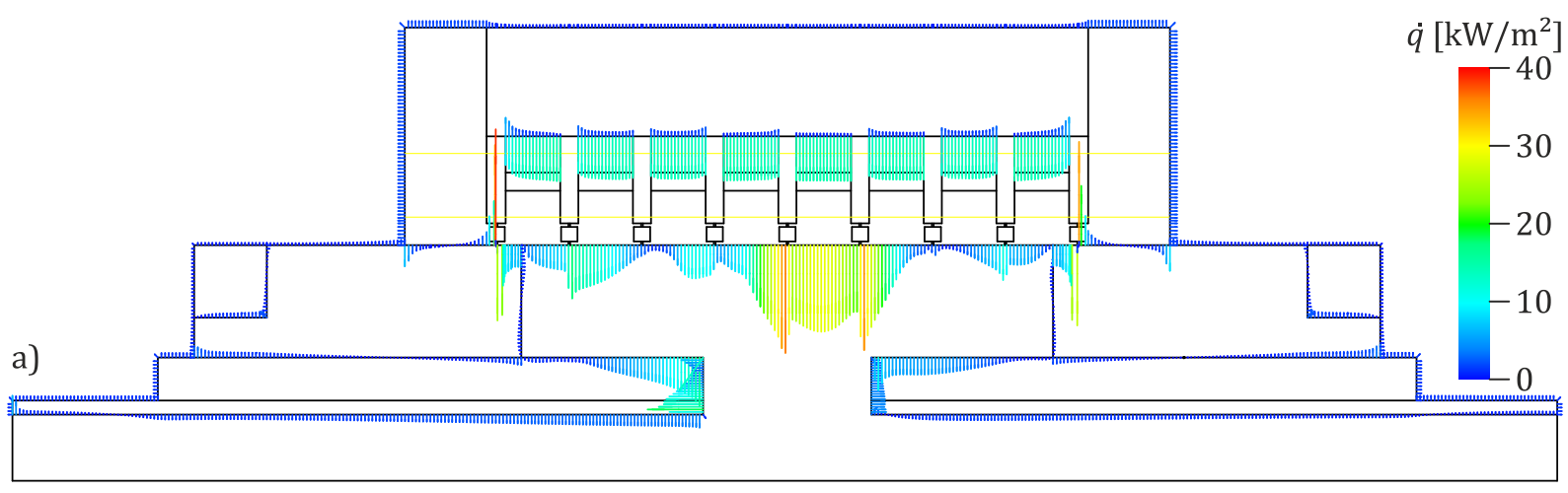

b)
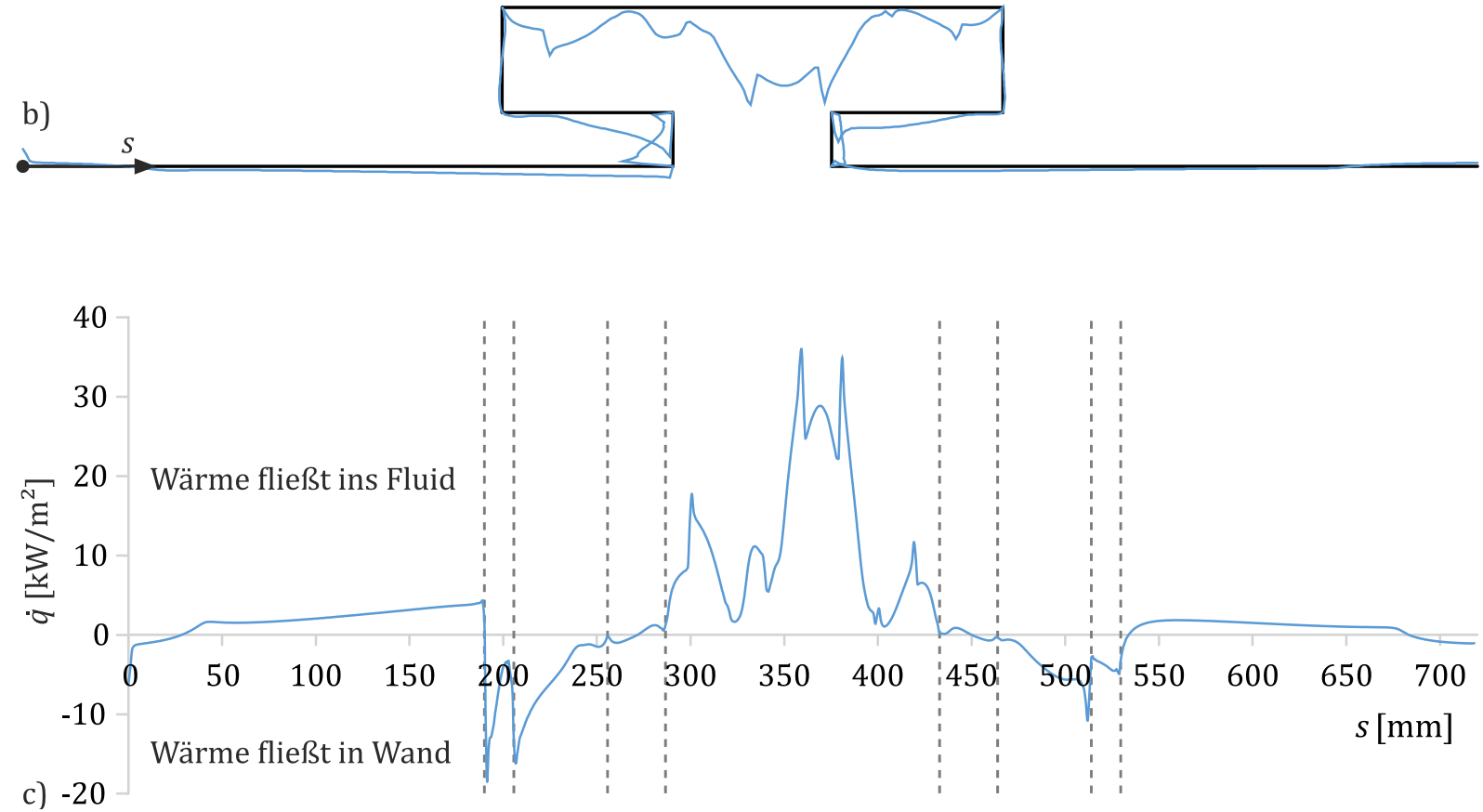

Abb. A.32 Wärmestromdichtenverteilung an ausgewählten Oberflächen des konjugierten Modells;

a) an ausgewählten Oberflächen; b) an der Fluid-Solid-Interface, c) wie b), jedoch mit ausgeklappten Koordinaten über der Laufkoordinate der Interface $s$ (vgl. a. Abb. 4.27). 


\section{A16 VIERTELMODELL DER SAUGROHRSTRECKE}

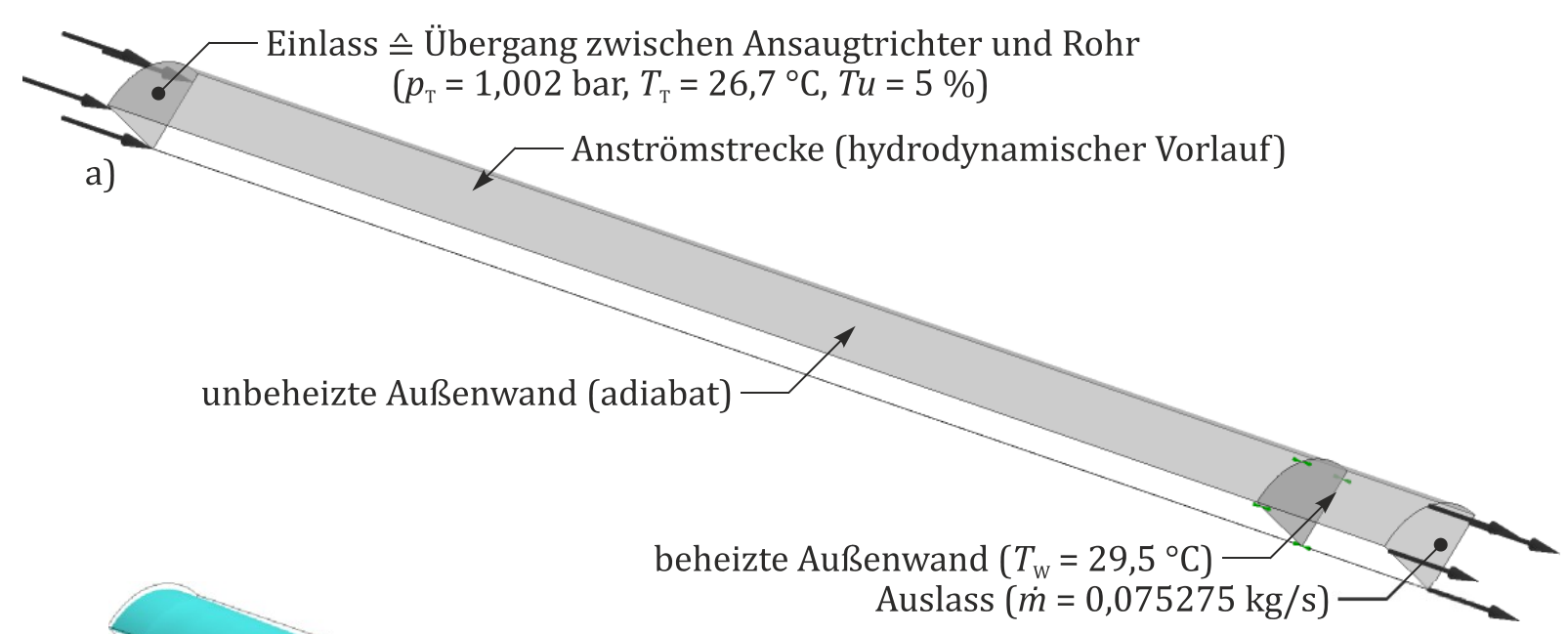

b)

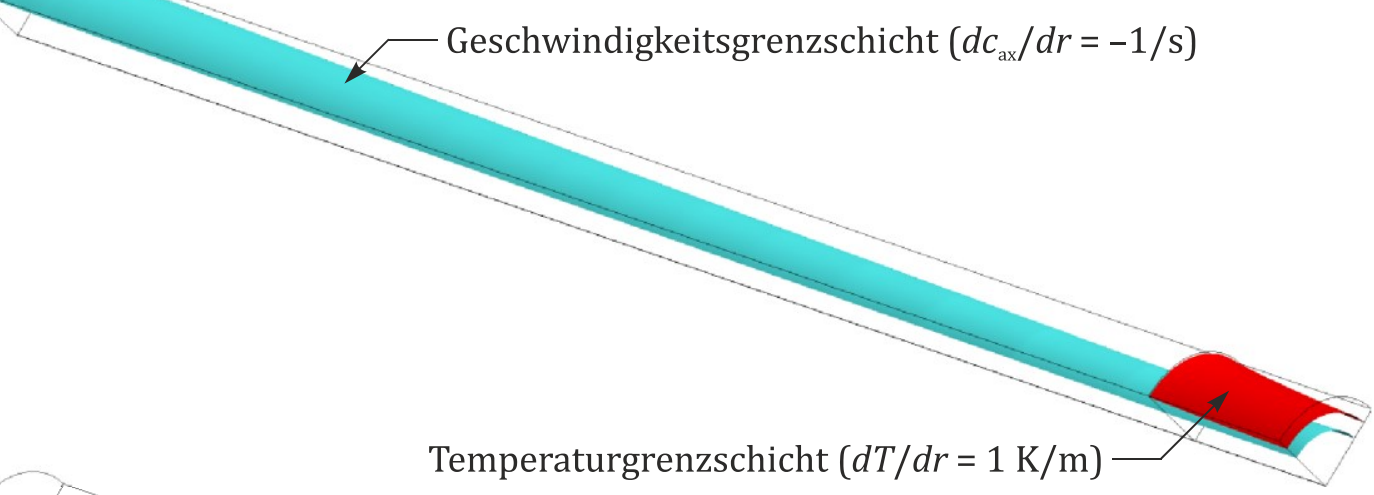

c)

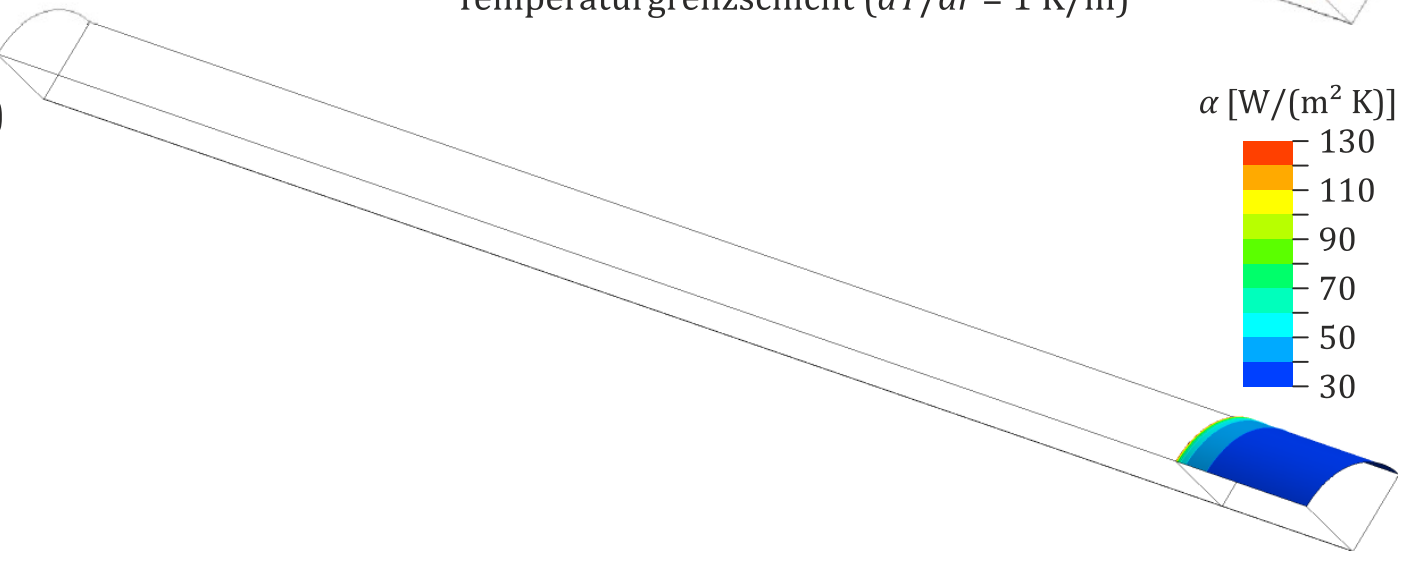

Abb. A.33 CFD-Modell zur Nachrechung der Kalibrierversuche an der Saugrohrstrecke für den Fall $R e=104.700$.

a) Geometrie und Randbedingungen; b) Temperatur- und Geschwindigkeitsgrenzschicht;

c) lokaler WÜK an der beheizten Außenwand. 


\section{A17 VIERTELMODELL DES VERSUCHSSTANDES OHNE TOTRAUM}

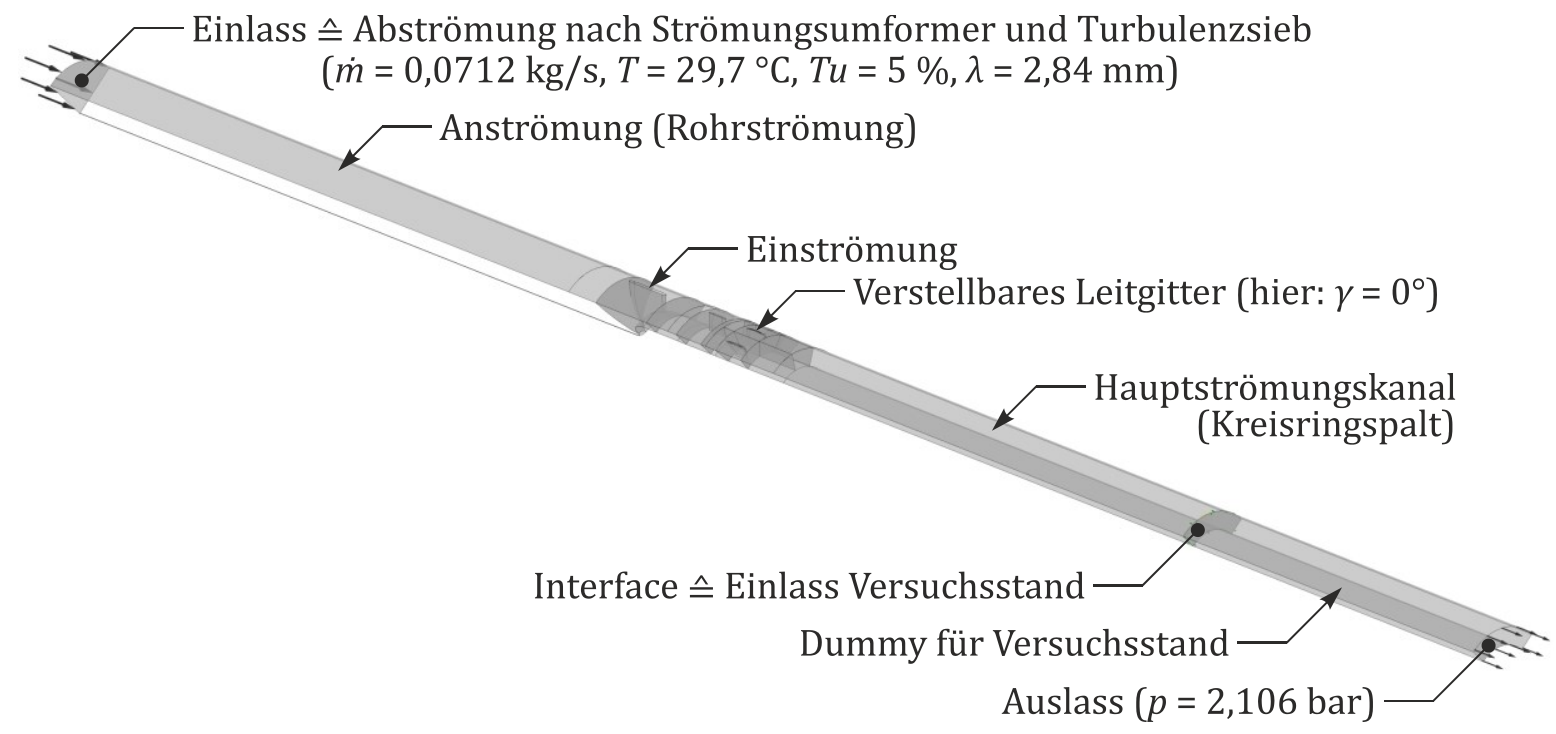

Abb. A.34 Bestandteile (Teilnetze) und ausgewählte Randbedingungen des CFD-Modells des kompletten Versuchsstandes ohne Totraum (Ansys CFX).

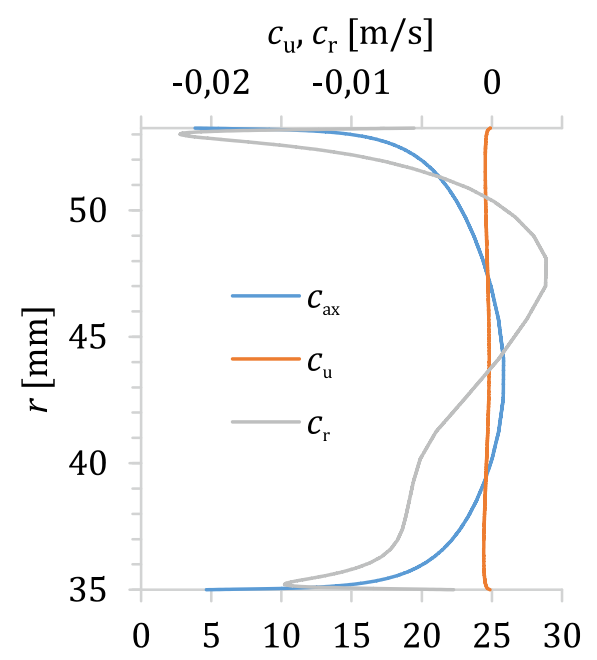

a)

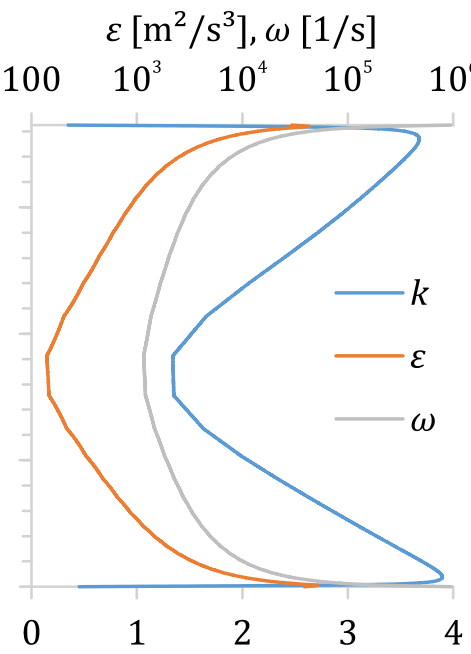

b) $\quad k\left[\mathrm{~m}^{2} / \mathrm{s}^{2}\right]$
$T\left[{ }^{\circ} \mathrm{C}\right], T_{\mathrm{T}}\left[{ }^{\circ} \mathrm{C}\right]$

$29,4 \quad 29,5 \quad 29,6 \quad 29,7 \quad 29,8$

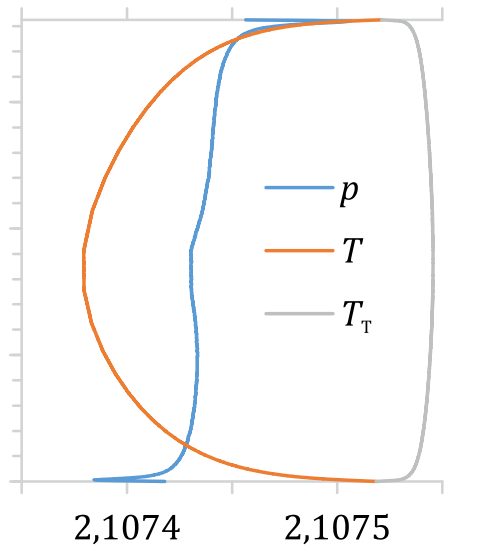

c) $\quad p[\mathrm{bar}]$

Abb. A.35 Radiale Verteilung umfangsgemittelter Strömungsparameter an der Interface zur Versuchsstandsektion (Ansys CFX).

a) Geschwindigkeitskomponenten, b) Turbulenzparameter, c) Druck und Temperatur. 


\section{A18 HERLEITUNG DER ZEITLICHEN MITTELUNG TRANSIENTER WÜK}

$$
\alpha=\frac{\hat{\dot{q}}_{\mathrm{W}}}{T_{\mathrm{W}}-T_{\mathrm{ref}}}
$$

\section{A18.1 Konstante Wandwärmestromdichte}

$$
\begin{aligned}
& \hat{\dot{q}}_{\mathrm{W}}=\text { konst. } \\
& T_{\text {ref }}=\text { konst. }
\end{aligned}
$$

Die Veränderung des WÜK bewirkt eine Veränderung der Wandtemperatur. Eine Mittelung des WÜK muss damit über Mittelung der Wandtemperatur erfolgen. Deren Mittelwert kann arithmetisch gemittelt werden, ist jedoch ebenfalls unbekannt. Um zusätzlichen Speicher zu sparen, der für das Herausschreiben der Temperatur benötigt wird, kann der über $n$ Iterationen gemittelte WÜK $\bar{\alpha}$ wie folgt aus den Einzelwerten $\alpha_{i}$ berechnet werden:

$$
\begin{array}{cc}
\bar{\alpha}=\frac{\hat{\dot{q}}_{\mathrm{W}}}{\bar{T}_{\mathrm{W}}-T_{\mathrm{ref}}} & \alpha_{i}=\frac{\hat{\dot{q}}_{\mathrm{W}}}{T_{\mathrm{W}, i}-T_{\mathrm{ref}}} \\
\bar{T}_{\mathrm{W}}=T_{\mathrm{ref}}+\frac{\hat{\dot{q}}_{\mathrm{W}}}{\bar{\alpha}} & T_{\mathrm{W}, i}=T_{\mathrm{ref}}+\frac{\hat{\dot{q}}_{\mathrm{W}}}{\alpha_{i}} \\
\bar{T}_{\mathrm{W}}=\frac{1}{n} \sum_{i=1}^{n} T_{\mathrm{W}, i} \\
T_{\mathrm{ref}}+\frac{\hat{\dot{q}}_{\mathrm{W}}}{\bar{\alpha}}=\frac{1}{n} \sum_{i=1}^{n}\left(T_{\mathrm{ref}}+\frac{\hat{\dot{q}}_{\mathrm{W}}}{\alpha_{i}}\right) \\
T_{\mathrm{ref}}+\hat{\dot{q}}_{\mathrm{W}} \frac{1}{\bar{\alpha}}=T_{\mathrm{ref}}+\hat{\dot{q}}_{\mathrm{W}} \cdot \frac{1}{n} \sum_{i=1}^{n} \frac{1}{\alpha_{i}} \\
\frac{1}{\bar{\alpha}}=\frac{1}{n} \sum_{i=1}^{n} \frac{1}{\alpha_{i}}
\end{array}
$$

Für konstante Wandwärmestromdichte müssen die WÜK also harmonisch gemittelt werden. 


\section{A18.2 Konstante Wandtemperatur}

$$
\begin{aligned}
& T_{\mathrm{W}}=\text { konst. } \\
& T_{\text {ref }}=\text { konst. }
\end{aligned}
$$

Die Veränderung des WÜK bewirkt eine Veränderung der Wandwärmestromdichte. Eine Mittelung des WÜK muss damit über Mittelung der Wandwärmestromdichte erfolgen. Deren Mittelwert kann arithmetisch gemittelt werden, ist jedoch ebenfalls unbekannt. Um zusätzlichen Speicher zu sparen, der für das Herausschreiben der Temperatur benötigt wird, kann der über $n$ Iterationen gemittelte WÜK $\bar{\alpha}$ wie folgt aus den Einzelwerten $\alpha_{i}$ berechnet werden:

$$
\begin{aligned}
\bar{\alpha}=\frac{\overline{\hat{\dot{q}}}_{\mathrm{W}}}{T_{\mathrm{W}}-T_{\mathrm{ref}}} & \alpha_{i}=\frac{\hat{\hat{q}}_{\mathrm{W}, i}}{T_{\mathrm{W}}-T_{\mathrm{ref}}} \\
\overline{\hat{\dot{q}}}_{\mathrm{W}}=\bar{\alpha} \cdot\left(T_{\mathrm{W}}-T_{\mathrm{ref}}\right) & \hat{\dot{q}}_{\mathrm{W}, i}=\alpha_{i} \cdot\left(T_{\mathrm{W}}-T_{\mathrm{ref}}\right) \\
\overline{\hat{\dot{q}}}_{\mathrm{W}}=\frac{1}{n} \sum_{i=1}^{n} \hat{\hat{q}}_{\mathrm{W}, i} & \\
\bar{\alpha} \cdot\left(T_{\mathrm{W}}-T_{\mathrm{ref}}\right) & =\frac{1}{n} \sum_{i=1}^{n}\left[\alpha_{i} \cdot\left(T_{\mathrm{W}}-T_{\mathrm{ref}}\right)\right] \\
\bar{\alpha} \cdot\left(T_{\mathrm{W}}-T_{\mathrm{ref}}\right) & =\left(T_{\mathrm{W}}-T_{\mathrm{ref}}\right) \cdot \frac{1}{n} \sum_{i=1}^{n} \alpha_{i} \\
\bar{\alpha} & =\frac{1}{n} \sum_{i=1}^{n} \alpha_{i}
\end{aligned}
$$

Für konstanten Wandtemperatur müssen die WÜK also arithmetisch gemittelt werden. 


\section{A18.3 Konjugierte Rechnung}

$$
\begin{aligned}
& \hat{\dot{q}}_{\mathrm{W}}, T_{\mathrm{W}} \neq \text { konst. } \\
& T_{\text {ref }}=\text { konst. }
\end{aligned}
$$

Die Veränderung des WÜK bewirkt eine Veränderung von Wandwärmestromdichte und Wandtemperatur. Eine Mittelung des WÜK kann damit entweder über Mittelung der Wandwärmestromdichte oder der Wandtemperatur erfolgen, jedoch nicht über beide. Somit kann nur der Speicher für eine der beiden Größen gespart werden. Der über $n$ Iterationen gemittelte WÜK $\bar{\alpha}$ kann demnach auf zwei verschiedene Arten wie folgt aus den Einzelwerten $\alpha_{i}$ berechnet werden:

$$
\begin{array}{cr}
\bar{\alpha}=\frac{\overline{\hat{\dot{q}}}_{\mathrm{W}}}{\bar{T}_{\mathrm{W}}-T_{\mathrm{ref}}} & \alpha_{i}=\frac{\hat{\dot{q}}_{\mathrm{W}, i}}{T_{\mathrm{W}, i}-T_{\mathrm{ref}}} \\
\overline{\hat{\dot{q}}}_{\mathrm{W}}=\frac{1}{n} \sum_{i=1}^{n} \hat{\dot{q}}_{\mathrm{W}, i} & \bar{T}_{\mathrm{W}}=\frac{1}{n} \sum_{i=1}^{n} T_{\mathrm{W}, i}
\end{array}
$$

Variante 1

Variante 2

$$
\begin{aligned}
& \bar{\alpha} \cdot\left(\frac{1}{n} \sum_{i=1}^{n}\left\{T_{\mathrm{W}, i}\right\}-T_{\mathrm{ref}}\right)=\frac{1}{n} \sum_{i=1}^{n}\left[\alpha_{i} \cdot\left(T_{\mathrm{W}, i}-T_{\mathrm{ref}}\right)\right] \quad \bar{\alpha} \cdot\left(\frac{1}{n} \sum_{i=1}^{n}\left\{T_{\mathrm{ref}}+\frac{\hat{\dot{q}}_{\mathrm{W}, i}}{\alpha_{i}}\right\}-T_{\mathrm{ref}}\right)=\frac{1}{n} \sum_{i=1}^{n} \hat{\dot{q}}_{\mathrm{W}, i} \\
& \bar{\alpha} \cdot\left(\frac{1}{n} \sum_{i=1}^{n}\left\{T_{\mathrm{W}, i}-T_{\mathrm{ref}}\right\}\right)=\frac{1}{n} \sum_{i=1}^{n}\left[\alpha_{i} \cdot\left(T_{\mathrm{W}, i}-T_{\mathrm{ref}}\right)\right] \quad \bar{\alpha} \cdot\left(T_{\mathrm{ref}}+\frac{1}{n} \sum_{i=1}^{n}\left\{\frac{\hat{\underline{q}}_{\mathrm{W}, i}}{\alpha_{i}}\right\}-T_{\mathrm{ref}}\right)=\frac{1}{n} \sum_{i=1}^{n} \hat{\dot{q}}_{\mathrm{W}, i} \\
& \bar{\alpha}=\frac{\sum_{i=1}^{n}\left[\alpha_{i} \cdot\left(T_{\mathrm{W}, i}-T_{\mathrm{ref}}\right)\right]}{\sum_{i=1}^{n}\left\{T_{\mathrm{W}, i}-T_{\mathrm{ref}}\right\}} \\
& \bar{\alpha}=\frac{\sum_{i=1}^{n} \hat{\dot{q}}_{\mathrm{W}, i}}{\sum_{i=1}^{n}\left\{\frac{\hat{\underline{q}}_{\mathrm{W}, i}}{\alpha_{i}}\right\}}
\end{aligned}
$$

temperaturdifferenzgewichtetes arithmetisches Mittel wärmestromgewichtetes

harmonisches Mittel 


\section{A19 WEITERE ERGEBNISSE DER LARGE-EDDY-SIMULATIONEN}

\section{A19.1 90-Modell, konstante Wandwärmestromdichte (Ansys CFX)}
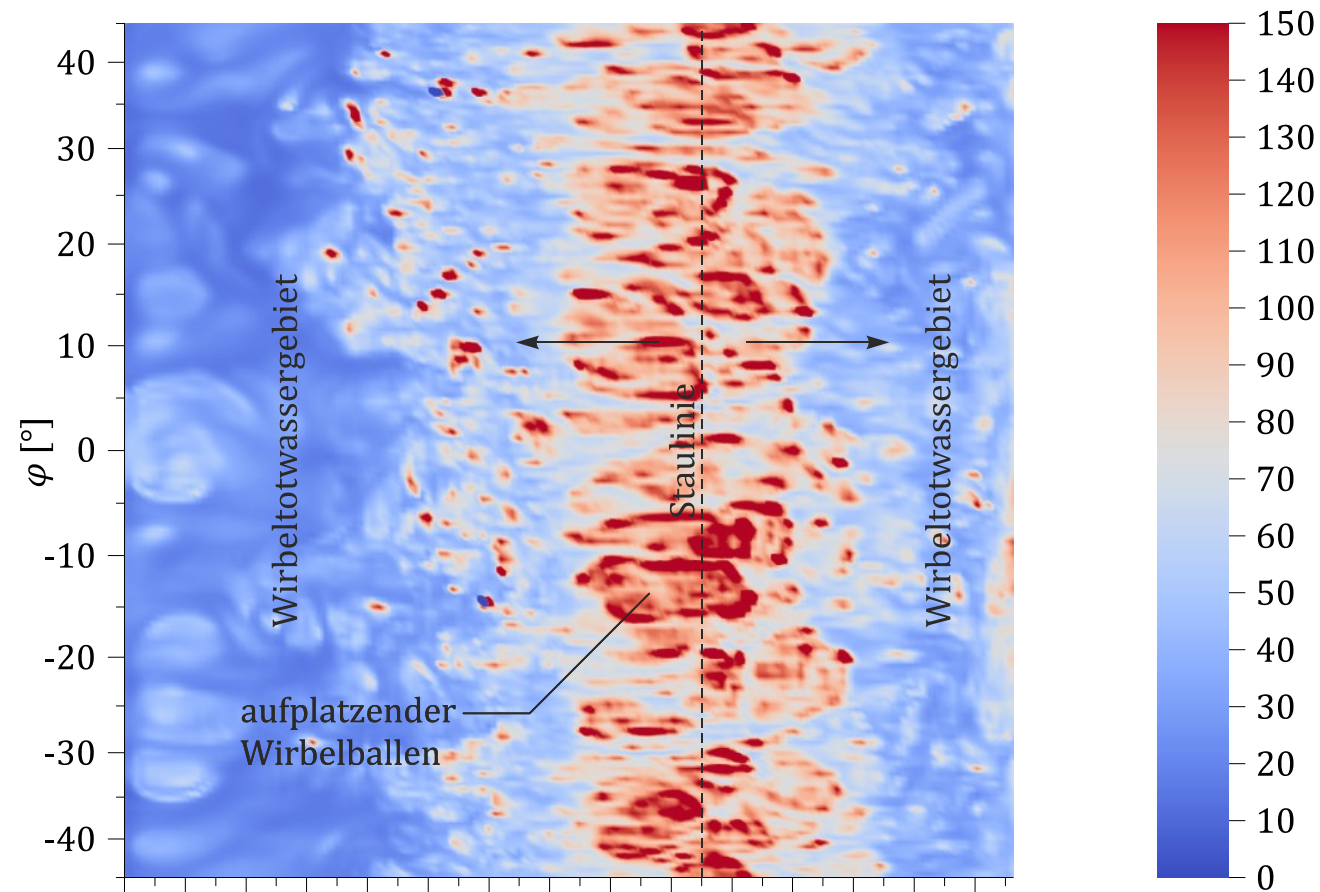

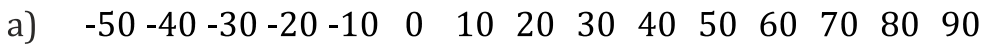
$\alpha\left[\mathrm{W} /\left(\mathrm{m}^{2} \mathrm{~K}\right)\right]$
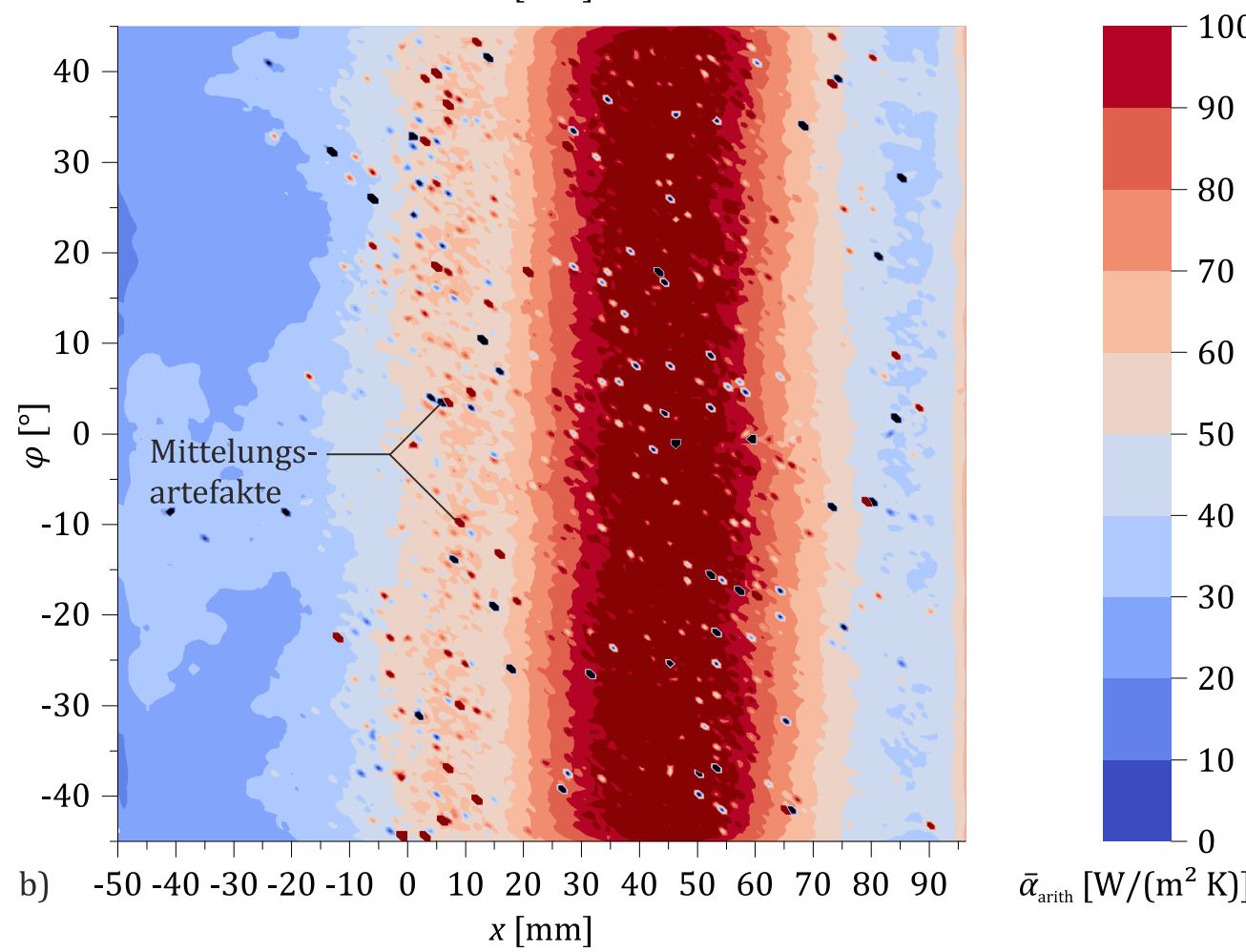

$\bar{\alpha}_{\text {arith }}\left[\mathrm{W} /\left(\mathrm{m}^{2} \mathrm{~K}\right)\right]$

Abb. A.36 Lokale WÜK-Verteilung an der Außenwand des $90^{\circ}$-LES-Modells. $\hat{\dot{q}}_{\mathrm{W}}=$ konst.

a) Momentaufnahme ( $t=1,9975 \mathrm{~s}$ ), b) (fälschlicherweise gebildeter) arithmetischer Mittelwert über die letzten $n=50.000$ Iterationen $(\Delta t=1 \mathrm{~s})$ 


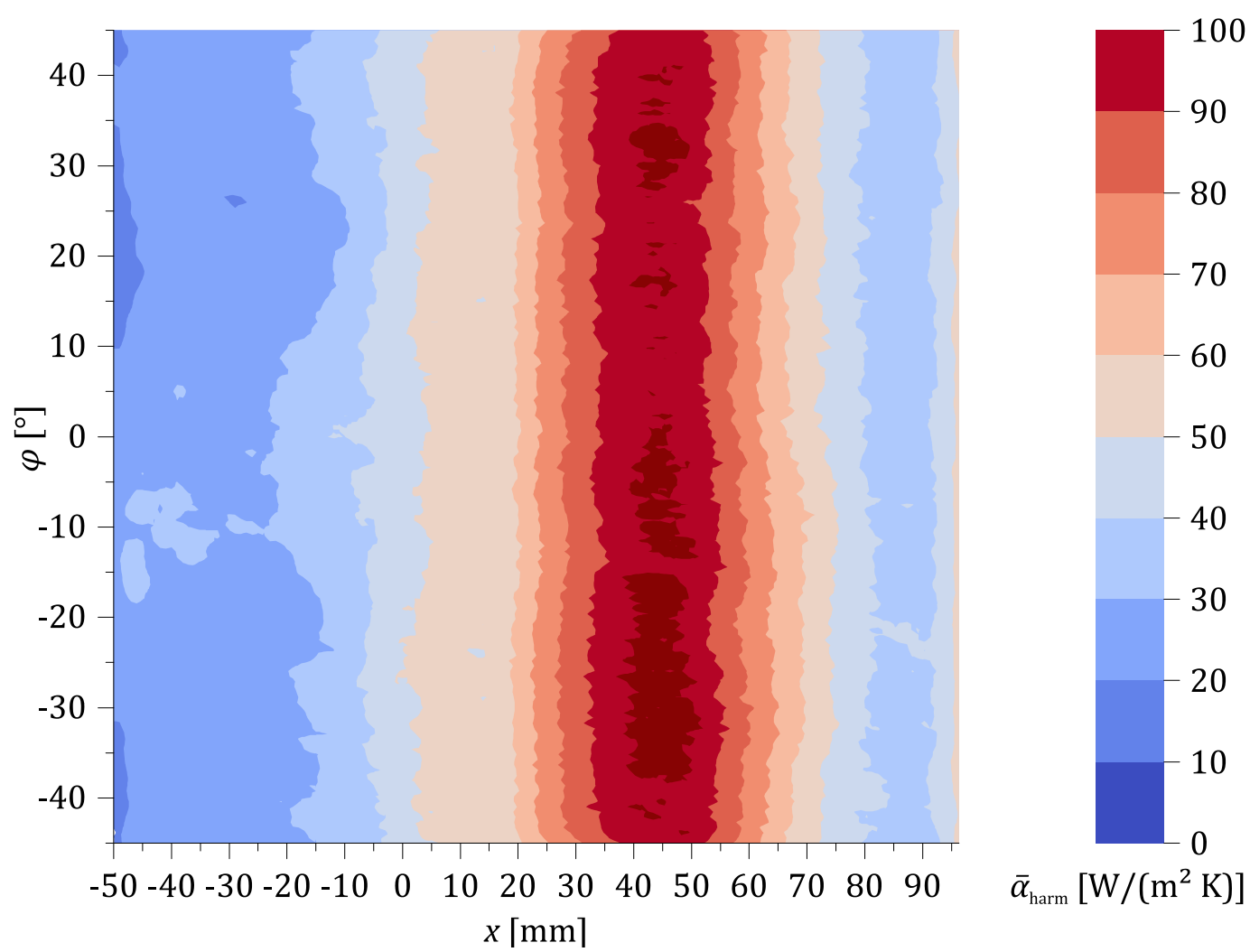

Abb. A.37 Harmonischer Mittelwert der lokalen WÜK-Verteilung an der Außenwand des $90^{\circ}$-LESModells über die letzten $\boldsymbol{n}=\mathbf{5 0 . 0 0 0}$ Iterationen. $\hat{q}_{\mathrm{W}}=$ konst. 

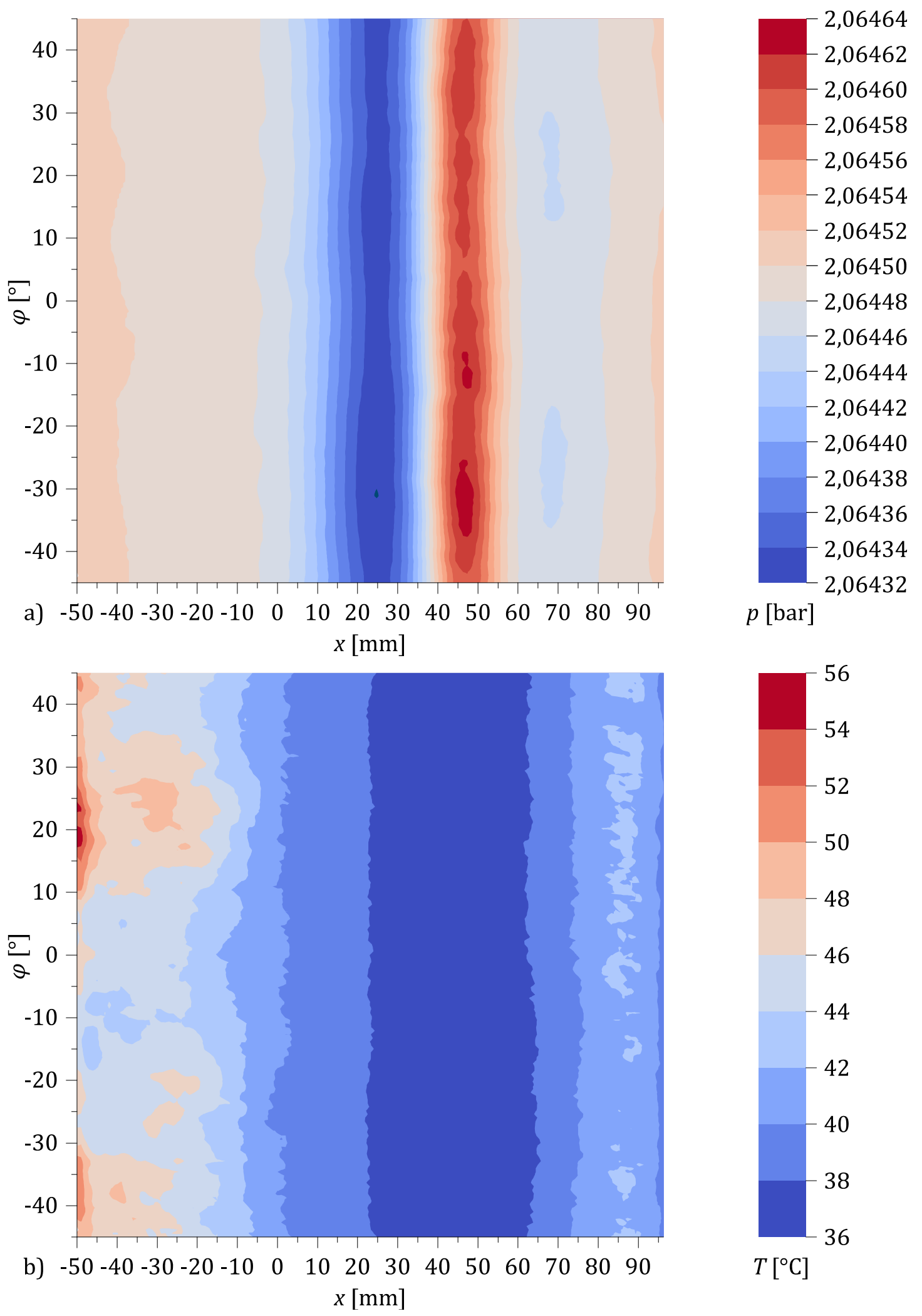

Abb. A.38 Arithmetischer Mittelwert ausgewählter Strömungsparameter an der Außenwand des $\mathbf{9 0}^{\circ}$-LES-Modells über die letzten $\boldsymbol{n}=\mathbf{5 0 . 0 0 0}$ Iterationen. $\hat{\boldsymbol{q}}_{\mathrm{W}}=$ konst.

a) Statischer = Totaldruck; b) Temperatur. 


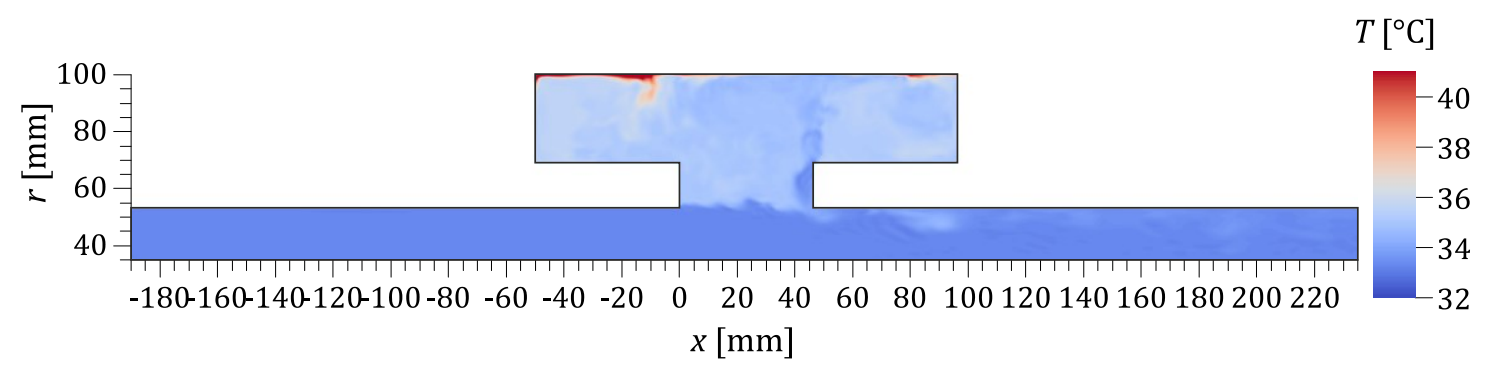

Abb. A.39 Momentaufnahme des Temperaturfeldes an der Symmetrieebene des $90^{\circ}$-LES-Modells. $\hat{\hat{q}}_{\mathrm{W}}=$ konst., $t=1,9969 \mathrm{~s}$.
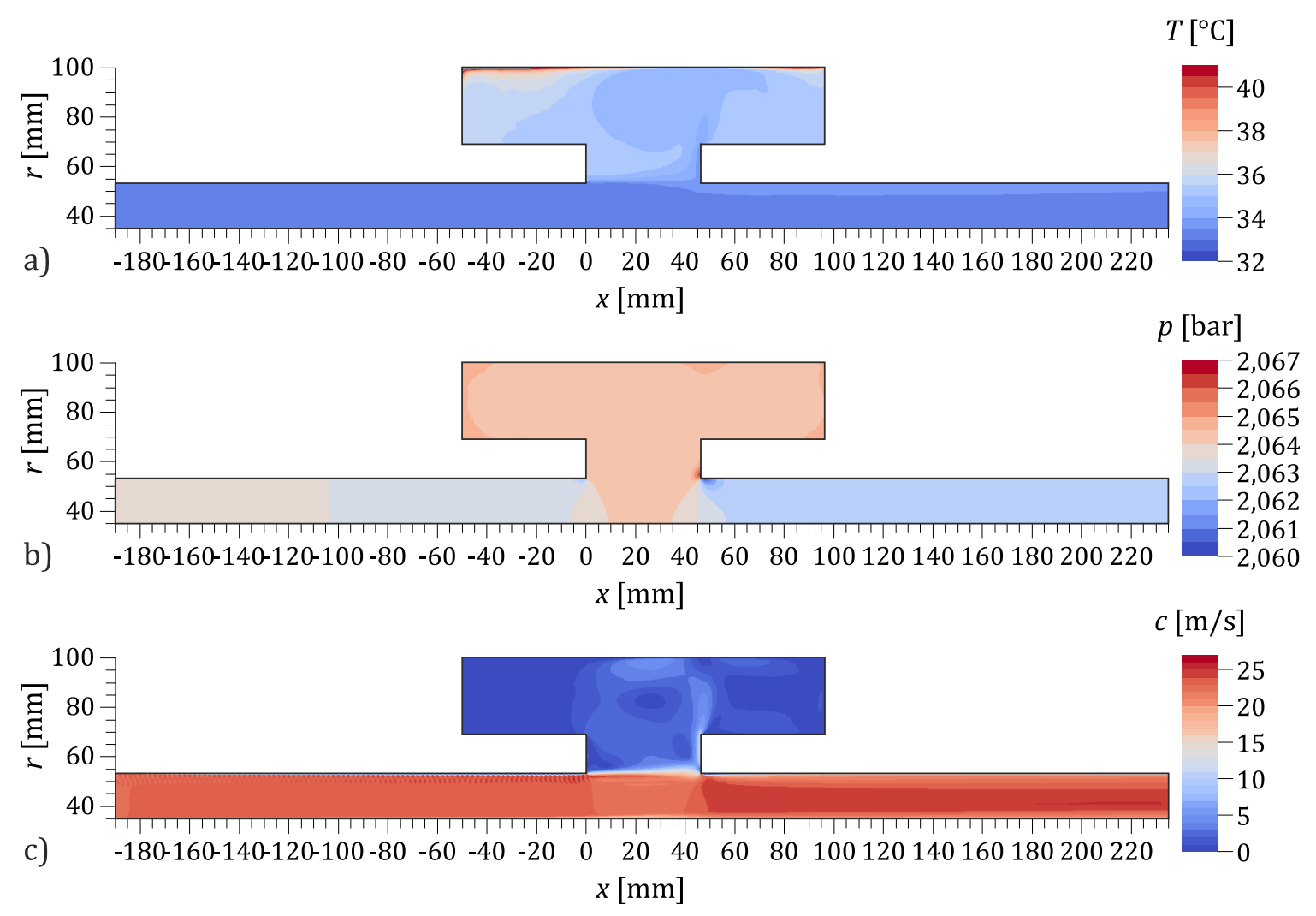

Abb. A.40 Arithmetischer Mittelwert ausgewählter Strömungsparameter an der Symmetrieebene des $90^{\circ}$-LES-Modells über die letzten $\boldsymbol{n}=\mathbf{5 0 . 0 0 0}$ Iterationen. $\hat{\dot{q}}_{\mathrm{w}}=$ konst.

a) Temperatur; b) statischer Druck; c) Geschwindigkeit. 


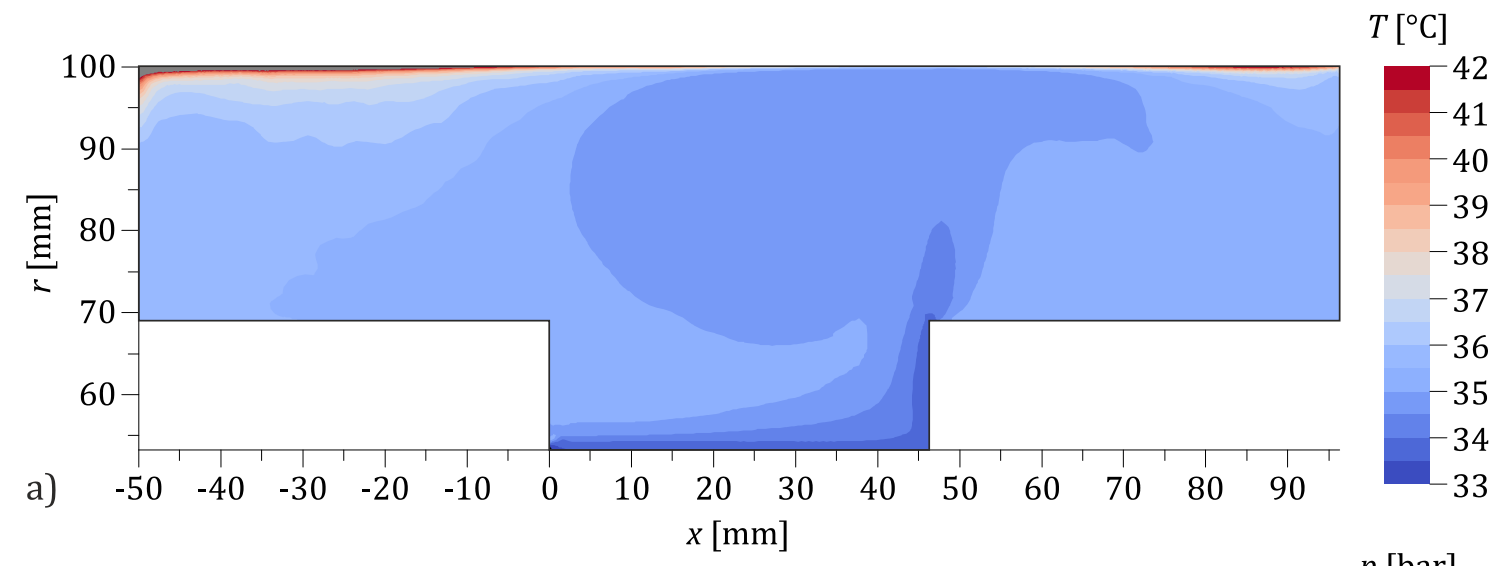

$T\left[{ }^{\circ} \mathrm{C}\right]$

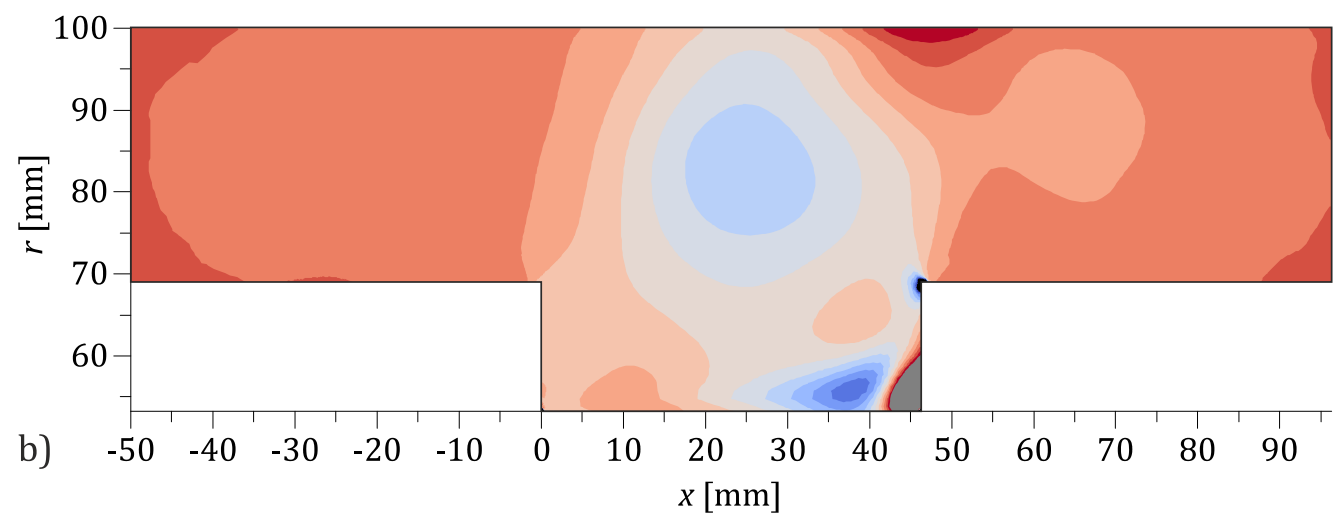

$p$ [bar]
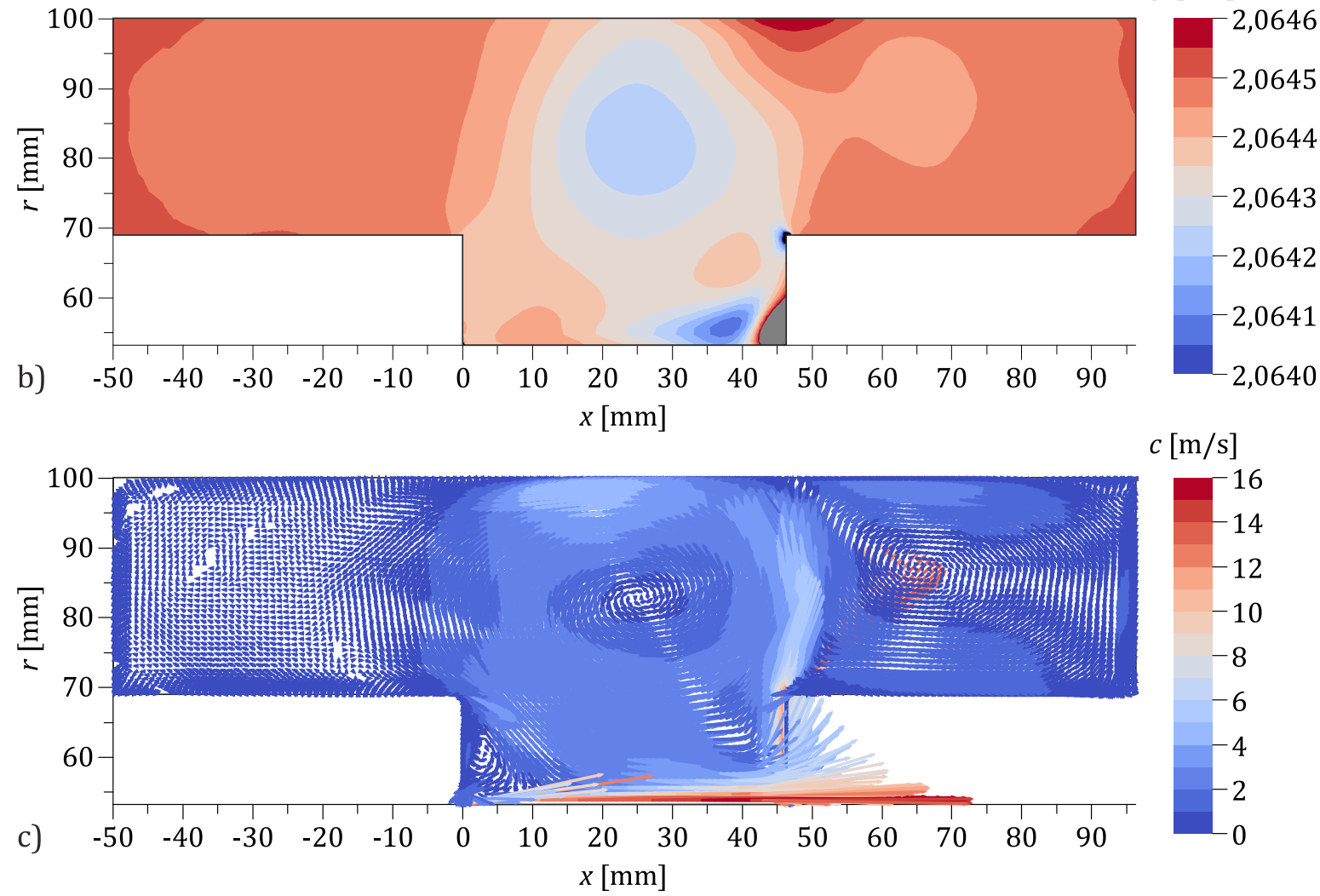

Abb. A.41 Arithmetischer Mittelwert ausgewählter Strömungsparameter im Seitenraum des $90^{\circ}$ LES-Modells über die letzten $\boldsymbol{n}=\mathbf{5 0 . 0 0 0}$ Iterationen. $\hat{q}_{\mathrm{W}}=$ konst.

a) Temperatur; b) statischer Druck; c) Geschwindigkeit. 


\section{A19.2 90-Modell, konstante Wandtemperatur (Ansys CFX)}

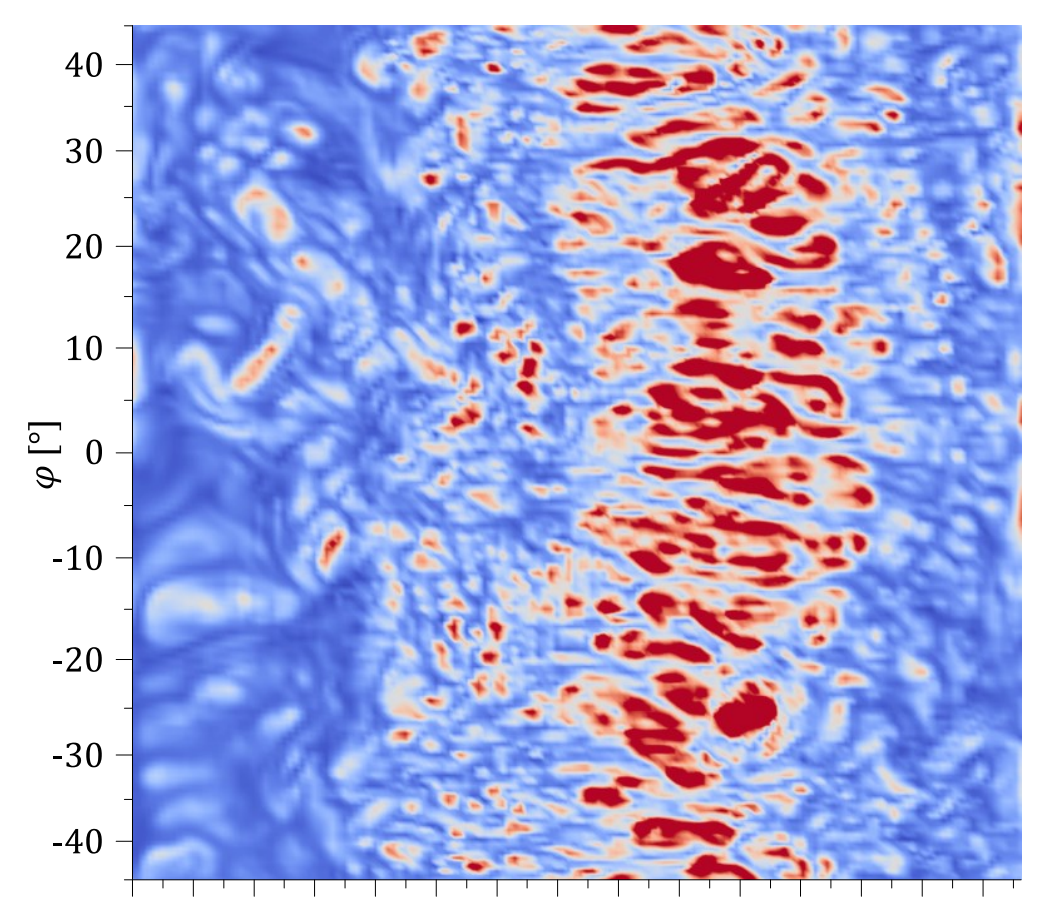

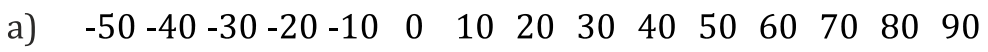
$x[\mathrm{~mm}]$

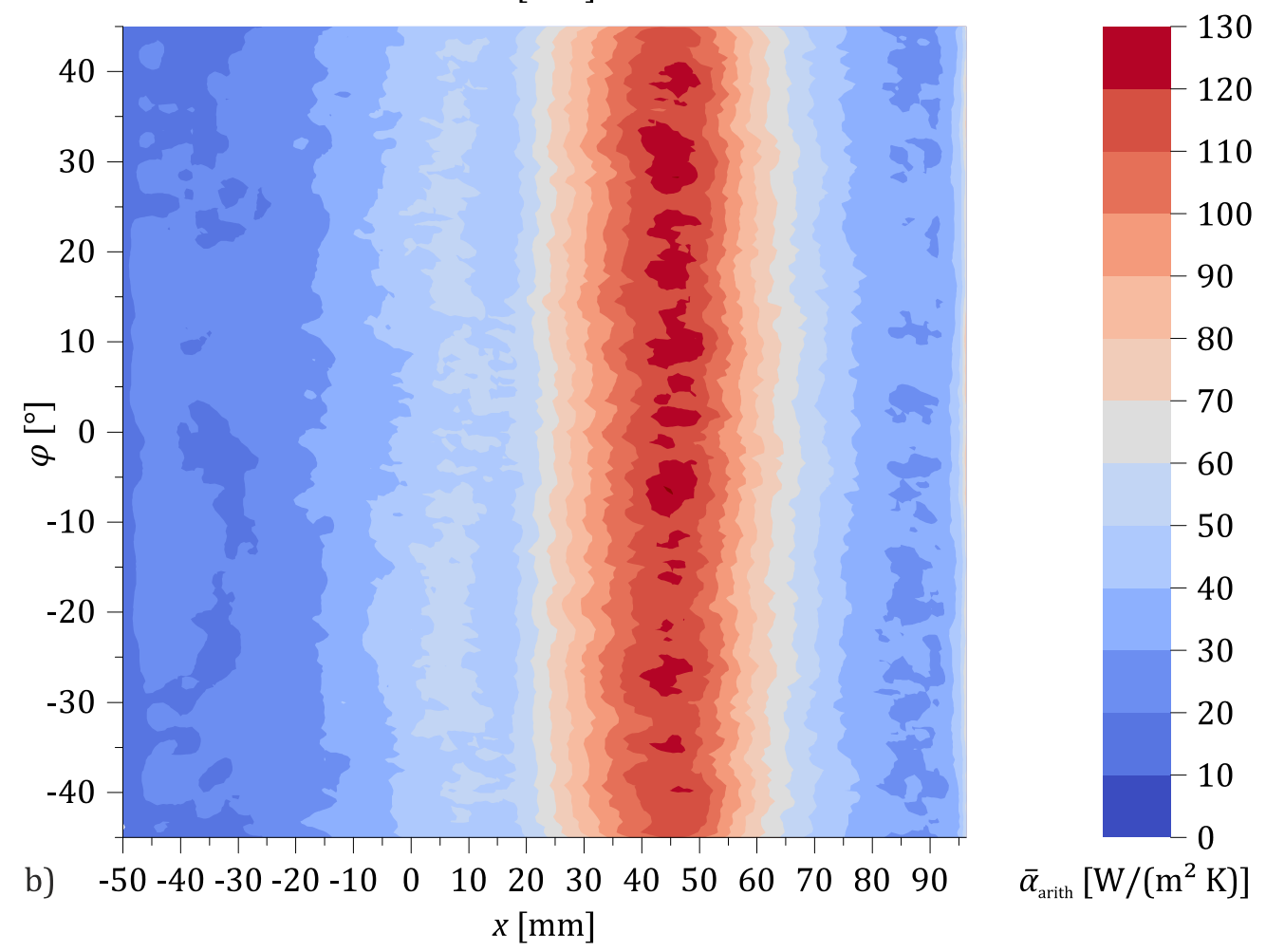

Abb. A.42 Lokale WÜK-Verteilung an der Außenwand des $90^{\circ}$-LES-Modells. $\hat{\dot{q}}_{\mathrm{W}}=$ konst.

a) Momentaufnahme ( $t=1,9998 \mathrm{~s})$, b) arithmetischer Mittelwert über die letzten $n=50.000$ Iterationen $(\Delta t=1 \mathrm{~s})$ 

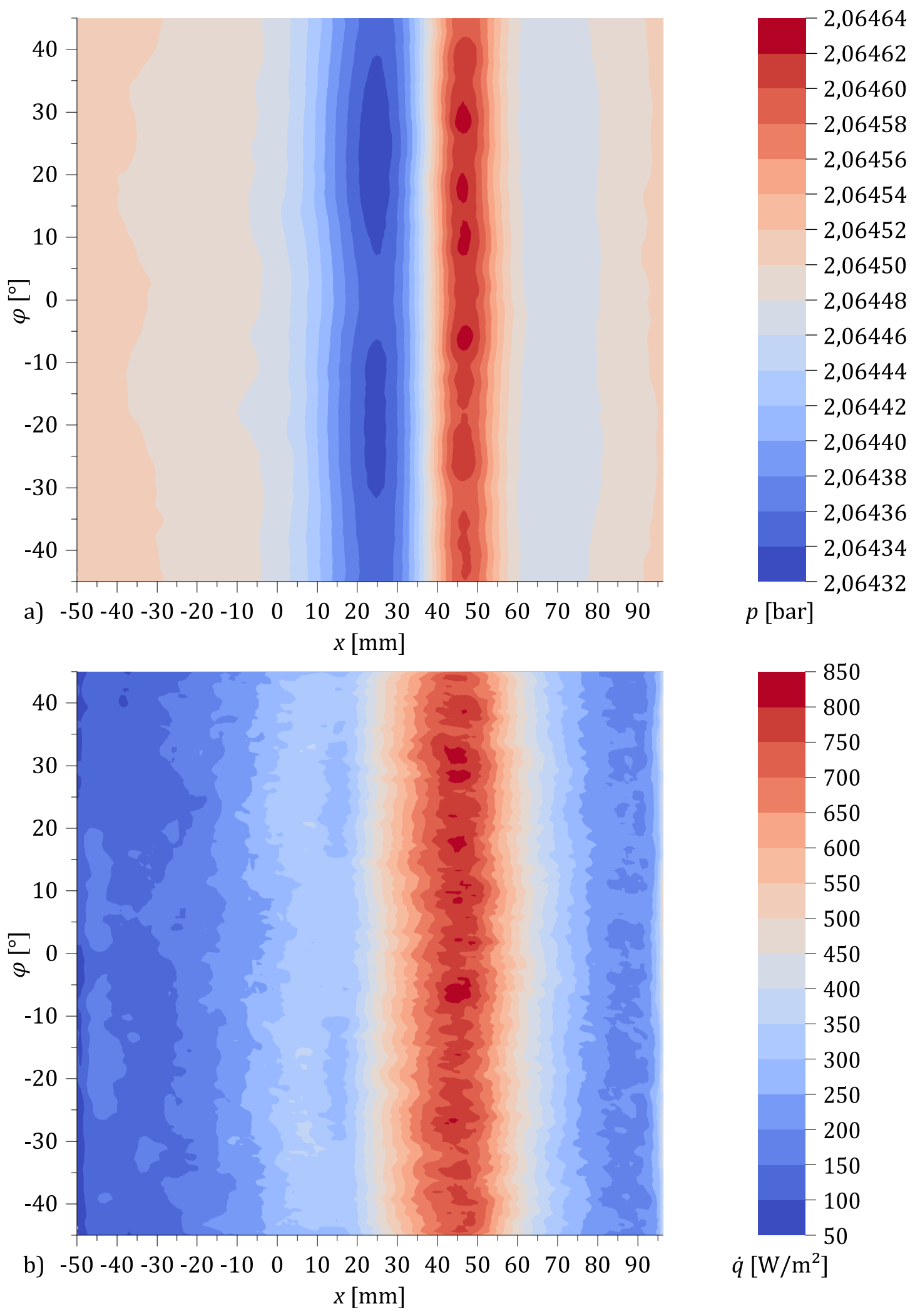

Abb. A.43 Arithmetischer Mittelwert ausgewählter Strömungsparameter an der Außenwand des $\mathbf{9 0}$-LES-Modells über die letzten $\boldsymbol{n}=\mathbf{5 0 . 0 0 0}$ Iterationen. $\mathrm{T}_{\mathrm{W}}=$ konst.

a) Statischer = Totaldruck; b) Wärmestromdichte. 


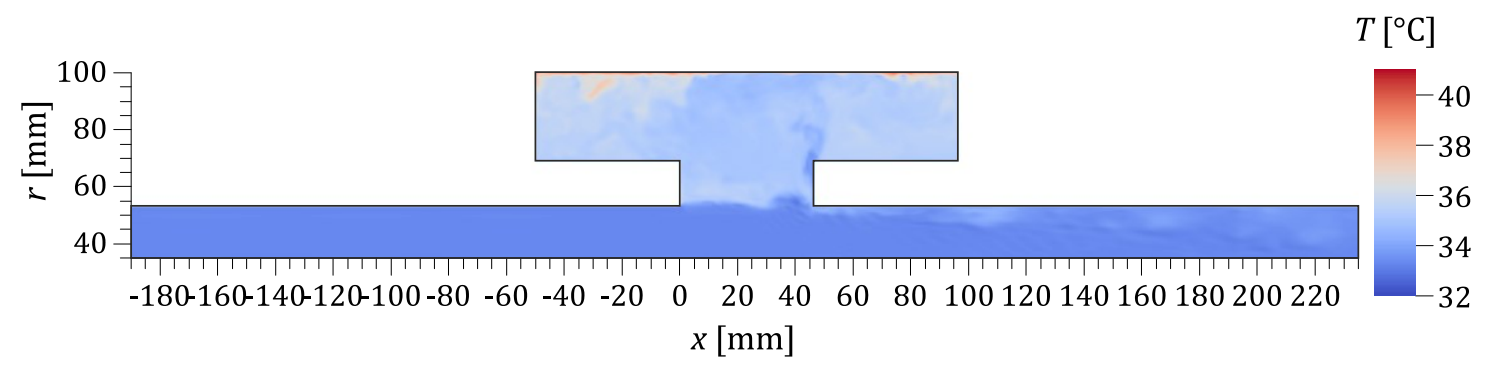

Abb. A.44 Momentaufnahme des Temperaturfeldes an der Symmetrieebene des $90^{\circ}$-LES-Modells. $\mathrm{T}_{\mathrm{W}}=$ konst., $t=1,9998 \mathrm{~s}$.
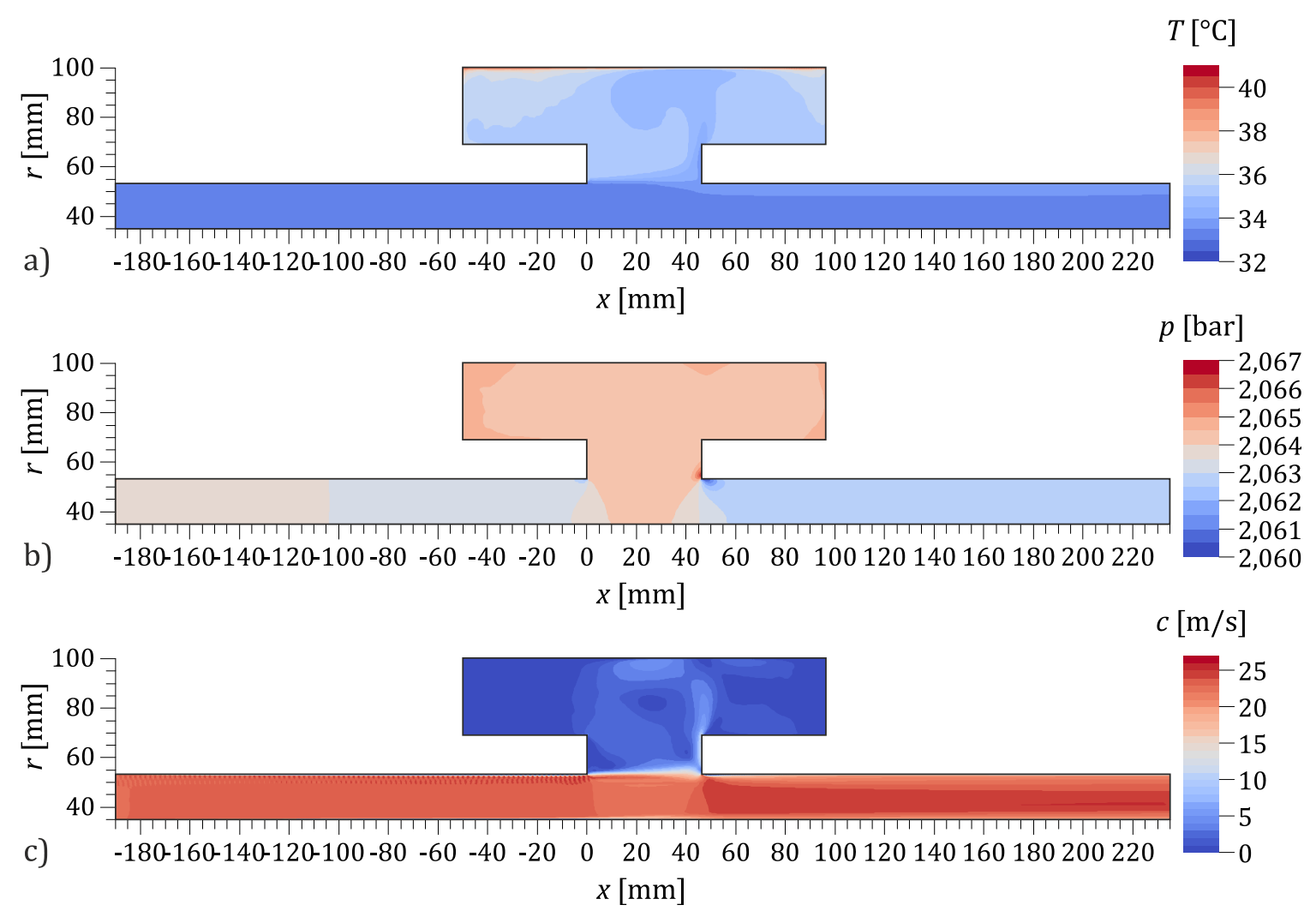

Abb. A.45 Arithmetischer Mittelwert ausgewählter Strömungsparameter an der Symmetrieebene des $\mathbf{9 0}{ }^{\circ}$-LES-Modells über die letzten $\boldsymbol{n}=\mathbf{5 0 . 0 0 0}$ Iterationen. $\mathrm{T}_{\mathrm{W}}=$ konst.

a) Temperatur; b) statischer Druck; c) Geschwindigkeit. 

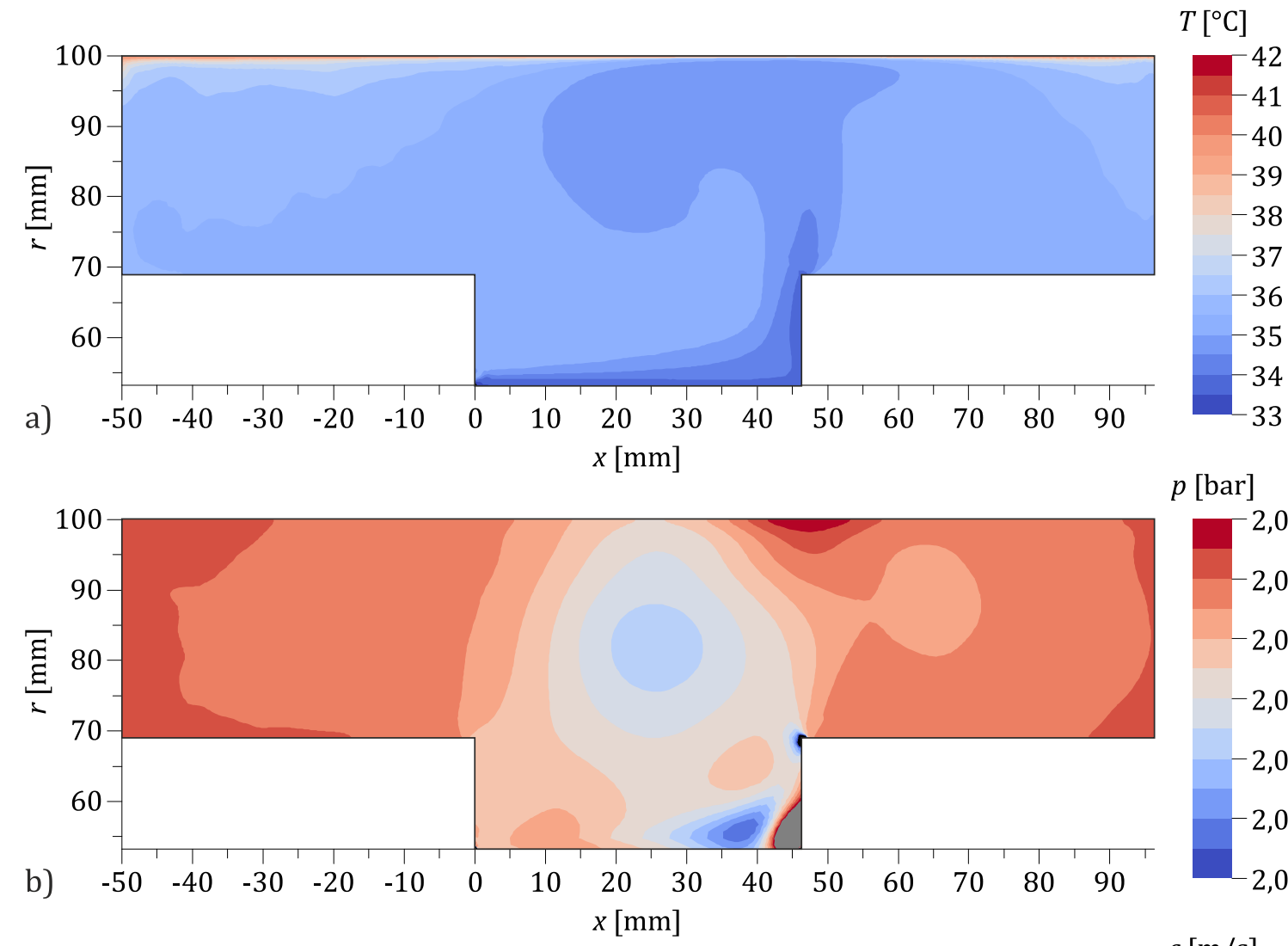

$p$ [bar]
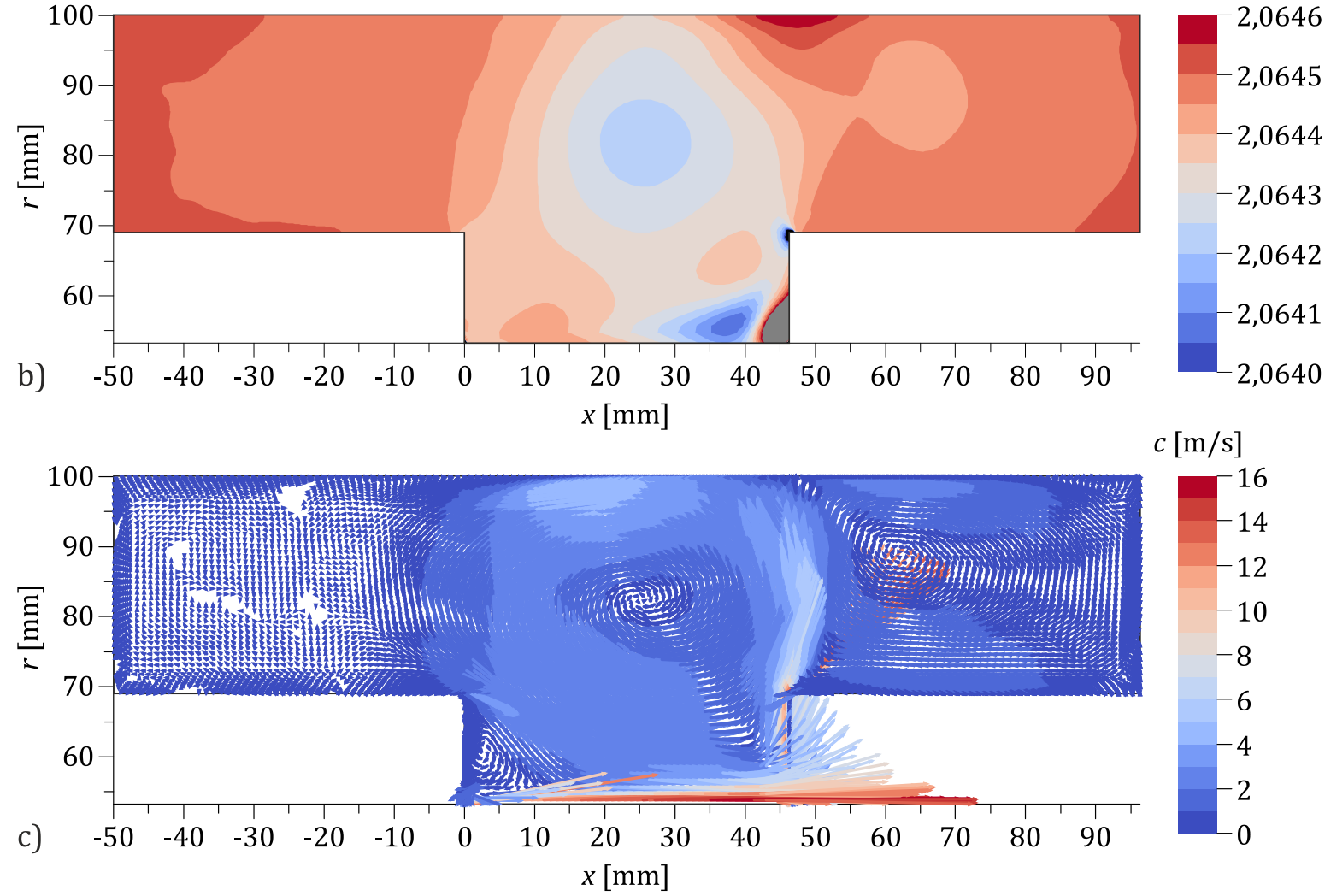

Abb. A.46 Arithmetischer Mittelwert ausgewählter Strömungsparameter im Seitenraum des $90^{\circ}$ LES-Modells über die letzten $\boldsymbol{n}=\mathbf{5 0 . 0 0 0}$ Iterationen. $\mathrm{T}_{\mathrm{W}}=$ konst.

a) Temperatur; b) statischer Druck; c) Geschwindigkeit. 


\section{A19.3 2D-Modell, konstante Wandwärmestromdichte (Ansys Fluent)}

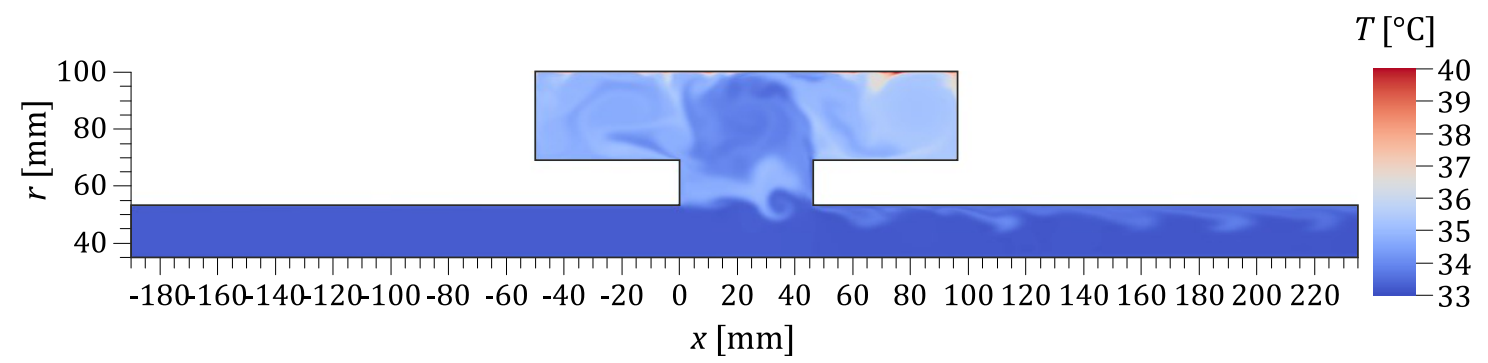

Abb. A.47 Momentaufnahme des Temperaturfeldes im 2D-LES-Modells. $\hat{q}_{\mathrm{W}}=$ konst., $t=1,9923 \mathrm{~s}$.
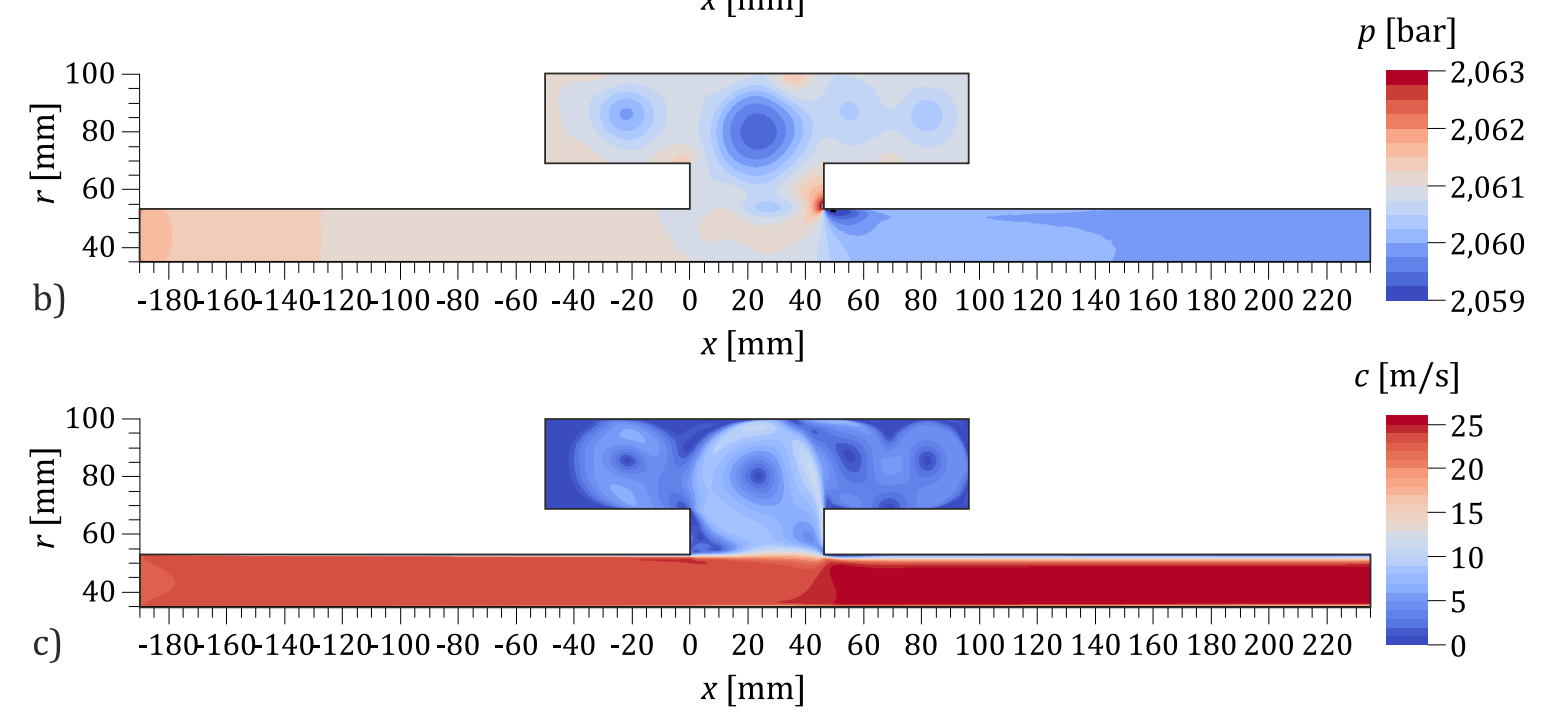

Abb. A.48 Arithmetischer Mittelwert ausgewählter Strömungsparameter im 2D-LES-Modells über die letzten $\boldsymbol{n}=\mathbf{5 0 . 0 0 0}$ Iterationen. $\hat{\dot{q}}_{\mathrm{W}}=$ konst.

a) Temperatur; b) statischer Druck; c) Geschwindigkeit. 


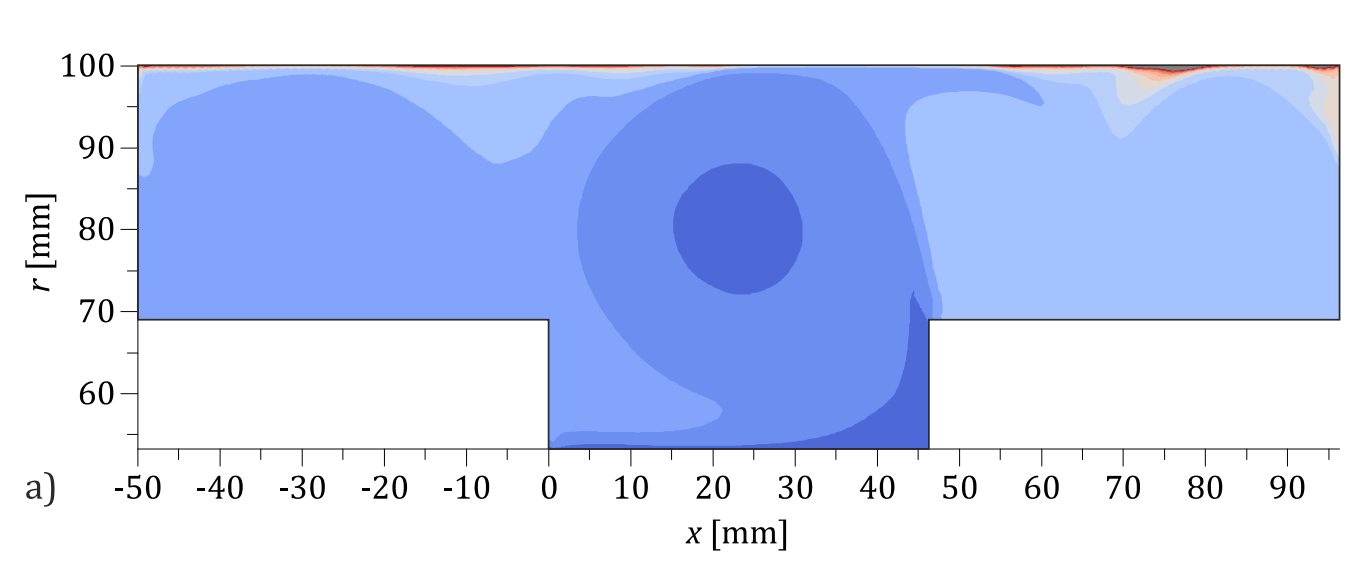

$T\left[{ }^{\circ} \mathrm{C}\right]$

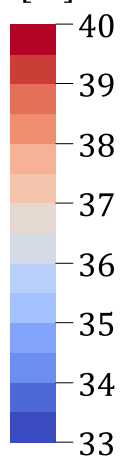

$p$ [bar]
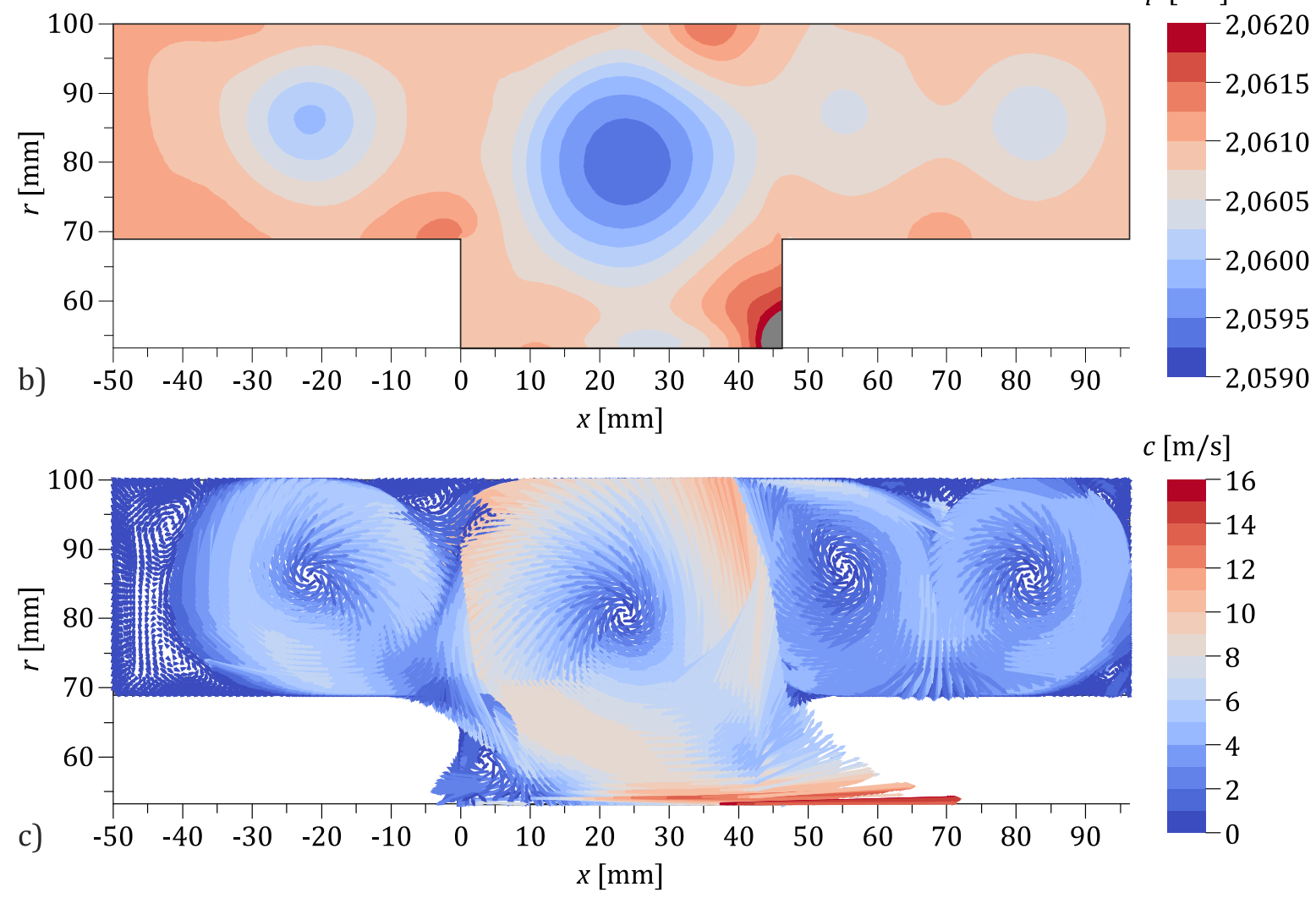

Abb. A.49 Arithmetischer Mittelwert ausgewählter Strömungsparameter im Seitenraum des 2DLES-Modells über die letzten $\boldsymbol{n}=\mathbf{5 0 . 0 0 0}$ Iterationen. $\hat{q}_{\mathrm{W}}=$ konst.

a) Temperatur; b) statischer Druck; c) Geschwindigkeit. 
A19.4 2D-Modell, konstante Wandtemperatur (Ansys Fluent)

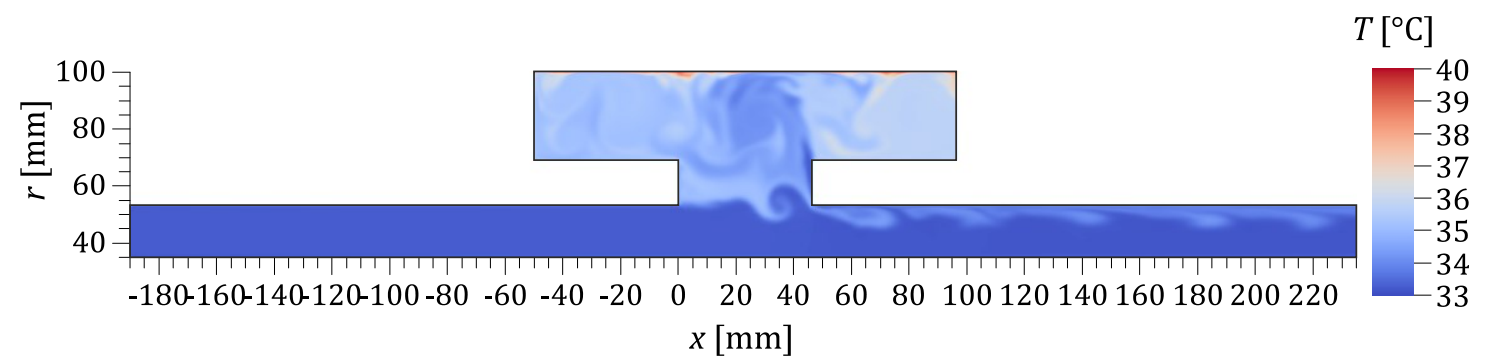

Abb. A.50 Momentaufnahme des Temperaturfeldes im 2D-LES-Modells. $T_{\mathrm{W}}=$ konst., $t=1,9995 \mathrm{~s}$.
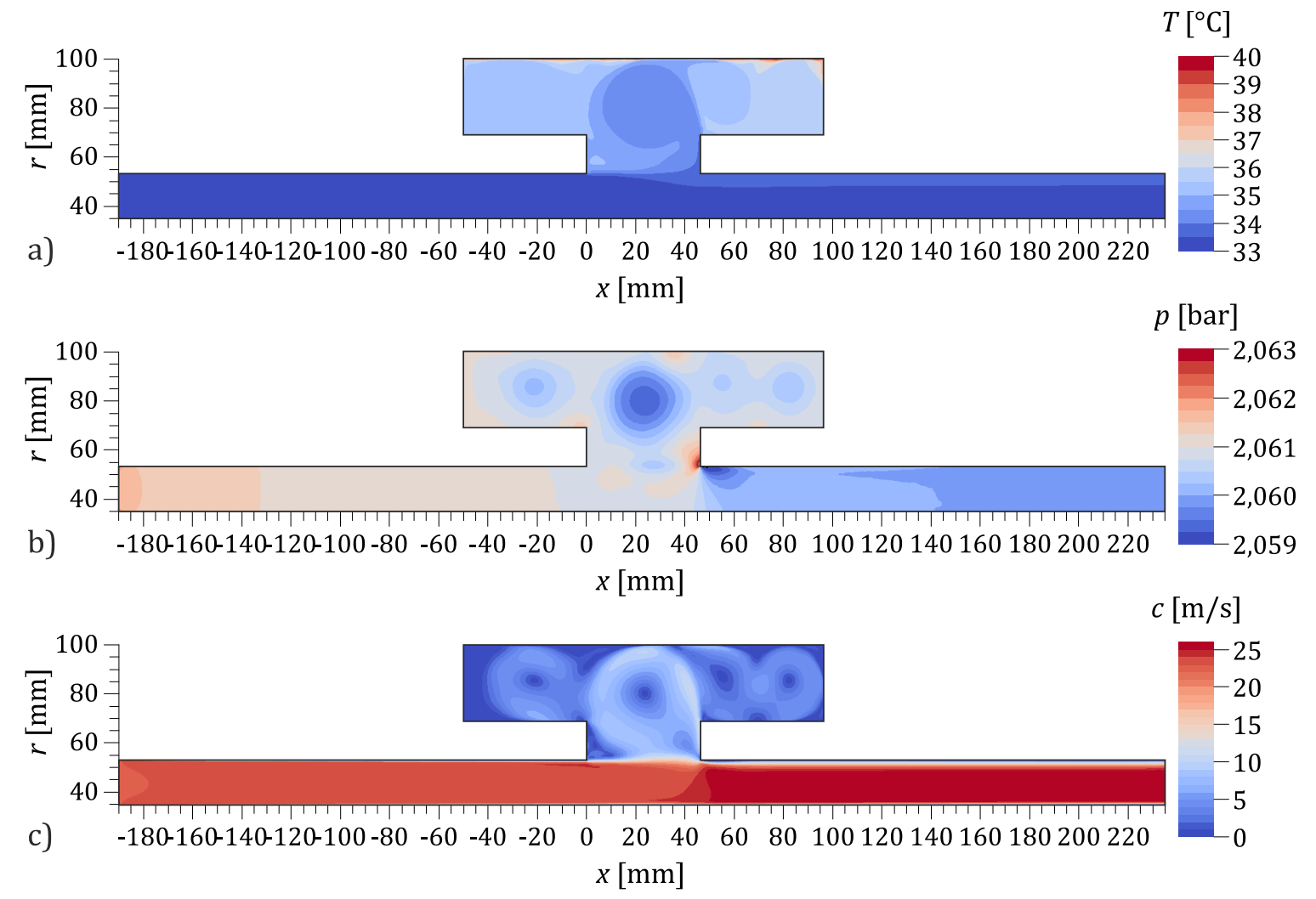

Abb. A.51 Arithmetischer Mittelwert ausgewählter Strömungsparameter im 2D-LES-Modells über die letzten $n=50.000$ Iterationen. $T_{\mathrm{W}}=$ konst.

a) Temperatur; b) statischer Druck; c) Geschwindigkeit. 


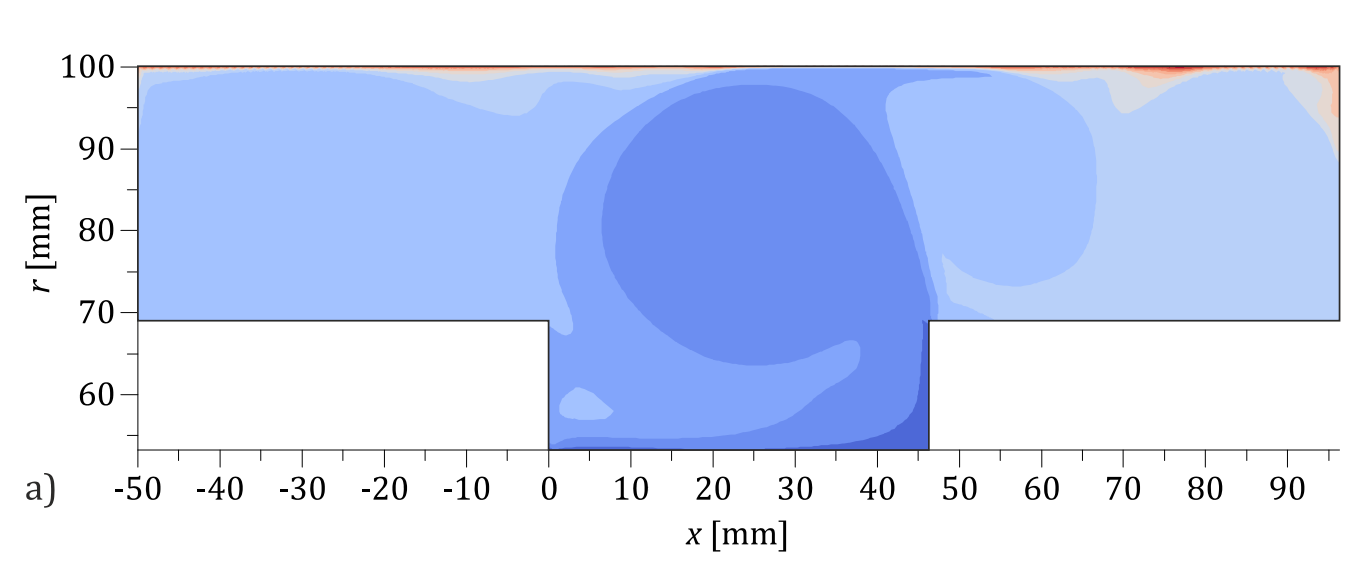

$T\left[{ }^{\circ} \mathrm{C}\right]$
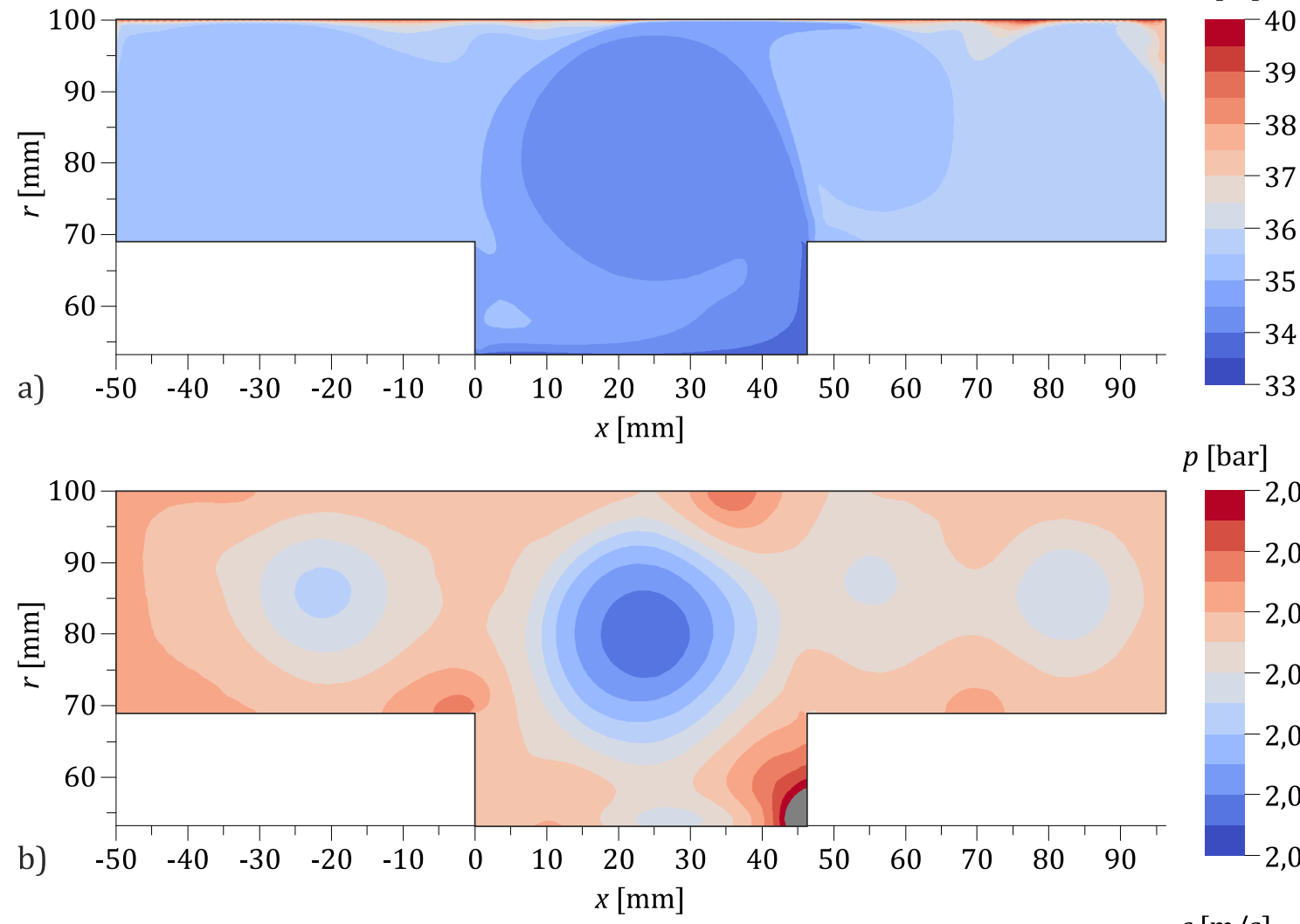

$p$ [bar]
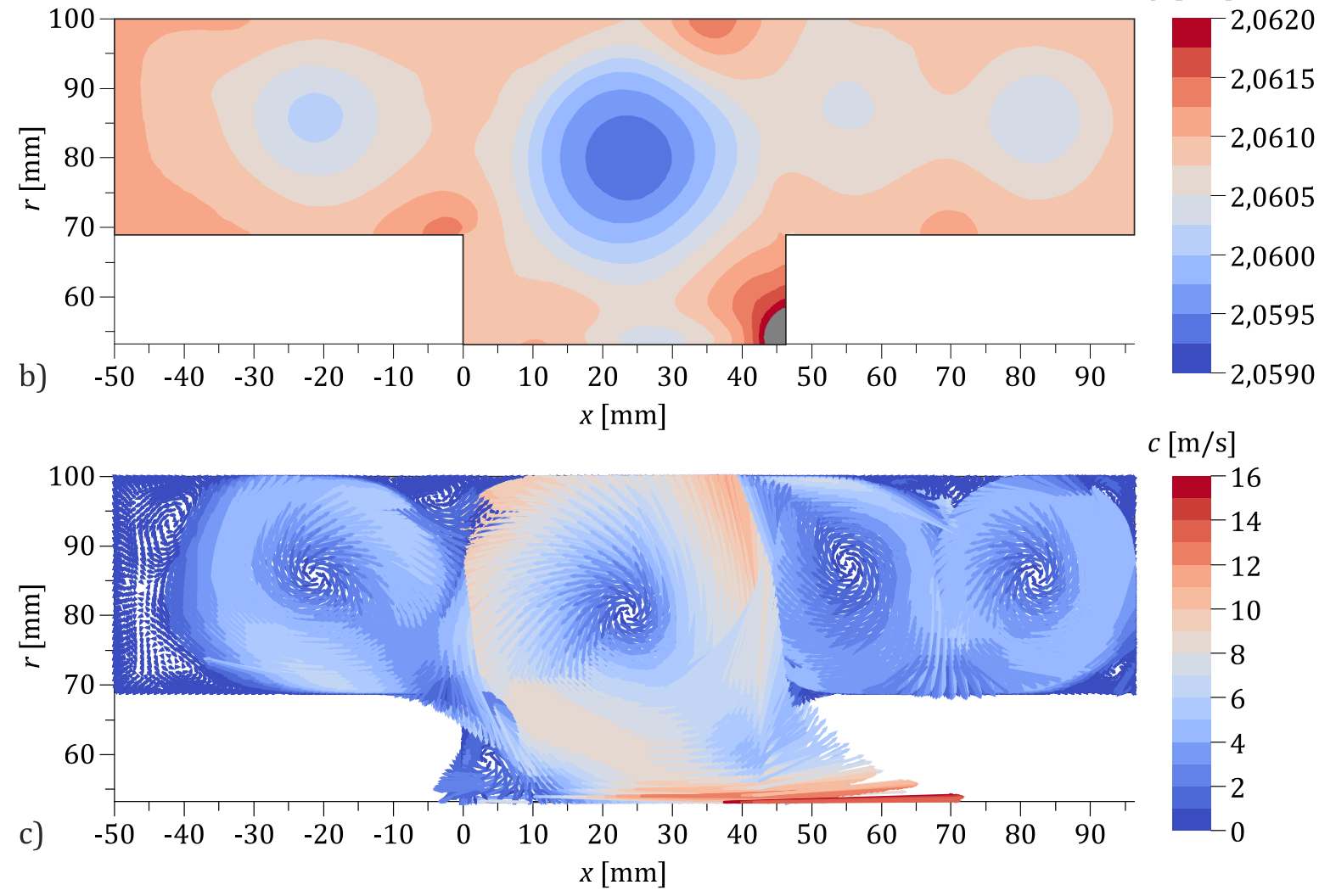

Abb. A.52 Arithmetischer Mittelwert ausgewählter Strömungsparameter im Seitenraum des 2DLES-Modells über die letzten $\boldsymbol{n}=\mathbf{5 0 . 0 0 0}$ Iterationen. $T_{\mathrm{W}}=$ konst.

a) Temperatur; b) statischer Druck; c) Geschwindigkeit. 


\section{A20 AUSBLICK AUF DEN DAMPFVERSUCHSSTAND „SISTER2“}

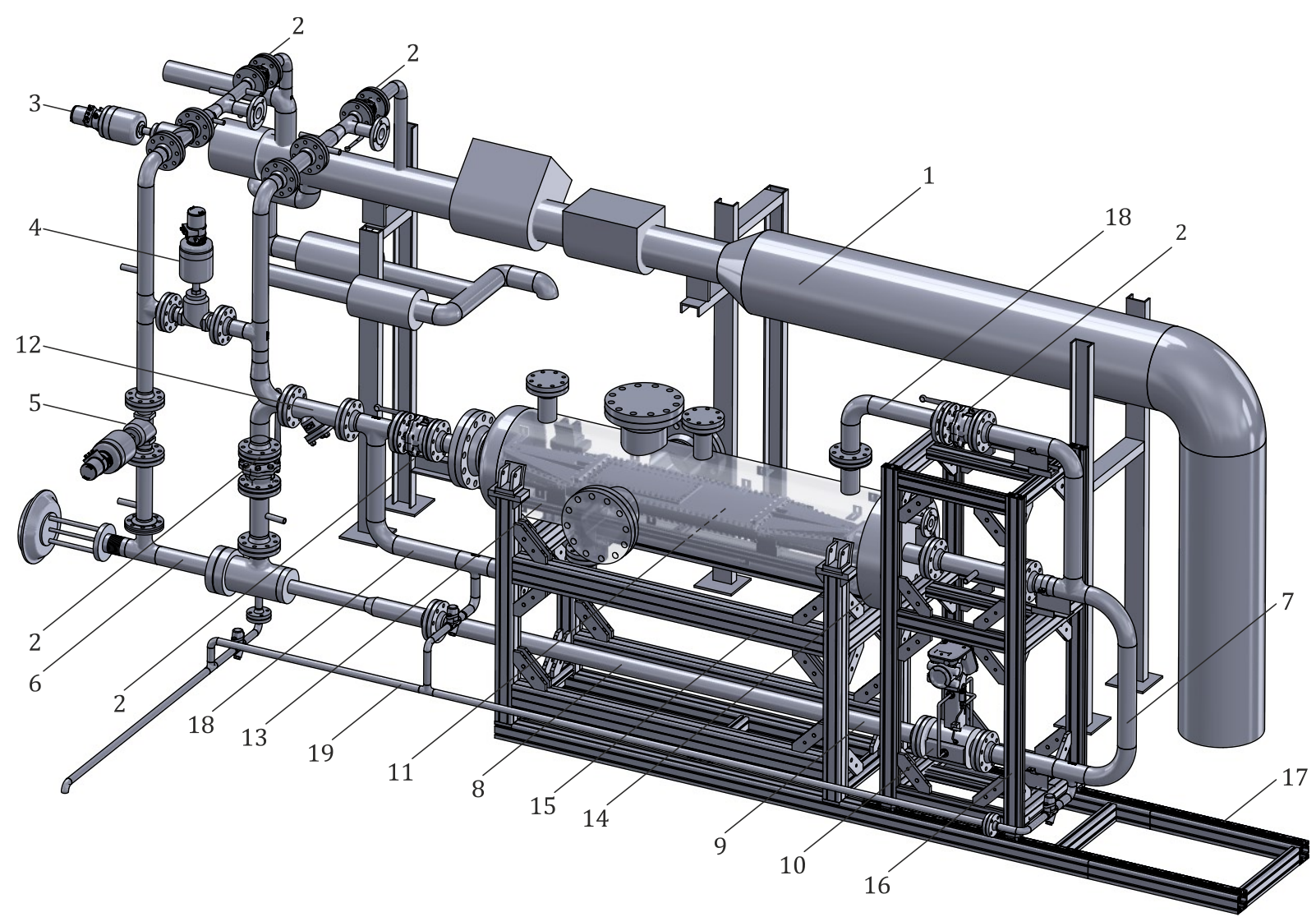

Abb. A.53 Konstruktionsentwurf der erweiterten Dampfversuchsanlage mit Dampfversuchsstand SiSTer2 (Quelle: Oliver Paulick).

1 - Bestandssystem; 2 - Absperrschieber; 3 - Regelventil 1 (Druck); 4 - Regelventil 2a (Hauptstrang);

5 - Regelventil 2b (Bypass); 6 - Dampfstrahlventilator; 7 - Rezirkulationsschleife;

8 - Anströmstrecke; 9 - Clamp-on-; 10 - Inline-Ultraschalldurchflussmessgerät;

11 - Versuchsstrecke (ausziehbar); 12 - Filtersieb; 13 - Druckbehälter („Dampfkessel“);

14 - Schnellverschluss („Deckel“); 15 - Gestell (fest); 16 - Wagen (axial verschiebbar);

17 - Schiene; 18 - Vorwärmdampfleitung; 19 - Entwässerung/ Kondensatableitung. 


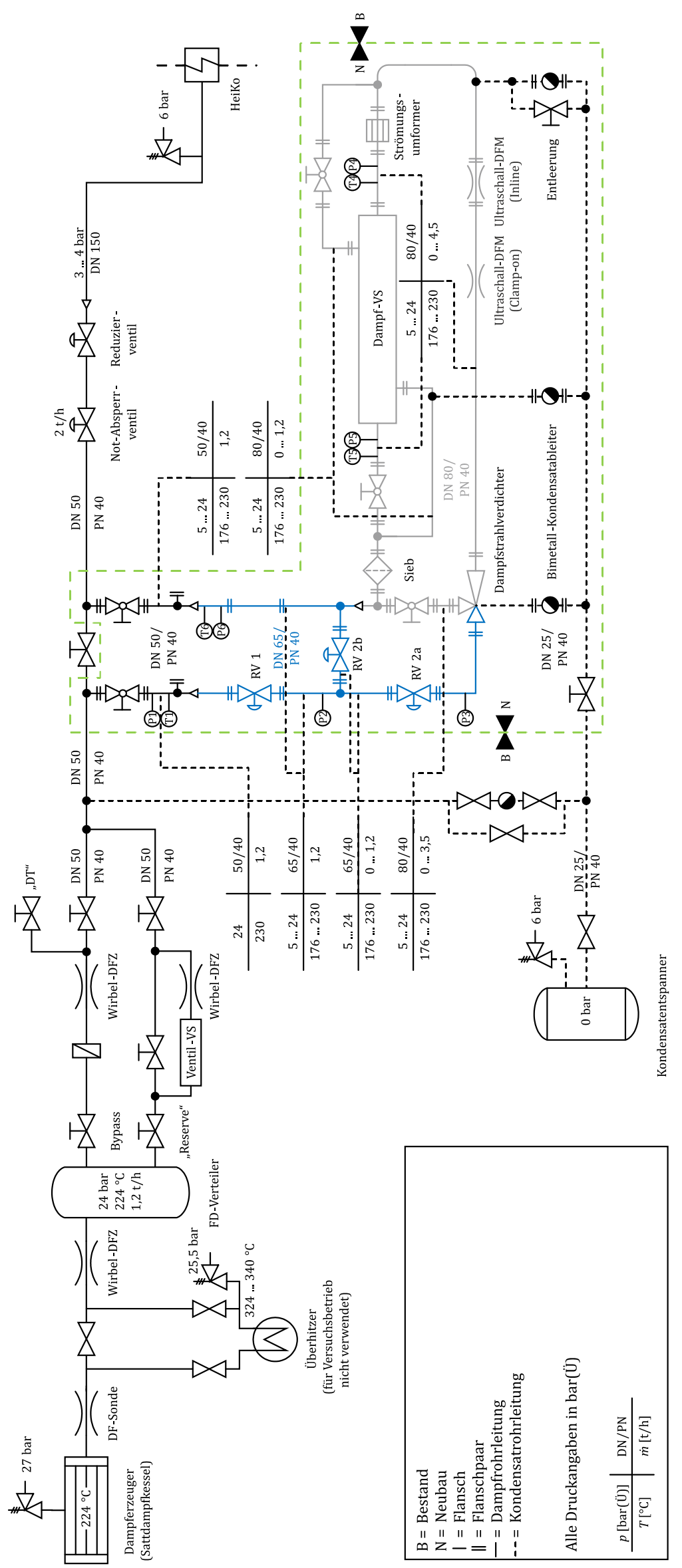

Abb. A.54 Rohrleitungs- und Instrumentenfließschema der erweiterten Dampfversuchsanlage. 